\title{
RISK STRATIFICATION FOR
}

ADVERSE EVENTS AFTER CAROTID ENDARTERECTOMY

\section{Nathalie Timmerman}

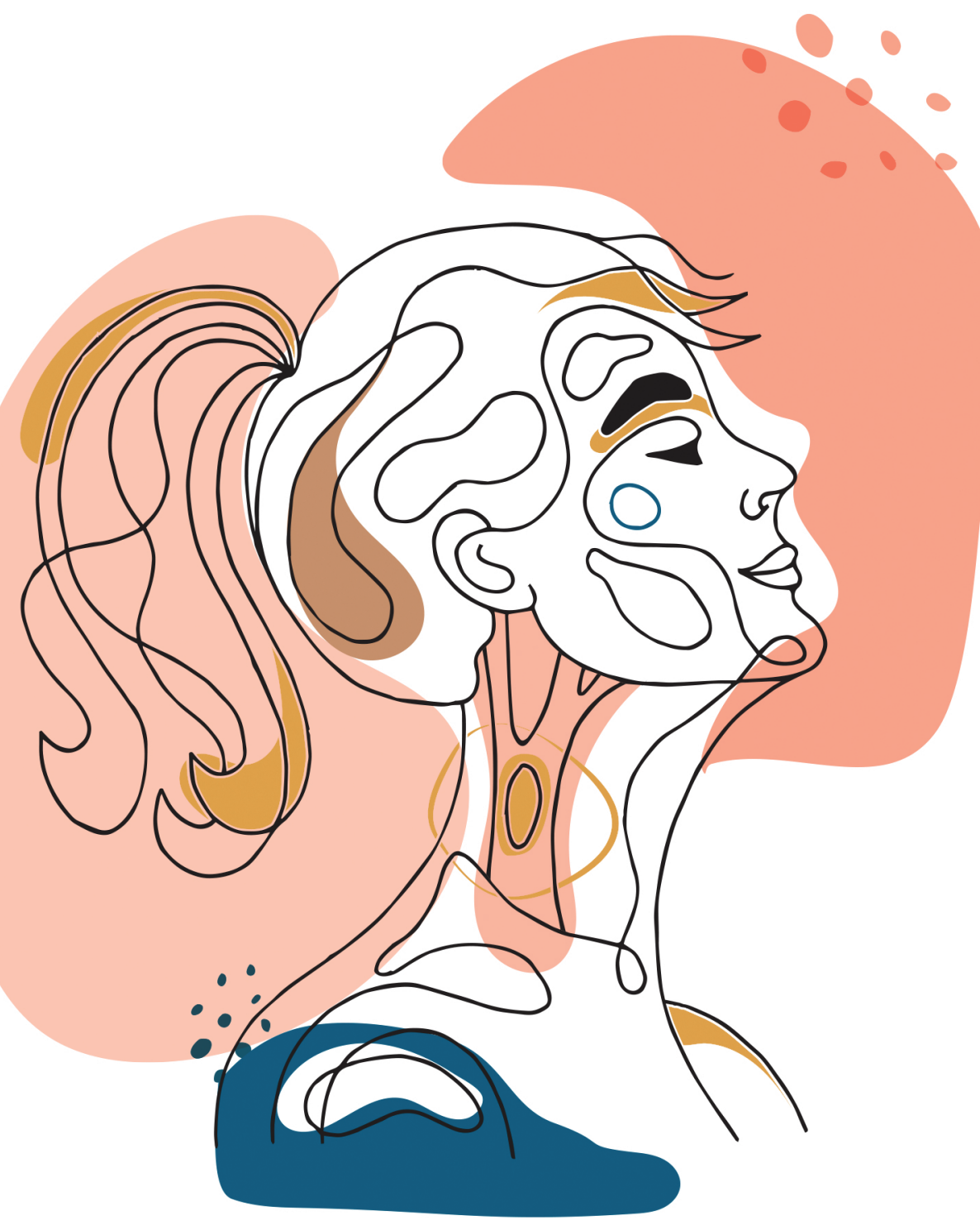



Risk Stratification for Adverse Events after Carotid Endarterectomy 


\section{Risk stratification for adverse events after carotid endarterectomy}

PhD thesis, Utrecht University, The Netherlands.

(c) Nathalie Timmerman, 2021. All rights reserved.

Financial support by the Dutch Heart Foundation for the publication of this thesis is gratefully acknowledged.

Publication of this thesis was additionally supported by Chirurgisch Fonds UMC Utrecht, Angiocare, Terumo Aortic Nederland, Stichting TWIN and Chipsoft.

ISBN 978-94-6416-730-6

Cover illustration Patricia Lotens | https://lotensatelier.nl

Cover design Joey Roberts | Publiss

Lay-out Wendy Schoneveld | www.wenzid.nl

Printing Ridderprint | www.ridderprint.nl 


\title{
Risk stratification for adverse events after carotid endarterectomy
}

Risico stratificatie voor cardiovasculaire complicaties na carotis endarterectomie (met een samenvatting in het Nederlands)

\author{
Proefschrift \\ ter verkrijging van de graad van doctor aan de \\ Universiteit Utrecht \\ op gezag van de \\ rector magnificus, prof. dr. H.R.B.M. Kummeling, \\ ingevolge het besluit van het college voor promoties \\ in het openbaar te verdedigen op \\ dinsdag 26 oktober 2021 des middags te 12:15 uur \\ door
}

Nathalie Timmerman

geboren op 11 september 1990

te Sögel, Duitsland 
Promotoren: Prof. dr. G.J. de Borst

Prof. dr. D.P.V. de Kleijn 
Aan mijn familie 


\section{Contents}

Chapter 1 General introduction and thesis outline

\section{Part I | Clinical characteristics and Genetics}

Chapter 2 Preoperative hypertension is associated with atherosclerotic intraplaque hemorrhage in patients undergoing carotid endarterectomy Atherosclerosis (2019)

Chapter 3 Family history and polygenic risk of cardiovascular disease: independent factors associated with secondary cardiovascular events in patients undergoing carotid endarterectomy Atherosclerosis (2020)

\section{Part II | Cerebral Imaging}

Chapter 4 Magnetic resonance imaging identified brain ischemia in

symptomatic patients undergoing carotid endarterectomy is related to histologically apparent intraplaque hemorrhage European Journal of Vascular and Endovascular Surgery (2019)

Chapter 5 Cerebral small vessel disease in standard pre-operative imaging reports is independently associated with increased risk of cardiovascular death following carotid endarterectomy European Journal of Vascular and Endovascular Surgery (2020)

\section{Part III | Circulating Biomarkers}

Chapter 6 Pre-operative plasma extracellular vesicle proteins are associated with a high risk of long term secondary major cardiovascular events in patients undergoing carotid endarterectomy European Journal of Vascular and Endovascular Surgery (in press)

Chapter 7 Elevated Lipoprotein(a) levels increase risk of 30-day MACE in patients following carotid endarterectomy

Stroke (2020) 
Chapter 8 High tenascin-C plasma extracellular vesicles levels are associated with an increased risk of major adverse cardiovascular events in carotid endarterectomy patients

Under review - Arteriosclerosis, Thrombosis, and Vascular Biology

Chapter 9 Ceramides and phospholipids are associated with high risk of major cardiovascular events after carotid endarterectomy

Under review - Scientific Reports

\section{Part IV | Risk Stratification Tools}

Chapter 10 Combined plasma Extracellular Vesicle biomarkers improve risk stratification for major adverse cardiovascular events after carotid endarterectomy Manuscript in preparation

Chapter 11 The Taxinomisis Project. A multidisciplinary approach for the development of a new risk stratification model for patients with asymptomatic carotid artery stenosis European Journal of Clinical Investigation (2020)

\section{Part V | Summary and Discussion}

Chapter 12 Summarizing discussion, future perspectives, and conclusions

Chapter 13 Summary in Dutch | Nederlandse samenvatting

\section{Appendices}

Review Committee

Authors and affiliations

List of publications 


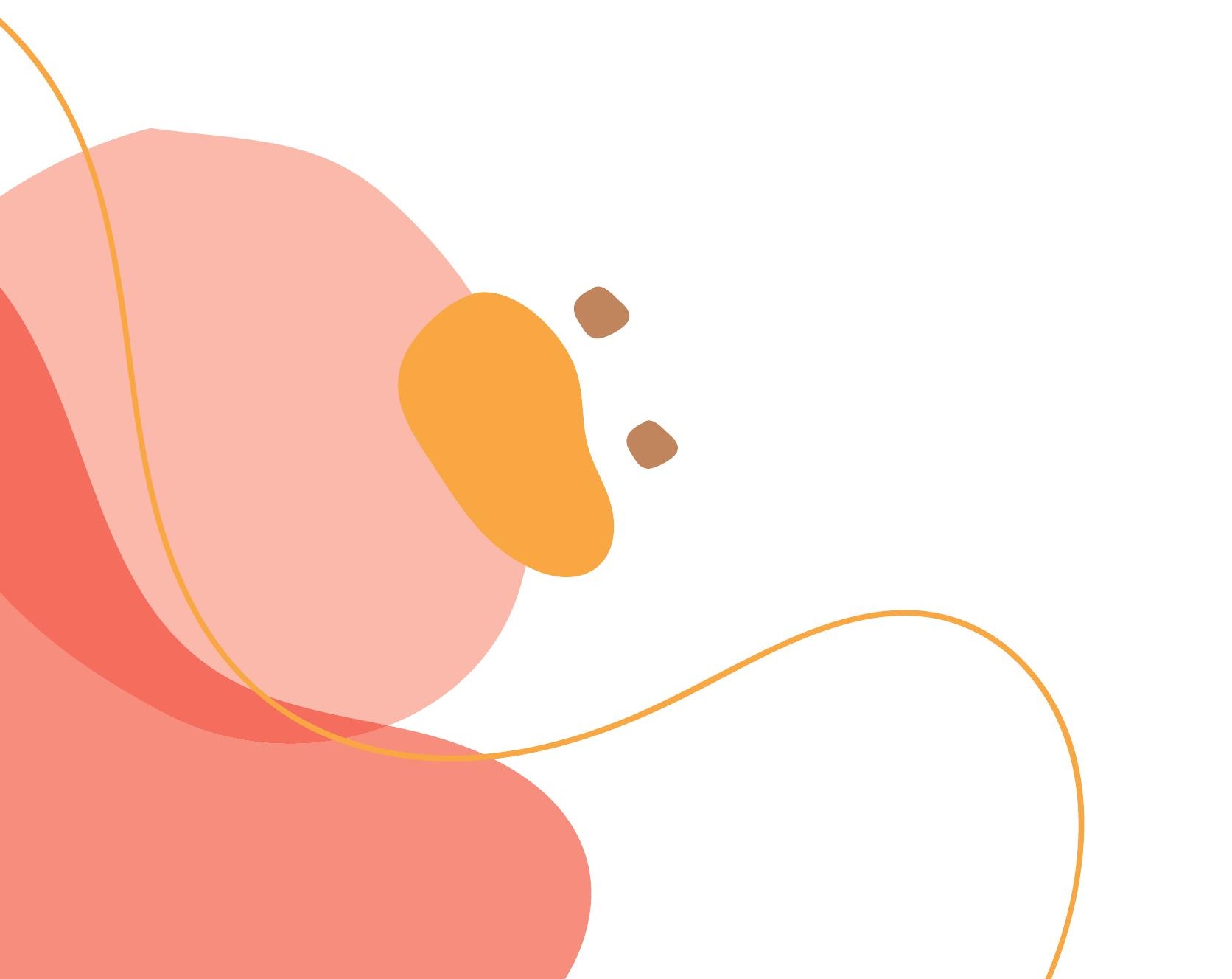


CHAPTER 1

General introduction and thesis outline 
Cardiovascular diseases (CVD) are the leading cause of death worldwide. In Europe, every minute eight people die as a result of CVD. ${ }^{1,2}$ In the Netherlands, 37,769 people died from CVD in 2018, representing $25 \%$ of all deaths. ${ }^{2}$ Of these, 8,434 deaths were attributed to ischemic heart disease and 5,365 to ischemic stroke. ${ }^{2} \mathrm{CVD}$ also causes long-term morbidity and disability leading to dependency on others for daily life activities. Altogether, CVD imposes a tremendous financial burden on our healthcare system.

\section{Atherosclerosis}

The majority of CVD is caused by atherosclerosis. Atherosclerosis is a chronic lipid and inflammation-driven disease resulting in the formation of plaques in arteries leading to luminal narrowing (stenosis). ${ }^{3}$ Plaques originate from the accumulation of lipids (lowdensity lipoprotein, LDL), inflammatory cells, smooth muscle cells, collagen and calcifications (Figure 1). ${ }^{3,4}$ Atherosclerosis starts early in life, but symptoms mostly occur decades later. Classical risk factors are age, family history of CVD, postmenopausal status, smoking, hypertension, abnormal cholesterol levels, obesity, diabetes, unhealthy diet and low physical activity. ${ }^{5}$

Atherosclerotic plaques can remain silent during life but may also give rise to clinical events in the affected vascular territory. Transient symptoms caused by stenosis can occur as a result of an oxygen-supply-demand mismatch such as during exercise. For example, in the coronary arteries this leads to chest pain and in the peripheral arteries of the legs this leads to a decreased walking distance. Rupture or erosion of the plaque surface leads to formation of thromboembolisms that can suddenly occlude the artery locally or embolize to distal vessels. ${ }^{6}$ This can lead to acute clinical events such as myocardial infarction, acute leg ischemia and stroke. Atherosclerotic plaques that are prone to rupture are called "vulnerable" or "unstable" plaques. These plaques are hallmarked by a thin-fibrous cap, few smooth muscle cells, a large lipid-rich necrotic core, high infiltration of inflammatory cells, neovessel formation and intraplaque hemorrhage. ${ }^{6}$ Vulnerable plaque characteristics have been related to preceding and future cardiovascular events. ${ }^{7,8}$ In particular, intraplaque hemorrhage has been associated with a higher risk of systemic cardiovascular events in patients with atherosclerotic disease. ${ }^{9}$

\section{Extracranial carotid artery stenosis: the current state of the art and challenges ahead}

The carotid arteries are two of the four main suppliers of oxygen-rich blood to the brain and are essential for adequate brain function. Extracranial carotid artery stenosis, due to atherosclerotic plaque formation, is an important cause of cerebrovascular events. Thromboembolisms may cause transient or permanent cerebral ischemia resulting in loss of neurological function. Transient neurological symptoms that last less than 24 hours are 

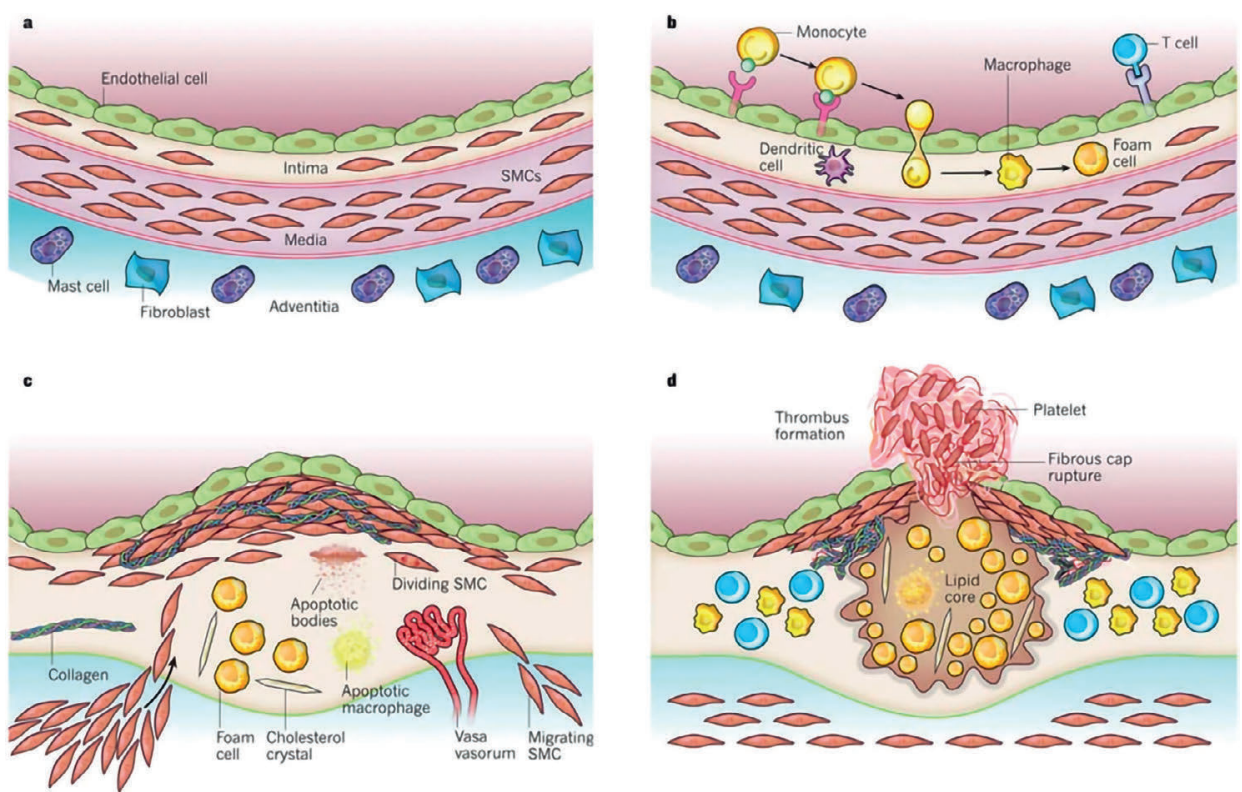

Figure 1. Mechanisms involved in atherosclerotic plaque development and progression

A normal artery contains of three layers: the intima (inner layer), media and adventitia (outer layer). Several mechanisms are involved in atherosclerosis. Initials steps include endothelial activation, recruitment of inflammatory cells that migrate into the intima. At the same time LDL crosses the endothelium where it becomes oxidized. Monocytes differentiate into macrophages. These ingest oxidized-LDL and transform into foam cells. During lesion progression, vascular smooth muscle cells (SMCs) migrate from the media layer to the intima and proliferate. SMCs produce extracellular matrix molecules such as collagen and proteoglycans and form a stabilizing overlying fibrous cap. A lipid-rich necrotic core is formed consisting of death foam cells and their debris. In the phase of thrombosis, fibrous cap rupture leads to thromboembolisms and atherosclerotic complications.

Figure copied and adapted from: Libby P, et al. Progress and challenges in translating the biology of atherosclerosis. Nature $2011^{4}$

defined as a transient ischemic attack (TIA), while neurological symptoms that persist longer are defined as a stroke. ${ }^{10}$ To prevent these devastating events, treatment of carotid artery stenosis consists of risk factor control, preventive medications and elimination of the atherosclerotic plaque by either surgical removal (carotid endarterectomy, CEA) or stenting (carotid artery stenting, CAS). Since these interventions carry a risk of procedural stroke, outweighing future stroke risks against procedural risks is essential for decisionmaking.

\section{Carotid intervention}

Current guidelines recommend CEA, or CAS as an alternative, for patients with symptomatic carotid artery stenosis (ipsilateral cerebrovascular symptoms have occurred in the prior 
six months). ${ }^{10}$ Asymptomatic patients (no ipsilateral cerebrovascular symptoms in the prior six months) are less likely to benefit from CEA or CAS as their risk for future stroke is thought to be low under contemporary medical therapy. The ongoing Asymptomatic Carotid Surgery Trial-2 (ACST-2) will provide more insight in the optimal treatment strategy of asymptomatic patients (clinicaltrials.gov; NCT00883402).

\section{Preventive medical therapy for new cardiovascular events}

Although CEA is an effective procedure to reduce the risk of future ipsilateral stroke, patients are still at high risk for future cardiovascular events as result of the systemic nature of atherosclerosis. About $24 \%$ of patients develop a major cardiovascular event within three years following CEA. ${ }^{9}$ This high residual cardiovascular risk remains even after optimal control of risk factors and best medical therapy (BMT) consisting of antiplatelet, statins and antihypertensive drugs. ${ }^{11}$ To lower this residual cardiovascular risk, novel medications in addition to standard BMT could be beneficial (so-called "add-on therapy"). To date, proprotein convertase subtilisin/kexin type-9 (PCSK-9) inhibitors are an established addon therapy used for aggressive LDL lowering but these come with high costs. ${ }^{5}$ Other addon therapies such as anti-inflammatory drugs and addition of anticoagulants next to antithrombotic therapy are options currently under investigation. ${ }^{12,13}$ Current European guidelines classify all CEA patients in one homogenous group at "very high risk" for secondary cardiovascular events and recommend PCSK-9 inhibitors. ${ }^{5}$ However it is known that at individual level the residual cardiovascular risk varies substantially. ${ }^{11}$ Considering that add-on therapies have deleterious side effects and high costs, a "one size fits all" treatment approach seems inappropriate. Therefore, risk stratification tools that discriminate CEA patients at high risk for future cardiovascular events qualifying for add-on therapy, from those at low risk are of great importance. To date, no risk stratification tools are available that accurately identify high risk patients for secondary cardiovascular events.

\section{Biomarkers}

Biomarkers have the potential to identify patients with carotid stenosis at higher risk for future cerebrovascular or cardiovascular events. The definition of a biomarker is "a characteristic that is measured as an indicator of normal biological processes, pathogenic processes or responses to an exposure or intervention". ${ }^{14}$ This thesis focusses on biomarkers of different origins: genetic, radiological and circulating.

\section{Genetic biomarkers}

CVD originates from the interplay of genetic inheritance (nature) and environmental factors (nurture). Studying genes help us understand underlying biological mechanisms but also provides information about disease risk. By comparing the genetic makeup of people with 
a certain disease to those without, genetic variations (single-nucleotide polymorphisms, SNPs) contributing to this disease can be discovered. Hundreds of SNPs have been identified for coronary artery disease ${ }^{15}$ and stroke ${ }^{16}$, emphasizing the polygenic nature of CVD. The genetic susceptibility of an individual patient for CVD can be captured by calculating a polygenic risk score which summarizes all SNPs with their associated effect sizes. Polygenic risk scores may be useful for individual risk estimation of future clinical events. ${ }^{17,18}$

\section{Radiographic biomarkers}

Cerebral lesions detected on brain imaging can provide information about cardiovascular prognosis. Periprocedural silent brain lesions detected on diffusion weighted imaging (DWI-lesions) are established markers for increased periprocedural and postprocedural stroke or TIA. ${ }^{19,20}$ White matter lesions (WMLs) on brain MRI or CT are surrogate markers for cerebral small vessel disease and have been associated with future cerebrovascular events and cognitive decline. ${ }^{21-23}$ DWI-lesions reflect acute moments of brain ischemia (either due to thromboembolisms or hypoperfusion) whether WMLs provide more insight in the natural course of atherosclerosis and long-term prognosis. ${ }^{24}$

\section{Circulating biomarkers in plasma and plasma extracellular vesicles (EVs)}

The composition of our blood plasma reflects ongoing physiological processes and can be considered a "liquid biopsy". Most studies in carotid artery stenosis patients have focused on biomarkers in plasma but biomarker discovery in extracellular vesicles (EVs), especially on EV content, is relatively unexplored. ${ }^{25} \mathrm{EV}$ s are small lipid-bilayer membrane particles - a nanometer in size- and are secreted by almost all cell types into body fluids such as plasma, saliva, tears and urine. ${ }^{25}$ Extracellular vesicles contain bioactive material of their parent cell including nucleic acids, lipids and proteins. EVs are vehicles for intercellular communication, and are involved in various (patho)physiological processes such as apoptosis, coagulation and inflammation. ${ }^{25}$ The content of EVs reflects the state of the parent cell, hereby providing a close look on ongoing pathophysiological processes. Therefore, we hypothesized that EV content may potentially hold accurate prognostic information for risk estimation. Most markers in this thesis are therefore studied in EVs, and few markers in plasma.

\section{Aim of this thesis}

The overall aim of this thesis is to investigate biomarkers that identify patients at high risk for future cardiovascular events after CEA. Since clinical factors have poor predictive value for future cardiovascular events ${ }^{26,27}$, biomarkers could improve risk stratification and ultimately help physicians select appropriate patients for specific treatments. All studies in this thesis are conducted in the ongoing Athero-Express biobank study; the world's largest 
atherosclerotic plaque biobank to date. ${ }^{28}$ This biobank collects preoperative blood samples and surgically removed atherosclerotic plaque tissue and follows patients during the postoperative three years for cardiovascular events (Figure 2).

\section{Thesis Outline}

This thesis consists of five parts. Part I focusses on clinical characteristics and genetics as biomarkers. In Chapter 2, we examined the association between preoperative blood pressure and histological carotid plaque characteristics, as both hypertension and histological plaque characteristics (mainly intraplaque hemorrhage) are established markers for future cardiovascular events. ${ }^{7,29}$ In Chapter 3, we investigated whether a polygenic risk score for first-ever coronary events was related to secondary cardiovascular events following CEA. Since a positive family history of CVD is an established proxy for genetic heritability in daily practice, we also explored the relation between family history and secondary cardiovascular events. Part II focusses on cerebral imaging biomarkers. In Chapter 4, we explored the association between recurrent (silent) brain ischemia, detected on MRI-DWI, in the waiting period before CEA and histological carotid intraplaque hemorrhage. We hypothesized that intraplaque hemorrhage may be a potential source of silent brain ischemia and might be a useful marker to prioritize patients for the timing of CEA. In Chapter $\mathbf{5}$ we described the relevance of preoperative cerebral small vessel disease documented in routine brain imaging reports (including WMLs and lacunar infarction) for the prediction of longterm postoperative outcome following CEA. Part III focusses on different types of circulating biomarkers; extracellular vesicle proteins (CD14, Cystatin C, Serpin F2, Serpin G1 and Serpin C1, Chapter 6), a lipoprotein (Lipoprotein(a), Chapter 7), an extracellular vesicle matrix glycoprotein (Tenascin-C, Chapter 8) and extracellular vesicle lipids (ceramides and phosphatidylcholines, Chapter 9). We studied whether preoperative levels of these biomarkers could be useful to predict postoperative cardiovascular events in CEA patients. Part IV describes risk stratification tools that combine multiple biomarkers. Our hypothesis was that different biomarkers may reflect different pathophysiological pathways and therefore hold complementary prognostic information. Combining biomarkers may be the holy grail in risk prediction for carotid artery stenosis patients. In Chapter 10, promising circulating biomarkers resulting from Chapters 6, 7, 8 and 9 were combined into a risk stratification model for secondary cardiovascular events after CEA. Chapter 11 reports the rationale and design of a multicenter observational study with the aim to develop a new risk stratification model for patients with asymptomatic carotid artery stenosis. For asymptomatic patients the challenge is to identify patients at high risk for future stroke under BMT as these patients may opt for a carotid intervention. Previous studies have 
proposed multiple biomarkers that indicate a high risk of future stroke in asymptomatic patients. ${ }^{10}$ A risk stratification model combining these biomarkers is therefore warranted. The final part provides a general discussion (Chapters 12) and a Dutch summary (Chapter 13).

\section{The Athero-Express Biobank}

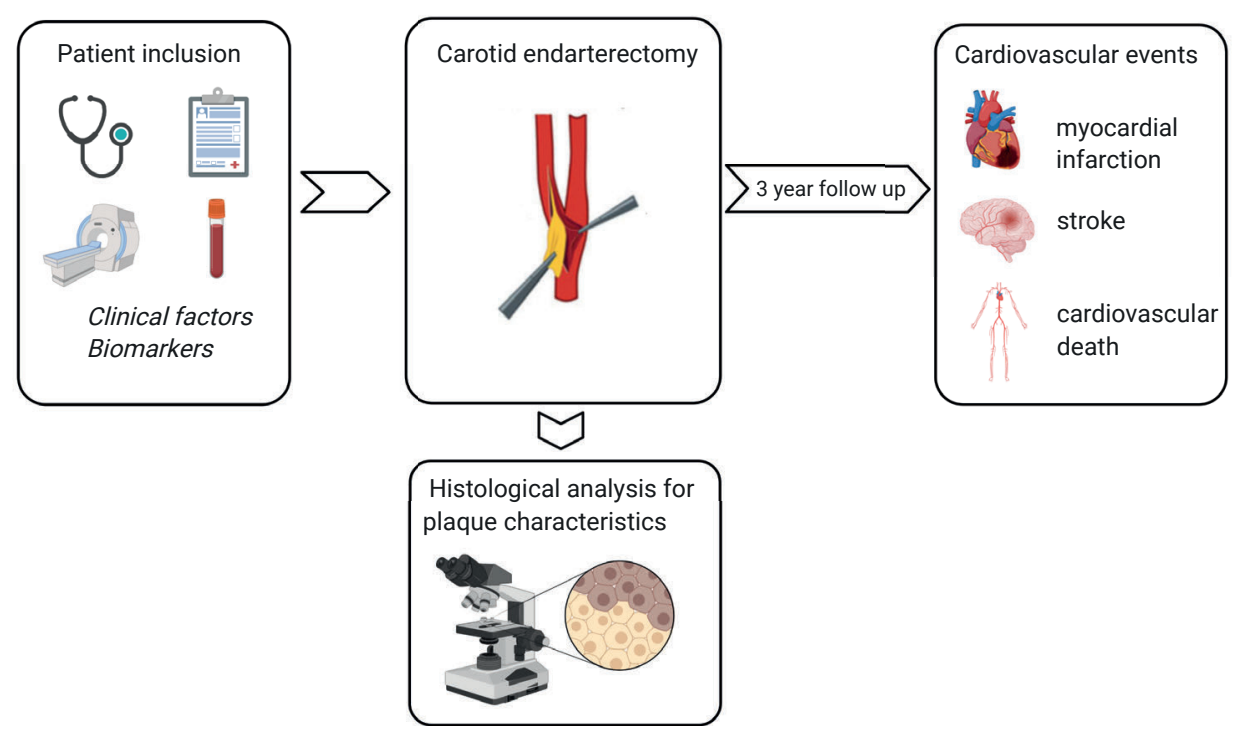

Figure 2. Design of the Athero-Express Biobank

Patients are included preoperatively with collection of baseline characteristics and blood samples for biomarker measurements. The plaque removed during carotid endarterectomy is processed in the laboratory for histological analysis. Patients are followed up for three years after surgery for the occurrence of cardiovascular events. Image created in BioRender.com 


\section{References}

1 Townsend N, Wilson L, Bhatnagar P, Wickramasinghe K, Rayner M, Nichols M. Cardiovascular disease in Europe: epidemiological update 2016. Eur Heart J 2016;37(42):3232-45.

2 Hartstichting, de Boer AR, Bots ML, van Dis I, Vaartjes I VF. Hart- en vaatziekten in Nederland 2019. Cijfers over incidentie, prevalentie, ziekte en sterfte. 2019.

3 Libby P, Ridker PM, Maseri A. Inflammation and Atherosclerosis. Circulation 2002;105(9):1135-43.

4 Libby P, Ridker PM, Hansson GK. Progress and challenges in translating the biology of atherosclerosis. Nature 2011;473(7347):317-25.

5 Mach F, Baigent C, Catapano AL, Koskinas KC, Casula M, Badimon L, et al. 2019 ESC/EAS Guidelines for the management of dyslipidaemias: lipid modification to reduce cardiovascular risk. Eur Heart $J$ 2020;41(1):111-88.

6 Bentzon JF, Otsuka F, Virmani R, Falk E. Mechanisms of Plaque Formation and Rupture. Circ Res 2014;114(12):1852-66.

7 Howard DPJ, Van Lammeren GW, Rothwell PM, Redgrave JN, Moll FL, De Vries JPPM, et al. Symptomatic carotid atherosclerotic disease: Correlations between plaque composition and ipsilateral stroke risk. Stroke 2015;46(1):182-9.

8 Verhoeven B, Hellings WE, Moll FL, de Vries JP, de Kleijn DPV, de Bruin P, et al. Carotid atherosclerotic plaques in patients with transient ischemic attacks and stroke have unstable characteristics compared with plaques in asymptomatic and amaurosis fugax patients. J Vasc Surg 2005;42(6):1075-81.

9 Hellings WE, Peeters W, Moll FL, Piers SRD, van Setten J, Van der Spek PJ, et al. Composition of Carotid Atherosclerotic Plaque Is Associated With Cardiovascular Outcome. Circulation 2010;121(17):1941-50.

10 Naylor AR, Ricco J-B, de Borst GJ, Debus S, de Haro J, Halliday A, et al. Editor's Choice - Management of Atherosclerotic Carotid and Vertebral Artery Disease: 2017 Clinical Practice Guidelines of the European Society for Vascular Surgery (ESVS). Eur J Vasc Endovasc Surg 2018;55(1):3-81.

11 Kaasenbrood L, Boekholdt SM, van der Graaf Y, Ray KK, Peters RJG, Kastelein JJP, et al. Distribution of Estimated 10-Year Risk of Recurrent Vascular Events and Residual Risk in a Secondary Prevention Population. Circulation 2016;134(19):1419-29.

12 Eikelboom JW, Connolly SJ, Bosch J, Dagenais GR, Hart RG, Shestakovska O, et al. Rivaroxaban with or without Aspirin in Stable Cardiovascular Disease. N Engl J Med 2017;377(14):1319-30.

13 Bonaca MP, Bauersachs RM, Anand SS, Debus ES, Nehler MR, Patel MR, et al. Rivaroxaban in Peripheral Artery Disease after Revascularization. N Engl J Med 2020;382(21):1994-2004.

14 Califf RM. Biomarker definitions and their applications. Exp Biol Med 2018;243(3):213-21.

15 Consortium the Cardi, Nikpay M, Goel A, Won H-H, Hall LM, Willenborg C, et al. A comprehensive 1000 Genomes-based genome-wide association meta-analysis of coronary artery disease. Nat Genet 2015;47(10):1121-30.

16 Traylor M, Farrall M, Holliday EG, Sudlow C, Hopewell JC, Cheng Y-C, et al. Genetic risk factors for ischaemic stroke and its subtypes (the METASTROKE Collaboration): a meta-analysis of genome-wide association studies. Lancet Neurol 2012;11(11):951-62.

17 Inouye M, Abraham G, Nelson CP, Wood AM, Sweeting MJ, Dudbridge F, et al. Genomic Risk Prediction of Coronary Artery Disease in 480,000 Adults. J Am Coll Cardiol 2018;72(16):1883-93.

18 Damask A, Steg PG, Schwartz GG, Szarek M, Hagström E, Badimon L, et al. Patients With High GenomeWide Polygenic Risk Scores for Coronary Artery Disease May Receive Greater Clinical Benefit From Alirocumab Treatment in the ODYSSEY OUTCOMES Trial. Circulation 2020;141(8):624-36.

19 Gensicke H, van der Worp HB, Nederkoorn PJ, Macdonald S, Gaines PA, van der Lugt A, et al. Ischemic brain lesions after carotid artery stenting increase future cerebrovascular risk. J Am Coll Cardiol 2015;65(6):521-9.

20 Traenka C, Engelter ST, Brown MM, Dobson J, Frost C, Bonati LH. Silent brain infarcts on diffusionweighted imaging after carotid revascularisation: A surrogate outcome measure for procedural stroke? A systematic review and meta-analysis. Eur Stroke J 2019;4(2):127-43.

21 Streifler JY, Eliasziw M, Benavente OR, Alamowitch S, Fox AJ, Hachinski VC, et al. Prognostic importance of leukoaraiosis in patients with symptomatic internal carotid artery stenosis. Stroke 2002;33(6):1651-5. 
22 van Dijk EJ, Prins ND, Vrooman HA, Hofman A, Koudstaal PJ, Breteler MMB. Progression of Cerebral Small Vessel Disease in Relation to Risk Factors and Cognitive Consequences. Stroke 2008;39(10):2712-9.

23 Debette S, Beiser A, DeCarli C, Au R, Himali JJ, Kelly-Hayes M, et al. Association of MRI Markers of Vascular Brain Injury With Incident Stroke, Mild Cognitive Impairment, Dementia, and Mortality. Stroke 2010;41(4):600-6.

24 Rots ML, van der Lugt A, de Borst GJ. Surrogate Markers and Reporting Standards for Outcome After Carotid Intervention. Eur J Vasc Endovasc Surg 2019;58(6):794-5.

25 Boulanger CM, Loyer X, Rautou P-E, Amabile N. Extracellular vesicles in coronary artery disease. Nat Rev Cardiol 2017;14(5):259-72.

26 Volkers EJ, Algra A, Kappelle LJ, Greving JP. Prediction models for clinical outcome after a carotid revascularisation procedure: A systematic review. Eur Stroke J 2018;3(1):57-65.

27 van Lammeren GW, Catanzariti LM, Peelen LM, de Vries J-PPM, de Kleijn DPV, Moll FL, et al. Clinical Prediction Rule to Estimate the Absolute 3-Year Risk of Major Cardiovascular Events After Carotid Endarterectomy. Stroke 2012;43(5):1273-8.

28 Verhoeven BAN, Velema E, Schoneveld AH, de Vries JPPM, de Bruin P, Seldenrijk CA, et al. Atheroexpress: differential atherosclerotic plaque expression of mRNA and protein in relation to cardiovascular events and patient characteristics. Rationale and design. Eur J Epidemiol 2004;19(12):1127-33.

29 Vrijenhoek JEP, Den Ruijter HM, De Borst GJ, de Kleijn DPV, De Vries J-PPM, Bots ML, et al. Sex Is Associated With the Presence of Atherosclerotic Plaque Hemorrhage and Modifies the Relation Between Plaque Hemorrhage and Cardiovascular Outcome. Stroke 2013;44(12):3318-23. 



\section{PART I}

\section{Clinical characteristics and Genetics}

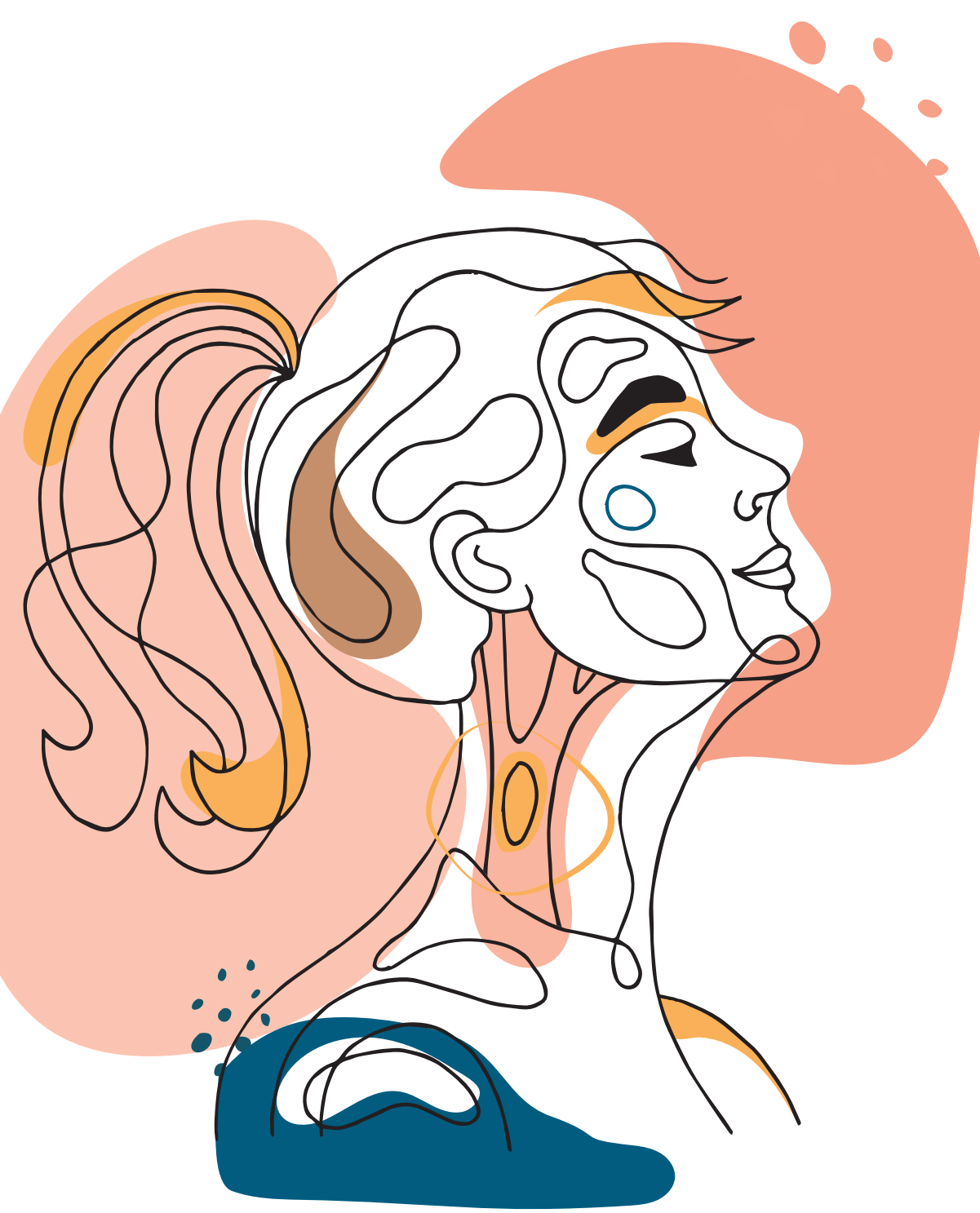




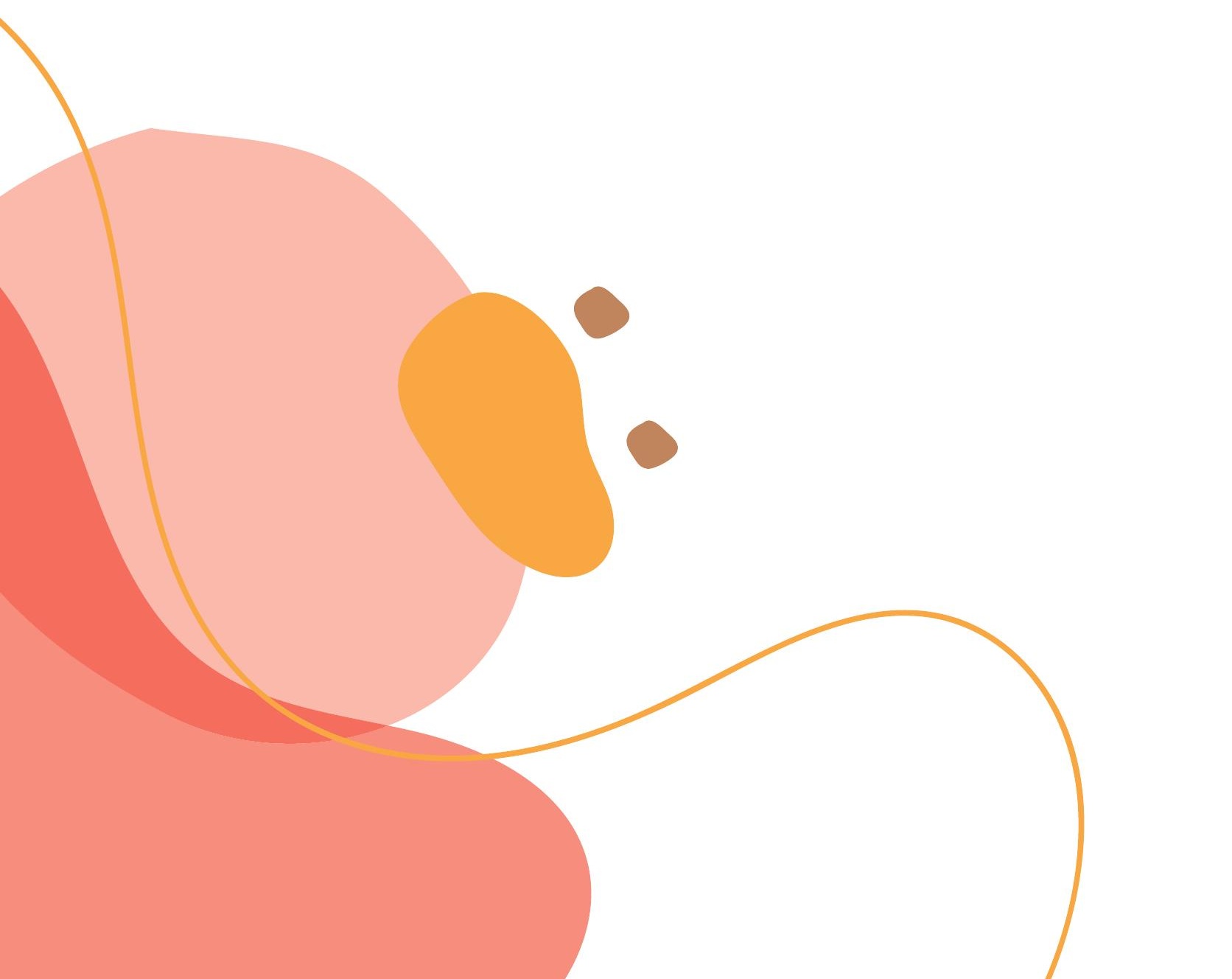


CHAPTER 2

\section{Preoperative hypertension is associated with atherosclerotic intraplaque hemorrhage in patients undergoing carotid endarterectomy}

Atherosclerosis 2019;290:214-221

Leonie M.M. Fassaert

Nathalie Timmerman

Ian D. van Koeverden

Gerard Pasterkamp

Dominique P.V. de Kleijn

Gert J. de Borst 


\section{Abstract}

\section{Background and aims}

Both hypertension and atherosclerotic plaque characteristics such as intraplaque hemorrhage (IPH) are associated with cardiovascular events (CVE). It is unknown if hypertension is associated with IPH. Therefore, we studied if hypertension is associated with unstable atherosclerotic plaque characteristics in patients undergoing carotid endarterectomy (CEA).

\section{Methods}

Prospectively collected data of CEA-patients (2002-2014) were retrospectively analyzed. Blood pressure (BP) was the mean of 3 preoperative measurements. Preoperative hypertension was defined as a systolic $\mathrm{BP}^{3} 160 \mathrm{mmHg}$. Post-CEA, carotid atherosclerotic plaques were analyzed for presence of calcifications, collagen, smooth muscle cells, macrophages, lipid core, IPH and microvessel density. Associations between BP (systolic and diastolic), patient characteristics and carotid plaque characteristics were assessed with univariate and multivariable analyses with correction for potential confounders. Results were replicated in a cohort of patients that underwent iliofemoral endarterectomy.

\section{Results}

Within CEA-patients ( $\mathrm{n}=1684), 708(42 \%)$ patients had preoperative hypertension. Increased systolic BP was associated with the presence of plaque calcifications (adjusted odds ratio (OR) 1.11 [95\%CI1.01-1.22], p=0.03), macrophages (adjusted OR 1.12 [1.041.21 ], $\mathrm{p}<0.01$ ), lipid core $>10 \%$ of plaque area (adjusted OR 1.15 [1.05-1.25], $\mathrm{p}<0.01$ ), IPH (adjusted OR 1.12 [1.03-1.21], $\mathrm{p}=0.01$ ) and microvessels (adjusted beta 0.04 [0.00-0.08], $\mathrm{p}=0.03$ ). Increased diastolic BP was associated with macrophages (adjusted OR 1.36 [1.171.58], $\mathrm{p}<0.01$ ), lipid core (adjusted OR 1.29 [1.10-1.53], $\mathrm{p}<0.01$ ) and IPH (adjusted OR 1.25 [1.07-1.46], $\mathrm{p}<0.01)$ but not with microvessels nor plaque calcifications. Replication in an iliofemoral-cohort $(n=657)$ showed that increased diastolic BP was associated with the presence of macrophages (adjusted OR 1.78 [1.13-2.91], $\mathrm{p}=0.01$ ), lipid core (adjusted OR1.45 [1.06-1.98], $\mathrm{p}=0.02$ ) and IPH (adjusted OR 1.48 [1.14-1.93], $\mathrm{p}<0.01$ ).

\section{Conclusion}

Preoperative hypertension in severely atherosclerotic patients is associated with the presence of carotid plaque macrophages, lipid core and IPH. IPH, as a plaque marker for CVE, is associated with increased systolic and diastolic BP in both the CEA and iliofemoral population. 


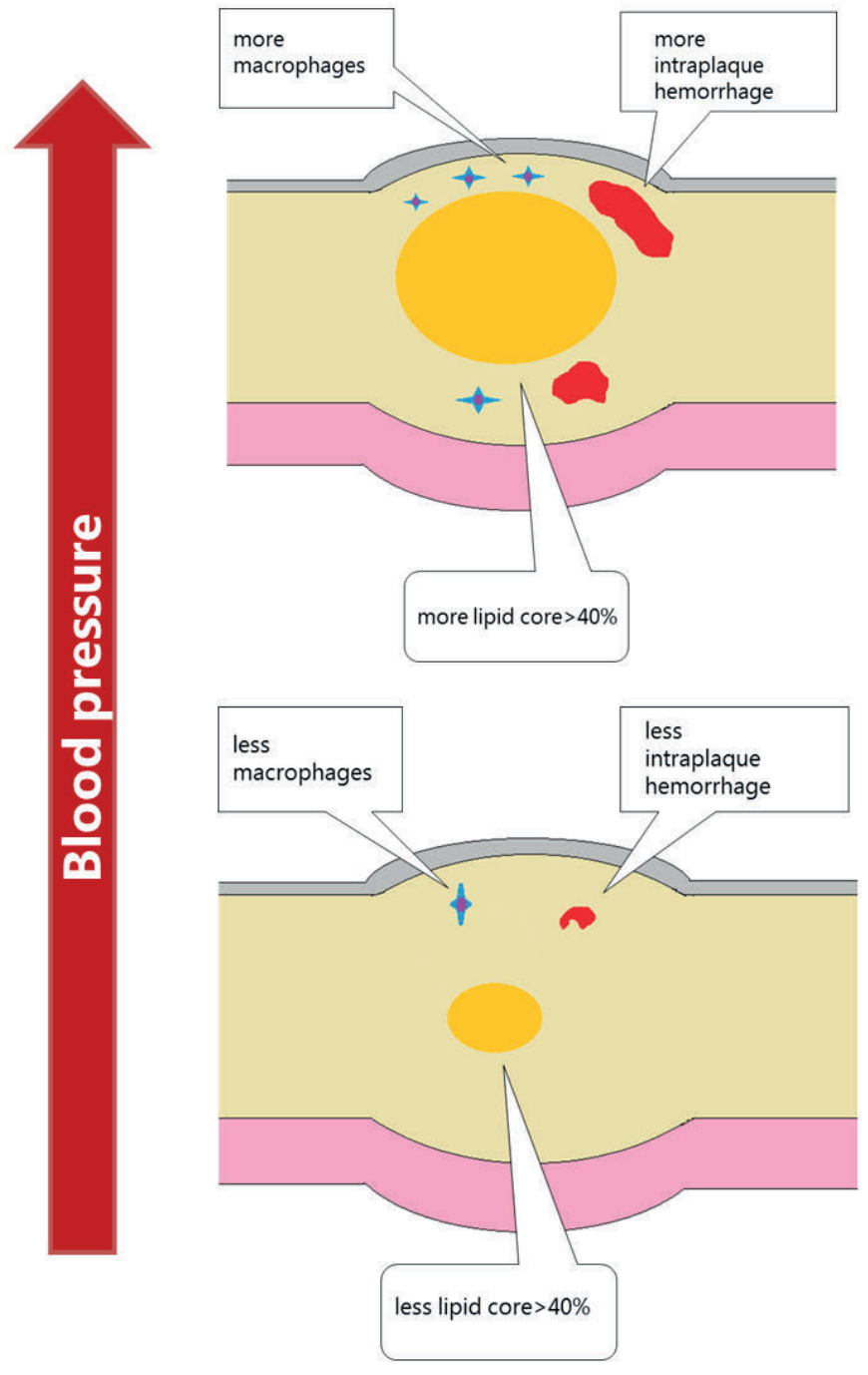

Graphical abstract 


\section{Introduction}

Hypertension is one of the most common risk factors that affects $20-30 \%$ of the world population. ${ }^{1}$ Hypertension is associated with cardiovascular events (CVE) such as myocardial infarction and stroke that have atherosclerosis as their underlying pathology. In primary prevention, intensive blood pressure (BP) lowering in high-risk patients strongly reduces myocardial infarction and stroke. ${ }^{2}$ Studies found a linear relation between high systolic BP levels and stroke risk, in which each $10 \mathrm{mmHg}$ reduction in systolic BP was associated with a one-third decrease of stroke incidence. ${ }^{3,4}$ The risk of cardiovascular death increased gradually with increased systolic and diastolic BP levels. ${ }^{5}$ Also in secondary prevention, BP lowering is associated with a reduction in stroke. ${ }^{6}$

CVE such as myocardial infarction and stroke mostly occur due to underlying atherosclerosis with activation of platelets on a ruptured or an eroded plaque surface. The association between BP and CVE is well established, but the association between BP and plaque progression is much less investigated. Lowering of systolic BP is associated with attenuation of coronary plaque progression while lowering of the diastolic BP is correlated with lower coronary plaque volume. ${ }^{7-9}$ Increased BP was also associated with progression of carotid intima media thickness. ${ }^{10}$ This shows that BP is associated with both plaque volume and plaque progression. However, no clear reversible process has been described.

On coronary plaque characteristics, a cross-sectional study demonstrated that increased diastolic BP was correlated with higher plaque volumes, more fibrous and more calcified plaques measured by CTA. ${ }^{9}$ No correlation between systolic BP and plaque volume or more fibrous and calcified plaques was found. These patients were well-controlled with systolic BP ranging from 95-154 mmHg. Two follow-up studies revealed that increased baseline systolic BP and diastolic BP were associated with an increase in atheroma volume of the coronary plaque after 1 or 2 years assessed with intravascular ultrasound. ${ }^{7,11}$ It is unknown, however, if high BP is associated with IntraPlaque Hemorrhage (IPH) in coronary plaques. Patients with carotid artery stenosis have an increased risk of CVE and death. Removal of the carotid plaque by carotid endarterectomy (CEA) is effective for severe symptomatic carotid artery stenosis in reducing the risk of future stroke and stroke-related death. ${ }^{12,13} \mathrm{~A}$ possible association between $\mathrm{BP}$ and carotid atherosclerotic plaque characteristics such as IPH has not been studied despite that carotid plaque IPH is associated with CVE. ${ }^{14}$ For this, we hypothesize that pre-operative hypertension is associated with vulnerable plaque characteristics such as IPH in carotid plaques.

In this study, we investigated the association of pre-operative systolic and diastolic BP, on histological carotid plaque characteristics including IPH in patients undergoing CEA. As atherosclerosis is considered a systemic disease, we replicated the results of this CEA cohort in a separate cohort of patients with peripheral artery disease undergoing iliofemoral endarterectomy (IFE). 


\section{Patients and methods}

This study was conducted in accordance with the declaration of Helsinki. Ethical approval for this study (TME/C01.18) was provided by the Medical Research Ethics Committee United (MEC-U) of St. Antonius Hospital Nieuwegein, The Netherlands on April 10, 2002.

\section{Patient population}

All patients in this study were included in the Athero-Express Biobank (AE biobank). The study protocol has been published before. ${ }^{15}$ In short, the AE biobank is a large ongoing prospective biobank study executed in two tertiary referral hospitals in The Netherlands, namely the St. Antonius Hospital in Nieuwegein and the University Medical Center in Utrecht. This biobank collects carotid atherosclerotic plaques and preoperative blood specimens of patients undergoing carotid and/or iliofemoral endarterectomy (IFE). All consecutive patients undergoing CEA or IFE were eligible for this study. Patients were recruited on ward during admission the day prior to surgery. Written and oral information concerning participation was provided, and informed consent was signed. The indication for CEA for asymptomatic patients was based on the recommendations published by the Asymptomatic Carotid Surgery Trial (ACST), and for symptomatic patients the indication was based on recommendations based on the European Carotid Surgery Trial and the North American Symptomatic Carotid Endarterectomy Trial (NASCET). ${ }^{12,13,16}$ Plaque removal was conducted by experienced vascular surgeons in accordance with local and international guidelines. Patients were followed for three years post-surgery for the occurrence of cardiovascular events by annual standardized questionnaires and by checking medical files. In case further information was required to define whether a cardiovascular event has occurred, the general practitioner was consulted. From the included patients, 2029 out of 2341 (87\%) had available follow-up data. Reasons for loss to follow-up included no response to follow-up questionnaires, referral to another hospital, unknown contact details because patients moved away or switch to different general practitioner of any reason.

\section{Inclusion \& exclusion criteria}

For the present study, patients undergoing CEA or IFE between 2002 to 2014 with available plaque histology and preoperative BP measurements were included. Clinical data were extracted from patient files and collected through standardized questionnaires. Biochemical data were obtained preoperatively as part of the standard preoperative work-up, either during the visit to the outpatient clinic or on the ward. Patients were excluded in case no preoperative BP or histologic plaque assessment were available. Patients operated for restenosis of the carotid or iliofemoral arteries were excluded for analysis. 


\section{Sample collection}

The sample collection protocol of the Athero-Express biobank had been described earlier. ${ }^{17}$ To summarize: preoperatively a blood sample was collected and stored at -80 degrees. Routine laboratory measurements of total cholesterol, triglycerides, HDL, LDL and creatinine were assessed. The atherosclerotic plaque was processed immediately after surgery and divided into segments of $5-\mathrm{mm}$ thickness. The section with the largest plaque burden was defined as the culprit lesion and was subjected to immunohistochemical staining. Segments were fixated in $4 \%$ formaldehyde, decalcified for one week in ethylenediaminetetraacetic acid (to soften the calcification in the plaque for handling purposes without fully dissolving it) and embedded in paraffin. This $5 \mathrm{~mm}$-segment of the culprit was cut into $5 \eta \mathrm{m}$ slices for histological analysis.

\section{Histological assessment}

Histological slides were assessed by a previously validated protocol. ${ }^{15}$ In short, plaque specimens were stained to examine the plaque characteristics as following: CD68 for macrophages, $\alpha$-actin to identify smooth muscle cells, Picro-Sirius Red (PSR) for collagen, hematoxylin eosin for general overview including calcifications, hematoxylin eosin and PSR for lipid core and CD34 for microvessels. Hematoxylin and eosin staining and fibrin by Mallory's phosphotungstic acid hematoxylin staining were used to identify the presence of luminal thrombi and intraplaque hemorrhage (IPH). Semi-quantitative scoring at $40 \times$ magnification was performed for the amount of collagen, calcification, macrophage infiltration and smooth muscle cell content and was scored as (1) no or minor staining along part of the luminal border of the plaque or (2) moderate or heavy staining along the entire luminal border or evident parts within the lesion. IPH was defined as the composite of luminal thrombi or intraplaque hemorrhages. The presence of either luminal thrombosis, intraplaque hemorrhage or both was considered as positive for IPH. IPH is scored as present or absent.

Polarized light was used to assess the area of the lipid core of the plaque, expressed as a percentage of the total plaque area. In addition, macrophages and smooth muscle cells were quantified as the percentage of plaque area with the use of computerized analyses using AnalySIS 3.2 software (Soft Imaging Systems GmbH, Münster, Germany). Microvessels were counted in 3 hotspots of the plaque and subsequently averaged per slide. All histologic slides were assessed by two independent dedicated experts, who were blinded for patient characteristics and outcomes. Good inter-observer and intra-observer similarities have been confirmed previously (K 0.6-0.9). ${ }^{18}$

\section{Study endpoints}

The primary endpoint of this study was to determine the relation between preoperative BP and the atherosclerotic plaque characteristics. Preoperative BP was defined as BP measured 
on the outpatient clinic or ward before surgery. BP measurements used in this study were the mean of three available preoperative BP measurements. Preoperative hypertension was defined as systolic BP $\geq 160 \mathrm{mmHg}$. Secondary, in order to obtain more information about the role of preoperative $\mathrm{BP}$ on the atherosclerotic plaque, results of the CEA-cohort were validated in an iliofemoral cohort. The secondary endpoint of this study was to determine the association of preoperative $\mathrm{BP}$ and secondary composite CVE during the three years after surgery. A composite endpoint of CVE included stroke, myocardial infarction, peripheral events or any cardiovascular death.

\section{Statistical analyses}

To evaluate whether an increased preoperative BP was associated with the presence of vulnerable atherosclerotic plaque characteristics, we analyzed our data for systolic and diastolic BP separately. Systolic and diastolic BP measurements were analyzed as a continuous variable, by steps of $20 \mathrm{mmHg}$. Data was inspected for missing data. Baseline characteristics of patients with preoperative measured hypertension (systolic BP $\geq 160 \mathrm{mmHg}$ ) were compared to those with normotensive preoperative BP measurement (systolic $\mathrm{BP}<160 \mathrm{mmHg}$ ). The chi-square test was used for categorical variables and an independent T-test or Mann-Whitney U test for continuous variables, as appropriate. Linear regression and logistic regression analysis were performed to investigate the correlation between plaque characteristics and BP, as appropriate. Non-normally distributed quantitative histological parameters, including macrophages, smooth muscle cells (SMC) and microvessels, required logarithmic transformation before entering into linear regression models. To adjust for potential confounders, multivariable logistic regression analysis and linear regression analysis was performed. Baseline characteristics that showed an association of $\mathrm{p}<0.20$ with BP levels as well as with the plaque characteristic of interest were considered as potential confounders for multivariable analyses (Supplemental Table 1,2). Since previous studies showed time-dependent trends in plaque characteristics and simultaneously improvement in risk factor management, year of inclusion was not included as a confounder because lowering of BP is suggested to be an underlying etiological factor (Table 1). Based on literature, symptom status was added to the model. ${ }^{17,19}$

Results of multivariable logistic regression analyses of CEA-cohort will be compared to the results of the iliofemoral cohort. Sub-analysis was performed to assess the relation between $\mathrm{BP}$ and secondary events within three years post-procedural. Cox-regression analysis was used. Values with a $\mathrm{p}<0.05$ were considered statistically significant. SPSS version 24.0 (SPSS Inc, Chicago, Illinois) was used for all statistical analyses. 


\section{Results}

\section{Patient population}

A total of 2383 patients who underwent carotid endarterectomy (CEA) and 720 patients

who underwent femoral iliac endarterectomy (IFE) were included. After exclusion of patients with missing preoperative $\mathrm{BP}$ measurements or missing plaque histology, and restenotic lesions, 1684 CEA-patients and 657 IFE-patients were included in the analysis. 26 CEA-patients (1.5\%) were also included in IFE-cohort. (See flowchart; Figure I)

\section{CEA-cohort}

Of the CEA-cohort, the majority of patients were male (68\%) with a median age of 70 years (62-76, interquartile range, IQR). At the moment of inclusion, diabetes mellitus was reported in $23 \%$ of the patients, smoking in $35 \%$ and preoperative hypertension in $42 \%$. In $87 \%$ of the patients, the carotid artery stenosis was symptomatic; these symptoms were mostly transient ischemic attacks (43\%). The median timing between index event and surgery was 30 days [IQR 61]. Patients with preoperative systolic hypertension were significantly older, were less often diabetic or had a history of coronary artery disease, had decreased kidney function and a more severe stenosis degree (Supplemental Table II). Moreover, these patients had higher total cholesterol levels, higher LDL levels and less often used statins. Patients with preoperative systolic hypertension were operated more often in the earlier years (2002-2005) than in the later years (2010-2015). Remarkably, 80\% of the patients with preoperative systolic hypertension used antihypertensive medications, for preoperative diastolic hypertension this was 75\% (Table 1).

Univariate logistic regression analyses showed a positive association between systolic BP (per $20 \mathrm{mmHg}$ ) and the presence of calcification, macrophage content, lipid core, IPH and the number of microvessels in the atherosclerotic plaque. After adjustment for potential confounders, the association between increased systolic BP and macrophages (OR 1.12, 95\% CI 1.04-1.21, $\mathrm{p}<0.01$ ), the presence of lipid core $\geq 10 \%$ and $\geq 40 \%$ (OR $1.15,95 \%$ CI $1.05-1.25, \mathrm{p}<0.01$ and OR $1.13,95 \%$ CI $1.03-1.23, \mathrm{p}=0.01$, for $10 \%$ and $40 \%$, respectively), calcification (OR 1.11, 95\% CI 1.01-1.22, p=0.03), IPH (OR 1.18, 95\% CI 1.03-1.21, p=0.01) and number of microvessels (OR $0.04,95 \%$ CI $0.00-0.08, \mathrm{p}=0.03$ ) remained statistically significant (Table 2).

In addition, for diastolic BP (per $20 \mathrm{mmHg}$ ) univariate analysis showed that the presence of more macrophages (OR 1.36, 95\% CI 1.17-1.58, $\mathrm{p}<0.01$ ), a lipid core $\geq 10 \%$ and $\geq 40 \%$ (OR1.29, 95\% CI 1.10-1.53, p<0.01 and OR 1.25, 95\% CI 1.05-1.49, $\mathrm{p}=0.01$ for $10 \%$ and $40 \%$, respectively) and IPH (OR 1.25, 95\% CI 1.07-1.45, p<0.01) were associated. After adjustment for potential confounders, these vulnerable plaque characteristics retained a significant association with high diastolic BP (Table 2). 


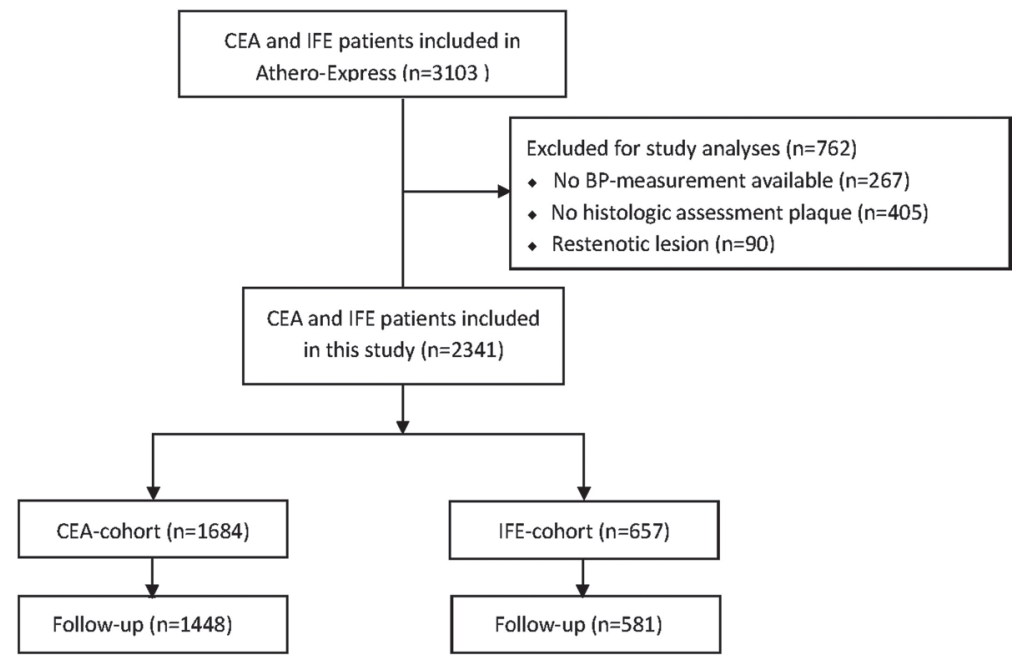

Figure 1. Flowchart of included patients in study

\section{Iliofemoral cohort}

Replication of results in 657 iliofemoral patients showed similar trends in baseline characteristics and BP levels (Supplemental Tables V and VI). Univariate and multivariable analyses revealed no significant associations between systolic BP levels and vulnerable plaque characteristics. Regarding diastolic BP, increased diastolic BP showed a strong association with the presence of IPH (OR 1.37, 95\% CI 1.07-1.76, p=0.01) (Table 3). After adjustment for potential confounders, diastolic BP remained associated with IPH (OR 1.48, 95\% CI 1.14-1.93, p<0.01). Also, increased diastolic BP levels were positively correlated with an increased number of macrophages and the presence of a lipid core $\geq 10 \%$ (OR 1.78 , 95\% CI 1.13-2.91, $\mathrm{p}=0.01$ and OR 1.45, 95\% CI 1.06-1.98, $\mathrm{p}=0.02$, respectively) (Table 3).

\section{Secondary events}

Three-year follow-up data of secondary composite CVE were available in 2029 patients of the total cohort of CEA $(n=1448)$ and IFE $(n=581)$. Secondary CVE within three years' post-procedural occurred in 669 patients (370 CVE in CEA cohort and 299 CVE in IFE cohort). Secondary CVE analyses corrected for cardiovascular risk factors showed a gradually increased risk for the composite endpoint with systolic BP (adjusted HR per 20mmHg increase 1.06, 95\% CI 1.00-1.13), $\mathrm{p}=0.04$, but not for diastolic BP (adjusted HR per $20 \mathrm{mmHg} 1.05,95 \%$ CI 0.93-1.17), $\mathrm{p}=0.43$. 
Table 1. Patient characteristics CEA and IFE cohort

\begin{tabular}{|c|c|c|}
\hline Patient characteristics & CEA $(n=1684)$ & IFE $(n=657)$ \\
\hline Systolic BP, mmHg, mean [SD] & $155[26]$ & $148[23]$ \\
\hline Diastolic BP, mmHg, mean [SD] & $82[13]$ & $78[13]$ \\
\hline Sex, male(\%) & $1153(69)$ & $484(74)$ \\
\hline Age, years, mean [SD] & $69[9]$ & $67[9]$ \\
\hline BMI, mean [IQR] & $26[4]$ & $26[4]$ \\
\hline Current smoker, $\mathrm{n}(\%)$ & $581(35)$ & $267(41)$ \\
\hline Alcohol use $>10$ units per week, $n(\%)$ & $416(25)$ & $228(35)$ \\
\hline Diabetes Mellitus, n (\%) & $392(23)$ & $202(31)$ \\
\hline Renal function, eGFR in $\mathrm{ml} / \mathrm{min} / 1.73 \mathrm{~m} 2$, median [IQR] & $72[27]$ & $76[27]$ \\
\hline History of CAD, n(\%) & $519(31)$ & $286(44)$ \\
\hline Treated hypertension, $\mathrm{n}(\%)$ & $1297(77)$ & $553(84)$ \\
\hline PAOD, yes, n(\%) & $361(21)$ & - \\
\hline \multicolumn{3}{|l|}{ Clinical presentation } \\
\hline Asymptomatic, n(\%) & $223(13)$ & - \\
\hline Ocular, n(\%) & $268(16)$ & - \\
\hline TIA, n(\%) & $722(43)$ & - \\
\hline Stroke, $\mathrm{n}(\%)$ & $464(28)$ & - \\
\hline \multicolumn{3}{|l|}{ Fontaine Classification } \\
\hline Fontaine IIb & - & $308(47)$ \\
\hline Fontaine III & - & $156(24)$ \\
\hline Fontaine IV & - & $109(17)$ \\
\hline \multicolumn{3}{|l|}{ Stenosis ipsilateral } \\
\hline $50-70 \%, \mathrm{n}(\%)$ & $118(7)$ & $51(8)$ \\
\hline $70-99, \mathrm{n}(\%)$ & $1528(91)$ & $458(70)$ \\
\hline \multicolumn{3}{|l|}{ Stenosis contralateral } \\
\hline $0-49 \%, n(\%)$ & $834(50)$ & $98(15)$ \\
\hline$>50 \%, \mathrm{n}(\%)$ & $696(41)$ & $167(25)$ \\
\hline \multicolumn{3}{|l|}{ Year of surgery } \\
\hline $2002-2003, n(\%)$ & $248(15)$ & $39(6)$ \\
\hline 2004-2005, n(\%) & $355(21)$ & $124(19)$ \\
\hline 2006-2007, n(\%) & $285(17)$ & $121(18)$ \\
\hline $2008-2009, \mathrm{n}(\%)$ & $195(12)$ & $114(17)$ \\
\hline $2010-2011, \mathrm{n}(\%)$ & $314(19)$ & $164(25)$ \\
\hline $2012-2013, \mathrm{n}(\%)$ & $211(13)$ & $95(15)$ \\
\hline $2014, \mathrm{n}(\%)$ & $76(4.5)$ & - \\
\hline Triglycerides in mg/dL, median [IQR) & $1.5[1.0]$ & $1.7[1.2]$ \\
\hline Total cholesterol in mg/dL, median [IQR] & $4.4[1.7]$ & $4.4[1.5]$ \\
\hline HDL in mg/dL, median [IQR] & $1.1[0.4]$ & $1.1[0.4]$ \\
\hline LDL in mg/dL, median [IQR] & $2.4[1.3]$ & $2.4[1.2]$ \\
\hline Statin use, yes n(\%) & $1295(77)$ & $493(75)$ \\
\hline
\end{tabular}


Table 1. Continued

\begin{tabular}{lll}
\hline Patient characteristics & CEA (n=1684) & IFE $(\mathbf{n = 6 5 7 )}$ \\
\hline Antiplatelet use, n(\%) & $1491(89)$ & $548(83)$ \\
Anti-coagulant use, n(\%) & $199(12)$ & $115(18)$ \\
Diuretic use, n(\%) & $590(35)$ & $300(46)$ \\
RAAS medication use, n(\%) & $854(51)$ & $417(64)$ \\
B-blocker use, $\mathrm{n}(\%)$ & $738(44)$ & $301(46)$ \\
\hline
\end{tabular}

Stenosis ipsilateral and stenosis contralateral refer to either carotid artery or iliofemoral artery. Degree of stenosis of the carotid artery was determined by the NASCET criteria. Baseline characteristics stratified for systolic and diastolic hypertension of CEA-cohort and IFE-cohort are presented in Supplemental Data. CAD, coronary artery disease; PAOD, peripheral artery occlusive disease; SD, standard deviation; IQR, interquartile range.
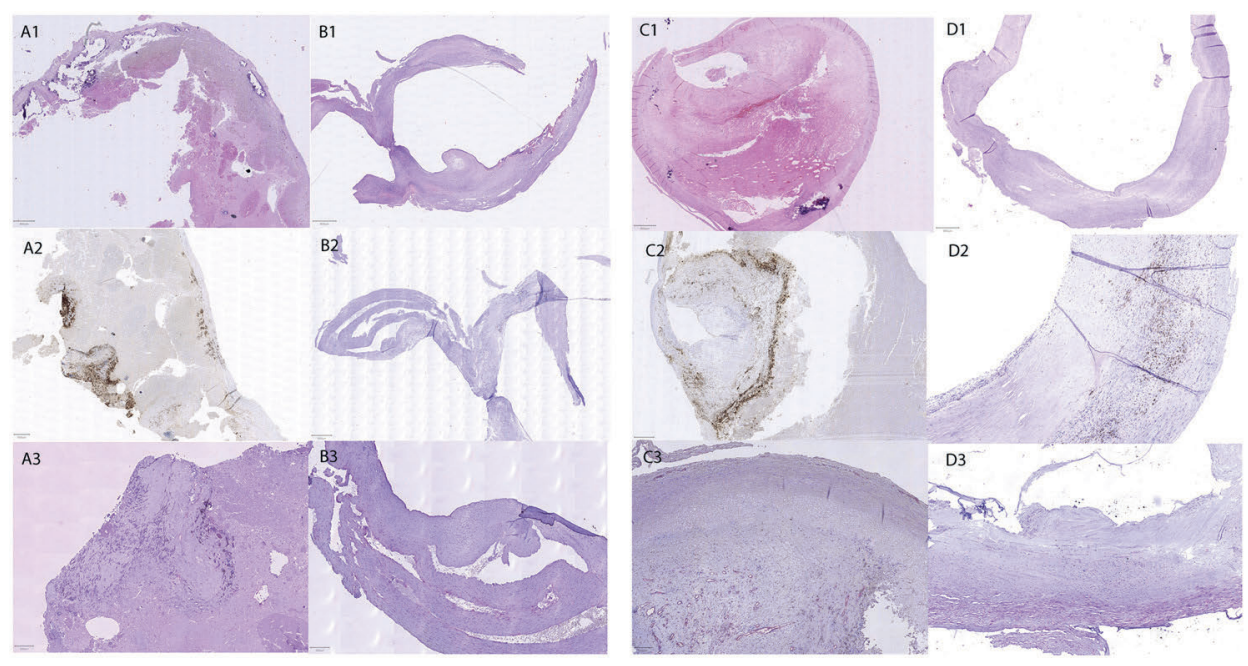

Figure 2. Histologic panel of symptomatic and asymptomatic patients stratified for blood pressure.

(A) Symptomatic patient with high blood pressure. (A1) HE staining. (A2) CD68 staining. (A3) CD34 staining. (B) Symptomatic patient with normal blood pressure. (B1) HE staining. (B2) CD68 staining. (B3) CD34 staining. (C) Asymptomatic patients with high blood pressure. (C1) HE staining. (C2) CD68 staining. (C3) CD34 staining. (D) Asymptomatic patient with normal blood pressure. (D1) HE staining. (D2) CD68 staining. (D3) CD34 staining. 
Table 2. CEA plaque characteristics \& systolic and diastolic BP

\begin{tabular}{|c|c|c|c|c|}
\hline \multicolumn{5}{|l|}{ Systolic BP (per $20 \mathrm{mmHg}$ ) } \\
\hline $\begin{array}{l}\text { Semi-quantitative plaque } \\
\text { characteristics }\end{array}$ & $\begin{array}{l}\text { OR unadjusted } \\
{[95 \% \mathrm{CI}]}\end{array}$ & $\begin{array}{l}p \text {-value } \\
\text { univariate }\end{array}$ & $\begin{array}{l}\text { OR adjusted } \\
{[95 \% \mathrm{CI}]}\end{array}$ & $\begin{array}{l}\text { p-value } \\
\text { multivariable }\end{array}$ \\
\hline Moderate/heavy calcification & $1.10[1.02-1.19]$ & 0.01 & $1.11[1.01-1.22]$ & $0.03^{a}$ \\
\hline Moderate/heavy collagen & $1.00[0.91-1.10]$ & 0.97 & $1.01[0.92-1.11]$ & $0.83^{\mathrm{b}}$ \\
\hline Moderate/heavy SMC & $1.00[0.92-1.08]$ & 0.90 & $0.95[0.86-1.04]$ & $0.26^{\mathrm{c}}$ \\
\hline Moderate/heavy macrophages & $1.10[1.02-1.19]$ & 0.01 & $1.12[1.04-1.21]$ & $<0.01^{\mathrm{d}}$ \\
\hline Presence of lipid core $>10 \%$ & $1.14[1.05-1.25]$ & $<0.01$ & $1.15[1.05-1.25]$ & $<0.01^{\mathrm{e}}$ \\
\hline Presence of lipid core $>40 \%$ & $1.12[1.03-1.21]$ & 0.01 & $1.13[1.03-1.23]$ & $0.01^{\mathrm{f}}$ \\
\hline Presence of IPH & $1.10[1.02-1.19]$ & 0.02 & $1.12[1.03-1.21]$ & $0.01^{\mathrm{g}}$ \\
\hline Continuous plaque characteristics & $\begin{array}{l}\text { Beta unadjusted } \\
{[95 \% \mathrm{CI}]}\end{array}$ & $\begin{array}{l}\text { p-value } \\
\text { univariate }\end{array}$ & $\begin{array}{l}\text { Beta adjusted } \\
{[95 \% \mathrm{CI}]}\end{array}$ & $\begin{array}{l}\text { p-value adjusted } \\
{[95 \% \mathrm{CI}]}\end{array}$ \\
\hline $\begin{array}{l}\text { Mean number of micro vessels per } \\
\text { hotspot }\end{array}$ & $0.04[0.01-0.07]$ & 0.01 & $0.04[0.00-0.08]$ & $0.03^{\mathrm{h}}$ \\
\hline
\end{tabular}

\begin{tabular}{|c|c|c|c|c|}
\hline \multicolumn{5}{|l|}{ Diastolic BP (per $20 \mathrm{mmHg}$ ) } \\
\hline $\begin{array}{l}\text { Semi-quantitative plaque } \\
\text { characteristics }\end{array}$ & $\begin{array}{l}\text { OR unadjusted } \\
{[95 \% \mathrm{CI}]}\end{array}$ & $\begin{array}{l}\text { p-value } \\
\text { univariate }\end{array}$ & $\begin{array}{l}\text { OR adjusted } \\
{[95 \% \mathrm{CI}]}\end{array}$ & $\begin{array}{l}\text { p-value } \\
\text { multivariable }\end{array}$ \\
\hline Moderate/heavy calcification & $1.05[0.91-1.21]$ & 0.54 & $1.08[0.90-1.28]$ & $0.41^{\mathrm{i}}$ \\
\hline Moderate/heavy collagen & $0.88[0.74-1.05]$ & 0.14 & $0.91[0.76-1.09]$ & $0.32^{j}$ \\
\hline Moderate/heavy SMC & $0.97[0.83-1.13]$ & 0.69 & $0.84[0.69-1.01]$ & $0.06^{\mathrm{k}}$ \\
\hline Moderate/heavy macrophages & $1.32[1.14-1.52]$ & $<0.01$ & $1.36[1.17-1.58]$ & $<0.01^{1}$ \\
\hline Presence of lipid core $>10 \%$ & $1.30[1.11-1.53]$ & $<0.01$ & $1.29[1.10-1.53]$ & $<0.01^{\mathrm{m}}$ \\
\hline Presence of lipid core $>40 \%$ & $1.22[1.04-1.44]$ & 0.01 & $1.25[1.05-1.49]$ & $0.01^{\mathrm{n}}$ \\
\hline Presence of IPH & $1.22[1.05-1.41]$ & 0.01 & $1.25[1.07-1.46]$ & $<0.01^{\circ}$ \\
\hline Continuous plaque characteristics & $\begin{array}{l}\text { Beta unadjusted } \\
{[95 \% \mathrm{CI}]}\end{array}$ & $\begin{array}{l}\text { p-value } \\
\text { univariate }\end{array}$ & $\begin{array}{l}\text { Beta adjusted } \\
{[95 \% \mathrm{CI}]}\end{array}$ & $\begin{array}{l}\text { p-value adjusted } \\
{[95 \% \mathrm{CI}]}\end{array}$ \\
\hline $\begin{array}{l}\text { Mean number of micro vessels per } \\
\text { hotspot }\end{array}$ & $0.04[-0.02-0.11]$ & 0.15 & $0.022[-0.51-0.10]$ & $0.55^{\mathrm{p}}$ \\
\hline
\end{tabular}

BP, blood pressure; SMC, smooth muscle cells; IPH, intraplaque hemorrhage; OR, odds ratio; CI, confidence interval. Bold values were considered statistically significant; $\mathrm{p}<0.05$.

a corrected for age, eGFR, CAD, ipsilateral stenosis, clinical presentation, total cholesterol, gender, HDL.

b corrected for ipsilateral stenosis, statins, clinical presentation.

c corrected for age, clinical presentation, total cholesterol, LDL, Gender, eGFR.

d corrected for clinical presentation, statins, gender.

e corrected for age, diabetes, CAD, statins, gender and clinical presentation.

${ }^{f}$ corrected for age, ipsilateral stenosis, statins, gender, clinical presentation.

g corrected for CAD, statins, gender, clinical presentation.

${ }^{\mathrm{h}}$ corrected for age, diabetes, ipsilateral stenosis, clinical presentation and triglycerides.

I corrected for age, CAD, ipsilateral stenosis, clinical presentation, total cholesterol, PAOD.

I corrected for ipsilateral stenosis, statins, clinical presentation, PAOD.

${ }^{k}$ corrected for age, clinical presentation, total cholesterol, LDL.

1 corrected for clinical presentation, statins, anti-coagulant use.

${ }^{\mathrm{m}}$ corrected for age, diabetes, CAD, statins, clinical presentation.

${ }^{\mathrm{n}}$ corrected for age, ipsilateral stenosis, statins, PAOD, BMI, clinical presentation.

${ }^{\circ}$ corrected for $\mathrm{CAD}$, statins, BMI, clinical presentation.

$\mathrm{p}$ corrected for age, diabetes, ipsilateral stenosis, clinical presentation, triglycerides, BMI. 
Table 3. Plaque characteristics of the iliofemoral cohort and systolic and diastolic BP

\begin{tabular}{|c|c|c|c|c|}
\hline \multicolumn{5}{|l|}{ Systolic BP (per 20 mmHg) } \\
\hline $\begin{array}{l}\text { Semi-quantitative } \\
\text { plaque characteristics }\end{array}$ & $\begin{array}{l}\text { OR unadjusted } \\
{[95 \% \mathrm{CI}]}\end{array}$ & $\begin{array}{l}\text { p-value } \\
\text { univariate }\end{array}$ & $\begin{array}{l}\text { OR adjusted } \\
{[95 \% \mathrm{CI}]}\end{array}$ & $\begin{array}{l}\text { p-value } \\
\text { multivariable }\end{array}$ \\
\hline Moderate/heavy calcification & $1.01[0.88-1.15]$ & 0.92 & $1.00[0.88-1.15]$ & $0.95^{\mathrm{a}}$ \\
\hline Moderate/heavy collagen & $0.97[0.81-1.16]$ & 0.72 & $1.03[0.86-1.24]$ & $0.73^{\mathrm{b}}$ \\
\hline Moderate/heavy SMC & $0.95[0.83-1.10]$ & 0.52 & $1.00[0.86-1.16]$ & $0.99^{c}$ \\
\hline Moderate/heavy macrophages & $0.97[0.81-1.16]$ & 0.75 & $1.03[0.81-1.32]$ & $0.79^{\mathrm{d}}$ \\
\hline Presence of lipid core, $>10 \%$ & $1.13[0.97-1.33]$ & 0.12 & $1.14[0.97-1.34]$ & $0.11^{\mathrm{e}}$ \\
\hline Presence of lipid core, $>40 \%$ & $1.13[0.74-1.71]$ & 0.57 & $1.10[0.74-1.36]$ & $0.63^{\mathrm{f}}$ \\
\hline Presence of IPH & $1.05[0.92-1.19]$ & 0.48 & $1.21[1.00-1.47]$ & $0.05^{\mathrm{g}}$ \\
\hline $\begin{array}{l}\text { Continuous } \\
\text { plaque characteristics }\end{array}$ & $\begin{array}{l}\text { Beta unadjusted } \\
{[95 \% \mathrm{CI}]}\end{array}$ & $\begin{array}{l}\text { p-value } \\
\text { univariate }\end{array}$ & $\begin{array}{l}\text { Beta adjusted } \\
{[95 \% \mathrm{CI}]}\end{array}$ & $\begin{array}{l}\text { p-value adjusted } \\
{[95 \% \mathrm{CI}]}\end{array}$ \\
\hline $\begin{array}{l}\text { Mean number of micro vessels per } \\
\text { hotspot }\end{array}$ & $-0.03[-0.12-0.07]$ & 0.61 & $0.39[-0.23-0.09]$ & $-0.07^{\mathrm{h}}$ \\
\hline
\end{tabular}

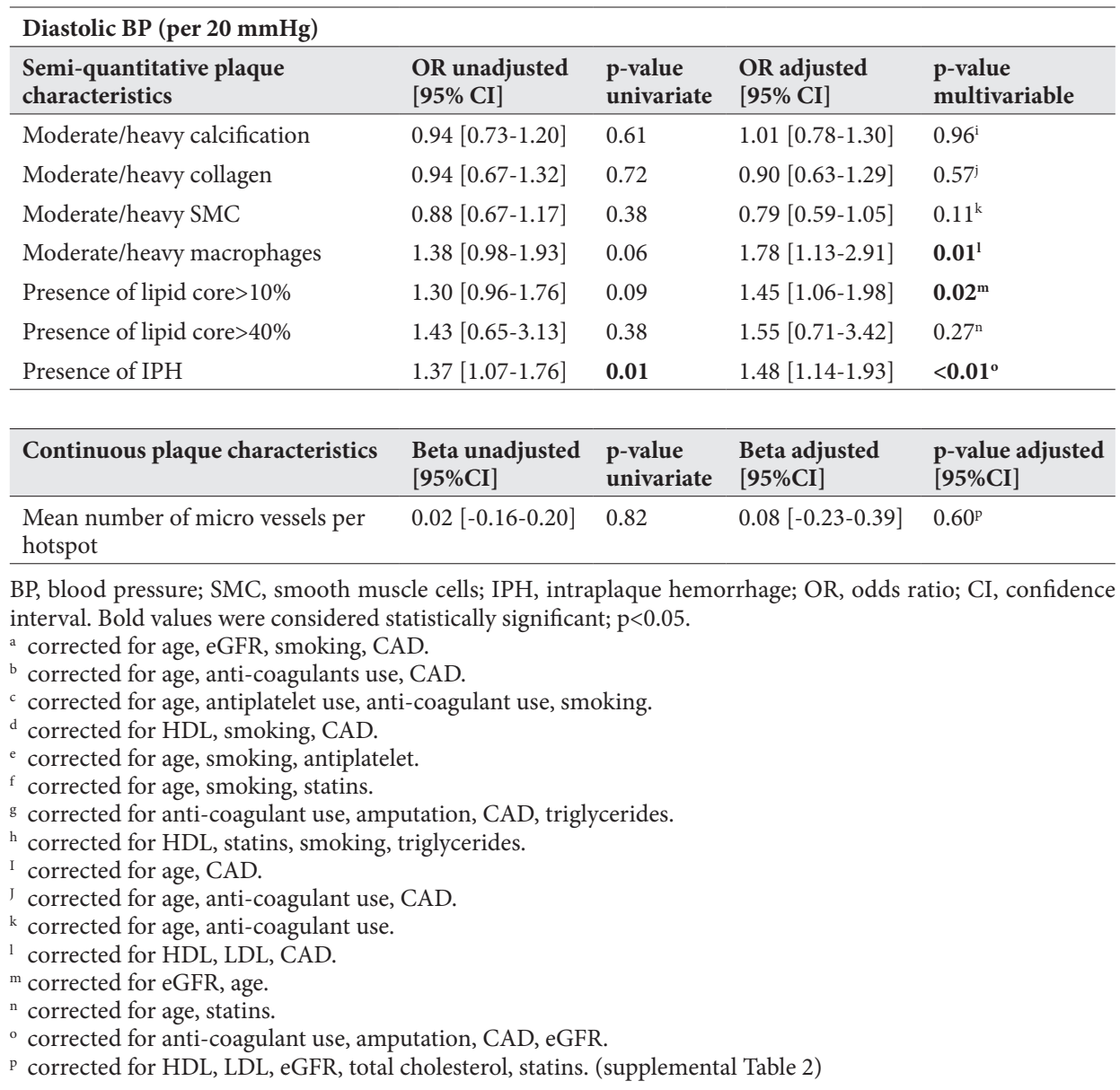




\section{Discussion}

The current study investigated the association between BP and carotid atherosclerotic plaque characteristics. Our results show that both increased systolic and diastolic BP levels were gradually associated with a more vulnerable plaque phenotype in patients with severe carotid artery stenosis undergoing CEA. Increased systolic BP and diastolic BP levels correlated with more macrophages, IPH and a larger lipid core. Similar trends were seen in the iliofemoral replication cohort as increased diastolic BP was associated with the presence of macrophages, IPH and a large lipid core.

Up to now, the relation between BP and carotid plaque characteristics has been unknown. Previous studies have mainly focused on carotid intima media thickness as determined by ultrasound. The only previous study that reported on histological carotid plaque characteristics in relation with BP was performed in a small asymptomatic hypertensive CEA cohort with specific patients that had a morning BP surge (defined as an increase of $\mathrm{SBP} \geq 50 \mathrm{mmHg}$ and/or DBP $\geq 22 \mathrm{mmHg}$ in the early morning) versus those without. Patients with a morning BP surge showed more unstable plaques with more inflammation compared with those without a morning BP surge. ${ }^{20}$ In contradiction to this previous study, our two cohorts are large with mainly symptomatic patients that are both hypertensive and non-hypertensive.

Although performed in a different study population and usage of different methods for plaque characterization, our results are in line with previous studies on the association of hypertension and coronary plaque characteristics. Increase in baseline BP was associated with coronary plaque atheroma (determined by intravascular ultrasound) which matches our association of high $\mathrm{BP}$ and a large lipid core in the carotid plaque. Intravascular ultrasound, however, does not allow detection of macrophages and IPH.

We replicated our findings in a cohort of patients undergoing IFE. Carotid and femoral plaques have been described to have different morphology. ${ }^{21}$ While carotid plaques show more atheromatous plaques with larger lipid cores and more macrophages and T-cells and with more metalloproteinase MMP-9 than the femoral plaques ${ }^{21-23}$, femoral plaques have more stable fibrocalcified lesions with a lower concentration of cholesterol and a higher concentration of calcium. ${ }^{21,22}$ Next to the vascular bed, this is probably due to the timing of surgery. In symptomatic patients undergoing carotid revascularization, treatment is performed preferably within the first two weeks after the index event. In contrast, in patients with intermittent claudication complaints surgery is often preceded by exercise training resulting in delayed iliofemoral surgery. ${ }^{24}$ Despite these intrinsic differences between carotid and femoral plaque morphology, we found that systolic and diastolic BP were in both cohorts associated with IPH, underlining the systemic importance of BP on IPH. 
IPH in coronary plaques has been described to contain glycophorin A and iron from erythrocytes. ${ }^{25}$ High levels of glycophorin A and iron have been associated with a large lipid core and high influx of macrophages suggesting that IPH represent a potent atherogenic stimulus. ${ }^{25}$ In our carotid plaques, IPH is increased with high BP together with an increased lipid core and macrophage accumulation that might point to IPH increase as a result of high BP. In accordance, IPH in carotid plaques was associated with accelerated plaque progression and increased lipid core. ${ }^{26}$

Leakage of plaque microvessel endothelium has been held responsible for IPH in advanced human coronary atherosclerosis. ${ }^{27}$ We only found an association of systolic BP and plaque microvessels in the CEA cohort and not in the iliofemoral cohort. One could hypothesize that increased BP could accelerate erythrocyte leakage from microvessels or cause rupture of these immature microvessels due to direct mechanic forces, both resulting in plaque instability with subsequent CVE.

Next to the association of high BP with vulnerable plaque characteristics, we found that patients despite treatment have residual hypertension, with respectively $86 \%$ and $82 \%$ of CEA and IFE patients having a systolic $\mathrm{BP} \geq 160 \mathrm{mmHg}$ while treated with antihypertensive medication. This could be either due to non-compliance of medications or unclose monitoring of the BP lowering effect by physicians. Surprisingly, diabetes and coronary artery disease were more frequently reported in patients with systolic BP $\leq 160 \mathrm{mmHg}$. This is probably induced by selection bias since patients with a history of diabetes or coronary artery disease will be subjected to more stringent secondary preventive strategies and therefore more often screened for hypertension and subsequently treated with antihypertensive medications.

Residual hypertension in our cohorts together with the association of high BP with more macrophages, lipid core and IPH as markers of the rupture-prone plaque and IPH as plaque marker associated with CVE strongly indicates that intensive BP monitoring and intensive anti-hypertensive therapy is needed for these severely atherosclerotic patients. ${ }^{14,28}$

\section{Limitations}

Some limitations should be addressed. First, BP measurements used in the current study are in-hospital preoperative measurements that were assessed on the nursing ward conform preoperative work-up. Stress-induced factors or white coat hypertension can influence these in-hospital BP measurements. To diminish the effect of these factors, the used BP measurements are the mean of three preoperative BP measurements measured on the ward on separate moments in time. These blood pressure measurements are conform BP measurements used in large clinical trials for management of arterial hypertension and 
most feasible in clinical practice. ${ }^{29,30}$ Second, although we found in both carotid and iliofemoral cohort a correlation between vulnerable plaque characteristics and increased $\mathrm{BP}$, no causal relation can be proven due to the observational study design. Future prospective trials should be addressed to investigate the causality of intensive BP treatment on atherosclerotic plaque characteristics. Third, as patients undergoing carotid artery stenting (CAS) were not included in these analyses, results cannot be extrapolated for CAS-patients. Finally, one out of five patients undergoing CEA have systolic BP inter-arm difference of $>15 \mathrm{mmHg} .{ }^{31}$ Nurses and healthcare takers in our hospitals are instructed to measure BP of patients undergoing CEA bilaterally, in which the side of the highest BP will be used for future BP measurements. However, there is no data available whether the mean of BP is solely based on single BP measurements of the highest arm. This might have influenced our results. ${ }^{31,32}$

\section{Conclusion}

In conclusion, increased systolic and diastolic BP levels are associated with more carotid plaque macrophages, lipid core and IPH in patients undergoing CEA. Replication in a separate iliofemoral cohort confirmed these associations.

\section{Financial support}

Funding was from the EU 755320 Taxinomisis grant to GP, DdK and GJdB. No competing interests declared.

\section{Conflicts of interest}

None 


\section{References}

1 Mackay J, Mensah GA. The atlas of heart disease and stroke. World Heal Organ 2004.

2 Xie X, Atkins E, Lv J, Bennett A, Neal B, et al. Effects of intensive blood pressure lowering on cardiovascular and renal outcomes : updated systematic review and meta-analysis. Lancet 2016;387:435-43.

3 Lawes CMM, Bennett DA, Feigin VL, Rodgers A. Blood Pressure and Stroke. An overview of published reviews. Stroke 2004;35:776-85.

4 Attar A, Sayadi M, Jannati M. Effect of intensive blood pressure lowering on cardiovascular outcomes based on cardiovascular risk : A secondary analysis of the SPRINT trial. Eur J Prev Cardiol 2019;26:238-45. Doi: $10.1177 / 2047487318800741$.

5 Prospective Studies Collaboration. Age-specific relevance of usual blood pressure to vascular mortality : a meta-analysis of individual data for one million adults in 61 prospective studies. Lancet 2002;360:1903-13.

6 Zhang H, Thijs L, Staessen JA. Blood Pressure Lowering for Primary and Secondary Prevention of Stroke. Hypertension 2006;48:187-95.

7 Chhatriwalla AK, Nicholls SJ, Wang TH, Wolski K, Sipahi I, et al. Low Levels of Low-Density Lipoprotein Cholesterol and Blood Pressure and Progression of Coronary Atherosclerosis. J Am Coll Cardiol 2009;53:1110-5.

8 Sipahi I, Tuzcu EM, Schoenhagen P, Wolski KE, Nicholls SJ, et al. Effects of Normal, Pre-hypertensive, and Hypertensive Blood Pressure Levels on Progression of Coronary Atherosclerosis. J Am Coll Cardiol 2006;48(4):4-9.

9 Saleh M, Alfaddagh A, Elajami TK, Ashfaque H, Haj-ibrahim H, et al. Diastolic blood pressure predicts coronary plaque volume in patients with coronary artery disease. Atherosclerosis 2018;277:34-41.

10 Chien K, Tu Y, Hsu H, Su T, Lin H, et al. Differential effects of the changes of LDL cholesterol and systolic blood pressure on the risk of carotid artery atherosclerosis. BMC Cardiovasc Disord 2012;12:1-7.

11 Garciá-Garciá MH, Klauss V, Gonzalo N, Garg S, Onuma Y, et al. Relationship between cardiovascular risk factors and biomarkers with necrotic core and atheroma size : a serial intravascular ultrasound radiofrequency data analysis. Internatial J Cardiovasc Imaging 2012;28:695-703.

12 North American Symptomatic Carotid Endarterectomy Trial Collaborators. Beneficial effect of carotid endarterectomy in symptomatic patients with high-grade carotid stenosis. N Engl J Med 1991;325(7):445-53.

13 Writing Group, Naylor AR, Ricco JB, de Borst GJ, Debus S, et al. Management of atherosclerotic carotid and vertebral artery disease: 2017 clinical practice guidelines of the european society for vascular surgery (ESVS). Eur J Vasc Endovasc Surg 2017;55(1):1-79.

14 Hellings WE, Peeters W, Moll FL, Piers SRD, van Setten J, et al. Composition of Carotid Atherosclerotic Plaque Is Associated. Circulation 2010;121:1914-50.

15 Verhoeven BAN, Velema E, Schoneveld AH, de Vries JPPM, de Bruin P, et al. Athero-express : Differential atherosclerotic plaque expression of mRNA and protein in relation to cardiovascular events and patient characteristics . Rationale and design. Eur J Epidemiol 2004;19:1127-33.

16 European Carotid Surgery Trialists' Collaborative Group. Randomised trial of endarterectomy for recently symptomatic carotid stenosis : final results of the MRC European Carotid Surgery Trial ( ECST ). Lancet 1998;351:13\79-87.

17 Verhoeven B, Hellings WE, Moll FL, de Vries JP,de Kleijn DPV, et al. Carotid atherosclerotic plaques in patients with transient ischemic attacks and stroke have unstable characteristics compared with plaques in asymptomatic and amaurosis fugax patients. J Vasc Surg 2005;42:1077-81.

18 Hellings WE, Pasterkamp G, Vollebregt A, Seldenrijk CA, de Vries JPM, et al. Intraobserver and interobserver variability and spatial differences in histologic examination of carotid endarterectomy specimens. J Vasc Surg 2007;46:1147-54.

19 Howard DPJ, vanLammeren GW, Redgrave JN, Moll FL, de Vries JPM, et al. Histological Features of Carotid Plaque in Patients With Ocular Ischemia Versus Cerebral Events. Stroke 2013;44:734-9.

20 Marfella R, Siniscalchi M, Portoghese M, Di Filippo C, Ferraraccio F, et al. Morning blood pressure surge as a destabilizing factor of atherosclerotic plaque: role of ubiquitin-proteasome activity. Hypertension 2007;49(4):784-91.

21 Herisson F, Heymann M-F, Chétiveaux M, Charrier C, Battaglia S, et al. Carotid and femoral atherosclerotic plaques show different morphology. Atherosclerosis 2011;216:348-54. 
22 Shaikh S, Brittenden J, Lahiri R, Brown PAJ, Thies F, et al. Macrophage Subtypes in Symptomatic Carotid Artery and Femoral Artery Plaques. Eur J Vasc Endovasc Surg 2012;44:491-7.

23 Orbe J, Fernandez L, Rodr JA, Rábago G, Belzunce M, et al. Different expression of MMPs/TIMP-1 in human atherosclerotic lesions . Relation to plaque features and vascular bed. Atherosclerosis 2003;170:26976.

24 Aboyans V, Ricco J-B, Bartelink MEL, Björck M, Brodmann M, et al. 2017 ESC Guidelines on the Diagnosis and Treatment of Peripheral Arterial Diseases, in collaboration with the European Society for Vascular Surgery ( ESVS ). Eur Heart J 2018;39:763-821.

25 Kolodgie FD, Gold HK, Burke AP, Fowler DR, Kruth HS, et al. Intraplaque Hemorrhage and Progression of Coronary Atheroma. N Engl J Med 2003;349:2316-25.

26 Takaya N, Yuan C, Chu B, Saam T, Polissar NL, et al. Presence of Intraplaque Hemorrhage Stimulates Progression of Carotid Atherosclerotic Plaques. Circulation 2005;111:2768-75.

27 Sluimer JC, Kolodgie FD, Bijnens APJJ, Maxfield K, Pacheco E, et al. Thin-Walled Microvessels in Human Coronary Atherosclerotic Plaques Show Incomplete Endothelial Junctions. J Am Coll Cardiol 2009;53(17):1517-27.

28 Schaar JA, Muller JE, Falk E, Virmani R, Fuster V, et al. Terminology for high-risk and vulnerable coronary artery plaques. Eur Heart J 2004;25:1077-82.

29 ADVANCE Collaborative Group. Effects of a fixed combination of perindopril and indapamide on macrovascular and microvascular outcomes in patients with type 2 diabetes mellitus (the ADVANCE trial): a randomised controlled trial. Lancet 2007;370:829-40.

30 Böhm M, Schumacher H, Teo KK, Lonn EM, Mahfoud F, et al. Achieved blood pressure and cardiovascular outcomes in high-risk patients: results from ONTARGET and TRANSCEND trials. Lancet 2017;389:2226-37.

31 Huibers A, Hendrikse J, Brown MM, Pegge SA, Arnold M, et al. Upper extremity blood pressure difference in patients undergoing carotid revascularisation. Eur J Vasc Endovasc Surg 2017;53(2):153-7.

32 Kranenburg G, Spiering W, de Jong PA, Kappelle LJ, de Borst GJ, et al. Inter-arm systolic blood pressure differences, relations with future vascular events and mortality in patients with and without manifest vascular disease. Int J Cardiol 2017;244:271-6. 


\section{Supplementary Materials}

The following supplementary material is omitted due to space limitation and can be found at the journal website:

- Supplemental Table I. CEA-cohort: confounders used for correction per plaque characteristic.

- Supplemental Table II. Iliofemoral-cohort: confounders used for correction per plaque characteristic.

- Supplemental Table IV. CEA-cohort: patient characteristics and diastolic BP.

- Supplemental Table VI. Iliofemoral-cohort: patient characteristics and diastolic BP.

- Supplemental Table VII. CEA-cohort: plaque distribution.

- Supplemental Table VIII. Iliofemoral-cohort: plaque distribution. 
Supplemental Table III. Patient characteristics CEA-cohort and systolic BP

\begin{tabular}{|c|c|c|c|}
\hline Patient characteristics & $\begin{array}{l}\text { Systolic BP }<160 \\
(\mathrm{n}=976)\end{array}$ & $\begin{array}{l}\text { Systolic BP } \geq 160 \\
(\mathrm{n}=708)\end{array}$ & p-value \\
\hline Systolic BP, mmHg, mean [SD] & $137[14]$ & $179[18]$ & $<0.01$ \\
\hline Diastolic BP, mmHg, mean [SD] & $76[11]$ & $90[12]$ & $<0.01$ \\
\hline Sex, male, n(\%) & $683(70)$ & $470(66)$ & 0.12 \\
\hline Age, years, mean $[\mathrm{SD}]$ & $68[9.5]$ & $70[8.8]$ & $<0.01$ \\
\hline BMI, mean [IQR] & {$[26 ; 5]$} & {$[26 ; 4]$} & 0.29 \\
\hline Current smoker, $\mathrm{n}(\%)$ & $340(35)$ & $241(35)$ & 0.78 \\
\hline Alcohol use $>10$ units per week, $n(\%)$ & $232(23.8)$ & $184(26.1)$ & 0.29 \\
\hline Diabetes Mellitus type II, n(\%) & 254(26) & $143(20)$ & 0.01 \\
\hline $\begin{array}{l}\text { Renal function, eGFR in } \mathrm{ml} / \mathrm{min} / 1.73 \mathrm{~m} 2 \text {, } \\
\text { median [IQR] }\end{array}$ & $74[25]$ & $70[26]$ & 0.04 \\
\hline History of CAD, n(\%) & $321(33)$ & 198(28) & 0.03 \\
\hline Treated hypertension, n(\%) & $734(75)$ & $563(80)$ & 0.03 \\
\hline PAOD, yes, n(\%) & $219(22)$ & $142(20)$ & 0.25 \\
\hline Clinical presentation & & & 0.08 \\
\hline Asymptomatic, n(\%) & $138(14)$ & $85(12)$ & \\
\hline Ocular, $\mathrm{n}(\%)$ & $146(15)$ & $122(17)$ & \\
\hline TIA, n(\%) & $402(41)$ & $320(45)$ & \\
\hline Stroke, $\mathrm{n}(\%)$ & 285(29) & $178(25)$ & \\
\hline Stenosis ipsilateral & & & 0.02 \\
\hline $50-70 \%, \mathrm{n}(\%)$ & $78(8)$ & $40(6)$ & \\
\hline $70-99, \mathrm{n}(\%)$ & $873(91)$ & $655(94)$ & \\
\hline Contralateral stenosis $>50 \%, \mathrm{n}(\%)$ & $405(46)$ & 291(45) & 0.91 \\
\hline Year of surgery, n(\%) & & & $<0.01$ \\
\hline $2002-2003$ & $117(12)$ & $131(19)$ & \\
\hline 2004-2005 & $195(20)$ & $160(23)$ & \\
\hline 2006-2007 & $161(17)$ & $124(18)$ & \\
\hline 2008-2009 & 104(11) & $91(13)$ & \\
\hline $2010-2011$ & $198(20)$ & $116(16)$ & \\
\hline $2012-2013$ & $145(15)$ & $66(9)$ & \\
\hline 2014-2015 & $56(6)$ & $20(3)$ & \\
\hline Triglycerides in mg/dL, median[IQR) & $1.4[1.0]$ & $1.5[0.9]$ & 0.15 \\
\hline Total cholesterol in mg/dL, median[IQR] & $4.2[1.6]$ & $4.4[1.8]$ & $<0.01$ \\
\hline $\mathrm{HDL}$ in $\mathrm{mg} / \mathrm{dL}$, median[IQR] & $1.1[0.4]$ & $1.1[0.4]$ & 0.12 \\
\hline $\mathrm{LDL}$ in $\mathrm{mg} / \mathrm{dL}$, median [IQR] & $2.3[1.3]$ & $2.4[1.4]$ & 0.08 \\
\hline Statin use, yes, n(\%) & $772(79)$ & $523(74)$ & 0.02 \\
\hline Antiplatelet use, n(\%) & $869(89)$ & $622(88)$ & 0.47 \\
\hline
\end{tabular}


Supplemental Table III. Continued

\begin{tabular}{llll}
\hline Patient characteristics & $\begin{array}{l}\text { Systolic BP }<\mathbf{1 6 0} \\
(\mathbf{n = 9 7 6 )}\end{array}$ & $\begin{array}{l}\text { Systolic BP } \geq \mathbf{1 6 0} \\
(\mathbf{n = 7 0 8 )}\end{array}$ & p-value \\
\hline Anti-coagulant use, n(\%) & $110(11)$ & $89(13)$ & 0.40 \\
Diuretic use, n(\%) & $341(35)$ & $249(35)$ & 0.89 \\
RAAS medication use, n(\%) & $479(49)$ & $375(53)$ & 0.10 \\
B-blocker use, n(\%) & $430(44)$ & $308(44)$ & 0.86 \\
\hline
\end{tabular}

Continue variables are analyzed with help of independent t-test, categorical variables with chi-square test or fishers exact test. Lipids were first log transformed and analyzed with t-test. Bold values were considered statistically significant; $\mathrm{p}<0.05$. Degree of stenosis was determined by the NASCET criteria. CAD, coronary artery disease; PAOD, peripheral artery occlusive disease; SD, standard deviation; IQR, interquartile range.

\section{Supplemental Table V. Patient characteristics iliofemoral-cohort and systolic BP}

\begin{tabular}{|c|c|c|c|}
\hline Patient characteristics & $\begin{array}{l}\text { Systolic } B P<160 \\
(n=453)\end{array}$ & $\begin{array}{l}\text { Systolic } B P \geq 160 \\
(n=204)\end{array}$ & p-value \\
\hline Systolic BP, mmHg, mean [SD] & $135.7[14.4]$ & $175.8[13.7]$ & $<0.01$ \\
\hline Diastolic BP, mmHg, mean [SD] & $74.7[12.0]$ & $84.3[12.2]$ & $<0.01$ \\
\hline Sex, male, $\mathrm{n}(\%)$ & $337(74.4)$ & $147(72.1)$ & 0.53 \\
\hline Age, years, mean [SD] & $66.1[9.4]$ & $69.6[8.9]$ & $<0.01$ \\
\hline BMI, mean [SD] & $26.1[3.9]$ & $25.9[3.7]$ & 0.66 \\
\hline Current smoker, $\mathrm{n}(\%)$ & $193(43.1)$ & $74(36.6)$ & 0.12 \\
\hline Alcohol use $>10$ units per week, $n(\%)$ & $161(35.6)$ & $67(32.8)$ & 0.49 \\
\hline Diabetes Mellitus type II, n(\%) & $144(31.8)$ & $58(28.4)$ & 0.39 \\
\hline $\begin{array}{l}\text { Renal function, eGFR in } \mathrm{ml} / \mathrm{min} / 1.73 \mathrm{~m} 2 \text {, } \\
\text { median [IQR] }\end{array}$ & $75.2[33.3]$ & $77.0[33.2]$ & 0.52 \\
\hline History of CAD, n(\%) & $206(45.5)$ & $80(39.2)$ & 0.13 \\
\hline Treated hypertension, $\mathrm{n}(\%)$ & $377(83.2)$ & $176(86.3)$ & 0.32 \\
\hline Fontaine Classification, $\mathrm{n}(\%)$ & & & 0.52 \\
\hline Fontaine IIb & $206(52.3)$ & $102(57.0)$ & \\
\hline Fontaine III & $109(27.7)$ & $47(26.3)$ & \\
\hline Fontaine IV & $79(20.1)$ & $30(16.8)$ & \\
\hline Stenosis contralateral $>50 \%, \mathrm{n}(\%)$ & $114(62.6)$ & $53(63.9)$ & 0.85 \\
\hline Operated Artery, n(\%) & & & 0.96 \\
\hline Femoral & $407(89.9)$ & $183(89.7)$ & \\
\hline Iliac & $46(10.2)$ & $21(10.3)$ & \\
\hline Operation Type, $\mathrm{n}(\%)$ & & & 0.90 \\
\hline REA & $106(25.3)$ & $49(25.8)$ & \\
\hline TEA & $313(74.7)$ & $141(74.2)$ & \\
\hline
\end{tabular}


Supplemental Table V. Continued

\begin{tabular}{|c|c|c|c|}
\hline Patient characteristics & $\begin{array}{l}\text { Systolic BP }<160 \\
(n=453)\end{array}$ & $\begin{array}{l}\text { Systolic } B P \geq 160 \\
(n=204)\end{array}$ & p-value \\
\hline Previous peripheral intervention, $\mathrm{n}(\%)$ & $222(49.1)$ & $102(50.2)$ & 0.79 \\
\hline Previous amputation & $24(5.5)$ & $4(2)$ & 0.06 \\
\hline Year of surgery & & & 0.09 \\
\hline $2002-2003$ & $24(5.3)$ & $15(7.4)$ & \\
\hline 2004-2005 & $75(16.6)$ & $49(24.0)$ & \\
\hline 2006-2007 & $92(20.3)$ & $29(14.2)$ & \\
\hline 2008-2009 & $78(17.0)$ & $36(17.6)$ & \\
\hline 2010-2011 & $120(26.5)$ & $44(21.6)$ & \\
\hline 2012-2013 & $64(14.1)$ & $31(15.2)$ & \\
\hline Triglycerides in mg/dL, median [IQR] & $1.8[1.2]$ & $1.6[1.2]$ & 0.13 \\
\hline Total cholesterol in mg/dL, median [IQR] & $4.4[1.5]$ & $4.4[1.5]$ & 0.43 \\
\hline $\mathrm{HDL}$ in $\mathrm{mg} / \mathrm{dL}$, median [IQR] & $1.0[0.4]$ & $1.2[0.4]$ & $<0.01$ \\
\hline LDL in mg/dL, median [IQR] & $2.4[1.1]$ & $2.3[1.3]$ & 0.78 \\
\hline Statin use, yes, n(\%) & $347(76.6)$ & $146(71.6)$ & 0.17 \\
\hline Antiplatelet use, n(\%) & $369(81.6)$ & $179(87.7)$ & 0.05 \\
\hline Anti-coagulant use, $\mathrm{n}(\%)$ & $92(20.3)$ & $23(11.3)$ & $<0.01$ \\
\hline Diuretic use, n(\%) & $213(47.0)$ & $87(42.6)$ & 0.30 \\
\hline RAAS medication use, $\mathrm{n}(\%)$ & $283(62.5)$ & $134(65.7)$ & 0.43 \\
\hline B-blocker use, n(\%) & $205(45.3)$ & $96(47.1)$ & 0.67 \\
\hline
\end{tabular}

Continue variables are analyzed with help of independent t-test, categorical variables with chi-square test or fishers exact test. Lipids were first log transformed and analyzed with t-test. Bold values were considered statistically significant; $\mathrm{p}<0.05$. CAD, coronary artery disease; PAOD, peripheral artery occlusive disease; $\mathrm{SD}$, standard deviation; IQR, interquartile range. 


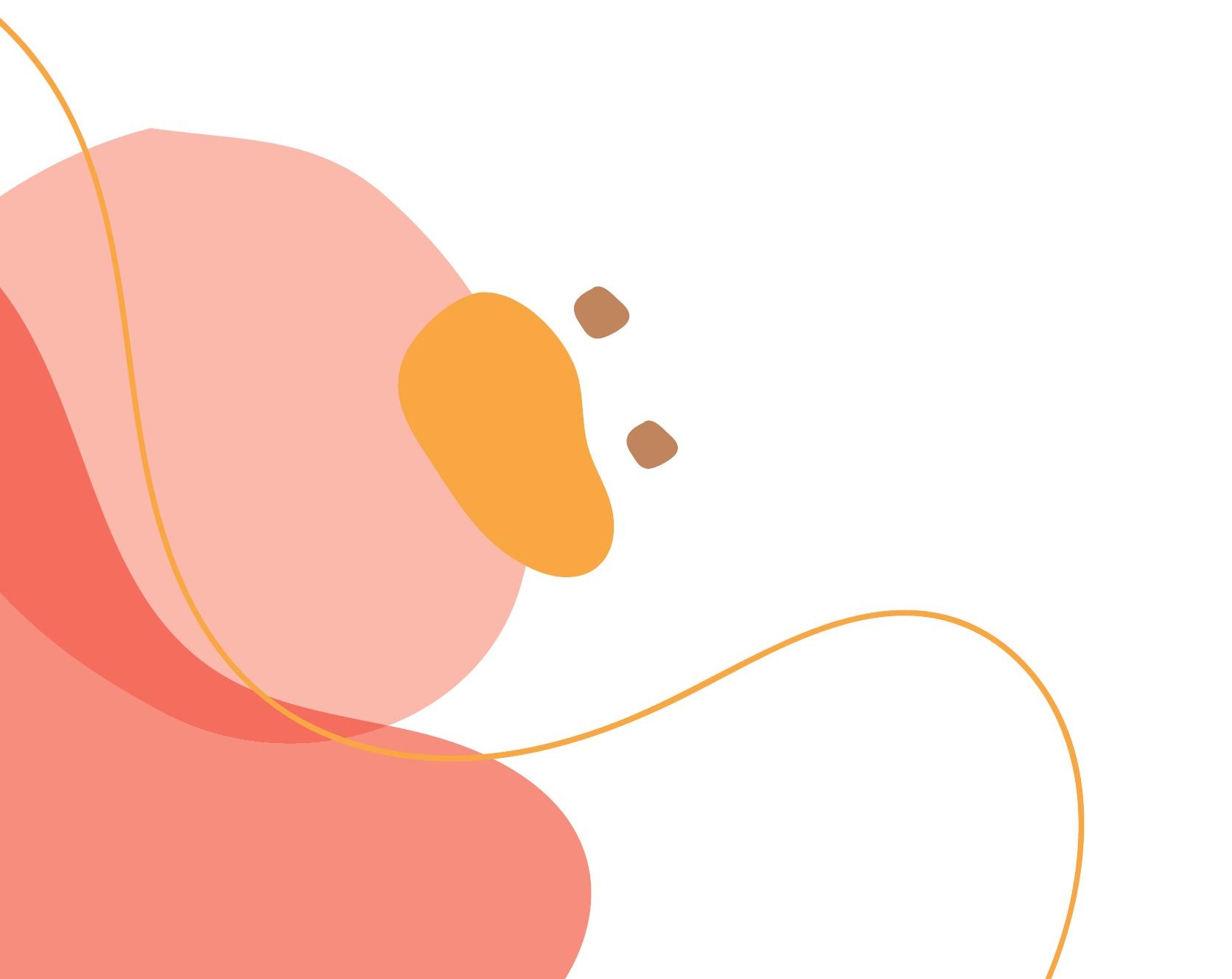


CHAPTER 3

\section{Family history and polygenic risk of cardiovascular disease: independent factors associated with secondary cardiovascular events in patients undergoing carotid endarterectomy}

Atherosclerosis 2020; 307:121-129

Nathalie Timmerman

Dominique P.V. de Kleijn Gert J. de Borst Hester M. den Ruijter

Folkert W. Asselbergs

Gerard Pasterkamp Saskia Haitjema* Sander W. van der Laan*

${ }^{*}$ Joint last authorship 


\section{Abstract}

\section{Background and aims}

Family history (FHx) of cardiovascular disease (CVD) is a risk factor for CVD and a proxy for cardiovascular heritability. Polygenic risk scores (PRS) summarizing $>1$ million variants for coronary artery disease $(\mathrm{CAD})$ are associated with incident and recurrent CAD events. However, little is known about the influence of FHx or PRS on secondary cardiovascular events (sCVE) in patients undergoing carotid endarterectomy (CEA).

\section{Methods}

We included 1,788 CEA patients from the Athero-Express Biobank. A weighted PRS for CAD including 1.7 million variants was calculated (MetaGRS). The composite endpoint of sCVE during three years follow-up included coronary, cerebrovascular and peripheral events and cardiovascular death. We assessed the impact of FHx and MetaGRS on sCVE and carotid plaque composition.

\section{Results}

Positive FHx was associated with a higher 3-year risk of sCVE independent of cardiovascular risk factors and MetaGRS (adjusted HR 1.40, 95\%CI 1.07-1.82, $\mathrm{p}=0.013$ ). Patients in the highest MetaGRS quintile had a higher 3-year risk of sCVE compared to the rest of the cohort independent of cardiovascular risk factors including FHx (adjusted HR 1.35, 95\% CI 1.01-1.79, $\mathrm{p}=0.043$ ), and their atherosclerotic plaques contained more fat (adjusted OR 1.59, 95\%CI, 1.11-2.29, $\mathrm{p}=0.013$ ) and more macrophages (OR 1.49, 95\%CI 1.12-1.99, $\mathrm{p}=0.006$ ).

\section{Conclusions}

In CEA patients, both positive FHx and higher MetaGRS were independently associated with increased risk of SCVE. Moreover, higher MetaGRS was associated with vulnerable plaque characteristics. Future studies should unravel underlying mechanisms and focus on the added value of PRS and FHx in individual risk prediction for SCVE. 
Family history and genetic risk score (MetaGRS) are independently associated with secondary cardiovascular events during 3-year follow-up

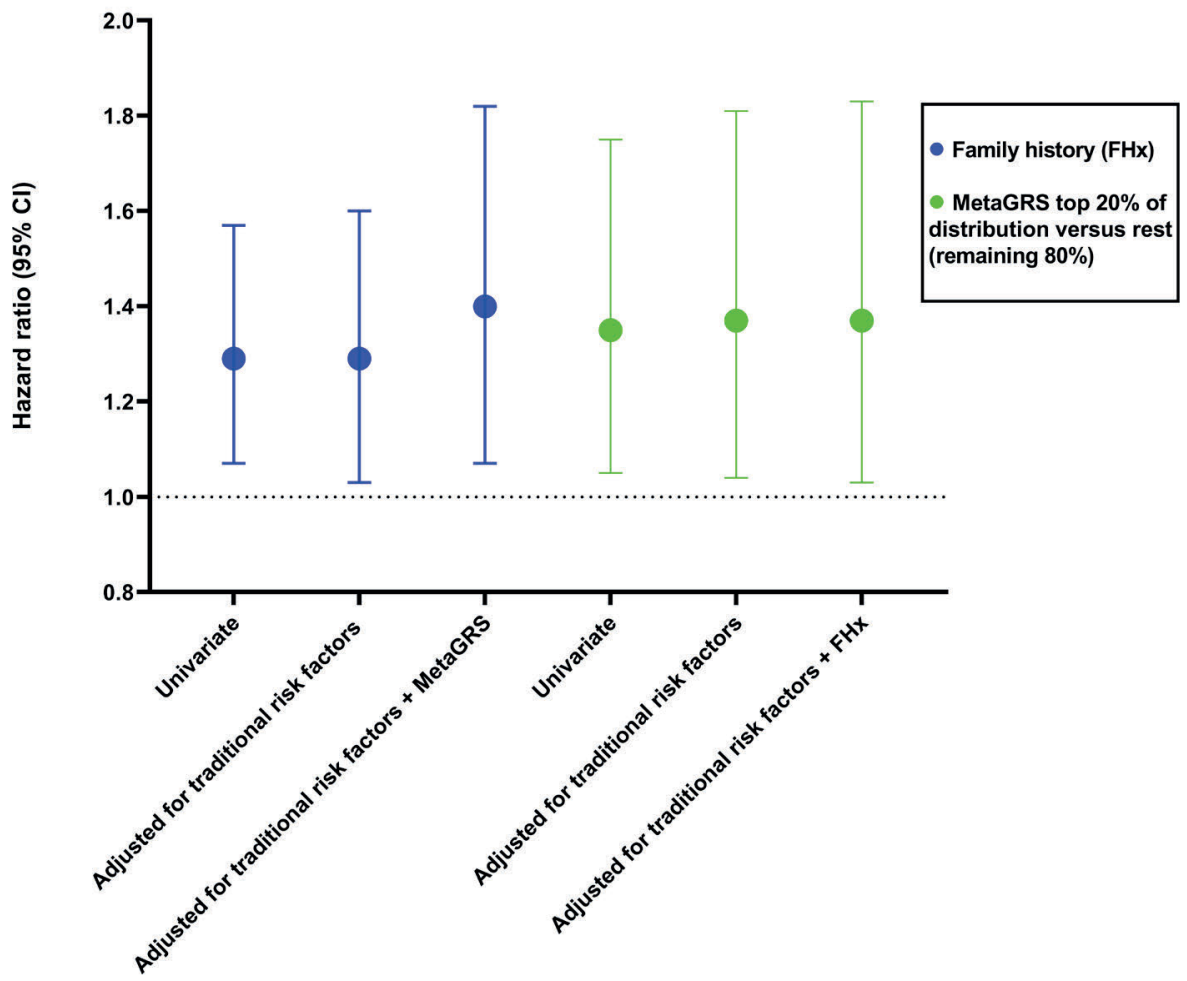

Summarizing illustration 


\section{Introduction}

Family history of cardiovascular disease (FHx) is a major risk factor for primary cardiovascular disease (CVD) and serves as a surrogate for genetic predisposition. ${ }^{1,2}$ Risk prediction for secondary cardiovascular events remains challenging as traditional risk factors have limited discriminative performance. ${ }^{3}$ The main underlying mechanism of CVD is atherosclerosis, and atherosclerotic plaque composition, exemplified by intraplaque hemorrhage (IPH), have been associated with adverse secondary cardiovascular events (sCVE). ${ }^{4}$ Yet, the relevance of FHx for secondary outcome of cardiovascular events is still unclear. ${ }^{5-9}$

Large-scale genome-wide association studies (GWAS) have identified hundreds of common genetic variants (single-nucleotide polymorphisms or SNPs) robustly associated with coronary artery disease $(\mathrm{CAD})^{10-14}$ and ischemic stroke $\mathrm{e}^{15-17}$ predisposition, albeit with small individual effects. Exact pathobiological mechanisms leading to cardiovascular symptoms are still poorly understood, but CAD- and ischemic stroke genetic variants were previously associated to atherosclerotic plaque composition. ${ }^{18}$

Polygenic risk scores (PRS) summarize the small individual genetic effects into a quantitative measure of genetic disease susceptibility. CAD-PRS were strongly correlated with prevalent and incident CAD independent of traditional risk factors including family history in the UK Biobank population. ${ }^{19,20}$ For example, individuals with higher scores of the MetaGRS (a PRS for CAD including 1.7 million SNPs) were at 1.7-4.2 fold higher risk for a first coronary event compared to individuals with lower MetaGRS scores. Two recent studies in CAD-patients showed that CAD-PRS was also associated with an increased risk of recurrent $\mathrm{CAD}$-events. ${ }^{21,22}$ However, no studies have investigated this relation in patients undergoing carotid endarterectomy (CEA), nor its impact on plaque composition. Therefore, we aimed to investigate the association between MetaGRS and sCVE in patients undergoing CEA and explore possible underlying pathophysiological mechanisms by studying the impact of MetaGRS on carotid histological plaque characteristics. Given that FHx is used in clinical practice as a derivative of genetic background, we also examined the association between $\mathrm{FHx}, \mathrm{SCVE}$ and plaque characteristics.

\section{Methods}

\section{Athero-Express Biobank}

All patients in this study were included in the Athero-Express Biobank (www.atheroexpress. $\mathrm{nl}$ ), a prospective cohort study that included consecutive patients with severe carotid artery 
stenosis undergoing CEA in two large tertiary referral hospitals in The Netherlands, the University Medical Center Utrecht (inclusion is ongoing) and the St. Antonius Hospital Nieuwegein (inclusion until 2014). ${ }^{23}$ The study design has been published before. ${ }^{23}$ In short, patient characteristics, such as demographics, cardiovascular risk factors including medical history, medication use, and FHx for cardiovascular disease, were obtained through standardized questionnaires and checked in electronic health records. Preoperative blood samples were drawn. The atherosclerotic plaque obtained during surgery was collected and immunohistochemically analyzed for plaque characteristics. Patients were followed up for three years after surgery for the occurrence of secondary cardiovascular events through standardized questionnaires and by checking electronic health records. General practitioners were consulted in case of no response to questionnaires or in order to obtain further information regarding reported cardiovascular events. Patients operated for restenosis $(6 \%$ of 2,044 eligible patients for this study) were excluded because these differ in future cardiovascular event risk. ${ }^{24}$ Thus for the current study, a total of 1,788 patients operated from March 2002 until July 2016 had available 3-year follow-up data and FHx data, and were included for analysis. Of these, 1,551/1,788 (87\%) patients had available histological carotid plaque data. A total of 1,319/1,788 (74\%) patients had available genotype data of whom 1,301 (98\%) also had histological carotid plaque data. This study was performed according to the Declaration of Helsinki and was approved by the local ethics committee of both hospitals. Patients provided written informed consent before study participation.

\section{Definitions}

A positive FHx was defined as having a first-degree relative (either a parent or sibling) with onset of cardiovascular disease (myocardial infarction (MI), coronary artery stenosis, stroke, abdominal aortic aneurysm (AAA), or cardiovascular death including sudden death) before the age of 60 years. The primary outcome of this study was defined as a composite secondary cardiovascular event (sCVE) within three years of follow-up including fatal or non-fatal MI, fatal or non-fatal stroke, ruptured AAA, fatal cardiac failure, coronary or peripheral interventions (either percutaneous or bypass surgery), leg amputation due to cardiovascular causes and cardiovascular death. Secondary outcomes were histological atherosclerotic carotid plaque characteristics.

\section{Genotyping}

Methods for genotyping, quality control and imputation in the Athero-Express biobank have been published elsewhere ${ }^{25,26}$. Briefly, DNA was extracted from EDTA whole blood samples or if not present from atherosclerotic plaque tissue according to validated protocols. Genotyping was performed with two commercially available chips: the first batch by Affymetrix Genome-Wide Human SNP array 5.0 (previously used in the Athero-Express 
Genomics Study 1 (AEGS1), covering samples obtained in 2002-2007) and the second batch by Affymetrix Axiom GW CEU 1 array (previously used in Athero-Express Genomics Study 2 (AEGS2), covering samples obtained in 2002-2013). Procedures for data quality control and data cleaning were in accordance with global standards. ${ }^{27}$ After genotype calling according to Affymetrix' specification, data was filtered on 1) individual call rate $>97 \%$, 2) genotype call rate $>97 \%, 3$ ) minor allele frequencies $>3 \%$, 4) average heterozygosity rate \pm 3.0 standard deviations, 5) relatedness (pi-hat $>0.20$ ), 6) Hardy-Weinberg Equilibrium $\mathrm{p}<1.0 \times 10^{-6}$ ), and 7) population stratification (based on HapMap 2, release 22, b36) by excluding samples deviating more than 6 standard deviations from the average in 5 iterations during principal component analysis and by visual inspection. ${ }^{25}$

After pre-phasing using SHAPEIT2 v2.644, a combined dataset of 1000 Genome (phase 3, version 5) and The Genome of the Netherlands Project release 5 was used as a reference for imputation with IMPUTE2 v2.3.0 to impute missing genotypes for 88,784,475 variants. ${ }^{28}$

\section{Polygenic risk score (MetaGRS)}

To estimate the polygenic cardiovascular disease susceptibility for included patients in our cohort, we used the previously published polygenic risk score for CAD (MetaGRS). ${ }^{19}$ Its construction was described elsewhere. ${ }^{19}$ Briefly, the MetaGRS comprises 1,745,179 genetic variants with a minor allele frequency $(\mathrm{MAF})>0.1 \%$ associated with $\mathrm{CAD}$ and was constructed through meta-analysis of three genomic risk scores: GRS46K (comprising 46,000 cardiometabolic genetic variants), FDR202 (including 202 genetic variants associated with CAD at false discovery rate $\mathrm{p}<0.05$ in the recent GWAS CARDIoGRAMplusC4D), and the 1000 Genomes genetic score also created with CARDIoGRAMplusC4D. The MetaGRS was internally and externally validated for the primary risk of prevalent and incident CAD in the UK Biobank. ${ }^{19}$

We matched the 1.7 million variants from the MetaGRS to 1,742,593 variants in our data (2,586 variants were not present in our data). Given that the median imputation quality was high (INFO = 0.978 [IQR 0.945-0.991]), and the variants included in the MetaGRS have MAF $>0.1 \%$ as described in the supplemental material of the original publication, we did not further filter on imputation quality. Moreover, since we used the imputed genotype probabilities to calculate the MetaGRS, rather than the hard-coded genotypes, bias arising from imputation error, i.e. low imputation quality, will only reduce predictive accuracy. Thus, we calculated the MetaGRS for each included patient in this study using PRSice-2. ${ }^{29}$ We standardized the MetaGRS to mean-zero and unit-variance for each genotyping batch separately, i.e. AEGS1 and AEGS2, respectively. 


\section{Sample handling}

After CEA, the atherosclerotic plaque was directly processed in the laboratory following standardized protocols. ${ }^{4,23,30,31}$ The plaque was cut in cross-sectional segments of $5 \mathrm{~mm}$. The segment with largest plaque burden was chosen as the culprit lesion and immunohistochemically analyzed for macrophages, smooth muscle cells (SMC), lipid core, calcification, collagen, intraplaque hemorrhage (IPH) and microvessel content. Extensive description of the standardized protocol for atherosclerotic plaque processing and analysis of plaque characteristics has been previously reported and is added to the Online Supplemental. ${ }^{4,23,30,31}$ To assess the overall vulnerability of the atherosclerotic plaque, a vulnerability score was created ranging from 0-5 with 1 point for plaque characteristics that are considered hallmarks of a vulnerable plaque (moderate/heavy macrophages, no/ minor collagen, no/minor SMC, lipid core $>10 \%$ and presence of IPH), based on a previous publication. ${ }^{32}$

\section{Statistical analysis}

Baseline characteristics were compared between patient groups (FHx and MetaGRS) by chi-square test for categorical variables and Student's t-test for continuous variables (lipid levels were log-transformed). We analyzed the association between FHx and MetaGRS and sCVE by Cox-proportional hazard regression models and the associations with plaque characteristics through logistic or linear regression models. To fully unravel the genetic association, the association of MetaGRS with sCVE and plaque characteristics was analyzed in three ways: (1) MetaGRS as a continuous quantification of genetic CAD susceptibility (2) patients in the top $20 \%$ of the MetaGRS distribution compared to the remaining $80 \%$, and (3) patients in the top $20 \%$ of the MetaGRS distribution compared to those in the bottom $20 \%$ of the distribution. Kaplan-Meier curves were constructed to graphically illustrate univariate associations. Confounders for multivariable analyses were selected based on literature ${ }^{19,32,33}$ (for sCVE these were age, sex, diabetes, BMI, smoking and hypercholesterolemia and for plaque characteristics these were age, sex, surgery year and type of cerebrovascular symptoms). Additional confounders were added when showing an association of $\mathrm{p}<0.20$ with the determinant (FHx or MetaGRS) and outcome of interest (sCVE or plaque characteristics). For MetaGRS models genotype array and principal components 1-4 were also added. Full model description is displayed in the Supplemental Tables S1 and S2. Because a previous study in our biobank showed that IPH is associated with $\mathrm{sCVE}^{4}$, IPH was added to multivariable models of FHx, MetaGRS and sCVE to explore whether IPH could be one possible underlying mechanism. Sex-stratified analyses were performed to unravel sex-dependent differences in associations. Values with $\mathrm{p}<0.05$ were considered statistically significant. All analyses were performed in IBM SPSS Statistics version 25.0. 


\section{Results}

Patient selection from the Athero-Express Biobank and characteristics of the study population are displayed in Figure 1 and Table 1. Patients had a mean age 69 years and $70 \%$ were men. The cohort represented a typically severe atherosclerotic cohort with high prevalence of traditional risk factors and atherosclerotic manifestations in other vascular beds (coronary or peripheral arteries, respectively $30 \%$ and 20\%). Baseline characteristics were similar between the total cohort with FHx data and the sub-cohort with genotyped data (Table 1).

\section{Patients with positive FHx have a higher risk of sCVE}

Patients with a positive FHx (744/1,788, 41.6\%) were younger and had on average more cardiovascular risk factors (Table 1). During a median follow-up of 2.9 years, 418 patients (23.4\%) reached the composite endpoint of sCVE (Figure 2A) of whom 105 (5.9\%) had stroke or fatal stroke, $119(6.7 \%)$ had MI or fatal MI, 29 (1.6\%) had cardiovascular death due to other causes (fatal cardiac failure, AAA rupture or sudden death) and 165 (9.2\%) had a peripheral intervention or leg amputation.

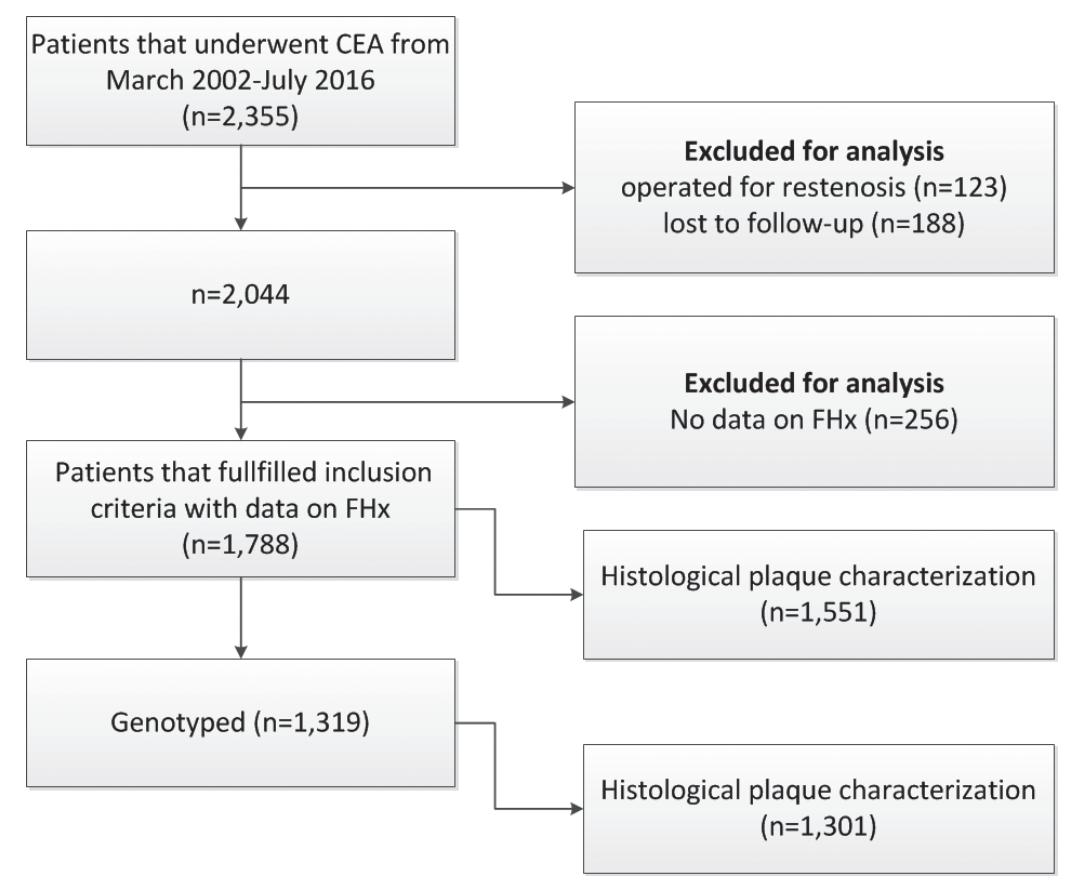

Figure 1. Flowchart 


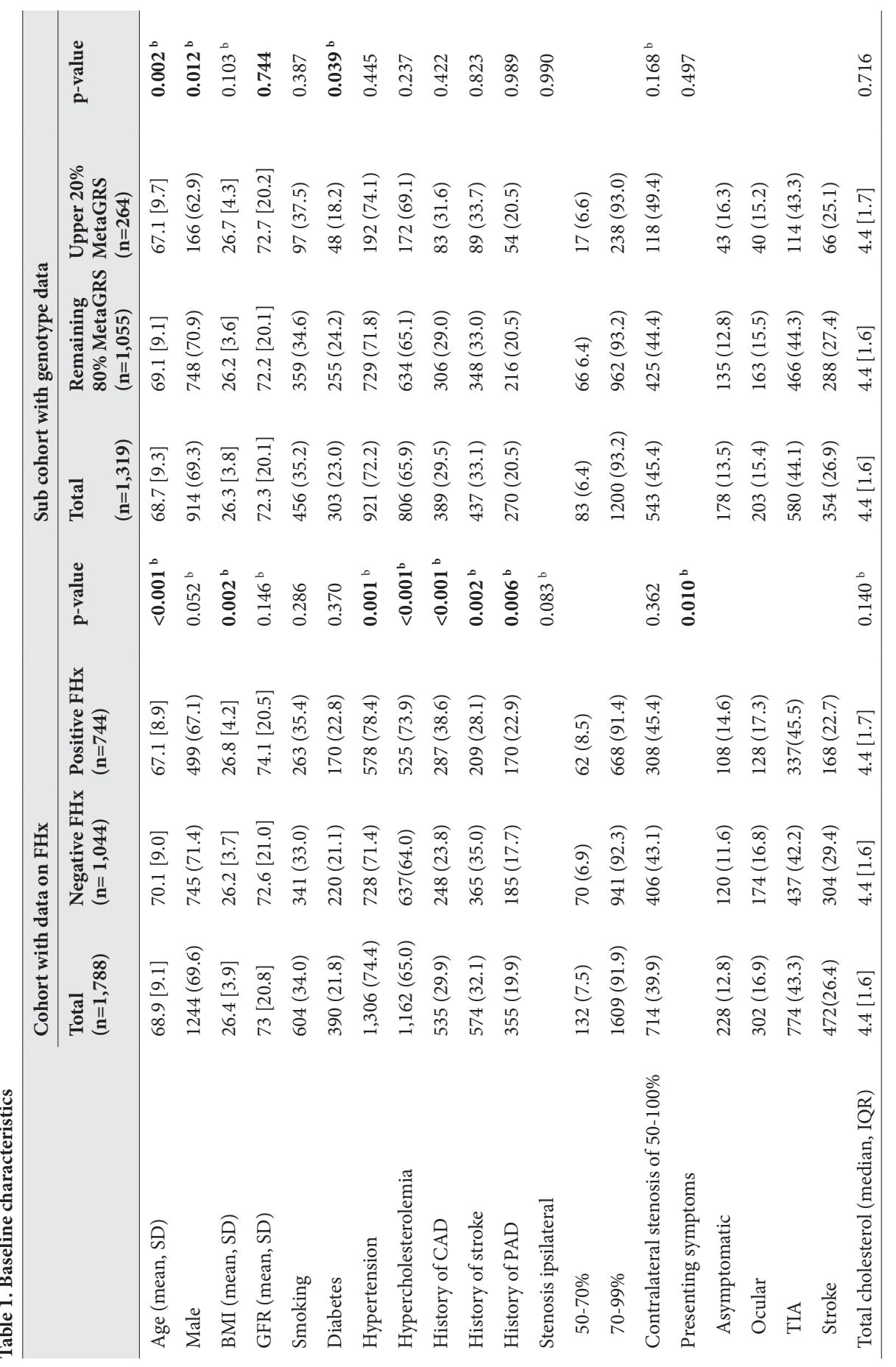




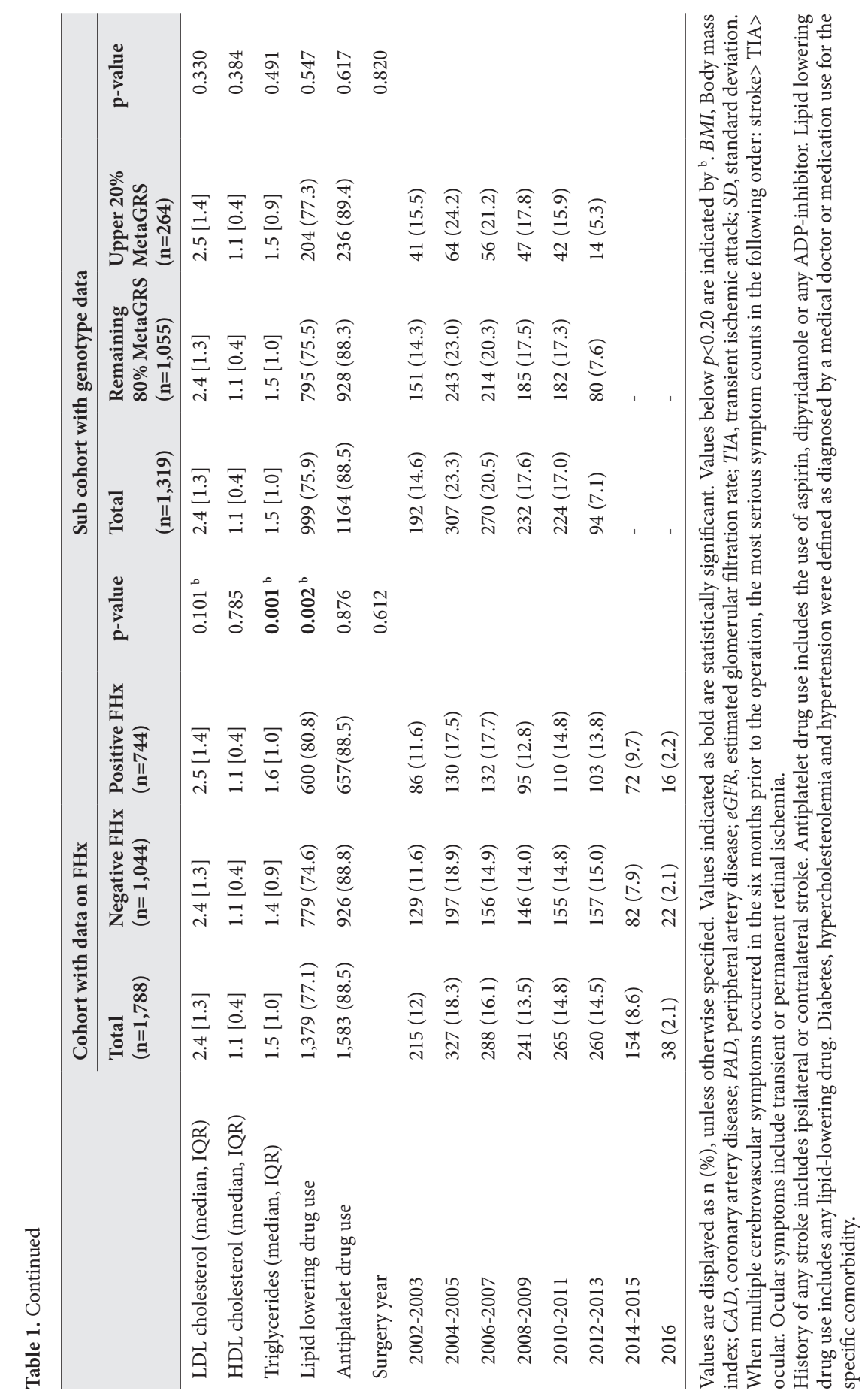


Patients with positive FHx had an increased risk of sCVE compared to those without (absolute 3-year risks of 26.5\% versus 21.2\% respectively, hazard ratio (HR) 1.292, 95\% confidence interval (CI), 1.066-1.566, p=0.009 (Figure 2A, Figure 3; Supplemental Table S3). This association remained significant after correction for confounders with adjusted HR 1.287, 95\% CI 1.033-1.604, p=0.024 (Figure 3; Supplemental Table S3) and was independent of genetic predisposition as measured by MetaGRS (adjusted HR 1.397, 95\% CI 1.074-1.819, $\mathrm{p}=0.013$, Figure 3; Supplemental Table S3). Sex-stratified analyses confirmed results in men (with adjusted HR after correction for confounders of 1.380, 95\% CI 1.068-1.783, p=0.014; adjusted HR after correction for confounders including MetaGRS was 1.513, 95\% CI 1.115$2.052, \mathrm{p}=0.008$ ). However, in women the univariate association between FHx and sCVE was not significant (unadjusted HR 1.187, 95\% CI 0.822-1.171, p=0.360) but multivariable analyses could not be performed because of limited power (Supplemental Table S3).

\section{Patients with higher MetaGRS have a higher risk of sCVE}

The MetaGRS, standardized to mean-zero and unit-variance, approximated a normal distribution in the study population (Supplemental Figure S1). Patients in the top 20\% of MetaGRS were relatively more often females, younger of age and had less often diabetes compared to the remaining $80 \%$ of the cohort (Table 1). Also, high genetic risk patients (highest quintile of MetaGRS) had higher LDL cholesterol levels compared to low genetic risk patients (lowest quintile of MetaGRS), see Supplemental Table S4. In the 3-year followup, a total of 326/1319 (24.7\%) patients reached the composite endpoint of sCVE of whom $96(7.3 \%)$ had stroke or fatal stroke, 85 (6.4\%) had MI or fatal MI, 21 (1.6\%) died of other cardiovascular causes (fatal cardiac failure, AAA rupture or sudden death) and 124 (9.4\%) had a peripheral intervention or leg amputation.

Patients in the top 20\% of MetaGRS had 1.4-fold increased risk of developing sCVE within the three years of follow-up when compared to the remaining $80 \%$ of the cohort (Figure $2 \mathrm{~B}$, absolute 3 -year risks of $29.5 \%$ versus $23.5 \%$ respectively, HR 1.353, 95\% CI 1.047-1.749, $\mathrm{p}=0.021$ ). After adjustment for possible confounders including FHx this association remained statistically significant (HR for top 20\% 1.345, 95\% CI 1.009-1.792, p=0.043). We found similar results when we compared patients in top 20\% to the bottom $20 \%$ of MetaGRS (in univariate analysis with HR 1.539, 95\% CI 1.086-2.181, $\mathrm{p}=0.015$ (Figure 2C) and for multivariable analysis adjusted HR including FHx 1.583, 95\% CI, 1.066-2.351, p=0.023), and when analyzing MetaGRS as a continuous quantity (adjusted for confounders with HR 1.150 per one SD increase in MetaGRS, 95\% CI 1.022-1.293, $\mathrm{p}=0.021$, adjusted HR including FHx 1.112 per one SD increase in MetaGRS, 95\% CI, 0.983-1.259, $\mathrm{p}=0.091$ ). Results are illustrated in Figures $2 \mathrm{~B}+\mathrm{C}$ and 3 and displayed in Supplemental Table S3. Confounders added to multivariable models are displayed in Supplemental Tables S1 and S2. Similar results were found in men (adjusted for confounders including FHx showed a HR 1.219 
Figures 2A-C. Kaplan-Meier graphs for 3-year risk of secondary CVE after CEA

The cumulative event-free survival from sCVE in years is displayed for (2A) Patients with positive FHx compared to those without, (2B) Patients in the top 20\% of MetaGRS compared to the rest of the patients (remaining $80 \%$ ), (2C)Patients in the top $20 \%$ of MetaGRS compared to those in the bottom $20 \%$ of MetaGRS. Vertical lines indicate censoring.

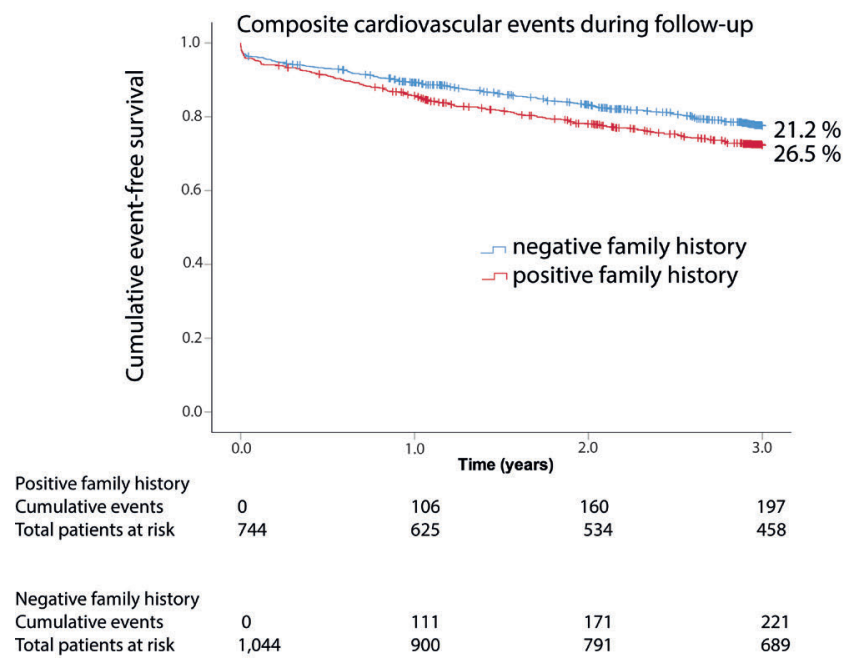

Figure 2A. Patients with positive FHx compared to those without

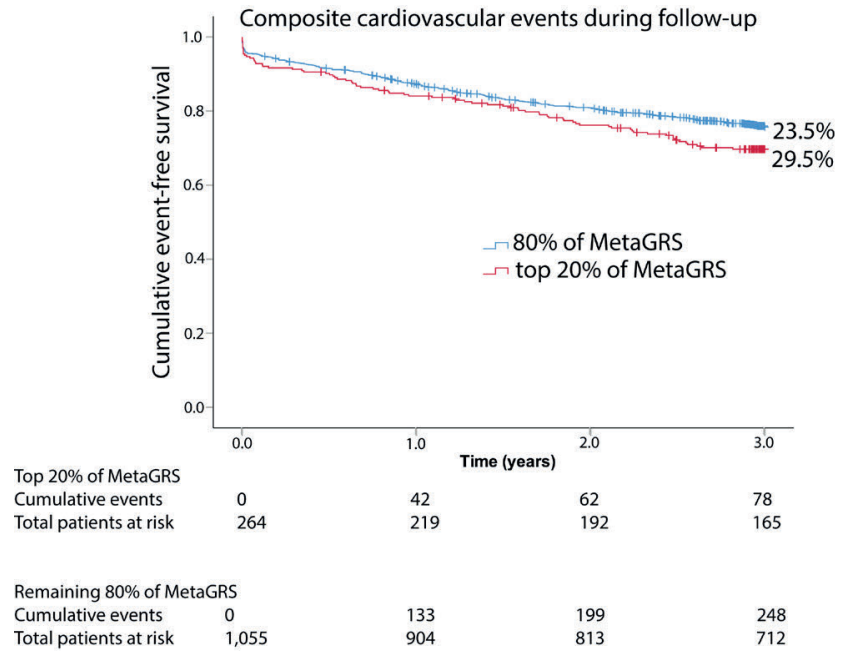

Figure 2B. Patients in the top $20 \%$ of MetaGRS compared to the rest of the patients (remaining $80 \%$ ) 
per one SD increase in MetaGRS, 95\% CI 1.056-1.408, p=0.006, Supplemental Table S3). In women univariate analyses showed no significant associations between MetaGRS and sCVE (HR 0.916 per one SD increase in MetaGRS, 95\% CI 0.743-1.129, p=0.413), yet multivariable analysis was not possible due to lack of power (Supplemental Table S3).

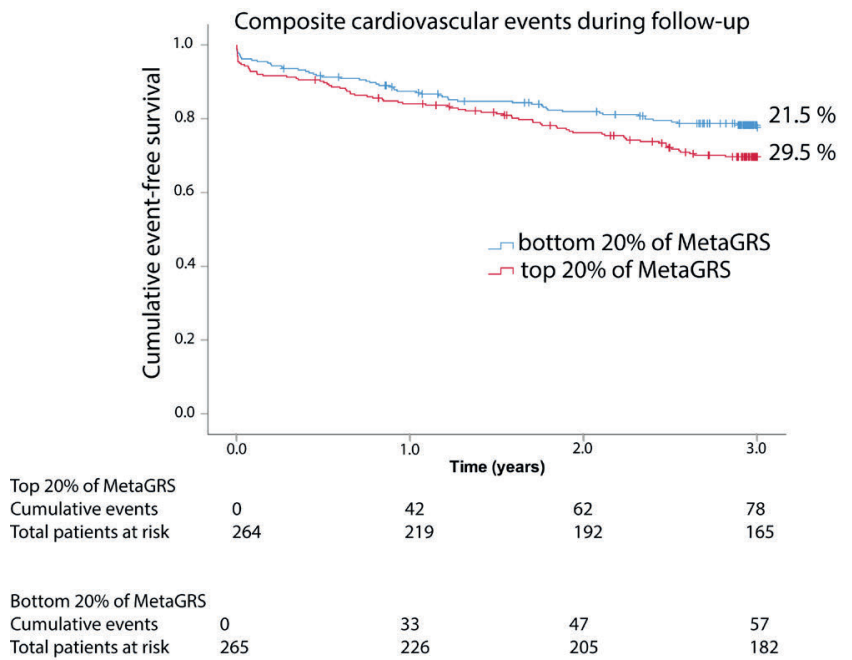

Figure 2C. Patients in the top $20 \%$ of MetaGRS compared to those in the bottom $20 \%$ of MetaGRS 


\section{MetaGRS is associated with vulnerable carotid plaque characteristics}

To unravel possible underlying pathophysiological mechanisms of the associations between MetaGRS, FHx and CVD, we explored the impact of FHx and the MetaGRS on atherosclerotic plaque characteristics. We found no associations between histological plaque characteristics and FHx in the total cohort or in women although not all multivariable analyses could be performed (Supplemental Table S5 and S6). However carotid plaques from men with a positive FHx contained less collagen and less SMC content compared to men with negative FHx (Supplemental table S6). MetaGRS was associated with significantly higher overall plaque vulnerability score (regression coefficient $\beta$ of 0.198 for top $20 \%$ of MetaGRS compared to the rest, 95\% CI, 0.003-0.364, $\mathrm{p}=0.004$, Table 2). To determine the plaque characteristics on which this association was based, plaque characteristics were analyzed separately. High genetic risk patients (in top 20\% of MetaGRS) more frequently had a lipid core $>10 \%$ of total plaque area (adjusted odds ratio (OR), 1.591, 95\% CI 1.1052.291, $\mathrm{p}=0.013$ ) and more macrophage infiltration (adjusted OR 1.490, 95\% CI 1.118-1.986, $\mathrm{p}=0.006$ ) compared to patients with MetaGRS in the remaining $80 \%$ (Table 2). For a lipid core $>10 \%$, we found the same association when comparing the top $20 \%$ with the bottom $20 \%$ of the MetaGRS (adjusted HR 1.887, 95\% CI, 1.188-2.997, p=0.007, Supplemental Table S7). Analyses of MetaGRS as a continuous quantity confirmed the association with lipid core $>10 \%$ (adjusted OR 1.171 per SD increase in MetaGRS, 95\% CI 1.026-1.337, $\mathrm{p}=0.019$, Supplemental Table S8). Sex-stratified analyses revealed a significant association of MetaGRS with macrophages in women (adjusted OR per SD increase in MetaGRS 1.238, 95\% CI, 1.007-1.521, $\mathrm{p}=0.043$ ) whereas a significant association of MetaGRS with IPH was found in men (adjusted OR per SD increase 1.220, 95\% CI 1.050-1.418, p=0.010, Supplemental Table S9).

Because IPH has been associated with increased risk of $\mathrm{sCVE}^{4}$, we added IPH to the multivariable models of FHx, MetaGRS and sCVE to unravel whether the association between FHx, MetaGRS and sCVE could be explained by IPH. We found that all associations of FHx and MetaGRS were independent of IPH given that adding IPH to multivariable models of sCVE did not alter the effect sizes (Supplemental Table S3). 


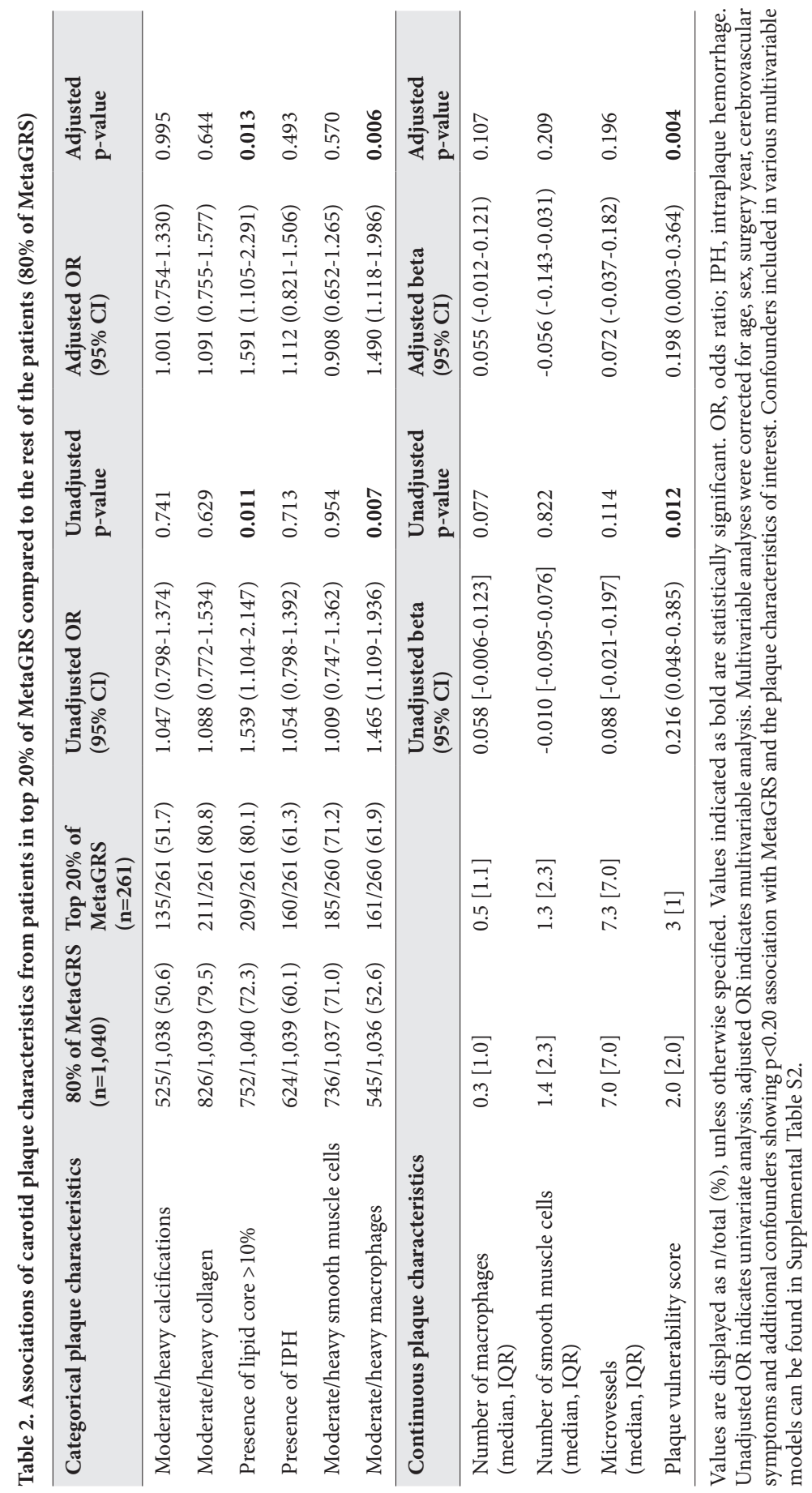




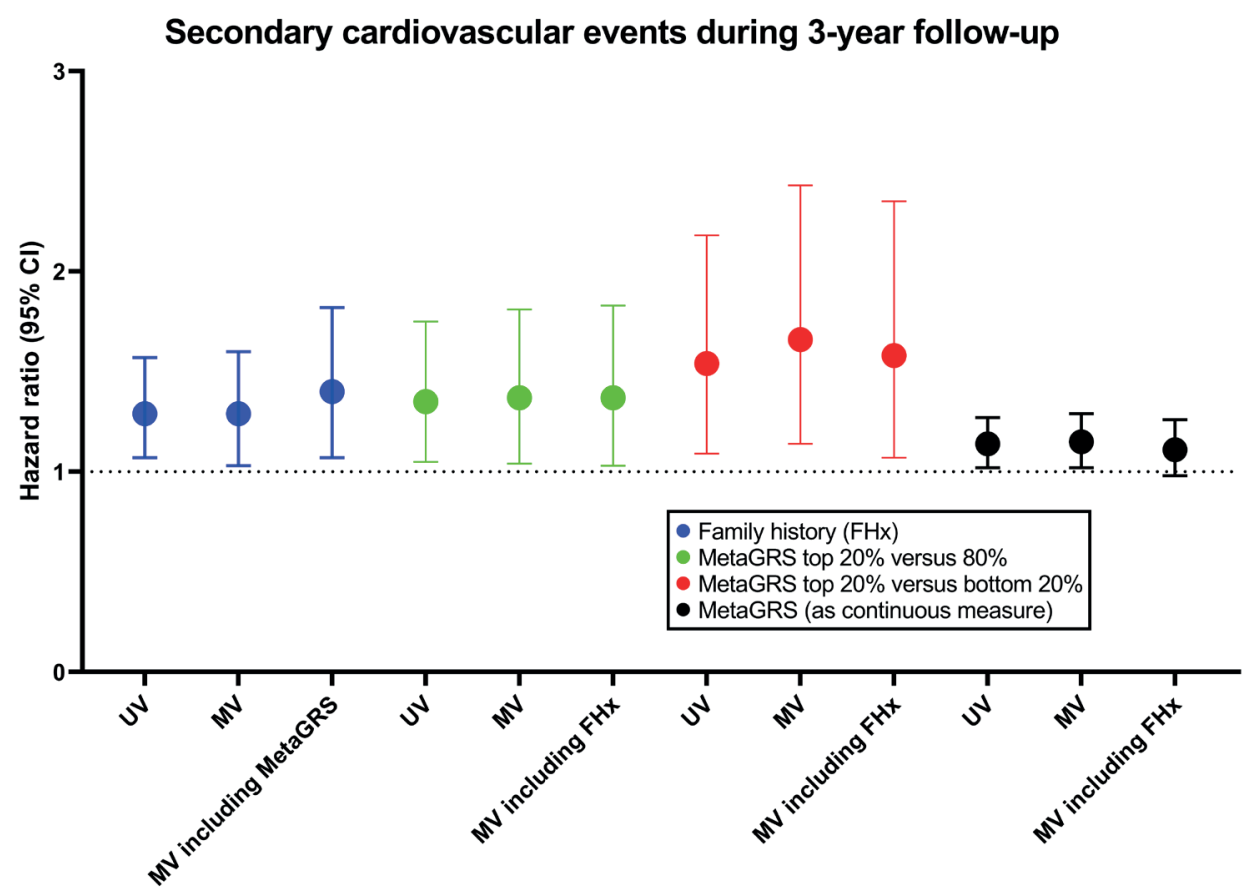

Figure 3. Cox-regression analyses of FHx and MetaGRs for sCVE after CEA

Hazard ratio's (HR) and 95\% confidence intervals (95\% CI) for the different univariate and multivariable Coxregression models of FHx or MetaGRS for sCVE. The HR for MetaGRS as a continuous quantity indicates the HR per one SD increase in MetaGRS.

UV, univariate model. The univariate model for MetaGRS included age, sex, PC1-4 and genotype array.

MV, multivariable model.

The multivariable model for FHx was corrected for traditional risk factors (age, sex, hypercholesterolemia, diabetes, hypertension, BMI and smoking) and additional confounders (history of CAD, history of PAD, cerebrovascular symptoms and eGFR).

The univariate model for MetaGRS included age, sex, PC1-4 and genotype array. For multivariable analyses of MetaGRS traditional risk factors (hypercholesterolemia, diabetes, hypertension, BMI and smoking) were added.

\section{Discussion}

We validated the polygenic risk score for CAD (MetaGRS) for sCVE in a severe atherosclerotic cohort of carotid artery stenosis patients undergoing CEA. We report two key findings. First, in CEA patients FHx and MetaGRS were both independently associated with an increased risk of sCVE. Second, high MetaGRS was associated with more vulnerable atherosclerotic plaque characteristics suggesting possible underlying pathobiological mechanisms through which genetic variants could affect CVD. 
Although positive FHx is a well-known risk factor for primary $\mathrm{CVE}^{1,2}$, previous studies assessing FHx and secondary outcome are inconsistent. ${ }^{5,7-9,34}$ In patients with first-MI ${ }^{7,8,34}$, studies have reported a protective effect of FHx on all-cause mortality, whereas others showed an increased risk of CVE. ${ }^{5,9}$ One can assume that patients with positive FHx are identified earlier as at-risk individuals through screening programs resulting in more intensive surveillance and preventive strategies leading to the benefit in overall survival. ${ }^{7}$ Indeed, in our cohort patients with positive FHx were also younger at timing of CEA. Of note, it is known that the sensitivity of self-reported FHx can be low (50\%-70\%) and might therefore be an unreliable estimate. ${ }^{35}$

Our results are in line with two recent studies in CAD-patients that showed that high PRS was associated with an elevated risk of recurrent CAD-events, of which one study used the MetaGRS. ${ }^{21,22}$ We now validate that MetaGRS is associated with an increased risk of sCVE in a different population consisting of CEA patients with high prevalence of other CVD comorbidities either in coronary or peripheral vascular beds. Our results therefore underscore the concept of atherosclerosis as a complex and systemic disease underlying CVD. Indeed, a 300-SNP-CAD-GRS has been previously associated with the development of stroke, peripheral artery disease (PAD) and AAA indicating shared genetic roots. ${ }^{36}$ Furthermore, we provide mechanistic insights by showing associations of MetaGRS with plaque characteristics (lipid core and macrophages content) indicative of an unstable plaque morphology.

Interestingly, the association of MetaGRS as continuous quantity became insignificant after addition of FHx but remained significant when the highest quintile of MetaGRS was compared with the remaining patients. Although one could argue that such cut-off limits may be arbitrary, it rather indicates that the effect of PRS on SCVE is not linear but either exponential. Indeed, the exponential relationship between PRS and CVD-risk has already been shown for primary CAD-event risk. ${ }^{20}$

Previous studies have demonstrated that PRS was associated with first- and recurrent CADevents independent from FHx. ${ }^{19-21,37,38}$ We now show that FHx is associated with increased risk of sCVE independent from MetaGRS. Several reasons could be hypothesized for the non-overlapping associations. MetaGRS includes common genetic variants associated with an increased risk for CAD in the general population, whilst CVD in families may arise in part from more rare genetic mutational events, and thus a positive FHx captures individual yet family specific rare variation. Another explanation could be that FHx reflects not only genetic factors but also non-genetic factors. Although we corrected for traditional risk factors, other environmental factors that were not taken into account such as social economic status or nutrition patterns could still be attributable to the risk of secondary events. 
We found that MetaGRS was associated with a more rupture-prone atherosclerotic plaque displayed by a higher plaque vulnerability score caused by more fat, IPH (in men) and macrophages (predominantly in women). Moreover, carotid plaques from men with positive FHx were associated with less SMC and less collagen, whereas the association in women remains unclear. Similarly, a previous AE study showed, that PRSs constructed based on summary statistics from a GWAS on CAD using increasingly liberal p-value thresholds were correlated with more fat, whereas large-artery stroke-PRSs were correlated with more IPH and SMC. ${ }^{18}$ However, the current MetaGRS is the result of a meta-analytic approach to identify 1.7 million variants capturing information from the full genome for CAD and was internally and externally validated making the MetaGRS more generalizable. ${ }^{19,22}$ Thus, the results presented here provide more evidence supporting the view that genetic variants could mediate their effect on CVD by influencing atherosclerotic plaque composition and morphology. Interestingly, IPH has been associated with sCVE in men independent of other clinical risk factors in our biobank ${ }^{4,39}$, yet we found that the associations of MetaGRS, FHx and sCVE were independent of IPH. It therefore remains unclear what the exact underlying mechanism is through which MetaGRS and FHx exert their increased sCVE risk. A previous study investigating a 50-CAD-SNP-GRS and positive FHx found that only small proportions $(<8 \%)$ of the effects were mediated through known metabolic pathways such as blood lipids and hypertension whereas the majority ( $>80 \%)$ was not. ${ }^{40}$ Although CAD-variants have been linked to pathways involved in atherosclerosis ${ }^{13}$, most CADvariants are situated outside protein-coding regions with unknown functions making them hard to map to pathophysiological mechanisms. ${ }^{13,41}$ Future studies should explore exact pathophysiological mechanisms how the genetic variants of MetaGRS influence plaque destabilization and CVE, for example through deep-phenotyping of atherosclerotic plaque characteristics by quantitative computerized analysis ${ }^{42}$ and mapping MetaGRS loci to specific CVE (MI, stroke or PAD). Also, potential sex-differences should be investigated. Although PRS could identify high-risk patients for CVE, one could conclude that due to their unfavorable genetic risk the CVE-risk is unchangeable. However, previous studies have suggested that high genetic risk is modifiable by lifestyle interventions or medication, thus not deterministic per se. ${ }^{21,43}$ Among individuals with high genetic risk (top 20\% of CAD-GRS), those adhering to a healthy lifestyle had lower risk of a first CAD-event compared to those with an unfavorable lifestyle. ${ }^{43}$ Moreover, post-hoc analyses of secondary prevention trials investigating statins ${ }^{37,44}$ and PCSK-9 inhibitor ${ }^{21}$ showed that high genetic risk patients had a greater absolute and relative risk reduction of recurrent CAD-events than those with lower genetic risk, despite equal LDL-level reductions. Prospective studies and implementation studies are needed to confirm PRS as a useful tool to predict the benefit of preventive medications and for selecting those patients that benefit most from such add-on therapies. 
Admittedly, our study has several limitations. First, although our results may suggest interesting sex-differences as we only observed the independent association of MetaGRS and $\mathrm{FHx}$ for sCVE in men, the association in women remains unclear because we were underpowered for multivariable analysis. Second, we did not have data regarding medication use during follow-up nor therapy compliance which could have interfered with observed sCVE rates. Second, owing to limited power we were unable to assess associations with separate CVE or determine the predictive value of MetaGRS above clinical risk factors. Last, most included patients are of European ancestries and generalizability to other ethnicities needs further attention. Yet, the Athero-Express Biobank is unique in its scope and major strengths are the unique population that is relatively unexplored in the field of FHx and PRS, and the extensive data on plaque morphology that enable us to identify putative pathological mechanisms.

In the future, PRS may be a useful tool for personalized risk prediction for primary or secondary CVE. Adding MetaGRS to a model with traditional risk factors improved prediction of first CAD-events, although model improvement was modest. ${ }^{19}$ The clinical utility of PRS for SCVE is still unknown because the incremental value of PRS above clinical factors still needs to be established. One study suggested an added predictive value of PRS above clinical factors but did not include $\mathrm{FHx}^{45}$, whereas other studies failed to demonstrate this. ${ }^{46-48}$ The power of these studies may have been limited due to limited number of CVE. Pooling data of several cohorts including detailed data on preventive strategies and medications during follow-up together with use of uniform outcome definitions for sCVE and uniform PRS composition could help elucidate the clinical value of PRS, for example within international collaborations such as the GENIUS-CHD Consortium. ${ }^{49}$ Furthermore, possible sex-differences in the role of risk prediction with PRS need to be further elucidated. In conclusion, both higher MetaGRS and positive FHx were independently associated with increased risk of sCVE in CEA patients. Higher MetaGRS was also associated with more vulnerable atherosclerotic plaque characteristics indicating possible underlying mechanisms how genetic variants influence CVD. PRS could identify high-risk individuals and may help selecting future study populations when investigating new therapeutic CVD prevention strategies. 


\section{Conflict of interest}

none

\section{Financial support}

Dr. van der Laan was funded through grants from the Netherlands CardioVascular Research Initiative of the Netherlands Heart Foundation (CVON 2011/B019 and CVON 2017-20: Generating the best evidence-based pharmaceutical targets for atherosclerosis [GENIUS I\&II]). Dr. Timmerman, Dr. De Borst, dr. De Kleijn, and dr. Pasterkamp are funded through the EU 755320 Taxinomisis grant. We acknowledge the European Research Area Network on Cardiovascular diseases (ERA-CVD, grant number 01KL1802). There are no competing interests declared.

\section{Acknowledgements}

We thank Sara van Laar, Sara Bregman, Bram Vermeulen, Evelyn Velema and Joëlle van Bennekom for their technical support in the Athero-Express Biobank. 


\section{References}

1 Lloyd-Jones DM, Nam B-H, D’Agostino, Sr RB, Levy D, Murabito JM, Wang TJ, et al. Parental cardiovascular disease as a risk factor for cardiovascular disease in middle-aged adults. JAMA. 2004;291:2204-2211.

2 Chow CK, Islam S, Bautista L, Rumboldt Z, Yusufali A, Xie C, et al. Parental history and myocardial infarction risk across the world. J Am Coll Cardiol. 2011;57:619-627.

3 van den Berg MJ, Bhatt DL, Kappelle LJ, de Borst GJ, Cramer MJ, van der Graaf Y, et al. Identification of vascular patients at very high risk for recurrent cardiovascular events: validation of the current ACC/AHA very high risk criteria. Eur Heart J. 2017;38:3211-3218.

4 Hellings WE, Peeters W, Moll FL, Piers SRD, van Setten J, Van der Spek PJ, et al. Composition of carotid atherosclerotic plaque is associated with cardiovascular outcome. Circulation. 2010;121:1941-1950.

5 Mulders TA, Meyer Z, van der Donk C, Kroon AA, Ferreira I, Stehouwer CDA, et al. Patients with premature cardiovascular disease and a positive family history for cardiovascular disease are prone to recurrent events. Int J Cardiol. 2011;153:64-67.

6 Canto JG, Kiefe CI, Rogers WJ, Peterson ED, Frederick PD, French WJ, et al. Atherosclerotic risk factors and their association with hospital mortality among patients with first myocardial infarction (from the national registry of myocardial infarction). Am J Cardiol. 2012;110:1256-1261.

7 Abdi-Ali A, Shaheen A, Southern D, Zhang M, Knudtson M, White J, et al. Relation between family history of premature coronary artery disease and the risk of death in patients with coronary artery disease. Am J Cardiol. 2016;117:353-358.

8 Harpaz D, Behar S, Rozenman Y, Boyko V, Gottlieb S. Family history of coronary artery disease and prognosis after first acute myocardial infarction in a national survey. Cardiology. 2004;102:140-146.

9 Kim C, Chang H-J, Cho I, Sung JM, Choi D, Jeong MH, et al. Impact of family history on the presentation and clinical outcomes of coronary heart disease: data from the Korea Acute Myocardial Infarction Registry. Korean J Intern Med. 2013;28:547-556.

10 Nikpay M, Goel A, Won H-H, Hall LM, Willenborg C, Kanoni S, et al. A comprehensive 1000 Genomesbased genome-wide association meta-analysis of coronary artery disease. Nat Genet. 2015;47:1121-30.

11 Howson JMM, Zhao W, Barnes DR, Ho W-K, Young R, Paul DS, et al. Fifteen new risk loci for coronary artery disease highlight arterial-wall-specific mechanisms. Nat Genet. 2017;49:1113-1119.

12 Verweij N, Eppinga RN, Hagemeijer Y, van der Harst P. Identification of 15 novel risk loci for coronary artery disease and genetic risk of recurrent events, atrial fibrillation and heart failure. Sci Rep. 2017;7:2761.

13 Erdmann J, Kessler T, Munoz Venegas L, Schunkert H. A decade of genome-wide association studies for coronary artery disease: the challenges ahead. Cardiovasc Res. 2018;114:1241-1257.

14 van der Harst P, Verweij N. Identification of 64 novel genetic loci provides an expanded view on the genetic architecture of coronary artery disease. Circ Res. 2018;122:433-443.

15 Traylor M, Farrall M, Holliday EG, Sudlow C, Hopewell JC, Cheng Y-C, et al. Genetic risk factors for ischaemic stroke and its subtypes (the METASTROKE Collaboration): a meta-analysis of genome-wide association studies. Lancet Neurol. 2012;11: 951-62.

16 Holliday EG, Maguire JM, Evans T-J, Koblar SA, Jannes J, Sturm JW, et al. Common variants at 6p21.1 are associated with large artery atherosclerotic stroke. Nat Genet. 2012;44:1147-51.

17 Malik R, Traylor M, Pulit SL, Bevan S, Hopewell JC, Holliday EG, et al. Low-frequency and common genetic variation in ischemic stroke. Neurology 2016;86:1217-1226.

18 van der Laan SW, Siemelink MA, Haitjema S, Foroughi Asl H, Perisic L, Mokry M, et al. Genetic susceptibility loci for cardiovascular disease and their impact on atherosclerotic plaques. Circ Genomic Precis Med. 2018;11:e002115.

19 Inouye M, Abraham G, Nelson CP, Wood AM, Sweeting MJ, Dudbridge F, et al. Genomic risk prediction of coronary artery disease in 480,000 adults. J Am Coll Cardiol. 2018;72:1883-93.

20 Khera A V., Chaffin M, Aragam KG, Haas ME, Roselli C, Choi SH, et al. Genome-wide polygenic scores for common diseases identify individuals with risk equivalent to monogenic mutations. Nat Genet. 2018;50:1219-1224.

21 Damask A, Steg PG, Schwartz GG, Szarek M, Hagström E, Badimon L, et al. Patients with high genomewide polygenic risk scores for coronary artery disease may receive greater clinical benefit from alirocumab treatment in the odyssey outcomes trial. [published online Nov 11, 2019] Circulation. 2019. https://www. 
ahajournals.org/doi/abs/10.1161/CIRCULATIONAHA.119.044434. Accessed January 11, 2019.

22 Wünnemann F, Sin Lo K, Langford-Avelar A, Busseuil D, Dubé M-P, Tardif J-C, et al. Validation of genome-wide polygenic risk scores for coronary artery disease in French Canadians. Circ Genomic Precis Med.2019;12(6):e002481.

23 Verhoeven BAN, Velema E, Schoneveld AH, de Vries JPPM, de Bruin P, Seldenrijk CA, et al. Atheroexpress: differential atherosclerotic plaque expression of mRNA and protein in relation to cardiovascular events and patient characteristics. Rationale and design. Eur J Epidemiol 2004;19(12):1127-1133.

24 Kumar R, Batchelder A, Saratzis A, AbuRahma AF, Ringleb P, Lal BK, et al. Restenosis after carotid interventions and its relationship with recurrent ipsilateral stroke: a systematic review and meta-analysis. Eur J Vasc Endovasc Surg. 2017;53:766-775.

25 van der Laan SW, Foroughi Asl H, van den Borne P, van Setten J, van der Perk MEM, van de Weg SM, et al. Variants in ALOX5, ALOX5AP and LTA4H are not associated with atherosclerotic plaque phenotypes: The Athero-Express Genomics Study. Atherosclerosis. 2015;239:528-538.

26 Siemelink MA, van der Laan SW, van Setten J, de Vries JPPM, de Borst GJ, Moll FL, et al. Common variants associated with blood lipid levels do not affect carotid plaque composition. Atherosclerosis. 2015;242:351-356.

27 Anderson CA, Pettersson FH, Clarke GM, Cardon LR, Morris AP, Zondervan KT. Data quality control in genetic case-control association studies. Nat Protoc. 2010;5:1564-1573.

28 Genome of the Netherlands Consortium, Francioli LC, Menelaou A, Pulit SL, Dijk F van, Palamara PF, et al. Whole-genome sequence variation, population structure and demographic history of the Dutch population. Nat Genet. 2014;46:818-825.

29 Choi SW, O’Reilly PF. PRSice-2: Polygenic Risk Score software for biobank-scale data. Gigascience 2019;8(7):giz082.

30 Vrijenhoek JEP, Nelissen BGL, Velema E, Vons K, de Vries JPPM, Eijkemans MJC, et al. High reproducibility of histological characterization by whole virtual slide quantification; an example using carotid plaque specimens. PLoS One. 2014;9(12):e115907.

31 Hellings WE, Pasterkamp G, Vollebregt A, Seldenrijk CA, De Vries JPPM, Velema E, et al. Intraobserver and interobserver variability and spatial differences in histologic examination of carotid endarterectomy specimens. J Vasc Surg. 2007;46:1147-1154.

32 Verhoeven B, Hellings WE, Moll FL, de Vries JP, de Kleijn DPV, de Bruin P, et al. Carotid atherosclerotic plaques in patients with transient ischemic attacks and stroke have unstable characteristics compared with plaques in asymptomatic and amaurosis fugax patients. J Vasc Surg. 2005;42:1075-1081.

33 van Lammeren GW, den Ruijter HM, Vrijenhoek JEP, van der Laan SW, Velema E, de Vries JPPM, et al. Time-dependent changes in atherosclerotic plaque composition in patients undergoing carotid surgery. Circulation.2014;129:2269-2276.

34 Canto JG, Kiefe CI, Rogers WJ, Peterson ED, Frederick PD, French WJ, et al. Number of coronary heart disease risk factors and mortality in patients with first myocardial infarction. JAMA.2011;306.

35 Murabito JM, Nam B-H, D’Agostino RB, Lloyd-Jones DM, O’Donnell CJ, Wilson PWF. Accuracy of offspring reports of parental cardiovascular disease history: The Framingham Offspring Study. Ann Intern Med. 2004;140:434-440.

36 Ntalla I, Kanoni S, Zeng L, Giannakopoulou O, Danesh J, Watkins H, et al. Genetic risk score for coronary disease identifies predispositions to cardiovascular and noncardiovascular diseases. J Am Coll Cardiol. 2019;73:2932-2942.

37 Mega JL, Stitziel NO, Smith JG, Chasman DI, Caulfield MJ, Devlin JJ, et al. Genetic risk, coronary heart disease events, and the clinical benefit of statin therapy: an analysis of primary and secondary prevention trials. Lancet. 2015;385:2264-2271.

38 Tada H, Melander O, Louie JZ, Catanese JJ, Rowland CM, Devlin JJ, et al. Risk prediction by genetic risk scores for coronary heart disease is independent of self-reported family history. Eur Heart J. 2016;37:561567.

39 Vrijenhoek JEP, Den Ruijter HM, De Borst GJ, de Kleijn DPV, De Vries JPPM, Bots ML, et al. Sex is associated with the presence of atherosclerotic plaque hemorrhage and modifies the relation between plaque hemorrhage and cardiovascular outcome. Stroke. 2013;44:3318-3323.

40 Fritz J, Shiffman D, Melander O, Tada H, Ulmer H. Metabolic mediators of the effects of family history and genetic risk score on coronary heart disease-findings from the Malmö Diet and Cancer Study. J Am Heart Assoc. 2017;6: e005254. 
41 Haitjema S, Meddens CA, van der Laan SW, Kofink D, Harakalova M, Tragante V, et al. Additional candidate genes for human atherosclerotic disease identified through annotation based on chromatin organization. Circ Cardiovasc Genet. 2017;10: e001664.

42 Nelissen BGL, van Herwaarden JA, Moll FL, van Diest PJ, Pasterkamp G. SlideToolkit: an assistive toolset for the histological quantification of whole slide images. PLoS One. 2014;9:e110289.

43 Khera A V., Emdin CA, Drake I, Natarajan P, Bick AG, Cook NR, et al. Genetic risk, adherence to a healthy lifestyle, and coronary disease. N Engl J Med. 2016;375:2349-2358.

44 Natarajan P, Young R, Stitziel NO, Padmanabhan S, Baber U, Mehran R, et al. Polygenic risk score identifies subgroup with higher burden of atherosclerosis and greater relative benefit from statin therapy in the primary prevention setting. Circulation . 2017;135:2091-2101.

45 Wirtwein M, Melander O, Sjögren M, Hoffmann M, Narkiewicz K, Gruchala M, et al. Relationship between selected DNA polymorphisms and coronary artery disease complications. Int J Cardiol. 2017;228:814-820.

46 Weijmans M, de Bakker PIW, van der Graaf Y, Asselbergs FW, Algra A, de Borst GJ, et al. Incremental value of a genetic risk score for the prediction of new vascular events in patients with clinically manifest vascular disease. Atherosclerosis. 2015;239(2):451-458.

47 Vaara S, Tikkanen E, Parkkonen O, Lokki M-L, Ripatti S, Perola M, et al. Genetic risk scores predict recurrence of acute coronary syndrome. Circ Cardiovasc Genet. 2016;9:172-178.

48 Labos C, Martinez SC, Leo Wang RH, Lenzini PA, Pilote L, Bogaty P, et al. Utility of a genetic risk score to predict recurrent cardiovascular events 1 year after an acute coronary syndrome: A pooled analysis of the RISCA, PRAXY, and TRIUMPH cohorts. Atherosclerosis. 2015;242:261-267.

49 Patel RS, Asselbergs FW. The GENIUS-CHD consortium. Eur Heart J. 2015;36:2674-2676. 


\section{Supplementary Materials}

The following supplementary material is omitted due to space limitation and can be found at the journal website:

- Table S1. Overview of confounders used in multivariable analyses.

- Table S2. Overview of confounders added to multivariable models of histological plaque characteristics.

- Table S3. Univariate and multivariate Cox-regression analyses for the association of FHx and MetaGRS with secondary CVE.

- Table S4. Baseline characteristics of genotyped patients (those in the top 20\% of MetaGRS distribution compared to those in the bottom 20\%).

- Table S5. Histological carotid plaque characteristics and associations with FHx in the total cohort.

- Table S6. Associations of histological carotid plaque characteristics and FHx in men and women.

- Table S7. Associations of histological carotid plaque characteristics and MetaGRS (patients in top 20\% compared to those in bottom 20\% of MetaGRS).

- Table S8. Associations of MetaGRS (as continuous measure) and histological carotid plaque characteristics.

- Table S9. Sex-stratified associations of MetaGRS with histological carotid atherosclerotic plaque characteristics.

- Figure S1. Distribution of MetaGRS in the Athero-Express Biobank. 



\section{PART II}

Cerebral Imaging

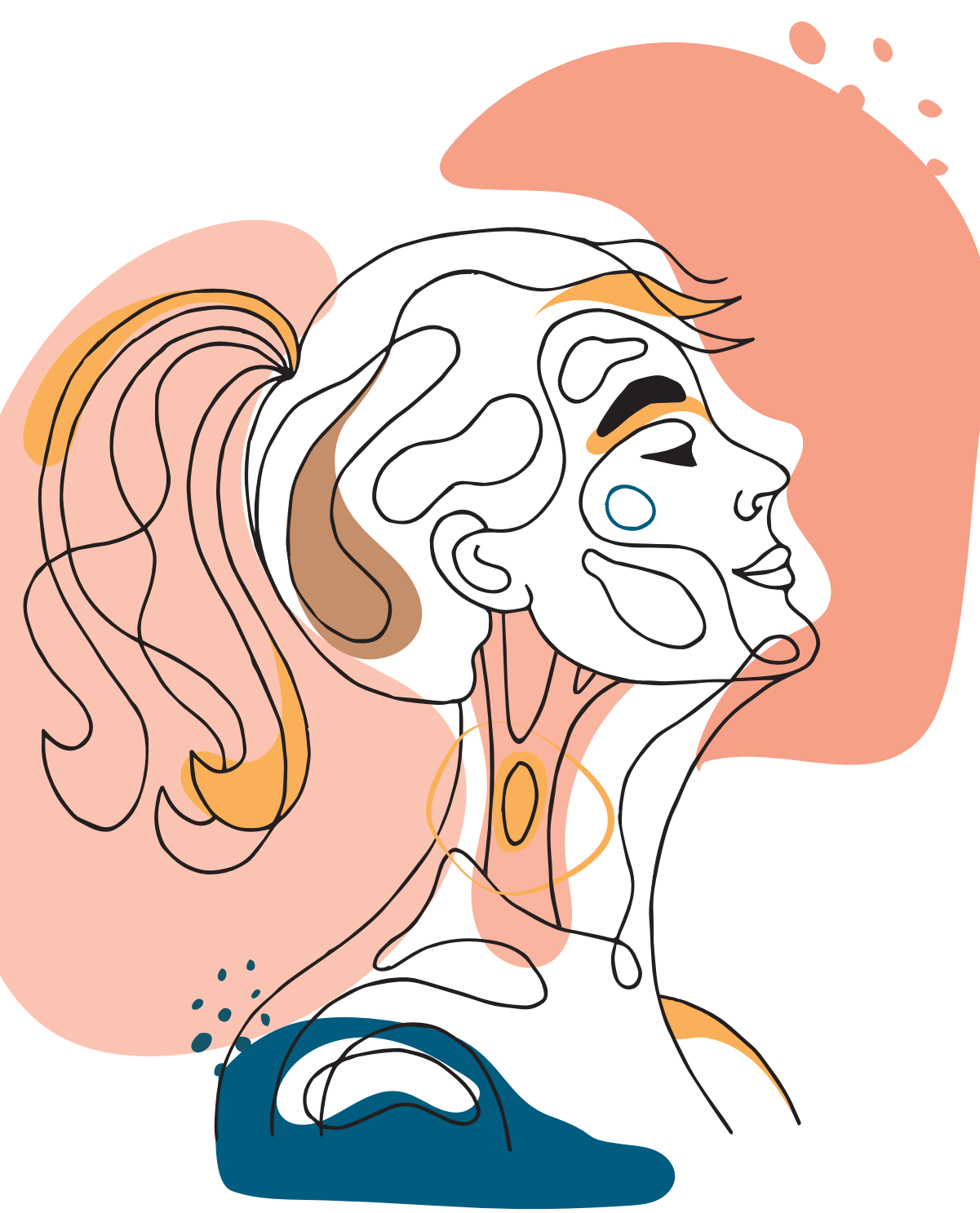




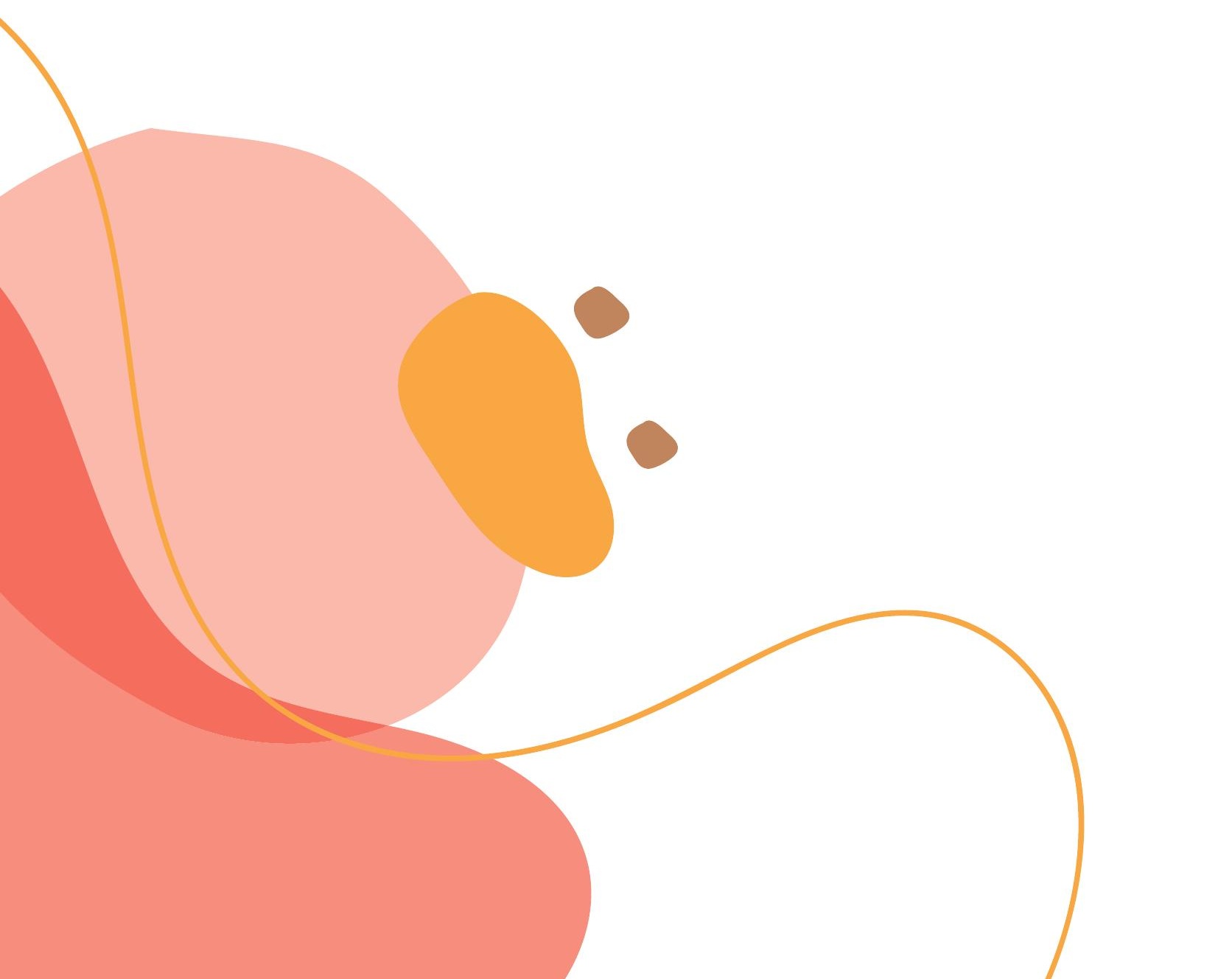


CHAPTER 4

\section{Magnetic resonance imaging identified brain ischemia in symptomatic patients undergoing carotid endarterectomy is related to histologically apparent intraplaque hemorrhage}

European Journal of Vascular and Endovascular Surgery 2019;58(6):796-804

Marjolijn L. Rots

Nathalie Timmerman

Dominique P.V. de Kleijn

Gerard Pasterkamp

Martin M. Brown

Leo H. Bonati

Gert J. de Borst 


\section{Abstract}

\section{Objective}

Intraplaque hemorrhage (IPH) has been independently associated with a higher risk of future ipsilateral stroke in patients with carotid artery stenosis. Evaluation of plaque characteristics may contribute to risk-assessment of recurrent (silent) cerebrovascular events in order to prioritize patients for timing of treatment. It is unknown if patients showing histologically apparent IPH also have increased risk of silent ischemic brain lesions in the waiting period between index event and revascularization.

\section{Methods}

A retrospective analysis was performed based on prospectively collected data of patients included simultaneously in the MRI-substudy of the International Carotid Stenting Study and Athero-Express biobank. Patients randomized for carotid endarterectomy (CEA) were operated between 2003-2008. Brain MRI was performed 1-7 days prior to CEA. Plaques were histologically examined for presence of IPH. Primary outcome parameter was presence of silent ipsilateral brain-ischemia on MR-DWI appearing hypo- or isointense on apparent diffusion coefficient.

\section{Results}

53 patients with symptomatic carotid stenosis meeting study criteria were identified of which 13 showed $\geq 1$ recent ipsilateral DWI lesion on pre-operative scan. Time between latest ipsilateral neurological event and revascularization was 45 days (range 6-200) in DWI-negative patients versus 34 days (range 6-74, $p=0.16$ ) in DWI-positive patients. IPH was present in 24/40 (60.0\%) DWI-negative patients versus 12/13 (92.3\%) DWI-positive patients (OR: 8.00; 95\% CI: 0.95-67.7, $\mathrm{p}=0.056$ ). Multivariable logistic regression analysis correcting for age and type of index-event revealed that IPH was independently associated with DWI lesions in the waiting period till surgery (OR:10.8; 95\% CI:1.17-99.9, $\mathrm{p}=0.036$ ).

\section{Conclusion}

Symptomatic patients with ipsilateral carotid stenosis and silent brain ischemia on preoperative MR-DWI, more often showed pathological evidence of IPH compared to those without ischemic lesions. This identifies carotid IPH as a marker for patients at risk for silent brain ischemia and possibly for future stroke and other arterial disease complications. Such patients may be more likely to benefit from CEA than those without evidence of ipsilateral carotid IPH. 


\section{What this study adds}

This study investigated the relation between histological apparent intraplaque hemorrhage (IPH) and presence of recent brain lesions on diffusion weighted imaging prior to carotid endarterectomy. We demonstrated that patients with IPH have an increased risk for development of silent brain ischemia in the waiting period between index event and surgery. These results qualify IPH as a potential marker for identifying patients at risk for recurrent events.

\section{Introduction}

The presence of carotid artery intraplaque hemorrhage (IPH) is considered an important maker of plaque instability and associated with a high risk for clinically relevant events such as transient ischemic attack (TIA) or stroke due to the tendency to rupture. ${ }^{1}$ Signs of presence of carotid artery IPH on magnetic resonance imaging (MRI) or duplex have been associated with an increased risk of future cerebrovascular events and IPH is more common in symptomatic patients compared to asymptomatic patients. ${ }^{2,3}$ Moreover, IPH is associated with increased risk of any type of secondary cardiovascular event in male patients such as (fatal) myocardial infarction (MI), (fatal) stroke, coronary and peripheral interventions, and cardiovascular death. ${ }^{4}$

Risk assessment of recurrent cerebrovascular events based on plaque characteristics may be helpful for prioritizing patients for the timing of carotid revascularization. Current guidelines recommend that symptomatic patients with carotid stenosis should be considered (in case of 50-69\% stenosis) or recommended (in case of 70-99\% stenosis) for treatment within 14 days of the index event. ${ }^{5}$ Nonetheless, these recommendations have been based on post hoc analyses of outdated randomized controlled trials (RCTs) and additionally may not always be feasible due to pre-hospital or in-hospital delay. ${ }^{6}$ Identification of IPH may help to select patients that are most at risk for recurrent events and may help to select those in which urgent revascularization may be appropriate.

Ischemic brain lesions on magnetic resonance diffusion weighted imaging (MR-DWI) are increasingly being used as a surrogate marker of ischemic events for postoperative outcome after revascularization as they are associated with an increased risk of future cerebrovascular events. ${ }^{7}$ To date, no studies have been performed that investigated the role of DWI lesions in the preoperative period specifically. One of the major advantages of assessing presence of new DWI lesions is that they may appear within few hours after a thrombo-embolic event and DWI is therefore sensitive to recent changes. By assessing diffusion restricted 
brain areas with MR-DWI in combination with apparent diffusion coefficient (ADC), recent infarction can be identified. ADC-maps may depict darkening within minutes of stroke onset and distinguishes stroke from "T2 shine through", which can be seen later after infarction and appears bright on DWI. Low signal intensity on ADC persists for about 7-10 days. ${ }^{8}$ Until now, it is unclear if carotid plaque characteristics are associated with these silent brain lesions identifying them as their potential source. For this, we investigated whether patients with characteristics of plaque instability have an increased risk of silent preprocedural ischemic brain lesions during their waiting period for carotid intervention.

Since thrombo-embolic events are the most common underlying cause of ischemic brain lesions in carotid patients, we hypothesize that patients showing histologically apparent signs of IPH in the atherosclerotic plaque (excised during carotid endarterectomy) are more likely to show (recurrent) silent ischemic brain lesions in the waiting period between index event and revascularization.

\section{Methods}

A retrospective analysis was performed based on prospectively collected data of patients included simultaneously in the MRI-substudy of the International Carotid Stenting Study $(\mathrm{ICSS})^{9}$ and the Athero-Express (AE) biobank study of the University Medical Center (UMC) Utrecht ${ }^{10}$. Patients that underwent carotid endarterectomy (CEA) between October 2003 and October 2008 were included with a median waiting time (defined as the number of days between the most recent event and surgery) of 44 days. Patients included in the ICSS were symptomatic (symptoms attributable to the randomized artery within twelve months before randomization) carotid stenosis patients with stenosis $>50 \%$ deemed to require treatment. Symptomatic patients included those with previous stroke (acute disturbance of focal neurological function attributed to vascular disease and of ischemic origin, lasting more than 24h), TIA (acute disturbance of focal neurological function attributed to vascular disease with recovery within $24 \mathrm{~h}$ ) or amaurosis fugax (transient monocular blindness attributed to vascular disease with recovery within $24 \mathrm{~h}$ ). The following exclusion criteria were asserted: previous revascularization in the randomized artery, contraindications for either treatment and planned major surgery. ${ }^{11}$ In course of the study protocol of the ICSS MRI-substudy, all patients underwent an additional MRI-scan 1-7 days before surgery for assessment of silent brain ischemia. Carotid plaques were collected within the AE-biobank. No additional exclusion criteria were asserted for the AE-biobank study other than that the patient had to be randomized for CEA. 


\section{MRI}

Primary outcome parameters include ipsilateral ischemic lesions on MR-DWI appearing hypo- or isointense on apparent diffusion coefficient (ADC), and it's correlation to intraplaque hemorrhage (see Figure 1 for an illustrative timeline). Secondary outcome parameters included presence of white matter lesions (WML) semi-quantitatively assessed on FLAIR sequences by use of the age-related-white matter changes (ARWMC) score. White matter lesions and basal ganglia lesions were both assessed on a 0-3 point scale for the ipsilateral hemisphere and a sumscore was used for further data analyses (see Supplemental Table SI for exact definitions). ${ }^{12}$

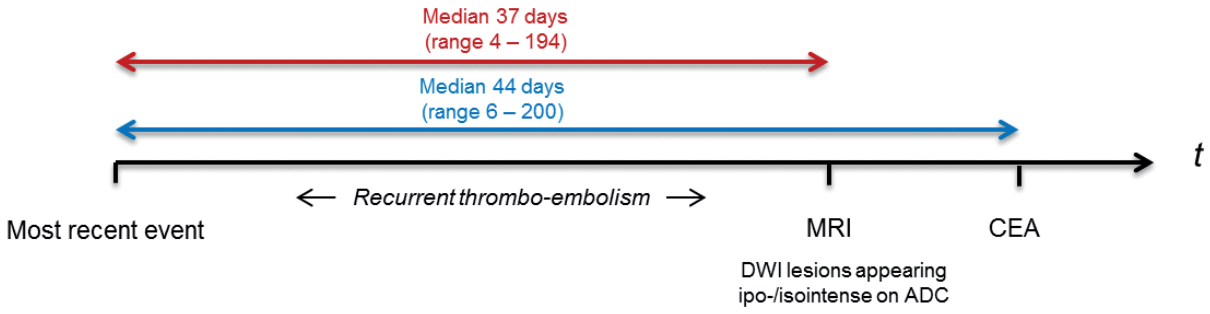

Figure 1. Timeline illustrating waiting time until revascularization

DWI, diffusion weighted imaging; ADC, apparent diffusion coefficient. Waiting time (indicated by blue arrow) was defined as the number of days between the most recent event (hemispheric/retinal stroke, TIA or amaurosis fugax) and CEA. The red arrow represents the time between latest symptom and preoperative MRI.

\section{Atherosclerotic plaque assessment}

After CEA, all atherosclerotic plaques were immediately processed. The carotid plaque was cut into segments of 5-mm thickness along the longitudinal axis. The segment with the largest plaque burden was chosen as a culprit lesion and subjected to histological examination. A more detailed description can be found in the online Supplemental Methods. The investigated plaque characteristics resulting from immunochemical staining were: presence of IPH, presence of lipid core $(\geq 40 \%)$, moderate/heavy calcifications, moderate/heavy collagen, mean number of microvessels per hotspot, percentage of positive macrophage staining per plaque and percentage of positive smooth muscle cell (SMC) staining per plaque. ${ }^{13} \mathrm{IPH}$ was defined as the composite of plaque bleeding at the luminal side of the plaque as a result of plaque disruption and hemorrhage within the tissue of the plaque. $^{14}$ 


\section{Clinical outcome}

Major cardiovascular events during 3-years of follow-up were reported, consisting of nonfatal MI, nonfatal stroke and cardiovascular death. MI was reported when at least two of the following criteria were present: (1) chest pain for $\geq 20$ minutes, not disappearing after administration of nitrates; (2) ST-elevation $>1 \mathrm{~mm}$ in two following leads or a left bindlebranch-block on the electrocardiogram; (3) CK elevation of at least two times the normal value of $\mathrm{CK}$ and a $\mathrm{MB}$ fraction $>5 \%$ of the total $\mathrm{CK}$.

\section{Statistics}

A sample size of at least of 48 patients was required on the basis of detecting a difference in proportion of patients with IPH in the DWI-positive group of two times compared to the DWI-negative group at a significance level of $0.05,80 \%$ power, assuming $25 \%$ of patients having preoperative DWI lesions and $80 \%$ of DWI-positive patients having IPH. Data were inspected for patterns of missing values. The proportion of randomly missing values for baseline characteristics did not exceed $2 \%$. Differences in binary characteristics were analyzed with Pearson's Chi square. Differences in continuous parameters were calculated with a student's t-test when data were normally distributed and otherwise using a MannWhitney $U$ test. For the ARWMC score a binary outcome parameter was used based on median ipsilateral ARWMC score (sum score $\leq 2$ compared to sum score $>2$ ). To investigate independent associations between histological plaque characteristics and presence of fresh DWI lesions as well as ARWMC score, we conducted a multivariable logistics regression analysis correcting for any baseline characteristics with $\mathrm{p}<0.1$ in univariate analysis. Age and type of qualifying event were also added to the multivariable models since these are considered as potential confounders based on earlier described associations with IPH.,15 In case of ARWMC score, estimated-packyears was identified as an additional potential confounder from univariate analysis and therefore included in multivariate analysis. Nonnormally distributed quantitative histological parameters including number of microvessels and percentages of macrophage and SMC staining required logarithmic transformation before entering into regression models. SPSS 25.0 (SPSS Inc, Chicago, Illinois, USA) was used for all statistical analysis.

\section{Results}

\section{Patient characteristics}

53 patients met the inclusion criteria of the ICSS-MRI substudy and were simultaneously included in the AE biobank study. Qualifying events were major stroke $(n=7)$, minor stroke $(n=11)$, cerebral TIA $(n=28)$ and amaurosis fugax $(n=7)$. Mean waiting time between the latest symptom (stroke/TIA) and MRI was 51 days (median 44, range 6-200 days). 
Table 1. Baseline characteristics of ipsilateral DWI-negative versus DWI-positive patients

\begin{tabular}{|c|c|c|c|}
\hline & $\begin{array}{l}\text { DWI - } \\
\mathrm{N}=40\end{array}$ & $\begin{array}{l}\mathrm{DWI}+ \\
\mathrm{N}=13\end{array}$ & p-value \\
\hline Age, mean (std) & $68.15(8.59)$ & $69.31(8.42)$ & 0.673 \\
\hline Male gender, n (\%) & $28(70.0)$ & $11(84.6)$ & 0.299 \\
\hline Hypertension, n (\%) & $26(65.0)$ & $11(84.6)$ & 0.181 \\
\hline Systolic blood pressure, mean (SD) & $162.5(29.26)$ & $158.4(21.39)$ & 0.716 \\
\hline Diastolic blood pressure, mean (SD) & $85.58(15.11)$ & $80.33(12.68)$ & 0.260 \\
\hline Blood pressure medication use, $\mathrm{n}(\%)$ & $29(72.5)$ & $12(92.3)$ & 0.138 \\
\hline Diabetes Mellitus, n (\%) & $9(22.5)$ & $3(23.1)$ & 0.966 \\
\hline Hypercholesterolemia, n (\%) & $26(65)$ & $8(61.5)$ & 0.821 \\
\hline LDL, median (range) & $2.50(0.91-5.80)$ & $1.84(0.57-4.50)$ & 0.188 \\
\hline Statin use, n (\%) & $35(87.5)$ & $13(100)$ & 0.180 \\
\hline Antiplatelet use, n (\%) & $36(90)$ & $13(100)$ & 0.236 \\
\hline Oral anticoagulants, $\mathrm{n}(\%)$ & $6(15)$ & $1(7.7)$ & 0.499 \\
\hline Currently smoking, n (\%) & $15(37.5)$ & $3(23.1)$ & 0.340 \\
\hline Estimated pack-years, median (range) & $15.0(0-86)$ & $12.5(0-65)$ & 0.461 \\
\hline BMI, mean (std) & $25.8(3.7)$ & $25.0(2.91)$ & 0.520 \\
\hline History of PAOD, $\mathrm{n}(\%)$ & $8(20.0)$ & $2(15.4)$ & 0.712 \\
\hline History of CAD, n (\%) & $12(30.0)$ & $5(38.5)$ & 0.570 \\
\hline Qualifying symptom $=$ hemispheric stroke, $\mathrm{n}(\%)$ & $13(32.5)$ & $5(38.5)$ & 0.693 \\
\hline Major stroke, n (\%) & $3(7.5)$ & $4(30.8)$ & \\
\hline Minor stroke, n (\%) & $10(25.0)$ & $1(7.7)$ & \\
\hline Cerebral TIA, n (\%) & $21(52.5)$ & $7(53.8)$ & \\
\hline Amaurosis fugax, $\mathrm{n}(\%)$ & $6(15.0)$ & $1(7.7)$ & \\
\hline Stenosis grade $\geq 70 \%, \mathrm{n}(\%)$ & $38(95.0)$ & $11(84.6)$ & 0.218 \\
\hline Waiting time (days), median (range) & $45.0(6-200)$ & $34.0(6-74)$ & 0.160 \\
\hline Time between event and MRI, median (range) & $40(5-198)$ & $33(4-73)$ & 0.218 \\
\hline
\end{tabular}

Data are given as proportion of the group (\%), as mean with standard deviation in case of normally distributed data, or as median with range in case of not normally distributed data. Hypertension; previously diagnosed by an MD or use of antihypertensive drugs. Systolic and diastolic blood pressure were measured at intake/hospital admission. Blood pressure medication use; use of any or more antihypertensive drugs. Diabetes mellitus; previously diagnosed by an MD or use of antidiabetic medication. LDL; mmol/L measured within 1 month prior to surgery. Antiplatelet use; dipyridamole, acetylsalicylic, carbasalate calcium or clopidogrel, anti-coagulation; coumarone or direct acting oral anticoagulant, PAOD; peripheral artery disease was defined as a history of peripheral interventions or intermittent claudication or ankle-brachial index $<0.7$. History of CAD; carotid artery disease was defined as a composite of angina pectoris, myocardial infarction, percutaneous coronary interventions or coronary bypass surgery. Stenosis grade $\geq 70 \%$; ipsilateral stenosis grade $\geq 70 \%$ as measured by NASCET criteria. Waiting time; number of days between the most recent event and CEA. Time between most recent event and MRI; number of days between the most recent preoperative cerebrovascular event and preoperative MRI. 


\section{DWI/ADC lesions}

Thirteen (25\%) of 53 included patients had preoperative ipsilateral DWI lesions that appeared hypo- or isointense on ADC. Baseline characteristics of patients with and without recent DWI lesions are presented in Table 1. No statistical differences in baseline features were found between the two groups. In patients with preoperative ipsilateral DWI lesions, mean number of DWI lesions was 2 (median 1, range 1-6). The mean time between the latest symptom and revascularization was 56 days (median 45, range 6-200 days) in the DWInegative group and 36 days (median 34, range 6-74 days, $\mathrm{p}=0.16$ ) in the DWI-positive group. Mean time between latest symptom and MRI was 52 days (median 40, range 5-298) in DWI-negative patients versus 34 (median 33, range 4-73, p=0.218) in DWI-negative patients. The investigated histological plaque characteristics are shown in Table 2 for DWI-negative and DWI-positive patients. 24 out of 40 (60.0\%) DWI-negative patients showed IPH on histological assessment, versus 12 out of 13 (92.3\%) in DWI-positive patients (see example in Figure 2). A lipid-core of $\geq 40 \%$ of the total plaque area was present more often in the DWI-negative group $(23 / 40 ; 57.7 \%)$ compared to the DWI-positive group $(3 / 13 ; 23.1 \%)$. For other plaque characteristics results were similar between DWI-positive and DWInegative patients. Of the total of 36 patients with IPH 12 (33.3\%) had preoperative DWI lesions. Of 17 patients without IPH 1 (6\%) showed DWI lesions. Univariate logistic regression showed an odds ratio $(\mathrm{OR})$ of 8.0 (95\% CI: $0.95-67.7, \mathrm{p}=0.056)$ for presence of DWI lesions in IPH-positive patients compared to IPH-negative patients. Multivariable logistic regression analysis with correction for age and type of index event revealed that IPH was independently associated with presence of DWI lesions in the waiting period till surgery (OR: 10.8; 95\% CI:1.17-99.9, p=0.036). Univariate analysis showed decreased odds for presence of DWI lesions in patients with a large (>40\%) lipid core (OR: 0.22; 95\% CI: $0.05-0.93, \mathrm{p}=0.040)$ which remained significant after correction for age and type of index event (OR:0.184; 95\%-CI: 0.041-0.836, $\mathrm{p}=0.028$ ). Other plaque characteristics did not show any significant association with development of DWI lesions in uni- or multivariate analysis. In 53 patients a total of 28 DWI lesions were detected of which 26 were ipsilateral and 2 were contralateral. Of the total cohort the mean number of DWI lesions was 0.5 (median 0 , range 0-6 lesions). Mean number of ipsilateral DWI lesions in IPH positive patients was 0.7 (median 0, range 0-6) versus 0.1 (median 0, range 0-1) DWI lesions in IPH negative patients $(\mathrm{p}=0.028)$. Of the two patients that had a (single) contralateral DWI lesion, one was IPH positive and one was IPH negative.

\section{ARWMC score}

Median ipsilateral ARMWC sum score was 2. Patients with an ipsilateral ARWMC sum score of $\leq 2(n=30)$ were compared to those with a score $>2(n=23)$. Patients with a high ARWMC sum score were significantly older $(72.5 \pm 6.46)$ compared to patients with a low score $(65.3 \pm 8.63, \mathrm{p}=0.002)$. Other baseline characteristics did not differ between the two 


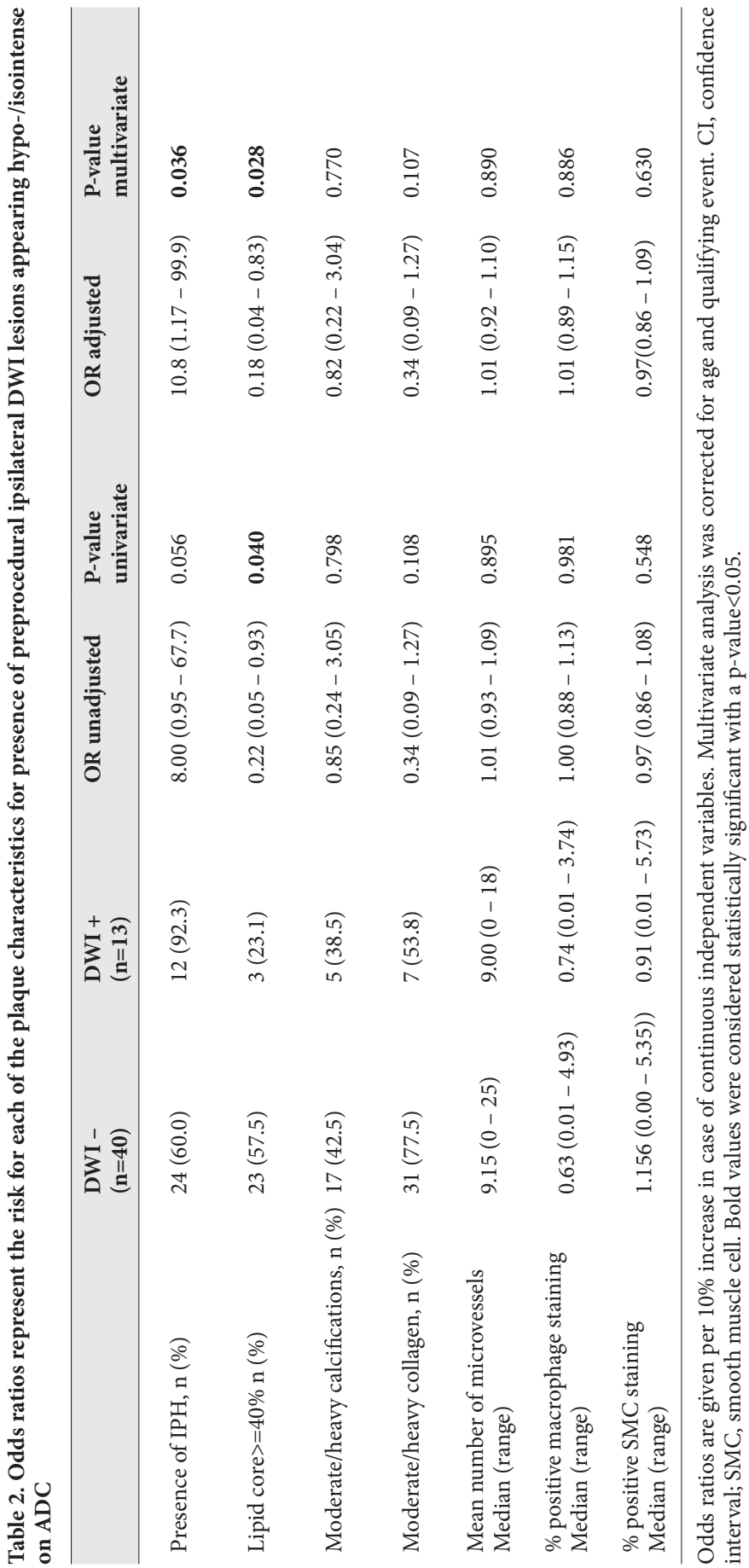


groups, although estimated-packyears was identified as a potential confounder $(\mathrm{p}=0.057$, see Supplemental Table SII). Univariate analysis showed fewer macrophage staining in the high-ARWMC group (OR: 0.19; 95\% CI: 0.05-0.75, p=0.017), which was no longer significant after adjustment for confounders in multivariate logistic regression (OR: 0.232 95\% CI: 0.052 - 10.02 p = 0.053), see Supplemental Table SIII.

\section{Postoperative course}

The median follow-up period was 3.0 years (IQR: 2.9-3.2). Of the 53 included patients two developed peri-operative (<30 days) stroke, one ischemic and one hemorrhagic, both were IPH-positive. No additional major peri-operative events (MI or death) occurred in both IPH-positive or IPH-negative patients. In six additional patients a major postoperative arterial disease complication (nonfatal stroke, nonfatal MI, vascular death) occurred during follow-up: two developed stroke (one ischemic and one hemorrhagic); three suffered from $\mathrm{MI}$ and one additional patient died of other cardiovascular origin (sudden cardiac death). All but one of these patients were IPH-positive; of the IPH-negative patients one patient had MI, no IPH-negative patients suffered from stroke or died of arterial disease complication during follow-up period.
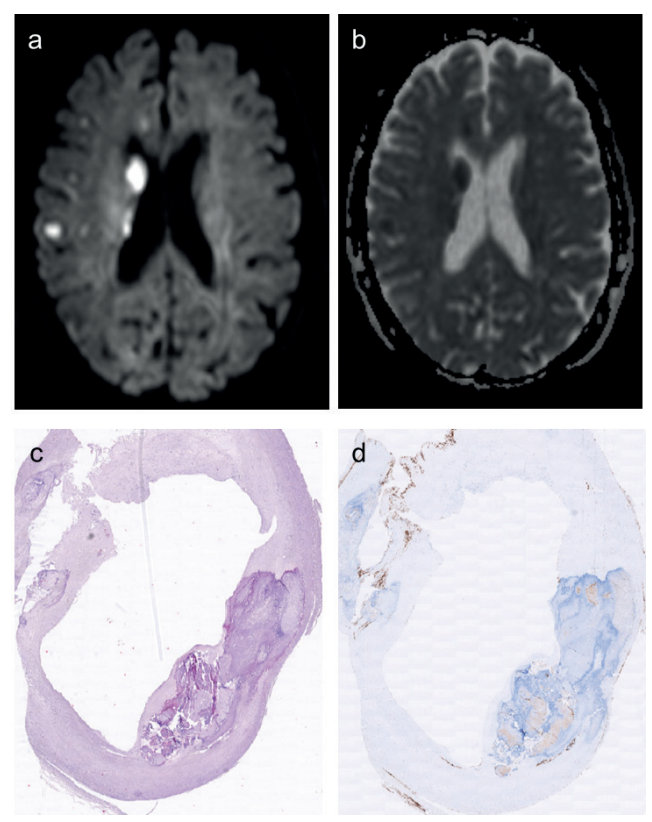

Figure 2. Typical example of patient presenting with minor stroke in right hemisphere and right-sited carotid stenosis. MRI just prior to CEA showed ipsilateral DWI lesions (a) appearing hypo-intense on ADC (b). Upon histological examination the culprit lesion showed intraplaque hemorrhage quantified using Hematoxylin and eosin staining (c, bar $1 \mathrm{~mm}$.) and glycophorine staining (d, bar $1 \mathrm{~mm}$.) 


\section{Discussion}

This study investigated the presence of recent silent ischemic lesions in patients with IPH compared to those without IPH. We demonstrated that carotid IPH is associated with presence of MR-DWI ischemic brain lesions in the waiting period between symptom onset and revascularization, identifying IPH as the potential pathological substrate for such lesions.

This finding is in agreement with imaging studies on carotid plaque characteristics that show that intraplaque hemorrhage is an independent risk factor for future stroke or TIA in patients with carotid artery atherosclerosis. ${ }^{16,17}$ This study, relating histological data to the development of silent ischemic lesions, provides unique insight in natural course and pathology up until revascularization. The results suggest that revascularization procedures of patients with IPH should be prioritized over patients without IPH in order to avert the risk of recurrent ischemia. It should be noted that this also applies in case of a pre- or in hospital delay of $>14$ days after the index event. Moreover, a broad consensus on early versus expedited treatment is still lacking. ${ }^{6,18-20}$ This study contributes to the ongoing discussion on this matter by stressing the importance of eliminating an unstable plaque prior to any (clinical or subclinical) recurrent event. Although IPH cannot specifically identify all patients at risk for recurrent ischemic events (silent or symptomatic) as IPH-rate is higher than DWI-rate, it can help to prioritize those that may benefit more from a rapid revascularization procedure. A more tailored therapy for symptomatic patients based on, amongst others, plaque imaging may be a solution for risk reduction of individual patients. One other clinical study showed that patients with symptomatic carotid stenosis with IPH are more likely to develop recurrent cerebrovascular events in the waiting period until revascularization. ${ }^{21}$ This study did not use histological data but MR T1-weighted 3-dimensional gradient echo sequence to demonstrate IPH. Reliable methods for preoperative assessment of IPH on imaging are essential for clinical applicability. Over the years, several studies investigating IPH-imaging on MR have been performed using various sequences and protocols. ${ }^{22,23}$ More recent articles propose magnetization-prepared rapid acquisition gradient-echo (MPRAGE) as one of the most promising for sequence identification of IPH, with high sensitivity, specificity and $\kappa$-values when validated with histological data. ${ }^{24}$ However, reliability of MR imaging of IPH in the plaque is still limited in case of smaller hemorrhages or coexisting calcifications. ${ }^{24}$

We found that presence of a large lipid core was negatively associated with DWI lesions whereas hypercholesterolemia, statin use and LDL-levels did not differ between groups. One might expect a lipid rich necrotic core to be positively associated with plaque instability and therefore recurrent events. Nonetheless, our results are in agreement with earlier 
research showing that large lipid core and macrophages are not associated with any vascular event, although no subanalyses for cerebrovascular events specifically were performed in this study. ${ }^{14}$ Another study comparing symptomatic to asymptomatic arteries within patients with unilateral symptomatic carotid stenosis found that IPH, but not a large lipid core, was more often seen on the symptomatic side. ${ }^{25}$ Perhaps the presence of a large lipid core shows that plaque rupture has not yet occurred and therefore at this time does not characterize an acute risk for thrombo-embolisms.

Our study used MR-DWI in combination with ADC imaging as our primary outcome parameter. Presence of DWI lesions as a surrogate marker for cerebral ischemia may become increasingly important, especially considering the evidence that DWI lesions are associated with a higher risk for recurrent cerebrovascular events. ${ }^{7}$ In light of the continuously decreasing stroke-rate under improvements in procedural timing and medical therapy, stroke-rate alone is no longer sufficient for determination of therapeutic strategies. A surrogate marker for stroke is warranted and use of DWI as surrogate marker is both biological plausible and clinical relevant. ${ }^{26}$ As the rate of recurrent cerebrovascular events in the period up until revascularization is as low as $1.6 \%{ }^{27}$, use of DWI lesions (seen in $25 \%$ of our population) provides the opportunity to detect statistically significant differences in relatively small patient cohorts.

We did not find a difference in ARWMC score between IPH positive and IPH negative patients. Although one might hypothesize that plaque instability can contribute to development of age related white matter lesions via embolic events ${ }^{21}$, convincing evidence of a causal relation between the two was not demonstrated in earlier studies either. ${ }^{28,29} \mathrm{~A}$ shared etiology for atherosclerosis and white matter lesions may be more likely. ${ }^{29}$

\section{Limitations}

Intraplaque hemorrhage was determined histologically after the plaque was excided during CEA. Considering the long time-interval between symptom onset and surgery, IPH was not necessarily already present at the moment of the index event. Relation to signs of IPH on preoperative imaging (MRI/duplex) may have improved the clinical applicability of our data. Additionally, we did not perform analyses to differentiate older (healed) IPH from acute IPH; although identification of healed IPH on histology is technically possible as shown in studies of coronary arteries. ${ }^{30}$ Furthermore, DWI cannot be used to reliably estimate the age of an ischemic lesion. Although ADC values can help to differentiate from older lesions by excluding T2-shinethrough, exact determination of the moment of onset of these lesions is impossible ${ }^{31}$ and we cannot rule out that the detected lesions were already present at hospital presentation. 
In the context of clinical relevance, the impact of DWI-lesions on future cerebrovascular events would have been of interest. However, MR-DWI performed within few days after revascularization revealed that several patients developed (new) periprocedural DWIlesions. Hence, any correlation between presence of DWI-lesions on preoperative imaging and future events is clouded by the carotid intervention shortly after and thus no statements can be made on the clinical impact of preprocedural silent ischemic lesions.

No information was available on the presence or lack of IPH in other sites of the arterial tree, such as the aortic arch and the contralateral carotid artery. Possibly, IPH in the carotid artery was a sign of general inflammation and although a revascularization procedure of the ipsilateral carotid artery contributes to averting the risk of ipsilateral stroke it does not necessarily reduce the risk of any stroke. Additionally, we were not able to exclude nonarterial sources of embolism and any differences between patients in quality of medical intervention prior to baseline is lacking.

We are aware of the chance of type II statistical error or overfitting of our statistical model as a result of the small patient cohort. Patients of this study were included between 2004 and 2007 when, under current standards, substantial delay between hospital presentation and intervention was common practice. We do believe that the obtained knowledge is of surplus value as prioritizing patients may be challenging when multiple patients qualify for treatment at the same time. Also, patients with a late hospital-presentation may sometimes be postponed in favor of patients with more recent events. This study emphasizes the relevance of prompt acting even after passing of 14 days since index events. This small patient cohort also limits statistical comparison of the clinical course of IPH-positive versus IPH-negative as well as DWI-positive versus DWI-negative patients. Future research should focus on validation of the found results in a large cohort using both histological data as well as MRI-plaque data to compare to development of future cerebrovascular events.

\section{Future perspectives}

Symptomatic patients with a low risk of future events based on several clinical and radiological features are currently under investigation in the ECST-2 trial randomizing between best medical therapy alone or an additional revascularization procedure. ${ }^{32}$ Assessment of IPH on plaque imaging can potentially help in stratification of these low-risk patients. Identification of histological IPH as a potential source of ischemic brain lesions also suggests that assessment of plaque characteristics may be useful in decision making on optimal treatment strategy in asymptomatic carotid stenosis patients. Studies on imaging of carotid plaque IPH and follow-up in asymptomatic patients are needed to confirm this. A meta-analysis including studies that performed MR plaque imaging on both symptomatic and asymptomatic patients demonstrated that patients with IPH have an annualized 
cerebrovascular event rate of $18 \%$ compared to $2 \%$ in those without $\mathrm{IPH} .{ }^{16}$ On the other hand, earlier research demonstrated that IPH is a common phenomenon in both symptomatic and asymptomatic patients ${ }^{33}$ suggesting that plaque complications such as IPH often heal without giving rise to symptoms and IPH alone may not be sufficient for risk stratification. We suggest that imminent clinical decision making-algorithms should include multiple risk factors as it has already been demonstrated that such models can reliably predict major cardiovascular events in patients with carotid artery disease. ${ }^{34}$ These algorithms should take into account not only IPH but also other promising diagnostic tools such as brain imaging for detection of silent infarction and embolus-detection. Prospective observational studies are running that assess amongst others baseline MRI characteristics (both plaque and brain) to develop such a clinically applicable tool for risk stratification. ${ }^{35}$

\section{Conclusion}

In this study, we demonstrated that MR-DWI identified silent brain ischemia in symptomatic patients undergoing CEA is associated with histologically apparent carotid plaque IPH. This qualifies IPH as a potential marker for identifying patients at risk for these ischemic brain lesions. 


\section{References}

1. Michel JB, Virmani R, Arbustini E, Pasterkamp G. Intraplaque haemorrhages as the trigger of plaque vulnerability. Eur Heart J. 2011;32(16):1977-85.

2. Takaya N, Yuan C, Chu B, Saam T, Underbill H, Cai J, et al. Association between carotid plaque characteristics and subsequent ischemic cerebrovascular events: A prospective assessment with MRI Initial results. Stroke. 2006;37(3):818-23.

3. Turc G, Oppenheim C, Naggara O, Eker OF, Calvet D, Lacour JC, et al. Relationships between recent intraplaque hemorrhage and stroke risk factors in patients with carotid stenosis: The HIRISC study. Arterioscler Thromb Vasc Biol. 2012;32(2):492-9.

4. Vrijenhoek JEP, Den Ruijter HM, De Borst GJ, De Kleijn DPV, De Vries JPPM, Bots ML, et al. Sex is associated with the presence of atherosclerotic plaque hemorrhage and modifies the relation between plaque hemorrhage and cardiovascular outcome. Stroke. 2013;44(12):3318-23.

5. Naylor AR, Ricco JB, de Borst GJ, Debus S, de Haro J, Halliday A, et al. Management of Atherosclerotic Carotid and Vertebral Artery Disease: 2017 Clinical Practice Guidelines of the European Society for Vascular Surgery (ESVS). Eur J Vasc Endovasc Surg. 2018;55(1):3-81.

6. Meershoek AJA, de Borst GJ. Timing of carotid intervention. Br J Surg. 2018;105(10):1231-3.

7. Gensicke H, van der Worp HB, Nederkoorn PJ, Macdonald S, Gaines PA, Van Der Lugt A, et al. Ischemic brain lesions after carotid artery stenting increase future cerebrovascular risk. J Am Coll Cardiol. 2015 Feb;65(6):521-9.

8. Allen LM, Hasso AN, Handwerker J, Farid H. Sequence-specific MR Imaging Findings That Are Useful in Dating Ischemic Stroke. RadioGraphics. 2012;32(5):1285-97.

9. Bonati LHLH, Jongen LM, Haller S, Flach HZ, Dobson J, Nederkoorn PJ, et al. New ischaemic brain lesions on MRI after stenting or endarterectomy for symptomatic carotid stenosis: a substudy of the International Carotid Stenting Study (ICSS). Lancet Neurol. 2010 Apr;9(4):353-62.

10. Verhoeven BAN, Velema E, Schoneveld AH, De Vries JPPM, De Bruin P, Seldenrijk CA, et al. Atheroexpress: Differential atherosclerotic plaque expression of mRNA and protein in relation to cardiovascular events and patient characteristics. Rationale and design. Eur J Epidemiol. 2004;19(12):1127-33.

11. Ederle J, Dobson J, Featherstone RL, Bonati LH, van der Worp HB, de Borst GJ, et al. Carotid artery stenting compared with endarterectomy in patients with symptomatic carotid stenosis (International Carotid Stenting Study): an interim analysis of a randomised controlled trial. Lancet. 2010;375(9719):985-97.

12. Wahlund LO, Barkhof F, Fazekas F, Bronge L, Augustin M, Sjögren M, et al. A New Rating Scale for AgeRelated White Matter Changes. Stroke. 2001;32(6):1318-22.

13. Wesseling M, van Koeverden ID, van Lammeren GW, van der Laan SW, Haitjema S, de Vries JPPM, et al. Impaired kidney function is associated with intraplaque hemorrhage in patients undergoing carotid endarterectomy. Atherosclerosis. 2017;266:128-35.

14. Hellings WE, Peeters W, Moll FL, Piers S, Van Setten J, Van Der Spek PJ, et al. Composition of carotid atherosclerotic plaque is associated with cardiovascular outcome: A prognostic study. Circulation. 2010;121(17):1941-50.

15. Singh N, Moody AR, Zhang B, Kaminski I, Kapur K, Chiu S, et al. Age-Specific Sex Differences in Magnetic Resonance Imaging-Depicted Carotid Intraplaque Hemorrhage. Stroke. 2017;48(8):2129-35.

16. Saam T, Hetterich H, Hoffmann V, Yuan C, Dichgans M, Poppert H, et al. Meta-analysis and systematic review of the predictive value of carotid plaque hemorrhage on cerebrovascular events by magnetic resonance imaging. J Am Coll Cardiol. 2013;62(12):1081-91.

17. Gupta A, Baradaran H, Schweitzer AD, Kamel H, Pandya A, Delgado D, et al. Carotid plaque MRI and stroke risk: A systematic review and meta-analysis. Stroke. 2013;44(11):3071-7.

18. Tsantilas P, Kuehnl A, König T, Breitkreuz T, Kallmayer M, Knappich C, et al. Short Time Interval Between Neurologic Event and Carotid Surgery Is Not Associated With an Increased Procedural Risk. 2016;2783-90.

19. Sharpe R, Sayers RD, London NJM, Bown MJ, Mccarthy MJ, Nasim A, et al. Procedural Risk Following Carotid Endarterectomy in the Hyperacute Period after Onset of Symptoms. Eur J Vasc Endovasc Surg. 2013;46(5):519-24.

20. Strömberg S, Gelin J, Österberg T, Bergström GML, Karlström L, Österberg K. Very urgent carotid endarterectomy confers increased procedural risk. Stroke. 2012;43(5):1331-5. 
21. Altaf N, MacSweeney ST, Gladman J, Auer DP. Carotid intraplaque hemorrhage predicts recurrent symptoms in patients with high-grade carotid stenosis. Stroke. 2007;38(5):1633-5.

22. Kerwin WS, Miller Z, Yuan C. Imaging of the high-risk carotid plaque : magnetic resonance imaging. Semin Vasc Surg. 2017;30(1):54-61.

23. Mcnally JS, Kim S, Mendes J, Hadley JR, Sakata A, Havenon AH De, et al. Magnetic Resonance Imaging Detection of Intraplaque Hemorrhage. 2017;

24. Ota H, Yarnykh VL, Ferguson MS, Underhill HR, DeMarco JK, Zhu DC, et al. Carotid Intraplaque Hemorrhage Imaging at 3.0-T MR Imaging: Comparison of the Diagnostic Performance of Three T1weighted Sequences. Radiology. 2010;254(2):551-63.

25. Saam T, Cai J, Ma L, Cai Y-Q, Ferguson MS, Polissar NL, et al. Comparison of symptomatic and asymptomatic atherosclerotic carotid plaque features with in vivo MR imaging. Radiol. 2006;240(2).

26. Traenka C, Engelter ST, Brown MM, Dobson J, Frost C, Bonati LH. Silent brain infarcts on diffusionweighted imaging after carotid revascularisation: A surrogate outcome measure for procedural stroke? A systematic review and meta-analysis. Eur Stroke J. 2019;

27. Shahidi S, Owen-Falkenberg A, Gottschalksen B, Ellemann K. Risk of early recurrent stroke in symptomatic carotid stenosis after best medical therapy and before endarterectomy. Int J Stroke. 2016;11(1):41-51.

28. Potter GM, Doubal FN, Jackson CA, Sudlow CLM, Dennis MS, Wardlaw JM. Lack of association of white matter lesions with ipsilateral carotid artery stenosis. Cerebrovasc Dis. 2012;33(4):378-84.

29. van den Bouwhuijsen QJA, Vernooij MW, Verhaaren BFJ, Vrooman HA, Niessen WJ, Krestin GP, et al. Carotid Plaque Morphology and Ischemic Vascular Brain Disease on MRI. Am J Neuroradiol. 2017;

30. Burke AP, Kolodgie FD, Farb A, Weber DK, Malcom GT, Smialek J, et al. Healed Plaque Ruptures and Sudden Coronary Death. Circulation. 2001;103(7):934 LP-940.

31. Abbott AL, Silvestrini M, Topakian R, Golledge J, Brunser AM, de Borst GJ, et al. Optimizing the definitions of stroke, transient ischemic attack, and infarction for research and application in clinical practice. Front Neurol. 2017;8(OCT):1-14.

32. Featherstone RL, Brown MM. The second european carotid surgery trial. Endovasc today. 2012;(October):75-7.

33. Bassiouny HS, Davis H, Massawa N, Gewertz BL, Glagov S, Zarins CK. Critical carotid stenoses: Morphologic and chemical similarity between symptomatic and asymptomatic plaques. J Vasc Surg. 1989;9(2):202-12.

34. Khanna NN, Jamthikar AD, Araki T, Gupta D, Piga M, Saba L, et al. Nonlinear model for the carotid artery disease 10-year risk prediction by fusing conventional cardiovascular factors to carotid ultrasound image phenotypes: A Japanese diabetes cohort study. Echocardiography. 2019;36(2):345-61.

35. TAXINOMISIS project. 2018. p. https://taxinomisis-project.eu/. 


\section{Supplementary Materials}

The following supplementary material is omitted due to space limitation and can be found at the journal website:

- Supplemental Table SI. The ARWMC score.

- Supplemental Table SII. Baseline characteristics between patients with ARWMC $\leq 2$ and ARWMC $>2$

- Supplemental Table SIII. Plaque characteristics compared between patients with ARWMC $\leq 2$ and $>2$ 


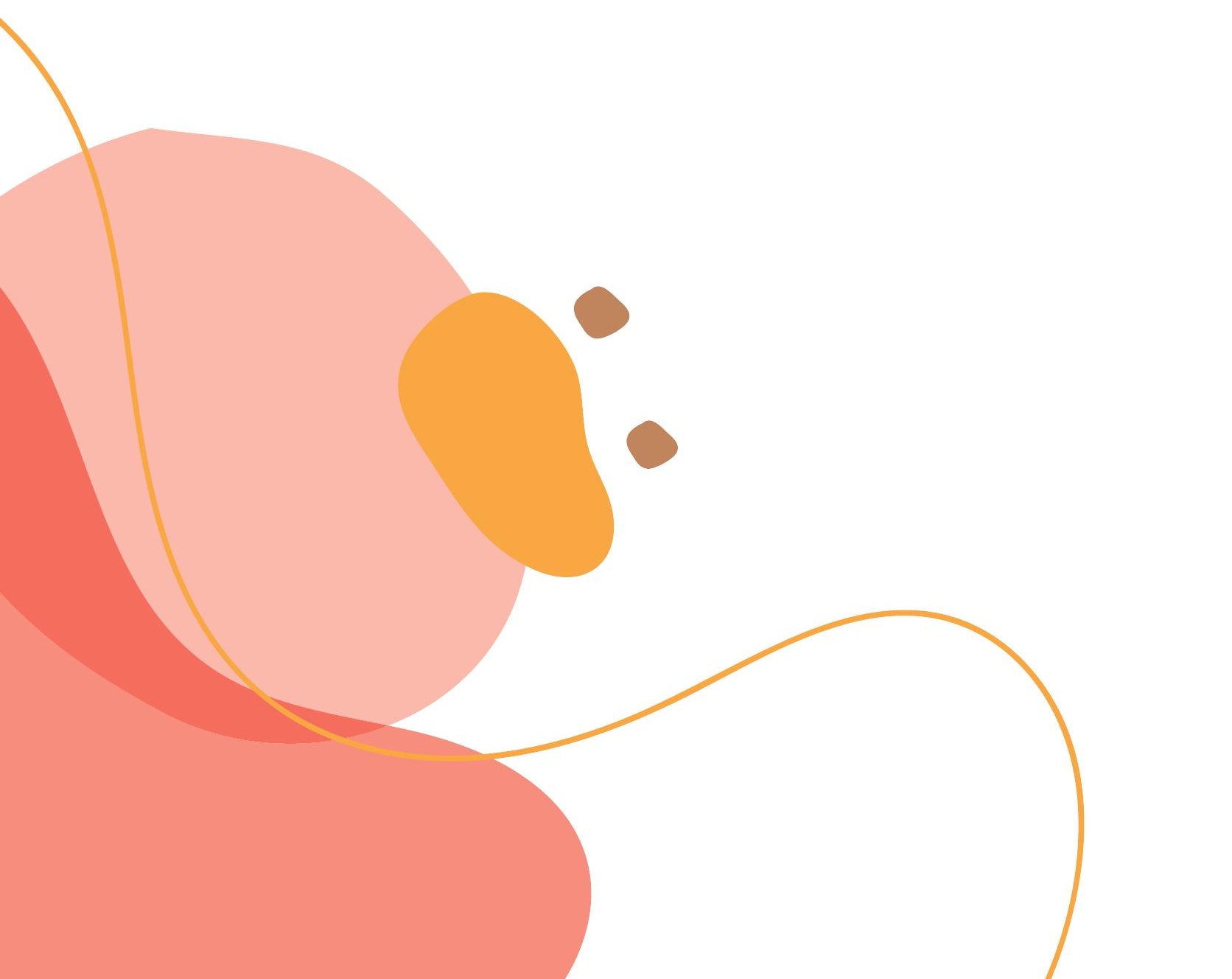




\section{Cerebral small vessel disease in standard pre-operative imaging reports is independently associated with increased risk of cardiovascular death following carotid endarterectomy}

European Journal of Vascular and Endovascular Surgery 2020;59(6):872-880

Nathalie Timmerman

Marjolijn L. Rots

Ian D. van Koeverden

Saskia Haitjema

Constance J.H.C.M. van Laarhoven Annemiek M. Vuurens Hester M. den Ruijter

Gerard Pasterkamp

L. Jaap Kappelle Dominique P.V. de Kleijn Gert J. de Borst 


\section{Abstract}

\section{Background}

Cerebral white-matter lesions (WMLs) and lacunar infarcts are surrogates of cerebral small vessel disease (SVD). WML severity as determined by trained radiologists predicts postoperative stroke or death in patients undergoing carotid endarterectomy (CEA). It is unknown if routine preoperative brain imaging reports as part of standard clinical practice also predicts short- and long-term risk of stroke and death after CEA.

\section{Methods}

Consecutive patients from the Athero-Express biobank study that underwent CEA for symptomatic high degree stenosis between March 2002 and November 2014 were included. Preoperative brain imaging (CT- or MRI) reports were reviewed for reporting of SVD, defined as WMLs or any lacunar infarcts. The primary outcome was defined as any stroke or any cardiovascular death during 3 years of follow-up. The secondary outcome was defined as the 30-day perioperative risk of stroke or cardiovascular death.

\section{Results}

1038 patients were included (34\% women), of whom 659 (63.5\%) had CT images and 379 (36.5\%) had MRI available. Of all patients, 697 (67\%) patients had SVD reported by radiologists. Patients with SVD had a higher 3-year risk of cardiovascular death compared to those without (6.5\% vs. $2.1 \%$, adjusted hazard ratio (HR) 2.52 (95\% CI: 1.12-5.67); $\mathrm{p}=0.026)$ but no association was observed for the 3 -year risk of stroke $(9.0 \%$ vs. $6.7 \%$, for patients with SVD versus those without, adjusted HR 1.24 (95\% CI: 0.76-2.02); $\mathrm{p}=0.395$ ). No differences in 30-day perioperative risk were observed for stroke (4.4\% vs. $2.9 \%$, for patients with versus those without SVD; adjusted HR 1.49 (95\% CI: 0.73-3.05); p=0.277), and for the combined stroke/cardiovascular death risk (4.4\% vs. 3.5\%, adjusted HR 1.20 (95\% CI: 0.61-2.35); $\mathrm{p}=0.592$ ).

\section{Conclusions}

Presence of SVD in preoperative brain imaging reports can serve as a predictor for the 3-year risk of cardiovascular death in symptomatic patients undergoing CEA but does not predict perioperative or long-term risk of stroke.

\section{What this paper adds}

This study investigated the clinical relevance of reported cerebral small vessel disease (SVD, including white-matter lesions and lacunar infarct) for postoperative stroke and death-risk in symptomatic patients undergoing carotid endarterectomy (CEA). The current study 
highlights that the presence of SVD in standard routine preoperative brain imaging reports identifies patients at high risk of cardiovascular death. Yet, reported SVD is not associated with procedural or long-term postoperative stroke risk and this should therefore not influence the decision whether CEA should be performed in symptomatic patients with high degree carotid artery stenosis.

\section{Introduction}

Carotid endarterectomy (CEA) is the preferred treatment for high degree carotid stenosis in symptomatic patients although procedural stroke may limit the benefit of the intervention. ${ }^{1}$ Patient-specific predictors of high procedural- or long-term stroke risk can be used to optimize patient selection for revascularization and individualized medical treatment. ${ }^{2}$ The presence of cerebral white-matter lesions (WMLs) on preoperative computed tomography (CT) or magnetic resonance imaging (MRI) has been previously suggested as a predictor for perioperative adverse events following CEA. ${ }^{3}$

WMLs are a common finding on baseline CT- and MRI brain scans. These are closely associated with age and risk factors for atherosclerosis and have been independently associated with increased risk for dementia, first-ever stroke, recurrent stroke, poor functional stroke outcome, and cardiovascular mortality. ${ }^{4,5}$ The pathogenesis of WMLs is multifactorial and suggested to be caused by small artery atherosclerosis leading to chronic cerebral hypoperfusion and white matter ischemia. ${ }^{6,7}$ Previous studies in symptomatic CEA patients suggested an increased peri- and postoperative stroke- and death risk for patients with more extensive WMLs. ${ }^{3,8,9}$ The association between WMLs and an increased risk for stroke may be related to impaired cerebral hemodynamics due to a reduced vessel density, impaired vessel autoregulation or a reduced baseline cerebral blood flow. ${ }^{6,10}$ Therefore, it can be hypothesized that a brain with more than age-appropriate WMLs may be more vulnerable to develop subsequent ischemic infarct when challenged by acute thromboembolic or hemodynamic events.

While previous studies investigated the predictive value of specific (semi-)quantitative WML severity-scores for adverse events in patients undergoing $\mathrm{CEA}^{3,8,9}$, thus far no studies have evaluated the clinical relevance of cerebral small vessel disease (SVD) as reported in daily practice. The current study aimed to assess the clinical relevance of baseline SVD in standard preoperative brain imaging reports in relation to future adverse cardiovascular events following CEA. We hypothesized that the presence of SVD is associated with increased stroke or cardiovascular death risk in symptomatic patients undergoing CEA. 


\section{Methods}

\section{Athero-Express}

All patients were recruited from the Athero-Express biobank study, of which the design has been published elsewhere. ${ }^{11,12}$ This ongoing prospective cohort study includes consecutive patients with carotid artery stenosis undergoing CEA in two large tertiary referral hospitals in the Netherlands, the University Medical Centre in Utrecht and the St. Antonius Hospital in Nieuwegein. Patient characteristics, including cardiovascular risk factors, history of cardiovascular disease, history of peripheral artery disease and medication use were obtained from standardized questionnaires and patient records at time of inclusion. Patients included in the study were symptomatic (stroke, hemispheric transient ischemic attacks (TIA) or monocular blindness $<6$ months prior to hospital presentation) with a high-grade carotid artery stenosis of 50-99\%. The indication for CEA was reviewed by a multidisciplinary vascular team and was based on local and international guidelines. ${ }^{13-17}$ Follow-up data were obtained for secondary cardiovascular and cerebrovascular events during the 3-year postoperative period. The study protocol was approved by the medical ethics committees of both hospitals and patients provided written informed consent before participation in the Athero-Express study. All consecutive patients included in the AtheroExpress and undergoing CEA for symptomatic carotid artery stenosis between January 2002 and November 2014 were included in the present analysis. Patients underwent routine preoperative brain CT- or MRI and/or CT- or MR-angiography, in concordance with international guidelines. ${ }^{13-17}$ Patients were excluded for analysis when baseline brain imaging reports were missing or incomplete (i.e. when only a concise imaging report was available from a referring center) or in case of lost to follow-up.

\section{Definition small vessel disease}

The routine preoperative brain CT and/or MRI reports were retrospectively analyzed for the presence or absence of SVD. SVD was defined as any reported WML (or a synonym, see Supplemental Table S1) or any old lacunar infarct. This definition was chosen due to the fact that both modalities have the same etiology and both have been proven to predict future stroke in the general population., ${ }^{4,7}$ To assess the accuracy of reporting SVD in standard clinical imaging reports, a subset of 30 scans were randomly selected from the total cohort for validation. Radiology images were meticulously evaluated for WML and lacunar infarct by a dedicated observer (MR) who was blinded for the standard clinical reports. Reliability (kappa-values) and proportion of agreement were calculated between reported WML and lacunar infarct in standard clinical imaging reports and those identified by re-evaluation of the radiology images of the blinded observer (MR). 


\section{Follow-up and outcome}

The primary outcome was defined as any stroke or any cardiovascular death during 3-year follow-up. Any stroke includes nonfatal ischemic stroke, nonfatal hemorrhagic stroke, fatal ischemic stroke and fatal hemorrhagic stroke. Any cardiovascular death includes death of presumed vascular origin such as fatal stroke, fatal myocardial infarction, fatal cardiac failure, fatal aneurysm rupture and sudden death. Secondary outcomes were all-cause death during the 3-year follow-up and the composite endpoint of any stroke or any cardiovascular death during the 30 -day perioperative period. The follow-up protocol was identical to the one previously described. ${ }^{11,12}$ Perioperative assessment was structurally done by a neurologist and/or vascular surgeon. Patient medical records were checked for perioperative adverse events. Patients were contacted annually for three years after CEA through standardized questionnaires asking whether they had experienced an adverse cardiovascular event or if they had been hospitalized. Questionnaires were verified with medical records; when a questionnaire is answered positively, additional data (medical records, electrocardiograms, laboratory and imaging studies) are retrieved and assessed for the occurrence of an outcome event. In case of non-response, the general practitioner was consulted. All available data to establish an outcome event were reviewed by two independent experts. In case of a disagreement, a third expert was consulted, and outcome was discussed until agreement was reached.

\section{Statistical analysis}

Baseline characteristics and survival-free and stroke-free rates were compared between patients with reported SVD and patients without reported SVD. For continuous baseline variables, the independent samples t-test was used for normally distributed variables and Mann Whitney U test for non-normally distributed variables. Categorical baseline variables were tested by the chi-squared test for equal distribution among both groups. The cumulative incidences of the outcome measures were estimated using a Kaplan-Meier analysis. To investigate the association between reported SVD and outcomes, Cox proportional-hazards regression modelling was used for the univariate and multivariable analysis. For baseline characteristics with an association of $\mathrm{p}<0.10$ with reported SVD, an association with outcome measures was also checked using Cox proportional-hazards regression modelling. Whenever statistical significance of $\mathrm{p}<0.10$ with the outcome of interest was also found, the baseline variable was added as a covariate in the multivariable models (Supplemental Table S2). Since contralateral carotid stenosis is considered as a strong predictor for future stroke, we included this as covariate in the multivariable analysis (full models are displayed in Supplemental Table S2). Missing data of covariates were imputed with multiple imputation to enable multivariable analysis (Supplemental Table S3). To quantify follow-up completeness in this study, the follow-up index (FUI) was calculated as described 
previously ${ }^{18}$ and compared between both study groups. All statistical analyses were performed with IBM SPSS statistics version 25.0 (SPSS Inc, Chicago, IL). A p-value $<0.05$ was considered as statistically significant.

\section{Results}

Patient selection for the current study is displayed in Figure A. Of the 1544 patients who met inclusion criteria, 1038 patients had available preoperative brain imaging reports and were included for analysis. Of those, 659 (63.5\%) had a CT-scan and $379(36.5 \%)$ had an MRI-scan preoperatively.

\section{Imaging characteristics}

Various CT- and MRI-scanners and scanning protocols were used. Slice thickness of CT scanning varied from 3 to $6 \mathrm{~mm}$. MRI T2- fluid-attenuated inversion-recovery (FLAIR) sequences were most often used, predominantly with field strengths of 1.5 Tesla (and few of 3.0 Tesla), and varying slice thickness from 4 to $6 \mathrm{~mm}$. Reasons for missing brain imaging (reports) included that only CT- or MRI angiography was performed (specifically in patients presenting with ocular symptoms) or referral from other hospitals from which imaging reports were not included in the referral data. Differences in baseline characteristics between included patients and patients with missing brain imaging or reports are displayed in Supplemental Table S4. Reliability of SVD identification from imaging reports compared with the SVD scoring of the radiology images by blinded observer was good ( $\kappa 0.74 \pm 0.14$, see Supplemental Table S5).

\section{Presence of SVD}

In the total cohort of 1038 patients, $34 \%$ were women, $18 \%$ had presented with transient or permanent retinal ischemia, 35\% with stroke and $43 \%$ with TIA (Table 1). SVD was reported in 697 patients (67\%). Of patients with SVD, 243 (35\%) were female versus 108 (32\%) without SVD ( $\mathrm{p}=0.307$ ). SVD was reported in $51 \%$ of patients $<60$ years, $61 \%$ of patients between $60-70$ years, $72 \%$ of patients between $70-80$ years and $82 \%$ of patients $>80$ years old $(\mathrm{p}<0.001)$. Detailed characteristics of SVD lesions are displayed in Figure A. Of all patients with reported SVD, 147/697 (21\%) had WMLs and lacunar infarct, 431/697 (62\%) had only WML and $119 / 697$ (17\%) had only lacunar infarct. SVD was located ipsilateral to the operated artery in $111 / 697$ patients (16\%), in 56/697 (8\%) contralateral and bilateral in 235/697 patients (34\%), whereas in $295 / 697$ patients (42\%) the location was not specified in the imaging report. Baseline characteristics of patients associated with SVD are shown in Table 1. Patients with SVD were older, more frequently hypertensive and diabetic, had decreased renal function and more frequently had a history of peripheral artery disease. 


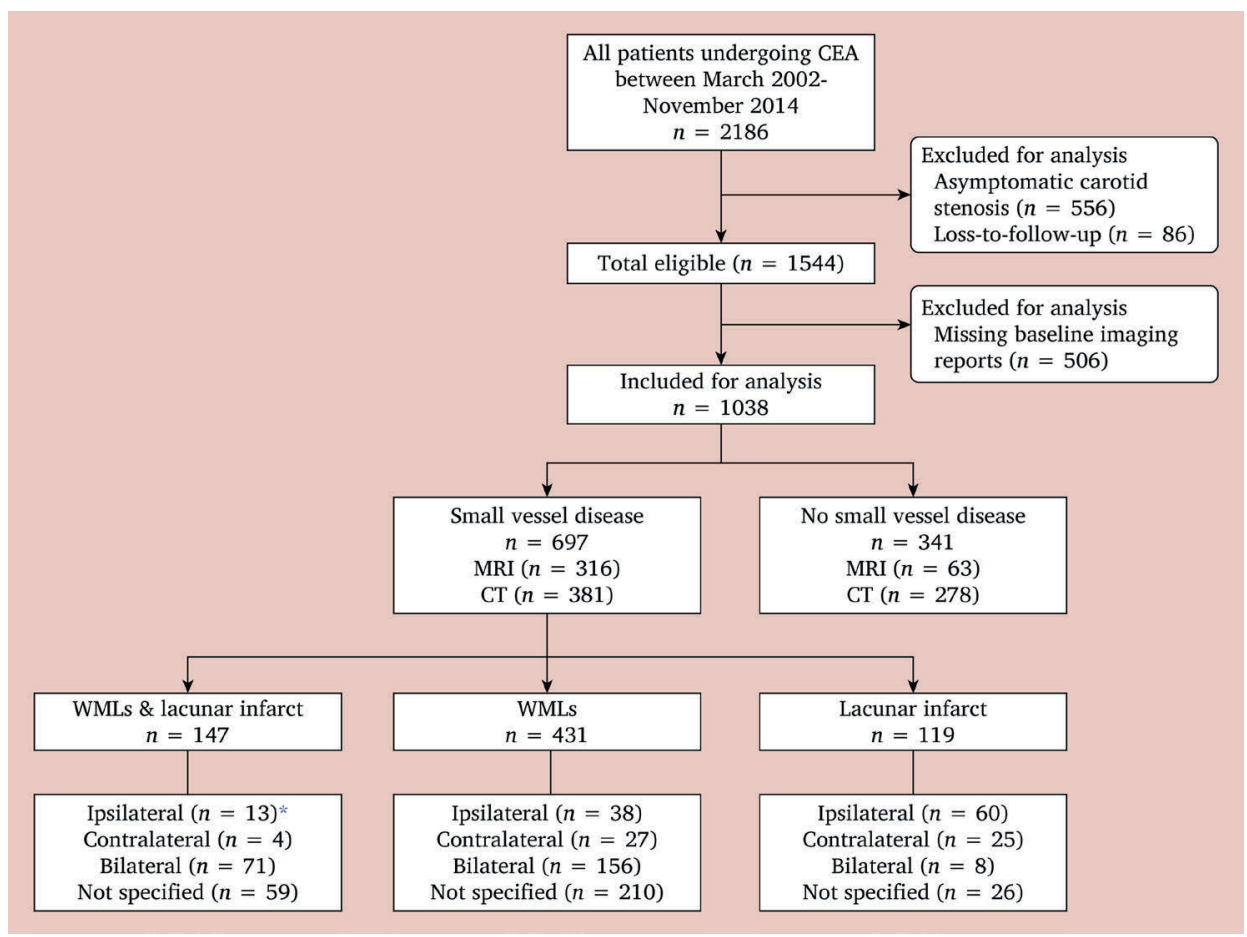

Figure A. Flowchart of included patients from the Athero-Express Biobank and type of SVD lesions Numbers in brackets indicate numbers of patients. Every patient can only belong in one category (numbers are mutually exclusive). * The location relates to any lesion including WML or lacunar infarct or both.

\section{Association between SVD and stroke or death}

The FUI for patients with SVD and those without were equal (mean FUI $0.95 \pm 0.15$ versus $0.97 \pm 0.11$ respectively, $\mathrm{p}=0.976$ ) indicating complete follow-up information for both study groups. The 3-year risk of the composite endpoint for stroke and cardiovascular death was similar for patients with SVD and those without SVD (12.6\% vs $8.2 \%$ respectively, adjusted hazard ratio (HR) 1.36 (95\% confidence interval (CI): 0.88-2.10); $\mathrm{p}=0.172$; Table 2, Figure B1) as was the risk of any stroke (9.0\% vs. 6.7\%, adjusted HR 1.24 (95\% CI: 0.76-2.02); $\mathrm{p}=0.395$; Table 2, Figure B2). The 3-year risk of cardiovascular death was significantly higher in patients with reported SVD compared to patients without (6.5\% vs. $2.1 \%$, adjusted HR 2.52 (95\% CI: 1.12-5.67); p=0.026; Table 2, Figure B3). The 3-year risk of all-cause death did not significantly differ between patients with reported SVD compared to those without (13.9\% vs. 8.2\%, adjusted HR 1.26 (95\% CI: 0.82-1.94); p=0.298; Supplemental Table S6). For the 30-day perioperative period, the combined stroke or cardiovascular death risks were similar for patients with and patients without SVD (4.4\% vs. 3.5\% respectively, with 
Table 1. Baseline characteristics of symptomatic CEA patients compared between those with reported cerebral small vessel disease and those without

\begin{tabular}{|c|c|c|c|}
\hline Baseline characteristics & $\begin{array}{l}\text { No small vessel disease } \\
(\mathbf{n}=341)\end{array}$ & $\begin{array}{l}\text { Small vessel disease } \\
(n=697)\end{array}$ & p-value \\
\hline Age, years (mean [SD]) & $67.2[9.3]$ & $71.7[9.3]$ & $<0.001^{\mathrm{b}}$ \\
\hline Women & $108(31.7)$ & $243(34.9)$ & 0.307 \\
\hline Hypertension & $231(70.2)$ & $530(79.1)$ & $0.002^{b}$ \\
\hline Diabetes & $65(19.1)$ & $189(27.1)$ & $0.005^{b}$ \\
\hline BMI, kg/m2 (mean [SD]) & $26.5[4.1]$ & $26.2[4.0]$ & 0.382 \\
\hline Hypercholesterolemia & $205(66.3)$ & $420(67.1)$ & 0.819 \\
\hline Current smoker & $125(37.8)$ & $213(31.2)$ & $0.039^{b}$ \\
\hline Packyears of smoking & & & 0.611 \\
\hline 0 & $50(16.9)$ & $116(19.9)$ & \\
\hline $1-10$ & $186(62.8)$ & $355(60.8)$ & \\
\hline $10-20$ & $40(13.5)$ & $84(14.4)$ & \\
\hline $20-30$ & $16(5.4)$ & $21(3.6)$ & \\
\hline $30-40$ & $4(1.4)$ & $8(1.4)$ & \\
\hline History of CAD & $90(26.4)$ & $213(30.6)$ & 0.157 \\
\hline History of PAD & $40(11.8)$ & $136(19.6)$ & $0.002^{b}$ \\
\hline eGFR, ml/min/1/1.73m2 (mean, [SD]) & $76.2[19.7]$ & $69.9[20.9]$ & $<0.001^{\text {b }}$ \\
\hline Ipsilateral carotid stenosis & & & 0.241 \\
\hline $50-70 \%$ & $43(12.6)$ & $71(10.2)$ & \\
\hline $70-99 \%$ & $298(87.4)$ & $626(89.8)$ & \\
\hline Contralateral carotid stenosis (50-100\%) & $127(42.5)$ & $248(39.2)$ & 0.347 \\
\hline Presenting cerebrovascular event & & & $0.079^{\mathrm{b}}$ \\
\hline stroke & $115(33.7)$ & $249(35.7)$ & \\
\hline TIA & $141(41.3)$ & $308(44.2)$ & \\
\hline ocular & $76(22.3)$ & $112(16.1)$ & \\
\hline unclear & $9(2.6)$ & $28(4.0)$ & \\
\hline History of any stroke & $133(39.0)$ & $308(44.2)$ & 0.112 \\
\hline Statin use & $263(77.4)$ & $525(75.5)$ & 0.520 \\
\hline Antiplatelet drug use & $294(86.7)$ & $611(88.0)$ & 0.547 \\
\hline Oral anticoagulant use & $39(11.5)$ & $74(10.6)$ & 0.690 \\
\hline
\end{tabular}

Data are displayed as $\mathrm{n}$ (\% of total) unless otherwise indicated. Numbers in bold indicate $\mathrm{p}<.05$.

${ }^{\mathrm{b}}$ Baseline characteristic showing $\mathrm{p}<0.10$ association with reported cerebral small vessel disease

$\mathrm{BMI}$, Body mass index; CAD, coronary artery disease; PAD, peripheral artery disease; eGFR, estimated glomerular filtration rate; TIA, transient ischemic attack; SD, standard deviation.

When multiple cerebrovascular symptoms occurred in the six months prior to the operation, the most serious symptom counts in the following order: stroke $>$ TIA $>$ ocular. Ocular symptoms include transient or permanent retinal ischemia. History of any stroke includes ipsilateral or contralateral stroke. Antiplatelet drug use includes the use of aspirin, dipyridamole or any ADP-inhibitor. 
adjusted HR 1.20 (95\% CI: 0.61-2.35); p=0.592; Table 3, Figure C1). Also, no difference in 30-day perioperative stroke risk was observed (4.4\% vs. 2.9\%; adjusted HR 1.49 (95\% CI: 0.73-3.05); $\mathrm{p}=0.277$; Table 3, Figure C2).

\section{Subanalyses}

Separate analyses for the CT- and MRI-cohort were performed and added to the supplemental materials (Supplemental Tables S7a-c). For those that had CT, 381 out of 659 (57.8\%) had SVD and for those that had MRI 316 out of 379 patients (83.3\%) had SVD. Similar trends in differences in baseline characteristics were observed for CT and MRIsubgroups. The independent association between SVD and 3-year risk of cardiovascular death was confirmed in the CT-subgroup analysis (absolute incidences of 7.9\% versus $2.5 \%$, respectively, with adjusted HR 2.56 (95\% CI:1.09-6.00); $\mathrm{p}=0.031$ ) but not in the MRI subgroup (absolute incidences of $4.7 \%$ versus $0.0 \%$ in patients with SVD versus those without). However, univariate analysis for MRI was inaccurate (unrealistic HR and wide CI) and multivariable analyses could not be performed, both because of limited power. Because the location of SVD was not specified in the reports in a large proportion of the patients (42\%), sub-analysis based on different location (ipsilateral versus contralateral versus bilateral) could not be performed.

\section{Discussion}

This study examined the clinical relevance of reported SVD in preoperative routine clinical brain imaging reports for perioperative and long-term adverse cardiovascular events in symptomatic patients undergoing CEA. Preoperative SVD was reported in two thirds of patients undergoing CEA and was associated with a 2.5-fold increased 3-year risk of postoperative cardiovascular death, independent of known cardiovascular risk factors. Our results indicate that reported SVD can identify patients with poor survival and accordingly a potentially lower long-term benefit of CEA. Yet, as reported SVD was not associated with procedural or postoperative stroke-risk, SVD should not be used to weigh up the indication for carotid intervention in symptomatic patients.

In line with our results, the International Carotid Stenting Study (ICSS) revealed no association between higher scores of age-related white matter changes (ARWMC) and 30day perioperative stroke risk in CEA patients. ${ }^{9}$ On the contrary, the North American Symptomatic Endarterectomy Trial (NASCET) observed increased 30-day combined stroke or death risks in patients with widespread WMLs compared to those without (13.9\% versus 
Figure B1-3. Survival analysis for the 3-year postoperative outcomes

B1, any stroke or cardiovascular death; B2, any stroke; B3, cardiovascular death. Red line indicates patients with cerebral small vessel disease, the blue line indicates patients without cerebral small vessel disease. Adjusted HR; adjusted hazard ratio calculated by multivariable Cox-proportional hazard analysis.

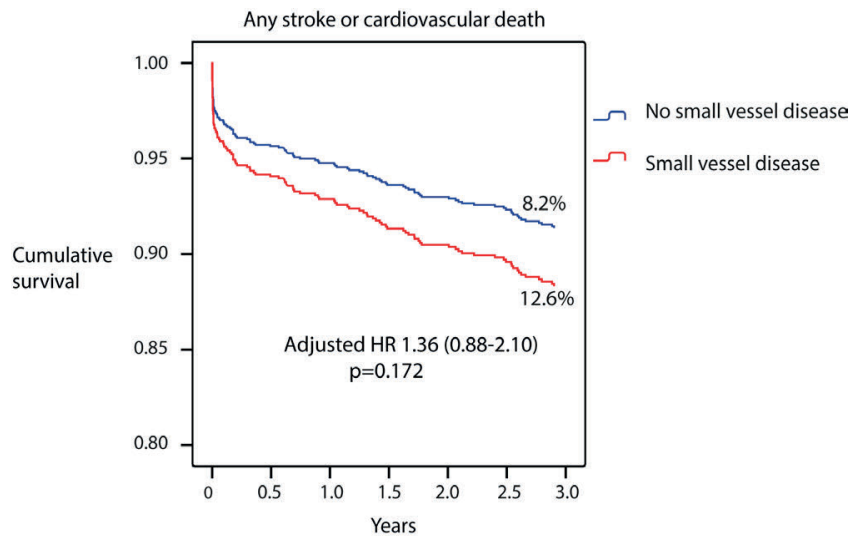

$\begin{array}{lllll}\text { Small vessel disease } & 697 & 618 & 565 & 502 \\ \text { No. of events } & 0 & 59 & 76 & 88 \\ \text { No small vessel disease } & 341 & 315 & 297 & 268 \\ \text { No. of events } & 0 & 20 & 25 & 28\end{array}$

Figure B1. Any stroke or cardiovascular death during 3-year follow-up

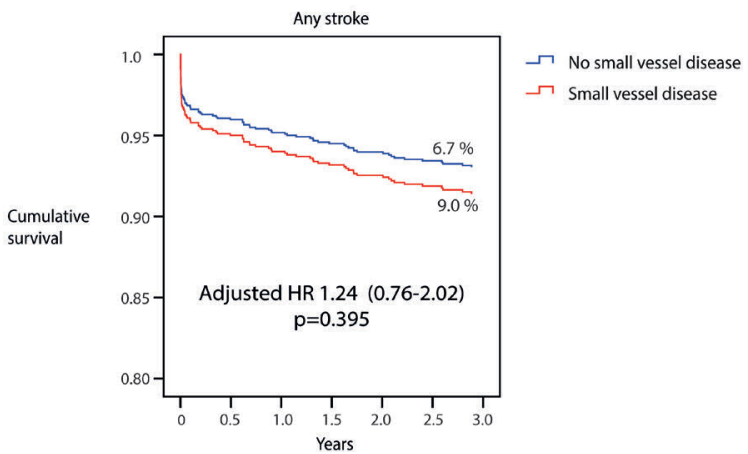

$\begin{array}{lcrrr}\text { Small vessel disease } & 697 & 618 & 565 & 503 \\ \text { Cumulative events } & 0 & 47 & 59 & 63 \\ \text { No small vessel disease } & 341 & 315 & 297 & 268 \\ \text { Cumulative events } & 0 & 19 & 22 & 23\end{array}$

Figure B2. Any stroke during 3-year follow-up 


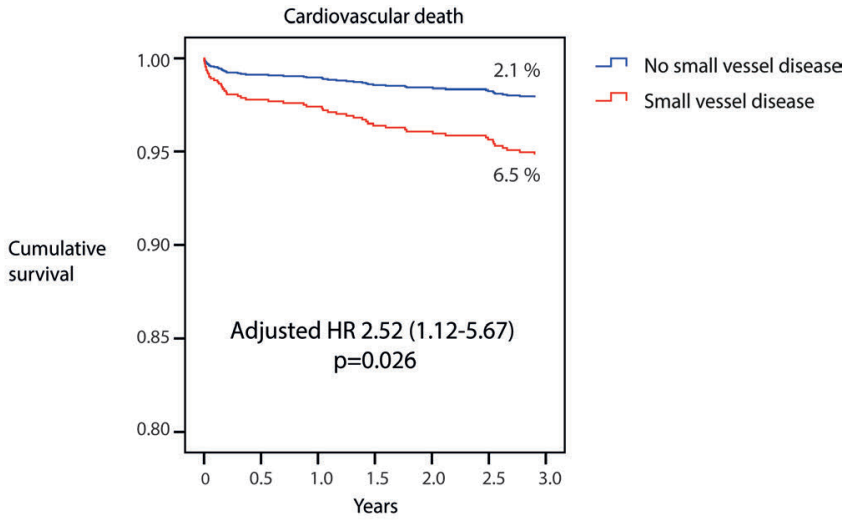

$\begin{array}{lcccc}\text { Small vessel disease } & 697 & 640 & 591 & 526 \\ \text { Cumulative events } & 0 & 28 & 37 & 45 \\ \text { No small vessel disease } & 341 & 329 & 313 & 284 \\ \text { Cumulative events } & 0 & 4 & 6 & 7\end{array}$

Figure B3. Cardiovascular death during 3-year follow-up

Table 2. Cox-proportional hazards analysis of 3-year risks for secondary adverse events after CEA

\begin{tabular}{|c|c|c|c|c|c|c|}
\hline \multirow[b]{2}{*}{$\begin{array}{l}\text { 3-year follow-up } \\
\text { outcome measure }\end{array}$} & \multirow[b]{2}{*}{$\begin{array}{l}\text { No small } \\
\text { vessel disease }\end{array}$} & \multirow[b]{2}{*}{$\begin{array}{l}\text { Small vessel } \\
\text { disease }\end{array}$} & \multicolumn{2}{|l|}{$\begin{array}{l}\text { Univariate } \\
\text { analysis }\end{array}$} & \multicolumn{2}{|l|}{$\begin{array}{l}\text { Multivariable } \\
\text { analysis }\end{array}$} \\
\hline & & & HR $[95 \% \mathrm{CI}]$ & p-value & $\mathrm{HR}[95 \% \mathrm{CI}]$ & p-value \\
\hline $\begin{array}{l}\text { Any stroke or } \\
\text { cardiovascular death a }\end{array}$ & $28 / 341(8.2 \%)$ & $88 / 697(12.6 \%)$ & $1.59[1.04-2.43]$ & 0.033 & $1.36[0.88-2.10]^{\mathrm{a}}$ & 0.172 \\
\hline Any stroke ${ }^{b}$ & $23 / 341(6.7 \%)$ & $63 / 697(9.0 \%)$ & $1.38[0.85-2.22]$ & 0.190 & $1.24[0.76-2.02]^{\mathrm{b}}$ & 0.395 \\
\hline Cardiovascular death ${ }^{c}$ & $7 / 341(2.1 \%)$ & $45 / 697(6.5 \%)$ & $3.29[1.48-7.30]$ & 0.003 & $2.52[1.12-5.67]^{\mathrm{c}}$ & 0.026 \\
\hline
\end{tabular}

$\mathrm{HR}$, hazard ratio. HR $>1.0$ indicates an increased risk for patients with cerebral small vessel disease compared to patients without. Numbers in bold indicate $\mathrm{p}<0.05$.

${ }^{a}$ Covariates included in multivariable model of the composite endpoint any stroke or cardiovascular death were age, cerebrovascular symptoms, diabetes, history of PAD, eGFR and contralateral stenosis (50-100\%).

${ }^{\mathrm{b}}$ Covariates included in multivariable model of any stroke were age, diabetes and contralateral stenosis (50-100\%).

${ }^{\mathrm{c}}$ Covariates included in multivariable model of cardiovascular death were age, history of PAD, eGFR and contralateral stenosis (50-100\%). 
Figure C1-2. Survival analysis for the 30-day perioperative outcomes

$\mathrm{C} 1$, any stroke or cardiovascular death; C2, any stroke. Red line indicates patients with cerebral small vessel disease, the blue line indicates patients without cerebral small vessel disease. Adjusted HR; adjusted hazard ratio calculated by multivariable Cox-proportional hazard analysis.

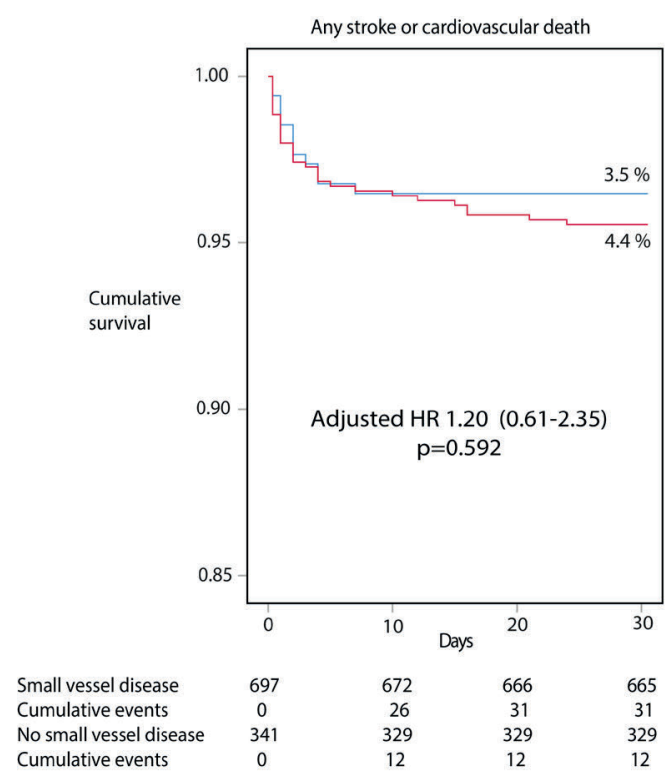

$\neg$ No small vessel disease

$\neg$ Small vessel disease

Figure C1. Any stroke or cardiovascular death during 30 days of follow-up

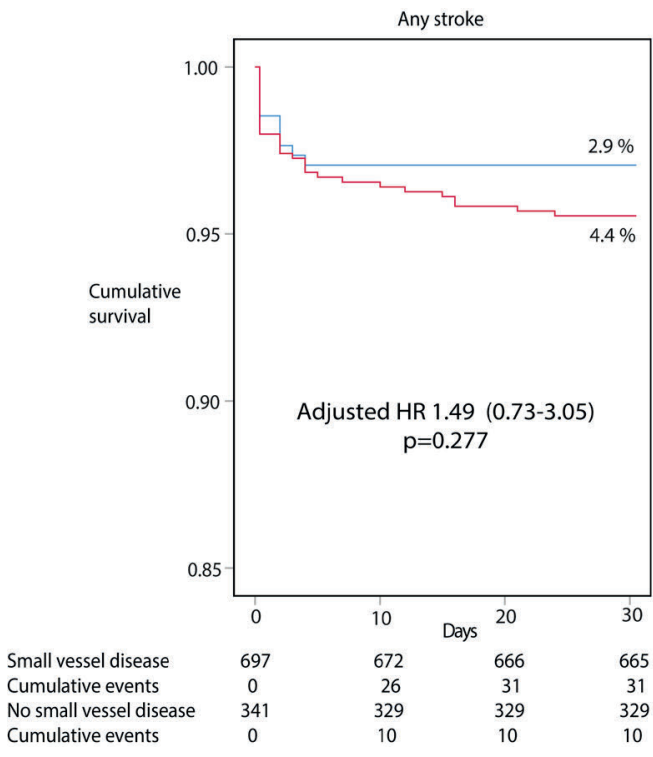

$\neg$ No small vessel disease

$\neg$ Small vessel disease

Figure C2. Any stroke during 30 days of follow-up 
Table 3. Cox-proportional hazards analysis of 30-day perioperative risks for secondary adverse events after CEA

\begin{tabular}{lllllll}
\hline & & & $\begin{array}{l}\text { Univariate } \\
\text { analysis }\end{array}$ & \multicolumn{3}{c}{$\begin{array}{l}\text { Multivariable } \\
\text { analysis }\end{array}$} \\
\cline { 4 - 7 } $\begin{array}{l}\text { Perioperative } \\
\text { outcome measure }\end{array}$ & $\begin{array}{l}\text { No small } \\
\text { vessel disease }\end{array}$ & $\begin{array}{l}\text { Small vessel } \\
\text { disease }\end{array}$ & HR [95\%CI] & p-value & HR [95\%CI] & p-value \\
\hline $\begin{array}{l}\text { Any stroke or } \\
\text { cardiovascular death }\end{array}$ & $12 / 341(3.5 \%)$ & $31 / 697(4.4 \%)$ & $1.27[0.65-2.47]$ & 0.484 & $1.20[0.61-2.35]$ & 0.592 \\
Any stroke $^{\mathrm{b}}$ & $10 / 341(2.9 \%)$ & $31 / 697(4.4 \%)$ & $1.52[0.75-3.11]$ & 0.248 & $1.49[0.73-3.05]$ & 0.277
\end{tabular}

HR, hazard ratio. HR > 1.0 indicates an increased risk for patients with cerebral small vessel disease compared to patients without. Numbers in bold indicate $\mathrm{p}<0.05$.

a Covariates included in multivariable model of any stroke or cardiovascular death were cerebrovascular symptoms, diabetes and contralateral stenosis (50-100\%).

${ }^{\mathrm{b}}$ Covariates included in multivariable model of any stroke were diabetes and contralateral stenosis (50-100\%)

$5.3 \%) .^{3}$ It is worth noting that the overall 30 -day perioperative stroke or death rate in the NASCET (6.7\%) was higher than the CEA cohort of the ICSS (3.4\%) and our cohort (4.1\%). These differences could be attributed to the improved surgical techniques and novel insights in anesthesiologic management. ${ }^{19}$ Moreover, it has been shown that concomitantly with improved cardiovascular prevention therapy, atherosclerotic plaques have stabilized over time. ${ }^{20}$ It could be hypothesized that due to plaque stabilization the occurrence of procedural embolisms has been reduced, resulting in lower stroke rates. Additionally, perioperative stroke is multifactorial, varying from thrombosis or thrombotic occlusion of the operated carotid artery, hyperperfusion syndrome, carotid-embolism or cardio-embolism and intraoperative hypoperfusion. ${ }^{21}$ Therefore SVD may not be one of the main predictors for these heterogeneous types of stroke with various pathophysiological mechanisms.

Regarding the long-term postoperative risk of stroke, the only available evidence results from the aforementioned NASCET that observed an increased 3-year postoperative stroke risk in patients with more extensive WMLs. ${ }^{3}$ Of note, increased long-term stroke risk was also observed in the medically treated group of this study. As discussed above, the NASCET cohort does not reflect the current stroke risk in patients undergoing CEA. Although WMLs predict recurrent stroke in patients with ischemic stroke in general ${ }^{5}$, these results cannot be directly extrapolated to patients with carotid stenosis because the risks of future cardiovascular events and survival varies between different etiologies of ischemic stroke. ${ }^{22,23}$ Furthermore, prevalence of WMLs in our study was higher than previously described ${ }^{3}$, likely as a result of more sensitive scanning techniques. Earlier studies may have selected patients with more severe WML, resulting in more pronounced differences between groups with, as a consequence, a stronger association between WML and short- and long-term 
outcome events. We cannot exclude that preoperative reported SVD may have a small predictive value for stroke but larger cohort studies, including assessment of WML load and localization, would be needed to confirm this.

Our finding that increased postoperative long-term risk of cardiovascular death in symptomatic patients undergoing CEA is associated with reported SVD is in line with a previous small study ${ }^{8}$, which showed that CEA patients (predominantly symptomatic) with any quantified WML on preoperative brain CT had an increased 5-year risk of mortality (predominantly of cardiovascular origin). We now show that, also based on standard brain imaging reports, SVD can be used as predictor for cardiovascular death. Preoperative SVD was associated with all-cause death in univariate analysis, yet this effect was diminished in multivariable analysis implying that SVD is not independently associated with all-cause mortality. This discrepancy with the previous study ${ }^{8}$ could be attributed to the shorter follow-up period or could be explained by the fact that the previous study did not fully adjust for confounders due to small sample size. The overall increased risk of cardiovascular death can be explained by the concept of systemic SVD due to generalized atherosclerotic disease, resulting in end organ manifestations such as kidney failure or cardiomyopathy. ${ }^{24,25}$ Furthermore, it is suggested that endothelial dysfunction in both cerebral arteries and systemic arteries is increased in patients with more severe WMLs. ${ }^{26} \mathrm{~A}$ shared etiology between SVD and cardiovascular death specifically is supported by the fact that no differences in all-cause death were found between patients with and those without SVD. Reliable estimates for long-term survival rates in patients qualifying for CEA are important to determine the absolute net-benefit of the procedure. As long-term recurrent stroke risk has decreased for symptomatic patients with carotid artery disease because of improved preventive medical strategies, the number-needed-to-treat of CEA may be higher than previously determined. When patients have a shorter life-expectancy due to severe systemic atherosclerotic disease, the duration of benefit of a revascularization procedure will be shorter. When assessment of SVD becomes part of standard clinical workup this can be used in future risk stratification algorithms that use multiple demographic, biological and imaging characteristics for decision making on optimal treatment strategy. ${ }^{27}$

\section{Strengths and limitations}

This study has several limitations. First, both CT and MRI brain scan reports were used, while MRI is more sensitive in detection of small WMLs. ${ }^{28}$ This could explain the higher prevalence of SVD found in the MRI subgroup (83.3\%) compared with the CT subgroup (57.8\%). Subgroup analyses confirmed the association of SVD with cardiovascular death in the CT-subcohort, but in the MRI-subcohort this could not be determined because of the limited sample size and limited number of events. However, both CT and MRI are 
interchangeably used in routine clinical practice and this study addresses its clinical relevance. Furthermore, differences in event-rates between patients with and without SVD are similar in CT and MRI subgroups and thus type of imaging modality does not seem to have large impact on the results. Yet, MRI may have led to the detection of minor lesions that may have been missed with CT. Patients with more severe lesions may have a higher risk of generalized atherosclerotic disease and are therefore at higher risk of CV death. Detection of all (minor and major) lesions in MRI-patients may have diluted the effect on outcome in these patients. As MRI is increasingly being used in clinical setting for diagnosis of stroke, future studies of larger sample size, also focusing on the difference between minor and major lesions, are needed to elaborate the association of preoperative SVD based on MRI and cardiovascular death. Second, CT and MRI imaging were performed with different scanners and different protocols due to the broad study time. This study therefore reflects routine clinical care. Third, in our analysis no distinction was made between ipsi- and contralateral or between mild and severe SVD. One can imagine that ipsilateral SVD is more likely to be attributable to thromboembolic events due to ipsilateral carotid stenosis, whereas bilateral WML may be an expression of generalized atherosclerotic disease. Semiquantitative methods for assessment of severity of WML are widely available and generally well-known but are not consistently used in standard practice. By pooling all patients with any reported WML we might have underestimated the correlation between SVD and outcome. Yet, previous studies that did investigate the correlation between (semi-) quantitative WML measures and outcome in patients undergoing CEA used trained radiologists for assessment of these study parameter which has the disadvantage of not being part of standard clinical practice. ${ }^{3,8,9}$ Fourth, we only included symptomatic patients whereas assessment of perioperative risk in asymptomatic patients may be even more relevant in the ongoing discussion on whether or not to perform revascularization procedures in these patients. Finally, stroke was less frequently observed for excluded patients compared to included patients (see Supplemental Table S4), mainly because imaging reports were not attached in the referral from other hospitals. Excluded patients from the present study could be considered as lower risk patients, which would have rather led to an overestimation of the association between SVD and stroke and would not have changed our conclusion.

Despite limitations, the present study includes a large CEA cohort reflecting current incidences of perioperative and postoperative adverse events. Additionally, the study design mimics daily practice in which clinicians evaluate the presence of SVD based on brain imaging reports constructed by radiologists through standardized protocols and not using non-standard quantification techniques. Future research should focus on how preoperative baseline imaging can be used for determining optimal treatment strategy. ${ }^{29}$ Such studies 
should also focus on the relation between WML and other promising imaging markers such as (silent) ischemic brain lesions, which have been associated with poor outcome and WMLs. ${ }^{30,31}$ Studies should report predictive value of both ipsi- and contralateral cerebral lesions, should investigate methods for easy identification and quantification of lesions, and should stratify symptomatic from asymptomatic patients in their analysis.

\section{Conclusion}

In clinical practice, SVD in standard preoperative brain imaging reports is independently associated with general increased risk of long-term cardiovascular death for symptomatic patients undergoing CEA. Yet, presence of preoperative SVD does not seem valuable to predict the 30-day perioperative stroke nor long-term stroke-risk in these patients.

\section{Acknowledgements}

We thank Sara van Laar, Evelyn Velema and Joelle van Bennekom for their technical support in the Athero-Express Biobank.

\section{Conflict of interest}

none

\section{Financial support}

Funding was obtained from the EU 755320 Taxinomisis grant to GP, DdK and GJdB. There are no competing interests declared. 


\section{References}

1 International Carotid Stenting Study investigators, Ederle J, Dobson J, Featherstone RL, Bonati LH, van der Worp HB, et al. Carotid artery stenting compared with endarterectomy in patients with symptomatic carotid stenosis (International Carotid Stenting Study): an interim analysis of a randomised controlled trial. Lancet 2010;375(9719):985-97.

2 Naylor AR, Ricco J-B, de Borst GJ, Debus S, de Haro J, Halliday A, et al. Editor's Choice - Management of Atherosclerotic Carotid and Vertebral Artery Disease: 2017 Clinical Practice Guidelines of the European Society for Vascular Surgery (ESVS). Eur J Vasc Endovasc Surg 2018;55(1):3-81.

3 Streifler JY, Eliasziw M, Benavente OR, Alamowitch S, Fox AJ, Hachinski VC, et al. Prognostic importance of leukoaraiosis in patients with symptomatic internal carotid artery stenosis. Stroke 2002;33(6):1651-5.

4 Debette S, Beiser A, DeCarli C, Au R, Himali JJ, Kelly-Hayes M, et al. Association of MRI Markers of Vascular Brain Injury With Incident Stroke, Mild Cognitive Impairment, Dementia, and Mortality. Stroke 2010;41(4):600-6.

5 Chutinet A, Rost NS. White matter disease as a biomarker for long-term cerebrovascular disease and dementia. Curr Treat Options Cardiovasc Med 2014;16(3):292.

6 Black S, Gao F, Bilbao J. Understanding white matter disease: Imaging-pathological correlations in vascular cognitive impairment. Stroke, vol. 40. 2009. pp. S48-52.

7 Topakian R, Barrick T, Howe F, Markus H. Blood-brain barrier permeability is increased in normal appearing white matter in patients with lacunar stroke and leukoaraiosis. J Neurol Neurosurg Psychiatry 2010;81(2):1-26.

8 Oksala N, Jaroma M, Pienimaki J-P, Kuorilehto T, Vanttinen T, Lehtomaki A, et al. Preoperative white matter lesions are independent predictors of long-term survival after internal carotid endarterectomy. Cerebrovasc Dis Extra 2014;4(2):122-31.

9 Ederle J, Davagnanam I, van der Worp HB, Venables GS, Lyrer PA, Featherstone RL, et al. Effect of whitematter lesions on the risk of periprocedural stroke after carotid artery stenting versus endarterectomy in the International Carotid Stenting Study (ICSS): A prespecified analysis of data from a randomised trial. Lancet Neurol 2013;12(9):866-72.

10 O'Sullivan M, Lythgoe DJ, Pereira AC, Summers PE, Jarosz JM, Williams SCR, et al. Patterns of cerebral blood flow reduction in patients with ischemic leukoaraiosis. Neurology 2002;59(3):321-6.

11 Verhoeven BAN, Velema E, Schoneveld AH, de Vries JPPM, de Bruin P, Seldenrijk CA, et al. Atheroexpress: differential atherosclerotic plaque expression of mRNA and protein in relation to cardiovascular events and patient characteristics. Rationale and design. Eur J Epidemiol 2004;19(12):1127-33.

12 Hellings WE, Peeters W, Moll FL, Piers SRD, van Setten J, Van der Spek PJ, et al. Composition of Carotid Atherosclerotic Plaque Is Associated With Cardiovascular Outcome. Circulation 2010;121(17):1941-50.

13 North American Symptomatic Carotid Endarterectomy Trial Collaborators, Barnett HJM, Taylor DW, Haynes RB, Sackett DL, Peerless SJ, et al. Beneficial effect of carotid endarterectomy in symptomatic patients with high-grade carotid stenosis. N Engl J Med 1991;325(7):445-53.

14 Barnett HJ, Taylor DW, Eliasziw M, Fox AJ, Ferguson GG, Haynes RB, et al. Benefit of carotid endarterectomy in patients with symptomatic moderate or severe stenosis. North American Symptomatic Carotid Endarterectomy Trial Collaborators. N Engl J Med 1998;339(20):1415-25.

15 Halliday A, Mansfield A, Marro J, Peto C, Peto R, Potter J, et al. Prevention of disabling and fatal strokes by successful carotid endarterectomy in patients without recent neurological symptoms: randomised controlled trial. Lancet 2004;363(9420):1491-502.

16 Warlow C, Farrell B, Fraser A, Sandercock P SJ. Randomised trial of endarterectomy for recently symptomatic carotid stenosis: final results of the MRC European Carotid Surgery Trial (ECST). Lancet 1998;351(9113):1379-87.

17 Rothwell P, Eliasziw M, Gutnikov S, Fox A, Taylor D, Mayberg M, et al. Analysis of pooled data from the randomised controlled trials of endarterectomy for symptomatic carotid stenosis. Lancet 2003;361(9352):107-16.

18 von Allmen RS, Weiss S, Tevaearai HT, Kuemmerli C, Tinner C, Carrel TP, et al. Completeness of FollowUp Determines Validity of Study Findings: Results of a Prospective Repeated Measures Cohort Study. PLoS One 2015;10(10):e0140817. 
19 Kragsterman B, Pärsson H, Lindbäck J, Bergqvist D, Björck M. Outcomes of carotid endarterectomy for asymptomatic stenosis in Sweden are improving: Results from a population-based registry. J Vasc Surg 2006;44(1):79-85.

20 van Lammeren GW, den Ruijter HM, Vrijenhoek JEP, van der Laan SW, Velema E, de Vries J-PPM, et al. Time-Dependent Changes in Atherosclerotic Plaque Composition in Patients Undergoing Carotid Surgery. Circulation 2014;129(22):2269-76.

21 Huibers A, Calvet D, Kennedy F, Czuriga-Kovács KR, Featherstone RL, Moll FL, et al. Mechanism of Procedural Stroke Following Carotid Endarterectomy or Carotid Artery Stenting Within the International Carotid Stenting Study (ICSS) Randomised Trial. Eur J Vasc Endovasc Surg 2015;50(3):281-8.

22 Melkas S, Sibolt G, Oksala NKJ, Putaala J, Pohjasvaara T, Kaste M, et al. Extensive white matter changes predict stroke recurrence up to 5 years after a first-ever ischemic stroke. Cerebrovasc Dis 2012;34(3):191-8.

23 Ois A, Cuadrado-Godia E, Rodriguez-Campello A, Giralt-Steinhauer E, Jimenez-Conde J, Lopez-Cuina M, et al. Relevance of stroke subtype in vascular risk prediction. Neurology 2013;81(6):575-80.

24 Oksala NKJ, Salonen T, Strandberg T, Oksala A, Pohjasvaara T, Kaste M, et al. Cerebral small vessel disease and kidney function predict long-term survival in patients with acute stroke. Stroke 2010;41(9):1914-20.

25 Melkas S, Putaala J, Oksala NKJ, Pohjasvaara T, Oksala A, Kaste M, et al. Small-vessel disease relates to poor poststroke survival in a 12-year follow-up. Neurology 2011;76(8):734-9.

26 Zupan M, Šabović M, Zaletel M, Popovič KŠ, Žvan B. The presence of cerebral and/or systemic endothelial dysfunction in patients with leukoaraiosis - a case control pilot study. BMC Neurol 2015;15(1):158.

27 TAXINOMISIS Project Clinical Trials.gov (2018). Stratification of patient with carotid disease (TAXINOMISIS). [online] Available at: https://clinicaltrials.gov/ct2/show/NCT03495830 [Accessed 16 Aug. 2019]

28 Wahlund LO, Barkhof F, Fazekas F, Bronge L, Augustin M, Sjögren M, et al. A New Rating Scale for AgeRelated White Matter Changes Applicable to MRI and CT. Stroke 2001;32(6):1318-22.

29 Rots ML, van der Lugt A, de Borst GJ. Surrogate Markers and Reporting Standards for Outcome After Carotid Intervention. Eur J Vasc Endovasc Surg 2019;58(6):794-5.

30 Streifler JY, den Hartog AG, Pan S, Pan H, Bulbulia R, Thomas DJ, et al. Ten-year risk of stroke in patients with previous cerebral infarction and the impact of carotid surgery in the Asymptomatic Carotid Surgery Trial. Int J Stroke 2016;11(9):1020-7.

31 Pini R, Faggioli G, Longhi M, Vacirca A, Gallitto E, Freyrie A, et al. The detrimental impact of silent cerebral infarcts on asymptomatic carotid endarterectomy outcome. J Vasc Surg 2016;64(1):15-24. 


\section{Supplementary Materials}

The following supplementary material is omitted due to space limitation and can be found at the journal website:

- Supplemental I. Synonyms for SVD

- Supplemental II. Covariates added in multivariable analysis

- Supplemental III. Percentages of missing values in covariates

- Supplemental IV. Baseline characteristics of excluded patients with missing brain imaging reports

- Supplemental V. Validation of SVD reporting in standard clinical imaging reports

- Supplemental VI. 3-year risk for all-cause death after CEA

- Supplemental VII. Subgroup analyses (CT and MRI) 



\section{PART III}

\section{Circulating Biomarkers}

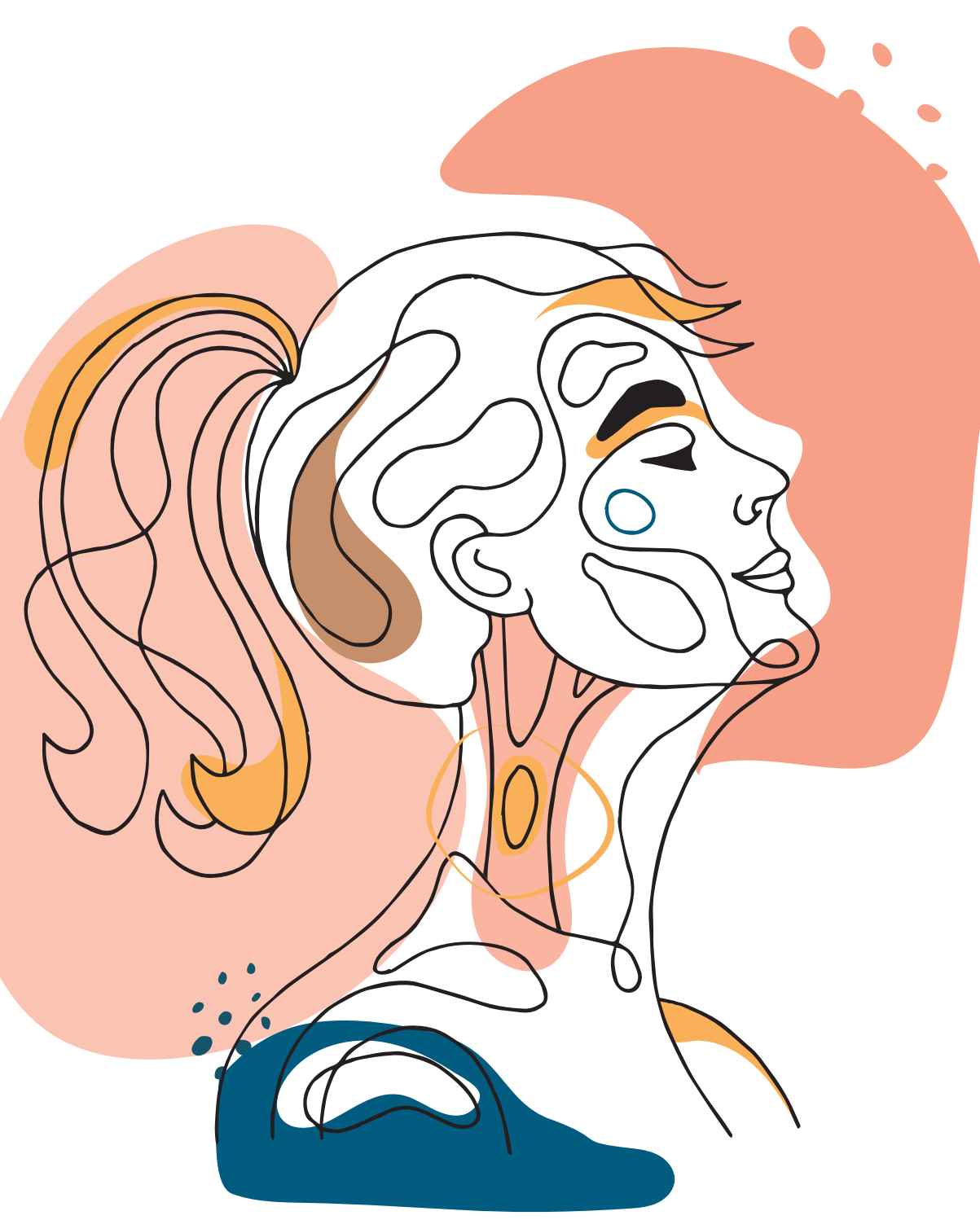




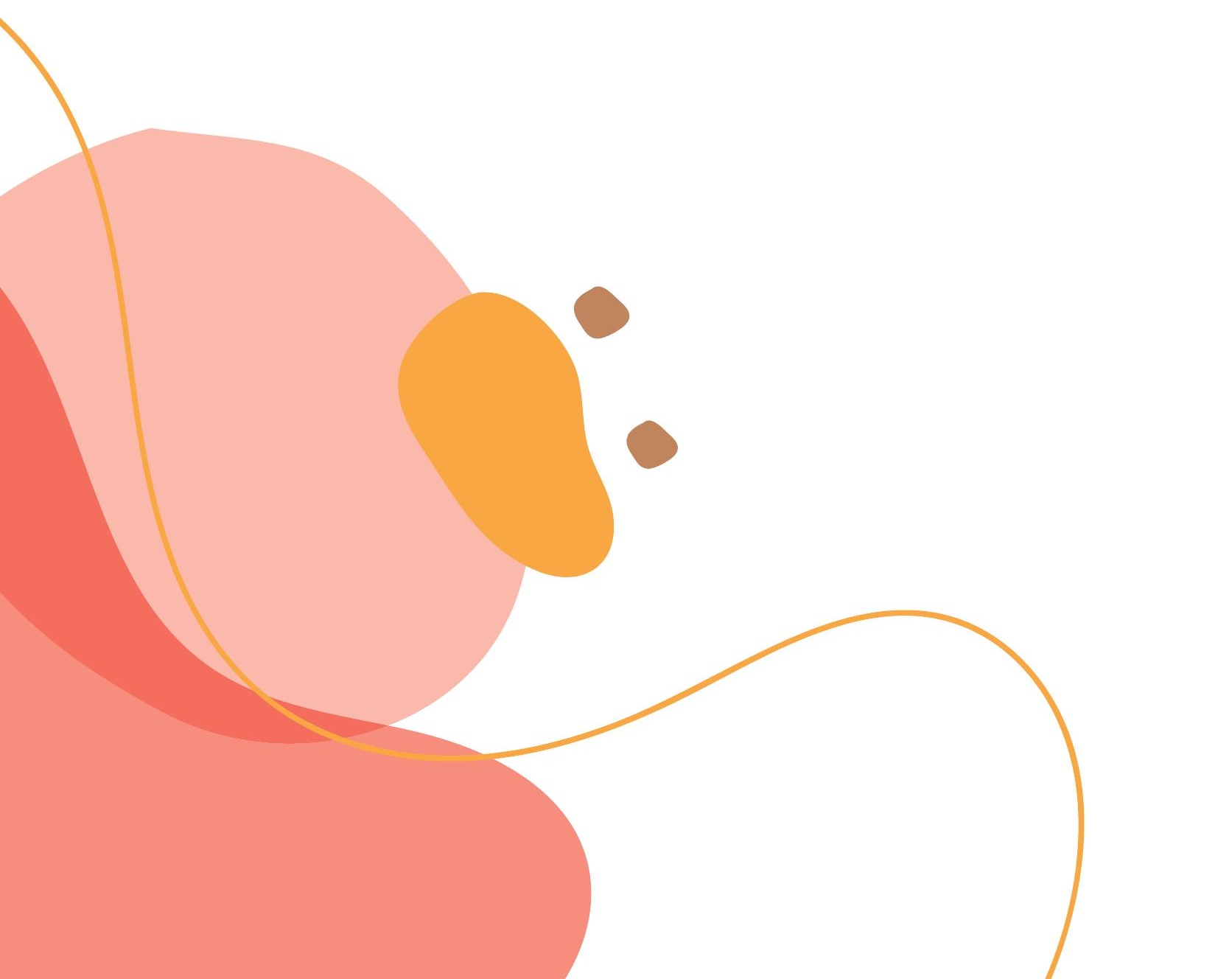




\section{Pre-operative plasma extracellular vesicle proteins are associated with a high risk of long term secondary major cardiovascular events in patients undergoing carotid endarterectomy}

European Journal of Vascular and Endovascular Surgery (in press)

Nathalie Timmerman* Farahnaz Waissi* Mirthe Dekker Qiu Ying van de Pol Joelle van Bennekom

Arjan Schoneveld Marjet J.M. klein Avink Robbert J. de Winter Gerard Pasterkamp Gert J. de Borst Dominique P.V. de Kleijn 


\section{Abstract}

\section{Background}

Patients undergoing carotid endarterectomy (CEA) maintain a substantial residual risk for major cardiovascular events (MACE). Improved risk-stratification is warranted to select high-risk patients qualifying for secondary add-on therapy. Plasma extracellular vesicles (EVs) are involved in atherothrombotic processes and their content has been related to the presence and recurrence of cardiovascular events. We assessed the association between preoperative levels of five cardiovascular disease related proteins in plasma EVs and the postoperative risk of MACE.

\section{Methods}

In 864 patients undergoing CEA from 2002-2016 included in the Athero-Express biobank, three plasma EV-subfractions (LDL, HDL and TEX) were isolated from preoperative blood samples. Five proteins were quantified in each EV-subfraction using an electrochemiluminescence immunoassay: Cystatin C, Serpin C1, Serpin G1, Serpin F2 and CD14. The association between EV protein levels and the three-year postoperative risk of MACE (any stroke, myocardial infarction or cardiovascular death) was evaluated using multivariable Cox-proportional hazard regression analyses.

\section{Results}

During a median follow-up of 3.0 years (IQR: 2.2-3.0), 137 (16\%) patients developed MACE. In the HDL-EV subfraction, increased levels of CD14, Cystatin C, Serpin F2 and Serpin C1 were associated with an increased risk of MACE (adjusted HRs per one SD increase [95\% CI] of 1.30 [1.15-1.48], 1.22 [1.06-1.42], 1.36 [1.16-1.61] and 1.29 [1.10-1.51], respectively), independently of cardiovascular risk factors. No significant associations were found for Serpin G1. CD14 improved the predictive value of the clinical model encompassing cardiovascular risk factors (net reclassification improvement, NRI $=0.16,95 \%$ CI 0.08 $0.25)$.

\section{Conclusion}

EV-derived preoperative plasma levels of Cystatin C, Serpin C1, CD14 and Serpin F2 were independently associated with an increased long-term risk of MACE following CEA and are thus markers for residual cardiovascular risk. EV-derived CD14 levels could improve identification of high-risk patients that may benefit from secondary preventive add-on therapy in order to reduce future MACE risk. 


\section{What this paper adds}

Patients treated with carotid endarterectomy (CEA) maintain a high risk for future major cardiovascular events (MACE), although this risk varies substantially across individual patients. With emerging novel options to intensify medical treatment, risk stratification for MACE is warranted. This study demonstrated that preoperative levels of four proteins (CD14, Cystatin C, Serpin C1, and Serpin F2) in plasma extracellular vesicles (EVs) were positively associated to increased risks of MACE after CEA. EV-CD14 improved risk stratification for MACE on top of cardiovascular risk factors. EV-CD14 may thus be a useful marker to select high-risk patients that qualify for intensified medical treatment.

\section{Introduction}

Carotid endarterectomy is an effective measure to reduce the risk of future ipsilateral stroke in patients with carotid stenosis. ${ }^{1,2}$ These patients, however, remain a high risk population with an estimated residual risk for future cardiovascular events (CVE) up to $20 \%$ due to the systemic nature of atherosclerosis. ${ }^{3}$ This residual risk persists even after adequate control of vascular risk factors by secondary preventive strategies. ${ }^{4}$ European guidelines recommend to intensify the medical treatment in selected very high-risk patients by increasing the dose of lipid-lowering drugs or adding "add-on therapies" (such as PCSK-9 inhibitors). ${ }^{5}$ Moreover, recent studies with anti-inflammatory drugs (e.g. colchicine) as potential add-on therapy showed promising results in reducing CVE. ${ }^{6,7}$ According to the European guidelines ${ }^{5}$, all CEA patients are currently classified in one very high-risk category despite it is known that the risk for future CVE varies significantly between individuals. ${ }^{4}$ Personalization of secondary preventive strategies and often expensive add-on therapies remains important to lower the rate of recurrent CVE by keeping control on health care costs and prevent patients from unnecessary drug side effects. Therefore, risk-stratification algorithms are warranted that accurately predict individuals at high risk for future CVE. Clinical prediction models have limited ability to identify those patients at high risk. ${ }^{8}$ For this, biomarkers are needed to improve the predictive value of these risk-stratification models.

Plasma extracellular vesicles (EVs) are raising interest as potential biomarkers for cardiovascular disease (CVD). ${ }^{9}$ EVs are small bilayer membrane vesicles including microvesicles, microparticles and exosomes, ranging from 50-1000 $\mathrm{nm}$ in diameter, and are released during cell activation or apoptosis. ${ }^{9}$ EVs contain bioactive content (proteins, lipids, RNA and microRNA) from their parent cell and are acknowledged messengers for intercellular communication. ${ }^{10}$ EVs have been related to (patho-)physiological processes 
including thrombosis, inflammation, cell survival, endothelial dysfunction, angiogenesis as well as atherosclerotic plaque progression. ${ }^{10}$ Since EVs carry information about cellular processes of the cell of origin, EVs are referred as liquid biopsies making them a promising biomarker source. Previously, protein levels in plasma EVs were associated with recurrent vascular events in patients with established CVD. ${ }^{11}$ However, these specific EV proteins are not yet investigated in patients undergoing CEA. Furthermore, it is known that EVs vary in size and properties and that their cargo differs across EV subsets. ${ }^{9,12,13}$ Investigating the composition of EV subsets may improve predictive power, yet previous studies on future CVE did not distinguish between EV subsets.

In this study, we investigated whether preoperative levels of CVD-related proteins in plasma EVs subsets are associated with major cardiovascular events in patients undergoing CEA.

\section{Methods}

\section{Study population}

All patients participated in the ongoing prospective Athero-Express Biobank Study that has been initiated in 2002 and includes consecutive patients undergoing CEA in two major vascular centers in the Netherlands (University Medical Center, Utrecht and St. Antonius Hospital Nieuwegein). All patients signed informed consent. Indications for surgery were based on international and local guidelines and judged in a multidisciplinary vascular team. ${ }^{2,14-18}$ Preoperatively, a blood sample was taken and stored at $-80^{\circ} \mathrm{C}$ until isolation of plasma EV subsets for measurements. Baseline characteristics (demographics, medical history, medication use and cardiovascular risk factors) were collected by standardized questionnaires and medical records. During CEA, the atherosclerotic plaque was collected and subsequently immunohistochemically analyzed. Patients were followed up for three years after CEA for CVE. For an extensive description of the Athero-Express study design we refer to previous publications., ${ }^{3,19}$

Patients that underwent CEA from April 1, 2002 until December 31, 2016 with available citrate plasma samples and follow-up data were considered eligible for the current study. Patients operated for restenosis were excluded. This study was approved by ethics committees of both study sites and was conducted following the ethical principles of the Declaration of Helsinki.

\section{Follow-up}

Patients were asked once per year to fill out standardized questionnaires regarding experienced CVE and hospital admissions. When a vascular event was suspected, additional documentation regarding the CVE were requested from hospital medical records and the 
general practitioner. In case a patient did not respond to the questionnaires, the general practitioner was contacted to provide follow-up information. All outcome events were evaluated by two independent observers. If disagreement existed, a third expert (GJdB) was asked. The criteria for outcome events were reported previously. ${ }^{19}$

\section{Study endpoints}

The primary endpoint of this study was the three-year postoperative risk of MACE defined as a composite of nonfatal or fatal myocardial infarction (MI), any nonfatal or fatal ischemic or hemorrhagic stroke, and cardiovascular death due to other causes (fatal heart failure, fatal abdominal aneurysm rupture and sudden cardiac death). Since histological atherosclerotic plaque characteristics are established markers for future $\mathrm{CVE}^{3}$, secondary endpoints of the study were associations between EV-subset proteins and atherosclerotic plaque characteristics in order to unravel underlying pathobiological mechanisms.

\section{Biomarkers}

The following EV proteins as potential biomarkers were selected based on previous studies: CD14, Cystatin C, Serpin F2, Serpin G1 and Serpin C1. ${ }^{11-13}$

\section{Isolation of extracellular vesicle subsets}

Three subsets of plasma extracellular vesicles (EVs) were isolated using previously published protocols. ${ }^{13,20}$ The five proteins were measured in all three subsets of plasma EVs. A more detailed description of the isolation procedure of the EV subsets and quantification of proteins is included in the Supplemental Materials.

In short, one plasma EV-subset co-precipitates with Low-Density Lipid (LDL) particles whilst another EV-subset co-precipitates with High-Density Lipid (HDL) particles allowing for isolation of two plasma EV-subsets. These EV-subsets are respectively named as the LDL-EV subfraction and HDL-EV subfraction. For precipitation of LDL-EV subfraction and HDL-EV subfraction solutions of Dextran Sulphate (MP Biomedicals) and Manganese (II) Chloride (Sigma-Aldrich) were used (Supplemental Materials Figure S1). A third EVsubfraction, named TEX-EV fraction (Tiny Extracellular vesicle fraction), was obtained without HDL- or LDL sub fractionation using Xtractt buffer, 1:4, Cavadis BV. Previous experiments that characterized EV size showed relatively large EVs in the HDL fraction (mean $120 \mathrm{~nm}$ ), slightly smaller EVs in LDL fraction (mean $101 \mathrm{~nm}$ ) and the smallest EVs in the TEX fraction (mean $84 \mathrm{~nm}){ }^{13}$

Isolation of EVs from plasma subfractions was performed with magnetic dextran nanoparticles (Nanomag ${ }^{\circledR}$-D plain, Nanomag ${ }^{\circledR}-\mathrm{D}$ PEG-OH, Micromod) and a bio-plex handheld magnet (Bio-Rad). The pellets with EVs were lysed with complete lysis-M buffer including protease inhibitors (Roche). 


\section{Quantification of protein levels in EVs}

Protein levels were quantified in all three EV-subsets by electrochemiluminescence immunoassay (Quickplex SQ120, Meso Scale) and original essay units were expressed as $\mathrm{pg} / \mathrm{mL}$. To be able to correct for loss of pellet during the isolation steps, a standard quantity of synthetic liposomes (coated with DSG-PEG2000, Sunbright and fluorescently labelled with 18:1 Liss Rhod PE, MERCK) was added to each plasma sample and quantified afterwards.

\section{Histological atherosclerotic plaque examination}

The atherosclerotic plaque was processed and immunohistochemically analyzed for the amount of macrophages, smooth muscle cells (SMCs), collagen, intraplaque hemorrhage (IPH), microvessels, lipid core and calcifications by the standardized protocol of the AtheroExpress. ${ }^{3,19} \mathrm{~A}$ detailed description can be found in the Supplemental Materials. The content of macrophages, SMCs and collagen was semi-quantitatively scored as no to minor staining and moderate to heavy staining. IPH was scored as absent or present. The size of the lipid core was visually estimated and scored as $<10 \%, 10-40 \%$ and $>40 \%$ of the total plaque area. Additional quantitative analyses of SMCs, macrophages and microvessels were performed using computerized software (AnalySIS 3.2, Soft Imaging Systems GmbH, Munster, Germany). SMCs and macrophages were expressed as percentages of total plaque area. Microvessels were counted in three hotspots and expressed as an averaged number per square millimeter.

\section{Statistical analyses}

All analyses were conducted in R statistical software version 3.6.2 (R Foundation for Statistical Computing, Vienna, Austria; https://www.r-project.org/). All statistical tests were two-sided and the significance level was set on $\mathrm{p}<0.05$.

\section{Associations with MACE}

Continuous baseline characteristics were compared using Student's t-test or Mann-Whitney $\mathrm{U}$ and categorical baseline characteristics by Chi-square tests. Univariable and multivariable Cox-proportional hazard models were applied to analyze the associations of the EV protein levels with MACE. Protein levels in EVs, baseline cholesterol and creatinine levels were logarithmically transformed to obtain normal distributions. To enable direct comparison between different proteins, protein levels in EVs were standardized to Z-scores and hazard ratios (HR) per one standard deviation (SD) increase in protein levels were calculated. Confounders for multivariable analyses were selected a priori based on existing literature 8,11,21-23: age, sex, history of coronary artery disease (CAD) and/or peripheral artery disease (PAD), cerebrovascular symptoms, current smoking, hypertension, diabetes, lipid-lowering 
drug use, creatinine and contralateral carotid artery stenosis of 50-100\%. Backward elimination based on Akaike information criterion (AIC) resulted in a clinical model consisting of age, history of CAD and/or PAD, cerebrovascular symptoms, current smoking and creatinine. Kaplan-Meier graphs of quartile levels of the proteins were constructed to explore the type of association (linear or non-linear) with MACE in order to explore possible clinically applicable cut-off values. Percentages of missing data were low $(<0.5 \%)$ so no imputation was performed (Supplemental Table S2).

\section{Prediction model development and performance}

A possible added predictive value of EV proteins to the clinical model was investigated using stepwise backward regression modelling based on AIC. Both the clinical model and the biomarker model were bootstrapped to adjust for overfitting and overoptimistic performance. Subsequently, calibration was evaluated by visual inspection of calibration plots and discriminative performance was analyzed by C-statistic and Net Reclassification Index (NRI). As no predefined cutoff points are available for risk prediction for secondary CVE, we defined four risk categories based on the overall incidence (cutoff points at half of the overall incidence, the overall incidence and twice the overall incidence) in accordance with previous publications..$^{21,24}$

\section{Associations with plaque characteristics}

To gain more insight in potential underlying biological mechanisms between the associated EV proteins with MACE, we examined the associations of these specific EV proteins with vulnerable histological plaque characteristics by logistic and linear regression analyses. Additional confounders to the above-mentioned multivariable model were added based on literature: $\operatorname{sex}^{25}$, year of surgery ${ }^{26}$, lipid-lowering drug use $\mathrm{e}^{27}$, hypertension ${ }^{28}$, anticoagulant drug use ${ }^{29}$ (for IPH and microvessels only).

\section{Results}

Of the 887 patients that fulfilled inclusion and exclusion criteria, 23 had insufficient sample material for EV isolation or failure of protein measurements (Figure 1). A final number of 864 patients with at least one valid protein measurement was left for analyses. The availability of protein measurements is displayed in the Supplemental (Table S3).

\section{Study population}

Baseline characteristics are stated in Table 1 . The mean age was $69 \pm 9.2$ and $70 \%$ were men. The cohort typically reflects a severe atherosclerotic cohort with a high prevalence of 
cardiovascular risk factors, history of cardiovascular diseases (31\% of the patients were previously diagnosed with CAD, 21\% with PAD and 33\% with any stroke), and use of secondary preventive medications (79\% of the patients were on lipid-lowering therapy, $88 \%$ on antiplatelets, $11 \%$ on anticoagulants). After a median follow-up time of 3.0 years [IQR: 2.2.-3.0], 137 (16\%) patients with MACE were observed of which 73 (8.4\%) had stroke as first event, 44 (5.1\%) had MI and 20 (2.3\%) died due to other cardiovascular causes. Patients that experienced a MACE were on average older (72.1 vs. 68.8), had higher baseline creatinine levels and lower HDL-cholesterol compared to patients that did not experience MACE. A history of CAD, PAD and diabetes were more common among those that experienced MACE.

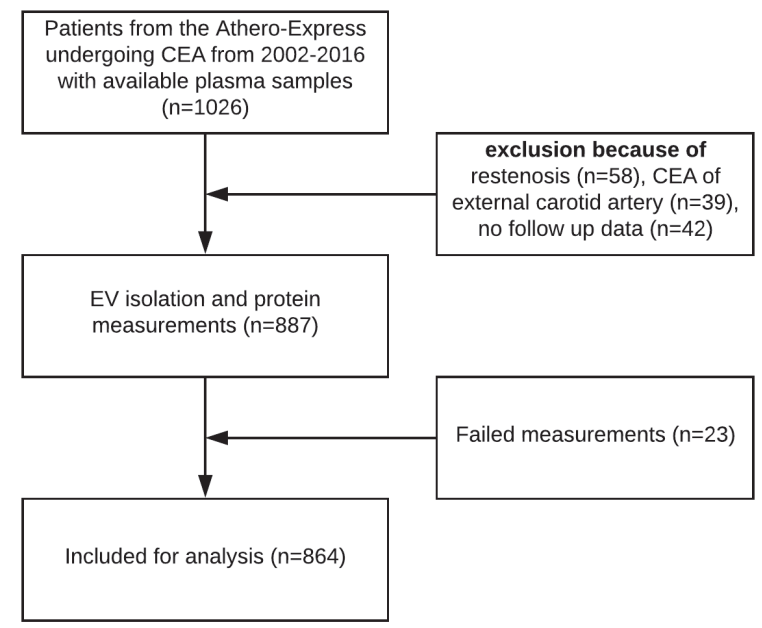

Figure 1. Flowchart

\section{EV protein levels and the risk of MACE}

Median and interquartile levels of the proteins in EV-subsets are stated in the Supplemental Table S3. Multivariable Cox-regression analyses corrected for cardiovascular risk factors (age, history of CAD and/or PAD, cerebrovascular symptoms, current smoking and creatinine levels) showed that four of the five proteins in the HDL-EV subfraction were independently associated with MACE (Table 2 and Supplemental Tables S4a-d). Increased levels of CD14, Cystatin C, Serpin F2 and Serpin C1 were associated with an increased risk of MACE (adjusted HRs per one SD increase [95\% CI] of 1.30 [1.15-1.48], 1.22 [1.06-1.42], 1.36 [1.16-1.61] and 1.29 [1.10-1.51] respectively). No associations were found in the LDL- 
Table 1. Baseline characteristics

\begin{tabular}{|c|c|c|c|}
\hline & $\begin{array}{l}\text { no MACE } \\
(\mathrm{n}=727)\end{array}$ & $\begin{array}{l}\text { MACE } \\
(n=137)\end{array}$ & p-value \\
\hline \multicolumn{4}{|l|}{ Demographics } \\
\hline Age, years, mean (SD) & $68.8(9.2)$ & $72.1(9.1)$ & $<0.001$ \\
\hline Male & $502(69.1)$ & $102(74.5)$ & 0.245 \\
\hline Ipsilateral carotid artery stenosis degree & & & 0.133 \\
\hline $50-70 \%$ & $62(8.8)$ & $6(4.5)$ & \\
\hline $70-99 \%$ & $646(91.2)$ & $127(95.5)$ & \\
\hline \multicolumn{4}{|l|}{ Risk factors } \\
\hline $\begin{array}{l}\text { Contralateral carotid artery stenosis } \\
\text { of } 50-100 \%\end{array}$ & $291(44.3)$ & $66(52.0)$ & 0.135 \\
\hline BMI, mean (SD) & $26.3(3.9)$ & $26.7(3.9)$ & 0.232 \\
\hline Hypertension & $601(82.7)$ & $118(86.1)$ & 0.384 \\
\hline Creatinine, median [IQR] & $87.0[75.0,104.0]$ & $95.0[79.0,114.0]$ & 0.002 \\
\hline GFR, mL/min/1.73 m2, mean (SD) & $74.1(20.3)$ & $67.6(21.4)$ & 0.001 \\
\hline Current smoking & $233(32.1)$ & $52(38.0)$ & 0.215 \\
\hline Diabetes & $156(21.5)$ & $42(30.7)$ & 0.025 \\
\hline Hypercholesterolemia & $463(69.7)$ & $80(68.4)$ & 0.854 \\
\hline Triglycerides levels, mmol/L, median [IQR] & $1.5[1.1,2.1]$ & $1.5[1.1,2.0]$ & 0.768 \\
\hline LDL-C levels, mmol/L, median [IQR] & $2.3[1.8,3.0]$ & $2.3[1.8,3.0]$ & 0.496 \\
\hline HDL-C levels, mmol/L, median [IQR] & $1.1[0.9,1.3]$ & $0.9[0.8,1.1]$ & $<0.001$ \\
\hline Total cholesterol levels, mmol/L, median [IQR] & $4.2[3.5,5.2]$ & $4.1[3.5,4.9]$ & 0.128 \\
\hline \multicolumn{4}{|l|}{ Medical history } \\
\hline Preprocedural cerebrovascular symptoms & & & 0.177 \\
\hline asymptomatic & $302(41.5)$ & $44(32.1)$ & \\
\hline ocular & $77(10.6)$ & $16(11.7)$ & \\
\hline TIA & $203(28.0)$ & $39(28.5)$ & \\
\hline stroke & $145(19.9)$ & $38(27.7)$ & \\
\hline History of stroke & $233(32.0)$ & $55(40.1)$ & 0.081 \\
\hline History of CAD or PAD & & & 0.004 \\
\hline no history of CAD and PAD & $428(58.9)$ & $65(47.4)$ & \\
\hline history of CAD or PAD & $248(34.1)$ & $52(38.0)$ & \\
\hline history of CAD and PAD & $51(7.0)$ & $20(14.6)$ & \\
\hline \multicolumn{4}{|l|}{ Drug therapy } \\
\hline Anticoagulants & $70(9.6)$ & $20(14.6)$ & 0.112 \\
\hline Antiplatelets & $642(88.6)$ & $117(86.7)$ & 0.632 \\
\hline Lipid lowering drugs & $587(80.9)$ & $102(74.5)$ & 0.110 \\
\hline
\end{tabular}

Values are displayed as frequency, $\mathrm{n}(\%)$ unless otherwise indicated. Values in bold are p-value $<0.05$.

$\mathrm{SD}$, standard deviation; IQR, interquartile range, BMI, Body mass index; CAD, coronary artery disease; PAD, peripheral artery disease; GFR, estimated glomerular filtration rate calculated by MDRD equation; TIA, transient ischemic attack; LDL-C, Low-density lipoprotein cholesterol; HDL-C, high-density lipoprotein cholesterol. History of CAD includes previously reported angina, myocardial infarction, percutaneous coronary interventions or coronary artery bypass grafting. Hypertension, diabetes and hypercholesterolemia were defined as diagnosed by a medical doctor or use of specific medication. Antiplatelet drug comprises the use of aspirin, dipyridamole or any ADP-inhibitor. Lipid lowering drug use comprises the use of any lipid lowering drug. History of stroke includes any ipsilateral or contralateral stroke. Cerebrovascular symptoms refer to the ipsilateral symptoms on which the indication for CEA was based. Asymptomatic is defined as no experience of ipsilateral cerebrovascular symptoms in the six months prior to CEA. In the MACE group, few patients received both anticoagulant as antiplatelet therapy. 
EV or TEX-EV subfractions. Serpin G1 levels were not associated with MACE (Table 2). Since the prevalence of diabetes and baseline HDL-levels were significantly different between patients who developed MACE and those who did not, additional multivariable analyses also corrected for diabetes and HDL-levels next to above mentioned cardiovascular risk factors was performed and showed similar results (Supplemental Materials, Table S5). In order to explore possible clinically applicable cut-off values of the associated proteins in HDL-EVs, protein quartiles levels were further analyzed by Kaplan-Meier analyses (Figures 2A-2E). Non-linear associations with MACE were found for CD14 and Serpin F2, as patients in $4^{\text {th }}$ quartile were at much higher risk compared to the rest of the patients

Table 2. Associations of protein levels in EVs with 3-year risk of MACE after CEA

\begin{tabular}{|c|c|c|c|c|}
\hline \multirow[t]{2}{*}{ Biomarker } & \multicolumn{2}{|l|}{ Univariable } & \multicolumn{2}{|l|}{ Multivariable } \\
\hline & HR (95\% CI) & p-value & HR (95\% CI) & p-value \\
\hline \multicolumn{5}{|l|}{ CD14 } \\
\hline$H D L-E V s$ & $1.37(1.21-1.54)$ & $<0.001$ & $1.30(1.15-1.48)$ & $<0.001$ \\
\hline$L D L-E V s$ & $1.17(1.04-1.31)$ & 0.009 & $1.12(1.00-1.26)$ & 0.050 \\
\hline TEX-EVs & $1.07(0.92-1.24)$ & 0.376 & $1.04(0.90-1.21)$ & 0.605 \\
\hline \multicolumn{5}{|l|}{ Cystatin C } \\
\hline$H D L-E V s$ & $1.34(1.18-1.52)$ & $<0.001$ & $1.22(1.06-1.42)$ & 0.007 \\
\hline$L D L-E V s$ & $1.21(1.08-1.37)$ & 0.002 & $1.14(1.00-1.30)$ & 0.057 \\
\hline TEX-EVs & $1.21(1.05-1.39)$ & 0.008 & $1.10(0.94-1.29)$ & 0.244 \\
\hline \multicolumn{5}{|l|}{ Serpin F2 } \\
\hline$H D L-E V s$ & $1.29(1.1-1.52)$ & 0.002 & $1.36(1.16-1.61)$ & $<0.001$ \\
\hline$L D L-E V s$ & $1.07(0.89-1.29)$ & 0.489 & $1.13(0.93-1.36)$ & 0.225 \\
\hline$T E X-E V s$ & $1.09(0.92-1.28)$ & 0.326 & $1.14(0.97-1.35)$ & 0.113 \\
\hline \multicolumn{5}{|l|}{ Serpin G1 } \\
\hline$H D L-E V s$ & $1.14(0.99-1.31)$ & 0.065 & $1.11(0.98-1.27)$ & 0.107 \\
\hline$L D L-E V s$ & $0.96(0.76-1.20)$ & 0.699 & $0.95(0.77-1.17)$ & 0.623 \\
\hline$T E X-E V s$ & $0.87(0.73-1.04)$ & 0.121 & $0.87(0.73-1.03)$ & 0.108 \\
\hline \multicolumn{5}{|l|}{ Serpin C1 } \\
\hline$H D L-E V s$ & $1.31(1.11-1.53)$ & 0.001 & $1.29(1.10-1.51)$ & 0.002 \\
\hline$L D L-E V s$ & $1.03(0.86-1.23)$ & 0.719 & $1.05(0.88-1.25)$ & 0.561 \\
\hline$T E X-E V s$ & $1.04(0.90-1.20)$ & 0.632 & $1.03(0.91-1.18)$ & 0.621 \\
\hline
\end{tabular}

Protein levels were logarithmically transformed and standardized per SD. HR indicates the hazard ratio for the 3-year postoperative risk of MACE per one standard deviation increase in concentration of protein levels in three subsets of extracellular vesicles EVs (LDL, HDL and TEX EV subfraction). Multivariable cox-regression was corrected for age, history of CAD, history of peripheral artery disease, cerebrovascular symptoms, creatinine and current smoking. Values in bold indicate $\mathrm{p}<0.05$. CI, confidence interval. 
(unadjusted HR of quartile 4 (Q4) versus quartile 1 (Q1) of 2.29 [1.41-3.71] and 1.56 [0.982.48 ], respectively) (Figures $2 \mathrm{~A}$ and $2 \mathrm{~B}$ ). Cystatin $\mathrm{C}$ was linearly associated with MACE (unadjusted HR of Q4 versus Q1 of 2.17 [1.29-3.65], Figure 2C). Q3 and Q4 of Serpin C1 also appeared to be linearly associated with MACE (unadjusted HR of Q4 versus Q1 of 1.89 [1.18-3.05], Figure 2D).

\section{Prediction model development and performance}

Based on likelihood estimates, addition of CD14 HDL-EV (as a continuous measure) improved the clinical model including age, history of CAD and/or PAD, cerebrovascular symptoms, current smoking and creatinine. Visual inspection of the calibration plots showed good calibration of the model with biomarker (see Supplemental Figure S2). The C-statistic for the clinical model was 0.660 and for the clinical model with CD14 HDL-EV 0.672 ( $\mathrm{p}=0.212$ for comparison). Addition of CD14 HDL-EV to the clinical model yielded a NRI of $0.16,95 \%$ CI $0.08-0.25$ (32\% improvement for events against $16 \%$ worsening for non-events). Reclassification tables are added to the Supplemental (Table S6).

\section{EV proteins levels and carotid atherosclerotic plaque characteristics}

As histological atherosclerotic plaque characteristics have previously been associated with future $\mathrm{CVE}^{3}$, we investigated whether Serpin C1, Serpin F2, Cystatin C and CD14 in the HDL-EV subfraction were associated with a more unstable plaque phenotype. Higher levels of Serpin C1 were associated with more IPH (adjusted odds ratio (OR) and 95\% CI; 1.23 [1.04-1.44]) and increased macrophage infiltration (adjusted OR for moderate/heavy staining was 1.23 [1.05-1.43]) (Table 3). Higher levels of Serpin F2 were also associated with more macrophage infiltration (adjusted OR for moderate/heavy staining was 1.31 [1.11-1.54] and adjusted beta for percentages of macrophage staining 0.11 [0.02-0.21]). Cystatin $\mathrm{C}$ was negatively associated with the amount of SMCs infiltration (adjusted beta for percentage of SMC staining: -0.18 [-0.35- -0.01]). CD14 levels were not associated with plaque characteristics. 
Figures 2A-2E. Kaplan-Meier estimates of major adverse cardiovascular event (MACE) for quartiles of multivariable significant associated EV-protein levels

The p-value depicts the overall difference in survival probabilities across quartiles calculated by the Log-rank test. Hazard ratios in the legends indicate the unadjusted quartile-specific hazard relative to the first quartile.
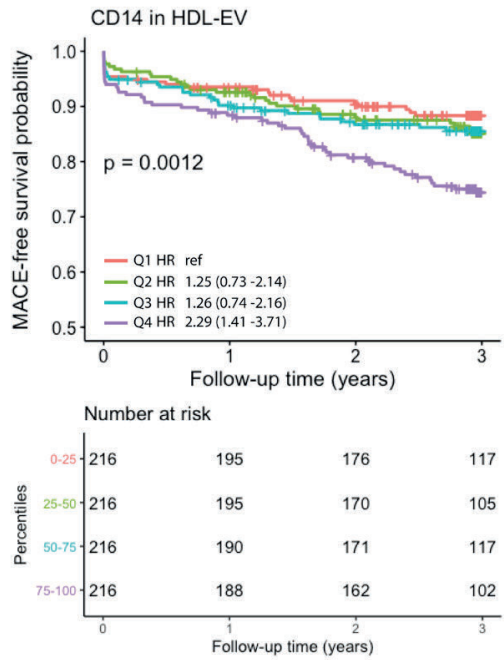

Figure 2A. The three-year MACE-free survival probability of CD14 quartile levels in the HDL-EV subfraction

Cystatin C in HDL-EV

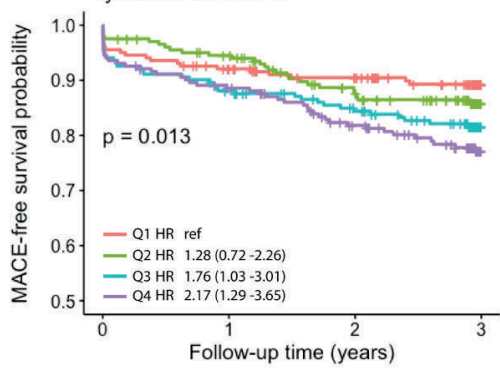

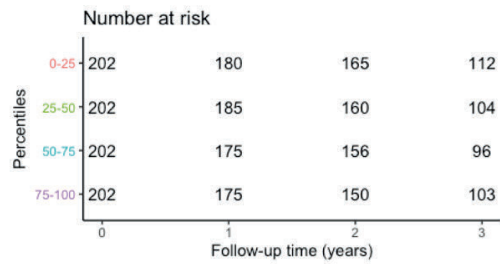

Figure 2C. The three-year MACE-free survival probability of Cystatin $\mathrm{C}$ quartile levels in the HDL-EV subfraction
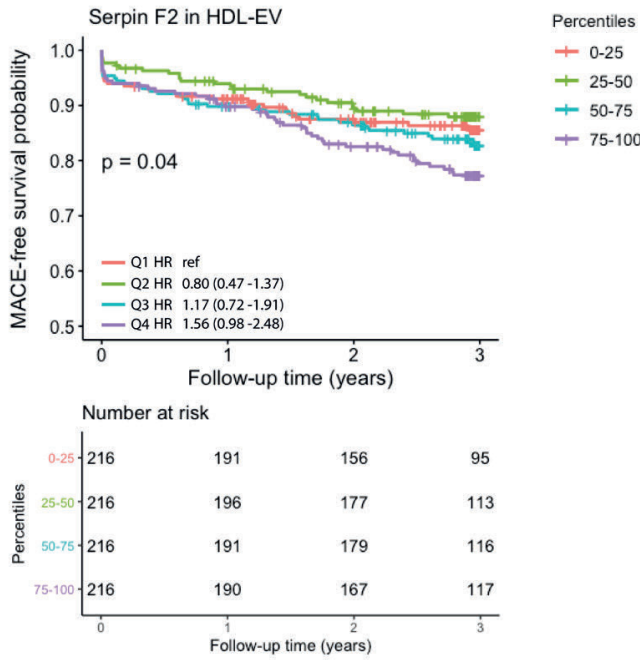

Figure 2B. The three-year MACE-free survival probability of Serpin F2 quartile levels in the HDL-EV subfraction

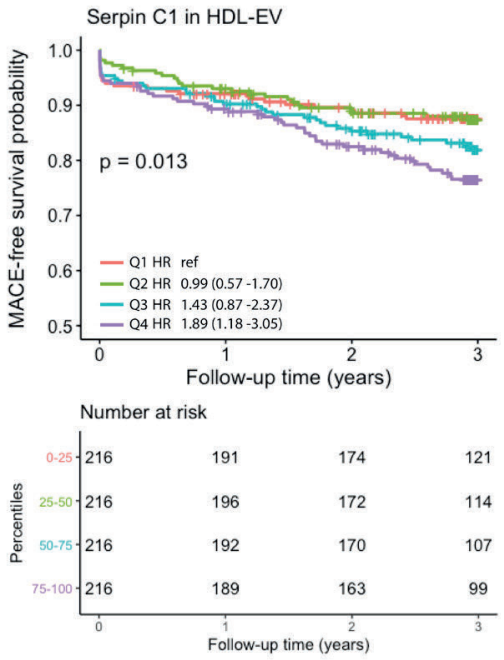

Figure 2D. The three-year MACE-free survival probability of Serpin C1 quartile levels in the HDL-EV subfraction 


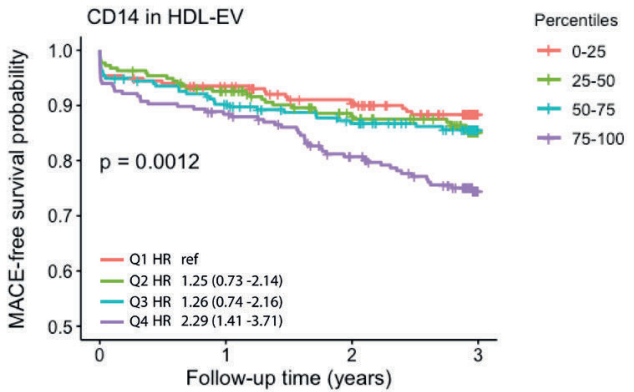

\begin{tabular}{l}
\multicolumn{5}{c}{ Number at risk } \\
\\
\end{tabular}

Figure 2E. The three-year MACE-free survival probability of CD14 quartile levels in the HDL-EV subfraction 


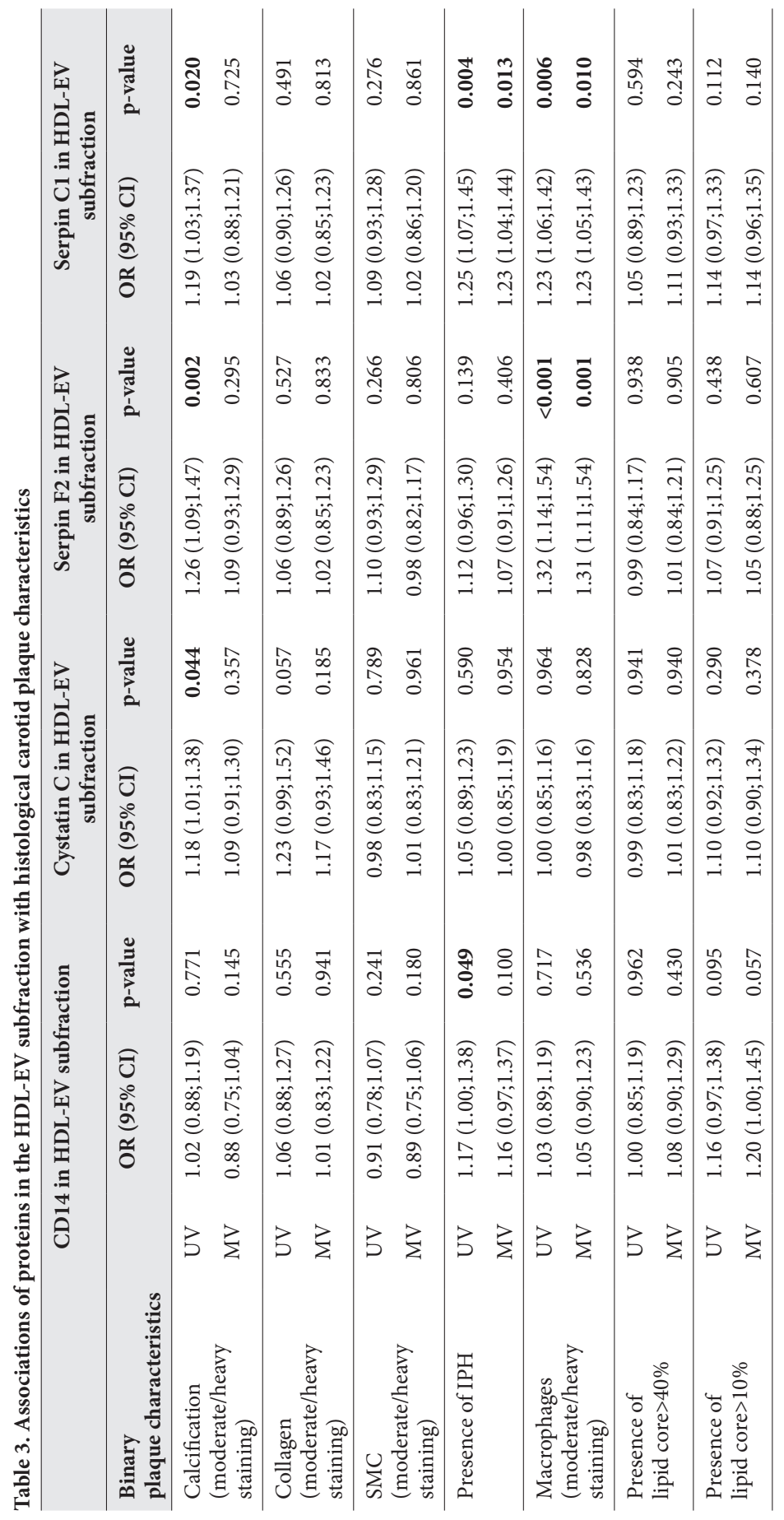




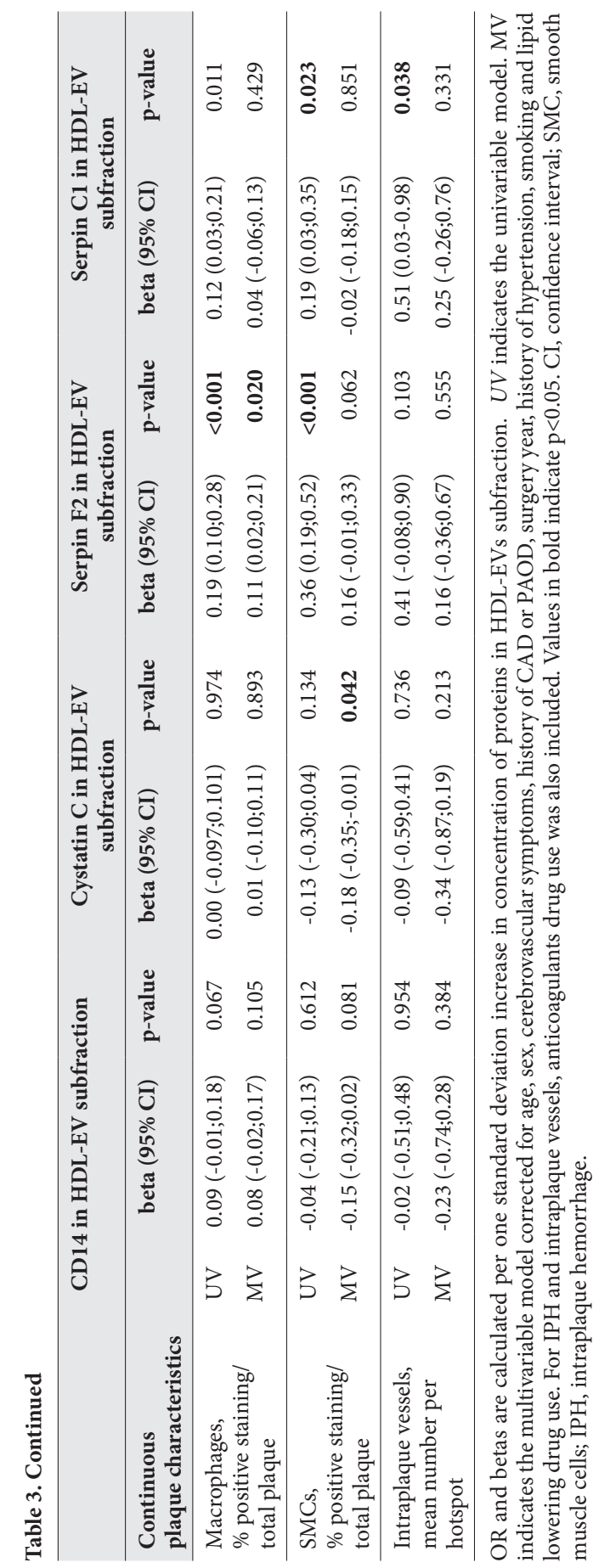




\section{Discussion}

In the present study, we assessed the association between preoperative levels of five CVDrelated proteins in three subsets of plasma EVs with the three-year risk of MACE after CEA. We showed that increased preoperative levels of CD14, Cystatin C, Serpin F2 and Serpin $\mathrm{C} 1$ in the HDL-EV subfraction were associated with an increased three-year postoperative risk of MACE, independent of cardiovascular risk factors. Moreover, CD14 HDL-EV levels showed incremental prognostic value for MACE prediction on top of cardiovascular risk factors with an improved reclassification of $16 \%$ of the patients. Protein levels in EV subfractions may thus be considered as markers to preoperatively identify patients at high residual risk of long-term CVE after CEA. These biomarkers might assist in timely identification of patients that may benefit from intensification of medical treatment by add-on therapy (e.g. PCSK-9 inhibitors or colchicine) next to standard preventive medications and CEA.

Already for two decades, it has become apparent that studying plasma EVs could provide crucial biological information about atherosclerotic diseases. ${ }^{9}$ Release of EV s from cells increases under inflammatory conditions, mechanical stress or hypoxia and their content reflect disease states. ${ }^{9}$ Previous studies showed that both the number and composition of EVs are associated with the presence and recurrence of CVD. ${ }^{11,13,22,23,30-32}$ The quantity of endothelial-derived microvesicles, considered as a marker for endothelial dysfunction, was an independent predictor for future CVE in patients with CAD. ${ }^{13,22,23,30-32}$ Moreover, higher protein levels of CD14, Cystatin C, Serpin G1 or Serpin C1 in EVs were related to the presence of heart failure, stable CAD, acute coronary syndrome or metabolic syndrome. . $3,22,23,32$ However, protein content of EVs in light of prognosis for future CVD have been relatively unexplored. The only prognostic study on EV protein content performed, showed that higher concentrations of CD14, Cystatin C and Serpin F2 (but not Serpin G1) were associated with a higher risk of future CVE in a mixed cohort of CVD. ${ }^{11}$ Our results now confirm these associations in patients with carotid stenosis. A recent study showed that Serpin C1 in HDL-EV is related to stress-induced myocardial ischemia. ${ }^{13}$ We now show that Serpin $\mathrm{C} 1$ is also associated with the risk of future MACE. Our results emphasize the potential use of these EV-proteins for CEA patients and that EV subpopulations provide more specific information since important associations were exclusively found in the HDL-EV subpopulation. A previous study suggested that the LDL-EV subpopulation contains procoagulant EVs ${ }^{12}$ but the properties of HDL-EVs are unknown and requires further research. Although exact biological and pathophysiological functions of EV subfractions remains to be elucidated, EV subfractions are known to have different subsets of EVs, each with its own protein content and related CVD 
manifestations ${ }^{12,13,32}$, which may reflect different cells and tissues involved and associated pathophysiological pathways.

It is important to emphasize that our results cannot prove causal relationships between $\mathrm{EV}$ proteins and MACE, thus exact underlying mechanisms remain unclear. Nevertheless, we studied associations of EV protein levels and histological characteristics of atherosclerotic plaques in order to generate hypotheses regarding underlying mechanisms. EV protein levels were positively related with a more unstable atherosclerotic plaque phenotype characterized by less SMC infiltration (for Cystatin C), more macrophage infiltration (for Serpin F2 and Serpin C1) and IPH (for Serpin C1). Since IPH has been previously established as a predictor for future CVE, we could hypothesize that EV proteins may affect atherosclerotic plaque instability and influencing cardiovascular disease risk. ${ }^{3}$ Cystatin $\mathrm{C}$, Serpin F2, Serpin C1 and CD14 have been previously related to key mechanisms involved in atherosclerosis such as coagulation, inflammation and endothelial dysfunction and therefore it is tempting to hypothesize contributing mechanisms. Plasma Cystatin C, a protease inhibitor, is known as a marker of kidney dysfunction and an independent predictor for primary and secondary CVE. ${ }^{33,34}$ Cystatin $\mathrm{C}$ has been related to inflammation and oxidative stress. ${ }^{34,35}$ A proposed mechanism of its proinflammatory role is the inhibition of TGF- $B$ cascade. TGF- $ß$ is an anti-inflammatory cytokine involved in extracellular matrix formation, inhibition of the expression of endothelial adhesion molecules and inhibition of SMCs proliferation and migration. ${ }^{11}$ Serpin F2, also known as a2-antiplasmin, inhibits fibrinolysis by inactivating plasmin. ${ }^{36}$ Serpin $\mathrm{C} 1$ is known as anti-thrombin and inhibits coagulation through inhibition of thrombin, factors Xa and IXa. ${ }^{37}$ Our finding that higher levels of Serpin C1 were associated with increased risk of MACE and plaque instability may be unexpected, but both low and high levels of Serpin C1 have been reported to increase the risk of CVD. ${ }^{38}$ Moreover, coagulation and fibrinolytic pathways including Serpin C1 and Serpin F2 are closely linked to inflammation. ${ }^{39}$ CD14 is a co-receptor of Toll-like receptor 4, known as a key receptor of the innate immune response, indicating its proinflammatory role. ${ }^{40}$ In vitro, monocyte derived-EVs, marked by CD14, induced endothelial cell thrombogenicity, endothelial cell apoptosis, and impaired anticoagulant pathways. ${ }^{41}$ In our study CD14-EV levels were not associated to plaque instability. It could be hypothesized that CD14 may not directly interfere in the process of plaque rupture or erosion, but once a thromboembolism is formed its procoagulant role may promote thrombus growth and prevent its degradation. Since these EV-proteins are involved in inflammatory processes, anti-inflammatory medications such as colchicine ${ }^{6,42,43}$ may be beneficial as add-on therapy in reducing the risk of future MACE in patients with manifest vascular disease. Future studies should unravel whether Cystatin C, Serpin F2, Serpin C1 and CD14 in plasma EVs are causally involved to atherosclerotic events rather than just 
being markers of CVE risk. An interesting approach would be to investigate the association between EV Cystatin C, Serpin F2, Serpin C1 and CD14 levels and the progression or regression atherosclerotic plaque burden (either in coronary or carotid plaques). ${ }^{44,45}$ If EV protein levels would be related to resistant atherosclerosis despite optimal preventive therapies, this would imply that EV proteins are specific markers for residual CVE risk. New therapeutic strategies that target EV-protein levels may be investigated in order to reduce CVE risk.

The determination of the cellular origin of EVs is challenging, since currently we do not know if well-known cell-type markers determined on EVs also indicate the cellular origin of these EVs. ${ }^{46}$ Therefore we were not able to distinguish the origin of EV subpopulations. All cell types, including cells of the vessel wall and circulating cells, are capable of producing EVs. ${ }^{9}$ A previous study showed that carotid atherosclerotic plaques contain EVs, predominantly with cellular markers from leukocytes (mostly macrophages) and to a lesser extent from erythrocytes, SMC and endothelial cells, while the EVs in plasma mainly had platelet surface markers. ${ }^{47}$ However, other studies have shown that EV s from plasma mainly possessed membrane proteins that were more typical for leukocytes and erythrocytes rather than for platelets. ${ }^{46}$ More insights in the cellular origin of EVs and its subpopulations could provide mechanistic insights in atherosclerotic pathophysiology.

Our study indicates that EV-derived CD14 levels may be a biomarker to improve risk stratification for future MACE. Addition of CD14 to a clinical model with cardiovascular risk factors resulted in a better reclassification concerning future MACE of $16 \%$ of all patients. More specifically, addition of CD14 led to a higher assigned risk category in 33\% of the patients who experienced MACE and only one patient was reclassified into a lower category. It should be noted that the correct reclassification of cases (MACE) came at the expense of a few controls (no MACE) that are wrongly classified as high-risk patients. If confirmed in an external validation study, such biomarker model would identify more accurate which patients could benefit from add-on therapy. As current guidelines suggest that all CEA patients should be given add-on therapy, fewer patients would be assigned to unnecessary add-on therapy leading to more personalized treatment. Nevertheless, these results should be interpreted with caution as external validation of this biomarker with determination of a clinically applicable cut-off value of CD14 HDL-EV is necessary. Future studies need to prove that using such a biomarker model improves clinical care by investigating whether high-risk patients identified by the biomarker model significantly benefit from add-on therapy. 
Our study has certain limitations. We were not able to analyze the outcomes of MACE separately because of low event numbers. In the Athero-Express, no follow-up data regarding medication use, medication compliance or the degree of risk factor control were available. Hence, we cannot exclude potential interferences of medication changes or risk factor management with observed associations with MACE. Other factors influencing MACE risk such as pre-existent or growing aortic aneurysms, coronary plaque burden, ipsilateral restenosis in the CEA trajectory, diet and metabolites of the intestinal microbiome ${ }^{48}$ could not be taken into account as data were not available. Moreover, due to the observational study design, our results do not prove a causal relationship between EVprotein levels and MACE. Last, plasma EVs were measured at one time point at baseline. Although this approach is similar to previous studies, some studies suggest that EVs numbers and content changes over time (possibly mediated by medication use or changes in disease progression). ${ }^{49,50}$ Multiple EVs measurements over time may be an interesting topic for future studies as change in EV content could potentially provide a more accurate reflection of cardiovascular disease risk. Strengths of this study include the relatively unexplored patient cohort of CEA patients in the field of EVs within a uniquely designed biobank including histological carotid plaque characterization data and validated study outcomes.

In conclusion, increased levels of CD14, Cystatin C, Serpin F2 and Serpin C1 in EV HDLsubfraction were associated with an increased risk of postoperative MACE after CEA. EV-HDL CD14 levels has added predictive value to a clinical risk prediction model for MACE. EV protein levels may be considered as markers for residual cardiovascular risk.

\section{Acknowledgements}

We thank Sara van Laar and Evelyn Velema for their technical support in the AtheroExpress Biobank.

\section{Financial support}

This work was supported by the EU Taxinomisis grant no 755320 (provided to Dr. De Borst, dr. De Kleijn and dr. Pasterkamp) and by the Dutch Heart Foundation, CVON 2017-05 pERSUASIVE (provided to Dr. de Kleijn and dr. de Winter). There are no competing interests declared.

\section{Conflict of interest}

None 


\section{References}

1 Barnett HJM, Taylor DW, Eliasziw M, Fox AJ, Ferguson GG, Haynes RB, et al. Benefit of carotid endarterectomy in patients with symptomatic moderate or severe stenosis. North American Symptomatic Carotid Endarterectomy Trial Collaborators. N Engl J Med 1998;339(20):1415-25.

2 Halliday A, Harrison M, Hayter E, Kong X, Mansfield A, Marro J, et al. 10-year stroke prevention after successful carotid endarterectomy for asymptomatic stenosis (ACST-1): a multicenter randomised trial. Lancet 2010;376(9746):1074-84.

3 Hellings WE, Peeters W, Moll FL, Piers SRD, van Setten J, Van der Spek PJ, et al. Composition of Carotid Atherosclerotic Plaque Is Associated With Cardiovascular Outcome. Circulation 2010;121(17):1941-50.

4 Kaasenbrood L, Boekholdt SM, van der Graaf Y, Ray KK, Peters RJG, Kastelein JJP, et al. Distribution of Estimated 10-Year Risk of Recurrent Vascular Events and Residual Risk in a Secondary Prevention Population. Circulation 2016;134(19):1419-29.

5 Mach F, Baigent C, Catapano AL, Koskinas KC, Casula M, Badimon L, et al. 2019 ESC/EAS Guidelines for the management of dyslipidaemias: lipid modification to reduce cardiovascular risk. Eur Heart $J$ 2020;41(1):111-88.

6 Tardif J-C, Kouz S, Waters DD, Bertrand OF, Diaz R, Maggioni AP, et al. Efficacy and Safety of Low-Dose Colchicine after Myocardial Infarction. N Engl J Med 2019;381(26):2497-505.

7 Nidorf SM, Fiolet ATL, Mosterd A, Eikelboom JW, Schut A, Opstal TSJ, et al. Colchicine in Patients with Chronic Coronary Disease. N Engl J Med 2020;383(19):1838-47.

8 Volkers EJ, Algra A, Kappelle LJ, Greving JP. Prediction models for clinical outcome after a carotid revascularisation procedure: A systematic review. Eur Stroke J 2018;3(1):57-65.

9 Boulanger CM, Loyer X, Rautou P-E, Amabile N. Extracellular vesicles in coronary artery disease. Nat Rev Cardiol 2017;14(5):259-72.

10 Loyer X, Vion A-C, Tedgui A, Boulanger CM. Microvesicles as Cell-Cell Messengers in Cardiovascular Diseases. Circ Res 2014;114(2):345-53.

11 Kanhai DA, Visseren FLJ, van der Graaf Y, Schoneveld AH, Catanzariti LM, Timmers L, et al. Microvesicle protein levels are associated with increased risk for future vascular events and mortality in patients with clinically manifest vascular disease. Int J Cardiol 2013;168(3):2358-63.

12 Wang J-W, Zhang Y-N, Sze S, van de Weg S, Vernooij F, Schoneveld A, et al. Lowering Low-Density Lipoprotein Particles in Plasma Using Dextran Sulphate Co-Precipitates Procoagulant Extracellular Vesicles. Int J Mol Sci 2017;19(1):94.

13 Dekker M, Waissi F, van Bennekom J, Silvis MJM, Timmerman N, Bank IEM, et al. Plasma extracellular vesicle proteins are associated with stress-induced myocardial ischemia in women presenting with chest pain. Sci Rep 2020;10(1):12257.

14 Warlow C, Farrell B, Fraser A, Sandercock P SJ. Randomised trial of endarterectomy for recently symptomatic carotid stenosis: final results of the MRC European Carotid Surgery Trial (ECST). Lancet 1998;351(9113):1379-87.

15 Barnett HJ, Taylor DW, Eliasziw M, Fox AJ, Ferguson GG, Haynes RB, et al. Benefit of carotid endarterectomy in patients with symptomatic moderate or severe stenosis. North American Symptomatic Carotid Endarterectomy Trial Collaborators. N Engl J Med 1998;339(20):1415-25.

16 North American Symptomatic Carotid Endarterectomy Trial Collaborators, Barnett HJM, Taylor DW, Haynes RB, Sackett DL, Peerless SJ, et al. Beneficial effect of carotid endarterectomy in symptomatic patients with high-grade carotid stenosis. N Engl J Med 1991;325(7):445-53.

17 Halliday A, Mansfield A, Marro J, Peto C, Peto R, Potter J, et al. Prevention of disabling and fatal strokes by successful carotid endarterectomy in patients without recent neurological symptoms: randomised controlled trial. Lancet 2004;363(9420):1491-502.

18 Fokkema M, Reichmann BL, den Hartog AG, Klijn CJ, Schermerhorn ML, Moll FL, et al. Selective external endarterectomy in patients with ipsilateral symptomatic internal carotid artery occlusion. J Vasc Surg 2013;58(1):145-151.e1.

19 Verhoeven BAN, Velema E, Schoneveld AH, de Vries JPPM, de Bruin P, Seldenrijk CA, et al. Atheroexpress: differential atherosclerotic plaque expression of mRNA and protein in relation to cardiovascular events and patient characteristics. Rationale and design. Eur J Epidemiol 2004;19(12):1127-33. 
20 Dekker M, Waissi F, van Bennekom J, Silvis MJM, Timmerman N, Schoneveld AH, et al. Extracellular Vesicle cystatin $\mathrm{c}$ is associated with unstable angina in troponin negative patients with acute chest pain. PLoS One 2020;15(8):e0237036.

21 van Lammeren GW, Catanzariti LM, Peelen LM, de Vries J-PPM, de Kleijn DPV, Moll FL, et al. Clinical Prediction Rule to Estimate the Absolute 3-Year Risk of Major Cardiovascular Events After Carotid Endarterectomy. Stroke 2012;43(5):1273-8.

22 Kranendonk MEG, de Kleijn DPV, Kalkhoven E, Kanhai DA, Uiterwaal CSPM, van der Graaf Y, et al. Extracellular vesicle markers in relation to obesity and metabolic complications in patients with manifest cardiovascular disease. Cardiovasc Diabetol 2014;13(1):37.

23 de Hoog VC, Timmers L, Schoneveld AH, Wang J-W, van de Weg SM, Sze SK, et al. Serum extracellular vesicle protein levels are associated with acute coronary syndrome. Eur Hear Journal Acute Cardiovasc Care 2013;2(1):53-60.

24 Cook NR, Paynter NP. Performance of reclassification statistics in comparing risk prediction models. Biometrical J 2011;53(2):237-58.

25 Hellings WE, Pasterkamp G, Verhoeven BAN, De Kleijn DP V, De Vries J-PPM, Seldenrijk KA, et al. Gender-associated differences in plaque phenotype of patients undergoing carotid endarterectomy. J Vasc Surg 2007;45(2):289-96.

26 van Lammeren GW, den Ruijter HM, Vrijenhoek JEP, van der Laan SW, Velema E, de Vries J-PPM, et al. Time-Dependent Changes in Atherosclerotic Plaque Composition in Patients Undergoing Carotid Surgery. Circulation 2014;129(22):2269-76.

27 Makris GC, Lavida A, Nicolaides AN, Geroulakos G. The effect of statins on carotid plaque morphology: A LDL-associated action or one more pleiotropic effect of statins? Atherosclerosis 2010;213(1):8-20.

28 Fassaert LMM, Timmerman N, van Koeverden ID, Pasterkamp G, de Kleijn DPV, de Borst GJ. Preoperative hypertension is associated with atherosclerotic intraplaque hemorrhage in patients undergoing carotid endarterectomy. Atherosclerosis 2019;290:214-21.

29 Derksen WJM, Peeters W, Tersteeg C, de Vries J-PPM, de Kleijn DPV, Moll FL, et al. Age and coumarintype anticoagulation are associated with the occurrence of intraplaque hemorrhage, while statins are associated less with intraplaque hemorrhage: A large histopathological study in carotid and femoral plaques. Atherosclerosis 2011;214(1):139-43.

30 Sinning J-M, Losch J, Walenta K, Bohm M, Nickenig G, Werner N. Circulating CD31+/Annexin V+ microparticles correlate with cardiovascular outcomes. Eur Heart J 2011;32(16):2034-41.

31 Nozaki T, Sugiyama S, Koga H, Sugamura K, Ohba K, Matsuzawa Y, et al. Significance of a Multiple Biomarkers Strategy Including Endothelial Dysfunction to Improve Risk Stratification for Cardiovascular Events in Patients at High Risk for Coronary Heart Disease. J Am Coll Cardiol 2009;54(7):601-8.

32 Zhang Y-N, Vernooij F, Ibrahim I, Ooi S, Gijsberts CM, Schoneveld AH, et al. Extracellular Vesicle Proteins Associated with Systemic Vascular Events Correlate with Heart Failure: An Observational Study in a Dyspnoea Cohort. PLoS One 2016;11(1):e0148073.

33 Shlipak MG, Sarnak MJ, Katz R, Fried LF, Seliger SL, Newman AB, et al. Cystatin C and the Risk of Death and Cardiovascular Events among Elderly Persons. N Engl J Med 2005;352(20):2049-60.

34 Woitas RP, Kleber ME, Meinitzer A, Grammer TB, Silbernagel G, Pilz S, et al. Cystatin C is independently associated with total and cardiovascular mortality in individuals undergoing coronary angiography. The Ludwigshafen Risk and Cardiovascular Health (LURIC) study. Atherosclerosis 2013;229(2):541-8.

35 Negrusz-Kawecka M, Poręba R, Hulok A, Ściborski K, Marczak J, Bańkowski T. Evaluation of the Significance of Cystatin C Levels in Patients Suffering from Coronary Artery Disease. Adv Clin Exp Med 2014;23(4):551-8.

36 Schaller J, Gerber SS. The plasmin-antiplasmin system: structural and functional aspects. Cell Mol Life Sci 2011;68(5):785-801.

37 Perry DJ. Antithrombin and its inherited deficiencies. Blood Rev 1994;8(1):37-55.

38 Meade TW, Cooper J, Miller GJ, Howarth DJ, Stirling Y. Antithrombin III and arterial disease. Lancet 1991;338(8771):850-1.

39 Schuliga M. The Inflammatory Actions of Coagulant and Fibrinolytic Proteases in Disease. Mediators Inflamm 2015;2015:1-9.

40 Reiner AP, Lange EM, Jenny NS, Chaves PHM, Ellis J, Li J, et al. Soluble CD14. Arterioscler Thromb Vasc Biol 2013;33(1):158-64. 
41 Aharon A, Tamari T, Brenner B. Monocyte-derived microparticles and exosomes induce procoagulant and apoptotic effects on endothelial cells. Thromb Haemost 2008;100(05):878-85.

42 Opstal TSJ, Hoogeveen RM, Fiolet ATL, Silvis MJM, The SHK, Bax WA, et al. Colchicine Attenuates Inflammation Beyond the Inflammasome in Chronic Coronary Artery Disease. Circulation 2020;142(20):1996-8.

43 Ashish K, Bandyopadhyay D, Mondal S, Ghosh RK. Colchicine in coronary artery disease: Role of antiinflammatory medications redefined. Int J Cardiol 2018;254:51.

44 Spence JD, Solo K. Resistant Atherosclerosis. Stroke 2017;48(6):1624-9.

45 Spence JD. Genetics of atherosclerosis: The power of plaque burden and progression. Atherosclerosis 2012;223(1):98-101.

46 van der Pol E, Böing AN, Gool EL, Nieuwland R. Recent developments in the nomenclature, presence, isolation, detection and clinical impact of extracellular vesicles. J Thromb Haemost 2016;14(1):48-56.

47 Leroyer AS, Isobe H, Lesèche G, Castier Y, Wassef M, Mallat Z, et al. Cellular Origins and Thrombogenic Activity of Microparticles Isolated From Human Atherosclerotic Plaques. J Am Coll Cardiol 2007;49(7):7727.

48 Bogiatzi C, Gloor G, Allen-Vercoe E, Reid G, Wong RG, Urquhart BL, et al. Metabolic products of the intestinal microbiome and extremes of atherosclerosis. Atherosclerosis 2018;273:91-7.

49 Verbree-Willemsen L, Zhang Y-N, Gijsberts CM, Schoneveld AH, Wang J-W, Lam CSP, et al. LDL extracellular vesicle coagulation protein levels change after initiation of statin therapy. Findings from the METEOR trial. Int J Cardiol 2018;271:247-53.

50 Chiva-Blanch G, Suades R, Crespo J, Vilahur G, Arderiu G, Padró T, et al. CD3+/CD45+ and SMA- $\alpha+$ circulating microparticles are increased in individuals at high cardiovascular risk who will develop a major cardiovascular event. Int J Cardiol 2016;208:147-9. 


\section{Supplementary materials}

The following supplemental materials are omitted due to space limitations and can be requested from the authors:

- Isolation procedure of extracellular vesicles

- Characterization of extracellular vesicles

- Quantification of proteins in extracellular vesicles

- Histological characterization of the carotid atherosclerotic plaque

- Supplemental Table S4a-d: multivariable models for MACE with all predictors

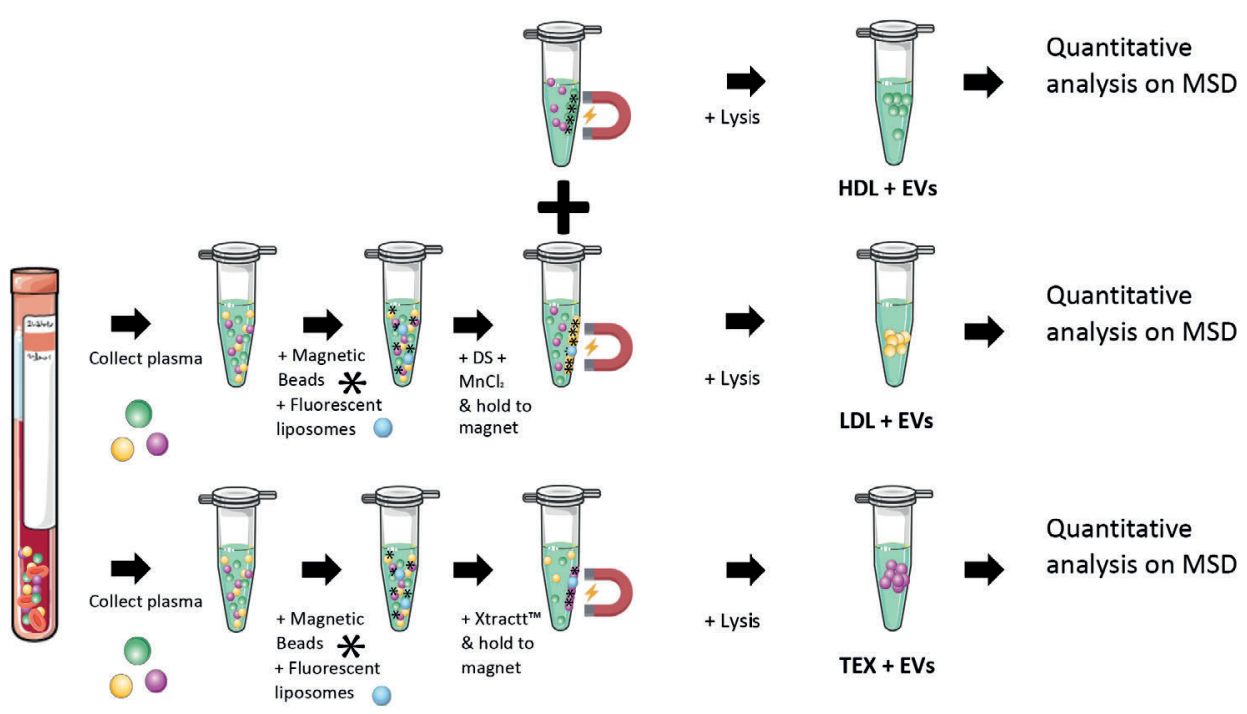

Supplemental Figure S1. The isolation procedure of plasma EVs. MSD refers to the assay used for quantification of the protein levels (electrochemiluminescence immunoassay Meso Scale). 
Supplemental Table S2. Percentages of missing covariates

\begin{tabular}{ll}
\hline Age & Missing (\%) \\
\hline Male sex & 0.0 \\
Current smoking & 0.0 \\
History of CAD or PAD & 0.1 \\
Cerebrovascular symptoms & 0.0 \\
Creatinine & 0.0 \\
Surgery year & 0.5 \\
Hypertension & 0.0 \\
Lipid-lowering drug use & 0.0 \\
Anticoagulant drug use & 0.2 \\
\hline
\end{tabular}

$C A D$, coronary artery disease; $P A D$, peripheral artery disease;

Supplemental Table S3. Median and interquartile ranges of protein levels in three EV subfractions

\begin{tabular}{llllll}
\hline Biomarker & & $\begin{array}{l}\text { No MACE } \\
(\mathbf{n = 7 2 7})\end{array}$ & $\begin{array}{l}\text { MACE } \\
(\mathbf{n}=\mathbf{1 3 7})\end{array}$ & p-value & Missing (\%) \\
\hline CD14 & HDL-EV & $0.12[0.08,0.18]$ & $0.15[0.10,0.22]$ & $<\mathbf{0 . 0 0 1}$ & 0.0 \\
& LDL-EV & $0.11[0.08,0.16]$ & $0.13[0.08,0.19]$ & $\mathbf{0 . 0 1 5}$ & 0.0 \\
& TEX-EV & $0.18[0.13,0.24]$ & $0.18[0.14,0.27]$ & 0.172 & 0.1 \\
Cystatin C & HDL-EV & $0.09[0.06,0.13]$ & $0.11[0.08,0.15]$ & $<\mathbf{0 . 0 0 1}$ & 6.5 \\
& LDL-EV & $0.15[0.10,0.22]$ & $0.15[0.10,0.25]$ & 0.128 & 0.2 \\
Serpin F2 & TEX-EV & $0.41[0.30,0.57]$ & $0.43[0.31,0.66]$ & 0.056 & 0.5 \\
& HDL-EV & $6.04[4.64,7.58]$ & $6.65[4.91,8.67]$ & $\mathbf{0 . 0 0 3}$ & 0.0 \\
& LDL-EV & $7.06[5.57,9.39]$ & $7.36[5.51,11.25]$ & 0.283 & 0.0 \\
Serpin G1 & TEX-EV & $7.87[6.27,10.54]$ & $8.11[6.28,11.21]$ & 0.370 & 0.2 \\
& HDL-EV & $0.05[0.04,0.07]$ & $0.06[0.04,0.09]$ & $\mathbf{0 . 0 0 9}$ & 0.0 \\
& LDL-EV & $0.04[0.03,0.06]$ & $0.04[0.03,0.06]$ & 0.412 & 0.1 \\
& TEX-EV & $1.29[0.79,1.83]$ & $1.13[0.70,1.74]$ & 0.094 & 0.1 \\
& HDL-EV & $3.42[2.07,5.42]$ & $4.52[2.52,6.20]$ & $\mathbf{0 . 0 0 1}$ & 0.0
\end{tabular}

Values are displayed as median [interquartile] protein concentrations in $\mathrm{pg} / \mathrm{mL}$. P-values originate from comparison of median levels by Mann-Whitney U test between patients that experienced MACE versus those who did not. Values in bold indicate $\mathrm{p}<0.05$. Missing measurements of proteins were randomly divided across all samples and were due to technical failures. HDL-EV indicates the HDL-EV subfraction, LDL-EV indicates the LDL-EV subfraction, TEX-EV indicates the TEX-EV subfraction. 
Supplemental Table S5. Multivariable cox-regression corrected for age, history of CAD, history of peripheral artery disease, cerebrovascular symptoms, creatinine, current smoking, diabetes and HDL levels

\begin{tabular}{|c|c|c|c|}
\hline & HR & $(95 \% \mathrm{CI})$ & p-value \\
\hline \multicolumn{4}{|l|}{ CD14 } \\
\hline HDL-EVs & 1.29 & $1.13-1.46$ & $<0.001$ \\
\hline LDL-EVs & 1.15 & $1.00-1.30$ & 0.050 \\
\hline TEX-EVs & 1.05 & $0.90-1.23$ & 0.512 \\
\hline \multicolumn{4}{|l|}{ Cystatin C } \\
\hline HDL-EVs & 1.20 & $1.03-1.39$ & 0.017 \\
\hline LDL-EVs & 1.16 & $1.00-1.33$ & 0.050 \\
\hline TEX-EVs & 1.09 & $0.92-1.29$ & 0.316 \\
\hline \multicolumn{4}{|l|}{ Serpin F2 } \\
\hline HDL-EVs & 1.33 & $1.12-1.57$ & 0.001 \\
\hline LDL-EVs & 1.10 & $0.91-1.34$ & 0.330 \\
\hline TEX-EVs & 1.15 & $0.97-1.36$ & 0.111 \\
\hline \multicolumn{4}{|l|}{ Serpin G1 } \\
\hline HDL-EVs & 1.13 & $0.98-1.30$ & 0.087 \\
\hline LDL-EVs & 0.96 & $0.77-1.20$ & 0.733 \\
\hline TEX-EVs & 0.88 & $0.73-1.05$ & 0.148 \\
\hline \multicolumn{4}{|l|}{ Serpin C1 } \\
\hline HDL-EVs & 1.30 & $1.11-1.52$ & 0.001 \\
\hline LDL-EVs & 1.07 & $0.90-1.29$ & 0.447 \\
\hline TEX-EVs & 1.05 & $0.91-1.21$ & 0.510 \\
\hline
\end{tabular}

Protein levels were logarithmically transformed and standardized per SD. HR indicates the hazard ratio for the 3-year postoperative risk of MACE per one standard deviation increase in concentration of protein levels in three subsets of extracellular vesicles EVs (LDL, HDL and TEX EV subfraction).

Values in bold indicate $\mathrm{p}<0.05$. CI, confidence interval. 

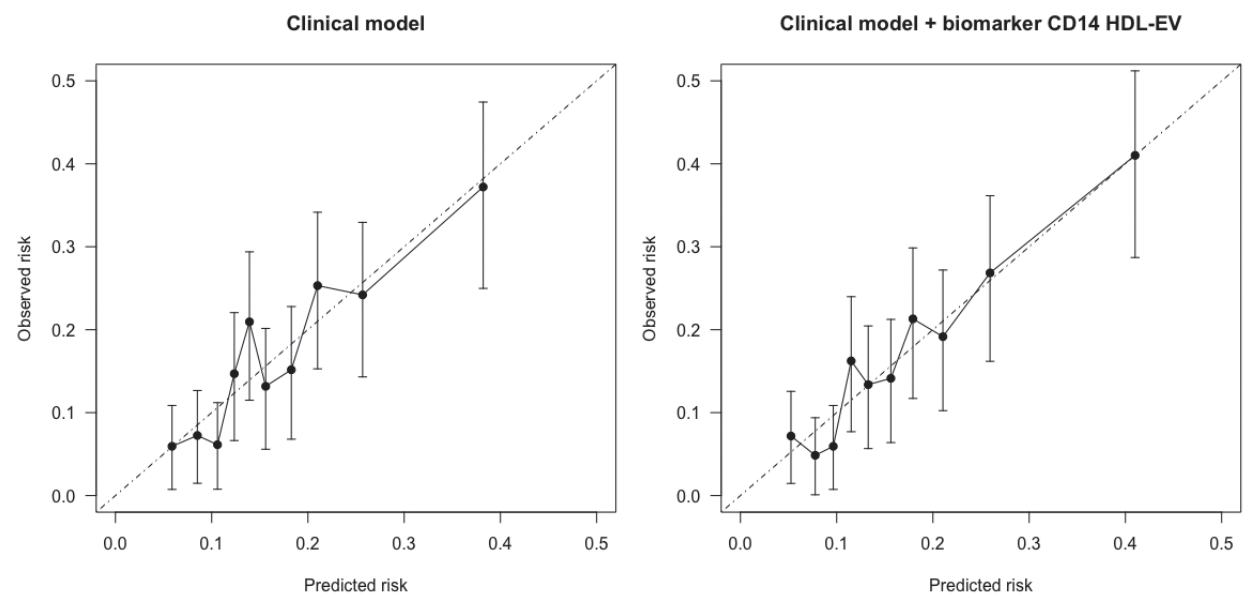

Supplemental Figure S2. Calibration plots

The clinical prediction model consisted of age, history of CAD and/or PAD, cerebrovascular symptoms, current smoking and creatinine levels. The biomarker model includes the clinical model with CD14 HDL-EV. 
Supplemental Table S6. Net reclassification improvement with addition of CD14 HDL-EV to the clinical model

\begin{tabular}{|c|c|c|c|c|c|}
\hline \multicolumn{2}{|l|}{ CASES $(n=137)$} & & & & \\
\hline & \multirow[b]{2}{*}{ MACE risk } & \multicolumn{4}{|c|}{ Clinical model + CD14 HDL-EV } \\
\hline \multirow{5}{*}{ Clinical model } & & $<8 \%$ & 8 to $<16 \%$ & 16 to $<32 \%$ & $\geq 32 \%$ \\
\hline & 0 to $<8 \%$ & 48 & 29 & 1 & 0 \\
\hline & 8 to $<16 \%$ & 0 & 41 & 11 & 2 \\
\hline & 16 to $<32 \%$ & 0 & 1 & 2 & 2 \\
\hline & $\geq 32 \%$ & 0 & 0 & 0 & 0 \\
\hline Reclassified up No. (\%) & & \multicolumn{4}{|c|}{$45(33 \%)$} \\
\hline Reclassified down No. (\%) & & \multicolumn{4}{|c|}{$1(0.7 \%)$} \\
\hline NRI (95\% CI) & & \multicolumn{4}{|c|}{$0.32(0.24 ; 0.40)$} \\
\hline \multicolumn{6}{|l|}{ CONTROLS $(n=722)$} \\
\hline & & \multicolumn{4}{|c|}{ Clinical model + CD14 HDL-EV } \\
\hline \multirow{5}{*}{ Clinical model } & MACE risk & $<8 \%$ & 8 to $<16 \%$ & 16 to $<32 \%$ & $\geq 32 \%$ \\
\hline & 0 to $<8 \%$ & 456 & 96 & 3 & 0 \\
\hline & 8 to $<16 \%$ & 9 & 123 & 26 & 0 \\
\hline & 16 to $<32 \%$ & 0 & 2 & 7 & 0 \\
\hline & $\geq 32 \%$ & 0 & 0 & 0 & 0 \\
\hline Reclassified up No. (\%) & & \multicolumn{4}{|c|}{$125(17 \%)$} \\
\hline Reclassified down No. (\%) & & \multicolumn{4}{|c|}{$11(1.5 \%)$} \\
\hline NRI $(95 \%$ CI) & & \multicolumn{4}{|c|}{$-0.16(-0.19 ;-0.13)$} \\
\hline OVERALL NRI (95\%CI) & & \multicolumn{4}{|c|}{$0.16(0.08 ; 0.25)$} \\
\hline
\end{tabular}

The clinical model includes age, history of CAD and/or PAD, cerebrovascular symptoms, current smoking and creatinine. The Net Reclassification Index (NRI) is calculated with addition of the biomarker CD14 HDL-EV to the clinical model.

95\% CI, 95\% confidence interval 


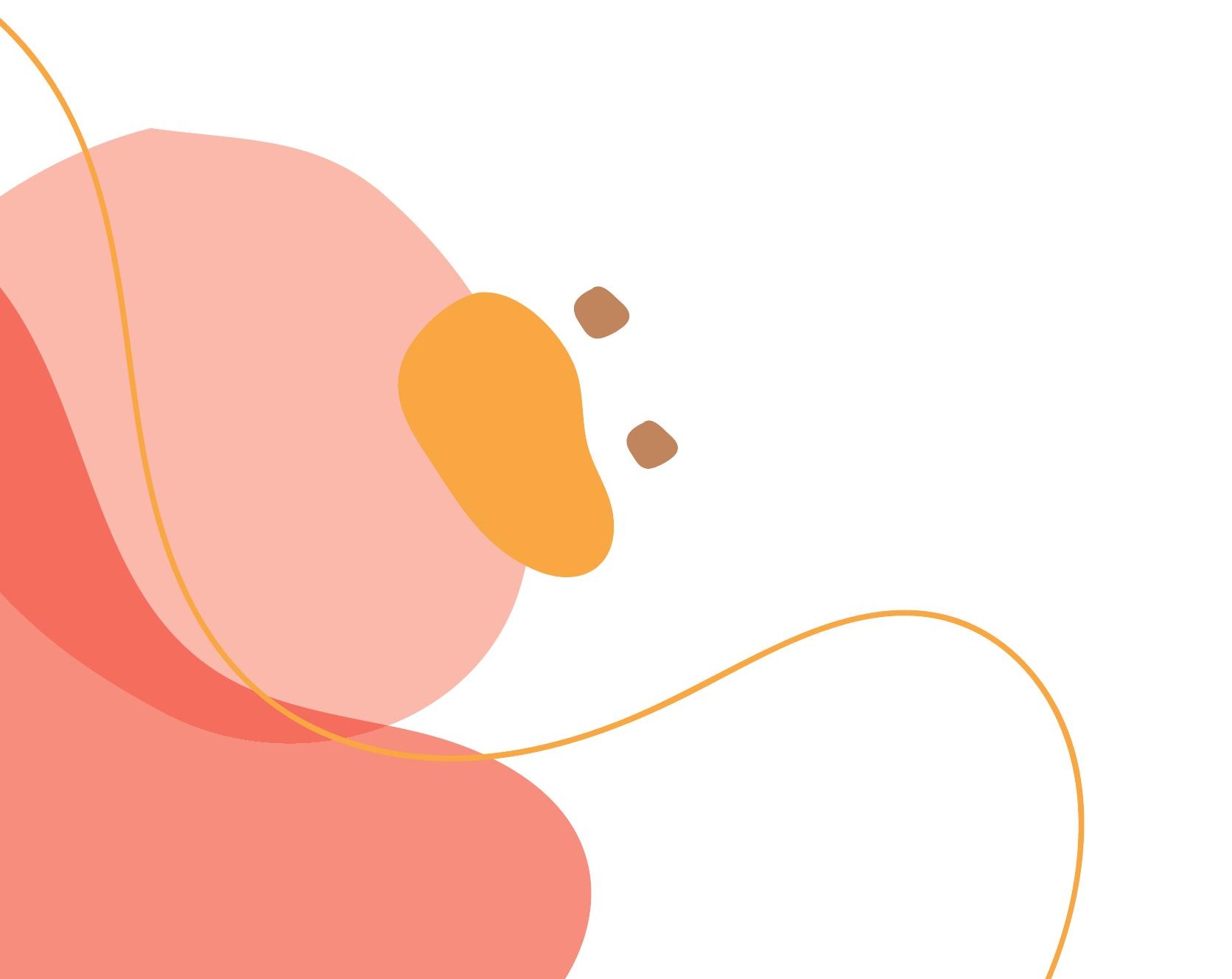




\title{
Elevated lipoprotein(a) levels increase risk of 30-day MACE in patients following carotid endarterectomy
}

Stroke 2020;51(10):2972-2982

\author{
Nathalie Timmerman ${ }^{*}$ \\ Farahnaz Waissi* \\ Mirthe Dekker* \\ Renate M. Hoogeveen \\ Joelle van Bennekom \\ Kim E. Dzobo \\ Johan G. Schnitzler \\ Gerard Pasterkamp \\ Diederick E. Grobbee \\ Gert J. de Borst \\ Erik S.G. Stroes \\ Dominique P.V. de Kleijn \\ Jeffrey Kroon
}




\section{Abstract}

\section{Background and Purpose}

General population studies have shown that elevated lipoprotein(a) $[\operatorname{Lp}(a)]$ levels are an emerging risk factor for cardiovascular disease and subsequent cardiovascular events (CVE). The role of $\mathrm{Lp}(\mathrm{a})$ for the risk of secondary major adverse cardiovascular events (MACE) in patients undergoing carotid endarterectomy (CEA) is unknown. Our objective is to assess the association of elevated Lp(a) levels with the risk of secondary MACE in patients undergoing CEA.

\section{Methods}

$\mathrm{Lp}$ (a) concentrations were determined in pre-operative blood samples of 944 consecutive CEA patients included in the Athero-Express Biobank Study. During 3-years follow-up, major adverse cardiovascular events (MACE), consisting of myocardial infarction, stroke and cardiovascular death, were documented.

\section{Results}

After 3 years follow-up, Kaplan-Meier cumulative event rates for MACE were $15.4 \%$ in patients with high $\mathrm{Lp}(\mathrm{a})$ levels $\left(>137 \mathrm{nmol} / \mathrm{L} ;>80^{\text {th }}\right.$ cohort percentile) and $10.2 \%$ in patients with low $\mathrm{Lp}$ (a) levels ( $\leq 137 \mathrm{nmol} / \mathrm{L} ; \leq 80^{\text {th }}$ cohort percentile) (log-rank test: $\left.\mathrm{p}=0.047\right)$. Cox regression analyses adjusted for conventional cardiovascular risk factors revealed a significant association between high Lp(a) levels and 3-year MACE with an adjusted HR of 1.69 (95\% CI 1.07-2.66). One third of MACE occurred within 30 days after CEA, with an adjusted HR for the 30-day risk of MACE of 2.05 (95\% CI 1.01-4.17). Kaplan-Meier curves from timepoint 30 days to 3 years onward revealed no significant association between high Lp(a) levels and MACE. Lp(a) levels were not associated with histological carotid plaque characteristics.

\section{Conclusions}

High Lp(a) levels (>137 nmol/L; > 80th cohort percentile) are associated with an increased risk of 30-day MACE after CEA. This identifies elevated Lp(a) levels as a new potential risk factor for secondary CVE in patients after carotid surgery. Future studies are required to investigate whether Lp(a) levels might be useful in guiding treatment algorithms for carotid intervention. 


\section{Introduction}

Carotid endarterectomy (CEA), an established treatment for patients with moderate to severe carotid artery stenosis, reduces the recurrent risk of ipsilateral stroke. These patients, however, are characterized by extensive polyvascular disease, reflecting the systemic nature of atherogenesis ${ }^{1}$. This results in a high residual cardiovascular risk following $\mathrm{CEA}^{2}$, with 3 -year major adverse cardiovascular event (MACE) incidence rates as high as $19-35 \%{ }^{1,3}$.

Although aggressive secondary prevention strategies have contributed to reduction of secondary MACE, the residual risk is still markedly elevated and a high number of patients on active secondary prevention still develop cardiovascular events (CVE). This suggests that other modifiable risk factors may contribute to the risk of secondary $\mathrm{CVE}^{4,5}$.

Genetic and observational data have convincingly demonstrated that elevated lipoprotein(a) $[\mathrm{Lp}(\mathrm{a})]$ is a causal and highly prevalent risk factor for cardiovascular diseases $(\mathrm{CVD})^{4-6}$. $\mathrm{Lp}(\mathrm{a})$ is a low-density lipoprotein (LDL)-like particle characterized by covalently bound apolipoprotein(a) to apolipoprotein B-100 of LDL. Lp(a), like LDL cholesterol, is able to accumulate in the subendothelial space, leading to progressive atherosclerosis. It has also been shown to exert a plethora of signalling effects exacerbating its atherogenic capacity. $\mathrm{Lp}$ (a) induces a systemic pro-inflammatory state, but in addition has prothrombotic as well as pro-oxidant effects ${ }^{4,6,7}$.

To date, available evidence on $\operatorname{Lp}(\mathrm{a})$ and the risk for cardiovascular events is based on studies on individuals in primary prevention setting. Evidence on whether Lp(a) is associated with risk of recurrent CVE remain scarce ${ }^{8}$ and only include patients with established coronary artery disease ${ }^{9-11}$. To date, the association between $\operatorname{Lp}(\mathrm{a})$ and recurrent CVE in CEA patients is unknown. This association is important in weighing up treatment strategies, including add-on medical therapy or an endovascular approach for carotid revascularisation. Hence, in the present study, we set out to address the impact of elevated $\mathrm{Lp}$ (a) levels and the risk of MACE in a large cohort of 944 CEA patients.

\section{Methods}

\section{Study population}

From the Athero-Express Biobank all patients undergoing CEA between 2002-2016 with available lipid profile measurements were included in the current study. Patients undergoing CEA for restenosis were excluded (Figure 1). The Athero-Express started in 2002 and is a 
prospective ongoing biobank study including all consecutive patients scheduled for CEA in two referral hospitals in the Netherlands (the St. Antonius hospital Nieuwegein and University Medical Center Utrecht). The study design has been described previously in more detail ${ }^{12}$. In short, preoperative blood and atherosclerotic plaque specimens of all patients undergoing CEA are collected. Indications for CEA were reviewed by a multidisciplinary vascular team and experienced surgeons performed the surgery in accordance with local and international guidelines ${ }^{2,13-16}$. Standardized preoperative questionnaires and hospital medical records were used to obtain baseline patient characteristics. Perioperative assessment was structurally done by a neurologist and/or

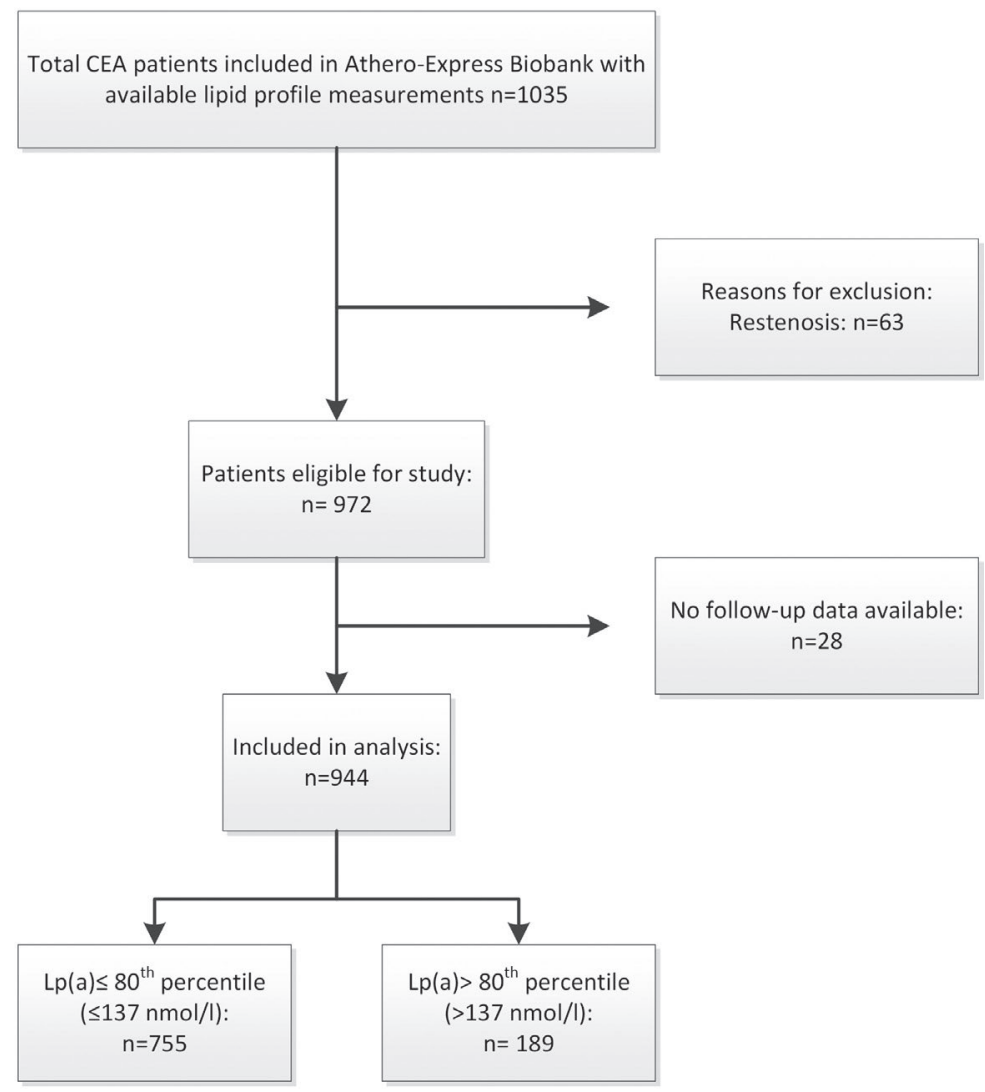

Figure 1. Flowchart of study population. A total of 1035 CEA patients with available lipid profile measurements were included in the Athero-Express Biobank

Following exclusion of patients with restenosis $(n=63)$ and no available follow-up data $(n=28), 944$ patients were eligible for this study. Patients were classified in low Lp(a) ( $\leq 80$ th cohort percentile, $\leq 137 \mathrm{nmol} / \mathrm{l}) \mathrm{n}=755 \mathrm{and}$ high Lp(a) (>80th cohort percentile, $>137 \mathrm{nmol} / \mathrm{l}) \mathrm{n}=189$. 
vascular surgeon. Hospital medical records were reviewed for perioperative events. All patients received questionnaires annually for the first 3 consecutive years after CEA to collect follow-up data on cardiovascular events or hospital admissions. If an outcome event occurred medical records were checked and relevant documentation was acquired by contacting the general practitioner or hospital. If patients did not respond to the follow-up questionnaire, the general practitioner was contacted for follow-up information. The medical ethics board of both hospitals approved the study and all study participants provided written informed consent. The study is conducted in accordance with the declaration of Helsinki. The data that support the findings of this study are available from the corresponding author upon reasonable request.

\section{Laboratory Measurements}

All venous blood samples were collected during hospital admission prior to surgery and stored at -80 degrees Celsius until further use. Patients with available lipid profile measurements (assessed as part of routine care) were selected for lipoprotein(a) measurements. Lipoprotein(a) concentrations were measured with a latex-enhanced particle immunoturbidimetric assay in serum samples using the Cobas c702 (Roche) and the LPA2 Tina-quant Lipoprotein(a) Gen.2 kit from Cobas (LPA2: CAN 8723). In short, Lp(a) was measured in nanomoles per liter by means of a particle enhanced immunoturbidimetric assay, where lipoprotein(a) agglutinates with latex particles coated with anti-Lp(a) antibodies. The precipitate is determined turbidimetrically at $800 / 660 \mathrm{~nm}$. The measuring rage of this assay is between $7-240 \mathrm{nmol} / \mathrm{L}$. More information regarding specific performance data, the repeatability and intermediate precision can be found in the manufacturers' instructions.

\section{Atherosclerotic plaque assessment}

Histological assessment of the atherosclerotic plaques was performed according to a standardized protocol on sections of the culprit segment and has previously been described in detail ${ }^{17}$. In short, plaques were stained with alpha-actin for smooth muscle cells (SMC), CD68 for macrophages, CD34 for microvessels, picrosirius Red (PSR) for collagen and lipid content, haematoxylin eosin (HE) for general overview including calcifications, and $\mathrm{HE}$ and fibrin for intraplaque. All stainings were semi-quantitatively scored by two independent observers. SMC, macrophages, collagen content, presence of calcifications, were scored either as no/minor (0) or moderate/heavy (1) staining. Microvessel density was determined by the average number of vessels of 3 hotspots within every plaque. Lipid content was estimated as a percentage of total plaque area and stratified into less than 10\%/40\% and higher than 10\%/40\%. Intraplaque hemorrhage was rated as absent or present. Intraobserver and interobserver variability were examined previously and showed good reproducibility 
$(\kappa 0.6-0.9)^{18}$. In addition, macrophage and SMC content were scored quantitatively using computerized analysis software AnalySIS 3.2 (Soft Imaging Systems GmbH, Münster, Germany), and reported as percentage positive staining per plaque area.

\section{Outcomes}

The primary outcome, MACE, was a composite encompassing myocardial infarction (MI), stroke or cardiovascular death. Cardiovascular death was defined as: fatal MI, fatal stroke (bleeding or ischemic), fatal ruptured abdominal aneurysm, fatal heart failure or sudden death. Patients who reached multiple endpoints during follow-up only the first manifestation of a cardiovascular event was used for analysis. End-point criteria have been described previously ${ }^{12}$; all were adjudicated by two members of an outcome assessment committee.

\section{Statistical analyses}

Based on the thresholds proposed in previous studies and guidelines ${ }^{4,19,20}$ for $\operatorname{Lp}(\mathrm{a}), \mathrm{Lp}(\mathrm{a})$ levels were stratified in high and low based on the $80^{\text {th }}$ cohort percentile as cut-off. Continuous variables are presented as mean $( \pm \mathrm{SD})$ or as median (IQR) when appropriate. Discrete data are presented as frequencies and percentages. To compare baseline characteristics and plaque composition of patients across high and low Lp(a) groups, Pearson's chi-squared test was used for categorical data, Student's t-test for continuous data and Mann-Whitney U-test for non-normally distributed variables. The MACE-free survival after 30 days and 3 years of follow-up of patients in high and low Lp(a) categories was estimated with Kaplan-Meier survival analysis. Survival was compared by performing the log-rank test. Cox proportional hazard regression was used to calculate the hazard ratio (HR) with 95\% confidence interval (95\% CI) for the association between $\operatorname{Lp}(\mathrm{a})$ and the occurrence of MACE during follow-up. Based on literature: age, sex, smoking status, systolic blood pressure, diabetes, LDL, HDL, history of peripheral artery disease (PAD) and/or coronary artery disease (CAD), and presence of contralateral carotid stenosis were identified as potential confounders. ${ }^{1,4,6,21} \mathrm{LDL}$ cholesterol was corrected for the $\mathrm{Lp}$ (a) contribution by subtracting $30 \%$ of total $\mathrm{Lp}$ (a) mass (corresponding to the cholesterol content in lipoprotein(a)) from LDL cholesterol. Across the selected confounders the proportion missing values was analyzed (Table 1 ). Missing values were imputed using a multiple imputation procedure. ${ }^{22,23}$ The selected confounders were included in a multivariable Cox proportional hazards model. All p values were 2 -tailed, with a value $<0.05$ considered to indicate statistical significance. Statistical analyses were performed with R Studio Version 1.1.456 (R Foundation for Statistical Computing, Vienna, Austria). ${ }^{24}$ 
Table 1. Baseline Characteristics of study population

\begin{tabular}{|c|c|c|c|c|c|}
\hline & & Lipoprotein( & ) $(\mathrm{nmol} / \mathrm{l})$ & & \\
\hline & Total & $\leq 137$ & $>137$ & p-value & $\begin{array}{c}\text { Missing } \\
\text { Values }\end{array}$ \\
\hline & $\mathrm{n}=944$ & $\mathrm{n}=755$ & $n=189$ & & n (\%) \\
\hline Age (years) & $69.7(9)$ & $69.7(9)$ & $69.4(10)$ & 0.63 & $0(0)$ \\
\hline Gender, women & $293(31)$ & $212(28)$ & $81(43)$ & $<0.01$ & $0(0)$ \\
\hline BMI $(\mathrm{kg} / \mathrm{m} 2)$ & $26.2(4)$ & $26.2(4)$ & $26.3(4)$ & 0.85 & $24(2.5)$ \\
\hline Hypertension & $668(73)$ & $536(73)$ & $132(70)$ & 0.51 & $27(2.9)$ \\
\hline Systolic BP (mmHg) & $151(25)$ & $152(25)$ & $148(26)$ & 0.08 & $115(12.2)$ \\
\hline Diastolic BP (mmHg) & $80(13)$ & $81(13)$ & $79(14)$ & 0.25 & $116(12.3)$ \\
\hline eGFR $\left(\mathrm{ml} / \mathrm{min}\right.$ per $\left.1.73 \mathrm{~m}^{2}\right)$ & $74[60,88]$ & $74[60,88]$ & $76[62,89]$ & 0.46 & $71(7.5)$ \\
\hline Current smoker & $321(34)$ & $265(35)$ & $56(30)$ & 0.17 & $9(1.0)$ \\
\hline Diabetes & $209(22)$ & $174(23)$ & $35(18)$ & 0.20 & $0(0)$ \\
\hline Insulin use & $59(6)$ & $54(7)$ & $5(3)$ & 0.02 & $1(0.1)$ \\
\hline Oral glucose inhibitor use & $149(16)$ & $125(17)$ & $24(13)$ & 0.22 & $1(0.1)$ \\
\hline Hypercholesterolaemia & $591(71)$ & $455(68)$ & $136(81)$ & $<0.01$ & $113(12.0)$ \\
\hline Triglycerides (mg/dl) & $1.5[1.1,2.0]$ & $1.5[1.1,2.0]$ & $1.5[1.1,2.0]$ & 0.61 & $4(0.42)$ \\
\hline $\mathrm{LDL}(\mathrm{mmol} / \mathrm{L})$ & $2.0[1.5,2.6]$ & $2.1[1.5,2.6]$ & $1.8[1.3,2.4]$ & $<0.01$ & $4(0.42)$ \\
\hline HDL (mg/dl) & $1.1(0.3)$ & $1.0(0.3)$ & $1.1(0.3)$ & $<0.01$ & $4(0.42)$ \\
\hline Total cholesterol (mg/dl) & $4.1(1.1)$ & $4.1(1.1)$ & $4.4(1.0)$ & $<0.01$ & $4(0.42)$ \\
\hline Anticoagulant use & $92(10)$ & $78(10)$ & $14(7)$ & 0.27 & $1(0.1)$ \\
\hline Antiplatelet use & $833(88)$ & $663(88)$ & $170(90)$ & 0.71 & $3(0.3)$ \\
\hline Statin use & $753(80)$ & $594(79)$ & $159(84)$ & 0.16 & $1(0.1)$ \\
\hline History of CAD & $268(28)$ & $207(28)$ & $61(32)$ & 0.24 & $1(0.1)$ \\
\hline History of Stroke & $314(33)$ & $254(34)$ & $60(32)$ & 0.65 & $0(0)$ \\
\hline History of PAD & $182(19)$ & $146(19)$ & $36(19)$ & 0.98 & $1(0.1)$ \\
\hline Preprocedural neurologic symptoms & & & & 0.87 & $12(1.3)$ \\
\hline Asymptomatic & $119(13)$ & $94(13)$ & $25(13)$ & & \\
\hline Ocular & $267(29)$ & $218(29)$ & $49(26)$ & & \\
\hline TIA & $160(17)$ & $126(17)$ & $34(18)$ & & \\
\hline Stroke & $387(42)$ & $308(41)$ & $79(42)$ & & \\
\hline Contralateral stenosis & & & & 0.14 & $122(12.9)$ \\
\hline $0-50 \%$ & $465(57)$ & $378(58)$ & $87(51)$ & & \\
\hline $50-100 \%$ & $358(44)$ & $275(42)$ & $83(49)$ & & \\
\hline $\begin{array}{l}\text { Time between last event and surgery } \\
\text { (days) }\end{array}$ & $23.0[12.0,50.8]$ & $24[12.0,50.0]$ & $22.5[11.7,51.0]$ & 0.87 & $175(18.5)$ \\
\hline
\end{tabular}

Data are presented as $\mathrm{n}(\%)$, mean \pm stand deviation $( \pm \mathrm{SD})$ or median [interquartile range] [IQR]. Abbreviations: BMI, Body Mass Index; BP blood pressure; eGFR, estimated Glomerular Filtration Rate; LDL, Low-density Lipoprotein; HDL, High-density Lipoprotein; CAD, coronary artery disease; PAD, peripheral artery disease; TIA, Transient Ischemic Attack. Bold values were considered statistically significant with a $\mathrm{p}<0.05$. 


\section{Results}

\section{Patient population \& Baseline characteristics}

944 Patients who underwent CEA with available lipid profile measurements were identified for the current study (Figure 1). Baseline characteristics of the study population are presented in Table 1. The mean age of all patients was 69.7 years $( \pm 9)$ and $31 \%(n=293)$ were women. Based on the $80^{\text {th }}$ percentile as cut-off, patients were stratified in to high $>137 \mathrm{nmol} / \mathrm{L}(\mathrm{n}=190)$ and low $\leq 137 \mathrm{nmol} / \mathrm{L}(\mathrm{n}=755) \mathrm{Lp}(\mathrm{a})$ levels. The number of women was higher in the high Lp(a) group ( $43 \%$ vs $28 \%$, p <0.001) compared to the low $\mathrm{Lp}$ (a) group. The mean follow-up time was $2.57( \pm 0.85)$ years, during which 102 out of $944(10.8 \%)$ patients reached the endpoint MACE. MACE consisted of 27 MI, 63 strokes and 12 cardiovascular-related deaths.

\section{Elevated Lp(a) levels are associated with MACE in 30 days follow-up}

Kaplan-Meier cumulative event rates for MACE after 3 years follow-up were $15.4 \%$ in patients with high $\mathrm{Lp}(\mathrm{a})$ levels and 10.2\% in patients with low $\mathrm{Lp}(\mathrm{a})$ levels ( $\mathrm{p}=0.047$ ) (Figure 2). High Lp(a) levels (above $80^{\text {th }}$ percentile; $>137 \mathrm{nmol} / \mathrm{L}$ ) were significantly associated with 3-year MACE with a HR of 1.54 (95\%CI 1.00-2.39), compared to patients with low Lp(a) levels (below $80^{\text {th }}$ percentile; $<137 \mathrm{nmol} / \mathrm{L}$ ). Adjustment for risk factors led to a more pronounced association with a HR of 1.69 (95\% CI 1.07-2.66) for patients with high Lp(a) levels compared to patients with low Lp(a) levels (Figure 3).

However, out of the 102 patients who reached the endpoint MACE, one-third of the patients (35 patients) had MACE within 30 days post-surgery. We therefore further analyzed the relation of elevated $\mathrm{Lp}(\mathrm{a})$ and the number of MACE that occurred during this 30-day perioperative period. In the subgroup of patients that had perioperative MACE, 6 patients underwent CEA for asymptomatic carotid stenosis and the remaining for symptomatic carotid stenosis. Presenting symptoms that were the reason for CEA were: 10 ocular symptoms (Amaurosis Fugax, ocular ischemic symptoms), 11 TIA, 7 stroke and 1 was unclear to localize. Median time from event to intervention was 23 days [IQR 12.5-46.5]. 30-Day MACE consisted of 28 strokes, 6 MI and 1 cardiovascular related death. KaplanMeier analysis for 30-day MACE revealed a significant difference in MACE-free survival between patients with high and low Lp(a) levels ( $\mathrm{p}=0.032$ ) (Figure 4A). After adjustment for sex and age a strong association was found between high $\mathrm{Lp}(\mathrm{a})$ levels and the risk of 30-day MACE, with a HR of 2.05 (95\% CI 1.01-4.17) (Figure 3). Since the association with 30-day MACE seemed much stronger compared to 3-year MACE, Kaplan-Meier curves were plotted excluding the cases within the first 30 days post-surgery. This revealed no significant association between high Lp(a) levels and MACE from timepoint 30 days to 3 years onward (Figure 4B). 

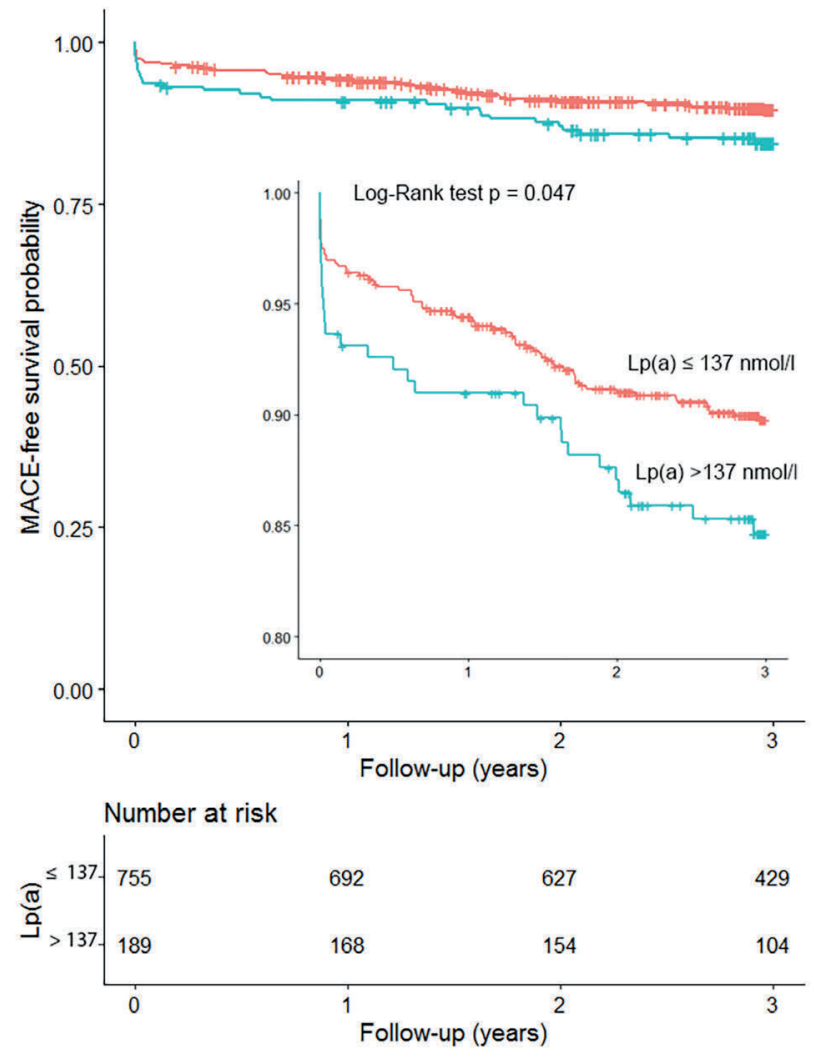

Figure 2. Kaplan-Meier curves according to high and low $\mathrm{Lp}(\mathrm{a})$ levels for the endpoint 3 years MACE Low Lp(a) corresponds to the value of $\leq 137 \mathrm{nmol} / \mathrm{L}$ ( $\leq 80$ th cohort percentile), high Lp(a) corresponds to the value of $>137 \mathrm{nmol} / \mathrm{L}$ ( $>80$ th cohort percentile). P-value of log-rank test. The inset shows the same data on an enlarged y-axis.

\section{Elevated $\mathrm{Lp}$ (a) levels are not associated with plaque characteristics}

To investigate whether high Lp(a) levels result in more MACE due to its association with more vulnerable plaque characteristics, we related plaque characteristics to Lp(a) levels. Using semi-quantitative analysis, plaque characteristics, (such as calcification, collagen, lipid core, intraplaque haemorrhage, macrophage and smooth muscle cell) were compared between patients with low and high levels of $\mathrm{Lp}(\mathrm{a})$. Quantitative measurement was done for vessel density, number of microvessels, macrophage staining and smooth muscle cell staining. For both semi-quantitative as quantitative analysis, no significant differences were found in plaque histology between both groups (Table 2). 


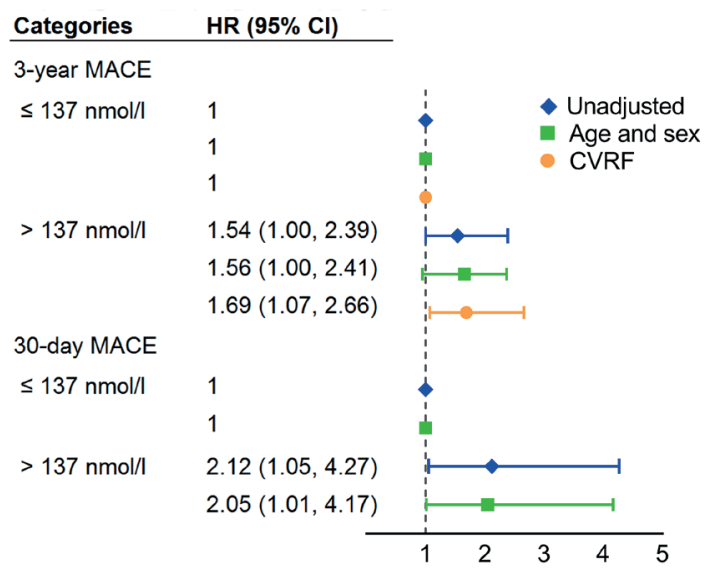

Figure 3. Cox regression analysis according to high $\mathrm{Lp}(\mathrm{a})(>137 \mathrm{nmol} / \mathrm{l},>80$ th cohort percentile) and low $\mathrm{Lp}(\mathrm{a})(\leq 137 \mathrm{nmol} / \mathrm{L}, \leq 80$ th cohort percentile) for the endpoint 3-year MACE and 30-day MACE

Unadjusted hazard ratios (blue rhombus), adjusted hazard ratios for age and sex (green square) and cardiovascular risk factors (CVRF) (orange circle) age, sex, systolic blood pressure, smoking status, diabetes, LDL, HDL, history of PAD and/or CAD and presence of contralateral carotid stenosis.

\section{Discussion}

In the present study, we show that elevated $\mathrm{Lp}$ (a) levels $\left(>137 \mathrm{nmol} / \mathrm{L} ;>80^{\text {th }}\right.$ cohort percentile) are associated with high risk of postoperative MACE, extending the impact of Lp(a) on overall CVD-risk from primary to secondary cardiovascular patients. Strikingly, the association between high Lp(a) and 3-year risk of MACE was completely driven by the events in the first 30 days after CEA.

Observational studies ${ }^{4-6,25}$ have established the association of $\mathrm{Lp}(\mathrm{a})$ with cardiovascular disease risk. The evidence on recurrent cardiovascular events and Lp(a), however, is limited. A general population study on individuals with pre-existing cardiovascular disease revealed that high concentrations of $\mathrm{Lp}$ (a) were associated with a high risk of recurrent cardiovascular events ${ }^{8}$. This association was also found in a cohort of patients with established CAD ${ }^{10}$. Carotid endarterectomy patients, however, were not included in both studies. Accordingly, we measured Lp(a) levels in a large cohort of 944 consecutive CEA patients. In this large cohort, more women were present in the high $\mathrm{Lp}(\mathrm{a})$ group, which is likely ascribed to the inclusion of older postmenopausal women (mean age total high Lp(a) group 69.4 years). This is in line with previous results from a cohort study among patients without ischemic stroke at baseline ${ }^{21}$. Earlier studies in carotid atherosclerosis have shown that high Lp(a) plasma levels are associated with the presence or progression of carotid atherosclerotic 

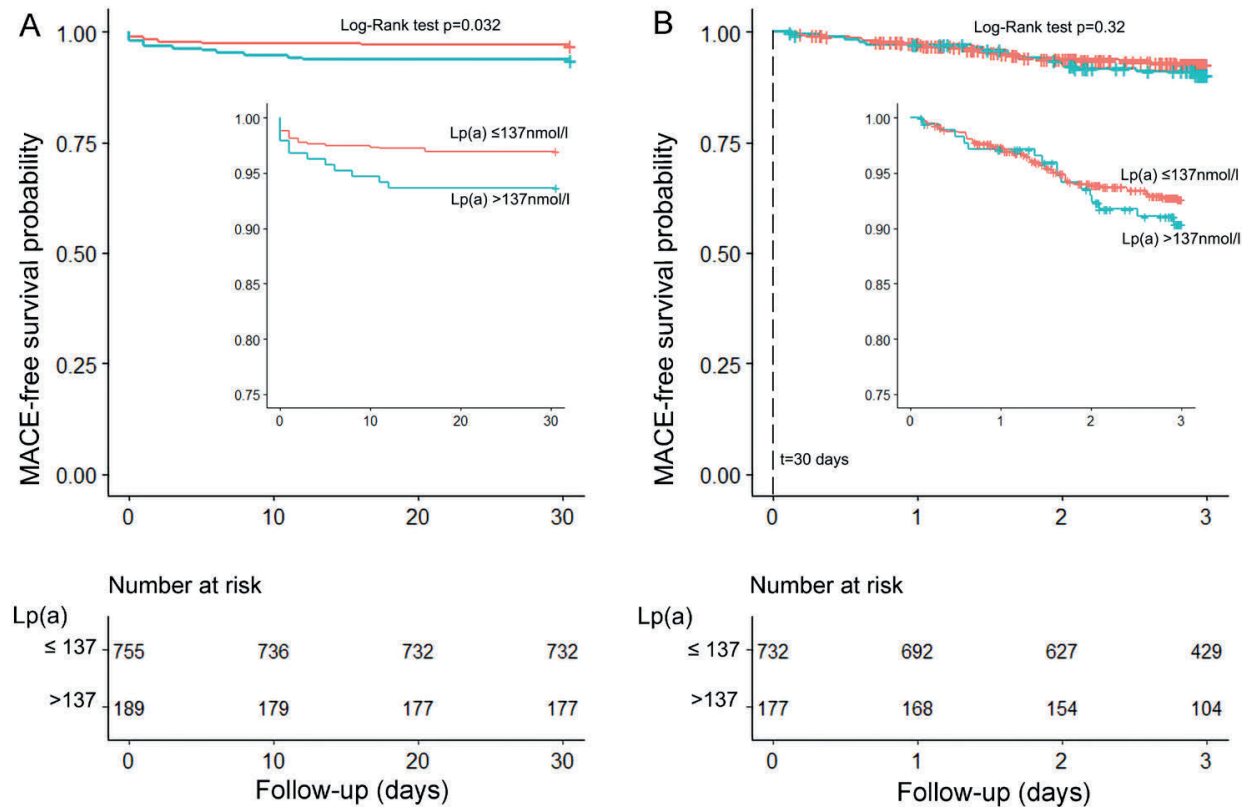

Figure 4. Kaplan-Meier curves according to high and low Lp(a) levels for the endpoint 30-day MACE (A) and 3-year MACE from timepoint 30 days to 3 years (B)

Low Lp(a) corresponds to the value of $\leq 137 \mathrm{nmol} / \mathrm{L}$ ( $\leq 80^{\text {th }}$ cohort percentile), high $\mathrm{Lp}$ (a) corresponds to the value of $>137 \mathrm{nmol} / \mathrm{L}\left(>80^{\text {th }}\right.$ cohort percentile). P-value of log-rank test. The inset shows the same data on an enlarged $y$ - axis.

plaque visualized by different imaging modalities in patients without a cardiovascular history ${ }^{26-29}$. In contrast, in other studies in young adults without cardiovascular risk factors $^{30,31}$, in statin-treated familial hypercholesterolemia patients $\mathrm{s}^{32}$ and in a cohort of patients with premature $\mathrm{CAD}^{33}$, no association was found between high $\mathrm{Lp}(\mathrm{a})$ levels and atherosclerotic changes in the carotid artery. We now show for the first time that elevated Lp(a) levels are associated with MACE 30-days after CEA.

Adjustment for cardiovascular risk factors resulted in an even stronger association between high $\mathrm{Lp}(\mathrm{a})$ levels and 3-year MACE, indicating that $\mathrm{Lp}$ (a) promotes atherosclerosis in the presence of other risk factor ${ }^{30}$. Evidence supporting this, arises from imaging studies that demonstrate an increase in intima-media thickness in patients with elevated Lp(a) levels, in a cohort of dialysis patient $\mathrm{s}^{34}$, and patients with concurrent severe hypercholesterolaemia ${ }^{35}$ or diabetes ${ }^{36}$. This is also supported by studies performed in young adults ${ }^{30,31}$ or in populations without risk factors ${ }^{32}$, where no association between $\mathrm{Lp}(\mathrm{a})$ levels and presence of atherosclerotic changes in the carotid artery were found. 
Table 2. Semi quantitative and continuous plaque characteristics stratified by high and low Lp(a) levels

\begin{tabular}{|c|c|c|c|}
\hline \multirow[b]{2}{*}{ Semi-quantitative plaque characteristics } & \multicolumn{2}{|c|}{ Lipoprotein(a) (nmol/l) } & \multirow[t]{2}{*}{ p-value } \\
\hline & $\begin{array}{l}\leq 137 \\
\mathbf{n}=755\end{array}$ & $\begin{array}{l}>137 \\
\mathrm{n}=189\end{array}$ & \\
\hline Moderate/heavy calcification (\%) & $264(42)$ & $66(48)$ & 0.32 \\
\hline Moderate/heavy collagen staining (\%) & $481(78)$ & $108(77)$ & 0.91 \\
\hline Presence of lipid core $\geq 10 \%(\%)$ & $438(70)$ & $98(70)$ & 0.91 \\
\hline Presence of intraplaque hemorrhage (\%) & $337(54)$ & $80(57)$ & 0.60 \\
\hline Moderate/heavy macrophage infiltration (\%) & $312(51)$ & $72(53)$ & 0.76 \\
\hline Moderate or heavy smooth muscle staining (\%) & $403(65)$ & $101(72)$ & 0.16 \\
\hline Vessel density $\geq$ median (\%) & $245(48)$ & $63(55)$ & 0.18 \\
\hline \multicolumn{4}{|l|}{ Continous quantified plaque characteristics } \\
\hline $\begin{array}{l}\text { Median number of microvessels per hotspot } \\
\text { (median [IQR]) }\end{array}$ & $6.30[3.00,11.00]$ & $7.00[3.67,13.00]$ & 0.10 \\
\hline $\begin{array}{l}\% \text { of positive macrophage staining per plaque } \\
\text { (median [IQR]) }\end{array}$ & $0.22[0.05,0.76]$ & $0.25[0.07,0.71]$ & 0.66 \\
\hline $\begin{array}{l}\% \text { of positive smooth muscle staining per plaque } \\
\text { (median }[\mathrm{IQR}] \text { ) }\end{array}$ & $1.23[0.48,2.44]$ & $1.13[0.50,2.71]$ & 0.75 \\
\hline
\end{tabular}

Abbreviations: IQR; interquartile range

The threshold to define $\mathrm{Lp}(\mathrm{a})$ elevation is a matter of debate. The cut-off in current study was based on the $80^{\text {th }}$ percentile, as proposed in several previous studies and guidelines. ${ }^{4,7,19,20}$ For patients with established coronary heart disease, a non-continuous relation with $\mathrm{Lp}(\mathrm{a})$ levels and secondary cardiovascular events has recently been reported wherein patients in the highest quintile had an increased risk. ${ }^{10}$ Furthermore, Lp(a) has a skewed concentration distribution with a tail toward extremely high levels. We believe Lp(a) measurements in patients with extreme levels have the potential to substantially improve risk prediction. This is supported by other study groups $s^{8,21,37-39}$, and underscored by our additional results for extreme Lp(a) levels (Supplemental Figure I and Supplemental Table I).

In this study, further analysis for 30-day risk of MACE revealed that the 3-year risk of MACE was driven by the events in the first 30 days. Furthermore, the association with elevated Lp(a) levels was much stronger compared to 3-year MACE. The main outcome, 3 -year MACE was mostly determined by stroke (63 out of 102), of these 28 where within 30 days post-surgery. Periprocedural stroke is an important complication of carotid endarterectomy and severely limits the absolute benefit of the procedure $e^{40,41}$. When excluding the cases within the first 30 days post-surgery, no significant association was found between high Lp(a) levels and MACE from 30 days to 3 years onward. These data 
imply that the association of elevated Lp(a) levels and MACE is driven by 30-day stroke outcome. To validate these important findings, studies in larger cohorts are warranted, since the number of perioperative events was low. In literature, the vast majority of studies report an increased risk of primary ischemic stroke in patients with elevated Lp(a) levels $s^{6,21,42-44}$, while only a small number of studies report contrasting results ${ }^{45,46}$. However, the reported association in these studies, is weaker compared to the risk associated with coronary heart disease. In the present study, a much stronger association between elevated $\mathrm{Lp}$ (a) levels and the composite endpoint MACE is observed, with HR 2.05 (95\% CI 1.014.17) for 30-day risk of MACE (Figure 3). Nevertheless, our findings extend to recurrent events, since patients included in the Athero-Express biobank all have an indication for CEA and thus already had their primary event. Furthermore, the risk of early recurrent stroke is related to the underlying pathology, where patients with large artery cerebrovascular disease have a higher early risk of recurrent stroke compared to etiologic subgroups such as small-vessel strokes. ${ }^{47} \mathrm{~A}$ higher risk of recurrent vascular events in patients with elevated $\mathrm{Lp}$ (a) levels (>30mg/dl), after first ischemic stroke has been reported in a previous study, with an adjusted HR of 2.60 (95\% CI, 1.19-5.67) ${ }^{48}$. We therefore extend the results from the previous study and highlight the importance of $\mathrm{Lp}(\mathrm{a})$ in patients with cerebrovascular symptoms caused by carotid stenosis.

Several pathophysiological mechanisms through which $\mathrm{Lp}(\mathrm{a})$ is able to mediate atherogenesis have been proposed. First, as with LDL, Lp(a) was shown to accumulate both in progressive lesions and in ruptured plaques ${ }^{49}$, suggesting that high levels of $\operatorname{Lp}(\mathrm{a})$ in plaque is associated with rupture-prone plaque phenotype. Second, a large body of evidence shows that $\mathrm{Lp}(\mathrm{a})$ exerts its pro-inflammatory properties by the oxidized phospholipids bound to apolipoprotein(a) [apo(a) $]^{7}$. Recent studies have shown that oxidized phospholipids bound to $\operatorname{Lp}(\mathrm{a})$ is able to elicit an inflammatory response in the endothelium, a process fueled by increased endothelial glycolysis and which are corroborated in patients from the Athero-Express biobank ${ }^{50}$. Finally, the structural homology of apolipoprotein(a) with plasminogen can reduce fibrinolytic activity, resulting in reduced thrombus degradation plaque rupture and subsequently larger number of ischemic events. It is proposed that high $\mathrm{Lp}$ (a) levels might promote cardiovascular events by its prothrombotic properties ${ }^{30,51}$. For this, we also assessed the association between high Lp(a) and plaque phenotype (Table 2). Specific plaque characteristics such as large lipid core, inflammation, thin fibrous cap and intraplaque hemorrhage (IPH) are associated with plaque rupture ${ }^{17,52-57}$. However, no statistically significant association between plaque phenotype and $\mathrm{Lp}(\mathrm{a})$ levels was found (Table 2), even though high Lp(a) levels were associated with MACE. In corroboration, the association between plaque characteristics and the outcome 3-year MACE did not show differences between both groups (Supplemental Table II). These results suggest that with 
this dichotomization, $\mathrm{Lp}(\mathrm{a})$ does not appear to affect plaque modification, but may play a role in enhancing a prothrombotic state. A mouse model of ischemic stroke showed that reduced blood plasminogen levels increase infarct size ${ }^{58}$. In humans it was shown that blood Tissue Plasminogen Activator and Plasminogen Activator Inhibitor-1 levels are higher in stroke patients versus controls, suggesting an altered fibrinolytic system in stroke patient ${ }^{59}$. Also the amount of plasminogen bound and plasmin formed at the surface of fibrin are directly related to in vivo variations in the circulating concentration of $\operatorname{Lp}(\mathrm{a})^{60}$. More studies are warranted to investigate if the role of elevated $\mathrm{Lp}(\mathrm{a})$ in modulating fibrinolytic activity leads to a higher risk of stroke within 30 days of surgery in CEA patients. Particular during this time period these patients are prone for carotid embolization: 1) as a consequence of manipulation of the carotid plaque during surgery, 2) the endarterectomized surface that can form emboli or 3 ) emboli that can originate from a loose intimal flap ${ }^{40}$.

If elevated $\mathrm{Lp}(\mathrm{a})$ levels are related to the risk of secondary MACE, this could have impact on clinical decision making. Pre-operatively, $\mathrm{Lp}(\mathrm{a})$ could be used as part of multiscale treatment algorithms to facilitate patient-specific treatment. For instance, patients with high Lp(a) levels in combination with other determinants, could be assigned to CEA as treatment strategy, over carotid stenting (CAS), since CEA is associated with a lower risk of perioperative stroke compared to CAS. However, in order for this to be implemented, future studies in stented carotid stenosis populations are warranted. In addition, this will also have marked consequences for secondary prevention. A recent study investigated whether lipid-lowering treatment with Evolocumab, an anti-Proprotein Convertase Subtilisin/Kexin type 9 monoclonal antibody, alters arterial wall inflammation in patients with elevated Lp(a). Therapy with Evolocumab leads to potent LDL-c reduction (-60\%) and a modest $\mathrm{Lp}(\mathrm{a})$ reduction $(-14 \%)$. Interestingly, this did not reduce arterial wall inflammation, possibly attributable to the residually elevated Lp(a) levels. Hence, Lp(a) lowering therapy, for example using Apo(a) antisense oligonucleotides, is strongly advised ${ }^{61}$, since only lowering the classical modifiable risk factors will leave a high residual risk of MACE in patient with elevated Lp(a) levels ${ }^{62}$.

\section{Strengths and limitations}

For proper interpretation of the study results, some limitations need to be considered. While the continuity of the Athero-Express is unparalleled, it should be noted that over the broad inclusion period, improvements of treatment options, in-hospital care and timing of the surgery have occurred. However, the distribution of MACE and Lp(a) levels, show no evident relationship with surgery year over time (Supplemental Table III and Supplemental Figure II).

Next, the changes in lipid levels or lipid-lowering treatments during the three years of follow-up could not be taken into account. As for the endpoints, we could not determine 
the proportion of strokes caused by thrombo-embolic events attributable to atrial fibrillation. However, all endpoints, were prespecified and assessed by two outcome assessors, ensuring clear differentiation of the outcomes. Even though, the number of stroke events was limited and stroke specific analysis could not be performed, this study provides evidence for future studies to investigate the association of $\mathrm{Lp}(\mathrm{a})$ and 30-day risk of stroke as this will be important in determining the indication for CEA or CAS. Finally, it has to be noted that this study population consisted of Caucasians, making these findings difficult to extrapolate to other ethnicities, since race is a modifying variable in $\mathrm{Lp}(\mathrm{a})$ related risk $^{27}$. A strength of this study is that we are the first to show a strong association between elevated Lp(a) levels and secondary MACE in CEA patients with available plaque histology in a large biobank that is unique in its kind.

\section{Conclusion}

In a carotid endarterectomy population, we demonstrated that elevated Lp(a) levels ( $>137$ $\mathrm{nmol} / \mathrm{L} ;>80^{\text {th }}$ cohort percentile) are associated with an increased risk of 30 -day MACE mainly composed of perioperative stroke. This study identifies elevated Lp(a) levels as a new and additional risk factor for secondary cardiovascular events, potentially identifying patients at highest preoperative risk. 


\section{References}

1 van Lammeren GW, Catanzariti LM, Peelen LM, de Vries J-PPM, de Kleijn DPV, Moll FL, et al. Clinical Prediction Rule to Estimate the Absolute 3-Year Risk of Major Cardiovascular Events After Carotid Endarterectomy. Stroke 2012;43(5):1273-8.

2 Halliday A, Mansfield A, Marro J, Peto C, Peto R, Potter J, et al. Prevention of disabling and fatal strokes by successful carotid endarterectomy in patients without recent neurological symptoms: randomised controlled trial. Lancet 2004;363(9420):1491-502.

3 van Koeverden ID, van Haelst STW, Haitjema S, de Vries JPPM, Moll FL, den Ruijter HM, et al. Timedependent trends in cardiovascular adverse events during follow-up after carotid or iliofemoral endarterectomy. Br J Surg 2017;104(11):1477-85.

4 Nordestgaard BG, Chapman MJ, Ray K, Borén J, Andreotti F, Watts GF, et al. Lipoprotein(a) as a cardiovascular risk factor: Current status. Eur Heart J 2010;31(23):2844-53.

5 Kamstrup PR, Tybjærg-hansen A, Steffensen R, Nordestgaard BG. Genetically Elevated Lipoprotein ( a ). J Am Med Assoc 2009;301(22):2331-9.

6 The Emerging Risk Factors Collaboration. Lipoprotein ( a ) Concentration and the Risk of Coronary Heart Disease, Stroke ,. JAMA 2009;302(4):412-23.

7 Tsimikas S. A Test in Context: Lipoprotein(a): Diagnosis, Prognosis, Controversies, and Emerging Therapies. J Am Coll Cardiol 2017;69(6):692-711.

8 Madsen CM, Kamstrup PR, Langsted A, Varbo A, Nordestgaard BG. Lp(a) (Lipoprotein[a])-Lowering by $50 \mathrm{mg} / \mathrm{dL}$ (105 nmol/L) May Be Needed to Reduce Cardiovascular Disease 20\% in Secondary Prevention. Arterioscler Thromb Vasc Biol 2019;(January):255-66.

9 Willeit P, Ridker PM, Nestel PJ, Simes J, Tonkin AM, Pedersen TR, et al. Baseline and on-statin treatment lipoprotein(a) levels for prediction of cardiovascular events: individual patient-data meta-analysis of statin outcome trials. Lancet 2018;392(10155):1311-20.

10 O’Donoghue ML, Morrow DA, Tsimikas S, Sloan S, Ren AF, Hoffman EB, et al. Lipoprotein(a) for risk assessment in patients with established coronary artery disease. J Am Coll Cardiol 2014;63(6):520-7.

11 Bittner VA, Szarek M, Aylward PE, Bhatt DL, Diaz R, Edelberg JM, et al. Effect of Alirocumab on Lipoprotein(a) and Cardiovascular Risk After Acute Coronary Syndrome. J Am Coll Cardiol 2020;75(2):133-44.

12 Verhoeven BAN, Velema E, Schoneveld AH, de Vries JPPM, de Bruin P, Seldenrijk CA, et al. Atheroexpress: differential atherosclerotic plaque expression of mRNA and protein in relation to cardiovascular events and patient characteristics. Rationale and design. Eur J Epidemiol 2004;19(12):1127-33.

13 Barnett HJM, Taylor DW, Eliasziw M, Fox AJ, Ferguson GG, Haynes RB, et al. Benefit of carotid endarterectomy in patients with symptomatic moderate or severe stenosis. N Engl J Med 1998;339(20):1415-25.

14 Warlow C, Farrell B, Fraser A, Sandercock P, Slattery J. Randomised trial of endarterectomy for recently symptomatic carotid stenosis: Final results of the MRC European Carotid Surgery Trial (ECST). Lancet 1998;351(9113):1379-87.

15 Norgren L, Hiatt WR, Dormandy JA, Nehler MR, Harris KA, Fowkes FG, et al. Inter-society consensus for the management of peripheral arterial disease. Int Angiol 2007;26(2):81-157.

16 Ferguson GG, Eliasziw M, Barr HWK, Clagett GP, Barnes RW, Wallace MC, et al. The North American Symptomatic Carotid Endarterectomy Trial. Stroke 1999;30(9):1751-8.

17 Hellings WE, Peeters W, Moll FL, Piers SRD, van Setten J, Van der Spek PJ, et al. Composition of Carotid Atherosclerotic Plaque Is Associated With Cardiovascular Outcome. Circulation 2010;121(17):1941-50.

18 Hellings WE, Pasterkamp G, Vollebregt A, Seldenrijk CA, De Vries J-PPM, Velema E, et al. Intraobserver and interobserver variability and spatial differences in histologic examination of carotid endarterectomy specimens. J Vasc Surg 2007;46(6):1147-54.

19 Waldeyer C, Makarova N, Zeller T, Schnabel RB, Brunner FJ, Jørgensen T, et al. Lipoprotein(a) and the risk of cardiovascular disease in the European population: Results from the BiomarCaRE consortium. Eur Heart J 2017;38(32):2490-8.

20 Mach F, Baigent C, Catapano AL, Koskinas KC, Casula M, Badimon L, et al. 2019 ESC/EAS Guidelines for the management of dyslipidaemias: lipid modification to reduce cardiovascular risk. Eur Heart $J$ 2020;41(1):111-88. 
21 Langsted A, Nordestgaard BG, Kamstrup PR. Elevated Lipoprotein(a) and Risk of Ischemic Stroke. J Am Coll Cardiol 2019;74(1):54-66.

22 Janssen KJM, Donders ART, Harrell FE, Vergouwe Y, Chen Q, Grobbee DE, et al. Missing covariate data in medical research: To impute is better than to ignore. J Clin Epidemiol 2010.

23 White IR, Royston P. Imputing missing covariate values for the Cox model. Stat Med 2009;28(15):1982-98.

24 Team RC. R: A language and environment for statistical computing. 2018.

25 Nordestgaard BG, Langsted A. Lipoprotein (a) as a cause of cardiovascular disease: insights from epidemiology, genetics, and biology. J Lipid Res 2016;57(11):1953-75.

26 Klein JH, Hegele RA, Hackam DG, Koschinsky ML, Huff MW, Spence JD. Lipoprotein(a) is associated differentially with carotid stenosis, occlusion, and total plaque area. Arterioscler Thromb Vasc Biol 2008;28(10):1851-6.

27 Steffen BT, Thanassoulis G, Duprez D, Stein JH, Karger AB, Tattersall MC, et al. Risk of Carotid Atherosclerosis The Multi-Ethnic Study of Atherosclerosis 2019:523-9.

28 Hippe DS, Phan BAP, Sun J, Isquith DA, O’Brien KD, Crouse JR, et al. Lp(a) (lipoprotein(a)) levels predict progression of carotid atherosclerosis in subjects with atherosclerotic cardiovascular disease on intensive lipid therapy an analysis of the AIM-HIGH (atherothrombosis intervention in metabolic syndrome with low HDL/h. Arterioscler Thromb Vasc Biol 2018;38(3):673-8.

29 Kamstrup PR, Tybjærg-Hansen A, Nordestgaard BG. Genetic evidence that lipoprotein(a) associates with atherosclerotic stenosis rather than venous thrombosis. Arterioscler Thromb Vasc Biol 2012;32(7):1732-41.

30 Grebe MT, Schoene E, Schaefer CA, Boedeker RH, Kemkes-Matthes B, Voss R, et al. Elevated Lipoprotein(a) does not promote early atherosclerotic changes of the carotid arteries in young, healthy adults. Atherosclerosis 2007;190(1):194-8.

31 Kivimäki M, Magnussen CG, Juonala M, Kähönen M, Kettunen J, Loo BM, et al. Conventional and Mendelian randomization analyses suggest no association between lipoprotein(a) and early atherosclerosis: The Young Finns Study. Int J Epidemiol 2011;40(2):470-8.

32 Bos S, Duvekot MHC, Touw-Blommesteijn AC, Verhoeven AJM, Mulder MT, Watts GF, et al. Lipoprotein (a) levels are not associated with carotid plaques and carotid intima media thickness in statin-treated patients with familial hypercholesterolemia. Atherosclerosis 2015;242(1):226-9.

33 Ooi EM, Ellis KL, Barrett PHR, Watts GF, Hung J, Beilby JP, et al. Lipoprotein(a) and apolipoprotein(a) isoform size: Associations with angiographic extent and severity of coronary artery disease, and carotid artery plaque. Atherosclerosis 2018;275:232-8.

34 Ohkuma T, Minagawa T, Takada N, Ohno M, Oda H, Ohashi H. C-reactive protein, lipoprotein(a), homocysteine, and male sex contribute to carotid atherosclerosis in peritoneal dialysis patients. Am J Kidney Dis 2003;42(2):355-61.

35 Baldassarre D, Tremoli E, Franceschini G, MichelagnoliS, SirtoriCR. Plasma lipoprotein(a) is an independent factor associated with carotid wall thickening in severely but not moderately hypercholesterolemic patients. Stroke 1996;27(6):1044-9.

36 Yamamoto M, Egusa G, Yamakido M. Carotid atherosclerosis and serum lipoprotein(a) concentrations in patients with NIDDM. Diabetes Care 1997;20(5):829-31.

37 Kamstrup PR, Tybjærg-Hansen A, Nordestgaard BG. Extreme lipoprotein(a) levels and improved cardiovascular risk prediction. J Am Coll Cardiol 2013;61(11):1146-56.

38 Kamstrup PR, Benn M, Tybjærg-Hansen A, Nordestgaard BG. Extreme lipoprotein(a) levels and risk of myocardial infarction in the general population: The Copenhagen City Heart Study. Circulation 2008;117(2):176-84.

39 Suk Danik J., Rifai N., Buring JE, Ridker PM. Independent of Apolipoprotein ( a ) Isoform Events Among Initially Healthy Women. JAMA 2006;296(11):1363-70.

40 Huibers A, Calvet D, Kennedy F, Czuriga-Kovács KR, Featherstone RL, Moll FL, et al. Mechanism of Procedural Stroke Following Carotid Endarterectomy or Carotid Artery Stenting Within the International Carotid Stenting Study (ICSS) Randomised Trial. Eur J Vasc Endovasc Surg 2015;50(3):281-8.

41 Riles TS, Imparato AM, Jacobowitz GR, Lamparello PJ, Giangola G, Adelman MA, et al. The cause of perioperative stroke after carotid endarterectomy. J Vasc Surg 1994;19(2):206-16.

42 Virani SS, Brautbar A, Davis BC, Nambi V, Hoogeveen RC, Sharrett AR, et al. Associations between lipoprotein(a) levels and cardiovascular outcomes in black and white subjects: The Atherosclerosis Risk in Communities (ARIC) study. Circulation 2012;125(2):241-9. 
43 Nave AH, Lange KS, Leonards CO, Siegerink B, Doehner W, Landmesser U, et al. Lipoprotein (a) as a risk factor for ischemic stroke: A meta-analysis. Atherosclerosis 2015;242(2):496-503.

44 Smolders B, Lemmens R, Thijs V. Lipoprotein (a) and stroke: A meta-analysis of observational studies. Stroke 2007;38(6):1959-66.

45 Canouï-Poitrine F, Luc G, Bard JM, Ferrieres J, Yarnell J, Arveiler D, et al. Relative contribution of lipids and apolipoproteins to incident coronary heart disease and ischemic stroke: The PRIME Study. Cerebrovasc Dis 2010;30(3):252-9.

46 Gurdasani D, Sjouke B, Tsimikas S, Hovingh GK, Luben RN, Wainwright NWJ, et al. Lipoprotein(a) and risk of coronary, cerebrovascular, and peripheral artery disease: The EPIC-norfolk prospective population study. Arterioscler Thromb Vasc Biol 2012;32(12):3058-65.

47 Lovett JK, Coull AJ, Rothwell PM. Early risk of recurrence by subtype of ischemic stroke in populationbased incidence studies. Neurology 2004;62(4):569-73.

48 Lange KS, Nave AH, Liman TG, Grittner U, Endres M, Ebinger M. Lipoprotein(a) Levels and Recurrent Vascular Events After First Ischemic Stroke. Stroke 2017;48(1):36-42.

49 Van Dijk RA, Kolodgie F, Ravandi A, Leibundgut G, Hu PP, Prasad A, et al. Differential expression of oxidation-specific epitopes and apolipoprotein(a) in progressing and ruptured human coronary and carotid atherosclerotic lesions. J Lipid Res 2012;53(12):2773-90.

50 Schnitzler JG, Hoogeveen RM, Ali L, Prange KHM, Waissi F, van Weeghel M, et al. Atherogenic Lipoprotein(a) Increases Vascular Glycolysis, Thereby Facilitating Inflammation and Leukocyte Extravasation. Circ Res 2020;126(10):1346-59.

51 Martínez C, Rivera J, Loyau S, Corral J, González-Conejero R, Lozano ML, et al. Binding of recombinant apolipoprotein(a) to human platelets and effect on platelet aggregation. Thromb Haemost 2001;85(4):686-93.

52 Golledge J, Cuming R, Ellis M, Davies AH, Greenhalgh RM. Carotid plaque characteristics and presenting symptom. Br J Surg 1997;84(12):1697-701.

53 Finn A V., Nakano M, Narula J, Kolodgie FD, Virmani R. Concept of vulnerable/unstable plaque. Arterioscler Thromb Vasc Biol 2010;30(7):1282-92.

54 De Kleijn DPV, Moll FL, Hellings WE, Ozsarlak-Sozer G, De Bruin P, Doevendans PA, et al. Local atherosclerotic plaques are a source of prognostic biomarkers for adverse cardiovascular events. Arterioscler Thromb Vasc Biol 2010;30(3):612-9.

55 van Lammeren GW, de Vries JPPM, Vink A, de Kleijn DPV, Moll FL, Pasterkamp G. New Predictors of Adverse Cardiovascular Events Following Vascular Surgery. Semin Cardiothorac Vasc Anesth 2010;14(2):148-53.

56 Naghavi M, Libby P, Falk E, Casscells SW, Litovsky S, Rumberger J, et al. From vulnerable plaque to vulnerable patient: A call for new definitions and risk assessment strategies: Part I. Circulation 2003;108(14):1664-72.

57 Libby P, Pasterkamp G. Requiem for the “vulnerable plaque.” Eur Heart J 2015;36(43):2984-7.

58 Singh S, Houng AK, Wang D, Reed GL. Physiologic variations in blood plasminogen levels affect outcomes after acute cerebral thromboembolism in mice: a pathophysiologic role for microvascular thrombosis. $J$ Thromb Haemost 2016;14(9):1822-32.

59 Lindgren A, Lindoff C, Norrving B, Åstedt B, Johansson BB. Tissue Plasminogen Activator and Plasminogen Activator Inhibitor-1 in Stroke Patients. Stroke 1996;27(6):1066-71.

60 ANGLÉS-CANO E, DÍAZ ADLP, LOYAU S. Inhibition of Fibrinolysis by Lipoprotein(a). Ann N Y Acad Sci 2006;936(1):261-75.

61 Boffa MB, Koschinsky ML. Therapeutic Lowering of Lipoprotein(a): A Role for Pharmacogenetics? Circ Genomic Precis Med 2018;11(2):e002052.

62 Stiekema LCA, Stroes ESG, Verweij SL, Kassahun H, Chen L, Wasserman SM, et al. Persistent arterial wall inflammation in patients with elevated lipoprotein(a) despite strong low-density lipoprotein cholesterol reduction by proprotein convertase subtilisin/kexin type 9 antibody treatment. Eur Heart $J$ 2019;40(33):2775-81. 


\section{Supplemental materials}

Supplemental Table I. Results of the cox-regression analysis for extreme elevated $\mathrm{Lp}(\mathrm{a})$ levels divided in three groups for the endpoint 3-year MACE

\begin{tabular}{lll}
\hline & $\begin{array}{l}\text { Univariate analysis } \\
\text { HR }(\mathbf{9 5 \%} \mathbf{C I})\end{array}$ & Number of MACE \\
\hline$<80$ & Reference & 74 \\
$80-90$ & $1.34(0.75-2.36)$ & 14 \\
$>90$ & $1.81(1.02-3.21)$ & 14 \\
\hline
\end{tabular}

$\mathrm{Lp}$ (a) $<80^{\text {th }}$ cohort percentile corresponds to the value of $<137 \mathrm{nmol} / \mathrm{L}, \mathrm{Lp}(\mathrm{a})$ between $80^{\text {th }}-90^{\text {th }}$ percentile corresponds to the value of $137-205 \mathrm{nmol} / \mathrm{L}$ - and $\mathrm{Lp}(\mathrm{a})>90^{\text {th }}$ cohort percentile corresponds to $>205 \mathrm{nmol} / \mathrm{L}$.

Supplemental Tables II-III and Supplemental Figures I-II can be found online. 


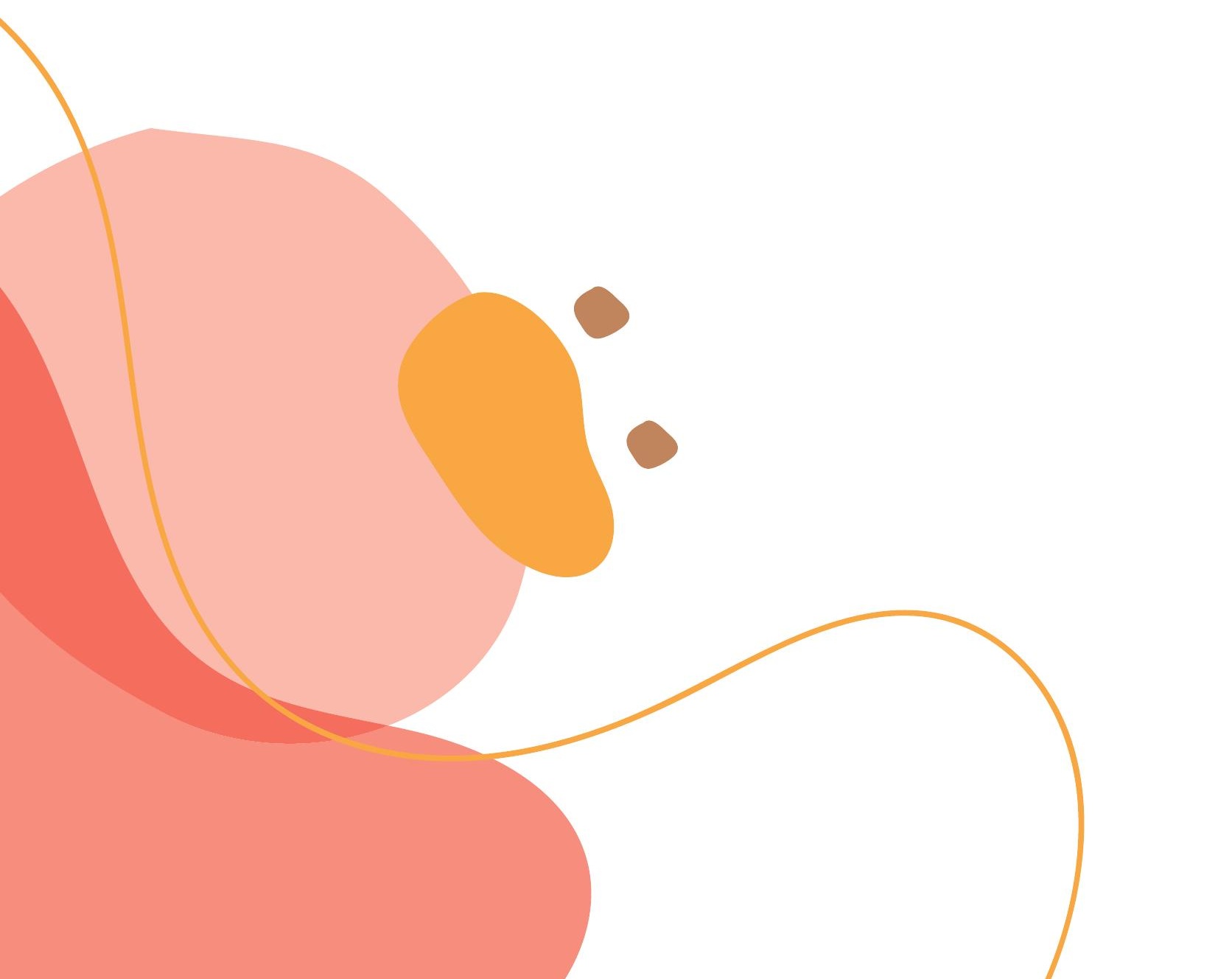




\section{High Tenascin-C plasma extracellular vesicles levels are associated with an increased risk of major adverse cardiovascular events in carotid endarterectomy patients}

Under review- Arteriosclerosis, Thrombosis, and Vascular Biology

Nathalie Timmerman*

Farahnaz Waissi* Mirthe Dekker Stella Bijkerk Joelle van Bennekom Arjan H. Schoneveld

Rong-Rong Zhao Robbert J. de Winter Gerard Pasterkamp Gert J. de Borst Dominique P.V. de Kleijn 


\section{Abstract}

\section{Objective}

Tenascin-C (TN-C) is a large extracellular matrix glycoprotein. TN-C expression in atherosclerotic plaques is associated with plaque rupture and localizes in areas of high macrophage infiltration. After carotid endarterectomy (CEA), patients remain at high risk for major adverse cardiovascular events (MACE). Previous studies identified plasma or serum levels of TN-C as a potential circulating biomarker for prognosis of cardiovascular events. The association of MACE and TN-C measured in plasma extracellular vesicles (EVs) has not been explored yet. We investigated whether plasma EV TN-C levels in CEA patients are associated with the 3 -year postoperative risk of MACE.

\section{Methods}

Plasma EV TN-C levels were determined in pre-operative blood samples of 837 symptomatic and asymptomatic CEA patients included in the Athero-Express Biobank study. The association of EV TN-C levels and MACE was analyzed with Cox-regression analysis adjusted for cardiovascular risk factors. Logistic and linear regression models were used to assess the association of EV TN-C levels and plaque characteristics.

\section{Results}

Cox-regression analysis revealed a significant association of plasma EV TN-C levels and future MACE with a HR of 1.30 (95\% CI 1.12-1.54) per SD increase of EV TN-C on a logarithmic scale. TN-C was associated with moderate/heavy collagen staining; OR 1.28 (95\% CI 1.03-1.60) and moderate/heavy macrophage content; OR 1.33 (95\% CI 1.11-1.60) and more microvessels with an adjusted beta of 0.07 (95\%CI 0.01-0.13).

\section{Conclusion}

Plasma EV TN-C levels are independently associated with an increased 3-year risk of MACE in patients undergoing CEA. This identifies EV TN-C as a possible prognostic aid in CEA patients.

\section{Highlights}

- TN-C is measurable in plasma extracellular vesicles

- Increased levels of circulating TN-C in plasma extracellular vesicles are independently associated with an increased 3-year risk of MACE

- High TN-C EV levels were independently associated with plaques composed of high collagen content, heavy macrophage infiltration and more microvessels.

- TN-C EV levels could aid as a prognostic biomarker in risk-stratification of CEA patients. 


\section{Introduction}

In symptomatic patients with high degree carotid stenosis, carotid endarterectomy (CEA) is the preferred treatment to prevent recurrent ischemic events. ${ }^{1}$ Successful CEA, however, does not fully eliminate the risk for future cardiovascular events (CVE). Due to the systemic nature of atherosclerosis, patients with manifest atherosclerotic disease are prone to develop secondary events. These events are often caused by plaque destabilization at other sites of the vascular bed. ${ }^{1,2}$ Identification of patients that are at high-risk for secondary events remains challenging as these patients often have a high atherosclerotic plaque burden that has been present for decades. Despite control of risk factors with secondary prevention therapy, the residual risk for secondary CVE after CEA is estimated to be up to $20 \%{ }^{3}$. According to current European guidelines ${ }^{4}$ all CEA patients are considered to be part of the very-high risk group and thus eligible for further intensification of secondary prevention therapies. Recent studies however, have established that the true risk of future secondary CVE varies among individuals. ${ }^{5}$ As intensification of secondary prevention is often costly there is a pressing need for prognostic biomarkers to identify patients that are truly part of the very-high risk group. Until now, clinical models and existing circulating biomarkers do not accurately predict the risk associated with secondary CVE. ${ }^{5,6}$

Extracellular vesicles (EVs) are nanometer size structures consisting of a lipid bilayer membrane, and include exosomes, microvesicles and microparticles. ${ }^{7}$ They are released in almost all biological fluids and contain proteins, lipids and nucleic acids from the cell of origin. For this, EVs are often referred to as liquid biopsies as pathological changes in the cell of origin are reflected in the number of circulating EVs and/or EV content released from this cell. ${ }^{7-9}$ Plasma EV protein content has already proven to be associated with future CVE in patients with clinically manifest vascular disease. ${ }^{8}$

Tenascin C (TN-C) is a large extracellular matrix (ECM) glycoprotein. In healthy tissue it has a restricted distribution ${ }^{10}$. It could, however, potentially be a useful marker for disease, as higher levels have been identified in areas of wound healing, cancer and cardiovascular disease. ${ }^{11}$ In atherosclerosis, plaque TN-C is associated with plaque formation, plaque rupture and localizes in areas of high macrophage infiltration. ${ }^{11-14}$ Elevated levels of TN-C have been detected in plasma and have been related to future CVE in cardiomyopathy patients, chronic kidney disease patients and patients after MI. ${ }^{10,15-17}$ These previous studies identified plasma or serum levels of TN-C as a potential circulating biomarker for prognosis of CVE. Only a few studies have demonstrated that TN-C is also measurable in EVs and is associated with the pathological state of the cell of origin. ${ }^{18-21}$ These studies were mainly performed in the field of oncology until date no studies in cardiovascular research have 
identified TN-C in EVs in relation to future CVE and no studies have explored this relation in CEA patients. Measuring TN-C in EVs has the potential to enhance our understanding of crosstalk between vascular cells and their atherosclerotic microenvironment, while also identifying potential biomarkers of patients with severe atherosclerotic disease. Therefore, in this study, we measured EV TN-C levels in a large cohort of CEA patients and determined whether there is an association with an increased risk of future MACE.

\section{Methods}

The data that support the findings of this study are available from the corresponding author upon reasonable request.

\section{Study population}

For the current study all patients undergoing CEA for symptomatic or asymptomatic stenosis between 2002-2016 from the Athero-Express Biobank with available citrate blood plasma samples and follow-up data, were considered eligible. Patients who underwent CEA for restenosis were excluded. A detailed description of the study design has been published previously. ${ }^{22}$ In summary, the Athero-Express Biobank is an ongoing prospective biobank study that includes consecutive patients scheduled for CEA in two tertiary referral hospitals (the St. Antonius hospital Nieuwegein (inclusions stopped after 2014) and University Medical Center Utrecht, the Netherlands). Indications for CEA were adjudicated by a multidisciplinary vascular team and surgery was performed by experienced surgeons in accordance with local and international guidelines. ${ }^{1,23-26}$ Blood samples were collected during hospital admission prior to surgery and stored in $-80^{\circ} \mathrm{C}$ freezer until further use. For the current study citrate plasma samples (collected $2000 \mathrm{~g}$ for $10 \mathrm{~min}$ at RT) were used. Plasma was aliquoted and samples were stored at $-80 \mathrm{C}$ within 2 hours after collection. The atherosclerotic plaque was obtained during CEA and transferred to the laboratory for further histological analyses. Baseline patient characteristics and basic laboratory parameters were collected through standardized preoperative questionnaires and medical records. Follow-up data on CVE or hospital admissions was collected through annual questionnaires for the first three consecutive years after CEA. If an outcome event was reported, this was validated with hospital medical records or discharge letters from the institution were the event occurred. In case of no response to questionnaires, the general practitioner was consulted for follow-up information. The medical ethics board of both hospitals approved the study and all study participants provided written informed consent. The study is conducted in accordance with the declaration of Helsinki. 


\section{Tissue processing and histopathological assessment}

As previously has been described, histological assessment was performed according to a standardized protocol on paraffin embedded consecutive sections of the culprit segment., ${ }^{3,22}$ Plaques were stained with haematoxylin eosin (HE) for a general overview including calcifications, CD68 for macrophages, alpha-actin for smooth muscle cells (SMC), picrosirius Red (PSR) for collagen and lipid content, CD34 for microvessels, and HE and fibrin for intraplaque hemorrhage. All stained sections were semi quantitatively assessed on microscope with $40 \mathrm{x}$ magnification by two independent observers according to predefined criteria. ${ }^{3}$ SMC, macrophages, collagen content and presence of calcifications, were scored either as no/minor (0) or moderate/heavy (1) staining. Lipid content was estimated as a percentage of total plaque area and stratified into less than $10 \%$ or above $10 \%$ and less than $40 \%$ or above $40 \%$. Intraplaque hemorrhage was defined as hemorrhage in the plaque or at the luminal border as a consequence of plaque disruption and rated as absent or present. Intraobserver and interobserver variability were examined previously and showed good reproducibility ( $\kappa$ 0.6-0.9). ${ }^{27}$ In addition, macrophage, SMC content and microvessel density were scored quantitatively using computerized analysis software AnalySIS 3.2 (Soft Imaging Systems GmbH, Münster, Germany). Macrophage and SMC content were reported as percentages of positive staining per plaque area. Microvessel density was determined by counting the number of CD34 positive vessels in 3 hotspots within every plaque and was expressed as the average number per square millimeter. ${ }^{28}$

\section{Study outcome}

The primary outcome was defined as the three-year postoperative risk of MACE. MACE was a composite endpoint encompassing fatal- or nonfatal myocardial infarction (MI), fatal- or nonfatal ischemic or hemorrhagic stroke, and CV death due to other causes, including sudden cardiac death, fatal aneurysm rupture and fatal cardiac failure. Endpoint criteria have been described previously ${ }^{22}$; all were adjudicated by two independent researchers. In case of disagreement, a third expert (GJdB) was consulted.

\section{Plasma extracellular vesicle isolation}

TN-C was measured in the EV subset that co-precipitated with Low-Density Lipid particles (LDL). This LDL subfraction was obtained from $25 \mathrm{uL}$ plasma diluted in $80 \mathrm{uL}$ phosphate buffered saline (PBS) (Gibco) and $5 \mu \mathrm{L}$ magnetic dextran nanoparticles (Nanomag ${ }^{\circledR}-\mathrm{D}$, $130 \mathrm{~mm}, 1: 25$ ) (Micromod). To be able to correct for loss of the pellet during isolation, $15 \mu \mathrm{L}$ of a standard amount of synthetic liposomes, coated with DSG-PEG2000 (Nanocs) and fluorescently labeled with 18:1 liss rhod pe (Merck), was added to each plasma sample. A total volume of $125 \mu \mathrm{l}$ was reached and Dextran Sulphate (DS, $0.05 \%$, MP Biomedicals) and Manganese II Chloride ( $\mathrm{MnCl} 2,0.05 \mathrm{M}$, Sigma-Aldrich) were added to the plasma samples. 
The mixture was incubated for $5 \mathrm{~min}$ at room temperature (RT). Subsequently, the samples were placed on a Bio-plex 200 systems handheld magnet (Bio-Rad) and incubated for 15 min at RT. The precipitate containing the EVs was lysed with $125 \mu \mathrm{L}$ Roche complete lysis-M with protease inhibitors (Roche). Samples were centrifugated at 3200xg for $10 \mathrm{~min}$ to remove magnetic beads and other debris. Fluorescence of the synthetic liposomes were measured with SpectraMax ${ }^{\oplus}$ Multi-Mode Microplate reader (Molecular Devices) directly after completion of the isolation protocol.

\section{EV characterization}

The extracellular vesicle characterization is described in detail in previously published papers. ${ }^{9,29,30}$ In short, density gradient centrifugation of the LDL subfraction, precipitated as described above, was performed to obtain 10 density gradient fractions. Previous studies already established with western blot analysis ${ }^{9,29,30}$ that the LDL density gradient fractions 1.02-1.08 contain the highest levels of CD9, an EV specific marker. Next, in the density gradient fractions LDL lipid particles were identified using $\mathrm{ApoB}$ and $\mathrm{TN}-\mathrm{C}$ was quantified with Human Tenascin C ELISA kit (AB213831). High TN-C was present in the $3^{\text {th }}$ density gradient fraction which also was positive for CD9 in the western blot analysis ${ }^{29}$ (Table I, supplemental materials), from which can be concluded that TN-C is indeed expressed in EVs. The peak value measured in the $7^{\text {th }}$ subfraction suggests that $\mathrm{TN}-\mathrm{C}$ release occurs in two formats: TN-C protein associated with EVs and soluble $\mathrm{TN}-\mathrm{C}$ protein. ${ }^{21}$ The presence of EVs was previously ${ }^{9,29,30}$ confirmed also with electron microscopy (EM) showing the typical bilayer EVs. ${ }^{29}$ To get easy access to these published data an EV-track ID was created: EV200044.

\section{Quantification of Tenascin-C in the plasma extracellular vesicle LDL subfraction}

Having established with the density gradient experiment and the Westernblot analysis that $\mathrm{TN}-\mathrm{C}$ is present in the EV fraction that co-precipitated with LDL particles, we quantified TN-C in the lysed precipitate of the LDL subfraction using an electrochemiluminescence immunoassay (Quickplex SQ120, Meso Scale Discovery, MSD) following manufacturer's protocol. These measurements were used for further analysis.

\section{Tenascin-C plasma measurements}

$\mathrm{TN}-\mathrm{C}$ levels in plasma were assessed in a 30 vs. 30 matched case-control study from the same study population. Patients were matched on: sex, age, history of PAD/CAD, diabetes, current smoking, GFR and hypertension. Tenascin-C was quantified using an electrochemiluminescence immunoassay (Quickplex SQ120, Meso Scale Discovery, MSD) following manufacturer's protocol in Human Tenascin C ELISA kit (AB213831). 
All research materials listed in the Materials and Methods can be found in the Major Resources Table in the Supplemental Materials.

\section{Statistical analysis}

Continuous variables are summarized as mean \pm standard deviation (SD) or median and interquartile range (IQR) where appropriate. Categorical data are expressed as frequencies and percentages. To compare baseline characteristics across patients with and without MACE during follow-up; Student's t-test was used for continuous data, Mann-Whitney U-test for non-normally distributed variables and Pearson's chi-squared test or Fisher's exact test was used for categorical data where appropriate. EV TN-C levels $(\mathrm{pg} / \mathrm{ml})$ were divided by the synthetic liposomes (SVs) to serve as internal control for the loss of pellet during isolation. To obtain a normal distribution the corrected EV TN-C levels and creatinine levels were logarithmically transformed. To facilitate the comparability between continuous variables in the models, age, log creatinine levels and log EV TN-C levels were standardized to Z-scores.

For more in depth exploration of the association of EV TN-C levels with the occurrence of MACE during the 3-year follow-up, EV TN-C levels were divided in quartiles and visualized with Kaplan-Meier survival analysis. For further insight in clinical applicability, the optimal cut-off value was determined, which was used to differentiate the risk of high and low EV TN-C levels. The overall MACE-free survival across quartiles and high and low levels of TN-C was compared by performing the log-rank test.

To assess the association between plasma EV TN-C levels in logarithmic scale and the occurrence of MACE during 3-year follow-up, multivariable Cox proportional hazards regression was used to calculate the hazard ratio (HR) expressed per SD with 95\% confidence interval (95\% CI). For this analysis EV TN-C levels were used as a continuous variable and as the categories mentioned above and added to a clinical model including only cardiovascular risk factors (CVRF). Based on literature following variables were identified as potential confounders: age, sex, current smoking, creatinine levels, diabetes, hypertension, antihypertension drug use, lipid lowering drug use, history of coronary artery disease (CAD) and/or history of peripheral artery occlusive disease (PAOD), history of stroke or transient ischemic attack (TIA), preprocedural cerebrovascular symptoms and presence of contralateral carotid artery stenosis of 50-100\%., ${ }^{2,6}$ Across the selected confounders the proportion missing values was analyzed (Table 1). As presence of missing values was minimal, no additional approaches to handle missing data were necessary. Selected confounders were included in a multivariable Cox proportional hazards model and model reduction using Akaike information criterion in a stepwise backward regression 
resulted in the final clinical model including cardiovascular risk factors (CVRF): age, creatinine levels, diabetes, current smoking, history of CAD/PAOD and preprocedural cerebrovascular symptoms.

To explore the model's potential as a prediction tool, model performance was assessed. First internal validation was performed with bootstrapping techniques. Calibration was evaluated by visual inspection of calibration plots. The $\mathrm{C}$-index ${ }^{31}$ and the net reclassification improvement $^{32,33}$ (NRI) were used to quantify the added predictive value of TN-C beyond a model including only CVRF. (Methods in Supplementary Data). Finally, considering high TN-C plaque expression previously has been associated with vulnerable plaque characteristics, ${ }^{13,34}$ we further investigated whether the same association could be found with circulating EV TN-C levels (Methods in Supplementary Data). All p-values were 2 -tailed, with a value $<0.05$ considered to indicate statistical significance. Statistical analyses were performed with R version 3.6.0 (R Foundation for Statistical Computing, Vienna, Austria). ${ }^{35}$

\section{Results}

\section{Study population and baseline characteristics}

In the current study, 837 patients who underwent CEA were included (Figure 1). Baseline characteristics for the overall study population are presented in Table 1, and stratified by occurrence of MACE in Table II, supplemental materials. Included patients were mainly operated for symptomatic, high degree (70-99\%) carotid stenosis. The overall mean age was $69( \pm 9)$ years and $70 \%(n=589)$ of the study population were male. Cardiovascular risk factors were highly prevalent since $35 \%$ of the patients had a history of CAD or PAD, also illustrated by the high number of patients on lipid lowering drugs (79\%) and antiplatelet therapy (88\%) at the time of study inclusion. The distribution of raw EV TN-C levels was right skewed with a median of $224.3 \mathrm{pg} / \mathrm{ml}$ [IQR 137.6,356.1 pg/ml] (Figure I, supplemental materials). Overall EV levels did not differ between patients with high and low TN-C EV levels (data not shown).

\section{Outcome analysis}

During a median follow-up time of 3.0 years [IQR 2.2-3.0], 137 patients (16.4\%) reached the endpoint MACE. MACE consisted of 73 stroke, $43 \mathrm{MI}$ and 21 other cardiovascularrelated deaths. EV TN-C levels were divided in quartiles for more in depth exploration of its association with the occurrence of MACE. As illustrated in the Kaplan-Meier survival analysis (Figure 2A), MACE rates increased in the upper quartile indicating a high-risk 
Table 1. Baseline characteristics of study population

\begin{tabular}{|c|c|c|}
\hline & Total & Missing \\
\hline & $\mathbf{n}=\mathbf{8 3 7}$ & $\%$ \\
\hline Age (years) & $69( \pm 9)$ & 0.0 \\
\hline Sex, male & $589(70)$ & 0.0 \\
\hline Ipsilateral carotid artery stenosis degree & & 2.4 \\
\hline $0-50 \%$ & $5(1)$ & \\
\hline $50-70 \%$ & $59(7)$ & \\
\hline $70-99 \%$ & $753(92)$ & \\
\hline BMI $(\mathrm{kg} / \mathrm{m} 2)$ & $26[24,28]$ & 3.8 \\
\hline Creatinine $(\mu \mathrm{mol} / \mathrm{L})$ & $88[76,106]$ & 0.5 \\
\hline eGFR $(\mathrm{ml} / \mathrm{min}$ per $1.73 \mathrm{~m} 2)$ & $73( \pm 21)$ & 5.7 \\
\hline Current smoker & $278(33)$ & 0.1 \\
\hline Diabetes & $192(23)$ & 0.0 \\
\hline Hypertension & $588(72)$ & 3.0 \\
\hline Hypercholesterolemia & $527(70)$ & 9.9 \\
\hline Triglycerides (mg/dl) & $1.5[1.1,2.1]$ & 4.1 \\
\hline $\mathrm{LDL}(\mathrm{mmol} / \mathrm{L})$ & $2.3[1.8,3.0]$ & 4.1 \\
\hline $\mathrm{HDL}(\mathrm{mg} / \mathrm{dl})$ & $1.0[0.9,1.3]$ & 3.5 \\
\hline Total cholesterol (mg/dl) & $4.2[3.5,5.1]$ & 3.1 \\
\hline Contralateral carotid artery stenosis of $50-100 \%$ & $349(46)$ & 9.1 \\
\hline Anticoagulants & $87(10)$ & 0.2 \\
\hline Antiplatelets & $734(88)$ & 0.5 \\
\hline Lipid lowering drugs & $663(79)$ & 0.2 \\
\hline Statins & $655(78)$ & 0.2 \\
\hline Hypertension drugs & $627(75)$ & 0.2 \\
\hline History of CAD or PAD & & 0.0 \\
\hline none & $475(57)$ & \\
\hline CAD or PAD & $294(35)$ & \\
\hline $\mathrm{CAD}$ and $\mathrm{PAD}$ & $68(8)$ & \\
\hline History of stroke or TIA & $643(77)$ & 0.0 \\
\hline Preprocedural cerebrovascular symptoms & & 0.0 \\
\hline asymptomatic & $338(40)$ & \\
\hline ocular & $90(11)$ & \\
\hline TIA & $232(28)$ & \\
\hline Stroke & $177(21)$ & \\
\hline Time between last event and surgery (days) & $24[13,51]$ & 44.7 \\
\hline
\end{tabular}

Data are presented as $\mathrm{n}(\%)$, mean \pm standard deviation (SD) or median [interquartile range] [IQR]. Abbreviations: BMI, Body Mass Index; eGFR, estimated Glomerular Filtration Rate; LDL, Low-density Lipoprotein; HDL, High-density Lipoprotein; CAD, coronary artery disease; PAD, peripheral artery disease; TIA, Transient Ischemic Attack. 
population. For further insight in clinical applicability, the optimal cut-off value was determined at $0.57 \mathrm{pg} / \mathrm{ml}$ (Figure II, supplemental materials). This cut-off was used to differentiate between the risk of high and low EV TN-C levels which is shown in figure $2 \mathrm{~B}$. Cox-regression analysis revealed a significant association of EV TN-C levels and future MACE with a HR of 1.30 (95\% CI 1.12-1.54) per SD increase of TN-C on a logarithmic scale after adjustment for CVRF (including age, creatinine, diabetes, current smoking, history of CAD/PAOD and preprocedural cerebrovascular symptoms). Cox-regression analysis for EV TN-C levels divided in quartiles and high and low values are displayed in figure $3 \mathrm{~A}$ and $3 \mathrm{~B}$, with respectively a $\mathrm{HR}$ of 2.30 for patients in the highest quartile ( $95 \%$ CI 1.39-3.78) compared to the lowest quartile and a HR of 1.93 (95\% CI 1.36-2.74) for high TN-C values compared to low values. Plasma measurements of TN-C (Figure III, supplemental materials) showed no association with MACE as cox-regression analysis revealed an HR of 1.00 (95\% CI 1.00-1.00).

Total CEA patients included in Athero-Express Biobank with available citrate plasma samples $(\mathrm{n}=987)$

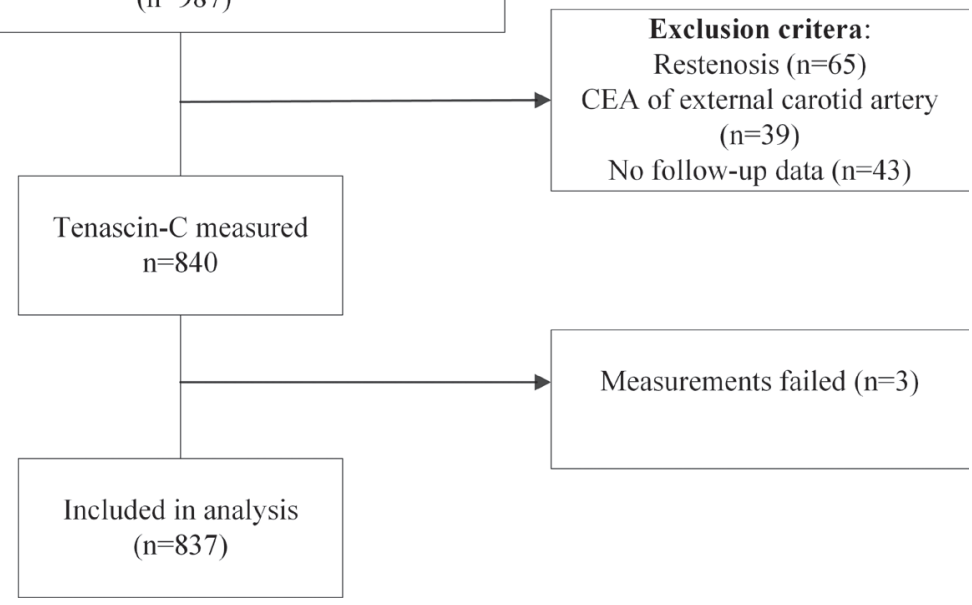

Figure 1. Flowchart of study population

\section{Tenascin-C and prediction of Major Adverse Cardiovascular Events}

The shrinkage factor calculated with bootstrapping was 0.86 for both models. Corrected regression coefficients of the final predictors for both the clinical model (including CVRF) and the biomarker model with addition of TN-C are presented in Table III, supplemental materials. After shrinkage of both models, visual inspection of the calibration plot for the 
model containing EV TN-C levels, showed better prediction for the 3-year MACE risks within the range of $0-22 \%$ and an overestimation of the risk among patients with a 3-year MACE risk of $>22 \%$ (Figure IV, supplemental materials) compared to the model with only clinical variables. The C-statistic for prediction of MACE after three years of follow-up marginally increased with addition of TN-C, C-index 0.685 (95\% CI 0.63-0.74), compared to the clinical model consisting of CVRF with C-index 0.667 (95\% CI 0.61-0.72). The difference however was not significant $(\mathrm{p}=0.06)$.

Addition of TN-C to the clinical model with CVRF led to an NRI of 0.083 (95\% CI -0.011-0.176), 0.044 (95\% CI -0.042-0.129) for MACE and 0.039 (95\% CI 0.002-0.076) for no MACE. Estimates of the expected number of reclassifications per risk category for MACE and no MACE are provided in the reclassification tables (Table IV, supplemental materials).

A

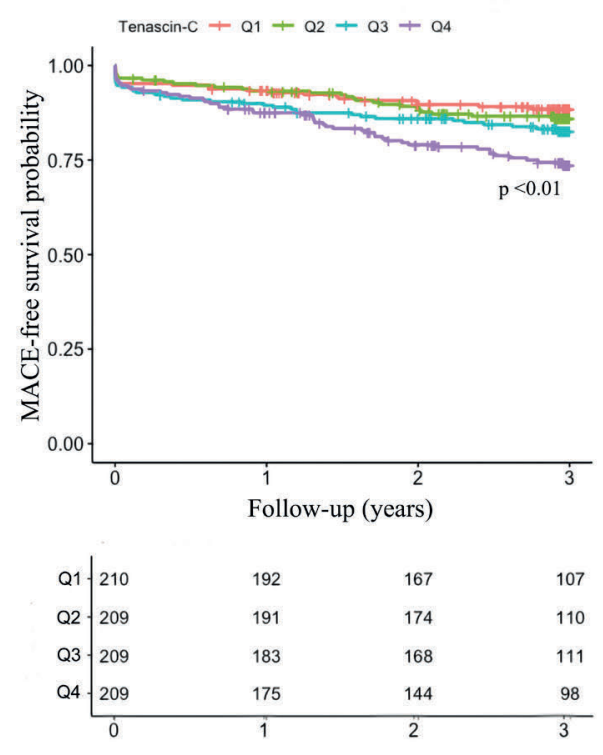

B

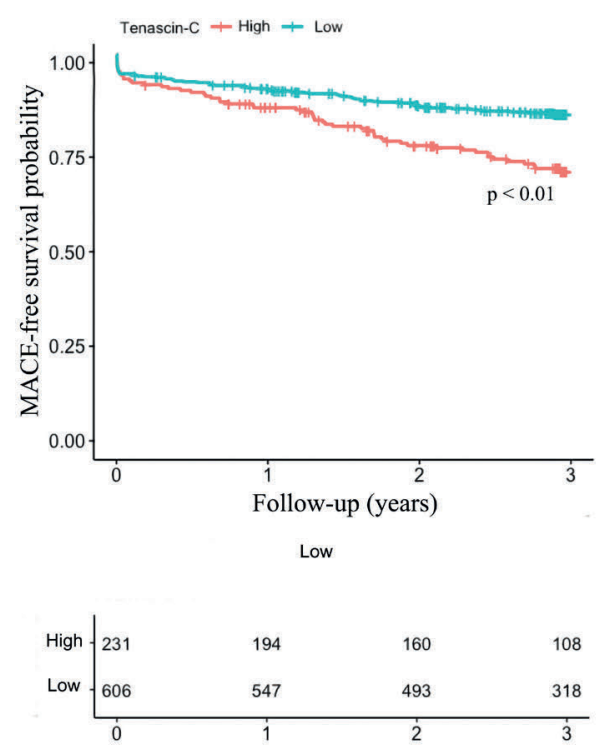

Figure 2. Kaplan Meier curves according to Tenascin-C quartiles (A) and optimal cut-off (B) for the endpoint MACE

\section{Plasma EV Tenascin-C levels and plaque characteristics}

Considering high TN-C expression in plaques has been associated with vulnerable plaque characteristics, we further investigated whether the same association could be found with EV TN-C as circulating biomarker. Table 2 summarizes the association of histological 
plaque characteristics with plasma EV TN-C. One SD increase in concentration of log Tenascin- $\mathrm{C}$ was associated with more collagen staining (adjusted OR for moderate/heavy staining of 1.28 (95\% CI 1.03-1.60; $\mathrm{p}=0.02$ ) and more macrophage content (adjusted OR for moderate/heavy staining of 1.33 (95\% CI 1.11-1.60; $\mathrm{p}<0.01$ ). TN-C was positively associated with a higher number of microvessels with an adjusted beta of 0.07 (95\% CI 0.01-0.13; $\mathrm{p}=0.03$ ) (Table 2). A positive association of TN-C and the percentage of macrophage infiltration (per plaque area) was found with an adjusted beta of 0.05 (95\% CI 0.02-0.09; $\mathrm{p}=0.01$ ) (Table 2).

A

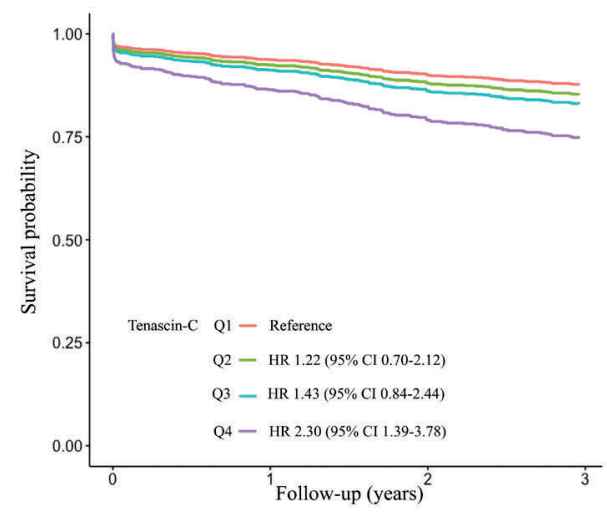

B

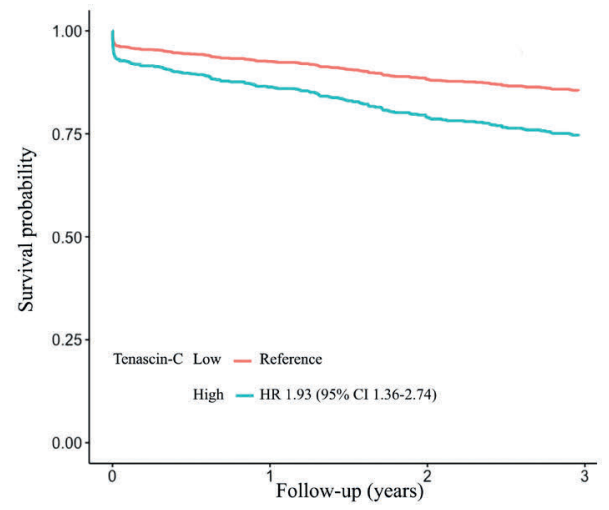

Figure 3. Adjusted survival curves for log transformed standardized version of Tenascin-C in quartiles (A) and according to the optimal cut-off (B) for the endpoint MACE

Cox regression analysis was adjusted for age, creatinine levels, diabetes, current smoking, history of CAD/PAOD and preprocedural cerebrovascular symptoms.

\section{Discussion}

In the present study, we show an independent association of plasma EV TN-C concentrations with a higher 3-year risk of postoperative MACE. Secondly, we also found positive associations of circulating plasma EV TN-C levels with histological plaque features such as the amount of collagen, macrophage infiltration and microvessels.

As the systemic nature of atherosclerotic disease is well established, we hypothesized that local plaque content, in this case TN-C, could be measured systemically and be an informative source for a prognostic biomarker. Circulating concentrations of TN-C have been reported to be increased in patients with a range of cardiovascular problems such as acute myocardial infarction ${ }^{17}$, cardiomyopathy ${ }^{36}$, and pulmonary thromboembolism ${ }^{37}$, 
Table 2. Univariable and multivariable measures of association of Tenascin- $C$ and vulnerable carotid plaque characteristics

\begin{tabular}{|c|c|c|c|c|}
\hline Binary plaque characteristics & & OR & 95\% CI & p-value \\
\hline Calcification & UV & 1.26 & $1.06,1.49$ & 0.01 \\
\hline (moderate/heavy staining) & MV & 1.20 & $1.00,1.44$ & 0.06 \\
\hline Collagen & UV & 1.31 & $1.07,1.60$ & 0.01 \\
\hline (moderate/heavy staining) & MV & 1.28 & $1.03,1.60$ & 0.02 \\
\hline Macrophages & UV & 1.33 & $1.12,1.58$ & $<0.01$ \\
\hline (moderate/heavy staining) & MV & 1.33 & $1.11,1.60$ & $<0.01$ \\
\hline SMC & UV & 1.11 & $0.92,1.34$ & 0.26 \\
\hline (moderate/heavy staining) & MV & 1.05 & $0.86,1.28$ & 0.62 \\
\hline Presence of & UV & 1.01 & $0.84,1.20$ & 0.95 \\
\hline lipid core $>10 \%$ & MV & 1.01 & $0.82,1.23$ & 0.94 \\
\hline Presence of & UV & 0.93 & $0.77,1.12$ & 0.46 \\
\hline lipid core $>40 \%$ & MV & 0.93 & $0.76,1.14$ & 0.48 \\
\hline \multirow[t]{2}{*}{ Presence of IPH } & UV & 1.00 & $0.85,1.18$ & 1.00 \\
\hline & MV & 0.96 & $0.80,1.15$ & 0.67 \\
\hline Continuous plaque characteristics & & beta & $95 \% \mathrm{CI}$ & p-value \\
\hline log Macrophages, & UV & 0.06 & $0.03,0.10$ & $<0.01$ \\
\hline$\%$ positive staining/total plaque & MV & 0.05 & $0.02,0.09$ & 0.01 \\
\hline $\log$ SMCs, & UV & 0.03 & $-0.02,0.08$ & 0.22 \\
\hline$\%$ positive staining/total plaque & MV & 0.01 & $-0.04,0.06$ & 0.75 \\
\hline log Intraplaque vessels, & UV & 0.10 & $0.04,0.16$ & $<0.01$ \\
\hline mean number per hotspot & MV & 0.07 & $0.01,0.13$ & 0.03 \\
\hline
\end{tabular}

OR and betas are calculated per one standard deviation increase in concentration of log Tenascin-C. Abbreviations: SMC, smooth muscle cell; IPH, intraplaque hemorrhage; UV, univariable; MV, multivariable. Logistic and linear regression models were used for the binominal and continuous variables, respectively. Multivariable measures of association (MV) represent the values corrected for age, gender, creatinine, diabetes, history of coronary artery disease and/or peripheral artery disease, current smoking, hypertension, anticoagulant use, statin use, preprocedural cerebrovascular symptoms and year of surgery. Values in bold indicate $\mathrm{p}<0.05$.

however the number of included patients in these studies was limited and varied from 20 44 patients. One study has reported high circulating TN-C concentrations in patients with large artery atherosclerotic stroke, however in this study patients with TIA were excluded. ${ }^{38}$ These results are in line with our findings and contribute to the concept of circulating plaque proteins as biomarkers for risk stratification of patients with higher risk of MACE. ${ }^{39}$ In this context, EVs are of particular interest. EVs are released in body fluids both under basal and pathological settings. They act as vectors of biological information from their cells of origin. ${ }^{7}$ It has been established that atherosclerotic lesions contain $\mathrm{EV} \mathrm{s}^{40,41}$ and that 
these EVs contribute to initial atherosclerotic lesion formation and progression as a result of their effect on inflammation, thrombosis, neoangiogenesis and hemostasis. In cardiovascular disease, circulating levels of EVs are increased, making EVs an even more interesting prognostic biomarker source. ${ }^{7}$ Aforementioned studies have examined serum or plasma levels of TN-C ${ }^{17,36,37,42-45}$, however, this is the first study that assessed TN-C in EVs related to cardiovascular disease and in a large cohort of CEA patients. For comparison, we also assessed plasma $\mathrm{TN}-\mathrm{C}$ levels. In plasma, we could not establish the same association with MACE as we found in EVs. We performed a Western blot analysis of plasma TN-C levels and TN-C in EVs to obtain more information on whether this difference could also be explained by difference in spliced variants of TN-C (Supplemental Methods). Previous studies have shown that there is considerable tissue and disease stage-specific variation in TN-C isoform expression. The Western blot, shows that TN-C in plasma and the LDL subfraction is different in separation by SDS-PAGE pointing to a different set of TN-C isoforms in EVs in the LDL subfraction. (Figure V, supplemental materials). Another possible explanation for this difference might be that previous studies performed in plasma are relatively small and do not study CEA patients.

Prediction tools in secondary prevention to identify patients at risk of MACE have been studied extensively, but have poor ability to stratify patients into groups with high or low risk for secondary events. ${ }^{46}$ Novel therapeutic options for risk reduction, such as proprotein convertase subtilisin/kexin type 9 inhibitors (PCSK-9 inhibitors) and anti-inflammatory agents (colchicine) ${ }^{5}$, are often costly or are accompanied with disabling side effects. Therefore, it is of utmost importance to identify those most likely to benefit from novel secondary prevention strategies. Currently, CEA patients are all considered as (very) high risk patients. ${ }^{4}$ However, it is established that there is great interindividual variations in the actual vascular prognosis. ${ }^{5}$ A one-size-fits-all secondary prevention approach is less appropriate, since all high risk patients would qualify for more intense medical treatment. ${ }^{4}$

Biomarkers reflecting different disease pathways, could improve the performance of clinical prediction tools. In the present study, an overestimation of the risk among patients with 3 -year MACE risk of approximately $>22 \%$ was seen when comparing the calibration of the model containing EV TN-C levels with the CVRF model only (figure S3). However, calibration of the model containing EV TN-C levels was better for the 3-year MACE risks within the range of $0-22 \%$ which is more relevant as this risk represents the majority of the patients seen in daily practice. ${ }^{2,46}$ Furthermore, an overestimation of the patients at high risk for MACE would not be problematic, as these patients already would qualify for intensified follow-up and secondary prevention. Addition of EV TN-C levels to a model consisting of CVRF yielded an NRI for no MACE of 0.04 (95\% CI 0.002-0.076). In this 
group $14.3 \%$ was reassigned to a lower risk category, which in practice could be useful as these patients would be identified as low risk and would not qualify for intensive follow-up and intensified secondary preventive therapies. The overall NRI and NRI for events were not significant, therefore further validation studies are warranted in a larger study population to prove this concept. Finally, novel biomarker risk stratification approaches may also be useful in guiding patient-specific treatment algorithms for carotid intervention, such as CEA or carotid stenting.

High TN-C EV levels were independently associated with plaques composed of high collagen content, heavy macrophage infiltration and more microvessels. Heavy macrophage infiltration and high microvessel content are plaque characteristics that are considered as hallmarks for vulnerable plaques. Studies in tissue levels of TN-C already revealed a strong association of TN-C with advanced plaques and plaque rupture. ${ }^{13,34} \mathrm{~A}$ strong association and co-localization with macrophages was also found previously in other study populations 34,47-49 and macrophages were identified as a source of TN-C expression in advanced atherosclerotic plaques. ${ }^{13}$ The observed association with microvessels is in line with previous studies, where TN-C was associated with endothelial cell activation and was important for angiogenesis. ${ }^{50}$ In patients with acute coronary syndrome, the same association was found for TN-C and microvessel formation. ${ }^{34}$ Although it has been proposed that organized intraplaque hemorrhage originates from rupture of microvessels $\mathrm{s}^{51}$ and that it is a feature associated with rupture prone plaques and secondary MACE ${ }^{28}$, in our study EV TN-C was not associated with intraplaque hemorrhage. This is in accordance with a study in Apo E/ $\mathrm{TN}-\mathrm{C}$ double knock-out mice revealing accelerated lesion and extensive intraplaque hemorrhage development suggesting a partly protective role for $\mathrm{TN}-\mathrm{C}$ in atherosclerosis. ${ }^{52}$

Our results also indicated an association of EV TN-C levels with plaques consisting of more collagen. Although plaque rupture and subsequent thrombosis are often the cause of events, an event can also be caused by a thrombus without plaque rupture, which is classified as plaque erosion. ${ }^{53,54}$ Eroded plaques are rich in proteoglycans at the plaque/thrombus interface. ${ }^{55}$ Proteoglycans, collagen and lipids are highly reactive platelet-adhesive materials that are expressed in atherosclerotic plaques and are absent in healthy vessel walls. One could therefore speculate that the increased accumulation of TN-C might also provide a high risk for thrombosis. This is supported by a previous study that proposed that upon erosion or rupture of an atherosclerotic plaque, platelets attach to TN-C through Von Willebrand factor and become activated, implicating a role for TN-C in platelet physiology and atherothrombosis. ${ }^{56}$

Although we consider our results to be valid, potential limitations need to be considered when interpreting them. First, it remains a challenge to identify the cellular origin of the 
EVs that are measured, as parent cell specific cellular surface markers cannot be used on EVs to determine its origin. ${ }^{57}$ Nevertheless, evidence of their cellular origin is also not provided when measuring TN-C in serum or plasma. We do not know if TN-C is inside or associated with EVs. The two peaks in the density gradient (Table I, supplemental materials) might suggest that in the LDL subfraction, we measure TN-C both associated in fractions not associated with CD9 and also inside EVs in fractions associated with CD9. For this study, however, we show that TN-C in de LDL subfraction is associated with MACE in contrast to TN-C in plasma. Furthermore, no data were available on alterations in risk factors due to risk factor management during follow-up, therefore this could not be considered in the analysis. Finally, external validation of the final prediction model consisting of EV TN-C levels and CVRF is warranted in another study population, before the model can be implemented in clinical practice. A strength of this study is that for the first time, we show a strong association between circulating TN-C EV levels and 3-year postoperative MACE in large cohort of CEA patients with systematically collected and scored histological and outcome data due to the unparalleled prospective design, continuity and outcome validation of the Athero-Express biobank.

\section{Conclusions}

Increased levels of circulating TN-C in plasma EVs are independently associated with an increased 3-year risk of major adverse cardiovascular events in a carotid endarterectomy population. High TN-C EV levels were also independently associated with plaques characteristics. These findings contribute to the concept of circulating plaque proteins in EVs for risk stratification of patients at high risk of secondary cardiovascular events.

\section{Sources of Funding}

Funding was obtained from the Dutch Heart Foundation, The Netherlands CardioVascular Research Committee [2017-05 pERSUASIVE], to D. de Kleijn. There are no competing interests declared. Funding was obtained from the European Union [755320 Taxinomisis] grant to D.P.V. de Kleijn, G.J. de Borst and G. Pasterkamp. There are no competing interests declared.

\section{Disclosures}

The authors have no disclosures that would be a potential conflict of interest. 


\section{References}

1. Halliday A, Mansfield A, Marro J, et al. Prevention of disabling and fatal strokes by successful carotid endarterectomy in patients without recent neurological symptoms: randomised controlled trial. Lancet (London, England). 2004;363(9420):1491-1502. doi:10.1016/S0140-6736(04)16146-1

2. Van Lammeren GW, Catanzariti LM, Peelen LM, et al. Clinical prediction rule to estimate the absolute 3-year risk of major cardiovascular events after carotid endarterectomy. Stroke. 2012;43(5):1273-1278. doi:10.1161/STROKEAHA.111.647958

3. Hellings WE, Peeters W, Moll FL, et al. Composition of carotid atherosclerotic plaque is associated with cardiovascular outcome: A prognostic study. Circulation. 2010;121(17):1941-1950. doi:10.1161/ CIRCULATIONAHA.109.887497

4. Mach F, Baigent C, Catapano AL, et al. 2019 ESC/EAS Guidelines for the management of dyslipidaemias: lipid modification to reduce cardiovascular risk. Eur Heart J. 2019:1-78. doi:10.1093/eurheartj/ehz455

5. Kaasenbrood L, Boekholdt SM, Van Der Graaf Y, et al. Distribution of Estimated 10-Year Risk of Recurrent Vascular Events and Residual Risk in a Secondary Prevention Population. Circulation. 2016;134(19):14191429. doi:10.1161/CIRCULATIONAHA.116.021314

6. Volkers EJ, Algra A, Kappelle LJ, Greving JP. Prediction models for clinical outcome after a carotid revascularisation procedure: A systematic review.EurStrokeJ.2018;3(1):57-65. doi:10.1177/2396987317739122

7. Boulanger CM, Loyer X, Rautou PE, Amabile N. Extracellular vesicles in coronary artery disease. Nat Rev Cardiol. 2017;14(5):259-272. doi:10.1038/nrcardio.2017.7

8. Kanhai DA, Visseren FLJ, Van Der Graaf Y, et al. Microvesicle protein levels are associated with increased risk for future vascular events and mortality in patients with clinically manifest vascular disease. Int $J$ Cardiol. 2013;168(3):2358-2363. doi:10.1016/j.ijcard.2013.01.231

9. Wang J-W, Zhang Y-N, Sze S, et al. Lowering Low-Density Lipoprotein Particles in Plasma Using Dextran Sulphate Co-Precipitates Procoagulant Extracellular Vesicles. Int J Mol Sci. 2017;19(1):94. doi:10.3390/ ijms 19010094

10. Chiquet-Ehrismann R, Chiquet M. Tenascins: Regulation and putative functions during pathological stress. J Pathol. 2003;200(4):488-499. doi:10.1002/path.1415

11. Golledge J, Clancy P, Maguire J, Lincz L, Koblar S. The role of tenascin C in cardiovascular disease. Cardiovasc Res. 2011;92(1):19-28. doi:10.1093/cvr/cvr183

12. Wallner K, Li C, Shah PK, Wu KJ, Schwartz SM, Sharifi BG. EGF-like domain of Tenascin-C is proapoptotic for cultured smooth muscle cells. Arterioscler Thromb Vasc Biol. 2004;24(8):1416-1421. doi:10.1161/01. ATV.0000134299.89599.53

13. Wallner K, Li C, Shah PK, et al. Tenascin-C Is Expressed in Macrophage-Rich Human. 1999:1284-1289. doi:10.1161/01.CIR.99.10.1284

14. Pedretti M, Rancic Z, Soltermann A, et al. Comparative immunohistochemical staining of atherosclerotic plaques using F16, F8 and L19: Three clinical-grade fully human antibodies. Atherosclerosis. 2010;208(2):382389. doi:10.1016/j.atherosclerosis.2009.07.043

15. Fujimoto N, Onishi K, Sato A, et al. Incremental Prognostic Values of Serum Tenascin-C Levels With Blood B-type Natriuretic Peptide Testing at Discharge in Patients With Dilated Cardiomyopathy and Decompensated Heart Failure. J Card Fail. 2009. doi:10.1016/j.cardfail.2009.06.443

16. Liabeuf S, Barreto D V., Kretschmer A, et al. High circulating levels of large splice variants of tenascin-C is associated with mortality and cardiovascular disease in chronic kidney disease patients. Atherosclerosis. 2011. doi:10.1016/j.atherosclerosis.2010.11.038

17. Sato A, Aonuma K, Imanaka-Yoshida K, et al. Serum Tenascin-C Might Be a Novel Predictor of Left Ventricular Remodeling and Prognosis After Acute Myocardial Infarction. J Am Coll Cardiol. 2006. doi:10.1016/j.jacc.2006.03.033

18. Mirzaei R, Sarkar S, Dzikowski L, et al. Brain tumor-initiating cells export tenascin-C associated with exosomes to suppress $T$ cell activity. Oncoimmunology. 2018;7(10):1-14. doi:10.1080/216240 2X.2018.1478647

19. Qian S, Tan X, Liu X, Liu P, Wu Y. $<\mathrm{p}>$ Exosomal Tenascin-c induces proliferation and invasion of pancreatic cancer cells by WNT signaling $</ \mathrm{p}>$. Onco Targets Ther. 2019; Volume 12:3197-3205. doi:10.2147/ott. s192218 
20. Zheng J, Hernandez JM, Doussot A, et al. Fluid Exosomes in Patients with Pancreatic Cancer. 2019;20(February 2017):597-604. doi:10.1016/j.hpb.2017.12.010.Extracellular

21. Mills JT, Schwenzer A, Marsh EK, et al. Airway epithelial cells generate pro-inflammatory tenascin-C and small extracellular vesicles in response to TLR3 stimuli and rhinovirus infection. Front Immunol. 2019;10(AUG):1-12. doi:10.3389/fimmu.2019.01987

22. Verhoeven BAN, Velema E, Schoneveld AH, et al. Athero-express: Differential atherosclerotic plaque expression of mRNA and protein in relation to cardiovascular events and patient characteristics. Rationale and design. Eur J Epidemiol. 2004;19(12):1127-1133. doi:10.1007/s10564-004-2304-6

23. Barnett HJM, Taylor DW, Eliasziw M, et al. Benefit of carotid endarterectomy in patients with symptomatic moderate or severe stenosis. N Engl J Med. 1998;339(20):1415-1425. doi:10.1056/NEJM199811123392002

24. Warlow C, Farrell B, Fraser A, Sandercock P, Slattery J. Randomised trial of endarterectomy for recently symptomatic carotid stenosis: Final results of the MRC European Carotid Surgery Trial (ECST). Lancet. 1998;351(9113):1379-1387. doi:10.1016/S0140-6736(97)09292-1

25. Norgren L, Hiatt WR, Dormandy JA, et al. Inter-society consensus for the management of peripheral arterial disease. Int Angiol. 2007;26(2):81-157. doi:10.1016/j.jvs.2006.12.037

26. Ferguson GG, Eliasziw M, Barr HWK, et al. The North American Symptomatic Carotid Endarterectomy Trial. Stroke. 1999;30(9):1751-1758. doi:10.1161/01.STR.30.9.1751

27. Hellings WE, Pasterkamp G, Vollebregt A, et al. Intraobserver and interobserver variability and spatial differences in histologic examination of carotid endarterectomy specimens. J Vasc Surg. 2007;46(6):11471154. doi:10.1016/j.jvs.2007.08.018

28. Derksen WJM, Peeters W, van Lammeren GW, et al. Different stages of intraplaque hemorrhage are associated with different plaque phenotypes: A large histopathological study in 794 carotid and 276 femoral endarterectomy specimens. Atherosclerosis. 2011;218(2):369-377. doi:10.1016/j.atherosclerosis.2011.07.104

29. Zhang YN, Vernooij F, Ibrahim I, et al. Extracellular vesicle proteins associated with systemic vascular events correlate with heart failure: An observational study in a dyspnoea cohort. PLoS One. 2016;11(1):119. doi:10.1371/journal.pone.0148073

30. Dekker M, Waissi F, van Bennekom J, et al. Plasma extracellular vesicle proteins are associated with stressinduced myocardial ischemia in women presenting with chest pain. Sci Rep. 2020;10(1):1-8. doi:10.1038/ s41598-020-69297-0

31. Kang L, Chen W, Petrick NA, Gallas BD. Comparing two correlated C indices with right-censored survival outcome: A one-shot nonparametric approach. Stat Med. 2015;34(4):685-703. doi:10.1002/sim.6370

32. Leening MJG, Vedder MM, Witteman JCM, Pencina MJ, Steyerberg EW. Net Reclassification Improvement: Computation, Interpretation, and Controversies. Ann Intern Med. 2014;160(2):122-131. doi:10.7326/M131522

33. Pencina MJ, D’Agostino RB, Steyerberg EW. Extensions of net reclassification improvement calculations to measure usefulness of new biomarkers. Stat Med. 2011;30(1):11-21. doi:10.1002/sim.4085

34. Kajiwara K, Ueda H, Yamamoto H, Imazu M, Hayashi Y, Kohno N. Tenascin-C is Associated with Coronary Plaque Instability in Patients with Acute Coronary Syndromes. Circ J. 2004;68(3):198-203. doi:10.1253/ circj.68.198

35. Team RC. R: A language and environment for statistical computing. 2018.

36. Aso N, Tamura A, Nasu M. Circulating tenascin-C levels in patients with idiopathic dilated cardiomyopathy. Am J Cardiol. 2004. doi:10.1016/j.amjcard.2004.07.156

37. Celik A, Kocyigit I, Calapkorur B, et al. Tenascin-C may be a predictor of acute pulmonary thromboembolism. J Atheroscler Thromb. 2011. doi:10.5551/jat.7070

38. Clancy P, Lincz LF, Maguire J, McEvoy M, Koblar SA, Golledge J. Tenascin-C is increased in atherothrombotic stroke patients and has an anti-inflammatory effect in the human carotid artery. BioFactors. 2014;40(4):448457. doi:10.1002/biof.1170

39. De Kleijn DPV, Moll FL, Hellings WE, et al. Local atherosclerotic plaques are a source of prognostic biomarkers for adverse cardiovascular events. Arterioscler Thromb Vasc Biol. 2010;30(3):612-619. doi:10.1161/ATVBAHA.109.194944

40. Leroyer AS, Isobe H, Lesèche G, et al. Cellular Origins and Thrombogenic Activity of Microparticles Isolated From Human Atherosclerotic Plaques. J Am Coll Cardiol. 2007;49(7):772-777. doi:10.1016/j. jacc.2006.10.053

41. Bobryshev Y V., Killingsworth MC, Orekhov AN. Increased shedding of microvesicles from intimal smooth muscle cells in athero-prone areas of the human aorta: Implications for understanding of the predisease stage. Pathobiology. 2012. doi:10.1159/000339430 
42. Schumann C, Lepper PM, Frank H, et al. Circulating biomarkers of tissue remodelling in pulmonary hypertension. Biomarkers. 2010;15(6):523-532. doi:10.3109/1354750X.2010.492431

43. Franz M, Berndt A, Altendorf-Hofmann A, et al. Serum levels of large tenascin-C variants, matrix metalloproteinase-9, and tissue inhibitors of matrix metalloproteinases in concentric versus eccentric left ventricular hypertrophy. Eur J Heart Fail. 2009. doi:10.1093/eurjhf/hfp128

44. Terasaki F, Okamoto $\mathrm{H}$, Onishi $\mathrm{K}$, et al. Higher serum tenascin-C levels reflect the severity of heart failure, left ventricular dysfunction and remodeling in patients with dilated cardiomyopathy. Circ J. 2007. doi:10.1253/circj.71.327

45. Hessel MHM, Bleeker GB, Bax JJ, et al. Reverse ventricular remodelling after cardiac resynchronization therapy is associated with a reduction in serum tenascin-C and plasma matrix metalloproteinase-9 levels. Eur J Heart Fail. 2007. doi:10.1016/j.ejheart.2007.07.007

46. Volkers EJ, Algra A, Jaap Kappelle L, et al. Prediction models for clinical outcome after a carotid revascularization procedure an external validation study. Stroke. 2018;49(8):1880-1885. doi:10.1161/ STROKEAHA.117.020486

47. Satta J, Soini Y, Pollanen R, Paakko P, Juvonen T. Tenascin expression is associated with a chronic inflammatory process in abdominal aortic aneurysms. J Vasc Surg. 1997;26(4):670-675. doi:10.1016/S07415214(97)70068-5

48. Le Poole IC, Van Den Wijngaard RMJGJ, Westerhof W, Das PK. Presence of T cells and macrophages in inflammatory vitiligo skin parallels melanocyte disappearance. Am J Pathol. 1996;148(4):1219-1228.

49. Gullberg D, Velling T, Sjöberg G, et al. Tenascin-C expression correlates with macrophage invasion in Duchenne muscular dystrophy and in myositis. Neuromuscul Disord. 1997;7(1):39-54. doi:10.1016/S09608966(96)00391-4

50. Zagzag D, Friedlander DR, Dosik J, et al. Tenascin-C expression by angiogenic vessels in human astrocytomas and by human brain endothelial cells in vitro. Cancer Res. 1996;56(1):182-189.

51. Kolodgie FD, Gold HK, Burke AP, et al. Intraplaque Hemorrhage and Progression of Coronary Atheroma. N Engl J Med. 2003;349(24):2316-2325. doi:10.1056/NEJMoa035655

52. Wang L, Wang W, Shah PK, Song L, Yang M, Sharifi BG. Deletion of tenascin-C gene exacerbates atherosclerosis and induces intraplaque hemorrhage in Apo-E-deficient mice. Cardiovasc Pathol. 2012;21(5):398-413. doi:10.1016/j.carpath.2011.12.005

53. Bentzon JF, Otsuka F, Virmani R, Falk E. Mechanisms of plaque formation and rupture. Circ Res. 2014;114(12):1852-1866. doi:10.1161/CIRCRESAHA.114.302721

54. Farb A, Burke AP, Tang AL, et al. Coronary Plaque Erosion Without Rupture Into a Lipid Core. Circulation. 1996;93(7):1354-1363. doi:10.1161/01.CIR.93.7.1354

55. Shah AD, Bartlett JW, Carpenter J, Nicholas O, Hemingway H. Comparison of random forest and parametric imputation models for imputing missing data using MICE: A CALIBER study. Am J Epidemiol. 2014;179(6):764-774. doi:10.1093/aje/kwt312

56. Schaff M, Receveur N, Bourdon C, et al. Novel function of tenascin-C, a matrix protein relevant to atherosclerosis, in platelet recruitment and activation under flow. Arterioscler Thromb Vasc Biol. 2011;31(1):117-124. doi:10.1161/ATVBAHA.110.206375

57. Yáñez-Mó $\mathrm{M}$, Siljander PRM, Andreu Z, et al. Biological properties of extracellular vesicles and their physiological functions. J Extracell Vesicles. 2015;4(2015):1-60. doi:10.3402/jev.v4.27066 


\section{Supplemental Materials}

\section{Supplemental Methods}

\section{Methods for internal validation and model performance}

Since the performance is calculated on the same dataset in which the models were derived, bootstrapping was used to control for optimism and overfitting. In each bootstrap sample, the modeling process was repeated, resulting in shrinkage of the regression coefficients. The C-index and the net reclassification improvement ${ }^{1,2}(\mathrm{NRI})$ were used to quantify the added predictive value of TN-C beyond a model including only classical CVRF. Discriminative ability of the model was measured with the C-statistic ${ }^{3}$. Differences in C-statistics after the addition of TN-C to the model consisting of only CVRF were computed. For NRI analysis risk categories were defined based on the overall incidence of MACE (p) which was divided into $0.5 \mathrm{p}, \mathrm{p}, 2 \mathrm{p}^{4}$, as there are no predefined clinical risk categories for secondary cardiovascular events.

\footnotetext{
Methods for the association of EV TN-C levels and vulnerable plaque characteristics High TN-C plaque expression has previously been associated with vulnerable plaque characteristics. ${ }^{5,6}$ We further investigated whether the same association could be found with circulating TN-C levels. Logistic and linear regression models were used for the binomial and continuous histological variables, respectively. Based on literature: age, sex, creatinine, diabetes, history of CAD/PAOD, current smoking, hypertension, anticoagulant use, statin use, preprocedural cerebrovascular symptoms and year of surgery were identified as potential confounders and were adjusted for. ${ }^{7-15}$
}

\section{Methods for the Western blot analysis}

Western blot analysis was performed on a $4-12 \%$ gradient Bis/Tris gel (NuPage, Invitrogen). After blotting, the blot was incubated with the following antibodies:1.Primary antibody: Human Tenascin C antibody (purified polyclonal goat anti-CD9 (AF3358) (R\&D systems). 2.Secondary antibody: Rabbit anti-Goat-HRP 1/1000) (Invitrogen).Visualization was done with a Super Signal enhanced luminol-based chemiluminescent kit (Thermoscientific) and image capture and analysis was done on a Biorad Chemidoc MP. 


\section{Supplemental Tables}

Table I. Distribution of TN-C in density sub-fractions of the LDL precipitate after density gradient centrifugation

After density gradient centrifugation of the LDL precipitate, the highest TN-C concentrations are present in fractions with density $1.00-1.04$ and in fractions with density $1.09-1.16$. We previously showed ${ }^{17}$ that CD9 bands were in the fractions with a density of 1.02-1.08. This shows that TN-C is indeed in EVs in the LDL precipitate. Since we also measured a peak value of TN-C in the 7th subfraction, suggesting that TN-C is not only in EVs. This is in accordance with a study showing that TN-C release occurs in two formats; TN-C protein associated with EVs and soluble TN-C protein. ${ }^{18}$

\begin{tabular}{lccc}
\hline Subfraction & Tenascin-C $(\mathbf{p g} / \mathbf{m l})$ & Density & CD9 Western $^{17}$ \\
\hline LDL UC 1 & 6.34 & 1.00 & - \\
LDL UC 2 & 20.30 & 1.00 & ++ \\
LDL UC 3 & 322.88 & 1.02 & +++ \\
LDL UC 4 & 37.60 & 1.04 & + \\
LDL UC 5 & 19.18 & 1.08 & - \\
LDL UC 6 & 23.03 & 1.09 & - \\
LDL UC 7 & 142.26 & 1.13 & - \\
LDL UC 8 & 30.74 & 1.16 & - \\
LDL UC 9 & 6.97 & 1.19 & - \\
LDL UC 10 & 3.93 & 1.28 & \\
\hline
\end{tabular}

Abbreviations: LDL UC: LDL subfraction from density gradient ultracentrifugation 
Table II. Baseline characteristics of study population stratified by MACE

\begin{tabular}{|c|c|c|c|}
\hline & No MACE & MACE & p-value \\
\hline & $\mathrm{n}=700$ & $\mathrm{n}=137$ & \\
\hline Age (years) & $69( \pm 9)$ & $72( \pm 9)$ & $<0.01$ \\
\hline Sex, male & $488(70)$ & $101(74)$ & 0.40 \\
\hline Ipsilateral carotid artery stenosis degree & & & 0.17 \\
\hline $0-50 \%$ & $4(1)$ & $1(1)$ & \\
\hline $50-70 \%$ & $54(8)$ & $5(4)$ & \\
\hline $70-99 \%$ & $626(92)$ & $127(96)$ & \\
\hline $\operatorname{BMI}\left(\mathrm{kg} / \mathrm{m}^{2}\right)$ & $26[24,28]$ & $26[24,28]$ & 0.33 \\
\hline Creatinine $(\mu \mathrm{mol} / \mathrm{L})$ & $87[75,104]$ & $95[79,114]$ & $<0.01$ \\
\hline eGFR $\left(\mathrm{ml} / \mathrm{min}\right.$ per $\left.1.73 \mathrm{~m}^{2}\right)$ & $74( \pm 20)$ & $67( \pm 21)$ & $<0.01$ \\
\hline Current smoker & $226(32)$ & $52(38)$ & 0.24 \\
\hline Diabetes & $149(21)$ & $43(31)$ & 0.01 \\
\hline Hypertension & $487(72)$ & $101(76)$ & 0.37 \\
\hline Hypercholesterolaemia & $446(70)$ & $81(69)$ & 0.95 \\
\hline Triglycerides (mg/dl) & $1.5[1.1,2.1]$ & $1.5[1.1,2.0]$ & 0.85 \\
\hline $\mathrm{LDL}(\mathrm{mmol} / \mathrm{L})$ & $2.3[1.8,3.0]$ & $2.3[1.8,3.0]$ & 0.57 \\
\hline HDL (mg/dl) & $1.1[0.9,1.3]$ & $0.9[0.8,1.1]$ & $<0.01$ \\
\hline Total cholesterol (mg/dl) & $4.2[3.5,5.2]$ & $4.1[3.5,5.0]$ & 0.14 \\
\hline Contralateral carotid artery stenosis of $50-100 \%$ & $283(45)$ & $66(52)$ & 0.16 \\
\hline Anticoagulants & $67(10)$ & $20(15)$ & 0.10 \\
\hline Antiplatelets & $617(88)$ & $117(87)$ & 0.67 \\
\hline Lipid lowering drugs & $561(80)$ & $102(75)$ & 0.20 \\
\hline Statins & $554(79)$ & $101(74)$ & 0.24 \\
\hline Hypertension drugs & $519(74)$ & $108(79)$ & 0.24 \\
\hline History of CAD or PAD & & & $<0.01$ \\
\hline none & $410(59)$ & $65(47)$ & \\
\hline CAD or PAD & $242(35)$ & $52(38)$ & \\
\hline CAD and PAD & $48(7)$ & $20(15)$ & \\
\hline History of stroke or TIA & $534(76)$ & $109(80)$ & 0.47 \\
\hline Preprocedural cerebrovascular symptoms & & & 0.10 \\
\hline asymptomatic & $294(42)$ & $44(32)$ & \\
\hline ocular & $74(11)$ & $16(12)$ & \\
\hline TIA & $193(28)$ & $39(29)$ & \\
\hline Stroke & $139(20)$ & $38(28)$ & \\
\hline Time between last event and surgery (days) & $24[13,49]$ & $28[13,56]$ & 0.55 \\
\hline
\end{tabular}

Data are presented as $\mathrm{n}(\%)$, mean \pm standard deviation $(\mathrm{SD})$ or median [interquartile range] [IQR]. Abbreviations: BMI, Body Mass Index; eGFR, estimated Glomerular Filtration Rate; LDL, Low-density Lipoprotein; HDL, Highdensity Lipoprotein; CAD, coronary artery disease; PAD, peripheral artery disease; TIA, Transient Ischemic Attack. 
Table III. Predictors in clinical model consisting of only CVRF and biomarker model consisting of CVRF from clinical model and Tenascin-C. Regression coefficients before and after internal validation through bootstrapping

\begin{tabular}{|c|c|c|c|c|c|c|}
\hline Predictors & $\begin{array}{l}\text { Coefficients } \\
\text { clinical model }\end{array}$ & p-value & $\begin{array}{c}\text { Shrunk } \\
\text { coefficients } \\
\text { clinical model }\end{array}$ & $\begin{array}{c}\text { Coefficients } \\
\text { biomarker } \\
\text { model }\end{array}$ & p-value & $\begin{array}{c}\text { Shrunk } \\
\text { coefficients } \\
\text { biomarker } \\
\text { model }\end{array}$ \\
\hline Ln of Tenascin-C level & $\mathrm{N} / \mathrm{A}$ & $\mathrm{N} / \mathrm{A}$ & N/A & 0.2707 & $<0.01$ & 0.2340 \\
\hline Age - y & 0.3464 & $<0.01$ & 0.2995 & 0.3626 & $<0.01$ & 0.3134 \\
\hline Ln of serum creatine level & 0.2071 & 0.02 & 0.1790 & 0.1695 & 0.06 & 0.1465 \\
\hline Current Smoking & 0.4756 & $<0.01$ & 0.4111 & 0.4439 & 0.02 & 0.3837 \\
\hline Diabetes & 0.4143 & 0.02 & 0.3582 & 0.4112 & 0.03 & 0.3554 \\
\hline \multicolumn{7}{|l|}{ History of CAD/PAD } \\
\hline none & \multicolumn{3}{|c|}{ reference } & \multicolumn{3}{|c|}{ reference } \\
\hline CAD or PAD & 0.2326 & 0.22 & 0.2011 & 0.2035 & 0.28 & 0.1759 \\
\hline $\mathrm{CAD}$ and $\mathrm{PAD}$ & 0.8033 & $<0.01$ & 0.6942 & 0.7639 & $<0.01$ & 0.6603 \\
\hline \multicolumn{7}{|c|}{ Preprocedural cerebrovascular symptoms } \\
\hline Asymptomatic & \multicolumn{3}{|c|}{ reference } & \multicolumn{3}{|c|}{ reference } \\
\hline ocular & 0.3593 & 0.22 & 0.3106 & 0.3792 & 0.2 & 0.3278 \\
\hline Transient ischemic attack & 0.2428 & 0.27 & 0.2099 & 0.2606 & 0.24 & 0.2253 \\
\hline Stroke & 0.6379 & $<0.01$ & 0.5514 & 0.6832 & $<0.01$ & 0.5906 \\
\hline
\end{tabular}

Abbreviations: $\mathrm{CAD}$, coronary artery disease; $\mathrm{PAD}$, peripheral artery disease

Table IV. Net reclassification improvement for endpoint MACE

\begin{tabular}{|c|c|c|c|c|c|c|c|}
\hline \multirow{2}{*}{$\begin{array}{l}\text { Model } \\
\text { consisting of } \\
\text { only CVRF }\end{array}$} & \multicolumn{7}{|c|}{ Model consisting of CVRF and TN-C } \\
\hline & $<8 \%$ & $\begin{array}{c}8 \text { to } \\
<16 \%\end{array}$ & $\begin{array}{l}16 \text { to } \\
<32 \%\end{array}$ & $\geq 32 \%$ & $\begin{array}{c}\text { Reclassified } \\
\text { up, } \mathbf{n}(\%)\end{array}$ & $\begin{array}{l}\text { Reclassified } \\
\text { down, } n(\%)\end{array}$ & NRI (95\% CI) \\
\hline MACE & & & & & $21(15.3)$ & $15(10.9)$ & $0.044(-0.042-0.129)$ \\
\hline$<8 \%$ & 4 & 0 & 0 & 0 & & & \\
\hline 8 to $<16 \%$ & 5 & 28 & 10 & 0 & & & \\
\hline 16 to $<32 \%$ & 0 & 7 & 61 & 11 & & & \\
\hline$\geq 32 \%$ & 0 & 0 & 3 & 8 & & & \\
\hline No MACE & & & & & $73(10.5)$ & $100(14.3)$ & $0.039(0.002-0.076)$ \\
\hline$<8 \%$ & 57 & 10 & 0 & 0 & & & \\
\hline 8 to $<16 \%$ & 38 & 283 & 45 & 0 & & & \\
\hline 16 to $<32 \%$ & 0 & 58 & 170 & 18 & & & \\
\hline$\geq 32 \%$ & 0 & 0 & 4 & 12 & & & \\
\hline Overall & & & & & & & $0.083(-0.011-0.176)$ \\
\hline
\end{tabular}




\section{Supplemental Figures}

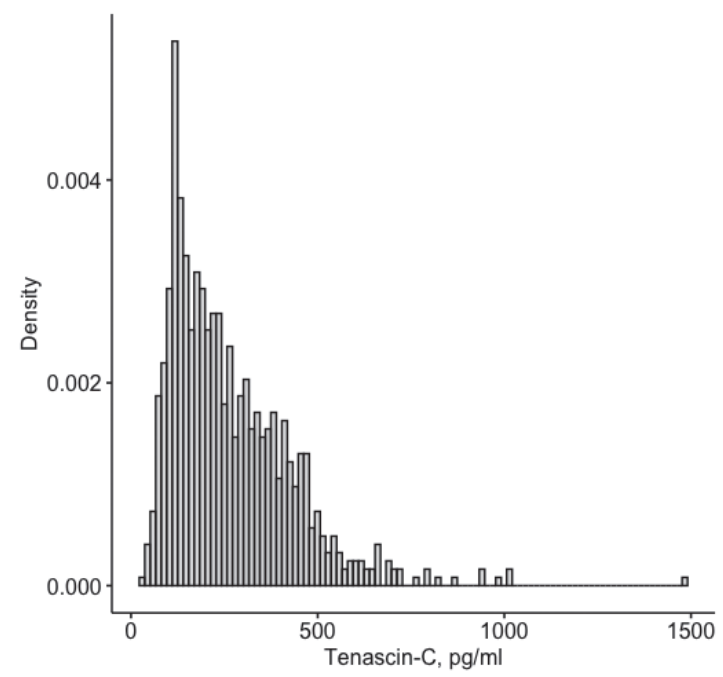

Figure I. Density of EV Tenascin-C levels in the entire study population

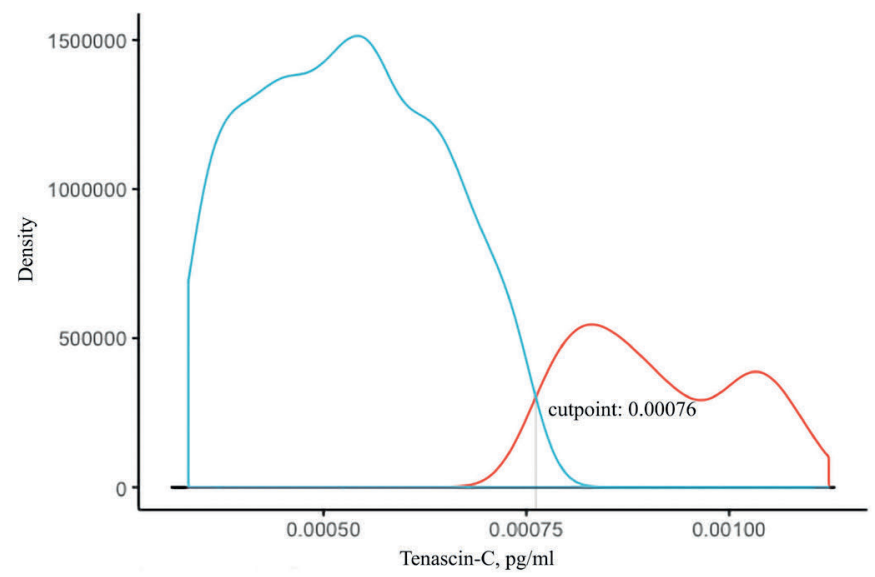

Figure II. Optimal cutpoint estimate of EV Tenascin-C, pg/ml. EV Tenascin-C levels are divided by synthetic liposomes, standardized and log transformed 


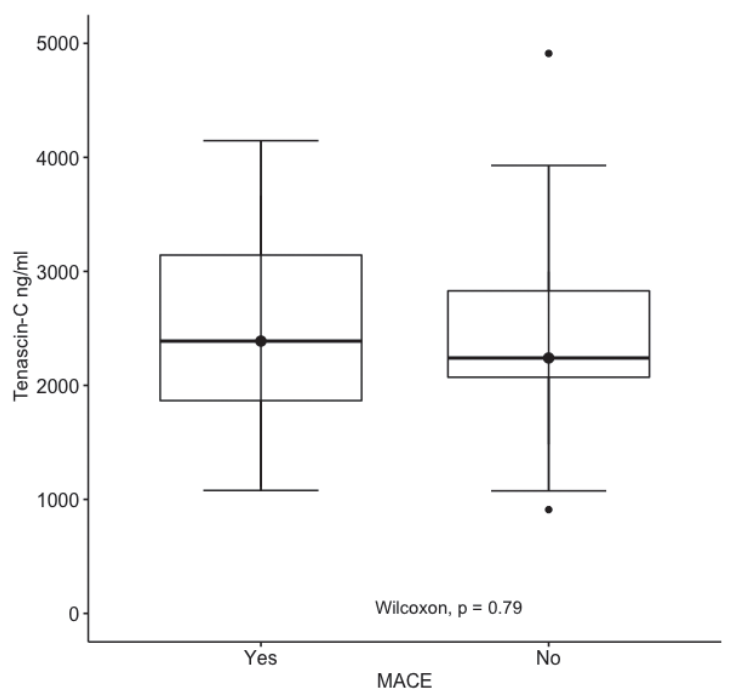

Figure III. Boxplot of Tenascin-C plasma levels in a matched case-control sample from the same study population

No significant difference was found between patients with (median $2388 \mathrm{ng} / \mathrm{ml}$; IQR1855-3295) and without (median 2240ng/ml;IQR 2046-2851) MACE after 3-years of follow-up.
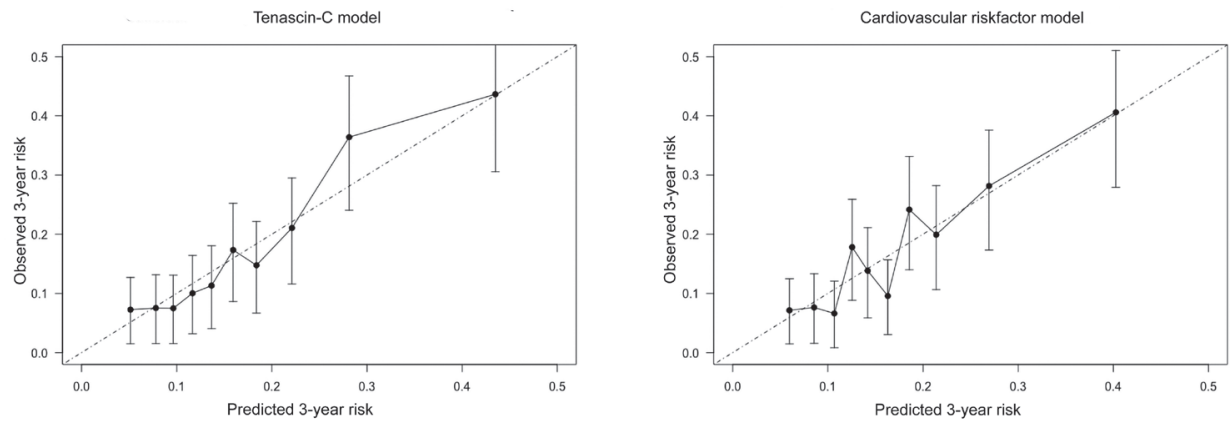

Figure IV. Calibration of model containing Tenascin-C and CVRF and model with only CVRF 
PART III | Chapter 8

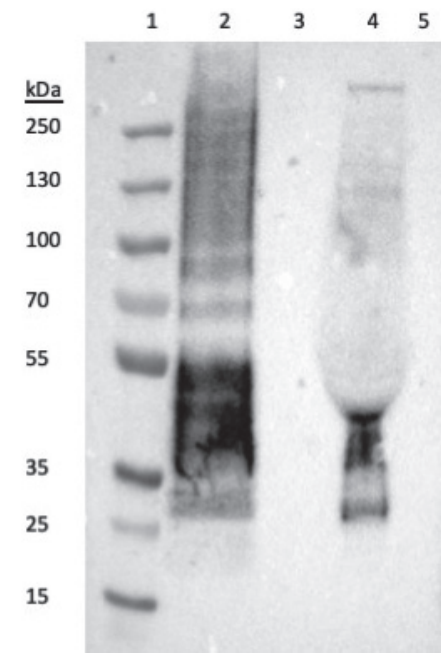

Figure V. Western blotting of circulating TN-C

Line 2 consists of measurements in EVs and line 4 consists of measurements in plasma. In line 4 a TN-C band of $320 \mathrm{kDa}$ was observed predominantly next to smaller isoforms. In line 2 bands of smaller molecular weight were present. Previous studies have shown that there is considerable tissue and disease stage-specific variation in TN-C isoform expression. TN-C can range in size from 180 to $330 \mathrm{kDa} .{ }^{16}$ The Western blot points to a different set of TN-C isoforms measure in EVs in the LDL subfraction. 


\section{Supplemental References}

1 Leening MJG, Vedder MM, Witteman JCM, Pencina MJ, Steyerberg EW. Net Reclassification Improvement: Computation, Interpretation, and Controversies. Ann Intern Med 2014;160(2):122-31.

2 Pencina MJ, D’Agostino RB, Steyerberg EW. Extensions of net reclassification improvement calculations to measure usefulness of new biomarkers. Stat Med 2011;30(1):11-21.

3 Kang L, Chen W, Petrick NA, Gallas BD. Comparing two correlated C indices with right-censored survival outcome: A one-shot nonparametric approach. Stat Med 2015;34(4):685-703.

4 Cook NR, Paynter NP. Performance of reclassification statistics in comparing risk prediction models. Biometrical J 2011;53(2):237-58.

5 Wallner K, Li C, Shah PK, Fishbein MC, Forrester JS, Kaul S, et al. Tenascin-C Is Expressed in MacrophageRich Human 1999:1284-9.

6 Kajiwara K, Ueda H, Yamamoto H, Imazu M, Hayashi Y, Kohno N. Tenascin-C is Associated with Coronary Plaque Instability in Patients with Acute Coronary Syndromes. Circ J 2004;68(3):198-203.

7 Hellings WE, Peeters W, Moll FL, Piers S, Van Setten J, Van Der Spek PJ, et al. Composition of carotid atherosclerotic plaque is associated with cardiovascular outcome: A prognostic study. Circulation 2010;121(17):1941-50.

8 van Lammeren GW, den Ruijter HM, Vrijenhoek JEP, van der Laan SW, Velema E, de Vries J-PPM, et al. Time-Dependent Changes in Atherosclerotic Plaque Composition in Patients Undergoing Carotid Surgery. Circulation 2014;129(22):2269-76.

9 Verhoeven B, Hellings WE, Moll FL, De Vries JP, De Kleijn DPV, De Bruin P, et al. Carotid atherosclerotic plaques in patients with transient ischemic attacks and stroke have unstable characteristics compared with plaques in asymptomatic and amaurosis fugax patients. J Vasc Surg 2005;42(6):1075-81.

10 Siemelink MA, van der Laan SW, Haitjema S, van Koeverden ID, Schaap J, Wesseling M, et al. Smoking is Associated to DNA Methylation in Atherosclerotic Carotid Lesions. Circ Genomic Precis Med 2018;11(9):e002030.

11 Wesseling M, van Koeverden ID, van Lammeren GW, van der Laan SW, Haitjema S, de Vries JPPM, et al. Impaired kidney function is associated with intraplaque hemorrhage in patients undergoing carotid endarterectomy. Atherosclerosis 2017.

12 Fassaert LMM, Timmerman N, van Koeverden ID, Pasterkamp G, de Kleijn DPV, de Borst GJ. Preoperative hypertension is associated with atherosclerotic intraplaque hemorrhage in patients undergoing carotid endarterectomy. Atherosclerosis 2019;290:214-21.

13 Derksen WJM, Peeters W, Tersteeg C, de Vries JPPM, de Kleijn DPV, Moll FL, et al. Age and coumarin-type anticoagulation are associated with the occurrence of intraplaque hemorrhage, while statins are associated less with intraplaque hemorrhage: A large histopathological study in carotid and femoral plaques. Atherosclerosis 2011;214(1):139-43.

14 Mujaj B, Bos D, Selwaness M, Leening MJG, Kavousi M, Wentzel JJ, et al. Statin use is associated with carotid plaque composition: The Rotterdam Study. Int J Cardiol 2018;260:213-8.

15 Van Lammeren GW, Reichmann BL, Moll FL, Bots ML, De Kleijn DPV, De Vries JPPM, et al. Atherosclerotic plaque vulnerability as an explanation for the increased risk of stroke in elderly undergoing carotid artery stenting. Stroke 2011;42(9):2550-5.

16 Page TH, Charles PJ, Piccinini AM, Nicolaidou V, Taylor PC, Midwood KS. Raised circulating tenascin-C in rheumatoid arthritis. Arthritis Res Ther 2012;14(6).

17 Zhang YN, Vernooij F, Ibrahim I, Ooi S, Gijsberts CM, Schoneveld AH, et al. Extracellular vesicle proteins associated with systemic vascular events correlate with heart failure: An observational study in a dyspnoea cohort. PLoS One 2016;11(1):1-19.

18 Mills JT, Schwenzer A, Marsh EK, Edwards MR, Sabroe I, Midwood KS, et al. Airway epithelial cells generate pro-inflammatory tenascin-C and small extracellular vesicles in response to TLR3 stimuli and rhinovirus infection. Front Immunol 2019;10(AUG):1-12. 


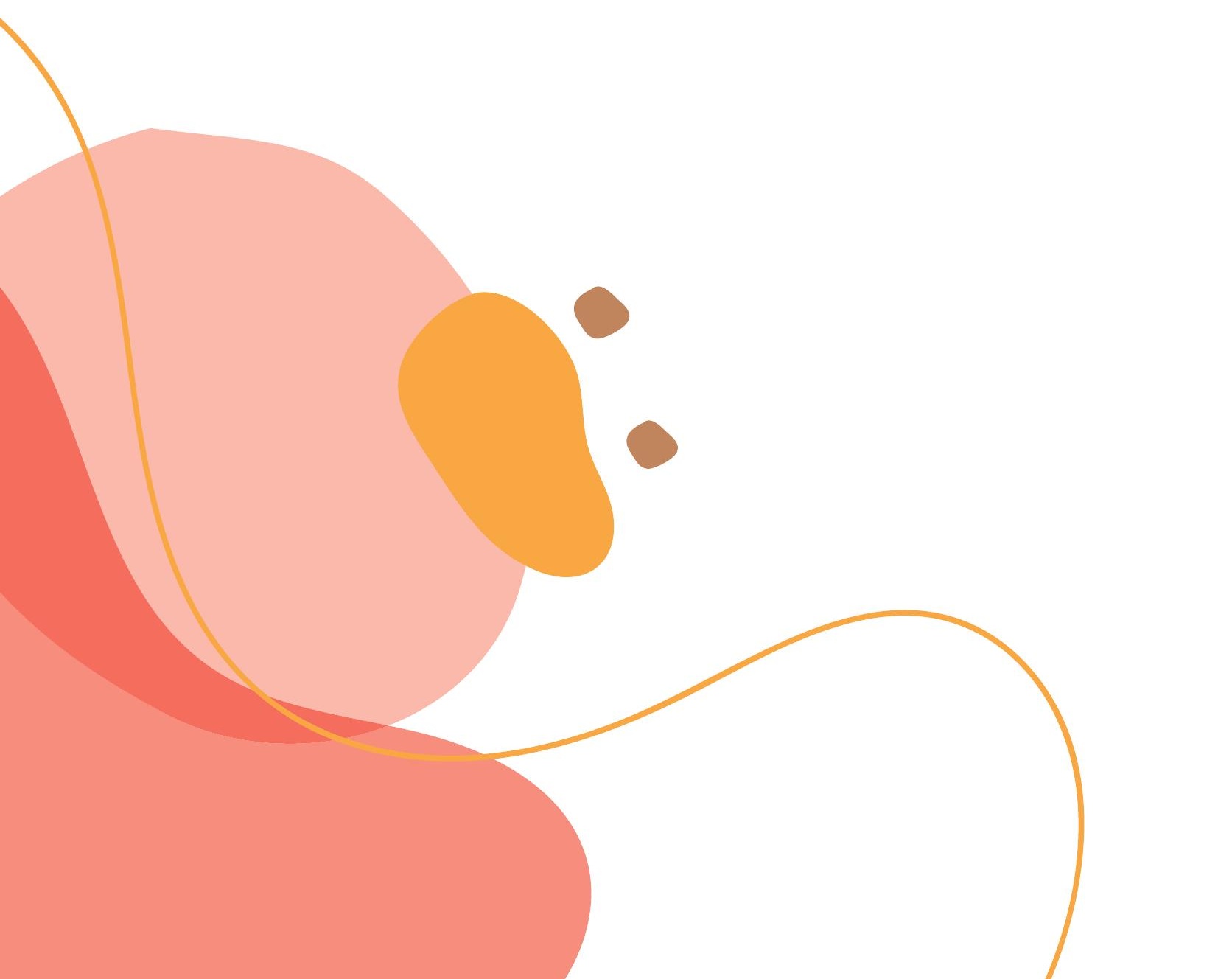




\section{Ceramides and phospholipids are associated with high risk of major cardiovascular events after carotid endarterectomy}

Under review- Scientific Reports

Nathalie Timmerman* Farahnaz Waissi* Mirthe Dekker Gert J. de Borst Joelle van Bennekom Robbert J. de Winter Mika Hilvo Antti Jylhä

Gerard Pasterkamp Dominique P.V. de Kleijn* Reijo Laaksonen* 


\section{Abstract}

\section{Introduction}

Ceramides and phosphatidylcholines (PCs) are bioactive lipids and lipid bilayer membrane components. Distinct ceramides/PCs (ratios) predict cardiovascular outcome in patients with coronary artery disease. Extracellular vesicles (EVs) are proposed biomarkers for cardiovascular disease and contain ceramides/PCs. Ceramides/PCs have not been studied in patients undergoing carotid endarterectomy (CEA) nor in EVs. We therefore investigated whether levels of ceramides/PCs in plasma and EVs are associated with postoperative risk of major adverse cardiovascular events (MACE) following CEA.

\section{Methods}

In 873 patients undergoing CEA of the Athero-Express biobank, we quantitatively measured seven ceramides/PCs in preoperative blood samples: Cer(d18:1/16:0), Cer(d18:1/18:0), Cer(d18:1/24:0), Cer(d18:1/24:1), PC(14:0/22:6), PC(16:0/16:0) and PC(16:0/22:5) in plasma and two plasma EV-subfractions (LDL and TEX). We analyzed the association of ceramides, PCs and their predefined ratios with the three-year postoperative risk of MACE (including stroke, myocardial infarction and cardiovascular death).

\section{Results}

A total of 138 patients (16\%) developed MACE during the three-year follow-up. In the LDL-EV subfraction, higher levels of Cer(d18:1/24:1) and Cer(d18:1/16:0)/PC(16:0/22:5) ratio were significantly associated with an increased risk of MACE (adjusted HR per SD [95\% CI] 1.24 [1.01-1.53] and 1.26 [1.04-1.52], respectively). In the TEX-EV subfraction, three ratios $\operatorname{Cer}(\mathrm{d} 18: 1 / 16: 0) / \operatorname{Cer}(\mathrm{d} 18: 1 / 24: 0), \operatorname{Cer}(\mathrm{d} 18: 1 / 18: 0) / \operatorname{Cer}(\mathrm{d} 18: 1 / 24: 0)$ and Cer(d18:1/24:1)/Cer(d18:1/24:0) were positively associated with MACE (adjusted HR per SD 1.34 [1.06-1.70], 1.24 [1.01-1.51] and 1.31 [1.08-1.58], respectively).

\section{Conclusion}

Distinct ceramides and PCs in plasma EVs determined in preoperative blood were independently associated with an increased three-year risk of MACE after CEA. These lipids are therefore potential markers to identify high-risk CEA patients qualifying for secondary preventive add-on therapy. 


\section{Introduction}

Carotid endarterectomy (CEA) is a common effective treatment to lower the risk of future ipsilateral stroke in patients with a high degree asymptomatic or symptomatic extracranial carotid artery stenosis. Despite CEA, the residual risk for future cardiovascular (CV) events after CEA is still markedly high with approximately $20 \%$ in the three years after CEA. ${ }^{1}$ CEA patients with high risk for secondary $\mathrm{CV}$ events qualify for more intensive medical treatment ${ }^{2}$, for instance by increasing the dose of statins or addition of PCSK-9 inhibitors. Also, anti-inflammatory drug therapies (such as colchicine) or addition of anticoagulants are potential options that are currently under investigation. ${ }^{3-7}$ These intensified treatments are often accompanied by high costs or detrimental side effects. Considering the varying risk of MACE across patients ${ }^{8}$, risk stratification tools that assist individualized secondary prevention are warranted. However, clinical prediction models including CV risk factors have poor predictive ability to discriminate between high and low risk CEA patients for future $\mathrm{CV}$ events. ${ }^{8,9}$ Biomarkers may improve risk stratification models to identify patients at high risk for future CV events.

Previous lipidomic studies have identified ceramides and phosphatidylcholines (PCs) as promising biomarkers for cardiovascular disease. ${ }^{10-16}$ Ceramides belong to sphingolipids consisting of a sphingosine backbone with an attached fatty acid. PCs are phospholipids that are characterized by a choline headgroup and two fatty acyl side chains. Both are main components of lipid bilayer membranes but also act as key signaling molecules. ${ }^{17}$ Ceramides and PCs are suggested to affect atherogenic processes and CV risk factors such as diabetes and obesity. ${ }^{17,18}$ In patients with established coronary artery disease (CAD), particular ceramides and PCs and their distinct ratios were predictive for $\mathrm{CV}$ death and future $\mathrm{CV}$ events. ${ }^{10-16}$ Until date, no studies have evaluated the role of ceramides and PCs for future $\mathrm{CV}$ events in patients following CEA.

Plasma extracellular vesicles (EVs) are a novel source of biomarkers for cardiovascular disease. ${ }^{19}$ EVs are lipid bilayer membrane microstructures that transfer biological information including lipids (e.g. ceramides and PCs), proteins and RNA over distance from cell to cell. EVs originate either from budding of the outer cell membrane or subcellular compartments hereby including parent-cell membrane lipids and enclosing cytosolic material that could affect remote cells and influence pathophysiological processes. ${ }^{19}$ The biological information in EVs is derived from the cell of origin and can reflect its status. It is known that EV subsets vary in size and in their biological content. ${ }^{19,20}$ Protein content in EVs have been associated with recurrent CV events. ${ }^{21}$ However, the content of ceramides and PCs in plasma EVs in relation with CV events have not yet been studied. 
We therefore aimed to investigate whether circulating levels of ceramides and PCs in plasma and in two plasma EV subsets (the LDL- and TEX subfraction) are associated with the postoperative risk of MACE in patients undergoing CEA.

\section{Methods}

\section{Study participants}

Study participants originated from the Athero-Express Biobank. This ongoing prospective biobank study includes consecutive patients undergoing CEA in two tertiary referral hospitals (University Medical Center Utrecht and St. Antonius Hospital Nieuwegein, The Netherlands). A comprehensive description of the study design has been published earlier. ${ }^{1,22}$ All patients undergoing CEA were asked for study participation. Indications for CEA were adjudicated by a multidisciplinary vascular team and based on recommendation from the European Carotid Surgery Trial ${ }^{23}$ and the North American Symptomatic Carotid Endarterectomy Trial ${ }^{24,25}$ for symptomatic patients and Asymptomatic Carotid Surgery Trial ${ }^{26,27}$ for asymptomatic patients. Patients included in the Athero-Express Biobank from April 1 $1^{\text {st }} 2002$ until December 31, 2016 were eligible for the current study. Inclusion criteria were availability of citrate blood plasma sample and follow-up data. Exclusion criteria were patients undergoing CEA for restenosis. All patients provided written informed consent. Ethical approval for study conduction was obtained from the ethical boards of both hospitals. Study conduction complied with the Declaration of Helsinki.

\section{Data collection}

Baseline characteristics were collected by standardized questionnaires that were verified against medical records including medical history, medication use, cardiovascular risk factors and basic laboratory parameters. A preoperative blood sample was taken and stored in -80C freezer until further use. The atherosclerotic plaque was freshly obtained during CEA and immediately transferred to the laboratory for further histological analyses.

\section{Follow-up}

Patients underwent yearly follow-up for a total duration of three years after CEA through standardized questionnaires send by post inquiring whether a patient had experienced any $\mathrm{CV}$ event or had been admitted to a hospital in the past year. Follow-up questionnaires were cross-checked with hospital medical records. In case of no response to questionnaires or when additional information regarding a CV event was necessary, the general practitioner was consulted to provide additional follow-up information consisting of medical records and hospital discharge letters from institutions where the event had occurred. All 
information regarding potential CV events were reviewed by two independent researchers. In case of disagreement, a third expert (GJdB) was consulted.

\section{Study outcomes}

The primary outcome was defined as the three-year postoperative risk of major adverse $\mathrm{CV}$ events (MACE) including fatal- or nonfatal ischemic or hemorrhagic stroke, fatal- or nonfatal myocardial infarction (MI) and any CV death also including sudden cardiac death, fatal aneurysm rupture and fatal cardiac failure. Secondary outcomes were histological atherosclerotic carotid plaque characteristics: content of macrophages, smooth muscle cells (SMCs), collagen, calcification, intraplaque hemorrhage (IPH), intraplaque vessels and lipid core size.

\section{Biomarker selection}

Selection of ceramides and phosphatidylcholines (PCs) was based on previous biomarker discovery and validation studies in CAD patients. ${ }^{10-13}$ Ratios of ceramide/ceramide or ceramide/PC were predefined based on proven associations with $\mathrm{CV}$ outcomes in CAD cohorts. ${ }^{10-13,15}$

\section{Measurement of ceramides/PCs in plasma and EV-subfractions}

Levels of ceramides and PCs were measured in unfractionated plasma and in two subpopulations of plasma extracellular vesicles (EV) called the LDL-EV subfraction and TEX-EV subfraction. A detailed overview of the EV isolation procedure has been reported previously ${ }^{20}$ and is described in the Supplemental Materials (Figure S1). In brief, the LDLEV subfraction was precipitated using Dextran Sulphate (DS, 0.05\%, MP Biomedicals) and Manganese II Chloride ( $\mathrm{MnCl} 2,0.05 \mathrm{M}$, Sigma-Aldrich), the TEX-EV fraction with Xtractt buffer (1:4, Cavadis BV). Magnetic dextran nanoparticles (nanomag ${ }^{\oplus}$-D plain for the LDLEV fraction and Nano-mag ${ }^{\oplus}-\mathrm{D}$ PEG-OH for the TEX-EV fraction) were added and EVs were isolated with use of a bio-plex handheld magnet. The pellet, containing the EVs, was lysed with lysis buffer to free its content. Magnetic nanoparticles and debris were separated and removed from the pellet by centrifugation. Ceramides and PC concentrations were quantified in plasma and in the LDL-EV and TEX-EV subfractions using liquid chromatography-mass spectrometry (LC-MS, Sciex TripleQuad 5500 mass spectrometer coupled to Sciex MPX LC system). Final concentrations of ceramides and PCs were expressed in $\mu \mathrm{M}$. Details of the LC-MS quantification analyses of ceramides and PCs are stated in the Supplemental Materials. EV characterization in plasma EV subfractions have been described in previous studies. ${ }^{20,28,29}$ Previous Nanoparticle Tracking Analyzer (NTA) experiments showed the smallest EVs in the TEX-fraction (mean $84 \mathrm{~nm}$ ) and relatively larger EVs in LDL-fraction (mean $101 \mathrm{~nm}$ ). ${ }^{20}$ Additional density gradient analyses of EV 
subfractions confirmed the presence of ceramides and PCs in EVs (see Supplemental Material for details; Figure S2, Figure S3 and Table S1).

\section{Histological atherosclerotic plaque characterization}

Histological examination of the carotid atherosclerotic plaque was performed according to the standardized Athero-Express biobank protocol. ${ }^{1,22}$ Details are described in the Supplemental Materials. ${ }^{1,22}$ The carotid plaque was cross-sectionally cut into segments of $5 \mathrm{~mm}$. The segment with the largest plaque volume was considered the culprit lesion was allocated to immunohistochemical analysis of collagen, smooth muscle cells (SMCs), macrophages, calcifications, intraplaque hemorrhage (IPH) and lipid core. Plaque characteristics were scored semi quantitatively as no/minor or moderate/heavy staining, except for IPH (scored as absent or present) and lipid core (size was visually estimated relative to the total plaque area and expressed as $<10 \%, 10-40 \%,>40 \%$ of the total plaque area). In addition, SMCs, macrophages and intraplaque vessels were quantified by computerized analysis software (AnalySIS 3.2, Soft Imaging Systems GmbH, Munster, Germany). SMCs and macrophage infiltration were expressed as the percentage of positive staining of the total plaque area. CD34 positive intraplaque vessels were counted in three hotspots with highest vessel density and the average number per square millimeter was calculated, as described previously. ${ }^{30}$

\section{Statistical analyses}

Continuous baseline characteristics and categorical baseline characteristics were respectively compared by Students t-test or Mann Whitney U test and Pearson's Chi-squared test. Cholesterol and creatinine levels were logarithmically transformed because of skewness. The associations of ceramides and PCs (ratio) levels with the three-year postoperative risk of MACE were analyzed by univariable, adjusted for LDL-C and HDL-C, and multivariable Cox proportional hazards models, similar to the previous CAD study. ${ }^{10}$ To facilitate easy comparison with existing literature ceramides and PCs (ratios) concentrations were standardized to Z-scores. ${ }^{10,11}$ Hazard ratios (HR) were expressed per one SD increase. Potential confounders for multivariable analyses were a priori selected based on available literature 9,10,13,15,31,32; age, history of coronary artery disease (CAD) and/or peripheral artery disease (PAD), preprocedural cerebrovascular symptoms, current smoking, hypertension, diabetes, lipid-lowering drug use, triglycerides, total cholesterol, creatinine and contralateral carotid artery stenosis of 50-100\%. Model reduction using Akaike information criterion in a stepwise backward regression resulted in the final model including age, history of CAD and/or PAOD, cerebrovascular symptoms, current smoking, LDL-C and HDL-C. Percentage of missing covariates was low (range 0.0-4.2\%), see Supplemental Table S2. To gain further insight in the association of ceramides and PCs (ratios) and the occurrence of MACE during 
three-year follow-up, multivariable significant ceramides/PCs were visualized in quartiles by Kaplan-Meier graphs.

To unravel potential underlying biological mechanisms, the association of multivariable significant ceramides and PCs (ratios) with histological atherosclerotic plaque characteristics were investigated by univariable and multivariable logistic or linear regression. Age, sex, LDL-C, HDL-C, triglycerides and total cholesterol were added as potential confounders for multivariable analyses of plaque characteristics. ${ }^{32,33}$ All p-values resulted from two-tailed hypothesis testing. A p-value $<0.05$ indicated statistically significance. Analyses were performed in R statistical software version 3.6.2 (R Foundation for Statistical Computing, Vienna, Austria; https://www.r-project.org/).

\section{Results}

\section{Study population}

A total of 887 patients were included in the current study (Figure 1). Measurement of ceramides and PCs failed in 14 patients leaving 873 patients for analyses. Patient characteristics are summarized in Table 1 . The overall mean age was 69 years, $70 \%$ were men and most were operated for high degree (70-99\%) symptomatic carotid stenosis. The prevalence of $\mathrm{CV}$ risk factors, history of $\mathrm{CAD}$ and $\mathrm{PAD}$ were high, exemplified by the high frequency of antiplatelet and lipid lowering drug use at study inclusion.

During a median postoperative follow-up duration of 3.0 years [IQR 2.2-3.0], 138 patients (15.8\%) experienced MACE consisting of 74 (8.5\%) fatal and non-fatal stroke, 44 (5\%) fatal and non-fatal MI and $20(2.3 \%) \mathrm{CV}$ death due to other causes. Patients that experienced MACE were at inclusion significantly older, more often had diabetes, had lower HDL-C levels, higher creatinine levels and were more likely to have a history of CAD and/or PAD compared to patients who remained free from MACE (Table 1). Availability of ceramide and PC measurements, as well as absolute concentrations compared between patients that experienced MACE and those that did not, are shown in Supplemental Table S3.

\section{Associations with the 3-year postoperative risk of MACE}

Univariable and multivariable associations of ceramides and PCs, measured in plasma and EV subfractions, with the 3-year risk of MACE are depicted in Table 2. After correction for all confounders (LDL-C, HDL-C, age, history of CAD and/or PAD, cerebrovascular symptoms and current smoking), one ceramide and four ceramide ratios remained significantly associated with MACE in the LDL-EV subfraction as well as in the TEX-EV subfraction (Table 2). 
Table 1. Baseline characteristics

\begin{tabular}{|c|c|c|c|}
\hline & $\begin{array}{l}\text { no MACE } \\
(n=735)\end{array}$ & $\begin{array}{l}\text { MACE } \\
(n=138)\end{array}$ & p-value \\
\hline \multicolumn{4}{|l|}{ Demographics } \\
\hline Age, mean (SD) & $68.8(9.2)$ & $72.1(9.0)$ & $<0.001$ \\
\hline Male & $508(69.1)$ & $103(74.6)$ & 0.231 \\
\hline \multicolumn{4}{|l|}{ Ipsilateral carotid artery stenosis degree } \\
\hline $50-70 \%$ & $62(8.7)$ & $6(4.5)$ & \\
\hline $70-99 \%$ & $654(91.3)$ & $128(95.5)$ & 0.119 \\
\hline \multicolumn{4}{|l|}{ Risk factors } \\
\hline $\begin{array}{l}\text { Contralateral carotid artery stenosis } \\
\text { of } 50-100 \%\end{array}$ & $292(44.0)$ & $66(51.6)$ & 0.138 \\
\hline BMI, mean (SD) & $26.3(3.9)$ & $26.7(3.9)$ & 0.250 \\
\hline Hypertension & $609(82.9)$ & $118(86.1)$ & 0.412 \\
\hline Creatinine, median [IQR] & $87.0[75.0,104.0]$ & $95.0[81.0,114.0]$ & 0.001 \\
\hline GFR, mL/min/1.73 m2, mean (SD) & $74.1(20.3)$ & $67.7(21.3)$ & 0.001 \\
\hline Current smoking & $235(32.3)$ & $52(38.2)$ & 0.210 \\
\hline Diabetes & $156(21.2)$ & $43(31.2)$ & 0.015 \\
\hline Hypercholesterolemia & $467(69.6)$ & $80(67.8)$ & 0.777 \\
\hline Triglycerides levels, mmol/L, median [IQR] & $1.5[1.1,2.1]$ & $1.5[1.1,2.0]$ & 0.803 \\
\hline LDL levels, mmol/L, median [IQR] & $2.3[1.8,3.0]$ & $2.3[1.8,3.0]$ & 0.496 \\
\hline HDL levels, mmol/L, median [IQR] & $1.1[0.9,1.3]$ & $0.9[0.8,1.1]$ & $<0.001$ \\
\hline $\begin{array}{l}\text { Total cholesterol levels, mmol/L, median } \\
\text { [IQR] }\end{array}$ & $4.2[3.5,5.2]$ & $4.1[3.5,4.9]$ & 0.125 \\
\hline \multicolumn{4}{|l|}{ Medical history } \\
\hline Cerebrovascular symptoms & & & 0.206 \\
\hline asymptomatic & $306(41.6)$ & $45(32.6)$ & \\
\hline ocular & $78(10.6)$ & $16(11.6)$ & \\
\hline TIA & $204(27.7)$ & $39(28.2)$ & \\
\hline stroke & $147(20.0)$ & $38(27.5)$ & \\
\hline History of stroke or TIA & $556(75.6)$ & $110(79.7)$ & 0.357 \\
\hline History of CAD or PAD & & & 0.005 \\
\hline no history of CAD and PAD & $429(58.7)$ & $65(47.1)$ & \\
\hline history of CAD or PAD & $249(34.1)$ & $53(38.4)$ & \\
\hline history of CAD and PAD & $53(7.3)$ & $20(14.5)$ & \\
\hline \multicolumn{4}{|l|}{ Drug therapy } \\
\hline Anticoagulants & $70(9.5)$ & $20(14.6)$ & 0.102 \\
\hline Antiplatelets & $648(88.4)$ & $118(86.8)$ & 0.690 \\
\hline Lipid lowering drugs & $591(80.5)$ & $102(74.5)$ & 0.133 \\
\hline
\end{tabular}

Values are displayed as frequency, $\mathrm{n}(\%)$ unless otherwise indicated. Values in bold are p-value $<0.05$. Categorical baseline characteristics were compared by Pearson's Chi-squared test. Continuous baseline characteristics were compared by Students t-test or Mann Whitney U based on the distribution of the data. Abbreviations: SD, standard deviation; $I Q R$, interquartile range, $B M I$, Body mass index; $C A D$, coronary artery disease; $P A D$, peripheral artery disease; GFR, estimated glomerular filtration rate calculated by MDRD equation; TIA, transient ischemic attack; $L D L$, Low-density lipoprotein; $H D L$, high-density lipoprotein. Hypertension, diabetes and hypercholesterolemia were defined as diagnosed by a medical doctor or use of specific medication. Antiplatelet drug comprises the use of aspirin, dipyridamole or any ADP-inhibitor. Lipid lowering drug use comprises the use of any lipid lowering drug. History of stroke or TIA includes ipsilateral or contralateral stroke or TIA. Cerebrovascular symptoms refer to the ipsilateral symptoms on which the indication for CEA was based. Asymptomatic is defined as no experience of ipsilateral cerebrovascular symptoms in the six months prior to CEA. 


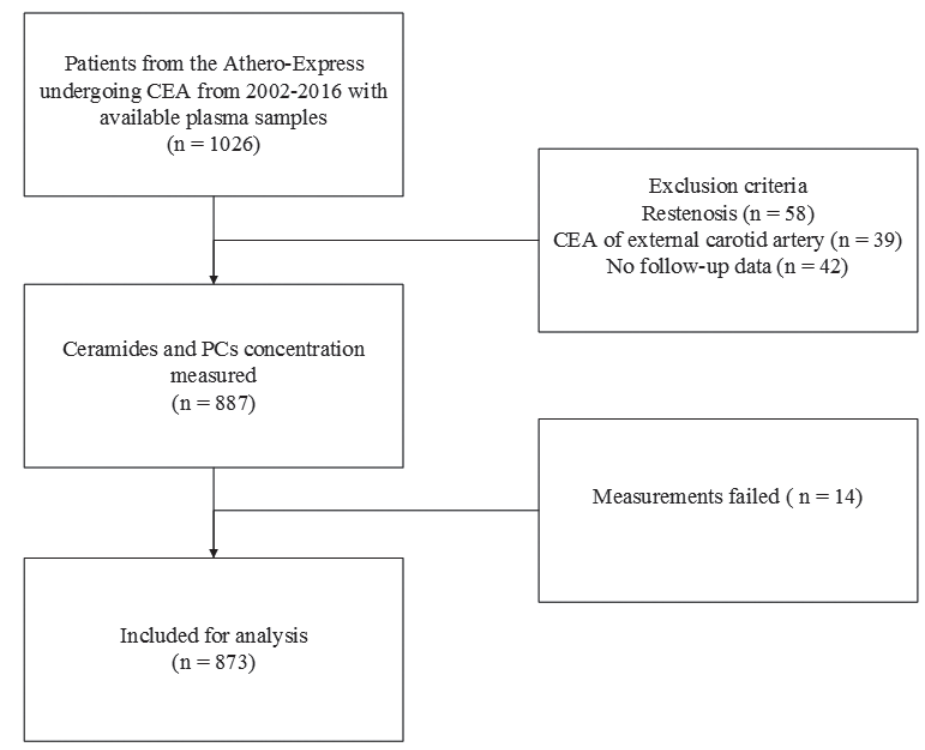

Figure 1. Flowchart of included patients in the present study

In the LDL-EV subfraction, higher levels of Cer(d18:1/24:1) were independently associated with a higher 3-year postoperative risk of MACE with adjusted HR 1.24 per SD, 95\% CI 1.01-1.53 ( $\mathrm{p}=0.040)$. A higher ratio of $\operatorname{Cer}(\mathrm{d} 18: 1 / 16: 0) / \mathrm{PC}(16: 0 / 22: 5)$ in the LDL-EV subfraction was independently associated with a higher 3-year risk of MACE with adjusted HR of 1.26 per SD, 95\% CI 1.04-1.52 ( $\mathrm{p}=0.016)$.

In the TEX-EV subfraction, three ceramide ratios were positively associated with the 3 -year risk of MACE, namely Cer(d18:1/16:0)/Cer(d18:1/24:0) with adjusted HR 1.34 per SD, 95\% CI 1.06-1.70 ( $\mathrm{p}=0.016), \operatorname{Cer}(\mathrm{d} 18: 1 / 18: 0) / \operatorname{Cer}(\mathrm{d} 18: 1 / 24: 0)$ with adjusted HR 1.24 per SD, 95\% CI, 1.01-1.51 ( $\mathrm{p}=0.042)$ and $\operatorname{Cer}(\mathrm{d} 18: 1 / 24: 1) / \operatorname{Cer}(\mathrm{d} 18: 1 / 24: 0)$ with adjusted HR 1.31 per SD, 95\% CI 1.08-1.58 ( $\mathrm{p}=0.005)$.

In plasma, multiple ceramide ratios were significantly associated with MACE in univariable analyses, however, these became insignificant after correction for confounders.

As statins are known to modify ceramide/PC levels ${ }^{13}$, sub analyses with addition of statin use to multivariable models were performed (Supplemental Table S4). All the lipids or lipid ratios in the LDL-EV and two in the TEX-EV subfractions remained significantly associated with MACE after CEA, but Cer(d18:1/18:0)/Cer(d18:1/24:0) in TEX-EV subfraction became borderline non-significant (Supplemental Table S4). 


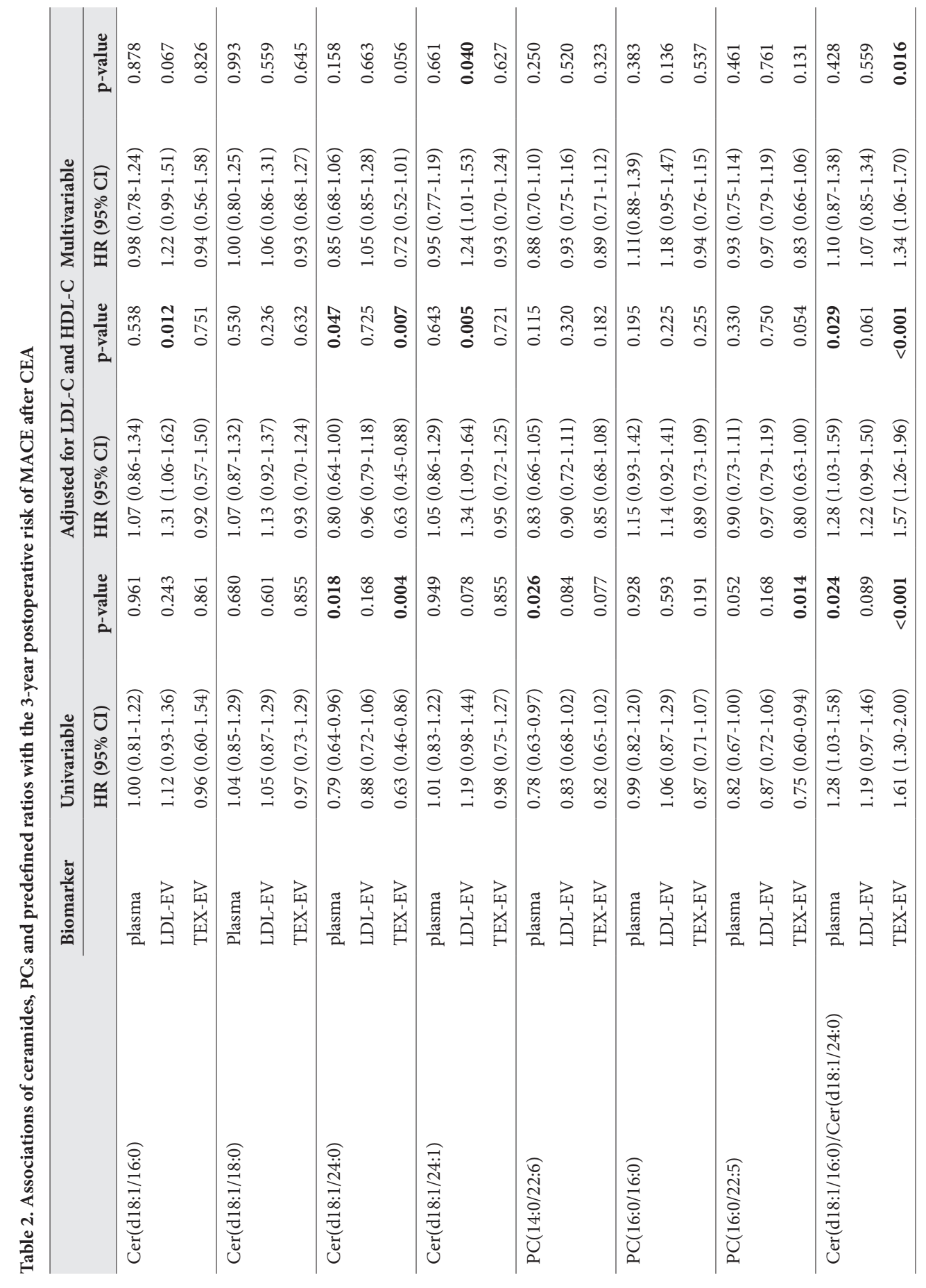




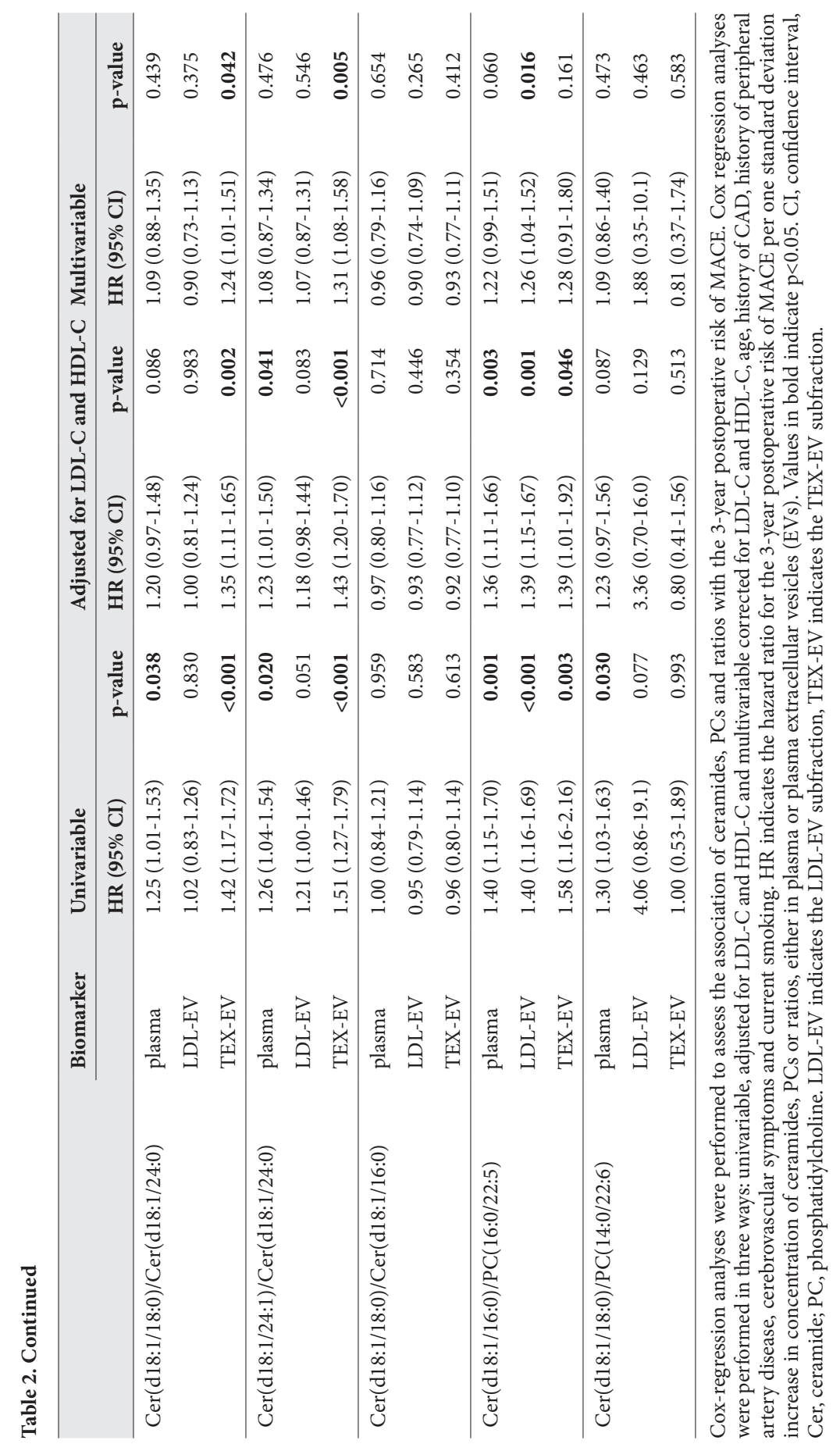


In order to gain further insight in the association of ceramide/PC ratios with MACE, we analyzed quartile levels by Kaplan-Meier plots and Cox-regression analyses (Figures 2A2E). Non-linearity was found for the ratios of $\operatorname{Cer}(\mathrm{d} 18: 1 / 16: 0) / \operatorname{Cer}(\mathrm{d} 18: 1 / 24: 0)$ and Cer(d18:1/18:0)/Cer(d18:1/24:0) in the TEX-EV fraction where patients in the $4^{\text {th }}$ quartile were at relatively high risk (respective HR for patients in the $4^{\text {th }}$ quartile versus those in the $1^{\text {st }}$ quartile were 2.15, 95\% CI 1.33-3.49 and 1.66, 95\% CI 1.05-2.63) (Figures 2A and 2B). The association of Cer(d18:1/24:1) in the LDL-EV fraction with MACE appeared to be dichotomous (Figure 2C). Patients with levels above the median had a HR of 1.44, 95\% CI 1.01-2.06, compared to patients with levels below the median. The $3^{\text {rd }}$ and $4^{\text {th }}$ quartiles of the Cer(d18:1/24:1)/Cer(d18:1/24:0) ratio in the TEX-EV subfraction and Cer(d18:1/16:0)/ $\mathrm{PC}(16: 0 / 22: 5)$ ratio in the LDL-EV subfraction seemed to be more linearly associated with increased risk of MACE (Figures 2D and 2E).

Figures 2A-2E. Kaplan-Meier estimates of major adverse cardiovascular event (MACE) for quartiles of multivariable significant associated ceramides/PC ratios

The p-value indicates the overall comparison of the MACE-free survival across quartile levels by the log-rank test. The hazard ratios in the legends indicate the univariable quartile-specific hazard relative to the first quartile.
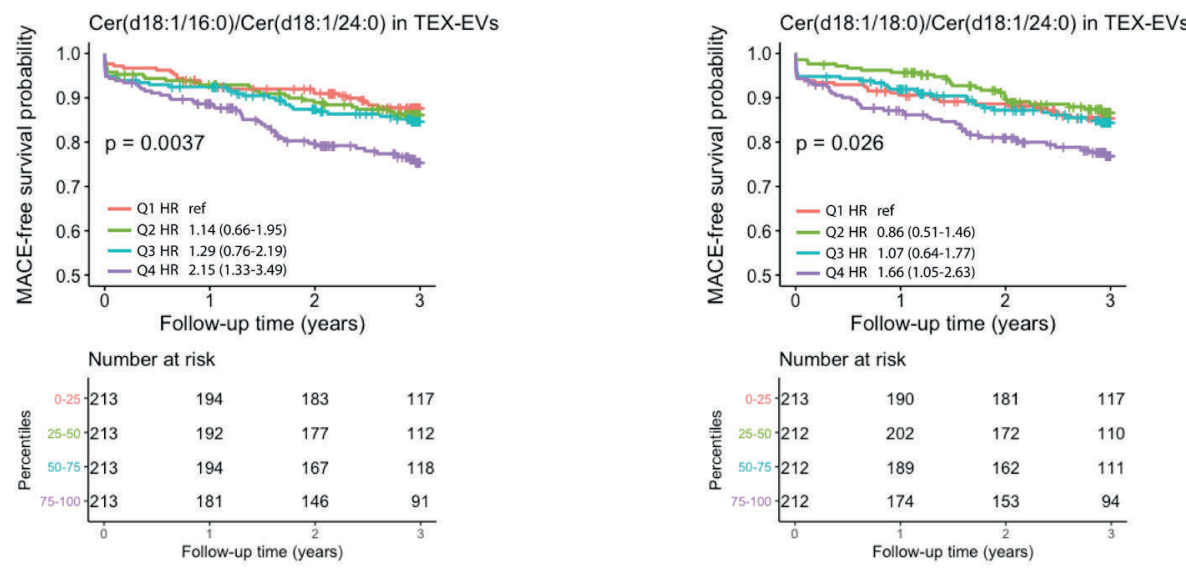

Figure 2A. The three-year MACE-free survival probability of Cer(d18:1/16:0)/

Figure 2B. The three-year MACE-free Cer(d18:1/24:0) in the TEX-EV subfraction

survival probability of $\operatorname{Cer}(\mathbf{d 1 8 : 1 / 1 8 : 0 ) /}$ Cer(d18:1/24:0) in the TEX-EV subfraction 


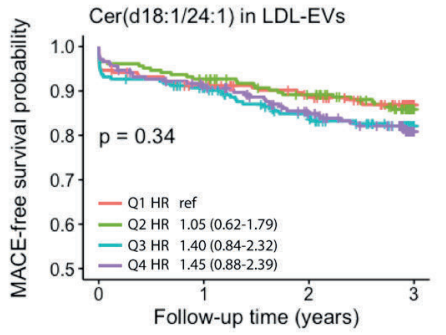

Number at risk

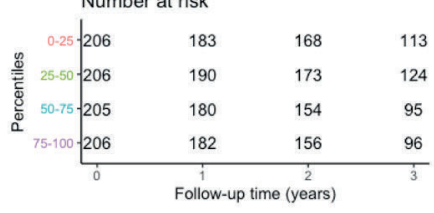

Figure 2C. The three-year MACE-free survival probability of $\operatorname{Cer}(d 18: 1 / 24: 1)$ in the LDL-EV subfraction

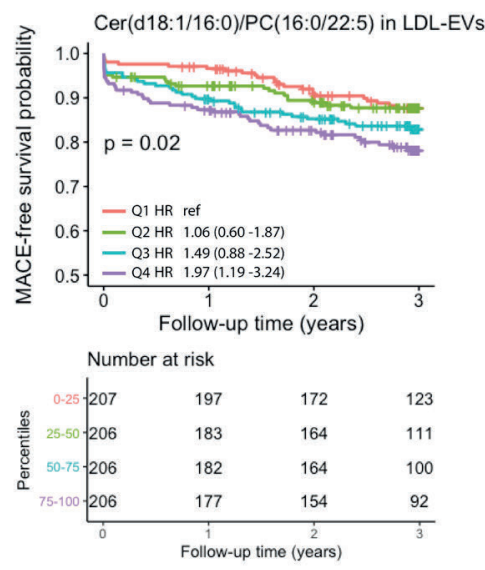

Figure 2E. The three-year MACE-free survival probability of Cer(d18:1/16:0)/PC(16:0/22:5) in the LDL-EV subfraction
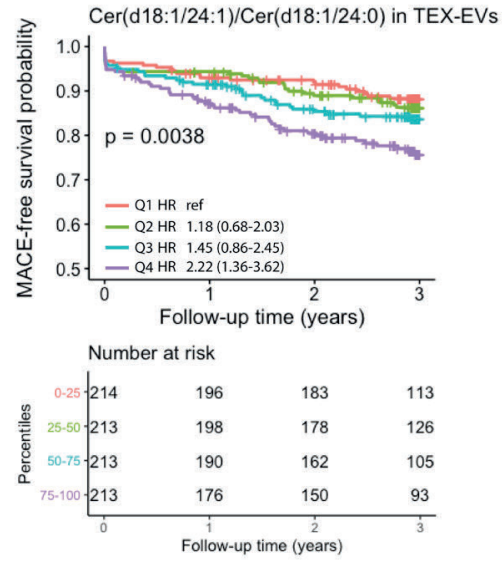

Figure 2D. The three-year MACE-free survival probability of $\operatorname{Cer}(\mathbf{d} 18: 1 / 24: 1) /$ Cer(d18:1/24:0) in the TEX-EV subfraction 


\section{Associations with histological atherosclerotic plaque characteristics}

Having established an association of ceramide/PC EV-levels with MACE and considering that EVs probably reflect the status of the cell of origin, we investigated the association of the multivariable significant ceramides and PCs with histological plaque characteristics that are considered as key markers for plaque vulnerability. This might unravel potential underlying mechanisms how these lipid ratios contribute to cardiovascular event risk. Increased ratios of Cer(d18:1/18:0)/Cer(d18:1/24:0) and Cer(d18:1/24:1)/Cer(d18:1/24:0) in the TEX-EV fraction were inversely associated with the amount of SMCs in the plaque (respective adjusted odds ratio (OR) 0.76 for moderate/heavy SMC staining, 95\% CI 0.61 $0.95, \mathrm{p}=0.015$ ) and adjusted beta of -0.26 for the percentage of SMCs staining, $95 \% \mathrm{CI}-0.47$ to $-0.04, \mathrm{p}=0.020$ ) (Table 3). An increased ratio of $\operatorname{Cer}(\mathrm{d} 18: 1 / 16: 0) / \mathrm{PC}(16: 0 / 22: 5)$ in the LDL-EV subfraction was positively associated with more macrophage infiltration (beta $0.16,0.05-0.28, \mathrm{p}=0.007)$. Neither Cer(d18:1/24:1) in the LDL-EV fraction nor the ratio Cer(d18:1/16:0)/Cer(d18:1/24:0) in TEX-EV subfraction were associated with histological plaque characteristics.

\section{Discussion}

Despite CEA, there remains a high residual risk for future CV events in these patients after surgery. Early identification of these high-risk CEA patients would allow early initiation of add-on therapy to reduce cardiovascular event risk. In this study, we examined the association of circulating molecular lipids, previously associated with CV outcome in CAD patients, with future CV events in patients undergoing CEA. In plasma EVs, elevated levels of $\operatorname{Cer}(\mathrm{d} 18: 1 / 24: 1)$ and the ratios $\operatorname{Cer}(\mathrm{d} 18: 1 / 16: 0) / \operatorname{Cer}(\mathrm{d} 18: 1 / 24: 0), \operatorname{Cer}(\mathrm{d} 18: 1 / 18: 0) /$ Cer(d18:1/24:0), Cer(d18:1/24:1)/Cer(d18:1/24:0) and Cer(d18:1/16:0)/PC(16:0/22:5) were associated with elevated risk of MACE during the three years follow-up independently of conventional cardiovascular risk factors. However, these associations were not found in unfractionated plasma. Thus, EV-derived ceramides/PCs ratios may be considered as biomarkers for high residual CV risk.

Previous studies investigated ceramides/PCs only in plasma and did show associations of particular ceramides/PCs (ratios) with an increased risk of CV death and future CV events in patients with acute coronary syndrome (ACS) or stable CAD. ${ }^{10-12,16}$ In the general population, elevated plasma levels of these specific ceramides were associated with both primary as well as recurrent MACE. ${ }^{15}$ Although we only found associations of these lipid ratios in plasma EVs subsets in a CEA population, our results further contribute to the concept of ceramides and PCs as markers for future CV events. 
This is the first study that examined levels of ceramides and PCs in plasma EVs whereas previous studies only investigated unfractionated plasma. ${ }^{10-12,16}$ Although univariable analysis showed significant associations of ceramides and PCs in both unfractionated plasma and plasma EVs, in multivariable analyses only the associations in plasma EVs remained significant. EV subpopulations differ in vesicle size and it is known that the biological composition and clearance mechanisms vary across subpopulations. ${ }^{19}$ Since unfractionated plasma contains all EV subpopulations next to other particles and complexes containing ceramides and PCs, differences in biological information between subpopulations might be masked and remain unnoticed. Therefore, the signal to noise ratio will probably be better for lipid levels in EV subpopulations than in unfractionated plasma. Our results suggest that lipid ratios in EVs may therefore be more potent biomarkers for MACE than in unfractionated plasma.

In general, the ratios of ceramides or ceramides/PCs species were more strongly associated with MACE than the individual lipid species, which is in line with prior studies in CAD patients. ${ }^{10-12}$ Very long chain ceramides are produced by the Ceramide Synthase enzyme isotype 2 (CerS2; C24:0 or 24:1), while long chain (C16:0) are produced by other isoforms, CerS6 and CerS5. ${ }^{34}$ CerS2 haplo-insufficiency, however leads to compensatory increase of C16:0 ceramides showing that, despite they are produced by different enzymes, changes in ceramide ratios are more pronounced when ceramide pathways are changing. ${ }^{34,35}$ For this, ratios of different ceramides and PCs species may serve as a better reflection of the complex ongoing metabolic pathways and CV disease risk than individual ceramide and PC species.

Ceramides and PCs are related to CV events and modification of lipid profiles by medical or dietary interventions aiming to reduce cardiovascular event risk has increasingly gaining attention. ${ }^{32,36-39}$ In patients, statins and PCSK-9 inhibitors reduced high-risk ceramide and PC levels. ${ }^{13,32}$ Interestingly, PCSK-9 inhibition by loss-of-function mutation lowered relatively more high-risk ceramide levels than LDL-C levels, suggesting that PCSK-9 inhibitors not solely act via reducing LDL-C but also through profound changes in the lipid metabolism. ${ }^{13}$ In patients with metabolic syndrome, treatment with pioglitazone (an antidiabetic drug) reduced high-risk ceramide levels and concomitantly enhanced insulin sensitivity. ${ }^{36}$ A post-hoc analyses indicated that in patients with elevated levels of high-risk ceramides, a Mediterranean diet may be favorable to reduce the cardiovascular event risk. ${ }^{37}$ Interestingly, higher levels of PC(16:0/22:5) seemed to be protective for cardiovascular death in patients with CAD which is in line with our results. ${ }^{14} \mathrm{PC}(16: 0 / 22: 5)$ belongs to omega-3 polyunsaturated fatty acids (PUFA) and are abundantly present in fatty fish. They are suggested to reduce the risk of cardiac death potentially by lowering resting heart rate, blood pressure, plasma triglycerides and improving endothelial function. ${ }^{40}$ Previous studies 


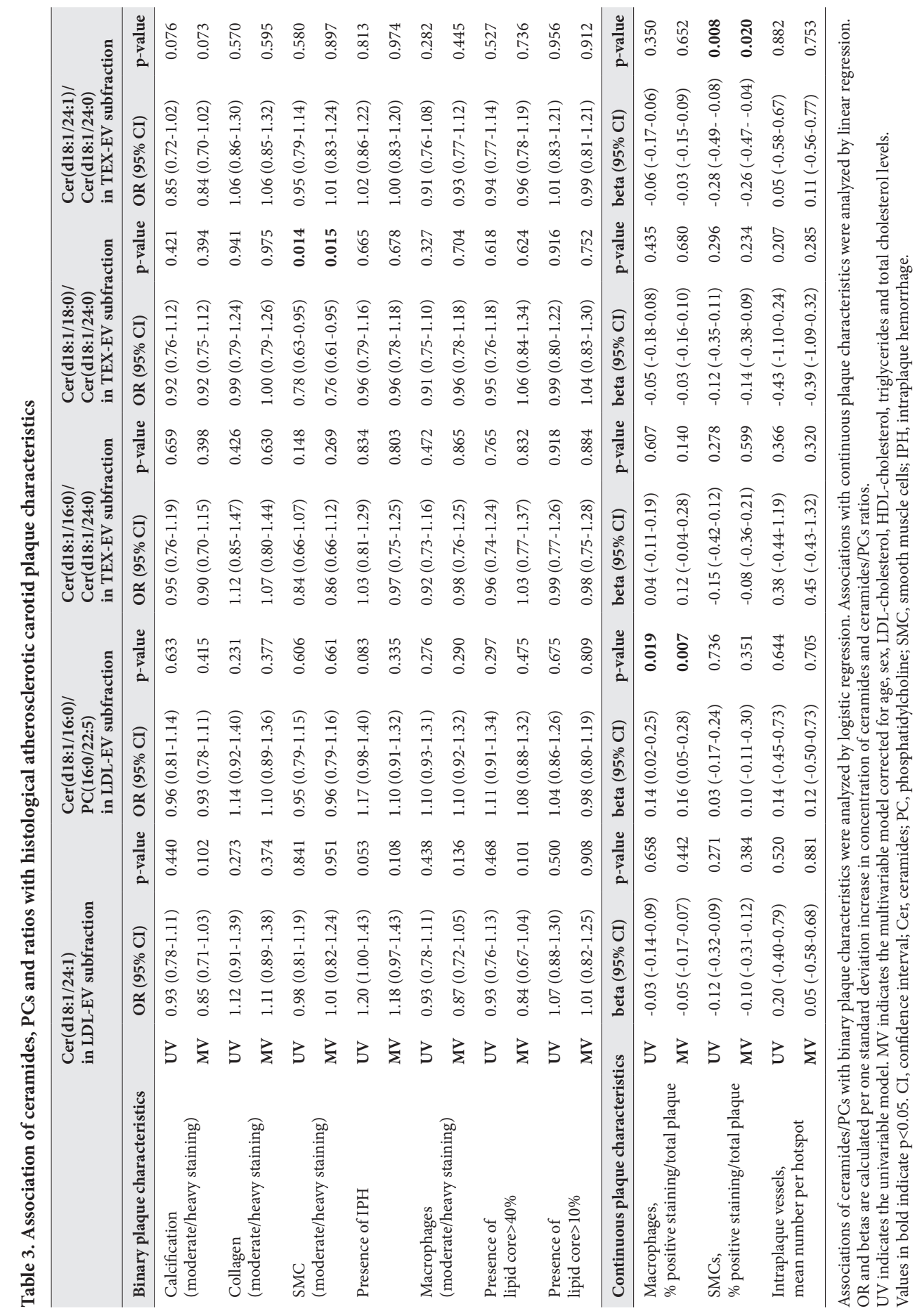


investigating omega-3 supplementation were inconsistent but two recent studies have shown a positive effect in reducing future $\mathrm{CV}$ event risk. ${ }^{38,39} \mathrm{An}$ interesting topic for future studies may be to examine whether ceramide/PC ratios could be useful in selecting patients that benefit most from dietary interventions or additional medical treatments in reducing cardiovascular risk.

Due to the observational study design, our findings cannot prove causality between ceramides/PCs and CV events. Based on evidence from experimental studies we could speculate, however, that these lipids contribute to atherosclerotic plaque vulnerability. We found that lipid ratios associated with MACE Cer(d18:1/24:1)/Cer(d18:1/24:0), $\operatorname{Cer}(\mathrm{d} 18: 1 / 18: 0) / \operatorname{Cer}(\mathrm{d} 18: 1 / 24: 0)$ and $\operatorname{Cer}(\mathrm{d} 18: 1 / 16: 0) / \mathrm{PC}(16: 0 / 22: 5)$ were also positively associated with a more vulnerable carotid atherosclerotic plaque phenotype suggesting putative underlying pathobiological mechanisms. Experimental studies have implied that ceramides drive main atherosclerotic processes such as LDL aggregation, LDL uptake across the endothelium, foam cell formation, production of reactive oxygen species, apoptosis, endothelial dysfunction and inflammatory processes. ${ }^{17}$ Studies on the relation of ceramides/ PCs and atherosclerotic plaque composition in patients are scarce. Two studies in CAD patients have revealed that increased levels of $\operatorname{Cer}(\mathrm{d} 18: 1 / 16: 0), \operatorname{Cer}(\mathrm{d} 18: 1 / 18: 0)$ and Cer(d18:1/24:1) were associated with higher coronary plaque vulnerability, characterized with a higher lipid volume, necrotic core and a thinner fibrous cap using intravascular ultrasound and optical coherence tomography. ${ }^{16,41}$ In our study, high-risk ceramides/PCs ratios were also associated with a more vulnerable atherosclerotic plaque composed of fewer SMCs and more macrophage infiltration. Experimental data has shown that ceramides induce the activity of matrix metalloproteinases (MMPs) and proinflammatory cytokines. ${ }^{17}$ In vitro, ceramides induced apoptosis of vascular smooth muscle cells (VSMCs). ${ }^{42}$ It is known that increase of MMPs and loss of VSMCs contributes to thinning of the fibrous cap and subsequent plaque disruption. Additionally, pharmacological inhibition of ceramide synthetic pathway reduced macrophage content and increased SMCs content in mice. ${ }^{43}$ Our results further contribute to evidence of the potential role of ceramides in plaque instability.

Another possible explanation for the association between EV-derived ceramides and MACE may be that high levels of ceramides reflect a general pathological state of the endothelium. The endothelium is an important source of plasma ceramides and are implicated in NO mediated vasodilatation. ${ }^{44}$ Moreover, it has been shown that the endothelium also generates EVs and, when activated by TNF $\alpha$, EVs are generated with increased ceramide content. ${ }^{45}$ Exact underlying mechanisms how EV-derived ceramides/PCs relate to increased risk of $\mathrm{CV}$ events remains to be elucidated. As the biological function of distinct ceramides and 
PCs in atherogenesis is still poorly understood, future studies should determine whether ceramides and PCs are real effectors or solely markers of progression of atherosclerosis and subsequent CV events. Understanding the role of ceramides/PCs and related synthesizing or degrading biosynthetic enzymes in atherosclerotic diseases may lead to new potential therapeutic targets. ${ }^{46}$

Reported correlations of ceramides/PCs in plasma EVs with plaque characteristics in our study do not establish the origin of EVs. Determination of the cellular origin of EVs is challenging since expression of cellular surface markers on EVs are not specific to the parent cell types. ${ }^{47}$ It is known that EVs can be released by almost all cell types such as endothelial cells, cardiomyocytes, platelets, red- or white blood cells. ${ }^{19}$ The role of EVs in atherothrombotic processes has been well acknowledged, although exact mechanisms in vivo are unclear. ${ }^{19}$ Future studies should explore the cellular origin of EVs because this would provide more insight in the origin of high-risk lipids and underlying processes related to atherosclerotic events.

Potential study limitations need to be addressed. First, although previous CAD cohorts found stronger associations of ceramide ratios with CV death compared to MACE ${ }^{10,11,14,16}$, we were not able to perform analyses on separate outcomes due to a limited number of events. Second, during follow-up no data regarding dietary patterns, medication use or adherence were available. This may have modified the observed associations between molecular lipids and MACE. Last, subgroup analyses in patients undergoing CEA for asymptomatic carotid stenosis could not be performed due to low patient numbers. Ceramides and PCs may be relevant for risk stratification in this particular subgroup in light of the ongoing debate whether or not to perform CEA, but this should be further explored in appropriately designed future studies. Major strengths include that this is the first study investigating ceramides and PCs in CEA patients and investigating these lipids in both plasma EVs and plasma. Another strength is the prospective design with validated $\mathrm{CV}$ outcomes. Due to the unique design of the Athero-Express biobank we were able to concomitantly investigate $\mathrm{CV}$ outcomes and histological atherosclerotic plaque characteristics to generate hypotheses regarding possible biological mechanisms.

To conclude, increased levels of ceramide and PCs ratios in plasma EVs, but not in unfractionated plasma, are independently associated with increased risk of MACE after $\mathrm{CEA}$. These EV-derived ceramide- and ceramide/PC ratio are therefore potential biomarkers for MACE. Ceramides/PCs ratios in EVs may be useful biomarkers for selecting high-risk patients in need for intensified secondary preventive therapy, such as add-on therapy. 


\section{Acknowledgements}

We thank Qiu Ying van de Pol, Sara van Laar and Evelyn Velema for their technical support in the Athero-Express Biobank.

\section{Funding}

This work was supported by the European Union [EU 755320 Taxinomisis grant to Dr. De Borst, dr. De Kleijn, dr. Pasterkamp and dr. Laaksonen]; and the Dutch Heart Foundation [CVON 2017-05 pERSUASIVE to Dr. de Kleijn and dr. De Winter]. There are no competing interests declared.

\section{Conflict of interest}

None. 


\section{References}

1 Hellings WE, Peeters W, Moll FL, Piers SRD, van Setten J, Van der Spek PJ, et al. Composition of Carotid Atherosclerotic Plaque Is Associated With Cardiovascular Outcome. Circulation 2010;121(17):1941-50.

2 Mach F, Baigent C, Catapano AL, Koskinas KC, Casula M, Badimon L, et al. 2019 ESC/EAS Guidelines for the management of dyslipidaemias: lipid modification to reduce cardiovascular risk. Eur Heart $\mathrm{J}$ 2020;41(1):111-88.

3 Tardif J-C, Kouz S, Waters DD, Bertrand OF, Diaz R, Maggioni AP, et al. Efficacy and Safety of Low-Dose Colchicine after Myocardial Infarction. N Engl J Med 2019;381(26):2497-505.

4 Ridker PM, Everett BM, Thuren T, MacFadyen JG, Chang WH, Ballantyne C, et al. Antiinflammatory Therapy with Canakinumab for Atherosclerotic Disease. N Engl J Med 2017;377(12):1119-31.

5 Nidorf SM, Fiolet ATL, Mosterd A, Eikelboom JW, Schut A, Opstal TSJ, et al. Colchicine in Patients with Chronic Coronary Disease. N Engl J Med 2020;383(19):1838-47.

6 Anand SS, Eikelboom JW, Dyal L, Bosch J, Neumann C, Widimsky P, et al. Rivaroxaban Plus Aspirin Versus Aspirin in Relation to Vascular Risk in the COMPASS Trial. J Am Coll Cardiol 2019;73(25):3271-80.

7 Bonaca MP, Bauersachs RM, Anand SS, Debus ES, Nehler MR, Patel MR, et al. Rivaroxaban in Peripheral Artery Disease after Revascularization. N Engl J Med 2020;382(21):1994-2004.

8 Kaasenbrood L, Boekholdt SM, van der Graaf Y, Ray KK, Peters RJG, Kastelein JJP, et al. Distribution of Estimated 10-Year Risk of Recurrent Vascular Events and Residual Risk in a Secondary Prevention Population. Circulation 2016;134(19):1419-29.

9 Volkers EJ, Algra A, Kappelle LJ, Greving JP. Prediction models for clinical outcome after a carotid revascularisation procedure: A systematic review. Eur Stroke J 2018;3(1):57-65.

10 Laaksonen R, Ekroos K, Sysi-Aho M, Hilvo M, Vihervaara T, Kauhanen D, et al. Plasma ceramides predict cardiovascular death in patients with stable coronary artery disease and acute coronary syndromes beyond LDL-cholesterol. Eur Heart J 2016;37(25):1967-76.

11 Hilvo M, Meikle PJ, Pedersen ER, Tell GS, Dhar I, Brenner H, et al. Development and validation of a ceramide- and phospholipid-based cardiovascular risk estimation score for coronary artery disease patients. Eur Heart J 2019;41(3):371-80.

12 Mundra PA, Barlow CK, Nestel PJ, Barnes EH, Kirby A, Thompson P, et al. Large-scale plasma lipidomic profiling identifies lipids that predict cardiovascular events in secondary prevention. JCI Insight 2018;3(17).

13 Tarasov K, Ekroos K, Suoniemi M, Kauhanen D, Sylvänne T, Hurme R, et al. Molecular Lipids Identify Cardiovascular Risk and Are Efficiently Lowered by Simvastatin and PCSK9 Deficiency. J Clin Endocrinol Metab 2014;99(1):E45-52.

14 Sigruener A, Kleber ME, Heimerl S, Liebisch G, Schmitz G, Maerz W. Glycerophospholipid and Sphingolipid Species and Mortality: The Ludwigshafen Risk and Cardiovascular Health (LURIC) Study. PLoS One 2014;9(1):e85724.

15 Havulinna AS, Sysi-Aho M, Hilvo M, Kauhanen D, Hurme R, Ekroos K, et al. Circulating Ceramides Predict Cardiovascular Outcomes in the Population-Based FINRISK 2002 Cohort. Arterioscler Thromb Vasc Biol 2016;36(12):2424-30.

16 Cheng JM, Suoniemi M, Kardys I, Vihervaara T, de Boer SPM, Akkerhuis KM, et al. Plasma concentrations of molecular lipid species in relation to coronary plaque characteristics and cardiovascular outcome: Results of the ATHEROREMO-IVUS study. Atherosclerosis 2015;243(2):560-6.

17 Bismuth J, Lin P, Yao Q, Chen C. Ceramide: A common pathway for atherosclerosis? Atherosclerosis 2008;196(2):497-504.

18 Meikle PJ, Summers SA. Sphingolipids and phospholipids in insulin resistance and related metabolic disorders. Nat Rev Endocrinol 2017;13(2):79-91.

19 Boulanger CM, Loyer X, Rautou P-E, Amabile N. Extracellular vesicles in coronary artery disease. Nat Rev Cardiol 2017;14(5):259-72.

20 Dekker M, Waissi F, van Bennekom J, Silvis MJM, Timmerman N, Bank IEM, et al. Plasma extracellular vesicle proteins are associated with stress-induced myocardial ischemia in women presenting with chest pain. Sci Rep 2020;10(1):12257.

21 Kanhai DA, Visseren FLJ, van der Graaf Y, Schoneveld AH, Catanzariti LM, Timmers L, et al. Microvesicle protein levels are associated with increased risk for future vascular events and mortality in patients with clinically manifest vascular disease. Int J Cardiol 2013;168(3):2358-63. 
22 Verhoeven BAN, Velema E, Schoneveld AH, de Vries JPPM, de Bruin P, Seldenrijk CA, et al. Atheroexpress: differential atherosclerotic plaque expression of mRNA and protein in relation to cardiovascular events and patient characteristics. Rationale and design. Eur J Epidemiol 2004;19(12):1127-33.

23 Warlow C, Farrell B, Fraser A, Sandercock P SJ. Randomised trial of endarterectomy for recently symptomatic carotid stenosis: final results of the MRC European Carotid Surgery Trial (ECST). Lancet 1998;351(9113):1379-87.

24 North American Symptomatic Carotid Endarterectomy Trial Collaborators, Barnett HJM, Taylor DW, Haynes RB, Sackett DL, Peerless SJ, et al. Beneficial effect of carotid endarterectomy in symptomatic patients with high-grade carotid stenosis. N Engl J Med 1991;325(7):445-53.

25 Barnett HJ, Taylor DW, Eliasziw M, Fox AJ, Ferguson GG, Haynes RB, et al. Benefit of carotid endarterectomy in patients with symptomatic moderate or severe stenosis. North American Symptomatic Carotid Endarterectomy Trial Collaborators. N Engl J Med 1998;339(20):1415-25.

26 Halliday A, Harrison M, Hayter E, Kong X, Mansfield A, Marro J, et al. 10-year stroke prevention after successful carotid endarterectomy for asymptomatic stenosis (ACST-1): a multicentre randomised trial. Lancet 2010;376(9746):1074-84.

27 Halliday A, Mansfield A, Marro J, Peto C, Peto R, Potter J, et al. Prevention of disabling and fatal strokes by successful carotid endarterectomy in patients without recent neurological symptoms: randomised controlled trial. Lancet 2004;363(9420):1491-502.

28 Zhang Y-N, Vernooij F, Ibrahim I, Ooi S, Gijsberts CM, Schoneveld AH, et al. Extracellular Vesicle Proteins Associated with Systemic Vascular Events Correlate with Heart Failure: An Observational Study in a Dyspnoea Cohort. PLoS One 2016;11(1):e0148073.

29 Wang J-W, Zhang Y-N, Sze S, van de Weg S, Vernooij F, Schoneveld A, et al. Lowering Low-Density Lipoprotein Particles in Plasma Using Dextran Sulphate Co-Precipitates Procoagulant Extracellular Vesicles. Int J Mol Sci 2017;19(1):94.

30 Derksen WJM, Peeters W, van Lammeren GW, Tersteeg C, de Vries J-PPM, de Kleijn DPV, et al. Different stages of intraplaque hemorrhage are associated with different plaque phenotypes: A large histopathological study in 794 carotid and 276 femoral endarterectomy specimens. Atherosclerosis 2011;218(2):369-77.

31 van Lammeren GW, Catanzariti LM, Peelen LM, de Vries J-PPM, de Kleijn DPV, Moll FL, et al. Clinical Prediction Rule to Estimate the Absolute 3-Year Risk of Major Cardiovascular Events After Carotid Endarterectomy. Stroke 2012;43(5):1273-8.

32 Hilvo M, Simolin H, Metso J, Ruuth M, Öörni K, Jauhiainen M, et al. PCSK9 inhibition alters the lipidome of plasma and lipoprotein fractions. Atherosclerosis 2018;269:159-65.

33 Hellings WE, Pasterkamp G, Verhoeven BAN, De Kleijn DPV, De Vries J-PPM, Seldenrijk KA, et al. Gender-associated differences in plaque phenotype of patients undergoing carotid endarterectomy. J Vasc Surg 2007;45(2):289-96.

34 Petrache I, Kamocki K, Poirier C, Pewzner-Jung Y, Laviad EL, Schweitzer KS, et al. Ceramide Synthases Expression and Role of Ceramide Synthase-2 in the Lung: Insight from Human Lung Cells and Mouse Models. PLoS One 2013;8(5):e62968.

35 Raichur S, Wang ST, Chan PW, Li Y, Ching J, Chaurasia B, et al. CerS2 Haploinsufficiency Inhibits $\beta$-Oxidation and Confers Susceptibility to Diet-Induced Steatohepatitis and Insulin Resistance. Cell Metab 2014;20(4):687-95.

36 Warshauer JT, Lopez X, Gordillo R, Hicks J, Holland WL, Anuwe E, et al. Effect of pioglitazone on plasma ceramides in adults with metabolic syndrome. Diabetes Metab Res Rev 2015;31(7):734-44.

37 Wang DD, Toledo E, Hruby A, Rosner BA, Willett WC, Sun Q, et al. Plasma Ceramides, Mediterranean Diet, and Incident Cardiovascular Disease in the PREDIMED Trial (Prevención con Dieta Mediterránea). Circulation 2017;135(21):2028-40.

38 Bhatt DL, Steg PG, Miller M, Brinton EA, Jacobson TA, Ketchum SB, et al. Cardiovascular Risk Reduction with Icosapent Ethyl for Hypertriglyceridemia. N Engl J Med 2019;380(1):11-22.

39 Manson JE, Bassuk SS, Cook NR, Lee I-M, Mora S, Albert CM, et al. Vitamin D, Marine n-3 Fatty Acids, and Primary Prevention of Cardiovascular Disease Current Evidence. Circ Res 2020;126(1):112-28.

40 Mozaffarian D, Wu JHY. Omega-3 Fatty Acids and Cardiovascular Disease. J Am Coll Cardiol 2011;58(20):2047-67.

41 Pan W, Dong H, Sun R, Zhao L, Sun M, Li L, et al. Plasma Ceramides in Relation to Coronary Plaque Characterization Determined by Optical Coherence Tomography. J Cardiovasc Transl Res 2020. 
42 Edsfeldt A, Dunér P, Ståhlman M, Mollet IG, Asciutto G, Grufman H, et al. Sphingolipids Contribute to Human Atherosclerotic Plaque Inflammation. Arterioscler Thromb Vasc Biol 2016;36(6):1132-40.

43 Park T, Rosebury W, Kindt E, Kowala M, Panek R. Serine palmitoyltransferase inhibitor myriocin induces the regression of atherosclerotic plaques in hyperlipidemic ApoE-deficient mice. Pharmacol Res 2008;58(1):45-51.

44 Cantalupo A, Sasset L, Gargiulo A, Rubinelli L, Del Gaudio I, Benvenuto D, et al. Endothelial Sphingolipid De Novo Synthesis Controls Blood Pressure by Regulating Signal Transduction and NO via Ceramide. Hypertension 2020;75(5):1279-88.

45 Serban KA, Rezania S, Petrusca DN, Poirier C, Cao D, Justice MJ, et al. Structural and functional characterization of endothelial microparticles released by cigarette smoke. Sci Rep 2016;6.

46 Yu Z, Peng Q, Huang Y. Potential therapeutic targets for atherosclerosis in sphingolipid metabolism. Clin Sci 2019;133(6):763-76.

47 Yáñez-Mó M, Siljander PR-M, Andreu Z, Bedina Zavec A, Borràs FE, Buzas EI, et al. Biological properties of extracellular vesicles and their physiological functions. J Extracell Vesicles 2015;4(1):27066. 


\section{Supplemental Tables and Figures}

Supplemental Table S2. Percentages of missing covariates

\begin{tabular}{ll}
\hline & Missing (\%) \\
\hline Age & 0.0 \\
Male & 0.0 \\
Current smoking & 1.0 \\
LDL-C & 4.2 \\
HDL-C & 3.4 \\
History of CAD or PAD & 0.5 \\
Cerebrovascular symptoms & 0.0 \\
Lipid lowering drug use & 0.2 \\
\hline
\end{tabular}

$\mathrm{CAD}$, coronary artery disease; PAD, peripheral artery disease; LDL-C, Low-density lipoprotein cholesterol; HDL-C, high-density lipoprotein cholesterol.

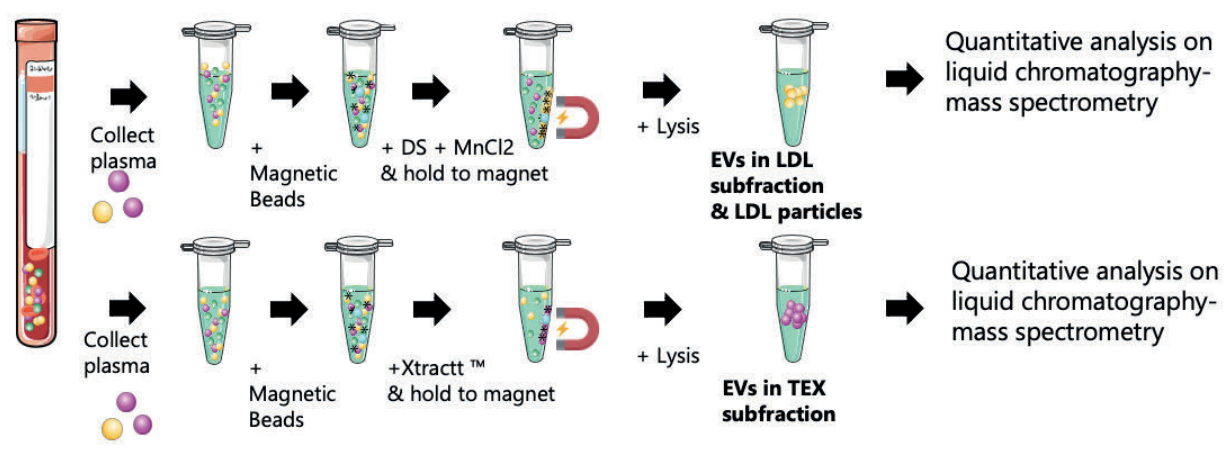

Supplemental Figure S1. Overview of the isolation procedure of plasma EVs. 
Supplemental Table S3. Median and interquartile ranges of ceramide/PC levels and the ratios in plasma and in the LDL- and TEX-EV subfractions

\begin{tabular}{|c|c|c|c|c|c|}
\hline Biomarker & & No MACE $(n=735)$ & MACE $(n=138)$ & p-value & Missing (\%) \\
\hline \multirow[t]{3}{*}{ Cer(d18:1/16:0) } & plasma & $0.200[0.168,0.238]$ & $0.198[0.170,0.238]$ & 0.902 & 5.5 \\
\hline & LDL-EV & $0.012[0.010,0.014]$ & $0.013[0.010,0.015]$ & 0.125 & 5.5 \\
\hline & TEX-EV & $0.009[0.007,0.012]$ & $0.009[0.007,0.011]$ & 0.523 & 1.8 \\
\hline \multirow[t]{3}{*}{ Cer(d18:1/18:0) } & plasma & $0.075[0.060,0.094]$ & $0.076[0.059,0.099]$ & 0.746 & 5.7 \\
\hline & LDL-EV & $0.004[0.003,0.005]$ & $0.004[0.003,0.005]$ & 0.953 & 5.7 \\
\hline & TEX-EV & $0.003[0.002,0.005]$ & $0.003[0.002,0.005]$ & 0.560 & 2.5 \\
\hline \multirow[t]{3}{*}{ Cer(d18:1/24:0) } & plasma & $2.208[1.736,2.683]$ & $2.071[1.616,2.522]$ & 0.025 & 5.2 \\
\hline & LDL-EV & $0.073[0.060,0.091]$ & $0.070[0.059,0.085]$ & 0.252 & 4.9 \\
\hline & TEX-EV & $0.115[0.086,0.157]$ & $0.096[0.073,0.134]$ & $<0.001$ & 3.1 \\
\hline \multirow[t]{3}{*}{ Cer(d18:1/24:1) } & plasma & $0.891[0.755,1.054]$ & $0.887[0.754,1.083]$ & 0.911 & 5.5 \\
\hline & LDL-EV & $0.043[0.035,0.053]$ & $0.046[0.039,0.058]$ & 0.056 & 5.7 \\
\hline & TEX-EV & $0.044[0.033,0.057]$ & $0.044[0.031,0.059]$ & 0.713 & 2.9 \\
\hline \multirow[t]{3}{*}{$\mathrm{PC}(14: 0 / 22: 6)$} & plasma & $0.411[0.285,0.565]$ & $0.341[0.272,0.488]$ & 0.021 & 5.4 \\
\hline & LDL-EV & $0.034[0.023,0.048]$ & $0.029[0.020,0.043]$ & 0.047 & 5.5 \\
\hline & TEX-EV & $0.010[0.006,0.017]$ & $0.008[0.004,0.015]$ & 0.033 & 8.7 \\
\hline \multirow[t]{3}{*}{$\mathrm{PC}(16: 0 / 16: 0)$} & Plasma & $9.073[7.590,11.04]$ & $9.295[7.630,11.08]$ & 0.914 & 5.2 \\
\hline & LDL-EV & $7.902[6.913,9.197]$ & $7.984[6.955,9.280]$ & 0.564 & 5.3 \\
\hline & TEX-EV & $24.66[21.54,27.80]$ & $24.39[20.84,27.02]$ & 0.140 & 2.1 \\
\hline \multirow[t]{3}{*}{$\mathrm{PC}(16: 0 / 22: 5)$} & plasma & $31.86[26.14,39.14]$ & $29.36[23.93,38.37]$ & 0.018 & 5.0 \\
\hline & LDL-EV & $2.706[2.090,3.374]$ & $2.520[1.998,3.269]$ & 0.174 & 5.2 \\
\hline & TEX-EV & $0.933[0.690,1.260]$ & $0.863[0.627,1.119]$ & 0.010 & 3.4 \\
\hline \multirow{3}{*}{$\begin{array}{l}\text { Cer(d18:1/16:0)/ } \\
\text { Cer(d18:1/24:0) }\end{array}$} & plasma & $0.091[0.078,0.107]$ & $0.094[0.079,0.111]$ & 0.081 & 5.5 \\
\hline & LDL-EV & $0.162[0.133,0.196]$ & $0.170[0.136,0.203]$ & 0.147 & 5.8 \\
\hline & TEX-EV & $0.075[0.062,0.091]$ & $0.084[0.067,0.107]$ & $<0.001$ & 2.4 \\
\hline \multirow{3}{*}{$\begin{array}{l}\text { Cer(d18:1/18:0)/ } \\
\text { Cer(d18:1/24:0) }\end{array}$} & plasma & $0.034[0.028,0.043]$ & $0.037[0.028,0.046]$ & 0.120 & 5.7 \\
\hline & LDL-EV & $0.052[0.040,0.067]$ & $0.054[0.039,0.070]$ & 0.737 & 5.6 \\
\hline & TEX-EV & $0.028[0.021,0.037]$ & $0.032[0.022,0.046]$ & 0.022 & 2.7 \\
\hline \multirow{3}{*}{$\begin{array}{l}\text { Cer(d18:1/24:1)/ } \\
\text { Cer(d18:1/24:0) }\end{array}$} & plasma & $0.410[0.339,0.490]$ & $0.420[0.365,0.518]$ & 0.070 & 5.4 \\
\hline & LDL-EV & $0.589[0.480,0.722]$ & $0.646[0.511,0.749]$ & 0.049 & 5.2 \\
\hline & TEX-EV & $0.379[0.303,0.463]$ & $0.421[0.330,0.524]$ & $<0.001$ & 2.3 \\
\hline \multirow{3}{*}{$\begin{array}{l}\text { Cer(d18:1/18:0)/ } \\
\text { Cer(d18:1/16:0) }\end{array}$} & plasma & $0.377[0.322,0.449]$ & $0.372[0.321,0.458]$ & 0.973 & 5.3 \\
\hline & LDL-EV & $0.323[0.267,0.390]$ & $0.300[0.252,0.388]$ & 0.345 & 5.2 \\
\hline & TEX-EV & $0.381[0.298,0.472]$ & $0.379[0.291,0.476]$ & 0.845 & 1.7 \\
\hline \multirow{3}{*}{$\begin{array}{l}\text { Cer(d18:1/16:0)/ } \\
\operatorname{PC}(16: 0 / 22: 5)\end{array}$} & plasma & $0.006[0.005,0.008]$ & $0.007[0.006,0.008]$ & 0.012 & 5.6 \\
\hline & LDL-EV & $0.005[0.004,0.005]$ & $0.005[0.004,0.006]$ & 0.002 & 5.5 \\
\hline & TEX-EV & $0.010[0.007,0.013]$ & $0.011[0.008,0.015]$ & 0.005 & 1.8 \\
\hline
\end{tabular}




\section{Supplemental Table S3. Continued}

\begin{tabular}{llllll}
\hline Biomarker & & No MACE $(\mathbf{n}=735)$ & MACE $(\mathbf{n}=138)$ & p-value & Missing $(\%)$ \\
\hline Cer(d18:1/18:0)/ & plasma & $0.182[0.122,0.271]$ & $0.205[0.135,0.336]$ & $\mathbf{0 . 0 3 5}$ & 5.8 \\
PC(14:0/22:6) & LDL-EV & $0.111[0.078,0.168]$ & $0.124[0.080,0.205]$ & 0.116 & 6.1 \\
& TEX-EV & $0.329[0.190,0.595]$ & $0.386[0.207,0.716]$ & 0.193 & 8.5 \\
\hline
\end{tabular}

Values are displayed as median [interquartile] concentrations in $\mu \mathrm{M}$. $P$-values originate from comparison of median levels by Mann-Whitney $U$ test between patients that experienced MACE versus those who did not. Values in bold indicate $\mathrm{p}<0.05$. Missing measurements of ceramides/PCs were randomly divided across all samples and were due to technical failures. Cer, ceramide; PC, phosphatidylcholine. LDL-EV indicates the LDL-EV subfraction, TEX-EV indicates the TEX-EV subfraction.

Supplemental Table S4. Subanalyses corrected for statin use

\begin{tabular}{|c|c|c|c|}
\hline Biomarker & & HR (95\% CI) & p-value \\
\hline \multirow[t]{3}{*}{$\operatorname{Cer}(\mathrm{d} 18: 1 / 16: 0)$} & plasma & $0.98(0.77-1.23)$ & 0.836 \\
\hline & LDL-EV & $1.22(0.98-1.51)$ & 0.069 \\
\hline & TEX-EV & $0.94(0.56-1.57)$ & 0.804 \\
\hline \multirow[t]{3}{*}{ Cer(d18:1/18:0) } & plasma & $1.00(0.80-1.24)$ & 0.985 \\
\hline & LDL-EV & $1.06(0.86-1.30)$ & 0.583 \\
\hline & TEX-EV & $0.93(0.68-1.26)$ & 0.618 \\
\hline \multirow[t]{3}{*}{ Cer(d18:1/24:0) } & plasma & $0.86(0.68-1.07)$ & 0.178 \\
\hline & LDL-EV & $1.05(0.86-1.28)$ & 0.648 \\
\hline & TEX-EV & $0.73(0.53-1.02)$ & 0.063 \\
\hline \multirow[t]{3}{*}{ Cer(d18:1/24:1) } & plasma & $0.94(0.76-1.18)$ & 0.607 \\
\hline & LDL-EV & $1.23(1.00-1.52)$ & 0.046 \\
\hline & TEX-EV & $0.92(0.69-1.23)$ & 0.588 \\
\hline \multirow[t]{3}{*}{$\mathrm{PC}(14: 0 / 22: 6)$} & plasma & $0.88(0.70-1.10)$ & 0.263 \\
\hline & LDL-EV & $0.94(0.76-1.16)$ & 0.557 \\
\hline & TEX-EV & $0.89(0.71-1.12)$ & 0.326 \\
\hline \multirow[t]{3}{*}{$\mathrm{PC}(16: 0 / 16: 0)$} & plasma & $1.09(0.86-1.37)$ & 0.481 \\
\hline & LDL-EV & $1.17(0.94-1.46)$ & 0.149 \\
\hline & TEX-EV & $0.94(0.76-1.15)$ & 0.540 \\
\hline \multirow[t]{3}{*}{$\operatorname{PC}(16: 0 / 22: 5)$} & plasma & $0.94(0.76-1.15)$ & 0.532 \\
\hline & LDL-EV & $0.98(0.80-1.20)$ & 0.867 \\
\hline & TEX-EV & $0.85(0.67-1.07)$ & 0.166 \\
\hline \multirow[t]{3}{*}{$\operatorname{Cer}(\mathrm{d} 18: 1 / 16: 0) / \operatorname{Cer}(\mathrm{d} 18: 1 / 24: 0)$} & plasma & $1.09(0.86-1.37)$ & 0.485 \\
\hline & LDL-EV & $1.06(0.85-1.33)$ & 0.592 \\
\hline & TEX-EV & $1.31(1.03-1.67)$ & 0.026 \\
\hline
\end{tabular}


PART III | Chapter 9

Supplemental Table S3. Continued

\begin{tabular}{llll}
\hline Biomarker & & HR (95\% CI) & p-value \\
\hline Cer(d18:1/18:0)/Cer(d18:1/24:0) & plasma & $1.08(0.87-1.34)$ & 0.470 \\
& LDL-EV & $0.90(0.72-1.12)$ & 0.349 \\
& TEX-EV & $1.22(1.00-1.50)$ & 0.052 \\
\hline Cer(d18:1/24:1)/Cer(d18:1/24:0) & plasma & $1.07(0.86-1.32)$ & 0.558 \\
& LDL-EV & $1.06(0.86-1.30)$ & 0.616 \\
& TEX-EV & $1.29(1.07-1.56)$ & $\mathbf{0 . 0 0 8}$ \\
\hline Cer(d18:1/18:0)/Cer(d18:1/16:0) & plasma & $0.96(0.79-1.16)$ & 0.657 \\
& LDL-EV & $0.90(0.74-1.09)$ & 0.269 \\
& TEX-EV & $0.93(0.78-1.11)$ & 0.427 \\
\hline Cer(d18:1/16:0)/PC(16:0/22:5) & plasma & $1.20(0.97-1.49)$ & 0.086 \\
& LDL-EV & $1.24(1.03-1.50)$ & $\mathbf{0 . 0 2 5}$ \\
& TEX-EV & $1.25(0.88-1.77)$ & 0.220 \\
\hline Cer(d18:1/18:0)/PC(14:0/22:6) & plasma & $1.10(0.86-1.41)$ & 0.453 \\
& LDL-EV & $1.86(0.35-9.99)$ & 0.467 \\
\hline
\end{tabular}

Multivariable analyses were corrected for statin use, LDL-cholesterol and HDL-cholesterol, age, history of coronary artery disease, history of peripheral artery disease, cerebrovascular symptoms and current smoking.

HR indicates the hazard ratio for the 3-year postoperative risk of MACE per one standard deviation increase in concentration of ceramides, PC or ratio either in plasma or plasma extracellular vesicles (EVs). Values in bold indicate $\mathrm{p}<0.05$. CI, confidence interval. Cer, ceramides; PC, phosphatidylcholine. LDL-EV indicates LDL-EV subfraction, TEX-EV indicates TEX-EV subfraction 


\section{Supplementary Methods}

The following supplemental materials were omitted due to space limitations and can be requested from the authors:

- Characterization of plasma extracellular vesicles subfractions including Figure S2, Figure S3 and Table S1.

- Quantification of ceramides and PCs concentrations by LC-MS/MS analysis

- Histological atherosclerotic plaque characterization 



\section{PART IV}

\section{Risk Stratification Tools}

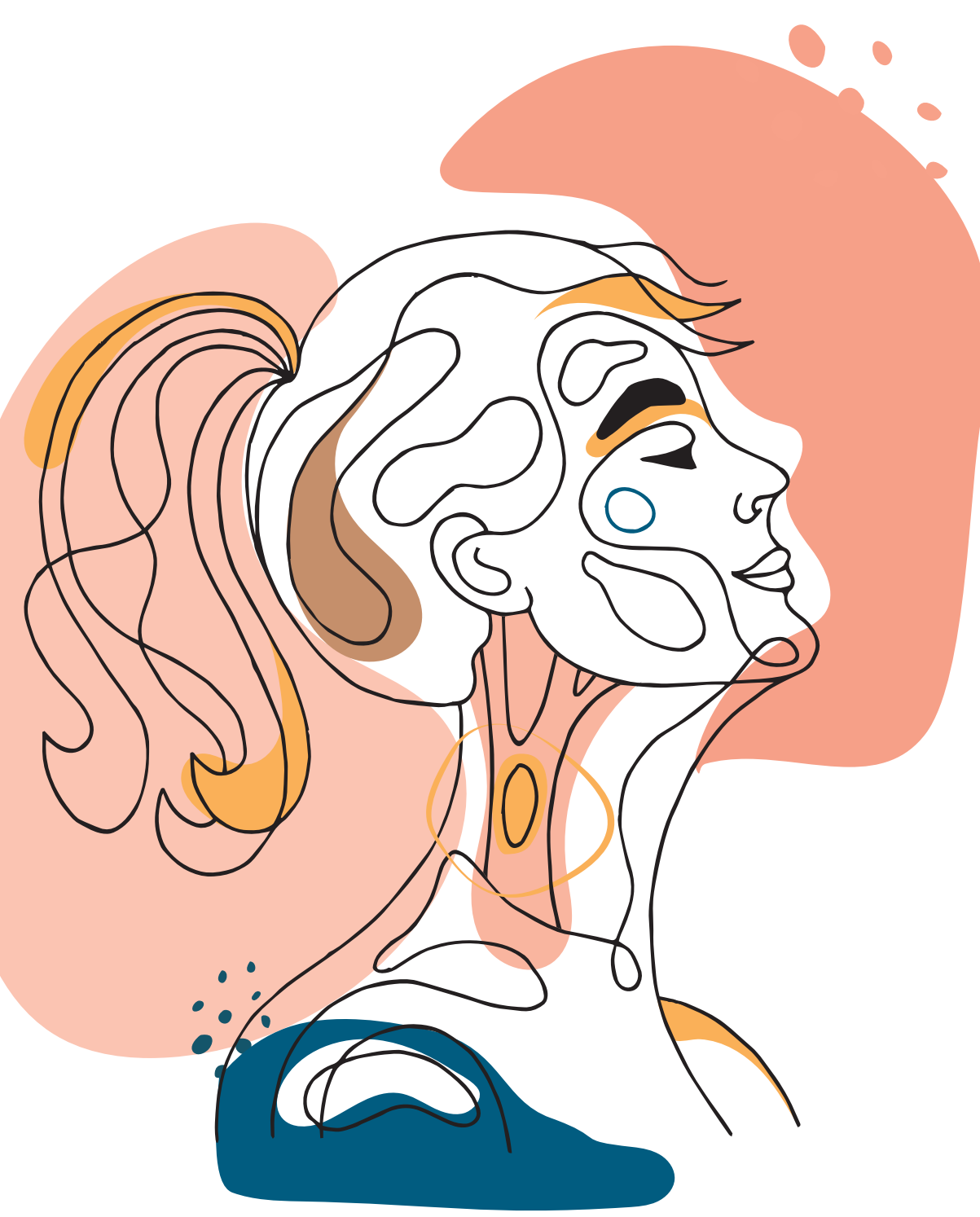




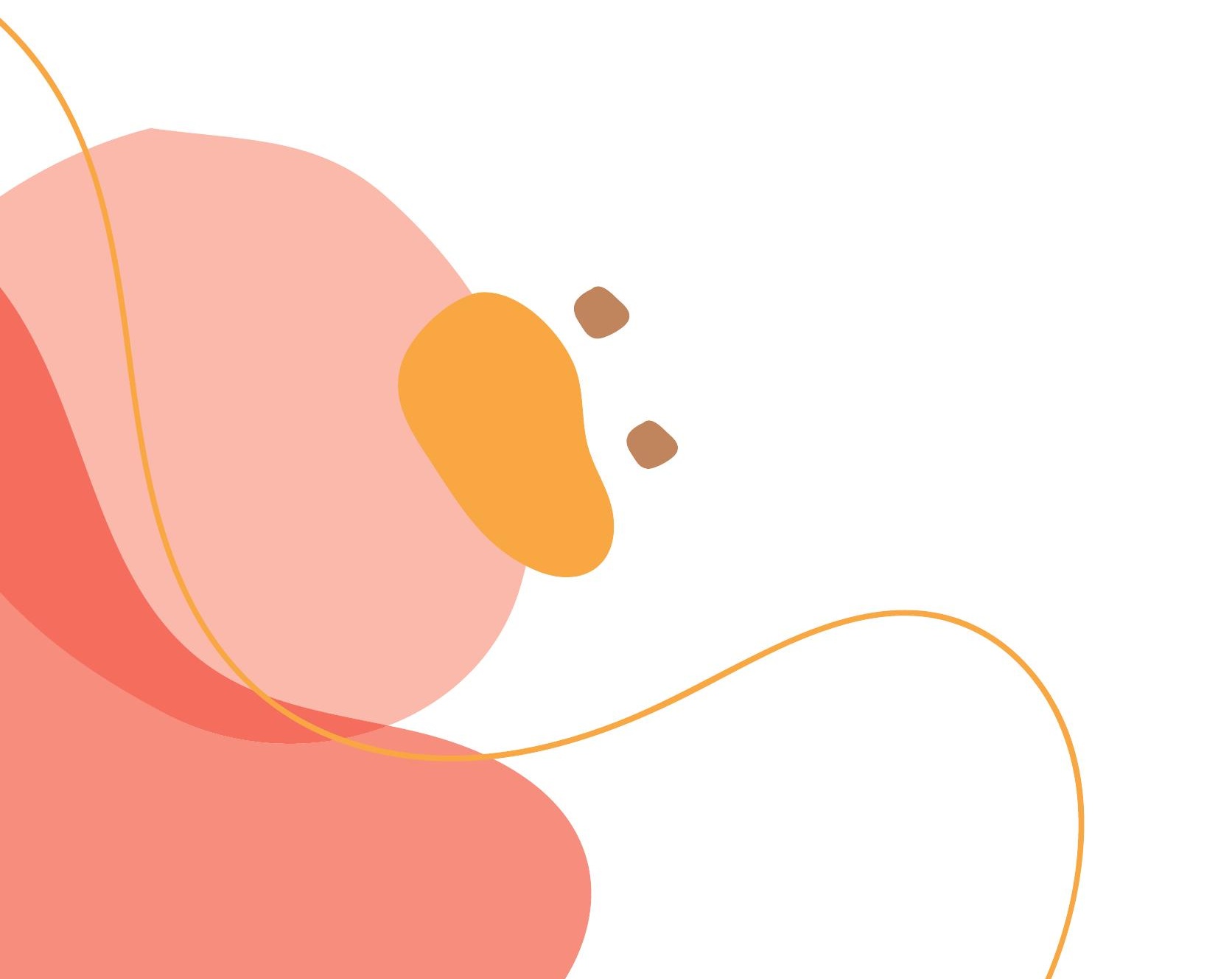




\section{Combined plasma extracellular vesicle biomarkers improve risk stratification for major adverse cardiovascular events after carotid endarterectomy}

Manuscript in preparation

Nathalie Timmerman* Farahnaz Waissi* Mirthe Dekker Jeffrey Kroon

Gert .J. de Borst Dominique P.V. de Kleijn 


\section{Abstract}

\section{Background}

Patients with carotid artery stenosis treated with carotid endarterectomy (CEA) remain at considerable residual risk for future cardiovascular (CV) events. With the emerging novel therapeutic options for secondary prevention in CV disease, accurate risk estimation tools identifying high-risk patients for secondary CV events are of major interest. Current clinical risk stratification tools for CEA patients have poor predictive value. Biomarkers could aid in optimizing risk stratification, especially when combining multiple biomarkers, using a multimarker approach. We recently reported different circulating markers in both plasma and extracellular vesicles (EVs) showing independent associations with postoperative CV events. In this study, we aimed to investigate whether a combined circulating biomarker strategy could improve the prediction of the 3-year risk of major adverse cardiovascular events (MACE) after CEA beyond predictions obtained from traditional CV risk factors only.

\section{Methods}

Measurements of 11 selected biomarkers in preoperative plasma were available in 847 patients undergoing CEA included between 2002-2016 in the Athero-Express Biobank. MACE was composed of myocardial infarction (MI), ischemic or hemorrhagic stroke and cardiovascular death. The prognostic model including biomarkers and established CV risk factors (age, creatinine, current smoking, history of coronary artery disease/ peripheral arterial occlusive disease and preprocedural cerebrovascular symptoms) was built using Cox proportional hazards regression with backward stepwise elimination. The model was internally validated with bootstrapping and model performance was assessed in terms of calibration and discrimination compared to a model containing the established CV risk factors only.

\section{Results}

A total of 133 MACE occurred during three years of follow-up. The final biomarker model consisted of the CV risk factors and two biomarkers: the EV-derived ceramide ratio Cer(d18:1/24:1)/Cer(d18:1/24:0) and EV-derived protein CD14. Combining the CV risk factors with our biomarker panel showed improved prediction. Discrimination in terms of C-statistic were 0.68 (95\% CI 0.63-0.73) for the biomarker model compared to 0.66 (95\% CI 0.61-0.71) for CV risk factors alone ( $\mathrm{p}=0.07$ for comparison). Addition of EVCer(d18:1/24:1)/Cer(d18:1/24:0) and EV-CD14 had additional prognostic value over CV risk factors indicated by an overall Net Reclassification Improvement of 0.147 (0.038$0.257)$. 


\section{Conclusion}

Addition of EV-derived ceramides and EV-derived CD14 on top of CV risk factors has superior predictive performance for future MACE in CEA patients. After external validation, this new prediction model could be used to optimize risk-stratification for management of individualized secondary prevention therapies.

\section{Introduction}

With the emerging novel therapeutic options for secondary cardiovascular (CV) risk reduction, identifying patients at high risk for secondary $\mathrm{CV}$ events after carotid endarterectomy (CEA) is becoming increasingly important. ${ }^{1}$ In the past few years, secondary prevention strategies have continued to evolve and guidelines recommend intensified management of risk factors in patients at high risk for secondary CV events, for example with "add-on therapy" such as proprotein convertase subtilisin/kexin type 9 (PCSK-9) inhibitors. ${ }^{2}$ Other options for add-on therapy in the future may be antiinflammatory medications (such as low-dose colchicine) or adding a direct oral anticoagulant (DOAC) next to aspirin..$^{3-7}$ Accurate identification of individuals at high risk for secondary events will lead to more risk reduction by timely intensification of medical treatment with these add-on therapies. However, add-on therapy is often expensive and has potentially hazardous side effects. Risk prediction models to estimate the probability of developing secondary CV events after CEA in individual patients are thus very important to guide and improve personalized treatment regiments. Previously, multiple studies attempted to develop clinical prediction models for prediction of secondary CV events after CEA. ${ }^{1,8}$ These models contain classical risk factors that have been established to contribute to secondary manifestations of atherosclerotic disease. ${ }^{9}$ However, due to high prevalence of these traditional CV risk factors among vascular patients, clinical models have low discriminative capability to identify patients at high risk for secondary CV events. This underpins the importance of improving risk prediction on top of the established CV risk factors in the individual patients.

Studies on biomarkers have emerged in attempt to improve these risk estimations. We recently identified elevated plasma lipoprotein(a) $[\operatorname{Lp}(\mathrm{a})]$ levels, a well-known risk factor and causal factor for primary cardiovascular disease $\mathrm{e}^{10-12}$, as an additional risk factor for secondary CV events in CEA patients. ${ }^{13}$ Another field with high potential are plasma extracellular vesicles (EVs) and their content. ${ }^{14-19}$ Now that EVs have proven useful as prognostic or diagnostic markers in the field of cancer $^{20}$, the era for EVs as biomarker source in the cardiovascular field has come. ${ }^{21} \mathrm{EVs}$ are nanometer-sized lipid-bilayer 
membrane vesicles shed by cells in the extracellular space under physiological and pathophysiological conditions. ${ }^{21}$ They contain proteins, lipids and nucleic acid content of their parent cells and are therefore considered as liquid-based biopsies. Recent studies within the Athero-Express Biobank showed that preoperative levels of inflammatory and coagulation proteins (CD14, Cystatin C, Serpin F2 and Serpin C1), the extracellular matrix glycoprotein Tenascin-C and lipids (ceramides, ceramide-ceramide ratios, ceramidephosphatidylcholine ratios) in plasma EVs were strongly associated with future postoperative risk of MACE following CEA. ${ }^{\mathrm{a}-\mathrm{c}}$

Although previous studies in CEA patients focused on the added predictive value of a sole biomarker, capturing the complex nature of atherosclerosis in one biomarker may seem a fallacy. Different biomarkers represent distinct biological pathways, thus may offer complementary information in risk estimation. Such synergistic effects of biomarkers in predicting cardiovascular outcome have been demonstrated in patients with coronary artery disease. ${ }^{22-25}$ Until now, there are no studies reported that evaluated a multiple biomarker strategy for predicting future MACE after CEA. Therefore, in the current study we aimed to investigate whether a combination of previously established circulating biomarkers could improve the prediction of the 3 -year risk of major adverse cardiovascular events (MACE) after CEA even further.

\section{Methods}

\section{Study population}

Biomarker data used for the current study were derived from four observational studies performed in CEA patients within the Athero-Express Biobank. ${ }^{13, a-c}$ All study participants provided written informed consent. The studies were conducted in accordance with the declaration of Helsinki and were approved by the local medical ethics board. Study design of the Athero-Express Biobank and the previous studies are described in the Supplementary Methods section.

\section{Candidate predictors}

Based on available literature ${ }^{1,8,13, \mathrm{a}-\mathrm{c}}$ we identified the following potential clinical predictors: age, current smoking, creatinine levels, diabetes, systolic blood pressure, use of antihypertensive drugs, history of coronary artery disease (CAD) and/or history of peripheral artery occlusive disease (PAOD), preprocedural cerebrovascular symptoms and presence of contralateral carotid stenosis. Based on four previous Athero-Express studies ${ }^{13, a-c}$ the following biomarkers were selected as candidates to study their added predictive value 
for MACE: a selection of ceramides, phosphatidylcholines (PCs) and their ratios in EVs (Cer(d18:1/24:1), Cer(d18:1/16:0)/PC(16:0/22:5), Cer(d18:1/16:0)/Cer(d18:1/24:0), $\operatorname{Cer}(\mathrm{d} 18: 1 / 18: 0) / \operatorname{Cer}(\mathrm{d} 18: 1 / 24: 0)$, Cer(d18:1/24:1)/Cer(d18:1/24:0)) ${ }^{\mathrm{a}}$; the proteins CD14, Cystatin C, Serpin F2 and Serpin $\mathrm{C}^{\mathrm{b}}$ in EVs; extracellular matrix glycoprotein Tenascin-C $\mathrm{C}^{\mathrm{c}}$ in EVs; and Lipoprotein(a) in unfractionated plasma ${ }^{13}$.

\section{Quantification of candidate biomarkers}

Supplemental Table S1 gives an overview of the sample matrix in which the biomarkers were measured. For the biomarkers measured in EVs, three subsets of EVs were isolated from plasma: the LDL-EV subfraction, the HDL-EV subfraction and the TEX-EV subfraction. Levels of ceramides $(\mu \mathrm{M})$ and PCs $(\mu \mathrm{M})$ were quantified in the LDL-EV subfraction and TEX-EV subfraction using liquid chromatography-mass spectrometry (SciexTripleQuad 5500 mass spectrometer coupled to Sciex MPX LC system). The protein levels in the HDL-EV subfraction and Tenascin-C levels in the LDL-EV subfraction were measured by electrochemiluminescence immunoassay (Quickplex SQ120, Meso Scale Discovery, MSD) and concentrations were expressed in $\mathrm{pg} / \mathrm{mL}$. Lp(a) concentrations were measured with a latex-enhanced particle immunoturbidimetric assay in serum samples using the Cobas c702 (Roche) and the LPA2 Tinaquant Lp(a) Gen.2 kit from Cobas (LPA2: CAN 8723). A detailed description of the isolation of EV subsets and quantification of the biomarkers is included in the Supplemental Methods section.

\section{Study outcome}

The primary outcome was defined as the three-year postoperative risk of MACE. MACE was composed of nonfatal or fatal myocardial infarction (MI), nonfatal or fatal ischemic or hemorrhagic stroke and cardiovascular death due to other causes (fatal heart failure, fatal abdominal aneurysm rupture and sudden cardiac death). Endpoints were adjudicated by two independent researchers; endpoint criteria have been described previously. ${ }^{26}$ In case of disagreement a third expert (GJdB) was consulted.

\section{Statistical analysis}

All analyses were performed with R version 3.6.2 (R Foundation for Statistical Computing, Vienna, Austria; https://www.r-project.org/). All statistical tests were two-sided, with a value $<0.05$ indicating statistical significance.

Baseline characteristics are described as mean \pm standard deviation (SD) or median and interquartile range (IQR) for continuous variables and frequencies and percentages for categorical data. To compare baseline characteristics across patients experiencing MACE and those not experiencing MACE, Student's t-test or Mann-Whitney U-test was used for 
continuous data and Pearson's chi-squared test or Fisher's exact test was used for categorical data. To obtain normal distributions creatinine levels, protein levels in EVs and EV TN-C levels were logarithmically transformed. To facilitate the comparability between continuous variables in the prediction model, age, log creatinine levels, ceramides and PCs (ratios) concentrations, $\log \mathrm{EV}$ protein levels and $\log \mathrm{EV} \mathrm{TN}-\mathrm{C}$ levels were standardized to Z-scores. Lipoprotein(a) was incorporated as binary variable, with the cut-off at $>137 \mathrm{nmol} / \mathrm{L}$; $>80$ th cohort percentile, in line with our previous publication. ${ }^{13}$ There were no missing values in the candidate predictors (Table 1), therefore no additional approaches to handle missing data were necessary.

\section{Prediction model development}

To assess the predictive value of the clinical model, we fitted a Cox proportional hazards model including age, current smoking, creatinine levels, diabetes, systolic blood pressure, use of antihypertensive drugs, history of coronary artery disease (CAD) and/or history of peripheral artery occlusive disease (PAOD), preprocedural cerebrovascular symptoms and presence of contralateral carotid stenosis as predictors and MACE occurrence at three year of follow up as the outcome. As incorporation of all these clinical variables in a model next to the biomarker candidates would lead to overcorrected models relative to the number of events in our cohort, we first reduced the clinical model using Akaike information criterion (AIC) in a stepwise backward regression. The final clinical model consisted of age, current smoking, creatinine levels, history of CAD/PAOD and preprocedural cerebrovascular symptoms (clinical model). Next, we fitted an extended Cox proportional hazards model by adding all candidate biomarkers simultaneously to the clinical model: $\operatorname{Cer}(\mathrm{d} 18: 1 / 24: 1)$, Cer(d18:1/16:0)/PC(16:0/22:5), Cer(d18:1/16:0)/Cer(d18:1/24:0), Cer(d18:1/18:0)/ Cer(d18:1/24:0), Cer(d18:1/24:1)/Cer(d18:1/24:0), CD14, Cystatin C, Serpin F2, Serpin $\mathrm{C} 1$, Tenascin-C and Lipoprotein(a). The final biomarkermodel was constructed using AIC based backward elimination.

\section{Internal validation and assessment of the incremental predictive value}

Bootstrapping methods were used for internal validation to adjust for overfitting and optimism in predictive performance of the developed prediction models. In each bootstrap sample, the modeling process was repeated, resulting in a shrinkage factor. With this factor, the estimated regression coefficients of the final models were adjusted for overfitting. The predictive performance of the bootstrap adjusted models was assessed by analyzing calibration and discrimination. Calibration was evaluated by visual inspection of the calibration plots. Discriminative performance was assessed by the $\mathrm{C}$-index ${ }^{27}$ and the net reclassification improvement (NRI). ${ }^{28,29}$ For NRI analysis, risk categories were predefined based on the overall incidence of MACE (p) which was divided into cutoff points at half 
Table 1. Baseline characteristics of the total study population and stratified by MACE

\begin{tabular}{|c|c|c|c|c|c|}
\hline & Total & No MACE & MACE & p-value & missing \\
\hline & $\mathrm{n}=\mathbf{8 4 7}$ & $\mathrm{n}=714$ & $\mathrm{n}=133$ & & \\
\hline Age (years) & $69(9)$ & $69(9)$ & $72(9)$ & $<0.01$ & 0 \\
\hline Gender, male & $594(70)$ & $493(69)$ & $101(76)$ & 0.14 & 0 \\
\hline Ipsilateral carotid artery stenosis degree & & & & 0.16 & 3 \\
\hline $50-70 \%$ & $66(8)$ & $60(9)$ & $6(5)$ & & \\
\hline $70-99 \%$ & $758(92)$ & $635(91)$ & $123(95)$ & & \\
\hline BMI $\left(\mathrm{kg} / \mathrm{m}^{2}\right)$ & $26[24,28]$ & $26[24,28]$ & $26[24,28]$ & 0.63 & 4 \\
\hline Creatinine $(\mu \mathrm{mol} / \mathrm{L})$ & $88[76,106]$ & $87[75,104]$ & $95[79,114]$ & $<0.01$ & 0 \\
\hline eGFR $\left(\mathrm{ml} / \mathrm{min}\right.$ per $\left.1.73 \mathrm{~m}^{2}\right)$ & $73(21)$ & $74(20)$ & $68(21)$ & $<0.01$ & 5 \\
\hline Current smoker & $280(33)$ & $228(32)$ & $52(39)$ & 0.13 & 0 \\
\hline Diabetes & $193(23)$ & $153(21)$ & $40(30)$ & 0.04 & 0 \\
\hline Hypertension & $588(72)$ & $491(71)$ & $97(75)$ & 0.37 & 3 \\
\hline Hypercholesterolemia & $535(70)$ & $455(70)$ & $80(69)$ & 0.95 & 9 \\
\hline Triglycerides (mg/dl) & $1.5[1.1,2.1]$ & $1.5[1.1,2.1]$ & $1.5[1.1,2.0]$ & 0.75 & 4 \\
\hline $\mathrm{LDL}(\mathrm{mmol} / \mathrm{L})$ & $2.3[1.8,3.0]$ & $2.3[1.8,3.0]$ & $2.3[1.8,3.0]$ & 0.48 & 4 \\
\hline $\mathrm{HDL}(\mathrm{mg} / \mathrm{dl})$ & $1.0[0.9,1.3]$ & $1.1[0.9,1.3]$ & $0.9[0.8,1.2]$ & $<0.01$ & 3 \\
\hline Total cholesterol (mg/dl) & $4.2[3.5,5.1]$ & $4.2[3.5,5.2]$ & $4.1[3.5,4.9]$ & 0.14 & 3 \\
\hline $\begin{array}{l}\text { Contralateral carotid artery stenosis of } \\
50-100 \%\end{array}$ & $351(46)$ & $286(44)$ & $65(52)$ & 0.12 & 9 \\
\hline Anticoagulants & $88(10)$ & $69(10)$ & $19(14)$ & 0.15 & 0 \\
\hline Antiplatelets & $746(88)$ & $631(89)$ & $115(88)$ & 0.93 & 0 \\
\hline Lipid lowering drugs & $677(80)$ & $577(81)$ & $100(75)$ & 0.17 & 0 \\
\hline Statins & $668(79)$ & $570(80)$ & $98(74)$ & 0.18 & 0 \\
\hline Hypertension drugs & $636(75)$ & $532(75)$ & $104(79)$ & 0.35 & 0 \\
\hline History of CAD or PAD & & & & $<0.01$ & 0 \\
\hline none & $483(57)$ & $420(59)$ & $63(47)$ & & \\
\hline Either CAD or PAOD & $294(35)$ & $244(34)$ & $50(38)$ & & \\
\hline Both CAD and PAOD & $70(8)$ & $50(7)$ & $20(15)$ & & \\
\hline History of stroke & $282(33)$ & $229(32)$ & $53(40)$ & 0.10 & 0 \\
\hline $\begin{array}{l}\text { Preprocedural cerebrovascular } \\
\text { symptoms }\end{array}$ & & & & 0.18 & 0 \\
\hline asymptomatic & $340(40)$ & $296(42)$ & $44(33)$ & & \\
\hline ocular & $93(11)$ & $77(11)$ & $16(12)$ & & \\
\hline TIA & $236(28)$ & $199(28)$ & $37(28)$ & & \\
\hline Stroke & $178(21)$ & $142(20)$ & $36(27)$ & & \\
\hline $\begin{array}{l}\text { Time between last event and surgery } \\
\text { (days) }\end{array}$ & $25[13,52]$ & $24[13,51]$ & $28[13,56]$ & 0.57 & 44 \\
\hline
\end{tabular}

Data are presented as $\mathrm{n}(\%)$, mean \pm stand deviation $( \pm \mathrm{SD})$ or median [interquartile range] [IQR]. Values in bold indicate $\mathrm{p}<0.05$. Abbreviations: BMI, Body Mass Index; eGFR, estimated Glomerular Filtration Rate; LDL, Lowdensity Lipoprotein; HDL, High-density Lipoprotein; CAD, coronary artery disease; PAOD, peripheral artery disease; TIA, Transient Ischemic Attack. 
of the overall incidence, the overall incidence and twice the overall incidence), since no predefined risk categories are available for risk prediction of secondary cardiovascular events. ${ }^{1,30}$

\section{Results}

\section{Patient characteristics}

In the current study 847 patients were included for analyses. Baseline characteristics of the total study population are shown in Table 1 . The mean age was $70( \pm 9)$ years and $70 \%$ of the patients were male. The cohort consisted mostly of patients with severe atherosclerotic disease: $30 \%$ has previously been diagnosed with CAD, $21 \%$ with PAOD and 33\% with any kind of stroke. There was a high prevalence of cardiovascular risk factors and concomitantly high prevalence of secondary preventive medication, such as anticoagulants (10\%), antiplatelet therapy (88\%) and lipid-lowering therapies (79\%). After 3 years of follow-up (with a median time-to-event of 3.0 [IQR 2.3-3.0]), 133 patients (15.7\%) reached the endpoint MACE. MACE consisted of 70 strokes, 43 MI, 20 other CV related deaths.

\section{Clinical model and biomarker model}

The clinical model consisted of age, current smoking, creatinine levels, history of CAD/ PAOD, preprocedural cerebrovascular symptoms. The biomarker model consisted of these clinical variables with addition of two circulating biomarkers Cer(d18:1/24:1)/ Cer(d18:1/24:0) measured in TEX-EV subfraction and the EV protein CD14 measured in the HDL-EV subfraction. Corrected regression coefficients of the clinical model and biomarker model before and after shrinkage by bootstrapping are shown in Table 2 . The clinical model showed slight underprediction at lower predicted probabilities (around the 3 -year MACE risk of 10\%) as well as overprediction for the range of 12-15\% (Figure 1).
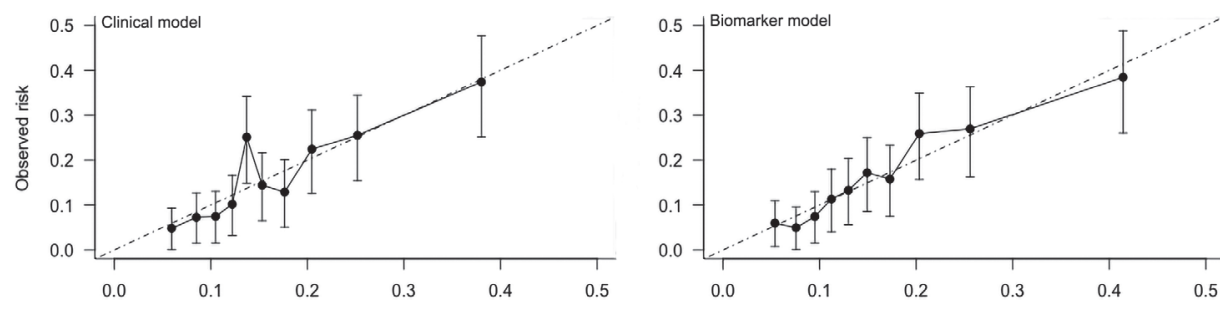

Figure 1. Calibration plots of the clinical model (left) and biomarker model (right) 
Table 2. Clinical model and biomarker model

\begin{tabular}{|c|c|c|c|c|c|c|c|c|}
\hline \multirow[t]{2}{*}{ Predictors } & \multicolumn{4}{|c|}{ Clinical model } & \multicolumn{4}{|c|}{ Biomarker model } \\
\hline & ن⿺辶ّ & 乩 & 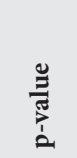 & 当 & 造 & 呫 & 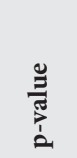 & 光 \\
\hline Age (year) & 0.3467 & 0.1007 & 0.001 & 0.3021 & 0.3113 & 0.1024 & 0.002 & 0.2724 \\
\hline Creatinine $(\mu \mathrm{mol} / \mathrm{L})$ & 0.2094 & 0.0835 & 0.012 & 0.1825 & 0.1650 & 0.0864 & 0.056 & 0.1444 \\
\hline \multicolumn{9}{|l|}{ History of CAD/PAOD } \\
\hline none & \multicolumn{4}{|c|}{ reference } & \multicolumn{4}{|c|}{ reference } \\
\hline Either CAD or PAOD & 0.2495 & 0.1931 & 0.196 & 0.2174 & 0.2377 & 0.1935 & 0.219 & 0.2079 \\
\hline Both CAD and PAOD & 0.9034 & 0.2634 & 0.001 & 0.7872 & 0.7513 & 0.2732 & 0.006 & 0.6573 \\
\hline Current smoking & 0.4901 & 0.1850 & 0.008 & 0.4271 & 0.3766 & 0.1882 & 0.045 & 0.3295 \\
\hline \multicolumn{9}{|l|}{$\begin{array}{l}\text { Preprocedural } \\
\text { neurological symptoms }\end{array}$} \\
\hline asymptomatic & \multicolumn{4}{|c|}{ reference } & \multicolumn{4}{|c|}{ reference } \\
\hline ocular & 0.3534 & 0.2950 & 0.231 & 0.3079 & 0.4121 & 0.2968 & 0.165 & 0.3605 \\
\hline TIA & 0.1972 & 0.2242 & 0.379 & 0.1718 & 0.2302 & 0.2265 & 0.310 & 0.2014 \\
\hline Stroke & 0.5859 & 0.2322 & 0.012 & 0.5105 & 0.5140 & 0.2384 & 0.031 & 0.4497 \\
\hline $\begin{array}{l}\operatorname{Cer}(\mathrm{d} 18: 1 / 24: 1) / \\
\operatorname{Cer}(\mathrm{d} 18: 1 / 24: 0)(\mu \mathrm{M})\end{array}$ & & & & & 0.2015 & 0.1001 & 0.044 & 0.1763 \\
\hline CD14 (pg/ml) & & & & & 0.2171 & 0.0677 & 0.001 & 0.1900 \\
\hline
\end{tabular}

S0(3) = 0.86 (3-year baseline survival). CAD, coronary artery disease; PAD, peripheral artery disease; TIA, Transient Ischemic Attack.

Coefficients are expressed for the condition present in dichotomous variables and per 1 SD increase for continuous variables. ${ }^{*}$ Regression coefficient multiplied with a shrinkage factor (obtained from bootstrapping procedure) of 0.868 for the clinical model and 0.875 for the biomarker model.

When extending the model with the two biomarkers, the accuracy of the predicted probabilities for the 3-year MACE risk within the range of 0-20\% improved. Above 20\% observed risk, the biomarker model showed modest overprediction (Figure 1). The C-index of the clinical model and the biomarker model was 0.66 (95\% CI 0.61-0.71) and 0.68 (95\% CI 0.63-0.73) ( $\mathrm{p}=0.07)$, respectively. Predicted probabilities based on the biomarker and clinical model are displayed in Supplemental Table S2. Supplemental Table S2 illustrated that most patients (approximately $80 \%$ ) fall within predicted risk category of $0-20 \%$, thereby the modest overprediction is acceptable. 


\section{Reclassification}

Results of the NRI analysis are summarized in Table 3. In 133 patients who developed MACE during 3-years of follow-up, the biomarker model improved reclassification of 30 patients $(23 \%)$ and incorrectly reclassify,ed 19 patients (14\%) downwards, with a net gain in reclassification of $8.3 \%$ (NRI $0.08395 \%$ CI $-0.020-0.185$ ). In the 714 patients that remained MACE-free during follow-up, the biomarker model reclassified 126 patients (18\%) correctly downwards and 80 patients $(11.2 \%)$ incorrectly upwards, with a net gain in reclassification of $6.4 \%$ (NRI $0.064,95 \%$ CI $0.025-0.104$ ). The overall NRI was $14.7 \%$ (NRI 0.147, 95\% CI 0.038-0.257), indicating that the biomarkers have additional prognostic value on top of the clinical predictors.

Table 3. Classification of 3-year MACE risk comparing the clinical model with an extended model

\begin{tabular}{|c|c|c|c|c|c|c|c|c|c|}
\hline \multirow{2}{*}{$\begin{array}{l}\text { Clinical } \\
\text { model }\end{array}$} & \multicolumn{5}{|c|}{ Biomarker model } & \multirow[b]{2}{*}{$\begin{array}{c}\text { Reclassified } \\
\text { up, } \mathbf{n}(\%)\end{array}$} & \multirow[b]{2}{*}{$\begin{array}{l}\text { Reclassified } \\
\text { down, } n(\%)\end{array}$} & \multirow[b]{2}{*}{ NRI (95\% CI) } & \multirow[b]{2}{*}{ p-value } \\
\hline & $<8 \%$ & $\begin{array}{c}8 \text { to } \\
<16 \%\end{array}$ & $\begin{array}{l}16 \text { to } \\
<32 \%\end{array}$ & $\geq 32 \%$ & Total & & & & \\
\hline MACE & & & & & & & & $0.083(-0.020-0.185)$ & 0.113 \\
\hline$<8 \%$ & 6 & 1 & 0 & 0 & 7 & $30(22.6 \%)$ & $19(14.3 \%)$ & & \\
\hline 8 to $<16 \%$ & 3 & 33 & 15 & 1 & 52 & & & & \\
\hline 16 to $<32 \%$ & 0 & 12 & 39 & 13 & 64 & & & & \\
\hline$\geq 32 \%$ & 0 & 0 & 4 & 6 & 10 & & & & \\
\hline Total & 9 & 46 & 58 & 20 & 133 & & & & \\
\hline No MACE & & & & & & & & $0.064(0.025-0.104)$ & 0.001 \\
\hline$<8 \%$ & 78 & 16 & 0 & 0 & 94 & $80(11.2 \%)$ & $126(17.6 \%)$ & & \\
\hline 8 to $<16 \%$ & 60 & 279 & 50 & 3 & 392 & & & & \\
\hline 16 to $<32 \%$ & 0 & 60 & 140 & 11 & 211 & & & & \\
\hline$\geq 32 \%$ & 0 & 0 & 6 & 11 & 17 & & & & \\
\hline Total & 138 & 355 & 196 & 25 & 714 & & & & \\
\hline Overall & & & & & & & & $0.147(0.038-0.257)$ & 0.008 \\
\hline
\end{tabular}

Marked in green are patients that are correctly reclassified if the combined biomarker model is used instead of the clinical model. Marked in red are patients that are wrongly reclassified if the combined biomarker model is used instead of the clinical model. In bold are the patients that remain in the same risk category using the biomarker or the clinical model.

\section{Discussion}

In the present study, we evaluated the incremental value of preoperative levels of circulating biomarkers on top of traditional CV risk factors as predictors of the three-year postoperative 
risk of MACE after CEA. The biomarker model containing a combination of two EV-derived biomarkers (Cer(d18:1/24:1)/Cer(d18:1/24:0) and CD14) next to CV risk factors (age, current smoking, creatinine levels, history of CAD/PAOD, preprocedural cerebrovascular symptoms) provided incremental prognostic information for MACE prediction compared to the clinical model containing only CV risk factors. The biomarker model showed improved performance metrics and resulted in a better reclassification of $14.7 \%$ of all patients according to their future postoperative risk of MACE compared to the clinical model. This prediction model can be used to stratify CEA patients more reliably in the preoperative phase for their future postoperative risk of MACE allowing for more personalized secondary prevention.

According to European guidelines ${ }^{2}$, patients with established clinical CV disease, such as CEA patients, are all classified in one group at very-high risk for secondary CV events. Variation in risk for secondary CV events, however, has been reported previously among individual patients with established CVD treated with secondary prevention according to guideline recommended targets. ${ }^{31}$ More accurate identification of individuals at high risk for secondary events will lead to more timely risk reduction by intensification of medical treatment with add-on therapies. Aggressive lowering of on-treatment cholesterol levels with proprotein convertase subtilisin/kexin type 9 (PCSK-9) inhibitors has already been implemented in the most recent ESC guidelines but have high costs. ${ }^{2}$ Anti-inflammatory medications (colchicine or canakinumab) have shown promising results in reduction of future cardiovascular events for coronary artery disease patients and might also become novel options as add-on therapy in the future. ${ }^{3-5}$ Addition of a direct oral anticoagulant (DOAC) to aspirin may be another future option to intensify medical therapy since studies have reported a positive effect on the reduction of future cardiovascular events but a major drawback is the increased bleeding risk. ${ }^{6,7}$ Hazardous side effects and high costs urge the need for careful selection of patients for add-on therapy. Prediction models or risk scores could aid to estimate the postprocedural long-term risk of patients undergoing CEA. An external validation study and a previous systematic review addressed that existing clinical risk predicting models have poor ability to predict postoperative CV events in CEA patients. ${ }^{8,32}$ We now show that addition of two EV-based biomarkers next to CV risk factors improve risk stratification in patients scheduled for CEA.

The EV-derived ceramide ratio Cer(d18:1/24:1)/Cer(d18:1/24:0) has been independently associated with the long-term risk of CV events in CEA patients as well as in patients diagnosed with coronary artery disease (CAD). ${ }^{33,34, a}$ Ceramides have been linked to central atherosclerotic processes such as LDL-uptake, LDL-aggregation, endothelial dysfunction, apoptosis and inflammation..$^{35}$ Previous studies have not reported an incremental predictive 
value of Cer(d18:1/24:1)/Cer(d18:1/24:0) as sole marker for CV prediction in CEA and CAD cohorts. On the other hand, two ceramide risk scores (CERT-1 and CERT-2) including plasma Cer(d18:1/24:1)/Cer(d18:1/24:0) showed incremental prognostic value for CV death and CV events in CAD patients. We now show EV-derived Cer(d18:1/24:1)/Cer(d18:1/24:0) and CD14 have synergistic prognostic effects. The idea of biomarker synergy has been well recognized in both coronary cohorts and vascular surgery patients. Different types of biomarkers related to various processes such as proinflammatory pathways, endothelial activation, renal function and cardiac function may simultaneously enhance risk estimation since each marker has the ability to provide incremental prognostic information. ${ }^{24,25,36-39}$

Previously we demonstrated that EV-derived CD14 levels have incremental predictive

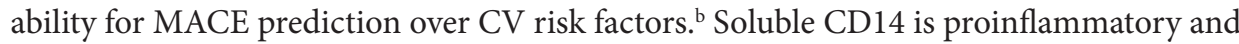
known to be implicated in the innate immune response, whereas monocyte-derived EVs containing CD14 possess procoagulant and prothrombotic properties. ${ }^{40,41}$ Increased inflammatory response through soluble CD14 has also been proposed to be genetically determined by specific polymorphisms in patients with carotid atherosclerosis. ${ }^{42}$ Addition of EV-derived CD14 to a clinical CV risk model improved overall reclassification (based on NRI) in 16\% of all CEA patients according to their future risk of MACE. ${ }^{b}$ However, this model underperformed in identification of patients that remain free from MACE since these were wrongfully reclassified in higher MACE risk categories. With the combined biomarker model composed of Cer(d18:1/24:1)/Cer(d18:1/24:0) and CD14, we propose a risk model that demonstrates improved discrimination and calibration. With this model reclassification of patients that remain MACE free improves significantly and in terms of calibration (the agreement in predicted risks by the model and observed risks) the combined biomarker model is superior to the biomarker model with EV-CD14 alone. The combined biomarker model showed slight overprediction in the 3-year risk of MACE above 20\%, however the majority of the CEA patients ( $80 \%$ of all patients) are part of the risk category below 20\%. Moreover, overprediction of the patients in high-risk categories would not have major impact in practice, as these patients would already qualify for aggressive secondary prevention and follow-up. Implementation of the biomarker model in clinical practice thus would have great advantage for patients in low risk categories as it would prevent overtreatment with add-on therapies, and for patients in high risk categories it will prevent undertreatment with add-on therapies.

Apart from improving cost-efficient allocation of add-on therapy, biomarker-based risk stratification may have other future implications. Accurate risk estimation tools could enable physicians to quantify the patients' future CV risk and this may promote awareness and motivation for patients' adherence to lifestyle changes or preventive medications. In 
general, therapy adherence of CV medications is a major issue and a substantial proportion of CVD events is likely to be attributed to poor adherence. ${ }^{43}$

Risk stratification models could also be used as a tool to select eligible patients for randomized-controlled trials investigating new medical interventions for CVD reduction. As patients at highest risk for CV events are most likely to benefit from novel preventive medications, inclusion of the highest risk patients may subsequently improve trial efficiency and power to detect clinically relevant effects of investigated interventions and reduce trial-related costs. Ceramides and CD14 are both related to inflammation ${ }^{35,42}$ and may be relevant to select patients that may benefit from potential anti-inflammatory drugs for CVD risk reduction (such as recent studies with low-dose colchicine or canakinumab). ${ }^{3,4}$ This could be extended in investigating whether multiple measurements of these biomarkers during follow-up could assist in monitoring the individual response of a patient to known or upcoming preventive medications.

Some limitations of this study should be mentioned. The goal of our study was to develop a combined biomarker-based risk prediction model consisting of preoperative characteristics to predict future risk of MACE for patients undergoing CEA. It is likely that modification of $\mathrm{CV}$ risk factors and medication changes during follow-up interfere with the risk of MACE. Unfortunately, changes in CV risk factors and medication use are not available in the AtheroExpress and therefore these factors could not be corrected for. Although pre-existing cardiac conditions such as atrial fibrillation and heart failure are important predictors for CV events in CEA patients ${ }^{8}$, these factors could not be considered in model development because this data was not available. It has been established that secondary CV event risk varies geographically ${ }^{44}$, therefore future studies should assess the generalizability of the current prediction model. In order to provide accurate and validated estimates, the biomarker model should be externally validated in other CEA populations. Furthermore, impact studies should be performed to investigate its effects on clinical decision-making. In order for it to be useful, clinical implementation of the biomarker model should improve therapeutic decision making, clinical outcome and cost-effectiveness of healthcare. Importantly, as no predefined risk scores in prevention for secondary CV events in CEA patients exist, we defined risk categories to calculate the NRI based on overall incidence, similar to previous studies. ${ }^{1,30}$ Future impact studies should unravel whether these risk categories are appropriate in improving clinical care. Finally, in recent years a widespread of biomarkers associated with CV outcome have been identified..$^{22,24,25,38,39,45}$ Independently the biomarkers estimate different aspects and reflect distinct biological processes involved in atherosclerosis and cardiovascular disease. It could be plausible that more biomarkers not under investigation in the present study may further improve the predictive value. Future collaboration of prospective biobanks or newly designed multicenter prospective studies in a sizeable study population may aid to 
investigate whether combining more biomarkers on top of CD14 and Cer(d18:1/24:1)/ Cer(d18:1/24:0) could improve MACE prediction.

Strengths include that this is the first large study in CEA patients showing that circulating biomarkers determined preoperatively have synergistic prognostic ability for estimation of future risk of MACE. After proven clinical relevance, this biomarker model could be easily incorporated into clinical practice as measurement methods are straightforward and could be robotized. After external validation, this model may more accurately identify patients at high risk for MACE and may be used to guide personalized secondary preventive management in order to reduce future MACE.

To conclude, extending the clinical model including CV risk factors with two preoperative circulating EV-derived biomarkers, CD14 and the ceramide ratio Cer(d18:1/24:1)/ Cer(d18:1/24:0), improved risk stratification for prediction of long-term postoperative risk of MACE in patients undergoing CEA.

\section{Funding}

Dr. De Borst, dr. De Kleijn, dr. Pasterkamp, are funded through the EU 755320 Taxinomisis grant. Dr. De Kleijn obtained funding from the Dutch Heart Foundation, CVON 2017-05 pERSUASIVE. There are no competing interests declared.

\section{Conflict of Interest}

None declared 


\section{References}

1 Van Lammeren GW, Catanzariti LM, Peelen LM, De Vries JPPM, De Kleijn DPV, Moll FL, et al. Clinical prediction rule to estimate the absolute 3-year risk of major cardiovascular events after carotid endarterectomy. Stroke 2012;43(5):1273-8.

2 Mach F, Baigent C, Catapano AL, Koskinas KC, Casula M, Badimon L, et al. 2019 ESC/EAS Guidelines for the management of dyslipidaemias: lipid modification to reduce cardiovascular risk. Eur Heart $J$ 2020;41(1):111-88.

3 Tardif J-C, Kouz S, Waters DD, Bertrand OF, Diaz R, Maggioni AP, et al. Efficacy and Safety of Low-Dose Colchicine after Myocardial Infarction. N Engl J Med 2019;381(26):2497-505.

4 Nidorf SM, Fiolet ATL, Mosterd A, Eikelboom JW, Schut A, Opstal TSJ, et al. Colchicine in Patients with Chronic Coronary Disease. N Engl J Med 2020:NEJMoa2021372.

5 Ridker PM, Everett BM, Thuren T, MacFadyen JG, Chang WH, Ballantyne C, et al. Antiinflammatory Therapy with Canakinumab for Atherosclerotic Disease. N Engl J Med 2017;377(12):1119-31.

6 Eikelboom JW, Connolly SJ, Bosch J, Dagenais GR, Hart RG, Shestakovska O, et al. Rivaroxaban with or without Aspirin in Stable Cardiovascular Disease. N Engl J Med 2017;377(14):1319-30.

7 Bonaca MP, Bauersachs RM, Anand SS, Debus ES, Nehler MR, Patel MR, et al. Rivaroxaban in Peripheral Artery Disease after Revascularization. N Engl J Med 2020;382(21):1994-2004.

8 Volkers EJ, Algra A, Kappelle LJ, Greving JP. Prediction models for clinical outcome after a carotid revascularisation procedure: A systematic review. Eur Stroke J 2018;3(1):57-65.

9 Van Lammeren GW, De Vries JPPM, Vink A, De Kleijn DPV, Moll FL, Pasterkamp G. New predictors of adverse cardiovascular events following vascular surgery. Semin Cardiothorac Vasc Anesth 2010;14(2):148-53.

10 Nordestgaard BG, Chapman MJ, Ray K, Borén J, Andreotti F, Watts GF, et al. Lipoprotein(a) as a cardiovascular risk factor: Current status. Eur Heart J 2010;31(23):2844-53.

11 Kamstrup PR, Tybjærg-hansen A, Steffensen R, Nordestgaard BG. Genetically Elevated Lipoprotein ( a ). J Am Med Assoc 2009;301(22):2331-9.

12 The Emerging Risk Factors Collaboration. Lipoprotein ( a ) Concentration and the Risk of Coronary Heart Disease, Stroke ,. JAMA 2009;302(4):412-23.

13 Waissi F, Dekker M, Timmerman N, Hoogeveen RM, van Bennekom J, Dzobo KE, et al. Elevated Lp(a) (Lipoprotein[a]) Levels Increase Risk of 30-Day Major Adverse Cardiovascular Events in Patients Following Carotid Endarterectomy. Stroke 2020.

14 Dekker M, Waissi F, van Bennekom J, Silvis MJM, Timmerman N, Bank IEM, et al. Plasma extracellular vesicle proteins are associated with stress-induced myocardial ischemia in women presenting with chest pain. Sci Rep 2020;10(1):1-8.

15 de Hoog VC, Timmers L, Schoneveld AH, Wang JW, Van de Weg SM, Sze SK, et al. Serum extracellular vesicle protein levels are associated with acute coronary syndrome. Eur Hear J Acute Cardiovasc Care 2013;2(1):53-60.

16 Kanhai DA, Visseren FLJ, Van Der Graaf Y, Schoneveld AH, Catanzariti LM, Timmers L, et al. Microvesicle protein levels are associated with increased risk for future vascular events and mortality in patients with clinically manifest vascular disease. Int J Cardiol 2013;168(3):2358-63.

17 Vrijenhoek JE, Pasterkamp G, Moll FL, De Borst GJ, Bots ML, Catanzariti L, et al. Extracellular vesiclederived CD14 is independently associated with the extent of cardiovascular disease burden in patients with manifest vascular disease. Eur J Prev Cardiol 2015;22(4):451-7.

18 Kranendonk MEG, de Kleijn DPV, Kalkhoven E, Kanhai DA, Uiterwaal CSPM, van der Graaf Y, et al. Extracellular vesicle markers in relation to obesity and metabolic complications in patients with manifest cardiovascular disease. Cardiovasc Diabetol 2014;13(1):1-11.

19 Dekker M, Waissi F, Van Bennekom J, Silvis MJM, Timmerman N, Schoneveld AH, et al. Extracellular vesicle cystatin $\mathrm{c}$ is associated with unstable angina in troponin negative patients with acute chest pain. PLoS One 2020;15(8 August):1-12.

20 Urabe F, Kosaka N, Kimura T, Egawa S, Ochiya T. Extracellular vesicles: Toward a clinical application in urological cancer treatment. Int J Urol 2018;25(6):533-43.

21 Boulanger CM, Loyer X, Rautou PE, Amabile N. Extracellular vesicles in coronary artery disease. Nat Rev Cardiol 2017;14(5):259-72. 
22 Pareek M, Bhatt DL, Vaduganathan M, Biering-Sørensen T, Qamar A, Diederichsen ACP, et al. Single and multiple cardiovascular biomarkers in subjects without a previous cardiovascular event. Eur J Prev Cardiol 2017;24(15):1648-59.

23 Nozaki T, Sugiyama S, Koga H, Sugamura K, Ohba K, Matsuzawa Y, et al. Significance of a Multiple Biomarkers Strategy Including Endothelial Dysfunction to Improve Risk Stratification for Cardiovascular Events in Patients at High Risk for Coronary Heart Disease. J Am Coll Cardiol 2009;54(7):601-8.

24 Wang TJ, Gona P, Larson MG, Tofler GH, Levy D, Newton-Cheh C, et al. Multiple biomarkers for the prediction of first major cardiovascular events and death. N Engl J Med 2006;355(25):2631-9.

25 Melander O, Newton-Cheh C, Almgren P, Hedblad B, Berglund G, Engström G, et al. Novel and conventional biomarkers for prediction of incident cardiovascular events in the community. JAMA - J Am Med Assoc 2009;302(1):49-57.

26 Verhoeven BAN, Velema E, Schoneveld AH, Vries JPPM de., de Bruin P, Seldenrijk CA, et al. Atheroexpress: Differential atherosclerotic plaque expression of mRNA and protein in relation to cardiovascular events and patient characteristics. Rationale and design. Eur J Epidemiol 2004;19(12):1127-33.

27 Kang L, Chen W, Petrick NA, Gallas BD. Comparing two correlated C indices with right-censored survival outcome: A one-shot nonparametric approach. Stat Med 2015;34(4):685-703.

28 Pencina MJ, D’Agostino RB, D’Agostino RB, Vasan RS. Evaluating the added predictive ability of a new marker: From area under the ROC curve to reclassification and beyond. Stat Med 2008;27(2):157-72.

29 Leening MJG, Vedder MM, Witteman JCM, Pencina MJ, Steyerberg EW. Net Reclassification Improvement: Computation, Interpretation, and Controversies. Ann Intern Med 2014;160(2):122-31.

30 Cook NR, Paynter NP. Performance of reclassification statistics in comparing risk prediction models. Biometrical J 2011;53(2):237-58.

31 Kaasenbrood L, Boekholdt SM, Van Der Graaf Y, Ray KK, Peters RJG, Kastelein JJP, et al. Distribution of Estimated 10-Year Risk of Recurrent Vascular Events and Residual Risk in a Secondary Prevention Population. Circulation 2016;134(19):1419-29.

32 Volkers EJ, Algra A, Jaap Kappelle L, Jansen O, Howard G, Hendrikse J, et al. Prediction models for clinical outcome after a carotid revascularization procedure an external validation study. Stroke 2018;49(8):1880-5.

33 Hilvo M, Meikle PJ, Pedersen ER, Tell GS, Dhar I, Brenner H, et al. Development and validation of a ceramide- And phospholipid-based cardiovascular risk estimation score for coronary artery disease patients. Eur Heart J 2020;41(3):371-80.

34 Laaksonen R, Ekroos K, Sysi-Aho M, Hilvo M, Vihervaara T, Kauhanen D, et al. Plasma ceramides predict cardiovascular death in patients with stable coronary artery disease and acute coronary syndromes beyond LDL-cholesterol. Eur Heart J 2016;37(25):1967-76.

35 Bismuth J, Lin P, Yao Q, Chen C. Ceramide: A common pathway for atherosclerosis? Atherosclerosis 2008;196(2):497-504.

36 Scrutinio D, Guido G, Guida P, Passantino A, Angiletta D, Santoro D, et al. Combined Use of Highsensitivity C-Reactive Protein and N-Terminal Pro-B-type Natriuretic Peptide for Risk Stratification of Vascular Surgery Patients. Ann Vasc Surg 2014;28(6):1522-9.

37 Goei D, Hoeks SE, Boersma E, Winkel TA, Dunkelgrun M, Flu W-J, et al. Incremental value of highsensitivity C-reactive protein and N-terminal pro-B-type natriuretic peptide for the prediction of postoperative cardiac events in noncardiac vascular surgery patients. Coron Artery Dis 2009;20(3):219-24.

38 Blankenberg S, McQueen MJ, Smieja M, Pogue J, Balion C, Lonn E, et al. Comparative impact of multiple biomarkers and $\mathrm{N}$-terminal pro-brain natriuretic peptide in the context of conventional risk factors for the prediction of recurrent cardiovascular events in the Heart Outcomes Prevention Evaluation (HOPE) study. Circulation 2006;114(3):201-8.

39 Schnabel RB, Schulz A, Messow CM, Lubos E, Wild PS, Zeller T, et al. Multiple marker approach to risk stratification in patients with stable coronary artery disease. Eur Heart J 2010;31(24):3024-31.

40 Reiner AP, Lange EM, Jenny NS, Chaves PHM, Ellis J, Li J, et al. Soluble CD14. Arterioscler Thromb Vasc Biol 2013;33(1):158-64.

41 Aharon A, Tamari T, Brenner B. Monocyte-derived microparticles and exosomes induce procoagulant and apoptotic effects on endothelial cells. Thromb Haemost 2008;100(05):878-85.

42 Giacconi R, Caruso C, Lio D, Muti E, Cipriano C, Costarelli L, et al. CD14 C (-260)T polymorphism, atherosclerosis, elderly: Role of cytokines and metallothioneins. Int J Cardiol 2007;120(1):45-51.

43 Chowdhury R, Khan H, Heydon E, Shroufi A, Fahimi S, Moore C, et al. Adherence to cardiovascular therapy: a meta-analysis of prevalence and clinical consequences. Eur Heart J 2013;34(38):2940-8. 
44 Ducrocq G, Bhatt D, Labreuche J, Corbalan R, Porath A, Gao R, et al. Geographic differences in outcomes in outpatients with established atherothrombotic disease: results from the REACH Registry. Eur J Prev Cardiol 2014;21(12):1509-16.

45 Patelis N, Kouvelos GN, Koutsoumpelis A, Moris D, Matsagkas MI, Arnaoutoglou E. An update on predictive biomarkers for major adverse cardiovascular events in patients undergoing vascular surgery. $J$ Clin Anesth 2016;33:105-16.

46 Randomised trial of endarterectomy for recently symptomatic carotid stenosis: final results of the MRC European Carotid Surgery Trial (ECST) n.d.

47 Barnett HJM, Taylor DW, Eliasziw M, Fox AJ, Ferguson GG, Haynes RB, et al. Benefit of carotid endarterectomy in patients with symptomatic moderate or severe stenosis. NEngl J Med 1998;339(20):141525.

48 Ferguson GG, Eliasziw M, Barr HWK, Clagett GP, Barnes RW, Wallace MC, et al. The North American Symptomatic Carotid Endarterectomy Trial. Stroke 1999;30(9):1751-8.

49 Halliday A, Mansfield A, Marro J, Peto C, Peto R, Potter J, et al. Prevention of disabling and fatal strokes by successful carotid endarterectomy in patients without recent neurological symptoms: randomised controlled trial. Lancet (London, England) 2004;363(9420):1491-502.

50 Halliday A, Harrison M, Hayter E, Kong X, Mansfield A, Marro J, et al. 10-year stroke prevention after successful carotid endarterectomy for asymptomatic stenosis (ACST-1): a multicentre randomised trial. Lancet 2010;376(9746):1074-84.

51 Hellings WE, Peeters W, Moll FL, Piers S, Van Setten J, Van Der Spek PJ, et al. Composition of carotid atherosclerotic plaque is associated with cardiovascular outcome: A prognostic study. Circulation 2010;121(17):1941-50.

52 Wang J-W, Zhang Y-N, Sze SK, Weg SM Van De. Lowering Low-density Lipoprotein Particles in Plasma Using Dextran Sulphate Co-precipitates Procoagulant Extracellular Vesicles n.d.;(V):1-16.

53 Dekker M, Waissi F, van Bennekom J, Silvis MJM, Timmerman N, Bank IEM, et al. Plasma extracellular vesicle proteins are associated with stress-induced myocardial ischemia in women presenting with chest pain. Sci Rep 2020;10(1):12257.

54 Zhang Y-N, Vernooij F, Ibrahim I, Ooi S, Gijsberts CM, Schoneveld AH, et al. Extracellular Vesicle Proteins Associated with Systemic Vascular Events Correlate with Heart Failure: An Observational Study in a Dyspnoea Cohort. PLoS One 2016;11(1):e0148073. 


\section{Unpublished references}

a Timmerman $\mathrm{N}^{\star}$,Waissi F*, Dekker M, De Borst GJ, Van Bennekom J, De Winter RJ, Hilvoc M, Jylhäc A, Pasterkamp G, De Kleijn DPV ${ }^{*}$, Laaksonen $\mathrm{R}^{*}$. Ceramides and phospholipids are associated with high risk of major cardiovascular events after carotid endarterectomy. Under review-Scientific Reports

b Timmerman $\mathrm{N}^{*}$,Waissi $\mathrm{F}^{*}$, Dekker M, Van der Pol QY, Van Bennekom J, Schoneveld A, Klein-Avink MJM, De Winter RJ, Pasterkamp G, De Borst GJ, De Kleijn DPV. Pre-operative plasma extracellular vesicle proteins are associated with a high risk of long term secondary major cardiovascular events in patients undergoing carotid endarterectomy. Eur J Vasc Endovasc Surg [in press]

c Waissi F*, Timmerman N*, Dekker M, Bijkerk S, Van Bennekom J, Schoneveld AH, Rong-Rong Z, De Winter R, Pasterkamp G, De Borst GJ, De Kleijn DPV. High Tenascin-C plasma extracellular vesicles levels are associated with vulnerable plaque characteristics and an increased risk of 3-year major adverse cardiovascular events in carotid endarterectomy patients. Under review-Arterioscler Thromb Vasc Biol 


\section{Supplementary Material}

Supplemental Table S1. Overview of the candidate biomarkers and the matrix in which these were measured

\begin{tabular}{ll}
\hline Candidate biomarkers & Sample Matrix \\
\hline $\operatorname{Cer}(\mathrm{d} 18: 1 / 24: 1)$ & LDL-EV subfraction \\
$\operatorname{Cer}(\mathrm{d} 18: 1 / 16: 0) / \operatorname{PC}(16: 0 / 22: 5)$ & LDL-EV subfraction \\
$\operatorname{Cer}(\mathrm{d} 18: 1 / 16: 0) / \operatorname{Cer}(\mathrm{d} 18: 1 / 24: 0)$ & TEX-EV subfraction \\
$\operatorname{Cer}(\mathrm{d} 18: 1 / 18: 0) / \operatorname{Cer}(\mathrm{d} 18: 1 / 24: 0)$ & TEX-EV subfraction \\
$\operatorname{Cer}(\mathrm{d} 18: 1 / 24: 1) / \operatorname{Cer}(\mathrm{d} 18: 1 / 24: 0)$ & TEX-EV subfraction \\
CD14 & HDL-EV subfraction \\
Cystatin C & HDL-EV subfraction \\
Serpin F2 & HDL-EV subfraction \\
Serpin C1 & HDL-EV subfraction \\
Tenascin-C & LDL-EV subfraction \\
Lipoprotein(a) & Unfractionated plasma \\
\hline
\end{tabular}

Supplemental Table S2. Predicted number of patients per risk category for MACE at three-year follow-up based on the final biomarker model and clinical model with only CV risk factors

\begin{tabular}{lcc}
\hline 3-year risk of MACE & $\begin{array}{c}\text { Predicted number of patients } \\
\text { according to the biomarker model }\end{array}$ & $\begin{array}{c}\text { Predicted number of patients } \\
\text { according to clinical model }\end{array}$ \\
\hline $0-10 \%$ & 236 & 202 \\
$10-20 \%$ & 443 & 476 \\
$20-30 \%$ & 115 & 132 \\
$30-40 \%$ & 39 & 27 \\
$40-50 \%$ & 7 & 7 \\
$50-60 \%$ & 2 & 1 \\
$60-100 \%$ & 5 & 2 \\
\hline Total patients & 847 & 847 \\
\hline
\end{tabular}




\section{Supplementary Methods}

\section{Description of study cohorts}

The Athero-Express Biobank

All previous biomarker studies were performed in the Athero-Express Biobank in CEA patients included from 2002-2016. The Athero-Express Biobank is an ongoing prospective cohort study initiated in 2002 in two tertiary referral hospitals (the St. Antonius Hospital Nieuwegein (inclusions stopped after 2014) and University Medical Center Utrecht, The Netherlands). Consecutive patients scheduled for CEA are asked for study participation. A multidisciplinary vascular team adjudicated indications for CEA in accordance with local and international guidelines and surgery was performed by experienced surgeons. ${ }^{26,46-50}$ During hospital admission, preoperative blood samples were collected and stored at $-80^{\circ} \mathrm{C}$ until further use. Baseline patient characteristics were collected by standardized preoperative questionnaires and medical records. Patients were followed up for the three consecutive years after CEA on cardiovascular events (CVE) and hospital admissions by standardized questionnaires. When an outcome event was reported, additional documentation regarding the event were requested from the institution where the event occurred and the general practitioner. In case a patient did not respond to questionnaires, the general practitioner was consulted for follow-up information. An extensive description of the Athero-Express study design has been published previously. ${ }^{26,51}$

\section{Ceramides and $P C s^{a}$}

This study analyzed 873 patients and measured seven ceramides and PC lipids: Cer(d18:1/16:0), Cer(d18:1/18:0), Cer(d18:1/24:0), Cer(d18:1/24:1), PC(14:0/22:6), PC(16:0/16:0) and PC(16:0/22:5) in plasma and two plasma EV-subfractions (LDL and TEX). The primary aim was to investigate whether circulating levels of ceramides and PCs in plasma and in two plasma EV subsets were associated with the postoperative risk of MACE in patients undergoing CEA. Inclusion criteria were availability of citrate blood plasma samples and follow-up data. Exclusion criteria were patients undergoing CEA for restenosis. During a median postoperative follow-up time of 3.0 years [IQR 2.2-3.0], 138 patients $(15.8 \%)$ experienced MACE consisting of $74(8.5 \%)$ fatal and non-fatal stroke, 44 (5\%) fatal and non-fatal MI and 20 (2.3\%) CV death due to other causes. Main results of the study were that in the LDL-EV subfraction, higher levels of Cer(d18:1/24:1) and Cer(d18:1/16:0)/PC(16:0/22:5) ratio were significantly associated with an increased risk of MACE. In the TEX-EV subfraction, three ratios Cer(d18:1/16:0)/Cer(d18:1/24:0), Cer(d18:1/18:0)/Cer(d18:1/24:0) and Cer(d18:1/24:1)/Cer(d18:1/24:0) were positively associated with an increased three-year risk of MACE after CEA. Details of the study design have been published earlier. ${ }^{a}$ 


\section{EV-proteins ${ }^{b}$}

The primary aim of this study was to investigate whether levels of CVD-related proteins (Cystatin C, Serpin C1, Serpin G1, Serpin F2 and CD14) in EV plasma subsets (LDL-, HDL- and TEX EV-subfraction) were associated with the 3-year risk of MACE after CEA. Final analyses were performed on 864 patients with at least one valid protein measurement. Inclusion criteria were availability of citrate plasma samples and follow-up data. Patients operated for restenosis were excluded. After a median follow-up time of 3.0 years [IQR: 2.2.-3.0 years], 137 (16\%) patients with MACE were observed of which 73 (8.4\%) patients had stroke as first event, 44 (5.1\%) had MI and 20 (2.3\%) died due to other cardiovascular causes. This study concluded that EV-derived plasma levels of Cystatin C, Serpin C1, CD14 and Serpin F2 determined in preoperative blood were independently associated with a higher long-term risk of MACE following CEA. An extensive description of the study design has been published previously. ${ }^{b}$

\section{Tenascin- $C^{c}$}

Plasma EV TN-C levels were determined in pre-operative blood samples of 837 symptomatic and asymptomatic CEA patients. Inclusion criteria were available citrate blood plasma samples and follow-up data. Patients who underwent CEA for restenosis were excluded. The primary aim was to investigate whether plasma EV TN-C levels in CEA patients were associated with the 3-year postoperative risk of MACE. Median follow-up time was 3.0 years [IQR 2.2-3.0], in which 137 patients (16.4\%) reached the endpoint MACE. MACE consisted of $73(8.7 \%)$ stroke, $43(5.1 \%) \mathrm{MI}$ and 21 (2.5\%) other cardiovascular-related deaths. Plasma EV TN-C levels were independently associated with an increased 3-year risk of MACE after CEA. A more detailed description of the study design has been published previously. ${ }^{c}$

\section{Lipoprotein $(a)^{13}$}

The primary aim of this study was to assess the association of elevated Lp(a) levels with the risk of secondary MACE in patients undergoing CEA. Lp(a) concentrations were determined in preoperative blood samples of 944 CEA patients included in the Athero-Express Biobank between 2002 and 2016 with available lipid profile measurements. Patients undergoing CEA for restenosis were excluded. The mean follow-up time was $2.57( \pm 0.85)$ years, during which $102(10.8 \%)$ patients reached the end point MACE. MACE consisted of $27(2.9 \%)$ myocardial infarction, 63 (6.7\%) strokes, and 12 (1.3\%) cardiovascular-related deaths. Elevated Lp(a) levels ( $>137 \mathrm{nmol} / \mathrm{L}$; $>80$ th cohort percentile) were associated with an increased risk of 30-day MACE mainly composed of perioperative stroke. This study identified elevated Lp(a) levels as a new and additional risk factor for secondary cardiovascular events, potentially identifying patients at highest periprocedural risk. 


\section{Extracellular vesicle isolation and characterization}

Detailed description of the isolation protocol can be found in previously published papers. ${ }^{52-54}$ In short, distinct EV subsets co-fractionate with Low-Density Lipid particles (LDL), High-Density Lipid particles (HDL) and with the independent TEX subfraction. The LDL and HDL subfractions are precipitated from plasma using sequential isolation with Dextran Sulphate (DS) (MP Biomedicals), Manganese (II) Chloride ( $\mathrm{MnCl}$ ) (SigmaAldrich) solutions. The TEX subfraction is obtained from plasma with Xtractt buffer (1:4) (Cavadis BV). Isolation of EVs were performed with magnetic dextran nanoparticles (Nanomag ${ }^{\oplus}$-D plain, Nanomag ${ }^{\oplus}$-D PEG-OH, Micromod) and a bio-plex handheld magnet (Bio-Rad). The pellets with EVs were lysed with complete lysis-M including protease inhibitors (Roche). To be able to correct for loss of pellet during the isolation steps, a standard quantity of synthetic liposomes (coated with DSG-PEG2000, Sunbright and fluorescently labelled with 18:1 Liss Rhod PE, MERCK) was added to each plasma sample and quantified afterwards.

\section{Biomarker quantification}

Quantification of ceramides and PCs

Ceramides and PCs concentrations were quantified in plasma, LDL-EV and TEX-EV subfractions using liquid chromatography-mass spectrometry (LC-MS/MS analysis) conducted on a Sciex TripleQuad 5500 mass spectrometer coupled to Sciex MPX LC system. Electrospray ionization in positive ion mode was used with multiple reaction monitoring. Instrument and data acquisition were controlled using Analyst ${ }^{\circledast}$ (version 1.7). The final concentrations of ceramides and PCs were expressed in $\mu \mathrm{M}$. A more detailed description can be found in the original publication. ${ }^{2}$

\section{Quantification of EV protein levels}

With an electrochemiluminescence immunoassay (Quickplex SQ120, Meso Scale Discovery) concentrations of CD14, Cystatin C, Serpin F2, Serpin G1 and Serpin C1 were quantified in all three EV-subfractions. For Cystatin C and Serpin F2, standard antibody sets of Meso Scale were used, the antibody set for Serpin C1, Serpin G1 and CD14 were custom-made. SoftMax Pro 7 Software (Molecular Devices) was used to analyze the data. Protein concentrations were expressed in $\mathrm{pg} / \mathrm{mL}$ and were divided by a standard quantity of synthetic liposomes to be able to correct for loss of pellet during the isolation steps. Additional information on specific antibodies can be found in the Supplemental Material section of our previous publication. ${ }^{\mathrm{b}}$ 


\section{Quantification of Tenascin-C EV levels}

Tenascin-C was quantified in the LDL-EV subfraction with a Human Tenascin C ELISA kit (AB213831) using an electrochemiluminescence immunoassay (Quickplex SQ120, Meso Scale Discovery, MSD) following manufacturer's protocol. EV TN-C levels were expressed in $\mathrm{pg} / \mathrm{ml}$ and were divided by the quantity measured synthetic liposomes which serves as internal control for the loss of pellet during isolation. ${ }^{c}$

\section{Quantification of $\operatorname{Lp}(a)$}

$\mathrm{Lp}$ (a) concentrations were measured with a latex-enhanced particle immunoturbidimetric assay in serum samples using the Cobas c702 (Roche) and the LPA2 Tinaquant Lp(a) Gen.2 kit from Cobas (LPA2: CAN 8723). Lp(a) was measured in nanomoles per liter with a particle enhanced immunoturbidimetric assay, where Lp(a) agglutinates with latex particles coated with anti-Lp(a) antibodies. The precipitate is determined turbidimetrically at 800/660 $\mathrm{nm}$. The measuring range of this assay is between 7 and $240 \mathrm{nmol} / \mathrm{L}$. More information regarding specific performance data, the repeatability, and intermediate precision can be found in the manufacturers' instructions. For more details on $\mathrm{Lp}$ (a) quantification we refer to the original publication. ${ }^{13}$ 


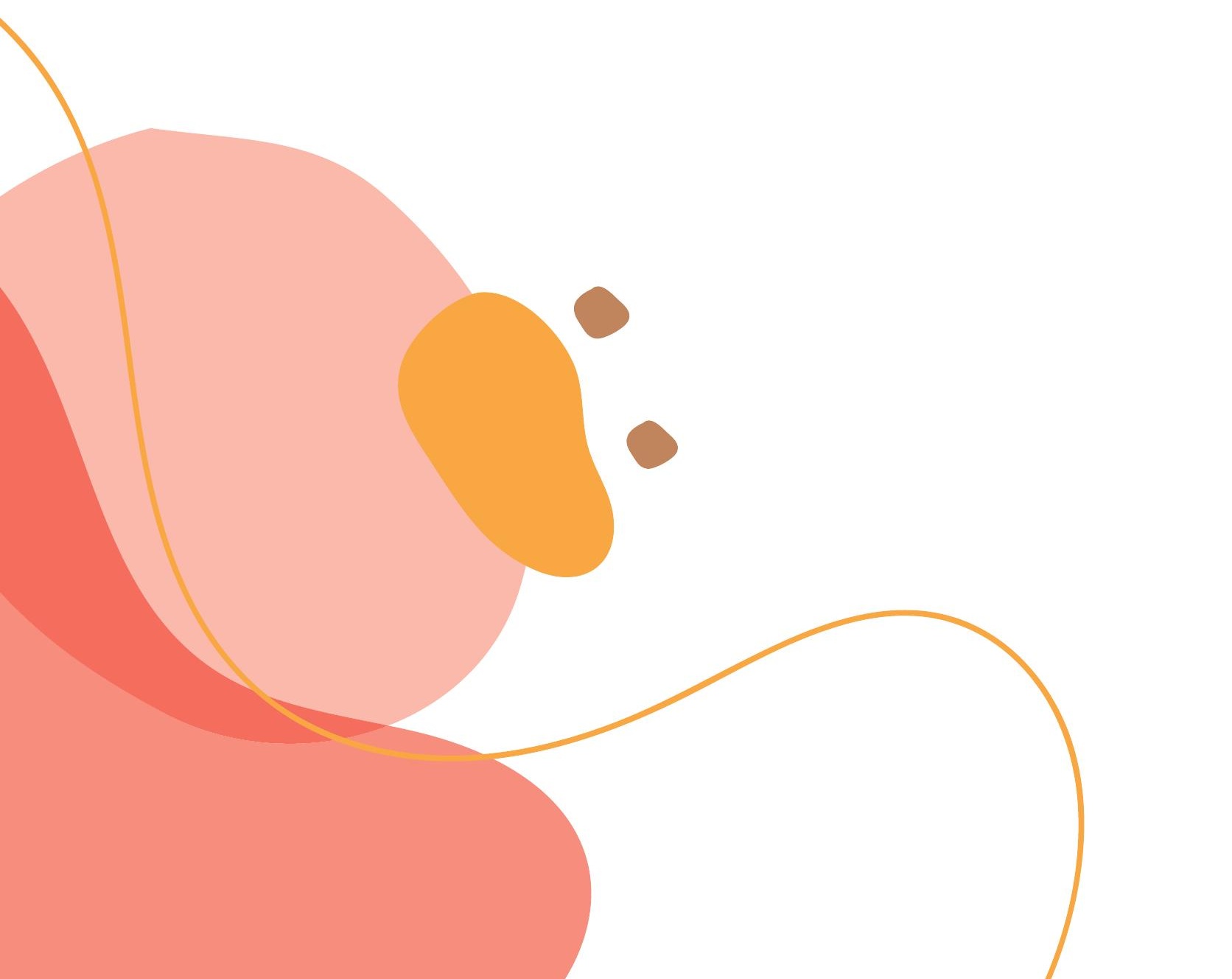




\section{The Taxinomisis Project. A multidisciplinary approach for the development of a new risk stratification model for patients with asymptomatic carotid artery stenosis}

Nathalie Timmerman

George Galyfos

Fragiska Sigala

Gert J. de Borst

Lazar Davidovic

Hans-Henning Eckstein

Dominique P.V. de Kleijn

Igor Koncar

Michalis D. Mantzaris

Gerard Pasterkamp

Vassiliki T. Potiska

Evangelos Andreakos

Dimitrios I. Fotiadis

All partners of the Taxinomisis Consortium 


\section{Abstract}

\section{Introduction}

Asymptomatic carotid artery stenosis (ACAS) may cause future stroke and therefore patients with ACAS require best medical treatment. Patients at high risk for stroke may opt for additional revascularization (either surgery or stenting) but the future stroke risk should outweigh the risk for peri/post-operative stroke/death. Current risk-stratification for patients with ACAS is largely based on outdated randomized controlled trials that lack the integration of improved medical therapies and risk factor control. Furthermore, recent circulating and imaging biomarkers for stroke have never been included in a risk stratification model. The TAXINOMISIS Project aims to develop a new risk stratification model for cerebrovascular complications in patients with ACAS and this will be tested through a prospective observational multicenter clinical trial performed in six major European vascular surgery centers.

\section{Methods and analysis}

The risk stratification model will compromise clinical-, circulating-, plaque- and imaging biomarkers. The prospective multicenter observational study will include 300 patients with 50-99\% ACAS. The primary endpoint is the three-year incidence of cerebrovascular complications. Biomarkers will be retrieved from plasma samples, brain MRI, carotid MRA and duplex ultrasound. The TAXINOMISIS Project will serve as a platform for the development of new computer tools that assess plaque progression based on radiology images and a lab-on-chip with genetic variants that could predict medication response in individual patients.

\section{Conclusion}

Results from the TAXINOMISIS study could potentially improve future risk stratification in patients with ACAS to assist personalized evidence-based treatment decision-making.

\section{Strengths and limitations of this study}

- This study will lead to the discovery of new biomarkers by investigating potential underlying pathobiological processes and combining existing data from European biobanks and cohort studies.

- Promising circulating biomarkers will be explored for carotid artery stenosis patients in the same biobanks.

- This is the first study that combines known and newly identified biomarkers of any kind (clinical-, imaging- and circulating biomarkers) in a multilevel risk stratification model.

- This new multilevel risk stratification model could potentially guide treatment decision for asymptomatic carotid artery stenosis. 
- Limitations include the need for external validation of this model in future studies that also take into account procedural risks of revascularization interventions. Thereafter, health technology assessment including feasibility and cost-effectiveness needs to be performed before implementation of the model in clinical practice.

\section{Introduction}

Stroke prevention is of utmost importance as it is associated with high mortality rates, accounting for 1.1 million deaths annually in Europe hereby being the third cause of death. ${ }^{1}$ Furthermore, stroke causes high morbidity with long-term disability and therefore accounts for a high socioeconomic burden. Moderate to severe carotid artery stenosis caused by atherosclerotic plaque formation resulting in progressive narrowing of the lumen is one of the major causes of ischemic stroke. Rupture or erosion of the plaque surface could lead to cerebrovascular thromboembolism and presentation of cerebral symptoms such as stroke, transient ischemic attack (TIA), or ocular symptoms such as amaurosis fugax or retinal infarction. Thromboembolisms are believed to account for $80 \%$ of all cerebrovascular symptoms while the remaining $20 \%$ is deemed to be of hemodynamic origin. ${ }^{1-3}$

Current treatment of patients with asymptomatic carotid artery stenosis (ACAS) consists of best medical treatment (BMT); including antiplatelet-, antihypertensive- and statin therapy, risk factor control and lifestyle coaching. ${ }^{1}$ If indicated, additional intervention with either surgery by carotid endarterectomy (CEA) or endovascular intervention (CAS, carotid artery stenting) may be considered. However, the criteria for defining a high-risk ACAS that necessitates revascularization are still not supported by high-level evidence according to the latest European Society for Vascular Surgery (ESVS) guidelines. ${ }^{1}$ Furthermore, as CEA and CAS are associated with a 30-day stroke/death rate of 1.7-3.1\% ${ }^{4}$, accurate patient risk stratification regarding future stroke risk is important for weighing up the indication for intervention.

For symptomatic patients, defined as those that presented with ipsilateral cerebrovascular symptoms in the previous six months, CEA and CAS have been proven to be effective in significant reduction of future stroke and stroke-related death. ${ }^{1}$ However, CEA or CAS for asymptomatic carotid artery stenosis (e.g. those that did not have ipsilateral cerebrovascular symptoms in the previous six months) remains controversial. ${ }^{1,4}$ Previous randomizedcontrolled trials (RCTs) suggested a modest benefit of CEA in asymptomatic patients, mainly in men younger than $75-80$ years with $\geq 60 \%$ stenosis with an average surgical risk (meaning no risk factors that increase procedural complications) ${ }^{4-7}$ However, these RCTs 
are outdated, with patient inclusion up to $1993^{5,7}$ or at maximum $2003^{6}$, when BMT did not consist of statins and antihypertensive therapy and more patients were active smokers. Therefore stroke risks in contemporary ACAS patients are considered to be lower, challenging the benefit of CEA., ${ }^{4,8}$

Previous studies have indicated subgroups of ACAS patients with high risk of stroke under BMT in which revascularization as addition to BMT may be beneficial. Markers for increased stroke risk on BMT include clinical factors (e.g. contralateral TIA or stroke ${ }^{9-11}$ ), cerebral imaging characteristics (e.g. silent infarction ${ }^{12}$, impaired cerebrovascular reserve ${ }^{13}$ ) as well as plaque characteristics on either duplex ultrasound (e.g. stenosis progression ${ }^{9,14}$, juxtaluminal black area ${ }^{15}$, plaque echolucency ${ }^{10,16}$ or plaque area ${ }^{10}$ ) or magnetic resonance angiography (MRA) (intraplaque hemorrhage ${ }^{17,18}$ ), as proposed in the latest ESVS guideline (2017). ${ }^{1}$ However, supporting evidence for these markers is based on small studies or nonprespecified subgroup analyses with BMT that is not analogous to current BMT. Furthermore, follow-up data regarding medication are often lacking and are thus not taken into account. ${ }^{11,19}$ More importantly, while combination of these markers could improve discriminative predicted ability for risk stratification ${ }^{10,15}$, thus far no studies have investigated the combination of all these high stroke risk markers. Furthermore, biobank initiatives across Europe ${ }^{20-24}$, analyzing blood samples and atherosclerotic plaque tissue, have identified potential circulating biomarkers which are associated with cardiovascular and cerebrovascular risk. ${ }^{25}$ However, current guidelines do not take these into account in advising patient risk stratification and treatment decision. Therefore, a prospective study in ACAS under current BMT is warranted to evaluate all potential predictors (clinical-, imaging characteristics and circulating biomarkers) combined in a risk stratification algorithm for stroke.

In addition to the change in pharmaceutical treatment and lifestyle management, recent reports also reveal a paradigm shift in the pathobiological concepts of the atherosclerotic plaque underlying the development of a major cardiovascular event. New pathobiological insights challenging the concept of the vulnerable plaque as sole underlying mechanism of symptomatic plaques have become available. ${ }^{26}$ Vulnerable plaques are characterized by a thin fibrous cap, a large necrotic core and abundant presence of inflammatory cells which have been shown to be the culprit lesions that rupture in fatal myocardial infarction and stroke. ${ }^{3}$ Studies have suggested that from all plaques with a vulnerable phenotype only a very small proportion (estimated at $<5 \%$ ) will rupture, whereas the remaining ones do not cause clinical events. ${ }^{27,28}$ Although vulnerable plaques are considered responsible for the majority of symptomatic patients, an increasing proportion of acute cerebrovascular events seems to originate from phenotypically stable plaques that lack these vulnerable 
characteristics with significant proteoglycan or glycosaminoglycan accumulation and higher collagen levels. ${ }^{3,26,29}$ Embolism originating from phenotypically stable plaque is probably caused by mechanisms of plaque erosion, yet such mechanisms are not fully understood. Conversely, it has been demonstrated that vulnerable plaques can lose their vulnerable characteristics, while stable plaques can become vulnerable over time depending on exogenous factors, such as medical treatment and lifestyle. ${ }^{30}$ Indeed, previous research showed a shift in carotid atherosclerotic plaque characteristics over the past decade. From 2002 to 2015, 50\% reduction of lipid-rich plaques and intraplaque hemorrhage was observed for both symptomatic and asymptomatic carotid plaques, concomitantly with improvement in cardiovascular risk factor management. ${ }^{31}$

The need for improvement in risk stratification to guide treatment for ACAS and new insights in underlying pathobiology have led to the initiation of the TAXINOMISIS project: "A multidisciplinary approach for risk stratification of patients with carotid artery disease". The Greek word TAXINOMISIS means "stratification". The overall aim of the project is to develop a novel multilevel risk stratification model for contemporary patients with ACAS in a prospective multicenter observational clinical trial (Objective 5, Table 1). This multilevel model will potentially integrate clinical-, plaque- and cerebral imaging characteristics as well as circulating biomarkers. During earlier phases of the TAXINOMISIS Project new potential biomarkers and risk stratification tools will be explored (Objectives 1-4, Table 1). This present report aims to describe the rationale and summarized objectives of the European TAXINOMISIS Project.

\section{Methods and Analysis}

The overall aim of the TAXINOMISIS project is to provide a novel multilevel risk stratification model for stroke in patients with ACAS. The main objectives of the project are stated in Table 1 and illustrated in Figure 1. First, underlying pathobiology of symptomatic carotid atherosclerotic plaques will be explored by studying gene expression profiles, cellular composition of plaques in combination with proteomic data (Objective 1). Second, new circulating biomarkers will be investigated with the aim to improve cardiovascular event risk prediction (Objective 1). Third, machine learning techniques will be used to combine available data from existing cohort- and biobank studies and explore additional susceptibility and protection factors for cerebrovascular symptoms (Objective 2). Fourth, the final novel multilevel risk stratification model will be developed in a prospective multicenter observational clinical trial (Objective 5). Fifth, new software tools will be developed including computerized models for plaque growth prediction (Objective 
3) and lab-on-chip pharmacogenomics (Objective 4) for assessment of preventive medication responsiveness. The plaque growth tool and lab-on-a-chip will be validated in the observational clinical trial (Objective 5). Finally, a cost-effectiveness analyses of the new risk stratification model as well as of novel computer tools will be performed (Objective 6). The project has been initiated at January $1^{\text {st }}, 2018$ and will last until December $31^{\text {st }}, 2023$. A total of 15 research centers and hospitals in 10 different European countries are involved in one or more of the objectives of the TAXINOMISIS Project (Table 1). The observational clinical study is performed in six major vascular centers (Athens, Barcelona, Belgrade, Genoa, Munich and Utrecht). Reporting of this study conforms to broad EQUATOR guidelines. $^{32}$

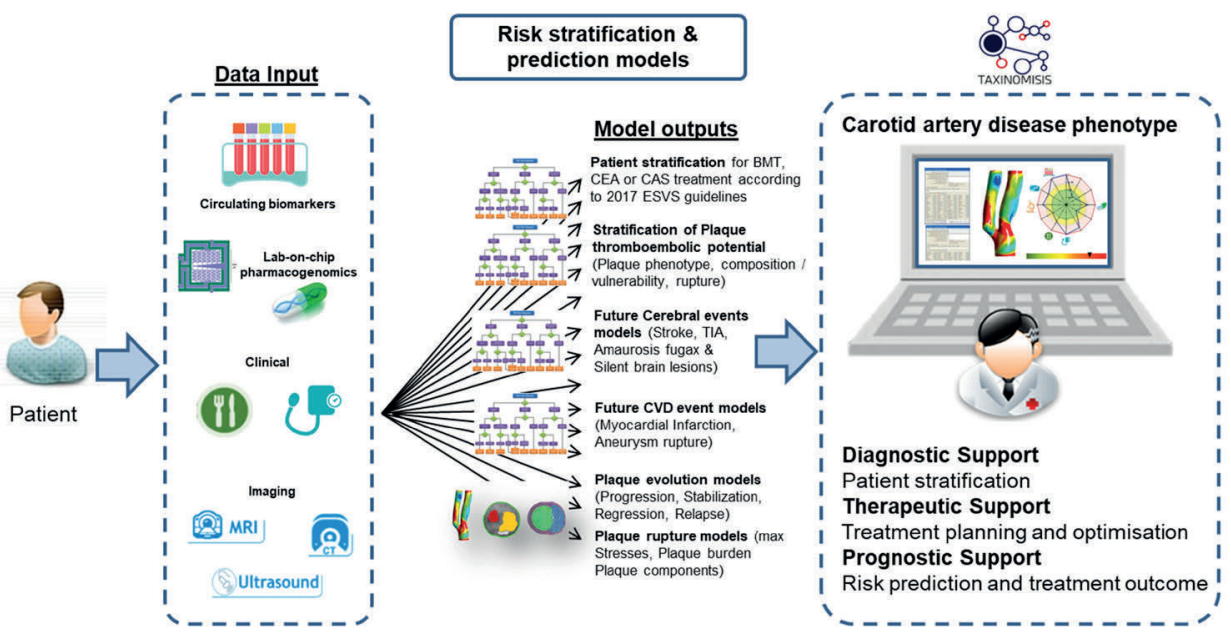

Figure 1. Concept of the TAXINOMISIS Project

\section{Unraveling the pathobiology underlying symptomatic plaques}

The gross histopathological classification of a vulnerable plaque is not sufficient to predict all cerebrovascular symptoms. ${ }^{26}$ It is therefore important to further identify molecular mechanisms that contribute to the thrombogenic potential of plaques. In Objective 1 the gene expression profiles (using bulk-RNA sequencing) and the cellular composition (using mass cytometry, CyTOF, in combination with single-cell RNA sequencing ${ }^{33}$ ) of symptomatic and asymptomatic plaques will be studied. This will be combined with available genomic, methylomic and proteomic data in order to identify key biological mechanisms of symptomatic plaques. These new insights could potentially lead to additional plaque- or circulating biomarkers that could be candidate markers for the risk stratification model (Objective 5). 
Table 1. Summary of the main objectives of the TAXINOMISIS Project

\begin{tabular}{ll}
\hline Objective 1 & $\begin{array}{l}\text { Exploring new risk and susceptibility factors for symptomatic carotid disease. } \\
\text { Unravel pathobiological mechanisms underlying symptomatic plaques by characterizing global } \\
\text { gene expression profiles and cellular composition of symptomatic and asymptomatic } \\
\text { atherosclerotic carotid lesions. } \\
\text { Assess the predictive ability of circulating biomarkers for carotid artery stenosis patients (plasma } \\
\text { ceramides and extracellular vesicles). }\end{array}$ \\
Objective 2 & $\begin{array}{l}\text { Integration of data from longitudinal cohort studies and biobanks (including clinical data, omics } \\
\text { data, plaque and circulating biomarkers) to dissect mechanisms mediating symptomatic carotid } \\
\text { artery disease for identification of susceptibility and protection factors by machine learning } \\
\text { techniques. }\end{array}$ \\
Objective 3 & $\begin{array}{l}\text { Development of novel computer tools for MRI and duplex ultrasound to predict plaque growth. } \\
\text { These tools will be validated in Objective 5. }\end{array}$ \\
Objective 4 & $\begin{array}{l}\text { Development of a lab-on-chip technology incorporating SNPs related to medical therapy } \\
\text { effectiveness and resistance to guide personalized medical treatment. Validation will be } \\
\text { performed in Objective 5. }\end{array}$ \\
Objective 5 & $\begin{array}{l}\text { Development of the multilevel risk stratification model in an observational multicenter clinical } \\
\text { trial including clinical, plaque- and brain imaging characteristics and circulating biomarkers } \\
\text { This multilevel risk stratification model will include possible predictors from Objective } 1 \text { and } 2 \\
\text { and known predictors from literature to evaluate the best combination of predictors for the risk } \\
\text { stratification model. } \\
\text { To perform cost-effective analyses of the multilevel risk stratification model, plaque growth } \\
\text { computer tools and lab-on chip device. }\end{array}$ \\
&
\end{tabular}

\section{New potential circulating biomarkers}

Plasma extracellular vesicles (EVs) and ceramides are potential circulating biomarkers for cardiovascular disease risk and will be specifically evaluated for carotid artery stenosis patients in Objective 1. EVs are bilayer membrane particles secreted by every cell of the body into body fluids such as blood. EVs contain lipids, nucleic acids and proteins of the cell of origin, therefore EVs are often referred to as a "liquid biopsy" of the cell/tissue of origin. ${ }^{34}$ EVs are involved in (patho)physiological processes including intercellular communication, coagulation and inflammation. Content of EVs was previously associated with acute coronary syndrome and metabolic syndrome. ${ }^{34,35}$. Also, four proteins in EVs (Cystatin C, Serpin G1, Serpin F2 and CD14) were associated with an increased risk of future vascular events in patients with manifest cardiovascular disease of any kind. ${ }^{36}$ In Objective 1 the predictive ability of these four EVs proteins for cerebrovascular disease risk will be investigated in patients with carotid artery stenosis.

Plasma ceramides are clinically relevant predictors for cardiovascular events. ${ }^{37}$ Elevated concentrations of circulating ceramides are associated with atherosclerotic plaque formation, ischemic heart disease, myocardial infarction, hypertension, type 2 diabetes mellitus, insulin resistance and obesity. In particular, specific plasma ceramides $\operatorname{Cer}(\mathrm{d} 18: 1 / 16: 0)$, $\operatorname{Cer}(\mathrm{d} 18: 1 / 18: 0)$ and $\operatorname{Cer}(\mathrm{d} 18: 1 / 24: 1)$ and its ratios with $\operatorname{Cer}(\mathrm{d} 18: 1 / 24: 0)$ 
were associated with cardiovascular death in three large coronary artery disease cohorts. ${ }^{38}$ Still, in carotid artery disease the potential predictive ability of plasma ceramides remains unexplored, and therefore, in Objective 1 the predictive ability of these circulating biomarkers for the risk of acute cerebrovascular complications in carotid artery disease will be evaluated. If EVs and/or plasma ceramides showed to be predictive for stroke, these will be incorporated as possible candidates for the final risk stratification model (Objective 5).

\section{Evaluating new biomarker signatures by integration of biobanks and longitudinal cohorts}

In Objective 2 available data from longitudinal cohort studies and biobanks (e.g. AtheroExpress $^{22}$, Munich Vascular Biobank ${ }^{20}$, Tampere Vascular Study ${ }^{23}$ and Young Finns Study ${ }^{24}$ ) will be combined and integrated. Types of available data are clinical, lifestyle, demographics, histopathological plaque data as well as "omics" data (including genomics, methylomics, transcriptomics and proteomics) and blood and serum biomarkers (proteins and lipids). Furthermore, data from Objective 1, also derived from the same patients in these biobanks, will be incorporated. ${ }^{22-24}$ Using machine learning techniques and mathematical models (e.g. data mining, cluster analysis, latent variable models and multivariate data analysis such as partial list squares), susceptibility and protection factors as well as molecular or biomarker signatures for cerebrovascular symptoms can be investigated. These could potentially be candidate predictors for the novel risk stratification model (Objective 5).

\section{Computational models for plaque growth}

The interest in advanced computational models as tools to predict plaque development and plaque growth has fast developed. ${ }^{39-43}$ Previously, 3D-reconstruction tools were developed using patient radiology imaging (MRA for carotid arteries ${ }^{42}$ or computed tomography angiography (CTA) for coronary arteries ${ }^{39}$ ) to analyze atherosclerotic plaque and morphology characteristics. Proof-of-concept studies have demonstrated that using mathematical models and simulations of blow flow as well as simulations of different stages contributing to atherosclerotic plaque formation (LDL transport into the vessel wall, recruitment and infiltration of monocytes, oxidation of LDL, differentiation of macrophages into foam cells and proliferation of smooth muscle cells), it was possible to predict regions that are prone to plaque progression. ${ }^{39-43}$ In Objective 3, using retrospective radiology data from TAXINOMISIS participating centers, a computational model for carotid arteries on CTA will be further developed based on the methodology previously used in coronary arteries ${ }^{39,44}$ and the MRA computational model for carotid arteries ${ }^{40-42}$ will be further refined for advanced carotid plaque lesions. Finally, both software tools will be validated for prediction of plaque growth in the prospective observational multicenter study (Objective 5) and its clinical utility in risk stratification will be established by relating plaque progression to future cerebrovascular symptoms. 


\section{Lab-on-chip pharmacogenomics}

Clopidogrel and statins are known for interpatient variability in efficacy and side effects. Part of this individual variability could be explained by genetic variations (single-nucleotide polymorphisms, SNPs) that influence pharmacokinetics and pharmacodynamics of drugs. Well-known are the CYP2C19*2/ CYP2C19*3 loss-of-function alleles for clopidogrel. The prodrug clopidogrel requires biotransformation into its active metabolite by the CYP2C19enzyme in the liver. Patients with these loss-of-function alleles have lower active metabolite concentrations, therefore diminished platelet inhibition and lower efficacy in preventing cardiovascular complications. ${ }^{45}$ For statins, multiple SNPs are identified that affect its pharmacokinetics, some influence protein transporters that are responsible for uptake or efflux of statins in the liver cell and others influencing the metabolism of statins (influencing CYP-isoenzymes). Some SNPs lead to decreased statin efficacy whereas others result in higher efficacy inducing toxicity (e.g. rhabdomyolysis). ${ }^{46}$ In Objective 4 a lab-on-chip device will be developed including the important SNPs for statins, clopidogrel and aspirin to assess drug response in the individual patient. A systematic literature search will be performed to identify most clinically relevant SNPs. A proof-of-concept lab-on-chip device has previously been developed and will be extended for more SNPs. ${ }^{47}$ This lab-on-chip could potentially guide personalized treatment by identifying non-responders and prevent unnecessary side effects. The lab-on-chip device will be validated in the observational clinical trial (Objective 5).

\section{Prospective observational multicenter clinical trial}

A prospective observational multicenter clinical trial will be performed to develop a new multilevel risk stratification model for cerebrovascular symptoms for patients with ACAS. Participating centers are six major vascular surgery clinics across Europe (Athens, Barcelona, Belgrade, Genoa, Munich and Utrecht). All hospitals have obtained ethical approval by their local ethics committees. The study will be conducted according to the Declaration of Helsinki. All patients must provide written informed consent before study participation. Patients with extracranial ACAS of 50-99\% are eligible for study participation. Full inclusion and exclusion criteria are displayed in Table 2. According to the 2017 ESVS guideline ${ }^{1}$, patients are defined as asymptomatic if they have not experienced ipsilateral cerebrovascular symptoms in the past six months. The recruitment period lasts from March 31, 2018 until December 31, 2020. Baseline characteristics of included patients by August 2020 are stated in Table 3.

\section{Candidate predictors for the risk stratification model}

The risk stratification model will potentially compromise clinical parameters, imaging (plaque and brain) characteristics and circulating biomarkers. Candidate predictors will 
be selected based on literature and new biomarkers identified in the earlier phase of the TAXINOMISIS project (Objective 1-2). Candidate predictors for the model based on previous studies include: (a) clinical parameters (demographics such as age, male sex ${ }^{48}$, medical comorbidities such as worse kidney function ${ }^{11,49}$, medical history of previous or contralateral stroke or TIA ${ }^{11,50}$, coronary artery disease, peripheral artery disease and cardiovascular risk factors ${ }^{50}$ such as diabetes, hypertension, smoking, hypercholesterolemia, high body mass index), (b) radiological characteristics of carotid plaque such as contralateral occlusion ${ }^{1}$, stenosis progression ${ }^{14}$, plaque morphology by duplex ultrasound (plaque echolucency ${ }^{16}$, grayscale median ${ }^{10}$, juxtaluminal black area ${ }^{15}$, plaque area ${ }^{10}$ ), plaque morphology by $\mathrm{MRI}^{17,18}$ (intraplaque hemorrhage, lipid-rich necrotic core, thinning/rupture of the fibrous cap) and, (c) circulating biomarkers such as LDL, HDL ${ }^{50}$, triglycerides, cholesterol, $\mathrm{Lp}(\mathrm{a}),{ }^{51}$ lipoprotein-associated phospholipase A2 activity $^{52}$, CRP ${ }^{53,54}$, homocysteine $e^{55}$, osteopontine ${ }^{23,56,57}$, matrix metalloproteinase ${ }^{20}$, plasma ceramides ${ }^{38}$, ApoA-1 auto-antibodies ${ }^{58}$, myeloperoxidase ${ }^{58}$, cystatin C, Serpin F2, Serpin G1 and CD14 ${ }^{36}$.

Table 2. An overview of the multicenter observational clinical study of TAXINOMISIS

\begin{tabular}{|c|c|}
\hline Primary aim & $\begin{array}{l}\text { To develop a new multilevel risk stratification model for the three-year risks of } \\
\text { cerebrovascular complications (ipsilateral stroke, TIA, transient or permanent retinal } \\
\text { infarction and silent brain ischemia) in patients with ACAS }\end{array}$ \\
\hline Secondary aim & $\begin{array}{l}\text { To validate computational models for plaque growth } \\
\text { To validate lab-on-chip pharmacogenomics for prediction drug response and side effects }\end{array}$ \\
\hline Study design & European multicenter observational clinical trial with three years of follow-up \\
\hline Inclusion criteria & $\begin{array}{l}\text { ACAS of } 50 \%-99 \% \text { (in the carotid bifurcation or internal carotid artery) assessed by } \\
\text { ultrasound (according to the NASCET criteria) }{ }^{62} \text {. } \\
\text { Patient is } 18 \text { years or older } \\
\text { Ability of the patient to participate in the follow-up examinations } \\
\text { Personally able and willing to give informed consent }\end{array}$ \\
\hline Exclusion criteria & $\begin{array}{l}\text { Carotid stenosis due to non-atherosclerotic causes (e.g. dissection, fibromuscular dysplasia, } \\
\text { carotid aneurysm or post-irradiation lesions) } \\
\text { Tandem carotid lesions (concomitantly stenosis of proximal common carotid artery or } \\
\text { brachiocephalic artery) } \\
\text { Restenosis after CEA or CAS } \\
\text { Increased risk of thromboembolic events (e.g. congenital or acquired hypercoagulability } \\
\text { conditions, active untreated cancer, atrial fibrillation, severe cardiomyopathy with ejection } \\
\text { fraction lower than 30\%) } \\
\text { Intracranial angioma or aneurysms } \\
\text { Previous hemorrhagic stroke } \\
\text { Planned any major surgery } \\
\text { Life expectancy < } 3 \text { years due to a pre-existing condition } \\
\text { Contra-indications to best medical therapy (statins, aspirin, clopidogrel) or contra- } \\
\text { indications for MRI examination }\end{array}$ \\
\hline
\end{tabular}




\begin{tabular}{|c|c|}
\hline & Total cohort $(n=275)$ \\
\hline \multicolumn{2}{|l|}{ Demographics } \\
\hline Male, n(\%) & $176(64.0)$ \\
\hline Age, mean (SD) & $70.0(8.5)$ \\
\hline \multicolumn{2}{|l|}{ Medical history } \\
\hline \multicolumn{2}{|l|}{ Ipsilateral carotid stenosis, n(\%) } \\
\hline $50-70 \%$ & $133(48.4)$ \\
\hline $70-99 \%$ & $142(51.6)$ \\
\hline \multicolumn{2}{|l|}{ Contralateral carotid stenosis, n(\%) } \\
\hline $0-50 \%$ & $160(58.2)$ \\
\hline $50-70 \%$ & $72(26.2)$ \\
\hline $70-99 \%$ & $28(10.2)$ \\
\hline $100 \%$ & $15(5.4)$ \\
\hline History of TIA or stroke, n (\%) & $65(24.3)$ \\
\hline History of stroke, n(\%) & $37(13.8)$ \\
\hline Previous contralateral CEA or CAS, n(\%) & $54(19.6)$ \\
\hline History of PAD, n(\%) & $52(18.9)$ \\
\hline History of CAD, n(\%) & $71(27.3)$ \\
\hline COPD, n(\%) & $23(8.4)$ \\
\hline Diabetes, n(\%) & $81(29.5)$ \\
\hline \multicolumn{2}{|l|}{ Risk factors } \\
\hline \multicolumn{2}{|l|}{ Smoking, n(\%) } \\
\hline Never & $75(27.3)$ \\
\hline Current & $74(26.9)$ \\
\hline Former & $126(45.8)$ \\
\hline LDL levels, mmol/L, median [IQR] & $2.4[1.9,3.0]$ \\
\hline HDL levels, mmol/L, median [IQR] & $1.3[1.1,1.5]$ \\
\hline Total cholesterol levels, mmol/L, median [IQR] & $4.3[3.6,5.0]$ \\
\hline Triglycerides levels, mmol/L, median [IQR] & $1.3[1.0,1.9]$ \\
\hline Hypertension, n(\%) & $218(83.2)$ \\
\hline Glomerular filtration rate, $\mathrm{mL} / \mathrm{min} / 1.73 \mathrm{~m} 2$, median $[\mathrm{IQR}]$ & $76.7[60.0,89.5]$ \\
\hline BMI, mean $(\mathrm{SD})$ & $26.7(3.9)$ \\
\hline \multicolumn{2}{|l|}{ Drug therapy } \\
\hline Antiplatelets, n(\%) & $247(89.8)$ \\
\hline Anticoagulants, n(\%) & $12(4.4)$ \\
\hline Lipid lowering drugs, n(\%) & $238(86.5)$ \\
\hline
\end{tabular}

History of stroke or TIA includes ipsilateral (more than six months prior to inclusion) or contralateral stroke, TIA, amaurosis fugax or retinal infarction. PAD is defined as atherosclerotic lesions in aorta-iliac or iliofemoral arteries, either treated conservatively or by intervention. CAD is defined as history of angina, myocardial infarction, percutaneous intervention or coronary bypass surgery. Hypertension and diabetes were defined as diagnosed by a medical doctor or use of specific medication. Antiplatelet drug comprises the use of aspirin, dipyridamole or any ADP-inhibitor. Lipid lowering drug use comprises the use of any lipid lowering drug.

Abbreviations: $\mathrm{n}(\%)$, frequencies; $\mathrm{SD}$, standard deviation; IQR, interquartile range; TIA, transient ischemic attack, CEA, carotid endarterectomy; CAS, carotid artery stenting; PAD, peripheral artery disease; CAD, coronary artery disease; COPD, chronic obstructive pulmonary disease; LDL, Low-density lipoprotein; HDL, high-density lipoprotein, BMI, Body mass index. 


\section{Outcomes}

The primary aim is to develop a risk stratification model for cerebrovascular symptoms for patients with ACAS. The primary endpoint is a composite endpoint including cerebrovascular symptoms (stroke, TIA, transient or permanent retinal ischemia and silent brain ischemia) during three years of follow-up. Secondary endpoints include periprocedural events, other cardiovascular events (myocardial infarction, abdominal aneurysm rupture or cardiovascular death), plaque progression determined by duplex or MRA and time to revascularization (CEA or CAS) during three years of follow-up.

\section{Sample size}

A total of 300 patients will be enrolled across all centers. The prespecified number of inclusion per center are: Utrecht (UMC), The Netherlands, 50 patients; University of Belgrade (UBEO), Serbia, 100 patients; Technischen Universität München (TUM), Germany: 50 patients; University of Genoa (USMI) Italy, 30 patients; University of Athens (NKUA) Greece, 50 patients; University of Barcelona (FCRB) Spain, 20 patients. A dropout of $10 \%$ is estimated. Based on previous studies, the combined incidence of TIA, stroke and ocular ischemia was estimated as $5 \%$ per year ${ }^{10,11,15}$ and silent brain ischemia as $10 \%$ per year ${ }^{12,59,60}$, resulting in a combined event-rate of $15 \%$ per year. After three year followup, 121 patients would reach the primary endpoint. According to the rule of thumb, 10 events per 1 predictor are needed for reliable prediction modelling. ${ }^{61}$ In the final prediction model a maximum of 12 predictors will be allowed which is considered sufficient and clinically applicable.

\section{Study procedures}

Study procedures include collection of clinical data (demographics, medical history and medication use), MRA and duplex ultrasound imaging of both carotid arteries to assess stenosis degree and carotid plaque characteristics, MRI brain for detecting silent brain ischemia and venous blood sampling for basic laboratory tests (creatinine, lipids, glucose, CRP, homocysteine, $\mathrm{Hb}$ and $\mathrm{Ht}$ ) and circulating biomarkers. These study procedures will be performed at study inclusion and once per year thereafter for a total duration of three years and will be combined with the regular follow-up visits at the outpatient clinic. Venous blood samples will be stored in freezers of participating centers at $-80^{\circ} \mathrm{C}$. At the end of the study, circulating biomarkers will be measured centrally to avoid measurement error across centers. During follow-up additional clinical data regarding cerebrovascular events and other cardiovascular events (including myocardial infarction, abdominal aneurysm rupture, cardiovascular death or all-cause mortality) and medication use will be collected. As the study is observational, this study will not interfere with physician's treatment policy for patients with ACAS. When the treating vascular surgeon decides that CEA or CAS is 
required, patients can still be included in this study. This will be taken into account in dataanalysis. Biomarker data (brain ischemia on MRI brain, carotid plaque characteristics on MRA and ultrasound of carotid arteries and circulating biomarkers) collected in the observational study is centrally analyzed by independent researchers. Biomarker data are blinded to the treating vascular surgeons to avoid interference with the treatment decision of their ACAS patients.

During CEA, the atherosclerotic plaque will be collected. Immunohistochemical staining for histological plaque characteristics will be performed to determine plaque vulnerability by assessing the amount of lipid core, smooth muscle cells, macrophages, calcification, intraplaque hemorrhage, intraplaque microvessels and collagen. The well-validated and appreciated standardized protocol of the Athero-Express Biobank will be used for plaque analyses. $^{22}$

\section{Data management}

Data will be collected in a predefined electronic case-report forms (eCRF) through a secured web-implementation in Open Clinica. Radiology images will be stored in DICOM format and will be collected in a secured online application, specially developed for TAXINOMISIS. Central evaluation of biomarker data (MRI, MRA, ultrasound) and measurements (regarding circulating markers) ensures uniformity of data analyses. Data will be regularly checked for completeness, consistency and quality. Participating centers will be contacted to complement missing or incomplete data. The TAXINOMISIS Project complies with General Data Protection Regulation (GDPR) of the European Union.

\section{Statistical analysis}

Cox-proportional hazard regression modelling will be used for model development. Potential biomarkers that show an independent association with cerebrovascular events will be included. This model will be further reduced using backward stepwise regression analyses based on Akaike information criterion. Calibration of the model will be evaluated through visual inspection of the calibration plot. The discriminative performance of the model will be expressed by Harrell's c-index and the net-reclassification index (NRI). Bootstrapping will be used for internal validation to estimate the final model's potential for optimism and overfitting. ${ }^{61}$ Association of biomarkers with secondary endpoints (including peri-procedural events, plaque progression and other cardiovascular events will be analyzed in sub analyses by Cox-proportional hazard regression analyses in case of time-to-event data or where appropriate by linear or logistic regression analyses. 


\section{Discussion}

In the TAXINOMISIS Project a novel multilevel risk stratification model for cerebrovascular complications of patients with ACAS will be developed. While previous studies have suggested clinical risk factors and imaging- and circulating biomarkers for high-risk patients, this is the first study that combines all these markers in one risk stratification model in patients receiving current standards of BMT. This model could potentially guide personalized treatment decision by identifying those at high risk of future cerebrovascular events that qualify for revascularization procedure in addition to BMT. The main limitation is that this risk stratification model will still need external validation in future studies. It should be noted that this model does not incorporate procedural risks of CEA or CAS, an important factor in determining the indication of revascularization. Procedural risks differ between CEA or CAS, with higher stroke/death rate after CAS, and depend on surgeons' or interventionists' experience as well as patient characteristics such as anatomy and comorbidities. ${ }^{1}$ Indeed, previous reports based on real-world registry data have noticed high variability in stroke/death rates across clinics. ${ }^{1}$ Since the number of revascularization procedures in this study is expected to be low as well as the observational study design, this factor could not be incorporated in the model and should require further attention in future studies. Before possible application of a model in clinical practice, health technology assessment including the feasibility and cost-effectiveness needs to be performed.

\section{Conclusions}

A new multilevel risk stratification model for patients with ACAS will be developed combining clinical-, imaging- and circulating biomarkers that have been associated with increased risk of future cerebrovascular symptoms. After external validation, this model aims to guide treatment decision making by identifying high-risk patients that opt for revascularization and prevent unnecessary procedural risks of revascularization in low-risk patients. 


\section{Financial support}

This work has received funding from the European Union's Horizon 2020 research and innovation program under grant agreement No 755320.

\section{Declarations of interest}

None

\section{Trial registration number}

The observational clinical trial has been registered at ClinicalTrials.gov (NCT03495830)

\section{Ethics and dissemination}

This work has received funding from the European Union's Horizon 2020 research and innovation program under grant agreement No 755320, as part of the TAXINOMISIS project. The observational clinical trial has been approved by the local ethics committees of all participating centers. Results will be presented at international conferences and published in peer-reviewed journals. 


\section{References}

1 Naylor AR, Ricco J-B, de Borst GJ, Debus S, de Haro J, Halliday A, et al. Editor's Choice - Management of Atherosclerotic Carotid and Vertebral Artery Disease: 2017 Clinical Practice Guidelines of the European Society for Vascular Surgery (ESVS). Eur J Vasc Endovasc Surg 2018;55(1):3-81.

2 Grotta JC. Carotid Stenosis. N Engl J Med 2013;369(12):1143-50.

3 Sigala F, Oikonomou E, Antonopoulos AS, Galyfos G, Tousoulis D. Coronary versus carotid artery plaques. Similarities and differences regarding biomarkers morphology and prognosis. Curr Opin Pharmacol 2018;39:9-18.

4 Abbott AL, Brunser AM, Giannoukas A, Harbaugh RE, Kleinig T, Lattanzi S, et al. Misconceptions regarding the adequacy of best medical intervention alone for asymptomatic carotid stenosis. J Vasc Surg 2020;71(1):257-69.

5 Mayberg MR. Endarterectomy for Asymptomatic Carotid Artery Stenosis. JAMA 1995;273(18):1459.

6 Halliday A, Harrison M, Hayter E, Kong X, Mansfield A, Marro J, et al. 10-year stroke prevention after successful carotid endarterectomy for asymptomatic stenosis (ACST-1): a multicentre randomised trial. Lancet 2010;376(9746):1074-84.

7 Hobson RW, Weiss DG, Fields WS, Goldstone J, Moore WS, Towne JB, et al. Efficacy of Carotid Endarterectomy for Asymptomatic Carotid Stenosis. N Engl J Med 1993;328(4):221-7.

8 Abbott AL, Paraskevas KI, Kakkos SK, Golledge J, Eckstein H-H, Diaz-Sandoval LJ, et al. Systematic Review of Guidelines for the Management of Asymptomatic and Symptomatic Carotid Stenosis. Stroke 2015;46(11):3288-301.

9 Hirt LS. Progression Rate and Ipsilateral Neurological Events in Asymptomatic Carotid Stenosis. Stroke 2014;45(3):702-6.

10 Nicolaides AN, Kakkos SK, Kyriacou E, Griffin M, Sabetai M, Thomas DJ, et al. Asymptomatic internal carotid artery stenosis and cerebrovascular risk stratification. J Vasc Surg 2010;52(6):1486-1496.e5.

11 Nicolaides AN, Kakkos SK, Griffin M, Sabetai M, Dhanjil S, Tegos T, et al. Severity of Asymptomatic Carotid Stenosis and Risk of Ipsilateral Hemispheric Ischaemic Events: Results from the ACSRS Study. Eur $J$ Vasc Endovasc Surg 2005;30(3):275-84.

12 Kakkos SK, Sabetai M, Tegos T, Stevens J, Thomas D, Griffin M, et al. Silent embolic infarcts on computed tomography brain scans and risk of ipsilateral hemispheric events in patients with asymptomatic internal carotid artery stenosis. J Vasc Surg 2009;49(4):902-9.

13 Gupta A, Chazen JL, Hartman M, Delgado D, Anumula N, Shao H, et al. Cerebrovascular Reserve and Stroke Risk in Patients With Carotid Stenosis or Occlusion. Stroke 2012;43(11):2884-91.

14 Kakkos SK, Nicolaides AN, Charalambous I, Thomas D, Giannopoulos A, Naylor AR, et al. Predictors and clinical significance of progression or regression of asymptomatic carotid stenosis. J Vasc Surg 2014;59(4):956-967.e1.

15 Kakkos SK, Griffin MB, Nicolaides AN, Kyriacou E, Sabetai MM, Tegos T, et al. The size of juxtaluminal hypoechoic area in ultrasound images of asymptomatic carotid plaques predicts the occurrence of stroke. $J$ Vasc Surg 2013;57(3):609-618.e1.

16 Gupta A, Kesavabhotla K, Baradaran H, Kamel H, Pandya A, Giambrone AE, et al. Plaque Echolucency and Stroke Risk in Asymptomatic Carotid Stenosis. Stroke 2015;46(1):91-7.

17 Gupta A, Baradaran H, Schweitzer AD, Kamel H, Pandya A, Delgado D, et al. Carotid Plaque MRI and Stroke Risk. Stroke 2013;44(11):3071-7.

18 Huibers A, de Borst GJ, Wan S, Kennedy F, Giannopoulos A, Moll FL, et al. Non-invasive Carotid Artery Imaging to Identify the Vulnerable Plaque: Current Status and Future Goals. Eur J Vasc Endovasc Surg 2015;50(5):563-72.

19 Abbott AL, Donnan GA. Does the 'High Risk' Patient with Asymptomatic Carotid Stenosis Really Exist? Eur J Vasc Endovasc Surg 2008;35(5):524-33.

20 Heider P, Pelisek J, Poppert H, Eckstein H-H. Evaluation of Serum Matrix Metalloproteinases as Biomarkers for Detection of Neurological Symptoms in Carotid Artery Disease. Vasc Endovascular Surg 2009;43(6):551-60.

21 Matic LP, Jesus Iglesias M, Vesterlund M, Lengquist M, Hong M-G, Saieed S, et al. Novel Multiomics Profiling of Human Carotid Atherosclerotic Plaques and Plasma Reveals Biliverdin Reductase B as a Marker of Intraplaque Hemorrhage. JACC Basic to Transl Sci 2018;3(4):464-80. 
22 Verhoeven BAN, Velema E, Schoneveld AH, de Vries JPPM, de Bruin P, Seldenrijk CA, et al. Atheroexpress: differential atherosclerotic plaque expression of mRNA and protein in relation to cardiovascular events and patient characteristics. Rationale and design. Eur J Epidemiol 2004;19(12):1127-33.

23 Sulkava M, Raitoharju E, Levula M, Seppälä I, Lyytikäinen L-P, Mennander A, et al. Differentially expressed genes and canonical pathway expression in human atherosclerotic plaques - Tampere Vascular Study. Sci Rep 2017;7(1):41483.

24 Raitakari OT, Juonala M, Ronnemaa T, Keltikangas-Jarvinen L, Rasanen L, Pietikainen M, et al. Cohort Profile: The Cardiovascular Risk in Young Finns Study. Int J Epidemiol 2008;37(6):1220-6.

25 Martinez E, Martorell J, Riambau V. Review of serum biomarkers in carotid atherosclerosis. J Vasc Surg 2020;71(1):329-41.

26 Pasterkamp G, den Ruijter HM, Libby P. Temporal shifts in clinical presentation and underlying mechanisms of atherosclerotic disease. Nat Rev Cardiol 2017;14(1):21-9.

27 Stone GW, Maehara A, Lansky AJ, de Bruyne B, Cristea E, Mintz GS, et al. A Prospective Natural-History Study of Coronary Atherosclerosis. N Engl J Med 2011;364(3):226-35.

28 Burke AP, Kolodgie FD, Farb A, Weber DK, Malcom GT, Smialek J, et al. Healed plaque ruptures and sudden coronary death: evidence that subclinical rupture has a role in plaque progression. Circulation 2001;103(7):934-40.

29 Arbab-Zadeh A, Fuster V. The Myth of the "Vulnerable Plaque": Transitioning From a Focus on Individual Lesions to Atherosclerotic Disease Burden for Coronary Artery Disease Risk Assessment. J Am Coll Cardiol 2015;65(8):846-55.

30 Mann J, Davies MJ. Mechanisms of progression in native coronary artery disease: role of healed plaque disruption. Heart 1999;82(3):265-8.

31 van Lammeren GW, den Ruijter HM, Vrijenhoek JEP, van der Laan SW, Velema E, de Vries J-PPM, et al. Time-Dependent Changes in Atherosclerotic Plaque Composition in Patients Undergoing Carotid Surgery. Circulation 2014;129(22):2269-76.

32 Simera I, Moher D, Hoey J, Schulz KF, Altman DG. A catalogue of reporting guidelines for health research. Eur J Clin Invest 2010;40(1):35-53.

33 Cole JE, Park I, Ahern DJ, Kassiteridi C, Danso Abeam D, Goddard ME, et al. Immune cell census in murine atherosclerosis: cytometry by time of flight illuminates vascular myeloid cell diversity. Cardiovasc Res 2018;114(10):1360-71.

34 Kranendonk ME, de Kleijn DP, Kalkhoven E, Kanhai DA, Uiterwaal CS, van der Graaf Y, et al. Extracellular vesicle markers in relation to obesity and metabolic complications in patients with manifest cardiovascular disease. Cardiovasc Diabetol 2014;13(1):37.

35 de Hoog VC, Timmers L, Schoneveld AH, Wang J-W, van de Weg SM, Sze SK, et al. Serum extracellular vesicle protein levels are associated with acute coronary syndrome. Eur Hear Journal Acute Cardiovasc Care 2013;2(1):53-60.

36 Kanhai DA, Visseren FLJ, van der Graaf Y, Schoneveld AH, Catanzariti LM, Timmers L, et al. Microvesicle protein levels are associated with increased risk for future vascular events and mortality in patients with clinically manifest vascular disease. Int J Cardiol 2013;168(3):2358-63.

37 Havulinna AS, Sysi-Aho M, Hilvo M, Kauhanen D, Hurme R, Ekroos K, et al. Circulating Ceramides Predict Cardiovascular Outcomes in the Population-Based FINRISK 2002 Cohort. Arterioscler Thromb Vasc Biol 2016;36(12):2424-30.

38 Laaksonen R, Ekroos K, Sysi-Aho M, Hilvo M, Vihervaara T, Kauhanen D, et al. Plasma ceramides predict cardiovascular death in patients with stable coronary artery disease and acute coronary syndromes beyond LDL-cholesterol. Eur Heart J 2016;37(25):1967-76.

39 Sakellarios A, Bourantas C V., Papadopoulou S-L, Tsirka Z, de Vries T, Kitslaar PH, et al. Prediction of atherosclerotic disease progression using LDL transport modelling: a serial computed tomographic coronary angiographic study. Eur Hear J - Cardiovasc Imaging 2017;18(1):11-8.

40 Filipovic N, Teng Z, Radovic M, Saveljic I, Fotiadis D, Parodi O. Computer simulation of three-dimensional plaque formation and progression in the carotid artery. Med Biol Eng Comput 2013;51(6):607-16.

41 Sakellarios AI, Bizopoulos P, Papafaklis MI, Athanasiou L, Exarchos T, Bourantas C V., et al. Natural History of Carotid Atherosclerosis in Relation to the Hemodynamic Environment. Angiology 2017;68(2):109-18.

42 Sakellarios AI, Stefanou K, Siogkas P, Tsakanikas VD, Bourantas C V., Athanasiou L, et al. Novel methodology for $3 \mathrm{D}$ reconstruction of carotid arteries and plaque characterization based upon magnetic resonance imaging carotid angiography data. Magn Reson Imaging 2012;30(8):1068-82. 
43 Sakellarios AI, Raber L, Bourantas C V., Exarchos TP, Athanasiou LS, Pelosi G, et al. Prediction of Atherosclerotic Plaque Development in an In Vivo Coronary Arterial Segment Based on a Multilevel Modeling Approach. IEEE Trans Biomed Eng 2017;64(8):1721-30.

44 Athanasiou L, Rigas G, Sakellarios AI, Exarchos TP, Siogkas PK, Bourantas C V., et al. Three-dimensional reconstruction of coronary arteries and plaque morphology using CT angiography - comparison and registration with IVUS. BMC Med Imaging 2016;16(1):9.

45 Mega JL, Simon T, Collet J-P, Anderson JL, Antman EM, Bliden K, et al. Reduced-Function CYP2C19 Genotype and Risk of Adverse Clinical Outcomes Among Patients Treated With Clopidogrel Predominantly for PCI. JAMA 2010;304(16):1821.

46 Guan Z, Wu K, Li R, Yin Y, Li X, Zhang S, et al. Pharmacogenetics of statins treatment: Efficacy and safety. J Clin Pharm Ther 2019;44(6):858-67.

47 Zhang L, Cai Q, Wiederkehr RS, Fauvart M, Fiorini P, Majeed B, et al. Multiplex SNP genotyping in whole blood using an integrated microfluidic lab-on-a-chip. Lab Chip 2016;16(20):4012-9.

48 Hellings WE, Pasterkamp G, Verhoeven BAN, De Kleijn DP V, De Vries J-PPM, Seldenrijk KA, et al. Gender-associated differences in plaque phenotype of patients undergoing carotid endarterectomy. J Vasc Surg 2007;45(2):289-96.

49 Wesseling M, van Koeverden ID, van Lammeren GW, van der Laan SW, Haitjema S, de Vries J-PPM, et al. Impaired kidney function is associated with intraplaque hemorrhage in patients undergoing carotid endarterectomy. Atherosclerosis 2017;266:128-35.

50 D’Agostino RB, Vasan RS, Pencina MJ, Wolf PA, Cobain M, Massaro JM, et al. General Cardiovascular Risk Profile for Use in Primary Care. Circulation 2008;117(6):743-53.

51 Rigamonti F, Carbone F, Montecucco F, Bonaventura A, Liberale L, Burger F, et al. Serum lipoprotein (a) predicts acute coronary syndromes in patients with severe carotid stenosis. Eur J Clin Invest 2018;48(3):e12888.

52 Oei H-HS, van der Meer IM, Hofman A, Koudstaal PJ, Stijnen T, Breteler MMB, et al. LipoproteinAssociated Phospholipase A2 Activity Is Associated With Risk of Coronary Heart Disease and Ischemic Stroke. Circulation 2005;111(5):570-5.

53 Corrado E, Rizzo M, Tantillo R, Muratori I, Bonura F, Vitale G, et al. Markers of Inflammation and Infection Influence the Outcome of Patients With Baseline Asymptomatic Carotid Lesions. Stroke 2006;37(2):482-6.

54 Ridker PM, Rifai N, Rose L, Buring JE, Cook NR. Comparison of C-Reactive Protein and LowDensity Lipoprotein Cholesterol Levels in the Prediction of First Cardiovascular Events. $N$ Engl J Med 2002;347(20):1557-65.

55 Duschek N, Ghai S, Sejkic F, Falkensammer J, Skrinjar E, Huber K, et al. Homocysteine Improves Risk Stratification in Patients Undergoing Endarterectomy for Asymptomatic Internal Carotid Artery Stenosis. Stroke 2013;44(8):2311-4.

56 de Kleijn DP V., Moll FL, Hellings WE, Ozsarlak-Sozer G, de Bruin P, Doevendans PA, et al. Local Atherosclerotic Plaques Are a Source of Prognostic Biomarkers for Adverse Cardiovascular Events. Arterioscler Thromb Vasc Biol 2010;30(3):612-9.

57 Carbone F, Rigamonti F, Burger F, Roth A, Bertolotto M, Spinella G, et al. Serum levels of osteopontin predict major adverse cardiovascular events in patients with severe carotid artery stenosis. Int J Cardiol 2018;255:195-9.

58 Vuilleumier N, Montecucco F, Spinella G, Pagano S, Bertolotto M, Pane B, et al. Serum levels of antiapolipoprotein A-1 auto-antibodies and myeloperoxidase as predictors of major adverse cardiovascular events after carotid endarterectomy. Thromb Haemost 2013;109(04):706-15.

59 Streifler JY, den Hartog AG, Pan S, Pan H, Bulbulia R, Thomas DJ, et al. Ten-year risk of stroke in patients with previous cerebral infarction and the impact of carotid surgery in the Asymptomatic Carotid Surgery Trial. Int J Stroke 2016;11(9):1020-7.

60 Bonati LH, Jongen LM, Haller S, Flach HZ, Dobson J, Nederkoorn PJ, et al. New ischaemic brain lesions on MRI after stenting or endarterectomy for symptomatic carotid stenosis: a substudy of the International Carotid Stenting Study (ICSS). Lancet Neurol 2010;9(4):353-62.

61 Moons KGM, Kengne AP, Woodward M, Royston P, Vergouwe Y, Altman DG, et al. Risk prediction models: I. Development, internal validation, and assessing the incremental value of a new (bio)marker. Heart 2012:683-90. 
62 Staikov IN, Arnold M, Mattle H, Remonda L, Sturzenegger M, Baumgartner RW, et al. Comparison of the ECST, CC, and NASCET grading methods and ultrasound for assessing carotid stenosis. J Neurol 2000;247(9):681-6. 



\section{PART V}

Summary and Discussion

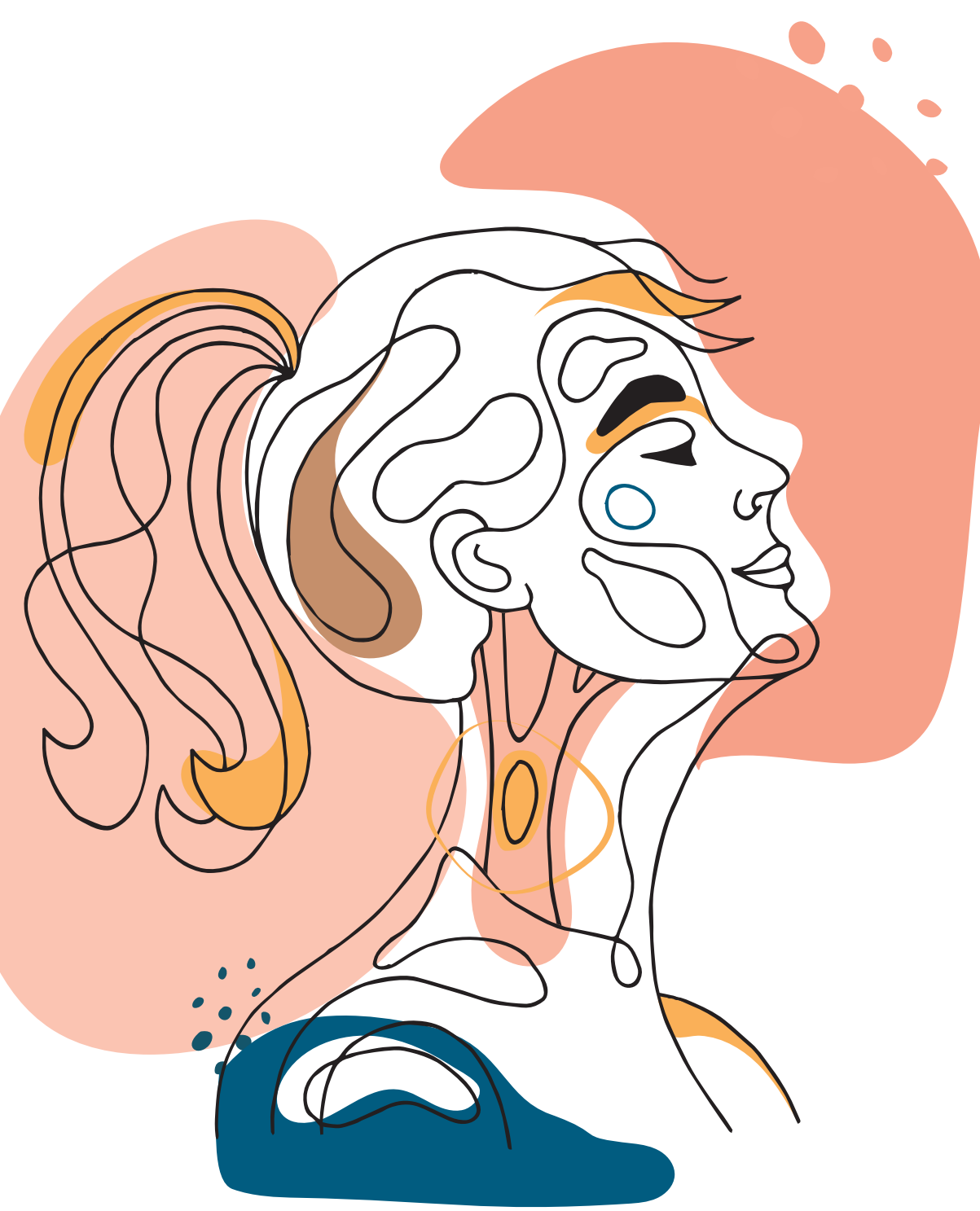




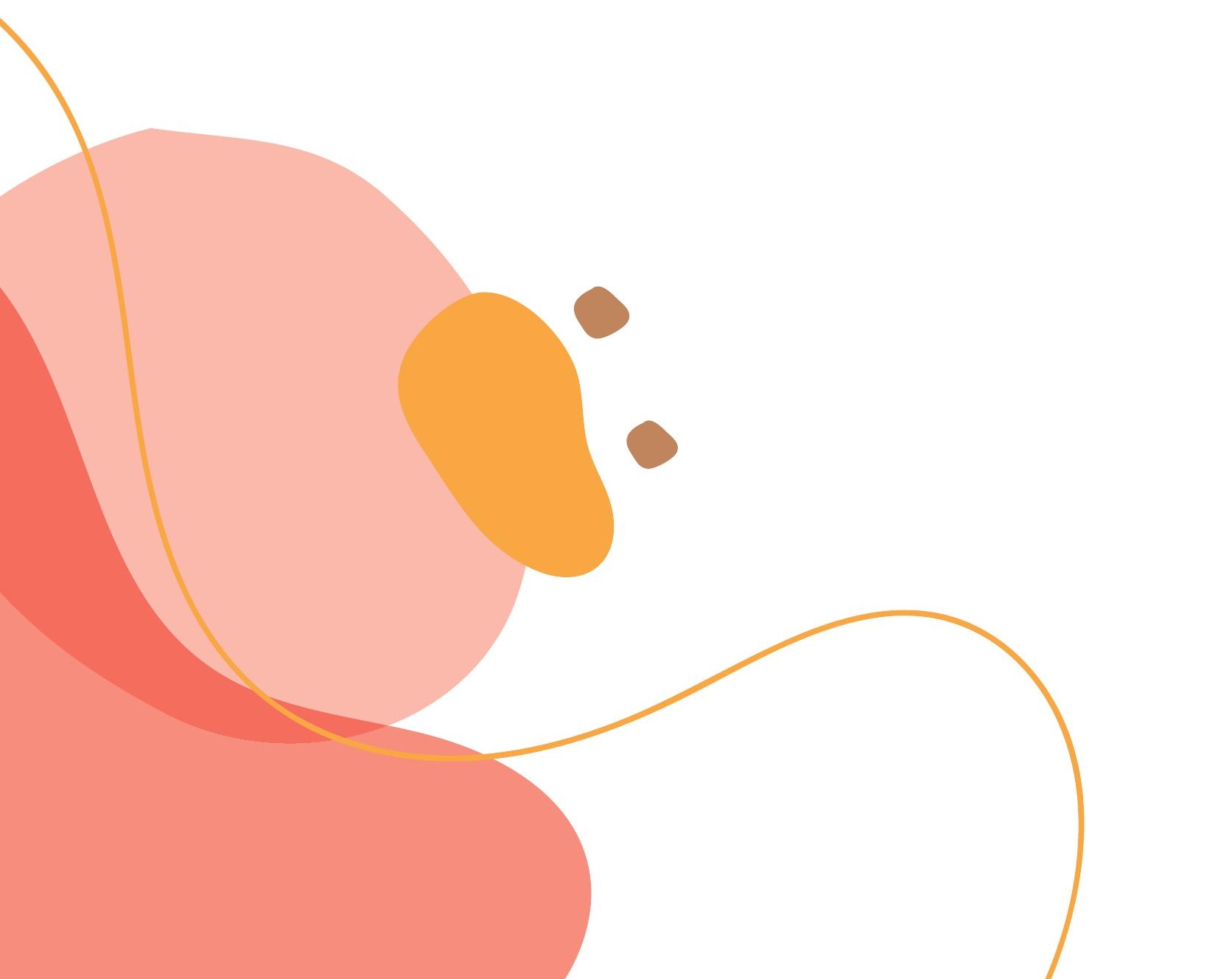


Summarizing discussion, future perspectives, and conclusions 
Identification of patients at highest risk for secondary cardiovascular events is becoming increasingly important considering the recent progress made in novel preventive therapies (add-on therapies). ${ }^{1-6}$ Since add-on therapies are accompanied by potentially hazardous side effects and high costs, patient-tailored treatment strategies are necessary. Risk stratification tools could assist physicians in selecting which patients benefit the most from intensive medical prevention.

The present thesis described different types of biomarkers for future cardiovascular events in patients undergoing carotid endarterectomy (CEA). These biomarkers were also associated to histological characteristics of the carotid plaque in order to increase our understanding of the complex mechanisms of atherosclerosis. This chapter provides a summarizing discussion and future perspectives in light of this thesis.

\section{A polygenic risk score as predictor for future cardiovascular events}

Recent studies have implied that a polygenic risk score (MetaGRS) summarizing 1.7 million genetic variants (SNPs) for coronary artery disease is predictive for first- and recurrent coronary events. ${ }^{7,8}$ In Chapter 3 we demonstrated that high genetic risk patients (high MetaGRS) had a higher 3-year risk for secondary cardiovascular events after CEA compared to low genetic risk patients. Surprisingly, this association was independent from traditional cardiovascular risk factors including a positive family history for cardiovascular disease (CVD). Moreover, we found that a positive family history for CVD was also related to an increased risk of secondary cardiovascular events, independent from MetaGRS. Our findings highlight that MetaGRS and family history, to some extent, capture different prognostic information regarding cardiovascular event risk. These non-overlapping associations may be explained by the hypothesis that the MetaGRS captures common genetic variants associated with CVD at population level, whereas CVD in families may arise from rare family-specific genetic variants. In addition, family history of CVD may also capture other non-genetic (environmental) factors influencing CVD risk. Future studies should explore the added prognostic value of MetaGRS for risk prediction of secondary events in CEA patients.

\section{Cerebral imaging characteristics in relation to recurrent or postoperative events}

Silent brain ischemia on MRI diffusion weighted imaging (DWI-lesions) and white matter lesions (WMLs) are proposed markers for perioperative and long-term cerebrovascular events. ${ }^{9}$ DWI-lesions become apparent within minutes after a hypoxic moment (either due to thromboembolisms or hypoperfusion) and resolve after days. DWI-lesions generally do not result in clinical neurological symptoms or permanent brain damage on imaging ${ }^{10}$, but these DWI-lesions are established markers for increased procedural and future cerebrovascular events. ${ }^{11,12}$ In Chapter 4 we showed that symptomatic patients who suffered from recurrent ischemia (detected on MR-DWI) during the waiting period before CEA, 
more frequently had histological intraplaque hemorrhage (IPH) in their carotid plaque compared to those without recurrent ischemia. This identifies IPH as a marker for recurrent brain ischemia, also illustrated by other studies that examined IPH in vivo on MR plaque imaging. ${ }^{13,14}$ However, the specificity of the marker IPH could be low, exemplified by the finding that a large proportion of patients without recurrent brain ischemia also showed histological IPH. Since different stages of IPH have been recognized ${ }^{15}$ and can be visualized by MR imaging ${ }^{16}$, it would be relevant for future studies to determine the predictive value of different IPH stages in order to increase its specificity. In the future, IPH (or specific stages of IPH) on MR plaque imaging at hospital presentation may be a useful marker to prioritize patients most at risk for recurrent ischemia in whom urgent or expedited CEA may be appropriate.

As opposed to MR plaque, cerebral imaging (MRI or CT) is part of the diagnostic work-up for CEA patients. Cerebral white matter lesions (WMLs) are surrogates for cerebral small vessel disease and have been associated with future cerebrovascular events, cognitive decline and dementia. ${ }^{17-19}$ In Chapter 5 we addressed the clinical relevance of reported cerebral small vessel disease (composed of WMLs and lacunar infarct) on routine preoperative imaging for the postoperative outcome of symptomatic CEA patients. Reported small vessel disease was related to a generally increased 3-year risk of cardiovascular death, but not specifically related to an increased stroke risk. Future studies should examine whether it is relevant to incorporate this easy obtainable marker in risk prediction algorithms estimating individual cardiovascular outcome for patient-tailored preventive treatment.

\section{Circulating biomarkers for future cardiovascular events}

Chapters 6, 7, 8, 9 and 10 highlighted several circulating markers, determined in preoperative plasma and plasma extracellular vesicles (EVs), that were associated with increased 3-year postoperative risk of major cardiovascular events (MACE) after CEA. Most markers have been previously associated with primary- or secondary events in coronary artery disease populations but have never been investigated in CEA patients or in plasma EVs. High levels of plasma lipoprotein (a), Lp(a), were associated with increased 3-year risk of MACE, mainly driven by the increased perioperative (30 day) risk of MACE (Chapter 7). In plasma EVs, increased protein levels (CD14, Cystatin C, Serpin F2, Serpin $\mathrm{C} 1$ and Tenascin-C) and bioactive lipids (1 ceramide and 4 ratios of ceramides) were associated with increased 3-year risk of MACE after CEA (Chapter 6, 8, 9 and 10). Since nonlinear associations with the risk of MACE were observed, future studies should validate clinically relevant cut-off values of these markers for risk prediction. In general, our studies highlight that particular biomarkers derived from EVs are more powerful than from unfractionated plasma because associations were exclusively found in plasma EVs and not in unfractionated plasma (Chapter 8 and 9). ${ }^{20}$ EVs consist of different subsets that vary in 
size, properties and composition. ${ }^{21}$ Although currently there is no consensus how to distinguish between EV subsets ${ }^{22,23}$, looking at specific subsets, rather than the entire EV population, improves the signal-to-noise ratio and could therefore provide more accurate prognostic information. Hence, we advocate the importance of EV subsets in biomarker research as observed associations were related to specific EV subsets (Chapter 6, 8, and 9). ${ }^{20}$ Few studies have suggested that EV content could change over time, possibly due to dietary or medical interventions or change in disease pathology. ${ }^{24,25}$ Therefore it would be highly interesting for future studies to explore the role of serial biomarker EV measurements in terms of risk estimation.

\section{Future perspectives}

\section{Multimarker algorithms to improve risk estimation}

To improve risk estimation for future cardiovascular events, future studies should focus on combining multiple biomarkers into one algorithm in addition to easily obtainable clinical characteristics. Since atherosclerosis is a complex multifactorial disease, it is intuitive that an approach of combining multiple biomarkers has more chance of success than focusing on the incremental prognostic value of a sole biomarker. Different biomarkers may represent distinct pathophysiological pathways and therefore may provide complementary prognostic information. The potency of combining biomarkers is illustrated in Chapter 10. After combining all established circulating biomarkers in this thesis, we found that the combination of two EV markers (CD14 and the ceramide ratio Cer(d18:1/24:1)/ Cer(d18:1/24:0)) significantly improved risk stratification on top of clinical risk factors for the 3-year risk of MACE after CEA (Figure 1). Future studies should also consider whether addition of radiological (carotid plaque and cerebral imaging), genetic or other circulating biomarkers previously identified in other cohorts and biobanks could further improve risk stratification. For asymptomatic carotid stenosis patients such a "multilevel biomarker approach" is currently investigated for the prediction of future stroke risk in a prospective multinational observational study (Chapter 11). Following a similar study design, a prediction algorithm for symptomatic patients undergoing CEA to estimate the risk of postoperative cardiovascular events can be developed. Another way to develop a prediction algorithm is through a collaboration of existing international vascular biobanks encompassing CEA patients (such as the Munich Vascular Biobank ${ }^{26}$, The Oxford Plaque Study $^{27}$, Biobank of Karolinska Endarterectomies ${ }^{28}$ ). To achieve this, existing vascular biobanks need to be harmonized regarding uniform indications to perform CEA, biomarker measurements, outcome definitions and follow-up protocols that include data regarding risk factor control and medication use. 
Before a prediction algorithm could be of clinical utility, it should meet several requirements. First, prediction algorithms should provide accurate and validated risk estimates. Therefore, external validation studies must be performed in other, similar, study populations. Since cardiovascular event rates vary geographically, geographic external validation is also important. ${ }^{29}$ Second, it is known that differences exist between men and women in cardiovascular disease symptomatology, prognosis and biological mechanisms which may affect the performance of biomarkers. ${ }^{30}$ Thus, potential sex-differences in prognostic performance of prediction models need to be determined. Taken all of the requirements together, these should help determine the generalizability of the prediction algorithm. Thoughtfully developed and investigated prediction algorithms should guide physician's clinical decision making that accordingly translates into improved clinical outcome of patients. Currently, no clinically relevant risk categories for secondary cardiovascular events exist. In this thesis we pragmatically defined "high risk patients" as those having twice the average risk of future cardiovascular events in our study population and "low risk patients" as those having half the average risk. Although this approach is common in research ${ }^{31}$, future studies should define absolute risk thresholds relevant to clinical decision making (such as allocation or withholding add-on therapy). Clinical impact studies are needed to prove that change in treatment, guided by these prediction algorithms, also leads to improved individual outcomes. Also, the cost-effectiveness of the prediction algorithm needs to be determined. Although a prediction algorithm can reduce overtreatment with add-on therapy thereby saving healthcare costs, this may be counterbalanced by required expensive or labor-intensive biomarker measurements.

Besides selecting appropriate patients for add-on therapy, risk prediction algorithms can have more purposes (Figure 1). Risk prediction algorithms could inform patients about their individual prognosis. Personalized risk estimates could increase awareness about their chronic disease and the accompanied risks. This could encourage patients to adopt a healthy lifestyle and improve therapy compliance. Poor compliance to cardiovascular medications remains a major issue and accounts for potentially preventable CVD events. ${ }^{32}$ Mobile apps providing personalized advices to patients about cardiovascular risk factor management are already available. ${ }^{33}$ Integrating risk prediction algorithms with these mobile apps may further contribute to improved secondary prevention of cardiovascular diseases in the future.

Risk stratification tools could also be useful in selecting study populations for clinical trials investigating new therapeutic strategies for CVD. As patients at highest risk for cardiovascular events most likely benefit most from new drugs, selective enrolment of high-risk patients will allow for smaller sample sizes to detect significant effects. This leads to higher efficiency and cost reduction of clinical trials. 
Clinical factors

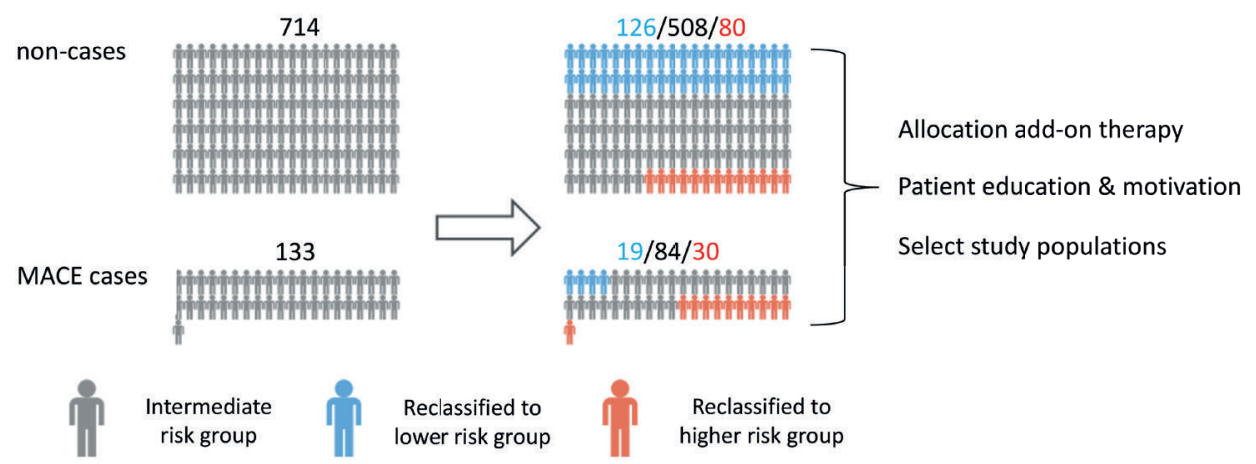

Figure 1. Biomarkers improve risk stratification on top of clinical risk factors

The daily clinical practice is displayed on the left. The potential future stratification by use of the biomarkerbased prediction algorithm is shown on the right. This figure illustrates a population of $847 \mathrm{CEA}$ patients. In the following 3 years after CEA, 133 patients develop a MACE (cases) whereas 714 patients do not suffer a MACE (non-cases).

Left: with use of clinical characteristics alone, both cases and controls are classified in one group (grey) at intermediate risk and receive the same treatment with add-on therapy.

Right: using biomarker measurements preoperatively, we could better predict which patients will suffer a MACE in the future (and need add-on therapy) and which will not suffer a MACE (and do not need add-on therapy). Using biomarkers, we see that patients with MACE are relatively more predicted as high risk individuals for MACE (red) whether patients without MACE (non-cases) are relatively more predicted to be at low risk for MACE (blue).

Adapted from: Tsimikas S, A Test in Context: Lipoprotein (a), JACC 2017

\section{Predicting treatment response in individuals}

Apart from identifying patients at high risk for MACE, biomarkers could also serve as a tool to predict the efficacy of medical or lifestyle interventions in certain individuals. This allows for further precision medicine. For example, a Mediterranean diet could be effective in patients with high levels of ceramides. ${ }^{34}$ Moreover, patients with a high genetic risk score for CVD appeared to have a greater benefit in terms of cardiovascular event reduction from proprotein convertase subtilisin/kexin type-9 (PCSK-9) inhibitors or statins than those with low genetic risk, independent of the extent of LDL reduction. ${ }^{35}$ Conversely, elevated levels of $\mathrm{Lp}(\mathrm{a})$ are not modified by statins ${ }^{36}$ and are only lowered modestly by PCSK-9 inhibitors ${ }^{37,38}$, underscoring the need for specific $\mathrm{Lp}(\mathrm{a})$ lowering treatments. Also, pharmacogenetics can assist personalized medicine in the future. Multiple SNPs influencing the pharmacokinetics or pharmacodynamics of clopidogrel, aspirin and statins have already been discovered. ${ }^{39,40}$ Future studies should determine whether genetic testing in individual patients could be used to identify drug resistance or drug intolerance and whether this has impact on better clinical outcomes. 


\section{Increase our understanding of atherosclerosis}

Studying biomarkers could help increase our understanding of the complex mechanisms of atherosclerosis. Ultimately, this may lead to the discovery of potential new drug targets to combat atherosclerotic diseases. In this thesis, biomarkers were related to carotid atherosclerotic plaque characteristics. Although we cannot derive conclusions about causality because plaques and biomarkers were cross-sectionally studied, our findings give rise to hypotheses about underlying mechanisms that could serve as a basis for future studies. Preoperative blood pressure was associated with a more vulnerable plaque phenotype including IPH, whilst IPH is a known predictor for secondary cardiovascular events $^{41}$ (Chapter 2). Other biomarkers were also related to a more vulnerable atherosclerotic plaque phenotype (e.g. the MetaGRS, the EV proteins Cystatin C, Serpin F2, Serpin C1 and Tenascin C, and EV ceramides, Chapters 3, 6, 8 and 9) suggesting that these may be somehow involved in atherosclerotic plaque development, progression or destabilization. Future experimental studies should unravel exact biological mechanisms underlying the increased risk of CVD. Mapping SNPs of the MetaGRS to pathophysiological functions may help, for instance, to elucidate contributing mechanisms to CVD. ${ }^{42}$ This is however hampered by the fact that many SNPs are located outside nonprotein coding regions with unknown functions. ${ }^{42}$ As for ceramides, animal models have already shown that specific targeting of key enzymes of the ceramide biosynthetic pathways can cause regression of atherosclerotic plaques. ${ }^{43}$ To date, no human studies with these specific ceramide drugs have been performed. Whether drugs modifying plasma ceramide levels translate to reduction in cardiovascular events has yet to be determined. Fortunately, more success has been achieved for $\operatorname{Lp}(\mathrm{a})$ as drug target. Accumulating insights in the genetic determination and metabolism of Lp(a) levels have contributed to the development of pharmacological treatments that specifically lower Lp(a). Recently, promising results were achieved with an antisense nucleotide drug targeting mRNA encoding for apo(a) (one of the main components of $\mathrm{Lp}(\mathrm{a})$ ). This drug caused significant lowering of $\mathrm{Lp}$ (a) levels in patients during a phase II clinical trial. ${ }^{44}$ Whether this drug is also effective in lowering cardiovascular events is being investigated in a phase III clinical trial (clinicaltrials.gov; NCT04023552).

\section{Conclusion}

This thesis highlights the great potential of biomarkers to improve risk estimation of future cardiovascular events for patients undergoing CEA, especially if different biomarkers are used in combination. In my point of view, plasma EVs as biomarker source hold the greatest promise as these showed incremental prognostic information on top clinical features. There 
PART V | Chapter 12

are definitely hurdles to overcome before physicians can use biomarker-based algorithms to optimize personalized treatment of patients in daily practice. But I am convinced that this era will arrive in the near future.

Nathalie Timmerman, May 2021 


\section{References}

1 Mach F, Baigent C, Catapano AL, Koskinas KC, Casula M, Badimon L, et al. 2019 ESC/EAS Guidelines for the management of dyslipidaemias: lipid modification to reduce cardiovascular risk. Eur Heart $J$ 2020;41(1):111-88.

2 Ridker PM, Everett BM, Thuren T, MacFadyen JG, Chang WH, Ballantyne C, et al. Antiinflammatory Therapy with Canakinumab for Atherosclerotic Disease. N Engl J Med 2017;377(12):1119-31.

3 Tardif J-C, Kouz S, Waters DD, Bertrand OF, Diaz R, Maggioni AP, et al. Efficacy and Safety of Low-Dose Colchicine after Myocardial Infarction. N Engl J Med 2019;381(26):2497-505.

4 Nidorf SM, Fiolet ATL, Mosterd A, Eikelboom JW, Schut A, Opstal TSJ, et al. Colchicine in Patients with Chronic Coronary Disease. N Engl J Med 2020;383(19):1838-47.

5 Anand SS, Eikelboom JW, Dyal L, Bosch J, Neumann C, Widimsky P, et al. Rivaroxaban Plus Aspirin Versus Aspirin in Relation to Vascular Risk in the COMPASS Trial. J Am Coll Cardiol 2019;73(25):3271-80.

6 Bonaca MP, Bauersachs RM, Anand SS, Debus ES, Nehler MR, Patel MR, et al. Rivaroxaban in Peripheral Artery Disease after Revascularization. N Engl J Med 2020;382(21):1994-2004.

7 Inouye M, Abraham G, Nelson CP, Wood AM, Sweeting MJ, Dudbridge F, et al. Genomic Risk Prediction of Coronary Artery Disease in 480,000 Adults. J Am Coll Cardiol 2018;72(16):1883-93.

8 Wünnemann F, Sin Lo K, Langford-Avelar A, Busseuil D, Dubé M-P, Tardif J-C, et al. Validation of Genome-Wide Polygenic Risk Scores for Coronary Artery Disease in French Canadians. Circ Genomic Precis Med 2019;12(6):e002481.

9 Rots ML, van der Lugt A, de Borst GJ. Surrogate Markers and Reporting Standards for Outcome After Carotid Intervention. Eur J Vasc Endovasc Surg 2019;58(6):794-5.

10 Hauth EAM, Jansen C, Drescher R, Schwartz M, Forsting M, Jaeger HJ, et al. MR and clinical follow-up of diffusion-weighted cerebral lesions after carotid artery stenting. Am J Neuroradiol 2005;26(9):2336-41.

11 Gensicke H, van der Worp HB, Nederkoorn PJ, Macdonald S, Gaines PA, van der Lugt A, et al. Ischemic brain lesions after carotid artery stenting increase future cerebrovascular risk. J Am Coll Cardiol 2015;65(6):521-9.

12 Traenka C, Engelter ST, Brown MM, Dobson J, Frost C, Bonati LH. Silent brain infarcts on diffusionweighted imaging after carotid revascularisation: A surrogate outcome measure for procedural stroke? A systematic review and meta-analysis. Eur Stroke J 2019;4(2):127-43.

13 Saam T, Hetterich H, Hoffmann V, Yuan C, Dichgans M, Poppert H, et al. Meta-Analysis and Systematic Review of the Predictive Value of Carotid Plaque Hemorrhage on Cerebrovascular Events by Magnetic Resonance Imaging. J Am Coll Cardiol 2013;62(12):1081-91.

14 Schindler A, Schinner R, Altaf N, Hosseini AA, Simpson RJ, Esposito-Bauer L, et al. Prediction of Stroke Risk by Detection of Hemorrhage in Carotid Plaques: Meta-Analysis of Individual Patient Data. JACC Cardiovasc Imaging 2020;13(2):395-406.

15 Derksen WJM, Peeters W, van Lammeren GW, Tersteeg C, de Vries J-PPM, de Kleijn DPV, et al. Different stages of intraplaque hemorrhage are associated with different plaque phenotypes: A large histopathological study in 794 carotid and 276 femoral endarterectomy specimens. Atherosclerosis 2011;218(2):369-77.

16 Chu B, Kampschulte A, Ferguson MS, Kerwin WS, Yarnykh VL, O’Brien KD, et al. Hemorrhage in the Atherosclerotic Carotid Plaque: A High-Resolution MRI Study. Stroke 2004;35(5):1079-84.

17 Streifler JY, Eliasziw M, Benavente OR, Alamowitch S, Fox AJ, Hachinski VC, et al. Prognostic importance of leukoaraiosis in patients with symptomatic internal carotid artery stenosis. Stroke 2002;33(6):1651-5.

18 van Dijk EJ, Prins ND, Vrooman HA, Hofman A, Koudstaal PJ, Breteler MMB. Progression of Cerebral Small Vessel Disease in Relation to Risk Factors and Cognitive Consequences. Stroke 2008;39(10):2712-9.

19 Debette S, Beiser A, DeCarli C, Au R, Himali JJ, Kelly-Hayes M, et al. Association of MRI Markers of Vascular Brain Injury With Incident Stroke, Mild Cognitive Impairment, Dementia, and Mortality. Stroke 2010;41(4):600-6.

20 Dekker M, Waissi F, van Bennekom J, Silvis MJM, Timmerman N, Bank IEM, et al. Plasma extracellular vesicle proteins are associated with stress-induced myocardial ischemia in women presenting with chest pain. Sci Rep 2020;10(1):12257.

21 Boulanger CM, Loyer X, Rautou P-E, Amabile N. Extracellular vesicles in coronary artery disease. Nat Rev Cardiol 2017;14(5):259-72. 
22 Yáñez-Mó M, Siljander PR-M, Andreu Z, Bedina Zavec A, Borràs FE, Buzas EI, et al. Biological properties of extracellular vesicles and their physiological functions. J Extracell Vesicles 2015;4(1):27066.

23 van der Pol E, Böing AN, Gool EL, Nieuwland R. Recent developments in the nomenclature, presence, isolation, detection and clinical impact of extracellular vesicles. J Thromb Haemost 2016;14(1):48-56.

24 Chiva-Blanch G, Suades R, Crespo J, Vilahur G, Arderiu G, Padró T, et al. CD3+/CD45+ and SMA- $a+$ circulating microparticles are increased in individuals at high cardiovascular risk who will develop a major cardiovascular event. Int J Cardiol 2016;208:147-9.

25 Verbree-Willemsen L, Zhang Y-N, Gijsberts CM, Schoneveld AH, Wang J-W, Lam CSP, et al. LDL extracellular vesicle coagulation protein levels change after initiation of statin therapy. Findings from the METEOR trial. Int J Cardiol 2018;271:247-53.

26 Pelisek J, Hegenloh R, Bauer S, Metschl S, Pauli J, Glukha N, et al. Biobanking: Objectives, Requirements, and Future Challenges-Experiences from the Munich Vascular Biobank. J Clin Med 2019;8(2):251.

27 Redgrave JNE, Lovett JK, Rothwell PM. Histological Features of Symptomatic Carotid Plaques in Relation to Age and Smoking. Stroke 2010;41(10):2288-94.

28 Razuvaev A, Ekstrand J, Folkersen L, Agardh H, Markus D, Swedenborg J, et al. Correlations Between Clinical Variables and Gene-expression Profiles in Carotid Plaque Instability. Eur J Vasc Endovasc Surg 2011;42(6):722-30.

29 Ducrocq G, Bhatt D, Labreuche J, Corbalan R, Porath A, Gao R, et al. Geographic differences in outcomes in outpatients with established atherothrombotic disease: results from the REACH Registry. Eur J Prev Cardiol 2014;21(12):1509-16.

30 Gerdts E, Regitz-Zagrosek V. Sex differences in cardiometabolic disorders. Nat Med 2019;25(11):1657-66.

31 Cook NR, Paynter NP. Performance of reclassification statistics in comparing risk prediction models. Biometrical J 2011;53(2):237-58.

32 Chowdhury R, Khan H, Heydon E, Shroufi A, Fahimi S, Moore C, et al. Adherence to cardiovascular therapy: a meta-analysis of prevalence and clinical consequences. Eur Heart J 2013;34(38):2940-8.

33 Neubeck L, Cartledge S, Dawkes S, Gallagher R. Is there an app for that? Mobile phones and secondary prevention of cardiovascular disease. Curr Opin Cardiol 2017;32(5):567-71.

34 Wang DD, Toledo E, Hruby A, Rosner BA, Willett WC, Sun Q, et al. Plasma Ceramides, Mediterranean Diet, and Incident Cardiovascular Disease in the PREDIMED Trial (Prevención con Dieta Mediterránea). Circulation 2017;135(21):2028-40.

35 Damask A, Steg PG, Schwartz GG, Szarek M, Hagström E, Badimon L, et al. Patients With High GenomeWide Polygenic Risk Scores for Coronary Artery Disease May Receive Greater Clinical Benefit From Alirocumab Treatment in the ODYSSEY OUTCOMES Trial. Circulation 2020;141(8):624-36.

36 Willeit P, Ridker PM, Nestel PJ, Simes J, Tonkin AM, Pedersen TR, et al. Baseline and on-statin treatment lipoprotein(a) levels for prediction of cardiovascular events: individual patient-data meta-analysis of statin outcome trials. Lancet 2018;392(10155):1311-20.

37 Bittner VA, Szarek M, Aylward PE, Bhatt DL, Diaz R, Edelberg JM, et al. Effect of Alirocumab on Lipoprotein(a) and Cardiovascular Risk After Acute Coronary Syndrome. J Am Coll Cardiol 2020;75(2):133-44.

38 Stiekema LCA, Stroes ESG, Verweij SL, Kassahun H, Chen L, Wasserman SM, et al. Persistent arterial wall inflammation in patients with elevated lipoprotein(a) despite strong low-density lipoprotein cholesterol reduction by proprotein convertase subtilisin/kexin type 9 antibody treatment. Eur Heart J 2019;40(33):2775-81.

39 Guan Z, Wu K, Li R, Yin Y, Li X, Zhang S, et al. Pharmacogenetics of statins treatment: Efficacy and safety. J Clin Pharm Ther 2019;44(6):858-67.

40 Ross S, Paré G. Pharmacogenetics of Stroke. Stroke 2018;49(10):2541-8.

41 Hellings WE, Peeters W, Moll FL, Piers SRD, van Setten J, Van der Spek PJ, et al. Composition of Carotid Atherosclerotic Plaque Is Associated With Cardiovascular Outcome. Circulation 2010;121(17):1941-50.

42 Erdmann J, Kessler T, Munoz Venegas L, Schunkert H. A decade of genome-wide association studies for coronary artery disease: the challenges ahead. Cardiovasc Res 2018;114(9):1241-57.

$43 \mathrm{Yu}$ Z, Peng Q, Huang Y. Potential therapeutic targets for atherosclerosis in sphingolipid metabolism. Clin Sci 2019;133(6):763-76.

44 Tsimikas S, Karwatowska-Prokopczuk E, Gouni-Berthold I, Tardif J-C, Baum SJ, Steinhagen-Thiessen E, et al. Lipoprotein(a) Reduction in Persons with Cardiovascular Disease. N Engl J Med 2020;382(3):244-55. 


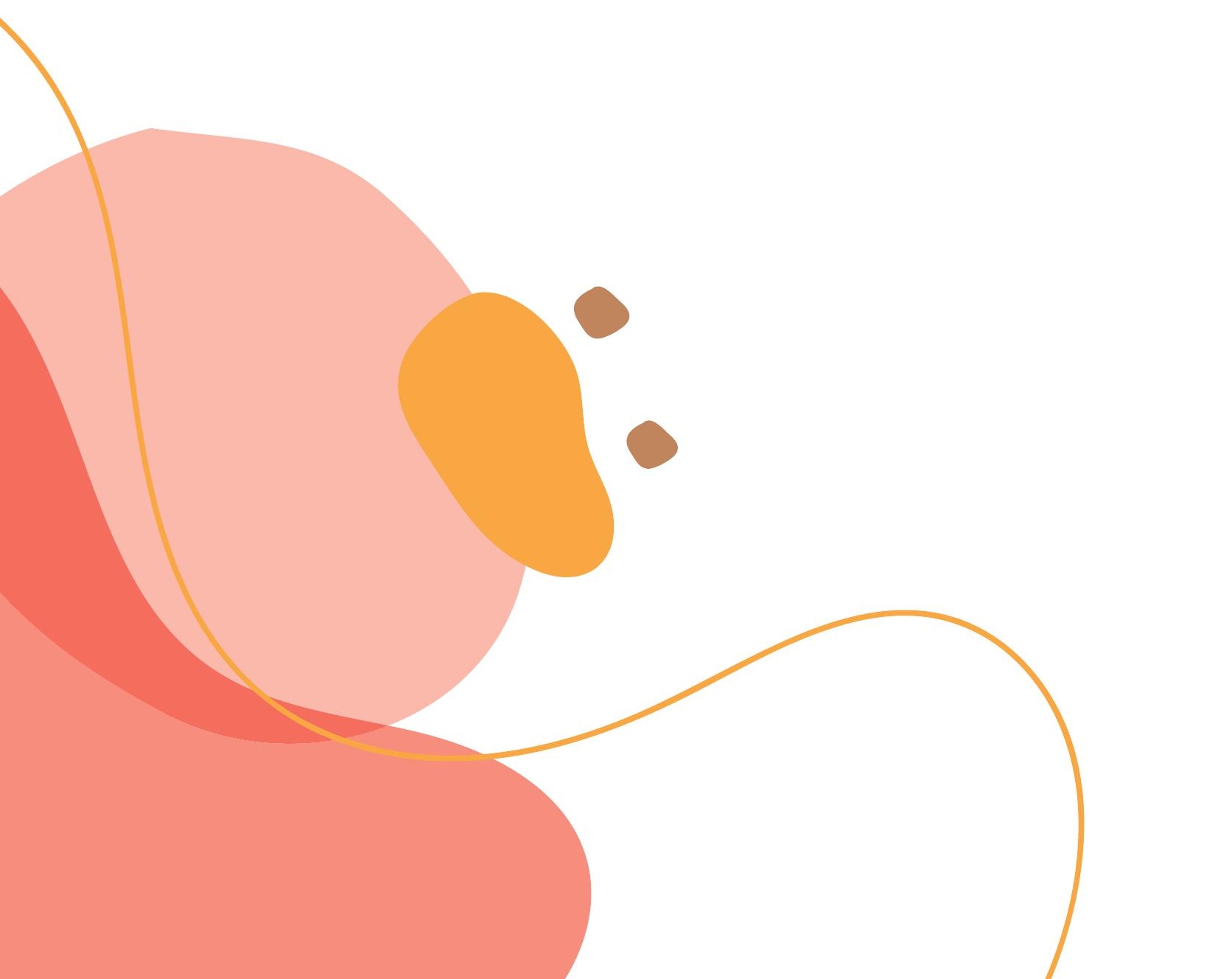


CHAPTER 13

Summary in Dutch

Nederlandse samenvatting 


\section{Hart- en vaatziekten \& atherosclerose}

Hart- en vaatziekten (HVZ) zijn de belangrijkste doodsoorzaak wereldwijd. In Nederland sterft 25\% van de mensen aan HVZ, bijvoorbeeld door een hartinfarct of een herseninfarct. Belangrijke risicofactoren zijn roken, een hoog cholesterol, een hoge bloeddruk, suikerziekte, overgewicht, weinig lichaamsbeweging, oudere leeftijd en erfelijke aanleg. De oorzaak van de meeste HVZ is atherosclerose (in de volksmond: slagaderverkalking). Atherosclerose is een complex proces waarbij ophopingen van onder andere vetten (cholesterol) en ontstekingscellen in de vaatwand ontstaan (atherosclerotische plaques). Deze plaques kunnen het bloedvat vernauwen. Tijdens inspanning kunnen klachten zoals pijn op de borst of pijn in de benen ontstaan omdat achterliggende organen te weinig bloed en zuurstof krijgen. Ook kan de atherosclerotische plaque plotseling scheuren (plaque ruptuur), wat leidt tot de vorming van bloedstolsels (trombus) die acuut een bloedvat occluderen. Achterliggende organen krijgen dan geen zuurstof meer en er ontstaat weefselschade. Zo ontstaat een hartinfarct, herseninfarct of ischemisch been afhankelijk van welk vat is aangedaan.

\section{Carotisstenose}

Een carotisstenose is een vernauwing van de halsslagader (arteria carotis) door een atherosclerotische plaque. De halsslagaderen vervoeren zuurstofrijk bloed naar de hersenen en zijn belangrijk voor een adequate hersenfunctie. Bloedstolsels vanuit een plaque in de carotis kunnen de bloedtoevoer naar delen van de hersenen blokkeren waardoor kortdurende, reversibele hersenschade (transient ischemic attack (TIA)) of permanente hersenschade (herseninfarct) kan ontstaan. Dit veroorzaakt neurologische uitvalsverschijnselen zoals eenzijdige verlammingen, gevoelsstoornissen aan de ledematen, een scheve mond of verwarde spraak of taal.

\section{Behandeling van een carotisstenose}

Ter preventie van een herseninfarct worden patiënten met een carotisstenose behandeld met medicijnen zoals cholesterolverlagers (statines), bloedverdunners (plaatjesaggregratieremmers, zoals clopidogrel of aspirine) en bloeddrukverlagers. Daarnaast kan de carotisplaque operatief worden verwijderd middels een carotis endarterectomie (CEA, Figuur 1) of worden uitgeschakeld door middel van het plaatsen van een stent (carotid artery stenting (CAS)). Interventies zijn helaas nooit zonder risico's. Een ernstige complicatie van een CEA of CAS is het perioperatief herseninfarct. Daarom is het belangrijk dat per patiënt het toekomstig risico en het perioperatief risico op een herseninfarct tegen elkaar worden afgewogen. 


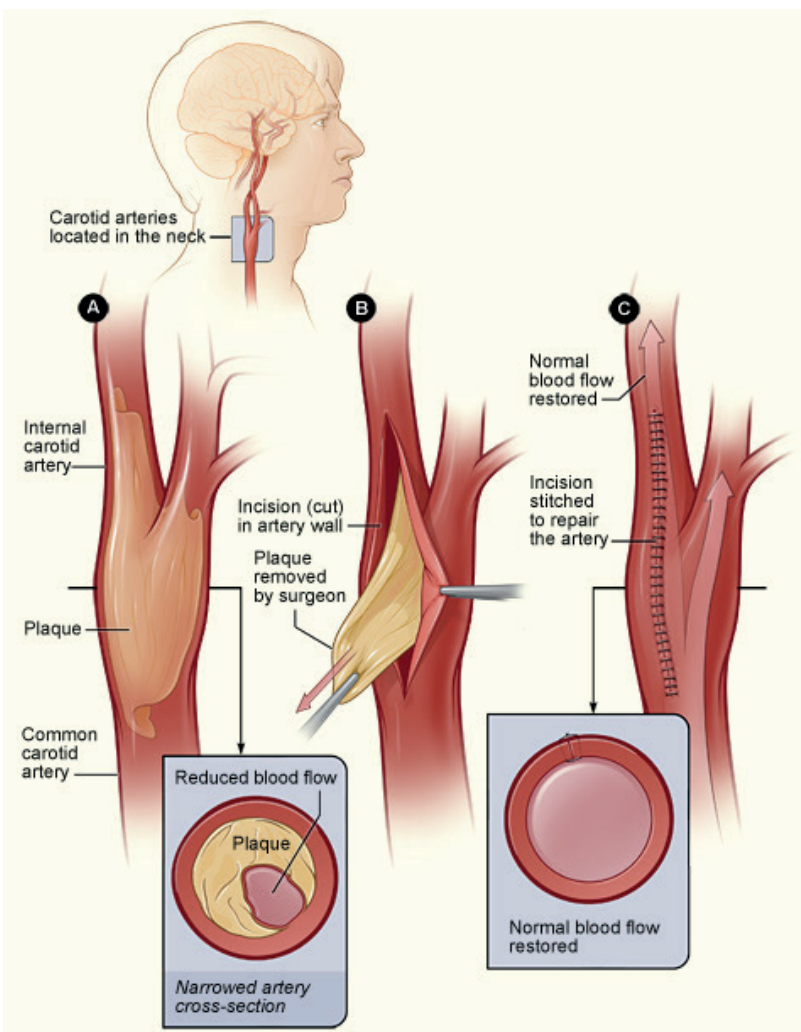

Figuur 1. De carotis endarterectomie operatie

\section{Preventie van secundaire cardiovasculaire (CV) events na een CEA}

Atherosclerose is een systemische ziekte die meerdere slagaderen van het lichaam aantast. Daarom hebben deze patiënten ook na operatie van de carotisstenose met een CEA nog steeds een hoog risico op het krijgen van een tweede cardiovasculair (CV) event, bijvoorbeeld vanuit een ander vaatbed. Ongeveer $25 \%$ van de patiënten krijgt een tweede $\mathrm{CV}$-event binnen drie jaar na een CEA- operatie. Het is belangrijk om vroegtijdig patiënten te identificeren met een hoog risico op een secundair CV-event omdat zij baat kunnen hebben bij frequentere controles en/of extra medicijnen (add-on therapy). Omdat zulke medicijnen bijwerkingen kunnen hebben of kostbaar zijn, zijn ze niet voor alle patiënten geschikt. Het is daarom van belang om alleen hoog-risico patiënten te behandelen met add-on therapy, waarbij het voordeel (verlaging van het cardiovasculair risico) opweegt tegen de nadelen (bijwerkingen en kosten). 


\section{Predictie van CV-events}

In dit proefschrift worden biomarkers onderzocht om beter te kunnen voorspellen welke CEA-patiënten een hoog risico hebben op een secundair CV-event. Biomarkers zijn biologische indicatoren voor (patho-)fysiologische processen. Voorbeelden zijn stoffen in het bloed, kenmerken op beeldvorming of genetische markers. De onderzoeken uit dit proefschrift zijn uitgevoerd in de Athero-Express Biobank-studie. De Athero-Express includeert patiënten die een CEA ondergaan. Van deze patiënten worden preoperatieve bloedsamples en het carotis plaqueweefsel (verwijderd tijdens de CEA-operatie) verzameld, tezamen met klinische informatie. Door het plaqueweefsel te bestuderen kan meer inzicht worden verkregen in onderliggende pathofysiologische mechanismen van HVZ.

\section{Deel I - Klinische factoren en genetica}

\section{Preoperatieve bloeddruk en atherosclerotische plaque karakteristieken}

In hoofdstuk 2 is de relatie tussen een preoperatieve hoge bloeddruk en histologische kenmerken van de carotisplaque onderzocht. Bij patiënten die preoperatief een hogere systolische en/of diastolische bloeddruk hadden, bleek de atherosclerotische plaque meer instabiele kenmerken (dat wil zeggen een hogere kans op plaque ruptuur) te vertonen. Eerdere studies hebben aangetoond dat met name intraplaque-bloedingen een voorspeller zijn voor het optreden van secundaire CV-events. Onze resultaten suggereren een relatie tussen een hoge bloeddruk en plaque-instabiliteit, waaruit mogelijk het hogere risico op $\mathrm{CV}$-events te verklaren is. Onze resultaten benadrukken het belang van behandeling van de bloeddruk. In toekomstige studies zou het interessant zijn te onderzoeken of intensievere bloeddrukverlaging in deze patiëntengroep leidt tot minder CV-events.

\section{Positieve familieanamnese en genetische predispositie}

Erfelijke aanleg is een belangrijke risicofactor voor HVZ. In de praktijk wordt een positieve familieanamnese (wanneer een eerstegraads familielid voor het $60^{\mathrm{e}}$ levensjaar HVZ doorgemaakt heeft) als surrogaat hiervoor gebruikt. Hoofdstuk 3 laat zien dat een positieve familieanamnese ook een onafhankelijke voorspeller is voor een hoger risico op secundaire CV-events na een CEA. Daarnaast werd de erfelijke aanleg bestudeerd aan de hand van genetische risicovarianten. Van elke patiënt zijn alle genetische risicovarianten voor HVZ bij elkaar opgeteld tot een zogenaamde polygene risico score (PRS). Een hoge PRS-score (hoog genetische risico voor HVZ) blijkt onafhankelijk gerelateerd aan een hoger risico op secundaire CV-events. Interessant genoeg blijken een hoge PRS en een positieve familieanamnese ook onafhankelijk van elkaar gerelateerd te zijn aan secundaire CV-events. Dit suggereert dat er mogelijk verschillende onderliggende mechanismen ten grondslag 
liggen. Onze hypothese is dat de PRS veelvoorkomende genetische varianten omvat terwijl een positieve familieanamnese waarschijnlijk ook zeldzame genetische varianten omvat die worden doorgegeven binnen families. Familieanamnese weerspiegelt daarnaast ook niet-genetische factoren, zoals sociale economische status of ongezonde levensstijl.

\section{Deel II - Radiologische markers}

Radiologische kenmerken op hersenscans kunnen prognostische informatie bevatten. Diffusion weighted imaging laesies (DWI-laesies) weerspiegelen afwijkingen in de hersenen met een tijdelijk zuurstoftekort. DWI-laesies komen niet tot uiting in klinische symptomen (stille ischemie). Uit eerdere studies weten we dat DWI-laesies wel een relatie hebben met een verhoogd risico op een toekomstig herseninfarct. De resultaten van hoofdstuk 4 tonen aan dat patiënten die nieuwe DWI-laesies ontwikkelden in de dagen tussen het eerste symptoom en het wachten op de CEA-operatie ook meer intraplaque bloedingen in het weefsel van de carotis hadden. Mogelijk kan in de toekomst detectie van intraplaque bloedingen op beeldvorming (bijvoorbeeld op MRI) bijdragen aan welke patiënten voorrang moeten krijgen voor een CEA. Hoofdstuk 5 beschrijft de klinische relevantie van een andere radiologische marker, namelijk wittestofafwijkingen. Patiënten die wittestofafwijkingen hadden op de preoperatieve hersenscan, beschreven door de radioloog in het verslag, bleken een hoger toekomstig risico te hebben om dood te gaan aan HVZ binnen drie jaar na de operatie. Wittestofafwijkingen bleken niet specifiek geassocieerd te zijn met een toekomstig herseninfarct.

\section{Deel III - Circulerende markers in plasma en extracellulaire vesicles (EVs)}

In hoofstukken $6 \mathrm{t} / \mathrm{m} 9$ onderzochten we verschillende circulerende biomarkers gemeten in preoperatieve bloedafnames. We onderzochten of deze biomarkers voorspellers konden zijn voor het optreden van secundaire major cardiovasculaire events (MACE) gedurende drie jaar na een CEA-operatie. MACE is een verzamelnaam voor het optreden van een herseninfarct, hartinfarct of cardiovasculaire dood. Biomarkers (eiwitten en lipiden) werden gemeten in plasma en in plasma extracellulaire vesicles (EVs). Plasma EVs zijn dubbelmembraanblaasjes die materiaal zoals eiwitten, lipiden, RNA en DNA transporteren tussen cellen. Ze zijn dus belangrijk voor de intercellulaire communicatie. De inhoud van EVs kan informatie geven over pathologische processen die gaande zijn. Het bestuderen van de inhoud van EVs geeft daarom mogelijk waardevolle prognostische informatie over ziektes. De resultaten van hoofdstuk 6 laten zien dat hogere concentraties van de eiwitten 
Cystatin C, Serpin C1, Serpin F2 and CD14 in EVs gerelateerd zijn aan een hoger postoperatief 3-jaars risico op MACE. Hoofdstuk 7 toont dat patiënten met hogere plasmaconcentraties van Lipoproteine a, $\mathrm{Lp}(\mathrm{a})$, een hoger risico op postoperatieve MACE hadden, met name gedurende de eerste 30 dagen na de CEA. Hoofdstuk $\mathbf{8}$ toont dat hogere concentraties van het extracellulaire matrix eiwit Tenascin-C in EVs gerelateerd zijn aan een hoger 3-jaars risico op postoperatieve MACE en aan een instabielere carotisplaque. In hoofdstuk 9 onderzochten we ceramides en phophatidylcholines (PCs), vetten die zich bevinden in celmembranen, maar ook fungeren als signaalmoleculen. Bepaalde typen ceramides en PCs in EVs blijken gerelateerd aan een hoger toekomstig risico op MACE en aan een instabielere atherosclerotische plaque. Ceramides en PCs gemeten in plasma blijken niet voorspellend te zijn voor MACE. Dit suggereert dat biomarkers specifiek gemeten in EVs mogelijk sterkere voorspellers zijn dan biomarkers gemeten in plasma.

\section{Deel IV- Risicostratificatie}

\section{Het verbeteren van de predictie op MACE na CEA}

$\mathrm{Na}$ het vinden van verschillende biomarkers die geassocieerd zijn aan een verhoogd risico op MACE na CEA was de vraag welke biomarkers in de praktijk het meest waardevol zijn om het individuele risico van de patiënt in te schatten. Hiervoor hebben we in hoofdstuk 10 onderzocht welke biomarkers samen met bekende klinische voorspellers (leeftijd, roken, nierfunctie, eerdere hartziekte of perifeer vaatlijden en cerebrovasculaire symptomen) de meest nauwkeurige risico-inschatting maken. Twee biomarkers, CD14 en de ceramide ratio Cer(d18:1/24:1)/Cer(d18:1/24:0), blijken een toegevoegde waarde te hebben naast de klinische factoren. Het voorspellingsmodel inclusief biomarkers blijkt 15\% nauwkeuriger dan het model zonder biomarkers. Na validatie van onze resultaten in toekomstige studies zou dit biomarkermodel nuttig kunnen zijn om hoog-risicopatiënten voor postoperatief MACE vroegtijdig (preoperatief aan de CEA) te identificeren. Deze patiënten zouden intensiever behandeld kunnen worden met extra medicijnen om zo hun risico op MACE te verkleinen en, hopelijk, MACE te voorkomen.

\section{Risico predictie voor patiënten met asymptomatische carotisstenose}

Hoewel CEA bewezen effectief is bij symptomatische patiënten met een carotisstenose (doorgemaakte TIA of herseninfarct), is het voordeel bij asymptomatische patiënten (gedefinieerd als geen neurologische klachten in het afgelopen half jaar) minder duidelijk. De meeste asymptomatische patiënten onder medicamenteuze behandeling hebben een laag risico op een toekomstig herseninfarct. Een interventie (CEA of CAS) met het risico op complicaties is daarom niet geïndiceerd. Eerdere studies hebben aangetoond dat 
asymptomatische patiënten met bepaalde risicofactoren of biomarkers een veel hoger risico hebben op een herseninfarct en mogelijk wel beter af zijn met een operatie. Hoofdstuk 11 beschrijft de opzet en rationale van een Europese, multicenter prospectieve observationele studie voor patiënten met een asymptomatische carotisstenose (TAXINOMISIS). Het doel van deze studie is het ontwikkelen van een voorspellingsmodel om de risico-inschatting op toekomstige cerebrovasculaire complicaties voor asymptomatische patiënten te verbeteren. In de praktijk zou deze tool gebruikt kunnen worden om individuele behandelstrategieën (een carotis interventie naast medicatie of alleen medicatie) af te stemmen. 


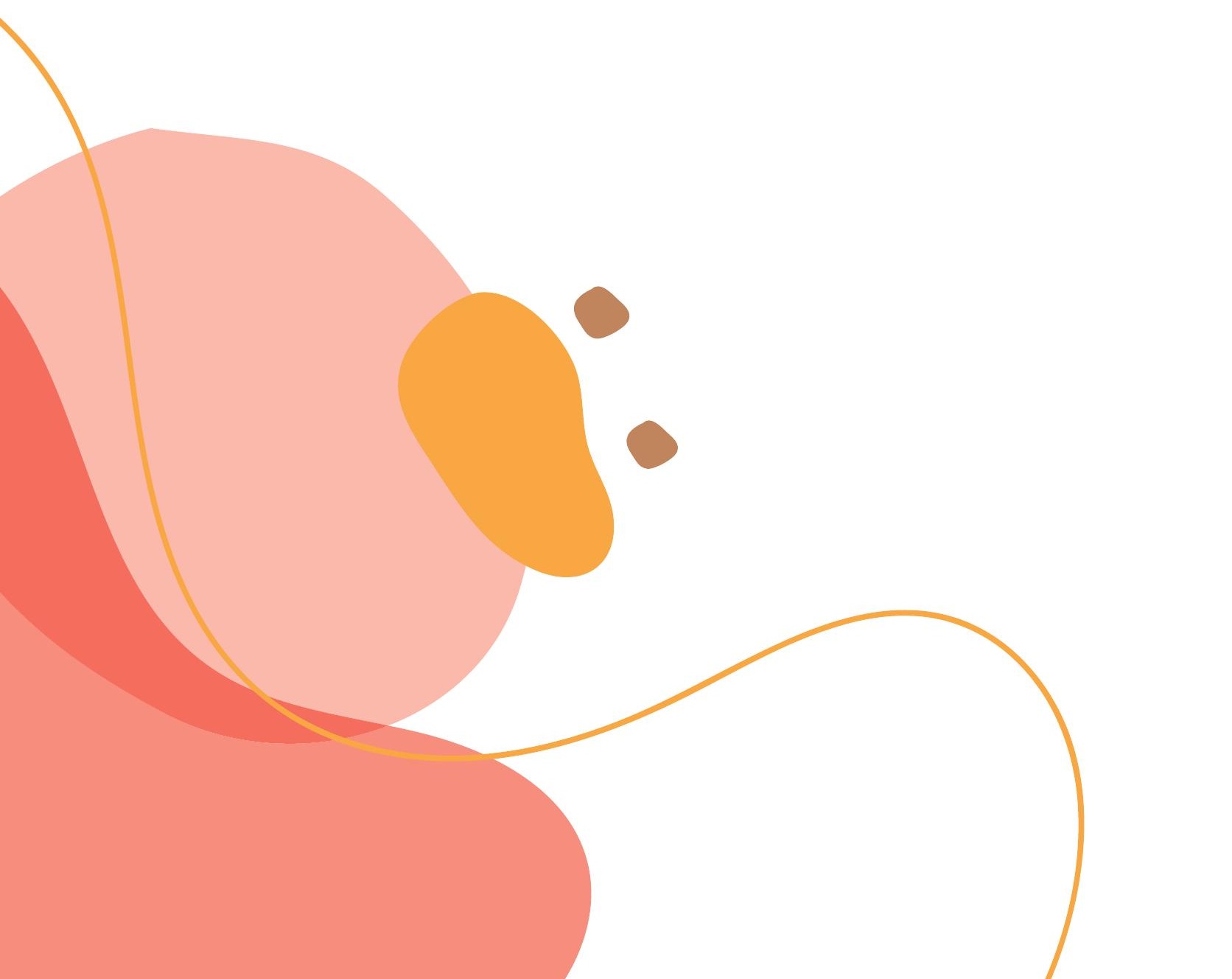




\title{
APPENDICES
}

\author{
Review Committee \\ Authors and affiliations \\ List of publications \\ Acknowledgements | Dankwoord \\ About the author
}




\section{Review Committee}

\section{Prof. dr. M.L. Bots}

Julius Center for Health Sciences and Primary Care

University Medical Center Utrecht

Utrecht, The Netherlands

Prof. dr. ir. H.M. den Ruijter

Department of Cardiology

University Medical Center Utrecht

Utrecht, The Netherlands

\section{Prof. dr. F.L.J. Visseren}

Department of Internal Medicine

University Medical Center Utrecht

The Netherlands

\section{Prof. dr. A. van der Lugt}

Department of Radiology \& Nuclear Medicine

Erasmus Medical Center

Rotterdam, the Netherlands

\section{Prof. dr. U. Hedin}

Department of Molecular Medicine and Surgery

Vascular Surgery Division

Karolinska University Hospital

Stockholm, Sweden 


\section{Authors and Affiliations}

Evangelos Andreakos, PhD

Laboratory of Immunobiology

Center for Clinical, Experimental Surgery and Translational Research, Biomedical Research

Foundation of the Academy of Athens,

Greece

Prof. Folkert W. Asselbergs, MD, PhD

Department of Cardiology

University Medical Center Utrecht

Utrecht, The Netherlands

Joelle van Bennekom, MSc

Department of Vascular Surgery

University Medical Center Utrecht

Utrecht, The Netherlands

Stella Bijkerk, BSc

Department of Vascular Surgery

University Medical Center Utrecht

Utrecht, The Netherlands

Prof. Leo H. Bonati, MD, PhD

Department of Neurology and Stroke Center,

University Hospital Basel

Basel, Switserland

Prof. Gert J. de Borst, MD, PhD

Department of Vascular Surgery

University Medical Center Utrecht

Utrecht, The Netherlands

Prof. Martin M. Brown, MD, PhD

Department of Neurology and Neurosurgery

University College London Hospitals

London, United Kingdom 
Prof. Lazar Davidovic, MD, PhD

Clinic for Vascular and Endovascular Surgery

Serbian Clinical Center, Belgrade

Belgrade, Serbia

Mirthe Dekker, MD

Department of Vascular Surgery

University Medical Center Utrecht; Department of Cardiology

Amsterdam UMC, The Netherlands

\section{Kim E. Dzobo, MSc}

Department of Experimental Vascular Medicine, Amsterdam University Medical Centers, Amsterdam, The Netherlands

\section{Prof. Hans-Henning Eckstein, MD}

Clinic and Policlinik for vascular and endovascular Surgery, Technical University Munich Germany

\section{Leonie M.M. Fassaert, MD, PhD}

Department of Vascular Surgery

University Medical Center Utrecht

Utrecht, The Netherlands

\section{Prof. Dimitrios I. Fotiadis, PhD}

Department of Materials Science and Engineering, University of Ioannina Ioannina, Greece.

\section{George Galyfos, MD, PhD}

First Propedeutic Department of Surgery

National and Kapodistrian University of Athens, Greece

\section{Prof. Diederick E. Grobbee, MD, PhD}

Julius Center for Health Sciences and Primary Care; University Medical Center Utrecht Utrecht, The Netherlands

\section{Saskia Haitjema, MD, PhD}

Laboratory of Clinical Chemistry and Hematology; University Medical Center Utrecht, The Netherlands 
Mika Hilvo, PhD

Zora Biosciences

Espoo, Finland

Renate M. Hoogeveen, $\mathrm{MD}, \mathrm{PhD}$

Department of Vascular Medicine

Amsterdam University Medical Centers

Amsterdam, the Netherlands

Antti Jylhä, MSc

Zora Biosciences

Espoo, Finland

Prof. L. Jaap Kappelle

Department of Neurology and Neurosurgery

University Medical Center Utrecht

Utrecht, the Netherlands

Prof. Dominique P.V. de Kleijn, PhD

Department of Vascular Surgery

University Medical Center Utrecht

Utrecht, The Netherlands

Marjet J.M. klein Avink, MSc

Department of Vascular Surgery

University Medical Center Utrecht

Utrecht, The Netherlands

Ian D. van Koeverden, $\mathrm{MD}, \mathrm{PhD}$

Department of Vascular Surgery

University Medical Center Utrecht

Utrecht, The Netherlands

Igor Koncar, $\mathrm{MD}, \mathrm{PhD}$

Clinic for Vascular and Endovascular Surgery

Serbian Clinical Center, Belgrade

Belgrade, Serbia 


\section{Jeffrey Kroon, PhD}

Department of Experimental Vascular Medicine; Amsterdam University Medical Centers, The Netherlands

\section{Reijo Laaksonen, MD, PhD}

Finnish Cardiovascular Research Center Tampere, Tampere University; Zora Biosciences, Espoo, Finland

\section{Sander W. van der Laan, PhD}

Laboratory of Clinical Chemistry and Hematology, University Medical Center Utrecht, The Netherlands

Constance J.H.C.M. van Laarhoven, MD, PhD

Department of Vascular Surgery

University Medical Center Utrecht

Utrecht, the Netherlands

\section{Michalis D. Mantzaris, $\mathrm{PhD}$}

Department of Materials Science and Engineering, University of Ioannina Ioannina, Greece

\section{Prof. Gerard Pasterkamp, MD, PhD}

Laboratory of Clinical Chemistry and Hematology, University Medical Center Utrecht, The Netherlands

\section{Qiu Ying van de Pol, BSc}

Department of Vascular Surgery

University Medical Center Utrecht

Utrecht, The Netherlands

\section{Vassiliki T. Potiska, PhD}

Department of Materials Science and Engineering, University of Ioannina Ioannina, Greece

Prof. Hester M. den Ruijter, PhD

Department of Cardiology

University Medical Center Utrecht

Utrecht, The Netherlands 
Marjolijn L. Rots, MD, PhD

Department of Vascular Surgery

University Medical Center Utrecht

Utrecht, The Netherlands

Johan G. Schnitzler, PhD

Department of Experimental Vascular Medicine, Amsterdam University Medical Centers, The Netherlands

\section{Arjan Schoneveld, BSc}

Division Laboratories and Pharmacy

University Medical Center Utrecht,

Utrecht, The Netherlands

\section{Fragiska Sigala, MD, PhD}

First Propedeutic Department of Surgery

National and Kapodistrian University of Athens, Greece

Prof. Erik S.G. Stroes, MD, PhD

Department of Vascular Medicine

Amsterdam University Medical Centers

Amsterdam, the Netherlands

\section{Annemiek M. Vuurens, BSc}

Department of Vascular Surgery

University Medical Center Utrecht

Utrecht, The Netherlands

\section{Farahnaz Waissi, MD}

Department of Vascular Surgery

University Medical Center Utrecht; Department of Cardiology

Amsterdam UMC, The Netherlands

\section{Prof. Robbert J. de Winter, MD, PhD}

Department of Cardiology

Amsterdam UMC

Amsterdam, The Netherlands 
Appendices

\section{Rong-Rong Zhao, PhD}

Cardiovascular Research Institute

National University of Singapore

Singapore, Singapore 


\section{List of publications}

Leonie M.M. Fassaert, Nathalie Timmerman, Ian D. van Koeverden, Gerard Pasterkamp, Dominique P.V. de Kleijn, Gert J. de Borst. Preoperative hypertension is associated with atherosclerotic intraplaque hemorrhage in patients undergoing carotid endarterectomy. Atherosclerosis 2019;290:214-221.

Nathalie Timmerman, Dominique P.V. de Kleijn, Gert J. de Borst, Hester M. den Ruijter, Folkert W. Asselbergs, Gerard Pasterkamp, Saskia Haitjema*, Sander W. van der Laan*. Family history and polygenic risk of cardiovascular disease: independent factors associated with secondary cardiovascular events in patients undergoing carotid endarterectomy. Atherosclerosis 2020; 307:121-129.

Marjolijn L. Rots, Nathalie Timmerman, Dominique P.V. de Kleijn, Gerard Pasterkamp, Martin M. Brown, Leo H. Bonati, Gert J. de Borst. Magnetic resonance imaging identified brain ischemia in symptomatic patients undergoing carotid endarterectomy is related to histologically apparent intraplaque hemorrhage. Eur J Vasc Endovasc Surg 2019;58(6):796804.

Nathalie Timmerman, Marjolijn L. Rots, Ian D. van Koeverden, Saskia Haitjema, Constance J.H.C.M. van Laarhoven, Annemiek M. Vuurens, Hester M. den Ruijter, Gerard Pasterkamp, L. Jaap Kappelle, Dominique P.V. de Kleijn, Gert J. de Borst. Cerebral small vessel disease in standard pre-operative imaging reports is independently associated with increased risk of cardiovascular death following carotid endarterectomy. Eur J Vasc Endovasc Surg 2020;59(6):872-880.

Nathalie Timmerman*, Farahnaz Waissi^, Mirthe Dekker, Qiu Ying van de Pol, Joelle van Bennekom, Arjan Schoneveld, Marjet J.M. klein Avink, Robbert J. de Winter, Gerard Pasterkamp, Gert J. de Borst, Dominique P.V. de Kleijn. Pre-operative plasma extracellular vesicle proteins are associated with a high risk of long term secondary major cardiovascular events in patients undergoing carotid endarterectomy. Eur J Vasc Endovasc Surg (in press)

Farahnaz Waissi ${ }^{\star}$, Nathalie Timmerman ${ }^{\star}$, Mirthe Dekker*, Renate M. Hoogeveen, Joelle van Bennekom, Kim E. Dzobo, Johan G. Schnitzler, Gerard Pasterkamp, Diederick E. Grobbee, Gert J. de Borst, Erik S.G. Stroes, Dominique P.V. de Kleijn, Jeffrey Kroon. Elevated lipoprotein(a) levels increase risk of 30-day MACE in patients following carotid endarterectomy. Stroke 2020;51(10):2972-2982. 
Nathalie Timmerman*, Farahnaz Waissi*, Mirthe Dekker, Stella Bijkerk, Joelle van Bennekom, Arjan H. Schoneveld, Rong-Rong Zhao, Robbert J. de Winter, Gerard Pasterkamp, Gert J. de Borst, Dominique P.V. de Kleijn. High Tenascin-C plasma extracellular vesicles levels are associated with an increased risk of major adverse cardiovascular events in carotid endarterectomy patients. (Under review-Arterioscler Thromb Vasc Biol)

Nathalie Timmerman ${ }^{\star}$, Farahnaz Waissi ${ }^{\star}$, Mirthe Dekker, Gert J. de Borst, Joelle van Bennekom, Robbert J. de Winter, Mika Hilvo, Antti Jylhä, Gerard Pasterkamp, Dominique P.V. de Kleijn*, Reijo Laaksonen*. Ceramides and phospholipids are associated with high risk of major cardiovascular events after carotid endarterectomy. (Under review-Scientific Reports)

Nathalie Timmerman ${ }^{\star}$, Farahnaz Waissi, Mirthe Dekker, Jeffrey Kroon, Gert .J. de Borst, Dominique P.V. de Kleijn. Combined plasma extracellular vesicle biomarkers improve risk stratification for major adverse cardiovascular events after carotid endarterectomy. (Manuscript in preparation)

Nathalie Timmerman, George Galyfos, Fragiska Sigala, Kalliopi Thanopoulou, Gert J. de Borst, Lazar Davidovic, Hans-Henning Eckstein, Nenad Filipovic, Roberto Grugni, Michael Kallmayer, Dominique P. V. de Kleijn, Igor Koncar, Michalis D. Mantzaris, Elisabeth Marchal, Miltiadis Matsagkas, Perica Mutavdzic, Domenico Palombo, Gerard Pasterkamp, Vassiliki T. Potsika, Evangelos Andreakos, Dimitrios I. Fotiadis, all partners of the TAXINOMISIS Consortium. The TAXINOMISIS Project: A multidisciplinary approach for the development of a new risk stratification model for patients with asymptomatic carotid artery stenosis. Eur J Clin Invest 2020;50(12):e13411.

\section{Other publications not included in this thesis}

Bastiaan M. Wallis de Vries, Nathalie Timmerman, Constance J.H.C.M. van Laarhoven, Linda Visser, Robert A. Pol, Mostafa El Moumni, Gerard Pasterkamp, Gert J. de Borst, Clark J. Zeebregts. The Effect of Metabolic Syndrome on the Occurrence of Restenosis After Carotid Endarterectomy. Eur J Vasc Endovasc Surg 2019;58(6):805-812.

Marie de Bakker, Nathalie Timmerman, Ian D. van Koeverden, Dominique P.V. de Kleijn, Gert J. de Borst, Gerard Pasterkamp, Eric Boersma, Hester M. den Ruijter. The age- and sex-specific composition of atherosclerotic plaques in vascular surgery patients. Atherosclerosis 2020;310:1-10. 
Mirthe Dekker, Farahnaz Waissi, Joelle van Bennekom, Max J.M. Silvis, Nathalie Timmerman, Ingrid E.M. Bank, Joan E. Walter, Christian Mueller, Arjan H. Schoneveld, Raymond M. Schiffelers, Gerard Pasterkamp, Diederick E. Grobbee, Robbert J. de Winter RJ, Arend Mosterd, Dominique P.V. de Kleijn, Leo Timmers. Plasma extracellular vesicle proteins are associated with stress-induced myocardial ischemia in women presenting with chest pain. Sci Rep 2020;10(1):12257.

Mirthe Dekker, Farahnaz Waissi, Joelle van Bennekom, Max J.M. Silvis, Nathalie Timmerman $_{2}$ Arjan H. Schoneveld, Diederick E. Grobbee, Robbert J. de Winter, Arend Mosterd, Leo Timmers, Dominique P.V. de Kleijn. Extracellular Vesicle cystatin c is associated with unstable angina in troponin negative patients with acute chest pain. PLoS One 2020;15(8):e0237036.

Mirthe Dekker, Farahnaz Waissi, Nathalie Timmerman, Max J.M. Silvis, Leo Timmers, Dominique P.V. de Kleijn. Extracellular vesicles in diagnosing chronic coronary syndromes: The bumpy road to clinical implementation. Int J Mol Sci 2020;21(23):9128.

Floor Groepenhoff, Ernest Diez Benavente, Arjan Boltjes, Nathalie Timmerman, Farahnaz Waissi, Robin JG Hartman, N. Charlotte Onland-Moret, Gerard Pasterkamp and Hester Den Ruijter. Plasma testosterone levels and atherosclerotic plaque gene expression in men with advanced atherosclerosis. Frontiers in Cardiovascular Medicine, section Sex and Gender in Cardiovascular Medicine (in press).

Joost M. Mekke, Daan Egbert, Farahnaz Waissi, Nathalie Timmerman, Ilze Bot, Johan Kuiper, Gerard Pasterkamp, Gert J. de Borst, Dominique P.V. de Kleijn. Mast cell Distribution in Human Carotid Atherosclerotic Plaque Differs Significantly per Histological Segment. (Eur J Vasc Endovasc Surg (in press))

Farahnaz Waissi, Mirthe Dekker, Nathalie Timmerman, Stella Bijkerk,Petra van der Kraak, Gerard Pasterkamp, Robbert J. de Winter, Gert J. de Borst, Aryan Vink, Dominique P.V. de Kleijn. Tenascin-C expression is associated with unstable carotid atherosclerotic plaques. (Under review - Scientific Reports)

Robin J. G. Hartman, Marten A. Siemelink, Saskia Haitjema, Koen F. Dekkers, Lotte Slenders, Arjan Boltjes, Michal Mokry, Nathalie Timmerman, Marie Depuydt, Gert J. de Borst, Bastiaan T. Heijmans, Folkert W. Asselbergs, Gerard Pasterkamp, Sander W. van der Laan, Hester M. den Ruijter. Sex-dependent gene regulation of human atherosclerotic plaques by DNA methylation and transcriptome integration points to smooth muscle cell involvement in women. (Submitted) 
Lotte Slenders, Lennart P. L. Landsmeer, Kai Cui, Marie A.C. Depuydt, Maarten Verwer, Joost Mekke, Nathalie Timmerman, Noortje A.M. van den Dungen, Johan Kuiper, Menno P.J. Winther, Koen H.M. Prange, Wei Feng Ma, Clint L. Miller, Redouane Aherrahrou, Mete Civelek, Gert J. de Borst, Dominique P.V. de Kleijn, Folkert W. Asselbergs, Hester M. den Ruijter, Arjan Boltjes, Gerard Pasterkamp, Sander W. van der Laan, Michal Mokry. Intersecting single-cell transcriptomics and genome-wide association studies identifies crucial cell populations and candidate genes for atherosclerosis. (submitted)

Michal Mokry, Arjan Boltjes, Kai Cui, Lotte Slenders, Joost Mekke, Marie A.C. Depuydt, Nathalie Timmerman, Farahnaz Waissi, Adam W. Turner, Mohammad Daud Khan, Chani J. Hodonsky, Ernest Diez-Benavente, Robin J.G. Hartman, Noortje A M van den Dungen, Nico Lansu, Emilia Nagyova, Koen H.M. Prange, Eleftherios Pavlos, Evangelos Andreakos, Heribert Schunkert, Gary K. Owens, Claudia Monaco, Aloke V Finn, Renu Virmani, Nicholas Leeper, Menno P.J. Winther, Johan Kuiper, Gert Jan de Borst, Erik S.G. Stroes, Mete Civelek, Dominique P.V. de Kleijn, Hester M. den Ruijter, Folkert W. Asselbergs, Sander W. van der Laan, Clint L. Miller, Gerard Pasterkamp. Transcriptomic-based clustering of advanced atherosclerotic plaques: Revisiting the lesion characteristics that identify the vulnerable patient. (submitted) 


\section{Acknowledgements | Dankwoord}

Promoveren doe je niet alleen; een cliché maar zo waar. Dit proefschrift was niet tot stand gekomen zonder hulp, steun en gezelligheid van collega's, vrienden en familie. Ik wil jullie allemaal bedanken voor deze mooie en leerzame tijd! Een aantal mensen wil ik graag extra in het zonnetje zetten.

Prof dr. G.J. de Borst, beste Gert Jan, bedankt voor jouw vertrouwen toen ik als $4^{\mathrm{e}}$-jaars geneeskunde student bij jou aanklopte omdat ik graag onderzoek wilde doen. Ik was helemaal gepassioneerd over de carotisoperatie die ik recent bij jou had mee gekeken (jij kon je eigenlijk niet herinneren wie ik was, logisch natuurlijk met al die tientallen coassistenten). Ik bewonder het hoe je de kliniek, research, en privé allemaal weet te combineren. Het bleef mij verbazen als ik op zondagmiddag een mail kreeg met feedback op het manuscript wat ik je enkele dagen geleden had gestuurd. Tijdens onze meetings gaf jij mij het gevoel dat je alle tijd had en ging ik altijd weer weg met nieuwe motivatie en inspiratie. Ook al kwamen er niet de resultaten uit die we van tevoren hadden verwacht, jij wist een andere invalshoek te vinden en mij te overtuigen dat het echt een mooi stuk ging worden. Het is knap hoe je samenhorigheid creëert binnen de vaatonderzoekersgroep; met z'n allen naar internationale congressen ( $\mathrm{al}$ is het voor een praatje van 2 minuten) en de jaarlijkse kerstborrels hebben daar zeker aan bijgedragen. Bedankt voor je enthousiasme, vertrouwen en excellente begeleiding!

Prof. dr. D.P.V. de Kleijn, beste Dominique, als bioloog en chemicus met jarenlange ervaring in de hart- en vaatziekten maakte jij het dreamteam compleet als tweede promotor. Bedankt dat ik altijd (soms wel dagelijks) kon binnen stappen in je kantoor om te sparren als ik vastliep met analyses, manuscripten of opzet van studies, of simpelweg om te spuien als ik lichtelijk geïrriteerd was over de gang van zaken in de multicenterstudie. Middels uitgebreide tekeningen kreeg ik uitleg over de lab experimenten die voor mij abracadabra waren. Jij leerde mij het schrijven van kwalitatief goede manuscripten waarbij ik zelfs kladversies mocht opsturen als ik de rode draad even kwijt was. "Let the data do the talking", "Stick to the message", "Let the reviewers do the rest" waren de beste adviezen. De congressen, buitenlandse reisjes eromheen en jaarlijkse etentjes met de onderzoeksgroep bij jou thuis zorgden voor een goede balans tussen ontspanning en werk. Heel veel dank voor je geduld, de motiverende gesprekken en fijne supervisie!

Hooggeleerde leden van de beoordelingscommissie, prof. dr. M.L. Bots, prof. dr. ir. H.M. den Ruijter, prof. dr. F.L.J. Visseren, prof. dr. A. van der Lugt en prof. dr. U. Hedin, hartelijk bedankt voor uw tijd en inspanning voor het beoordelen van dit proefschrift. Ik kijk ernaar uit om met u van gedachten te wisselen over de inhoud. 
Dear coauthors, many thanks for your critical view and feedback on various manuscripts. Your input greatly improved the contents of this thesis.

Dear TAXINOMISIS partners, many thanks for your collaboration during this large European project. I have gained a lot of experience and I will apply this in my future career.

Patiënten van de Athero-Express en Taxinomisis studie, bedankt voor uw deelname en tijd. Uw bijdrage aan de wetenschap om meer inzichten te verkrijgen in hart- en vaatziekten is zeer waardevol.

Alle medewerkers van de Athero-Express studie, in het bijzonder professor G. Pasterkamp, Ian van Koeverden, Sander van der Laan, Saskia Haitjema, Arjan Boltjes, Arjan Schoneveld, Marian Wesseling en Joelle van Bennekom. We mogen trots zijn hoe we de logistiek en organisatie van de grootste carotisplaque biobank ter wereld altijd weer rond weten te krijgen, bedankt! Sara van Laar, bedankt voor jouw vele jaren hulp bij het vergaren van de klinische data en administratieve taken, jij hebt enorm veel werk uit mijn handen genomen! Joelle van Bennekom, bedankt voor de honderden (soms geestdodende) uren voor het verrichten van de vesicle isolaties en metingen. Ik wens jou veel plezier in je loopbaan bij de forensische geneeskunde. Maarten en Joost; de beurt is nu aan jullie om het stokje over te nemen, ik heb er alle vertrouwen in, veel succes!

Dr. Ian van Koeverden; bedankt voor jouw begeleiding tijdens mijn wetenschapsstage, het vroegtijdig betrekken bij de projecten van de $\mathrm{AE}$ en de soepele overdracht toen ik jou mocht opvolgen. Door jou heb ik een vliegende start kunnen maken met mijn promotietraject. Dr. Saskia Haitjema; als student heb ik enorm veel van jou geleerd over hoe kwalitatief goed onderzoek uit te voeren en heb jij mij wegwijs gemaakt in de wondere wereld van statistiek en SPSS/R scriptjes. Ook erna tijdens mijn promotietraject heb ik erg veel gehad aan jouw begeleiding. Dr. Sander van der Laan, hetzelfde geldt voor jou. Jouw enthousiasme en ideeën over nieuwe vraagstukken (die soms midden in de nacht in mijn mailbox verschenen) wisten mij altijd te motiveren. Sander en Saskia, samen tilden jullie het family history stuk naar een hoger niveau en we mogen trots zijn op het resultaat.

Alle medewerkers van de polikliniek en afdeling Vaatchirurgie van het UMC Utrecht, in het bijzonder prof. dr. Joost van Herwaarden, dr. Stijn Hazenberg, dr. Eline van Hattum, dr. Raechel Toorop, dr. Bart-Jeroen Petri, dr. Martin Teraa, PA's Anouk Jansze en Trijntje Bloemert-Tuin. Veel dank voor de nuttige discussies tijdens de research meetings, kennisdeling en gezelligheid op congressen. Bedankt voor de gastvrijheid op de poli en verpleegafdeling voor het includeren van studie-patiënten. 
Beste Cobie, Cynthia en Susan, bedankt voor jullie hulp bij alle administratieve zaken. Zo fijn dat alles binnen no-time kon worden geregeld in een toch best wel bureaucratische omgeving zoals een ziekenhuis.

Mede-collegae Vaatonderzoekers, Farahnaz, Constance, Mirthe, Marjolijn, Marloes, Joost, Maarten, Simone, Robert, Fons, Michelle, Jurre, Michiel, Nicolaas, Ian, Leonie, Evelien, Armelle, Aarent, Bernard en Joep. Bedankt voor alle gezelligheid, zinvolle discussies over projecten en koffiepauzes om stoom af te blazen. Hoogtepunten waren zeker de congressen en natuurlijk de borrels en tripjes eromheen. Het is bijzonder hoe iedereen voor elkaar klaar staat om te helpen en elkaar het beste gunt. Farahnaz, Mirthe, Ian, Marjolijn en Leonie bedankt voor de fijne samenwerking bij onze manuscripten.

Arts-onderzoekers, arts-assistenten en stafleden van de afdeling Heelkunde uit het UMC Utrecht, bedankt voor alle activiteiten zoals interessante wetenschapsdagen, chirurgendagen, skireis en $\mathrm{PhD}$ weekenden die mijn promotietijd tot een toptijd hebben gemaakt.

Arts-onderzoekers van Experimentele Cardiologie; jullie waren altijd behulpzaam in het lab om mij te helpen om de samples te verwerken of met experimenten waar ik vanuit mijn geneeskunde achtergrond nauwelijks verstand van heb. Bedankt!

Arts-assistenten en stafleden van de Anesthesiologie in het St. Antonius Ziekenhuis, ik ga elke dag met veel plezier naar werk en de afgelopen maanden hebben mijn keuze voor de Anesthesiologie alleen maar meer bevestigt. Ik kijk uit naar de leerzame tijd die komen gaat!

Qiu Ying van de Pol en Claire Frissen, jullie enthousiasme en gedrevenheid zorgden dat ik met veel plezier jullie heb begeleid. Jullie zijn enorm gegroeid tijdens jullie stages en ik weet zeker dat jullie affiniteit met onderzoek goed van pas gaat komen in de rest van jullie carrière. Super bedankt voor jullie hulp met vesicle metingen (ook tijdens jullie vakantie).

Lieve VaCa kamergenootjes; Mirth, Far, Connie, Nyn, Max, Joost, Fons, bedankt voor gezellige koffie- en lunch breaks, spar-momentjes, hulp bij de velen R-struggles, plank challenges en VrijMiBo's. Het kon zeker soms iets te gezellig zijn in de kamer, maar wat ik heb het laatste jaar terugverlangd om weer met z'n allen in onze kamer te mogen werken. Far, Mirth en Connie; onze promotietijd heeft bijzondere vriendschappen opgeleverd; ik kijk uit naar nog vele kitesurf vakanties en fietsuitjes!

Lieve vriendinnen, Chapeau'tjes, Sjaakies, Elcke, Rosanne, Inge, Lotte, Eveline; ik voel mij erg gelukkig met jullie allemaal om mij heen. Bedankt voor alle gezelligheid! En ook 
voor jullie begrip als dokter Nath er niet altijd bij kon (of eigenlijk kan) zijn. Ik kijk uit naar alle leuke dingen die nog komen gaan. Snow, Fen en Juul, bedankt voor jullie inhoudelijke en taalkundige feedback!

Lieve schoonfamilie, Patricia, Ben, Francette, Tom, Margreet, Aveline, Floralie; bedankt voor het warm onthaal in jullie familie. Je schoonfamilie heb je niet voor het uitkiezen maar ik heb er zeker geluk mee. Patricia; door jou is de voorkant van dit proefschrift uniek geworden, bedankt voor al jouw tijd en energie!

Lieve Jan en Marjan Visser, jullie zijn altijd als een opa en oma voor ons geweest. Bedankt dat jullie altijd klaar staan voor ons gezin.

Lieve Kimmie, niet alleen zusjes, maar ook (oud)huisgenoten en vriendinnen. Ik ben erg blij dat we zo'n speciale band hebben. We kunnen altijd bij elkaar terecht, ik ben trots op je!

Lieve Papa en Mama, bedankt voor jullie onvoorwaardelijke steun, vertrouwen en liefde. Zolang mijn inzet maar duidelijk was, maakten jullie alles mogelijk. Ik ben jullie erg dankbaar voor wat jullie allemaal voor mij hebben gedaan. Jullie aanmoediging zorgt dat ik het beste uit mijzelf haal. Ik ben erg gelukkig met zo'n hecht gezin als de onze.

Lieve Matthisk, bedankt dat ik altijd bij jou mag klagen, lachen, huilen of gewoon lekker mezelf kan zijn. Bedankt voor jouw flexibiliteit om samen te zijn met een dokter met geheel andere werktijden dan de jouwe. Ik hoop nog heel veel mooie avonturen met jou te beleven! 


\section{About the author}

Nathalie Timmerman was born on the 11th of September 1990 in Sögel (Germany) as the oldest daughter of Nicole and Raymond. She grew up together with her younger sister Kimberley in Amsterdam and The Hague. After graduating cum laude from the Huygens Lyceum Voorburg in 2008, she took a gap year and worked as a ski instructor in Austria. Hereafter, she obtained a bachelor's degree in Pharmacy and a cum laude medical degree at the University of Utrecht. During her medical study, she developed an interest in clinical research and cardiovascular diseases and she contributed in projects of the Athero-Express study. Professor G.J. de Borst and professor D.P.V. de Kleijn gave her the opportunity to continue her work in a $\mathrm{PhD}$ programme at the Department of Vascular Surgery at the UMC Utrecht. She investigated new biomarkers for adverse events after carotid surgery. The results of her studies are presented in this thesis and were discussed at several international conferences. She received the best oral presentation award at the Munich Vascular Conference 2019, $3^{\text {rd }}$ best oral Young Researcher presentation award at the ESVB Conference 2019 and the best poster presentation award on the ESVS Annual meeting in 2019. She enjoys to go kitesurfing, cycling and spend time with friends and family. Currently she is working as a resident (not in training) at the Department of Anaesthesiology in the St. Antonius Hospital Nieuwegein and aspires to become an Anaesthesiologist. 

